US Army Corps

of Engineers ${ }_{\circledast}$

Engineer Research and

Development Center

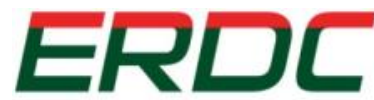

INNOVATIVE SOLUTIONS

for a safer, better world

Integrated Climate Assessment for Army Enterprise Planning

\title{
Estimating Resistance and Resilience of Military Lands Using Vegetation Indices
}

Ryan R. Busby, Dick L. Gebhart, Steven J. Oxley,

May 2017

William D. Tarantino, and Wade A. Wall

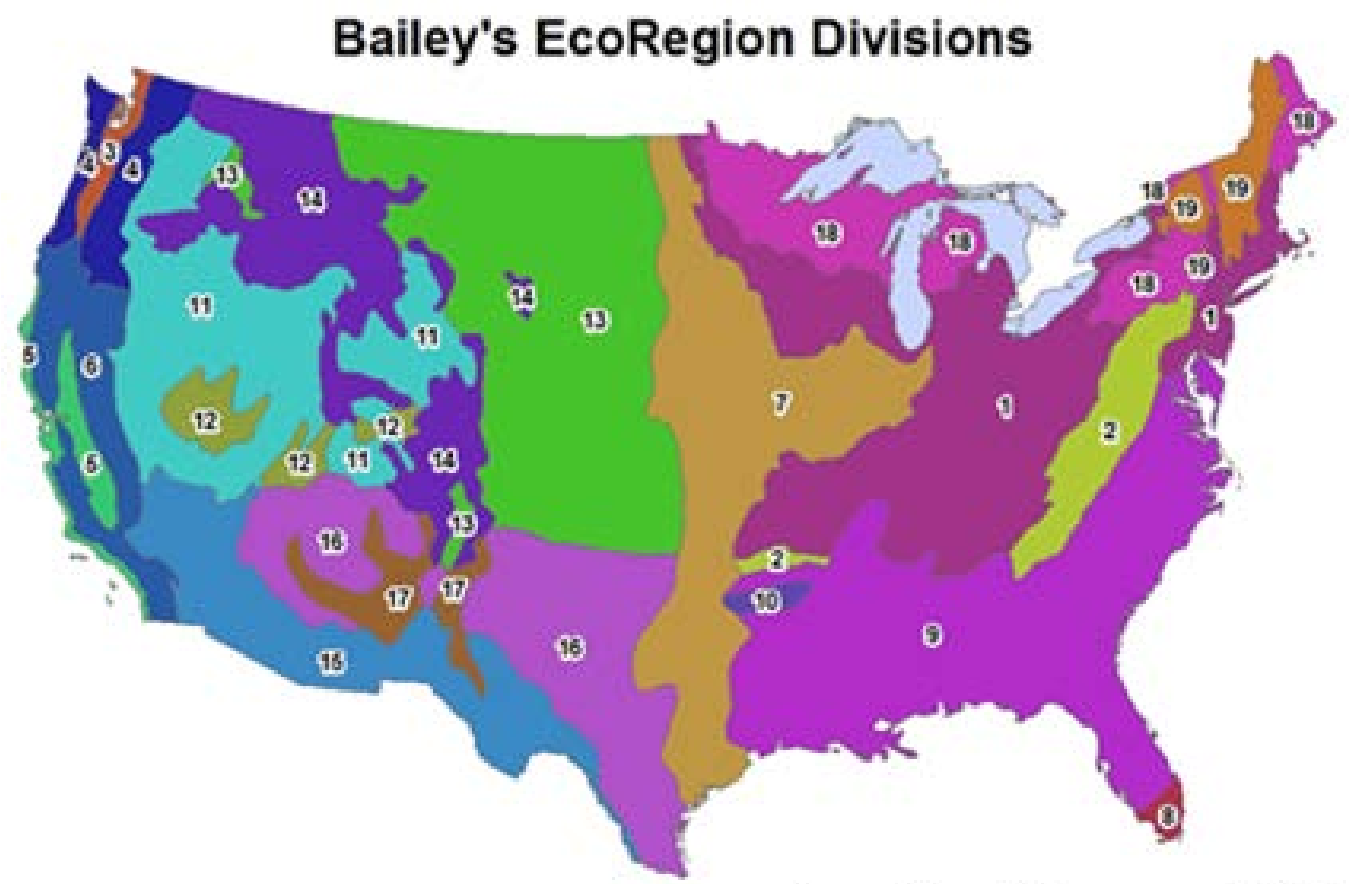


The U.S. Army Engineer Research and Development Center (ERDC) solves the nation's toughest engineering and environmental challenges. ERDC develops innovative solutions in civil and military engineering, geospatial sciences, water resources, and environmental sciences for the Army, the Department of Defense, civilian agencies, and our nation's public good. Find out more at www.erdc.usace.army.mil.

To search for other technical reports published by ERDC, visit the ERDC online library at http://acwc.sdp.sirsi.net/client/default. 


\section{Estimating Resistance and Resilience of Military Lands Using Vegetation Indices}

Ryan R. Busby, Dick L. Gebhart, Steven J. Oxley, William D. Tarantino, and Wade A. Wall

Construction Engineering Research Laboratory

U.S. Army Engineer Research and Development Center 2902 Newmark Drive

Champaign, IL 61822

Final Report

Approved for public release; distribution is unlimited.

Prepared for Headquarters, U.S. Army Corps of Engineers

Washington, DC 20314-1000

Under Program 622720896, Project 402188, “Integrated Climate Assessment for Army Enterprise Planning" 


\section{Abstract}

Military training inevitably leads to land degradation; however, some ecosystems have higher resistance and resilience to training based on the functional traits of existing vegetation, making them preferred for longterm use. This work estimated resistance and resilience for the continental United States using dominant plant species for numerous plant communities, resistance and resilience values for plant functional groups, and national community vegetation maps. Two datasets were combined to obtain greater detail and values for all land area. Results indicate that graminoid communities have the highest resistance values, and shrublands the lowest; and that eastern deciduous forests and prairies have the highest resilience values, and evergreen forests and shrublands the lowest. This lists the resistance and resilience values of a selection of Army installations using both datasets and a new combined metric. This new method will help the Army determine the portfolio of installations that will best meet its future training land requirements.

DISCLAIMER: The contents of this report are not to be used for advertising, publication, or promotional purposes. Citation of trade names does not constitute an official endorsement or approval of the use of such commercial products. All product names and trademarks cited are the property of their respective owners. The findings of this report are not to be construed as an official Department of the Army position unless so designated by other authorized documents. 


\section{Contents}

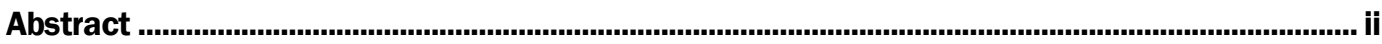

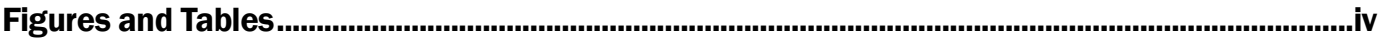

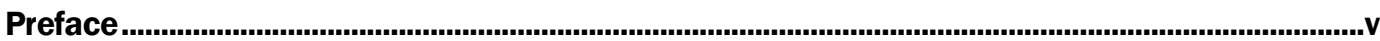

1 Introduction ................................................................................................................................ 1

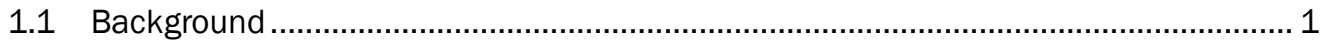

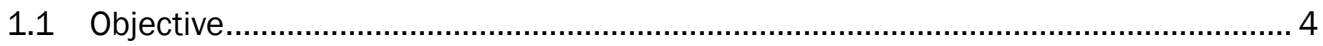

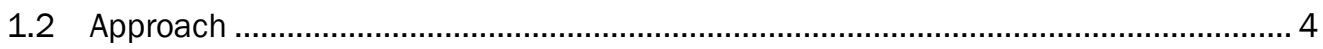

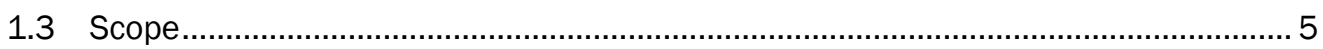

2 Resistance and Resilience in Natural Plant Communities .................................................... 6

3 Methods and Materials.............................................................................................................. 9

3.1 Estimation of resistance and resiliency values................................................. 9

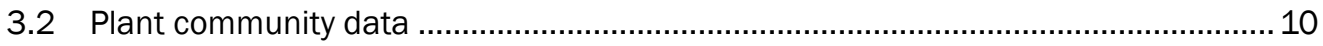

3.3 Data analysis.............................................................................................. 11

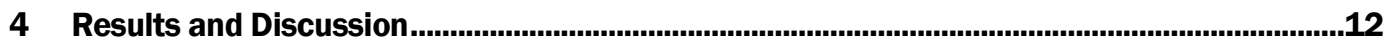

4.1 General results.......................................................................................... 12

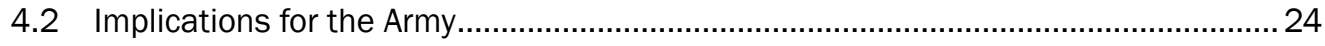

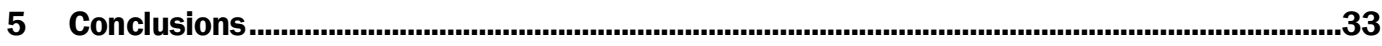

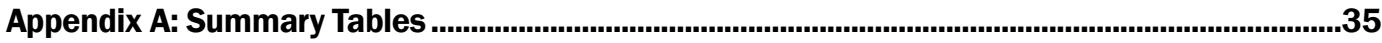

Appendix B: Dominant Species' Resistance and Resilience Values From USGS

National Vegetation Classification Community Types.................................................................39

Appendix C: Dominant Species' Resistance and Resilience Values from Küchler

Potential Vegetation Community Types .................................................................................126

Appendix D: Resistance and Resilience Values from USGS National Vegetation

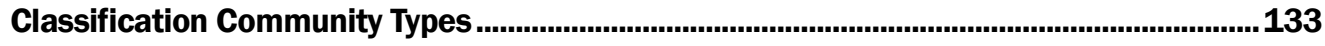

Appendix E: Resistance and Resilience Values from Küchler Potential Vegetation

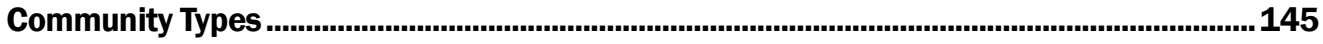

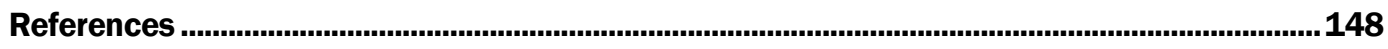

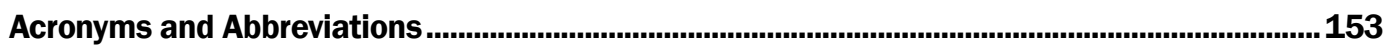

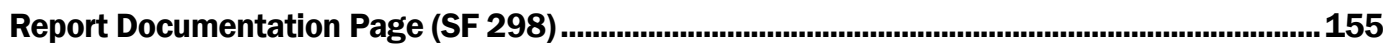




\section{Figures and Tables}

\section{Figures}

1 Bailey Ecoregion Divisions

2 Küchler vegetation resistance and resilience map for the United States. High values denote resistance and resilience to trampling and vehicular disturbance.

3 NatureServe vegetation resistance and resilience map for the United States. High values denote resistance and resilience to trampling and vehicular disturbance

4 Average vegetation resistance and resilience values based on the Combined Küchler and NatureServe Vegetation maps

5 Küchler vegetation resistance and resilience maps for the United States. High values denote resistance to trampling and vehicular disturbance.

6 NatureServe vegetation resistance and resilience maps for the United States. High values denote resistance to trampling and vehicular disturbance

7 Combined Küchler and NatureServe Vegetation resistance and resilience maps.

High values denote resistance to trampling and vehicular disturbance

8 Correlation between the Küchler and NatureServe RRVAL and the ecosystem resilience values as estimated using subject matter expert opinion in Doe et al. (1999). Numbers refer to Bailey Ecoregions (Bailey 1980: See Fig. 1). Correlation is 0.39. After the removal of three outlier ecoregions $(3,4$, and 13 ; data not shown.) correlation was 0.83

9 Average vegetation resistance and resilience values for Fort Benning Military Reservation (Georgia). Separate resistance and resilience values are included in Appendices $\mathrm{D}$ and $\mathrm{E}$

10 Combined Küchler and NatureServe Vegetation resistance and resilience maps for Fort Benning Military Reservation (Georgia).

\section{Tables}

1 Resilience and resistance values assigned to plant functional groups.....

2 Mean RRVAL for the Küchler, NatureServe, and Combined vegetation maps across Bailey Ecoregions (Bailey 1980). Doe values refer to average subject matter value. Multiple Doe values represent ecoregions that contained multiple resiliency values (see Doe et al. 1999)

3 Installation Doe values and Combined resistance and resilience values (current study)

4 Top 10 installations ranked according to the Combined RRVAL values; Küchler and NatureServe are provided for reference. Full data are available in Appendices $\mathrm{D}$ and $\mathrm{E}$ 


\section{Preface}

This study was conducted for Headquarters, U.S. Army Corps of Engineers (HQUSACE) under Program 622720896, "Base Facility Environmental Quality," Project 402188, "Integrated Climate Assessment for Army Enterprise Planning." The technical monitors were Ms. Sarah Harrop and Ms. Nancy Zoller, Center for Army Analysis (CAA).

The work was performed by the Ecological Processes Branch (CNN) of the Installations Division (CN), U.S. Army Engineer Research and Development Center - Construction Engineering Research Laboratory (ERDCCERL). At the time of publication, Dr. Chris Rewerts was Chief, CEERDCNN; Ms. Michelle J . Hanson was Chief, CEERD-CN; and Mr. Alan B. Anderson was the Technical Director for Environmental Quality/ Sustainable Lands and Ranges. The Deputy Director of ERDC-CERL was Dr. Kirankumar Topudurti and the Director was Dr. Ilker Adiguzel.

COL Bryan S. Green was Commander of ERDC, and Dr. David W. Pittman was the Director. 
THIS PAGE INTENTIONALLY LEFT BLANK 


\section{Introduction}

\subsection{Background}

Available training area is one of the U.S. Army's most valued resources in general and on installations in particular, as reflected by the high importance placed on maneuver area capacity when performing an installation level military value analysis (MVA) (DoD 2014). Training areas satisfy a most basic Army requirement and are defined as those installation areas designated for impact and detonation of all ordnance or those areas required for land-intensive training at the installation (HQDA 2013). Department of Defense Policy states that "The Department of Defense shall demonstrate stewardship of natural resources in its trust by protecting and enhancing those resources for mission support, biodiversity conservation, and maintenance of ecosystem services" (DoD 2011). One of the most important benefits of maintaining natural vegetation is that vegetation cover deters erosion that can degrade training areas to the point where training suitability is diminished, or where the areas become unusable.

Military training generally results in some level of vegetation disturbance. Since disturbance is one of the primary factors that structure plant communities in terrestrial ecosystems (Pickett and White 1985; Papaik and Canham 2006), understanding the impacts of military training on vegetation resilience to these types of disturbance is required to appropriately estimate maneuver area capacity (MAC).

Under the Army's most recent MVA, "Range Sustainability" is a metric that represents the percentage of training lands that are available for use without restrictions (Zoller 2016). The metric is scaled by a multiplicative factor (range 0-1) that reflects the resiliency value of the vegetation on an installation. Restrictions include wetlands (as defined by the National Wetland Inventory [USFWS 2015]) and areas with slopes greater than $30 \%$. The resiliency value for an installation is derived by determining the Bailey Ecoregion (1980) where the installation occurs and applying the corresponding resiliency value based on the ecosystem (Figure 1). Ecosystem resilience values are from Doe et al. (1999) and are based on subject matter expert opinion as applied to Bailey Ecoregions. MVA resiliency values reflect the number of passes from an M1A1 tank over the course of a 
year that an area can withstand before being considered at a critical disturbance level. Resiliency values range from 2 to 12 , with possible values being $2,3,4,6$, and 12 . Resiliency values are converted into multiplicative factors that scale the aerial estimates of the installation. For example, if an installation's resiliency value is 12 , the multiplicative value would be 1 , whereas if an installation's resiliency value is 6 , its multiplicative value would be 0.5 .

While the current Range Sustainability metric may capture an installation's resilience, it is an atemporal metric that does not account for or adjust for projected shifts in temperature and precipitation. It is well-known that temperature and precipitation patterns are one of the main determinants of vegetation patterns (Whittaker 1975). This being the case, it is expected that projected climate change will have an impact on the spatial distribution of vegetation, both of individual species and community assemblages. These projected shifts in vegetation could lead to changes in training area resilience and recovery capabilities. For example, a reduction in annual rainfall could lead parts of the Midwest to shift from tall grass to shortgrass prairie, which would reduce rooting depth and thus also reduce the area's resiliency. In addition to changes in temperature and precipitation trends, changes in weather variability are probable (Fischer and Knutti 2015). Both changes in long-term trends and variability will be spatially and temporally heterogeneous. For example, higher latitude regions are projected to continue to experience greater impacts (Duarte et al. 2012). Due to these possible influences, a more nuanced metric that captures these dependencies is needed to inform Army decision processes as it determines the lands that will best meet future training land requirements.

Although numerous studies have estimated resistance and resilience values (hereafter referred to as "RRVAL") of individual species to mechanical disturbance, none have quantified these concepts at the plant community or ecosystem level to a point where they could then be used to evaluate a training area or training range. Although range-specific RRVAL estimates could be developed for major Army training ranges, such a development would likely be cost prohibitive because it would require the need for individual study site experiments and because it would likely produce an unnecessarily complex model. 


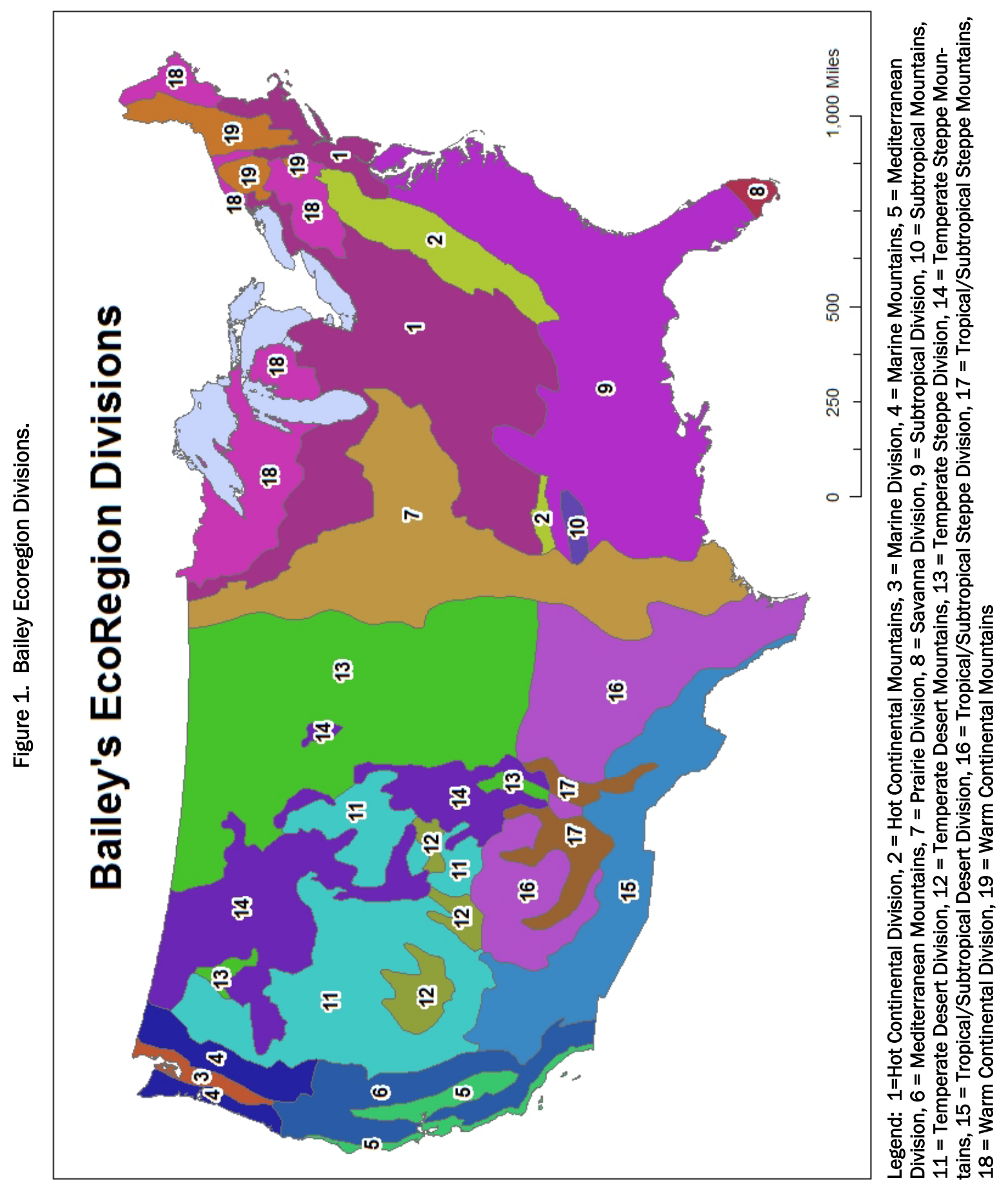


Because, for all the major Army installations and training ranges, MAC is dependent at the most basic level on vegetation cover and generic plant community types, a reasonable first estimate for vegetation recovery could use a simple model that predicts net plant community type RRVAL. The model would take advantage of existing large databases assembled from experimental studies of vegetation RRVAL to traffic, trampling, and other types of physical or mechanical disturbances. One such database was compiled in 1997 for 737 species from 403 separate experimental studies (Yorks et al. 1997) and was used to develop RRVAL for plant functional groups, discussed later in the document. Additional data from other more recently published reviews were included to develop plant functional group and plant community type RRVAL (Monz et al. 2010; Barros, Gonnet, and Pickering 2013; Pescott and Stewart 2014). This work was undertaken to provide a scientifically defensible RRVAL estimate for natural vegetation communities across the United States, and to characterize the RRVAL for Continental U.S. (CONUS) military installations.

\subsection{Objective}

The overall objective of this research was to provide a scientifically defensible RRVAL estimate for natural vegetation communities across the United States. The specific objective was to characterize the RRVAL for CONUS military installations so that, by using the methods proposed here, the Army could use the resulting RRVAL metrics for comparative installations studies to determine the portfolio with the greatest training potential and MAC.

\subsection{Approach}

The objectives of this technical report were met through the following steps:

1. Resistance and resilience values for plant functional groups (e.g., $\mathrm{C}_{4}$ grasses, shrubs) were identified based on a peer-reviewed database.

2. Dominant plant species on CONUS military installations were identified based on two national vegetation maps (NatureServe and Küchler natural vegetation maps).

3. Resistance and resilience values were assigned to these species based on functional group membership and average for the two vegetation maps. 
4. RRVAL was determined for the selected CONUS installations with the study results from Steps 1-3.

5. Results from Step 4 were compared to previous attempts at estimating resistance and resilience values for military installations.

\subsection{Scope}

It is anticipated that the results of this work will be applicable to all CONUS military installations. With some modification, it is further anticipated that the study methods proposed here could also be applicable to installation in Alaska and Hawaii, and to Outside Continental United States (OCONUS) installations. In addition, results are applicable to other CONUS Federal lands where off-road vehicle usage is prevalent. Note that this effort focused on mechanized training where vehicles are prevalent because that level of training causes the greatest disruption. Separate results for high levels of foot traffic usage common to some forms of light infantry training are not needed as current conclusions on MAC are applicable across all training types. 


\section{Resistance and Resilience in Natural Plant Communities}

Disturbance is one of the primary factors that structure plant communities in terrestrial ecosystems (Pickett and White 1985; Papaik and Canham 2006). Physical disturbances such as mechanized and dismounted training are highly visible and frequently result in the loss of vegetation cover and height, damage to soil quality and structure (Lucas-Borja et al. 2011), unexpected or undesirable changes in plant community composition (Ballantyne and Pickering 2013; Newsome, Moore, and Dowling 2012), and increased risks of invasive plant establishment and dominance (Potito and Beatty 2005; Crisfield, Macdonald, and Gould 2012; Barros, Gonnet, and Pickering 2013). Understanding the impacts and relationships between mechanized training and the sensitivity of vegetation to these types of disturbance is essential for effectively managing these interactions (Liddle 1997; Hamberg et al. 2010) and is required to appropriately and accurately estimate MAC.

Vegetation recovery is somewhat abstract and is often considered a component of the more common ecological concepts of vegetation resistance and resilience where resistance is often defined as the ability of the vegetation to withstand disturbance and resilience is defined as the ability to recover to the pre-disturbed state (Bernhardt-Römermann et al. 2011). For MAC analyses, the following definitions seem appropriate. Resistance is the relative ability of an individual plant species to withstand mechanized training, repeated trampling, or similar types of physical disturbance before being injured or impaired, while resilience is the capacity of an individual plant species, or more commonly, a plant community, to survive or regenerate following mechanized disturbance (Kuss and Hall 1991; Yorks et al. 1997). Therefore, resilience is considered the more important concept in the context of mechanized training disturbance because it operates at the plant community or landscape level rather than the individual plant level.

Resistance and resilience concepts have evolved over the past 20 years and several variations in their definitions exist (Vetter 2009). Resilience is often thought to be higher in species-rich communities, likely due to diversity of individual species' responses to perturbation, which minimizes effects on ecosystem functioning (Elmqvist et al. 2003; Speed et al. 2010). 
Resistance and resilience of vegetation to disturbance is also strongly associated with plant morphology and functional traits (Bratton 1985; Roovers, Baeten, and Hermy 2004). Species such as grasses and grass-like plants are considered more resistant to maneuver damage because they have flexible, horizontal, and branching stems, narrow leaves, below-ground reproductive structures, low crown positions, and protective culm sheaths (Tolvanen et al. 2001). Conversely, shrubs, ferns, and forbs have broad leaves and vertical stems with reproductive capacity more elevated than the graminoids, which make them far more sensitive to maneuver training impacts (Tolvanen et al. 2001).

Life form and plant height may provide some predictive capability for inferring plant tolerance to mechanized disturbance (Sun and Liddle 1993; Roovers, Baeten, and Hermy 2004). Rosette growth forms are more resilient than erect forms (Cole 1995) and those with higher growth rates and $\mathrm{C}_{4}$ photosynthetic pathways are also generally more resilient and recover faster from disturbance (MacGillivray and Grime 1995; Fortunel et al. 2009). In general terms, forbs are the most sensitive life form to disturbance followed by ferns, shrubs, and finally graminoids (Pickering 2010). Furthermore, Hill and Pickering (2009) suggested that a common pattern of resistance across generic plant community types was sand dune grasslands $>$ grasslands $>$ sand dune heaths $>$ forest understory $>$ heaths $>$ herb fields. In fact, many types of dune communities recover quickly from disturbance and exhibit high resistance and resilience (Greenberg et al. 1995; Kutiel, Eden, and Zhevelev 2000; Mangan et al. 2004), providing some support for Hill and Pickering's (2009) ordering of plant community resistance to disturbance. Polar, alpine, and tundra plant community types are generally considered sensitive and fragile to mechanical disturbance with long recovery times following disturbance and low resistance and resilience due to low productivity, short growing seasons, and generally poor soil conditions (Bell and Bliss 1973; Forbes et al. 2005; Willard, Cooper, and Forbes 2007; Pertierra et al. 2013).

These studies have contributed to a suggested order of resistance across ecosystems developed by Pickering (2010) that is as follows: subtropical $>$ alpine $>$ subalpine $>$ arctic $>$ temperate $>$ montane. Perennial species that have the ability to resprout or those with below-ground growing points are usually more resilient than annuals or species that possess above-ground 
growth points (Liddle 1975). Shrub-dominated communities with sclerophyllous tissues are frequently classified as one of the most susceptible plant communities to mechanical disturbances and exhibit low RRVAL (Liddle 1997; Whinam and Chilcott 2003; Pickering and Hill 2007; Bernhardt-Römermann et al. 2011; Mason et al. 2015).

Under a given set of mechanical/physical disturbance regimes, vegetation resistance and resilience can also be impacted by a number of abiotic factors. Timing of disturbance can alter vegetation response to damage primarily from the interruption of reproductive processes like flowering and seed set (Cole 2004; Barros, Gonnet, and Pickering 2013). Dzwonko and Loster (1997), Gallet and Rozé (2001), and Lane, Coffin, and Lauenroth (1998) have related resistance and resilience to water availability, soil texture, and level of irradiance. Plant communities adapted to drought are often highly resistant to mechanical disturbance (Andrés-Abellán et al. 2006), whereas plant communities adapted to more mesic conditions appear to be much less resistant (Grime and Campbell 1991; Francis et al. 2005). Several studies suggest that plant communities adapted to clayey soil types are more resistant to mechanical disturbances than those adapted to loamy soil types (Sala et al. 1988; Ludwig et al. 2001). Furthermore, Bernhardt-Rommermann et al. (2011) and Mangan et al. (2004) suggest that ecosystem types with long histories of frequent disturbance (grasslands, and one could argue military training lands) will be more resistant and resilient to mechanical disturbance than those where frequent disturbances are uncommon (mature forest understories, bogs, etc.).

The concepts of vegetation resistance and resilience as they relate to recovery are complex, interacting, and depend not only on the individual species attributes (growth form, photosynthetic pathway, growing point location, leaf size, leaf orientation, reproductive strategy, etc.) in the disturbed plant community, but also on a number of abiotic factors that influence ecosystem structure and function. Across a number of studies spanning many years, continents, and ecosystems, it appears that vegetation resistance to mechanical disturbance is dependent on the functional attributes of individual species, which assemble into plant communities and is strongly affected by disturbance history, whereas resilience is more controlled by abiotic factors such as soil texture and water holding capacity, which directly influence regrowth rates and productivity and can be affected by changes and/ or variation in climate. 


\section{Methods and Materials}

\subsection{Estimation of resistance and resiliency values}

A database of vegetation resistance and resilience to traffic was compiled for 737 species from 403 separate references (Yorks et al. 1997), where resistance is defined as the ability of a plant species to withstand traffic before being impaired, and resilience is defined as the capacity of a plant species to regenerate following traffic damage. Values were assigned for each species for each observation on a scale of 1 to 5 , where 5 represented an increase in plant cover or biomass and 1 represented disappearance of the species (Yorks et al. 1997).

For comparison, plant species were then grouped into life form, root form, lifespan, phenology, and reproductive pathway. For the purposes of this work, broad functional groups determined from these groupings consisted of deciduous trees, evergreen trees, deciduous shrubs, evergreen shrubs, shrubs (if deciduous or evergreen), forbs, $C_{3}$ graminoids and $C_{4}$ graminoids. Membership in functional groups was determined from the PLANTS Database (USDA 2015) for non-graminoids, and from Waller and Lewis (1979) and Li, Wedin, and Tieszen (1999) for graminoids. The data provided in Yorks et al. (1997) was then used to develop mean resistance and resilience values for these functional groups (Table 1).

Table 1. Resilience and resistance values assigned to plant functional groups

\begin{tabular}{|l|c|c|}
\hline Plant Functional Group & Resistance & Resilience \\
\hline Deciduous Tree & 3.0 & 4.2 \\
\hline Evergreen Tree & 3.2 & 2.2 \\
\hline Deciduous Shrub & 2.7 & 3.0 \\
\hline Evergreen Shrub & 2.7 & 2.4 \\
\hline Shrub & 2.7 & 2.8 \\
\hline Forb & 3.0 & 3.5 \\
\hline $\mathrm{C}_{3}$ Graminoid & 3.6 & 3.6 \\
\hline $\mathrm{C}_{4}$ Graminoid & 3.6 & 4.1 \\
\hline
\end{tabular}

Along with the plant community types for each respective vegetation community grouping, dominant plant species were also obtained (Appendices A and B). Each plant species was assigned a RRVAL based on its functional grouping (Yorks et al. 1997), and then all dominant species in each 
community type were averaged to produce a mean RRVAL for each community type (Appendices $\mathrm{C}$ and $\mathrm{D}$ ). Values ranged from 2.83 to 3.6 for the U.S. Geological Survey (USGS) resistance, 2.2 to 4.2 for USGS resilience, 2.7 to 3.6 for Küchler resistance, and 2.2 to 4.2 for Küchler resilience (Appendices $\mathrm{C}$ and $\mathrm{D}$ ). These mean values were then scaled on a 0 to 1 scale to allow for simplified comparison and averaging across datasets, and to provide a relative measure of community responses to anthropogenic mechanical disturbance. This was computed by subtracting the lowest value from all values for resilience and resistance in each dataset and then by dividing by the difference between the highest and lowest values (Appendices $C$ and $D$ ). These scaled RRVAL were summed to provide a net persistence value, which is a mean measure of the dominant species in a plant community's relative resistance and resilience responses to anthropogenic mechanical disturbance forces (Appendices $\mathrm{C}$ and $\mathrm{D}$ ).

\subsection{Plant community data}

Plant community types were obtained for the USGS National Vegetation Classification System (NatureServe 2015) and Küchler Potential Vegetation (Küchler 1964). The National Vegetation Classification System data provides a snapshot of existing vegetation at a single time point and is a raster-based Geographic Information System (GIS) layer provided by NatureServe. The 30 x 30 meter cell raster dataset provides land cover classification for 574 different land cover classes for the continental United States. The dataset is highly detailed and includes disturbed vegetation areas such as agricultural land, non-native plant dominated community types, and heavily urbanized areas for which RRVAL could not be computed. Therefore, although it provides high resolution in many locations, large areas that have undergone land conversion to agriculture or urbanization have no values assigned and were not assigned a RRVAL. The Küchler Potential Vegetation data provides a best case scenario for the native vegetation communities most likely to occur in an area regardless of past or current land use or plant invasions. It is a vector dataset digitized from a 1964 Küchler Potential Vegetation paper map that contains 116 vegetation types across the contiguous United States. While not as highly detailed as the NatureServe classification, it supports RRVAL calculations across the entire region. 
Pre-processing of the two GIS datasets was performed using ArcGIS 10.3 (ESRI 2011). First, the Küchler spatial layer was converted to a raster with the same cell size as the Nature Serve raster file by snapping the Küchler spatial layer to the Nature Serve raster file. To simplify analyses, the Küchler and NatureServe raster files were combined into a single raster.

The RRVAL for the NatureServe and the Küchler vegetation communities were joined to the Combined raster layer. Each raster cell of the Combined raster data included information on the NatureServe and Küchler vegetation communities, as well as their corresponding RRVAL. Because of the differences between the Küchler and NatureServe datasets, the resistance, resilience, and RRVAL were averaged from the two datasets. In this way, the higher resolution of the National Vegetation Classification System was combined with the complete coverage of the Küchler Potential Vegetation.

\subsection{Data analysis}

To compare the Combined RRVAL to the values in Doe et al. (1999), the average values for the Küchler, NatureServe, and Combined maps were calculated for each Bailey Ecoregion. The Combined RRVAL were compared to the values in Doe et al. by calculating the correlation between the derived values. In addition, the Combined RRVAL and assigned values were binned based on the distribution of values in Doe et al. across Ecoregions. Doe et al. assigned three ecoregions to the Category 1, 10 to the Category 2, 4 to the Category 3, and 3 to the Category 4.

The Combined RRVAL for 56 installations were estimated using the same methods outlined above. As for the ecoregions, the correlation between both the raw RRVAL for the installations and the Doe et al. values were calculated, and the installation RRVAL were binned with the same distribution. In this case, Doe et al. (1999) assigned six installations to Category 1, 17 to Category 2, 28 installations to Category 3, and 5 to the Category 4. 


\section{Results and Discussion}

\subsection{General results}

This chapter provides maps that illustrate the different resistance and resiliency evaluations, describe correlations between the different methods for estimating RRVAL, and describe possible implications to the Army and its installations' ability to provide MAC.

The Küchler, NatureServe, and Combined RRVAL all identified the Temperate Steppe and Prairie Divisions as the two divisions with the highest RRVAL (Figures 2, 3, and 4). These areas are in the interior of the continent with relatively gentle topography, loamy soils, and graminoid dominated plant communities. The Division with the lowest RRVAL was the Temperate Desert Mountains. Additionally, Marine Mountains and the Marine Division were identified as having low RRVAL, although the Küchler RRVAL identified the Tropical/ subtropical Desert Division as having a low RRVAL as well. The following paragraphs discuss the individual results from the maps.

For the the Küchler Potential Vegetation Map, the ecoregions with the highest resistance values were Temperate Steppe Division, Prairie Division, and the Mediterranean Division (Table 2). These areas are widespread across the United States (Figure 5). The lowest resistance values were found in the Temperate Desert Mountains and Tropical/Subtropical Desert. The Hot Continental, Prairie, and Hot Continental Mountain Divisions had the highest resilience values. The lowest resilience values, which were in the Marine Mountain and Temperate Desert Mountain Divisions (Table 2), covered a larger area than the low resistance values in the Southwest (Figure 5). The Temperate Steppe, Prairie, and Hot Continental Divisions had the highest the Küchler RRVAL (Table 2). 


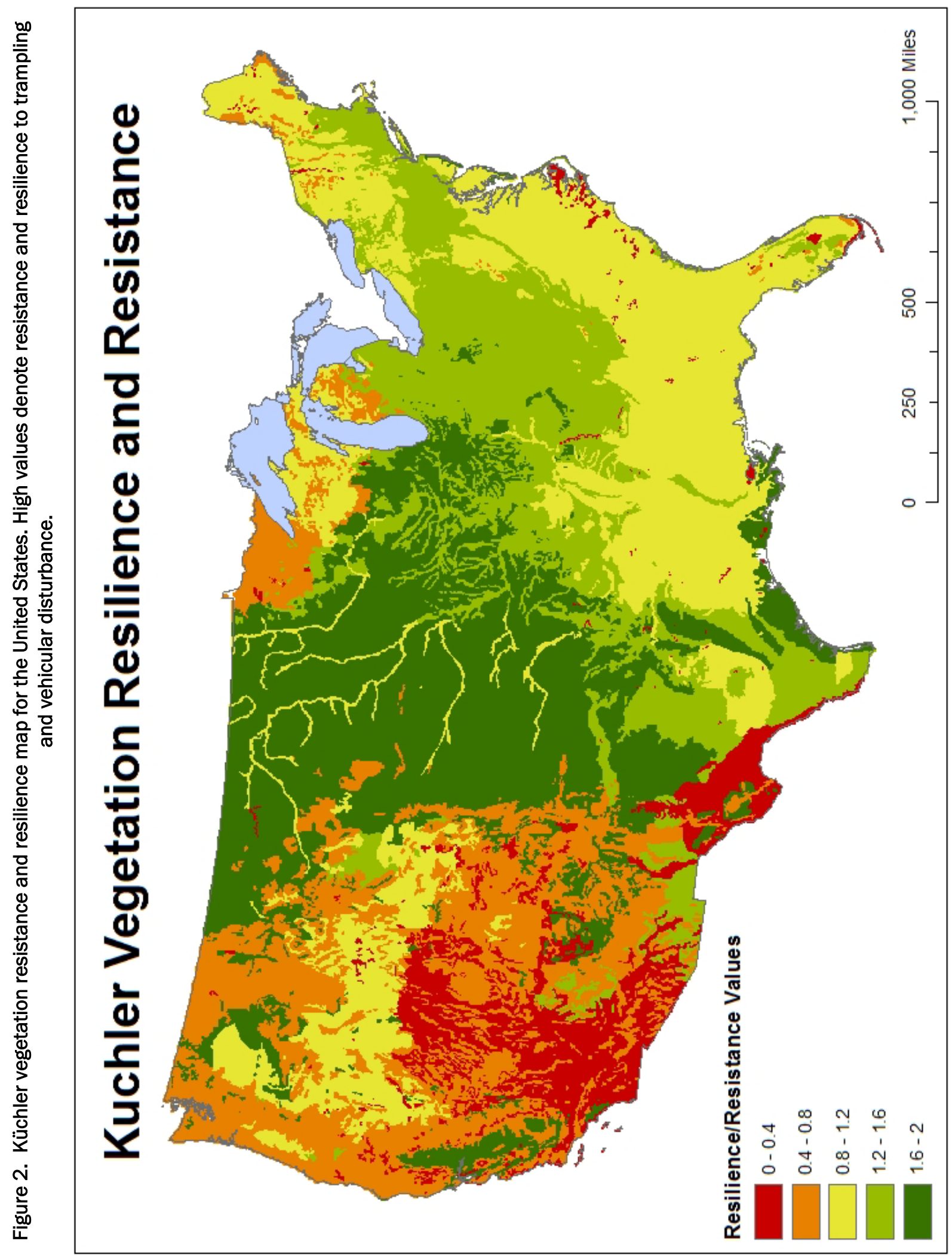




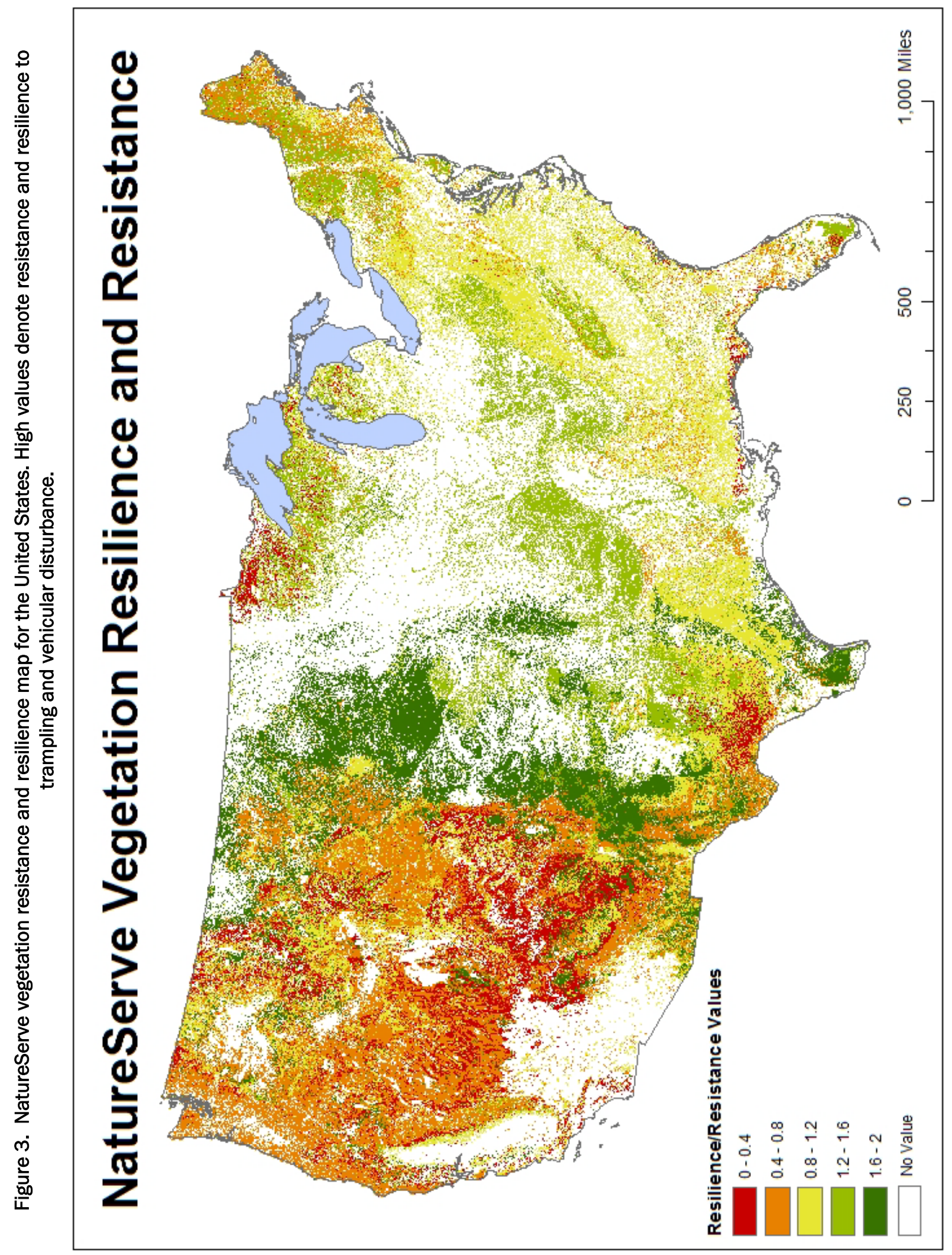




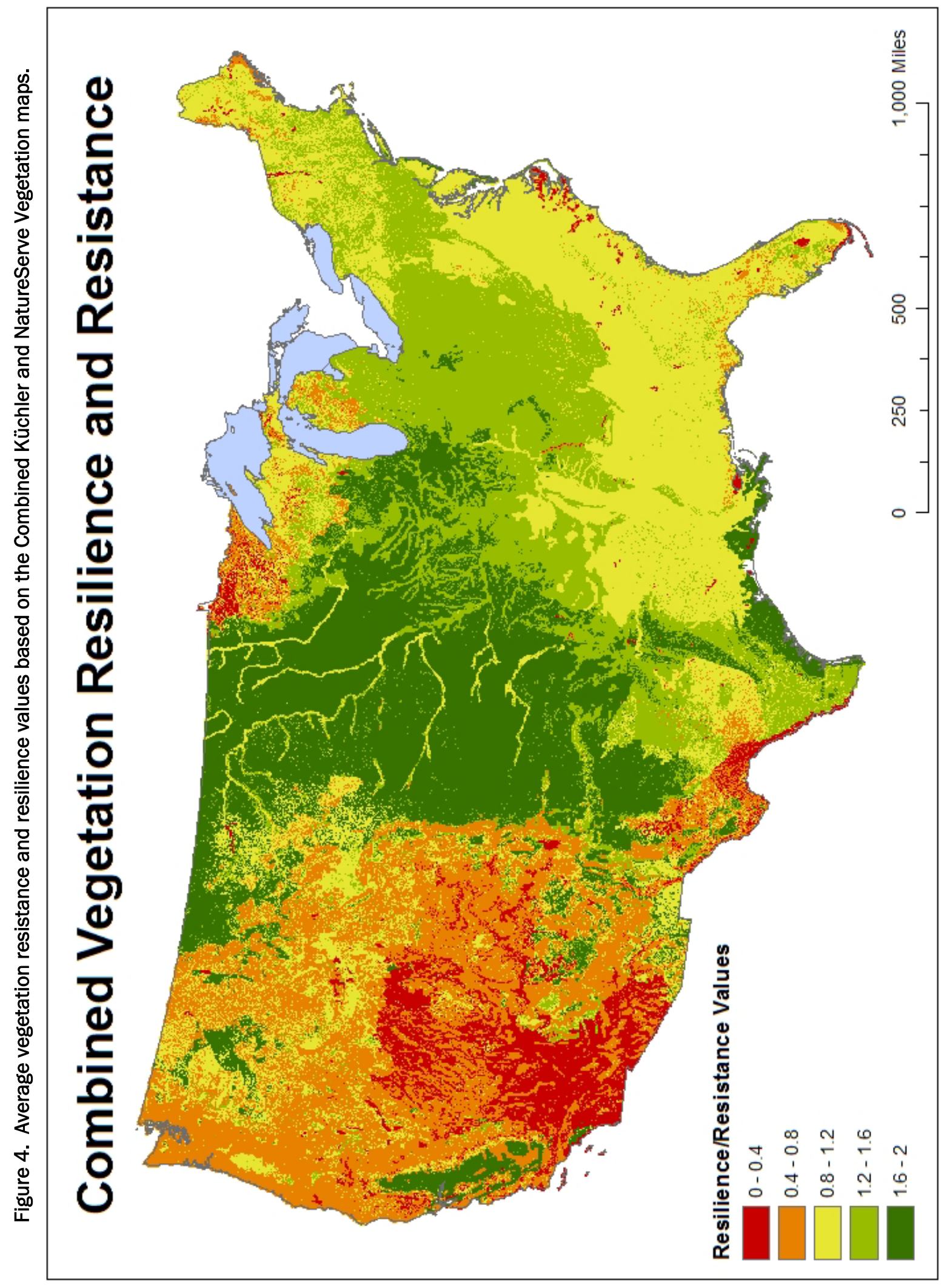


Table 2. Mean RRVAL for the Küchler, NatureServe, and Combined vegetation maps across Bailey Ecoregions (Bailey 1980). Doe values refer to average subject matter value. Multiple Doe values represent ecoregions that contained multiple resiliency values (see Doe et al. 1999).

\begin{tabular}{|l|c|c|c|c|c|}
\hline & & Küchler & NatureServe & Average & Doe \\
\cline { 3 - 5 } Bailey Ecoregion & Code & RRVAL & RRVAL & RRVAL & Value \\
\hline Hot Continental & 1 & 1.36 & 1.15 & 1.25 & 3 \\
\hline Hot Continental Mountains & 2 & 1.25 & 1.09 & 1.17 & 3 \\
\hline Marine & 3 & 0.65 & 0.60 & 0.63 & 4 \\
\hline Marine Mountains & 4 & 0.66 & 0.58 & 0.62 & 4 \\
\hline Mediterranean & 5 & 1.13 & 0.62 & 0.88 & 2 \\
\hline Mediterranean Mountains & 6 & 0.62 & 0.64 & 0.63 & 2 \\
\hline Prairie & 7 & 1.65 & 1.38 & 1.51 & 3,4 \\
\hline Savanna & 8 & 0.84 & 1.05 & 0.94 & 2 \\
\hline Subtropical & 9 & 1.04 & 1.02 & 1.03 & 2,3 \\
\hline Subtropical Mountains & 10 & 1.01 & 1.20 & 1.10 & 2 \\
\hline Temperate Desert & 11 & 0.69 & 0.65 & 0.67 & 1 \\
\hline $\begin{array}{l}\text { Temperate Desert } \\
\text { Mountains }\end{array}$ & 12 & 0.37 & 0.56 & 0.46 & 1 \\
\hline Temperate Steppe & 13 & 1.72 & 1.51 & 1.62 & 2,4 \\
\hline $\begin{array}{l}\text { Temperate Steppe } \\
\text { Mountains }\end{array}$ & 14 & 0.78 & 0.81 & 0.79 & 2 \\
\hline Tropical/Subtropical Desert & 15 & 0.52 & 1.00 & 0.76 & 1 \\
\hline Tropical/Subtropical Steppe & 16 & 1.28 & 1.13 & 1.20 & 2 \\
\hline $\begin{array}{l}\text { Tropical/Subtropical Steppe } \\
\text { Mountains }\end{array}$ & 17 & 0.83 & 0.94 & 0.89 & 2 \\
\hline Warm Continental & 18 & 0.92 & 0.94 & 0.93 & 3 \\
\hline $\begin{array}{l}\text { Warm Continental } \\
\text { Mountains }\end{array}$ & 19 & 1.00 & 1.02 & 1.01 & 3 \\
\hline
\end{tabular}




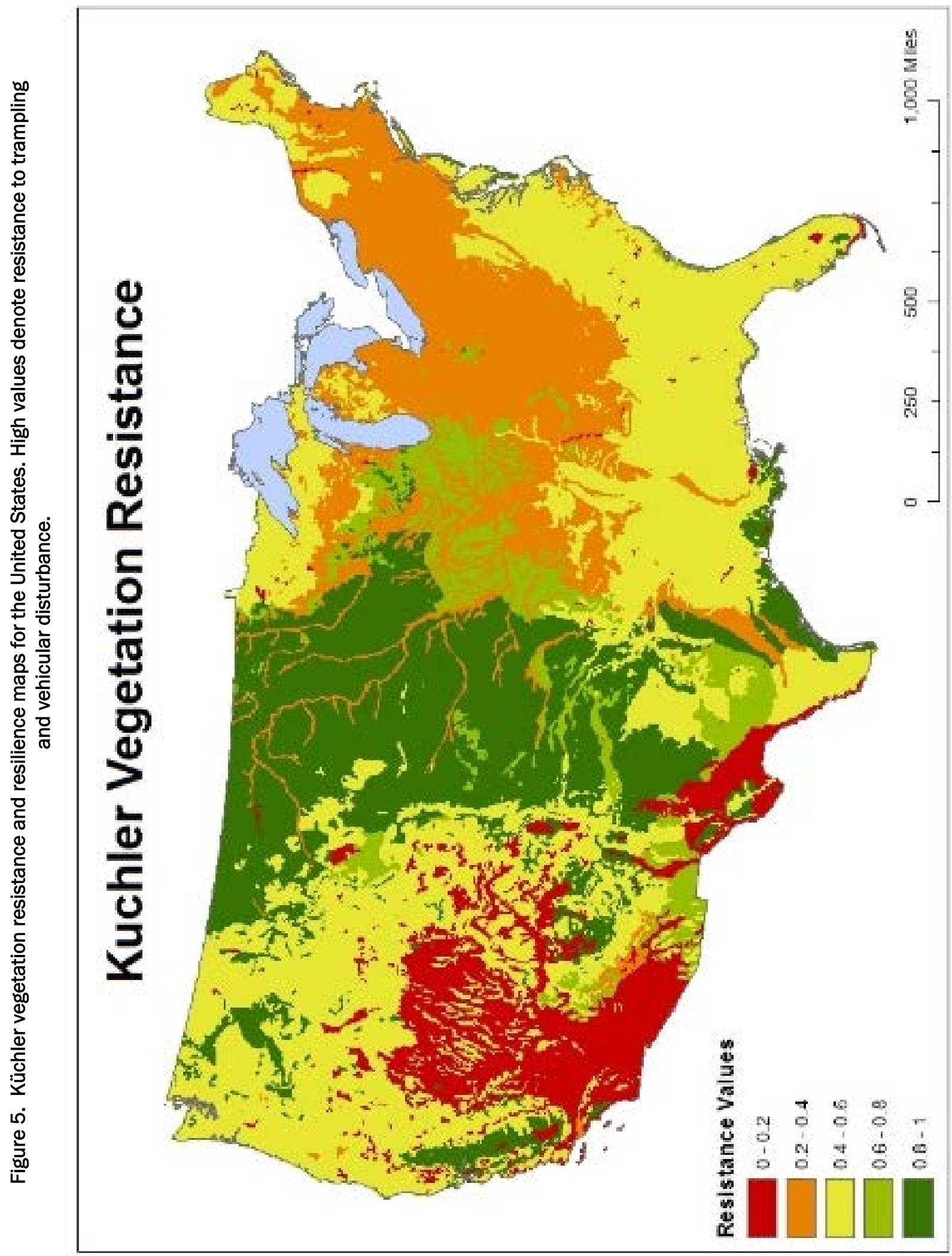




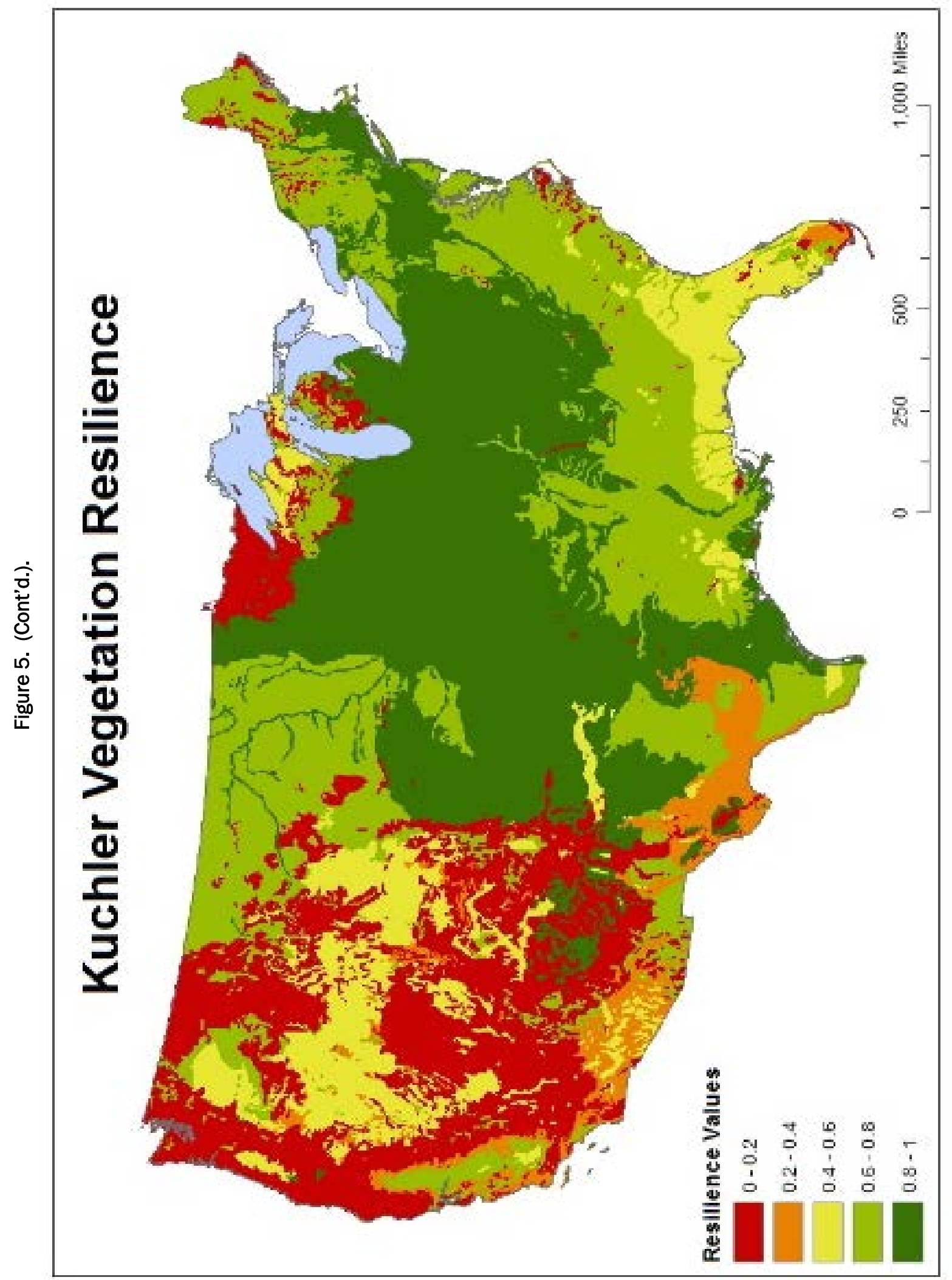


Resistance values based on the NatureServe natural vegetation map of the United States identified the Temperate Steppe, Prairie, and Savanna Divisions as having the highest resistance values; and the Warm Continental, Temperate Desert Mountains and Hot Continental Divisions as having the lowest resistance values (Figure 6). Resilience values based on the NatureServe natural vegetation map of the United States identified the Hot Continental, Subtropical Mountains, and Prairie Divisions as the three regions with the highest resistance values (Appendix E, Table E-1, p 145).

The correlation between the NatureServe and Küchler resistance values was 0.15 ; the correlation between the resilience values was 0.03 . These low correlation values should not come as an unexpected surprise given that the Küchler dataset represents potential natural plant communities irrespective of past disturbance history, whereas NatureServe is a contemporary snapshot of current plant communities, both disturbed and undisturbed, without regard to potential. In addition, the Küchler Potential Vegetation Map is drawn at a much coarser scale and does not capture the full range of vegetation communities. For example, riparian areas in the Prairie and Savanna Divisions would receive the same resistance and resilience values as would the actual prairie and savanna communities.

The average of the Küchler and NatureServe resistance maps (hereafter referred to as "Combined") identified the Temperate Steppe, Prairie, and Tropical/ Subtropical Steppe Divisions as having the highest resistance values (Figure 7). The Hot Continental, Prairie, and Hot Continental Mountains Divisions had the highest resilience values, while the Temperate Desert, Marine, and Marine Mountains Divisions had the lowest (Appendix $\mathrm{E}$, Table E-1, p 145). The Combined RRVAL identified the Temperate Steppe, Prairie, and Hot Continental Divisions as having the highest values; and the Temperate Desert Mountains, Marine Mountains, and the Mediterranean Mountains as having the lowest values (Figure 4). 


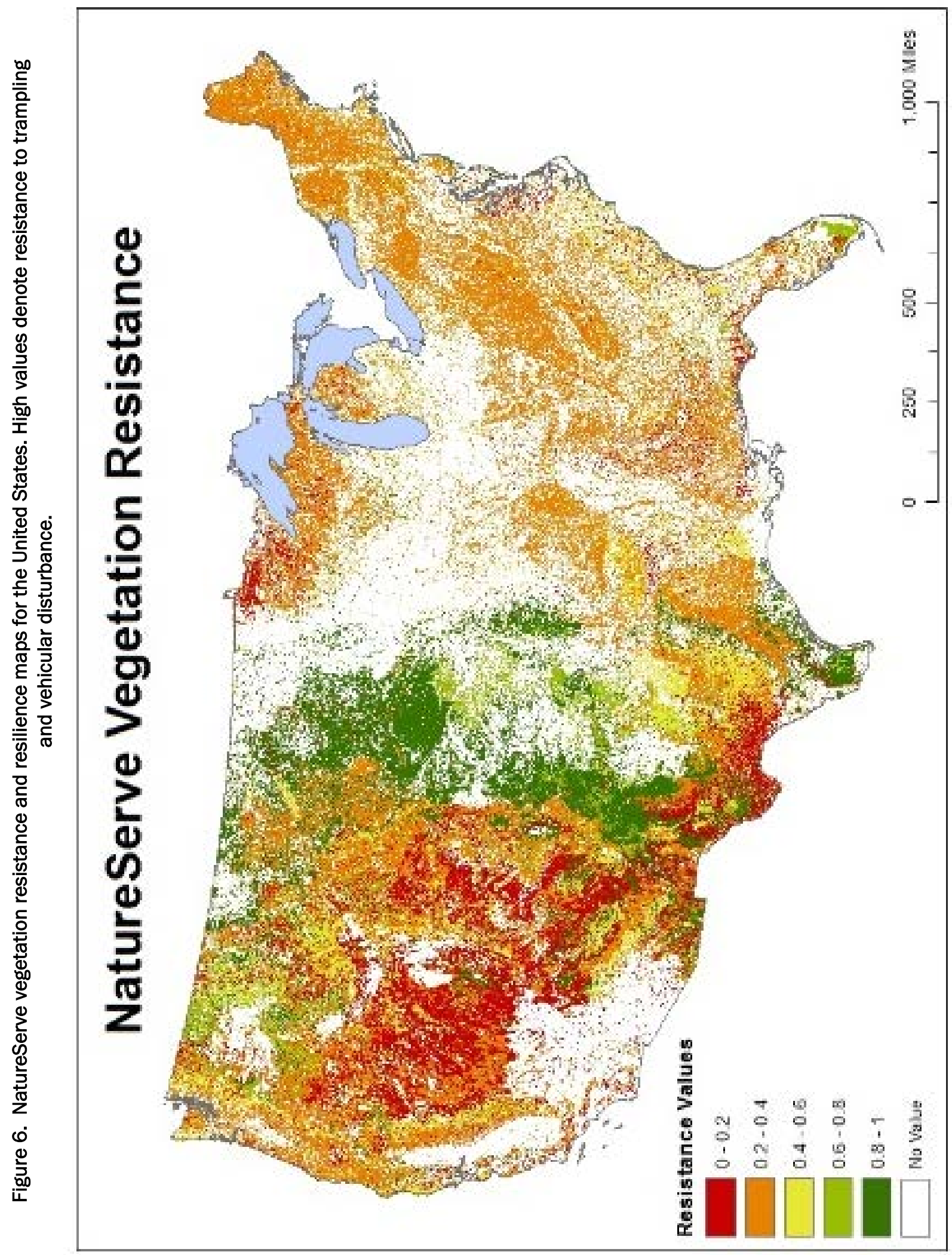




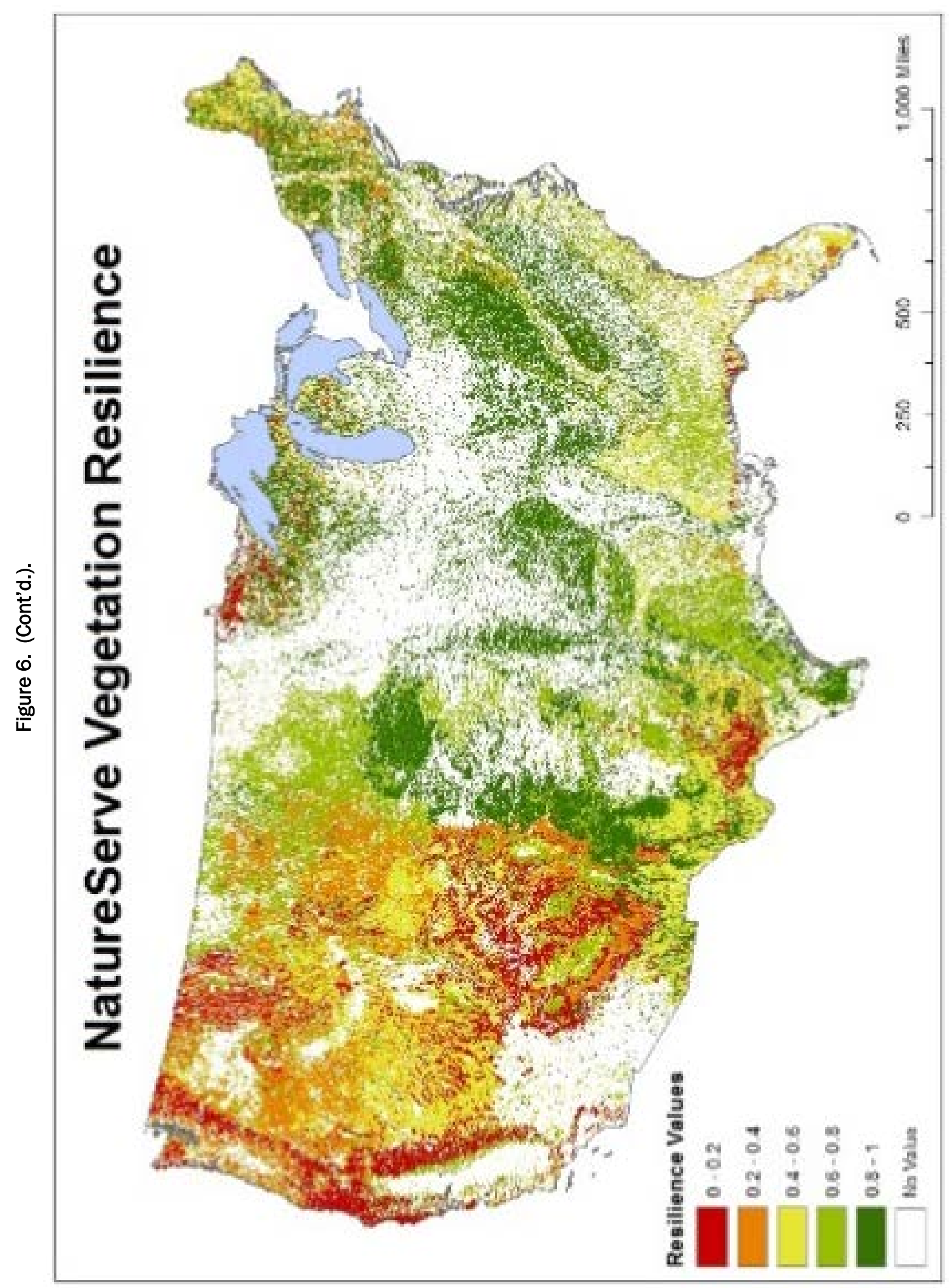




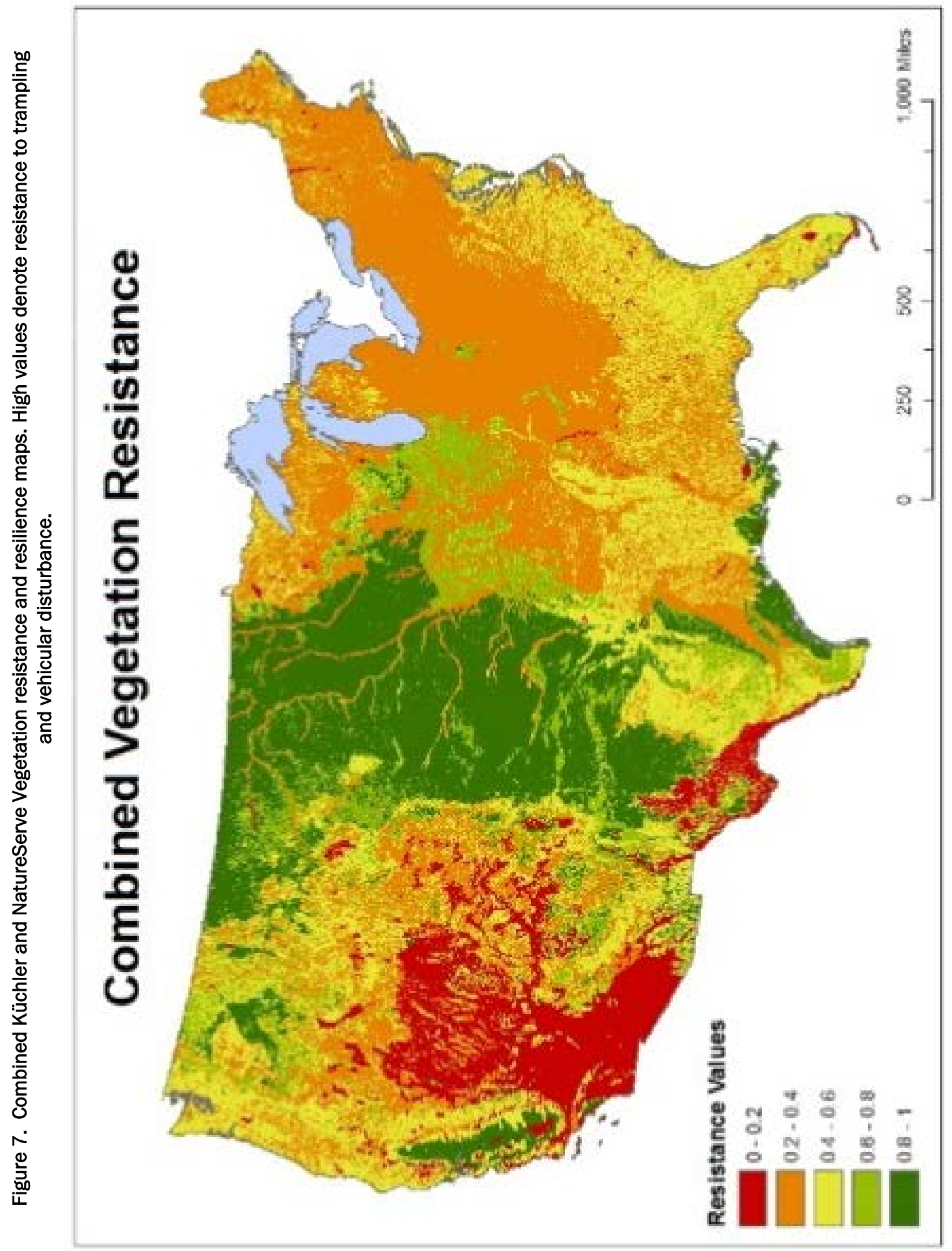




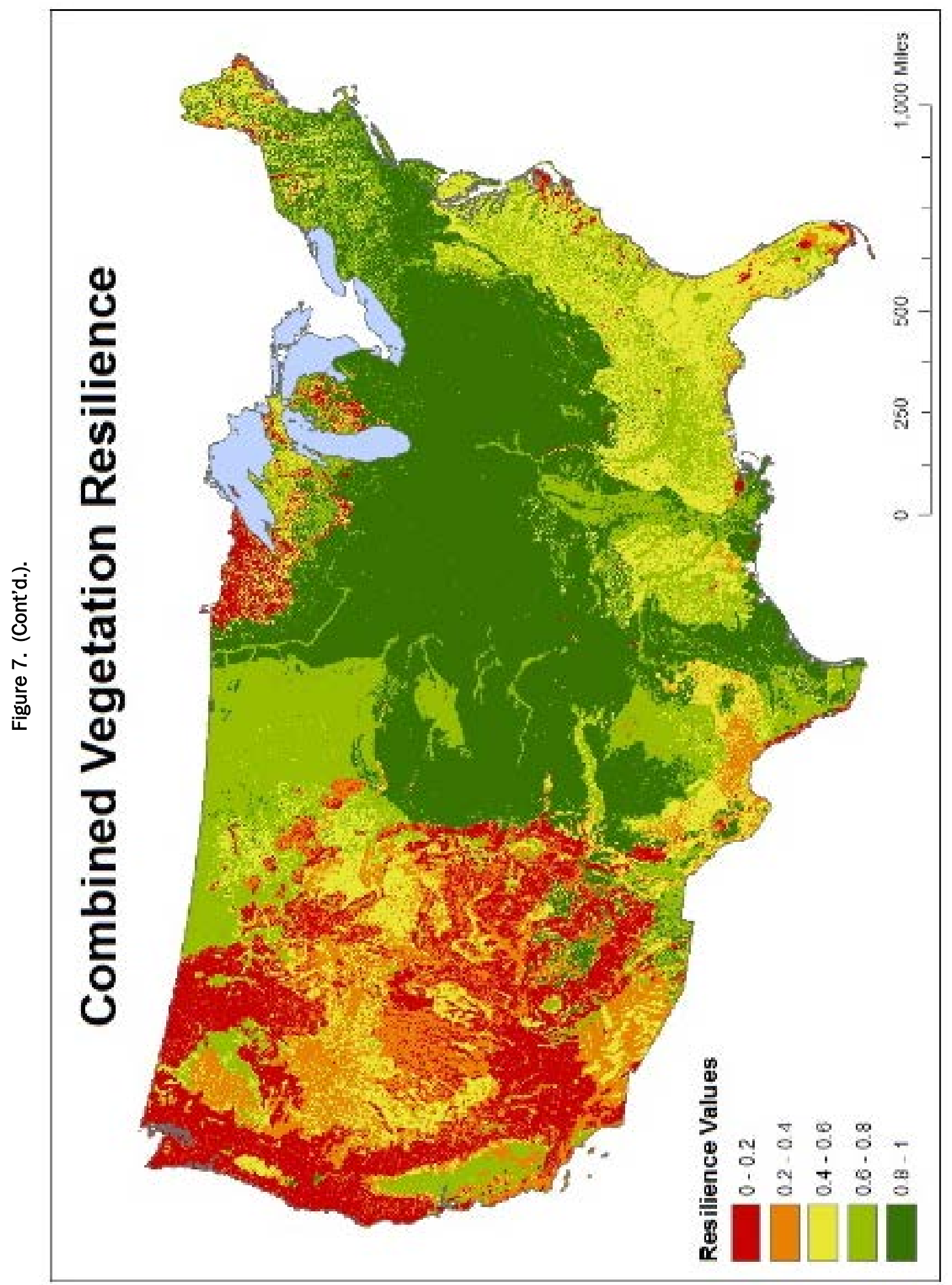


The correlation between the average RRVAL and the Doe (1999) values was 0.36 before the removal of three outlier regions. The relatively low correlation between the two maps was the result of three outlier Divisions. Doe et al. (1999) estimated the Marine Division and the Marine Mountains as being of high resilience (with a value of 4). Doe et al. also provided two resilience values $(2,4)$ to the Temperate Steppe Division. Values from the current study estimated these as having low RRVAL (in the case of the Marine and Marine Mountains Division) and high RRVAL (in the case of the Temperate Steppe Division).

It is likely that this current model underestimates Marine Division. The soils are silty loams in a relatively flat landscape that were historically prairies between the Coastal and Cascade Ranges in Pacific Northwest. It is also likely that Doe et al. (1999) overestimated the resilience of the Marine Mountains by combining the Marine Division with the Marine Mountains. Finally, there is a precipitation gradient across the Temperate Steppe Division, in which the western half is drier relative to the eastern half. By dividing it, Doe et al. were likely trying to incorporate this information. When these three outliers were removed, the correlation between the values in Doe et al. (1999) and the RRVAL was 0.82 (Figure 8), suggesting that the different sources were generally consistent between evaluations. Comparison of the binned values and the values in Doe et al. (1999) yielded a correlation of 0.33 , similar to correlation between the RRVAL and the Doe values.

\subsection{Implications for the Army}

The Army is interested in how installations compare across training metrics as it determines the portfolio of installations that can best meet its future training requirements. Historically, the Army uses multiple metrics to compare installations, for example, training, cost, facilities, and quality of life metrics (Ewing, Tarantino, and Parnell 2006). These metrics are static in that they do not provide a defensible way to account for change over time. This work has shown that lands have non-static RRVAL, which will help the Army evaluate training lands over time as installation conditions change. 
Figure 8. Correlation between the Küchler and NatureServe RRVAL and the ecosystem resilience values as estimated using subject matter expert opinion in Doe et al. (1999). Numbers refer to Bailey Ecoregions (Bailey 1980: See Fig. 1). Correlation is 0.39. After the removal of three outlier ecoregions $(3,4$, and 13 ; data not shown.) correlation was 0.83 .

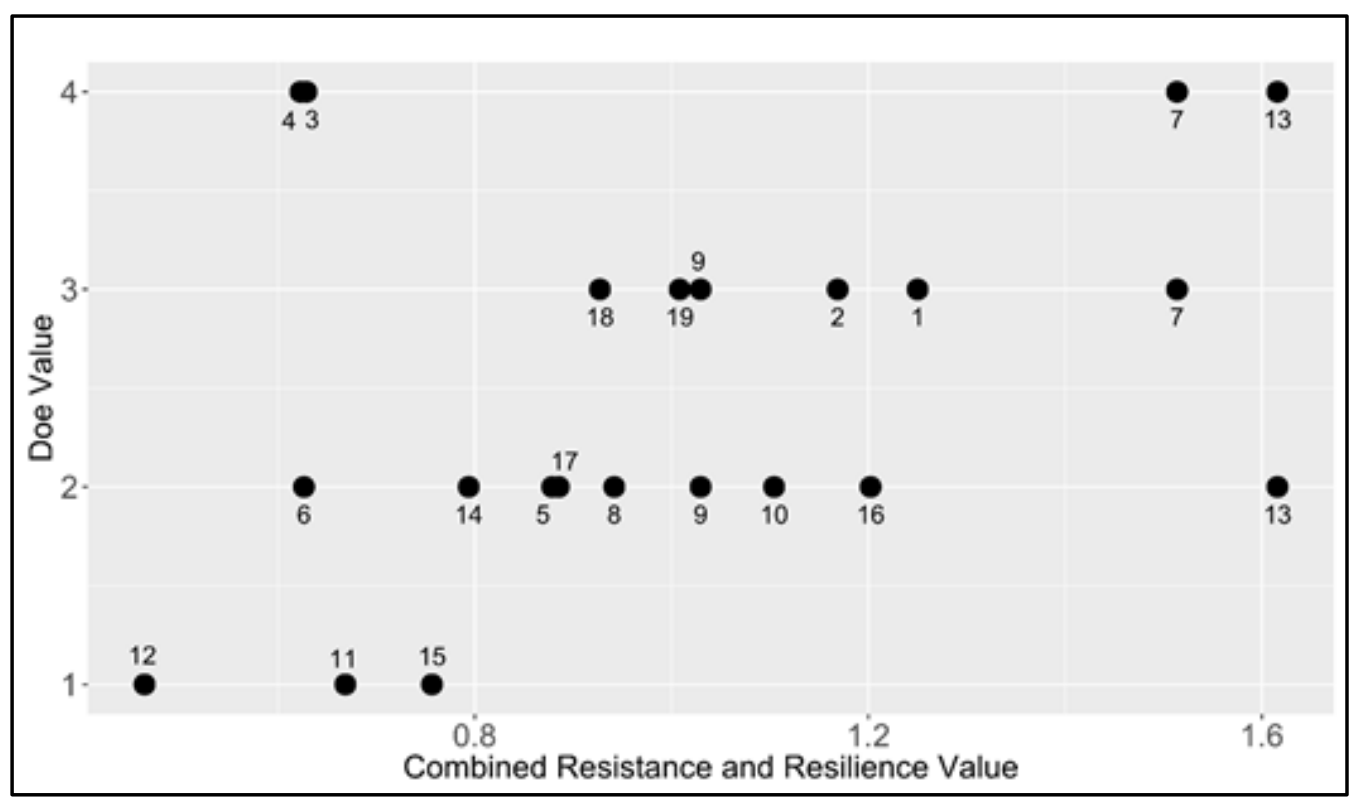

Army installations have a history of land disturbances due to training impacts. It appears that vegetation resistance to such disturbance is dependent on the functional attributes of individual species, which assemble into plant communities and are strongly affected by this disturbance history. The ability of Army lands to recover from disturbances, i.e., their resilience, is more controlled by abiotic factors such as soil texture and water holding capacity, which directly influence regrowth rates and productivity and can be affected by changes and/ or variation in climate.

Pinon Canyon, CO, Fort Riley, KS, Fort Sill, OK, and Parks Reserve Forces Training Area, CA were in the top 10 of all installations, regardless of the data source, while National Training Center (NTC) and Fort Irwin, CA, Infantry Training Camp (ITC) Camp San Luis Obisbo, CA, Fort Lewis, WA, Florence Military Reservation (FMR), AZ, Fort Hunter-Liggett, CA, and Maneuver Training Center-H (MTC-H) Camp Grayling, MI were in the bottom (Appendix E, Table E-1, p 145). Overall, 42 of the 56 installations had a change in rank of six or less between the Küchler, NatureServe, and Combined maps. Fort McCoy, WI, Fort Carson, CO, Camp Ashland, NE, and Fort Bragg, NC had the greatest change in rank, averaging 29, 27, 14, and 13 , respectively. 
The correlation of the resistance values between the Küchler Potential Vegetation Map and the NatureServe Vegetation Map was 0.63, higher than the ecoregion-level correlation. This is likely due to the reduced vegetation heterogeneity at the installation scale, compared to the entire ecoregion. Parks Reserve Forces Training Area, CA, Pinon Canyon, CO, and Fort Riley, KS had the highest resistance values based on the Küchler Potential Vegetation Map, while the top three resistance values based on the NatureServe Vegetation Map were Pinon Canyon, CO, Camp Perry, OH, and Fort Riley, KS. Several outliers were identified. For example, Camp Perry, $\mathrm{OH}$ had a resistance value of 0.92 according to NatureServe, but a relatively low value of 0.33 based on the Küchler potential vegation map. The correlation of the resilience values between the Küchler Potential Vegetation Map and the NatureServe Vegetation Map was 0.66, higher than for the resistance values. However, the NatureServe resilience values had a lower kurtosis relative to Küchler values. Küchler resilience values were 1 (the highest) for 15 installations, while NatureServe resilience values were highest for Fort Campbell, KY, Camp Atterbury, IN, and Camp Crowder, MO. The Combined resistance values identified Pinon Canyon, CO, Fort Riley, KS, and Parks Reserve Forces Training Area, CA, while the Combined resilience values identified five installations as having an average re sistance value of 1 .

While many of the installation level rankings match subject matter expert opinion, there are notable exceptions. Fort Lewis, WA in the Marine Division, was assigned low RRVAL by both the Küchler and NatureServe vegetation maps, even though the installation is considered one of the highest ranking installations in terms of resistance to mechanical disturbance and vegetation resilience. As discussed above, there are likely reasons for the low RRVAL for the Marine Division and, by extension, Fort Lewis, WA. The current study estimated Fort Sill, OK to have a high RRVAL and rank (Table 3), yet Doe et al. (1999) gave the installation a value of 2 (low to moderate resistance). Table 4 lists the top 10 installations ranked according to the Combined RRVAL values.

One of the benefits of incorporating the NatureServe resistance and resilience values into the index is that it allows for the estimation of higher resolution maps, thus allowing for intra-installation level exploration of RRVALs (Figures 9 and 10). For example, RRVAL based on the Küchler map for Fort Benning, GA would be a single value because of the relatively 
coarse resolution of the data. Using the NatureServe RRVAL correctly identifies wetland areas that have lower RRVAL relative to the upland areas. These maps can be quickly generated and would allow land managers to spatially identify training lands that would be more or less resilient.

Table 3. Installation Doe values and Combined resistance and resilience values (current study).

\begin{tabular}{|c|c|c|c|c|}
\hline Installation & Division & Doe & Combined & Change \\
\hline Camp Ashland & Prairie Division & 4 & 3 & -1 \\
\hline Camp Atterbury & Hot Continental Division & 3 & 3 & 0 \\
\hline Camp Blanding & Subtropical Division & 2 & 2 & 0 \\
\hline Camp Bowie & $\begin{array}{l}\text { Tropical/Subtropical Steppe } \\
\text { Division }\end{array}$ & 2 & 2 & 0 \\
\hline Camp Bullis & $\begin{array}{l}\text { Tropical/Subtropical Steppe } \\
\text { Division }\end{array}$ & 2 & 2 & 0 \\
\hline Camp Clark & Prairie Division & 4 & 3 & -1 \\
\hline Camp Dawson & Hot Continental Mountains & 3 & 3 & 0 \\
\hline Camp Maxey & Prairie Division & 3 & 3 & 0 \\
\hline $\begin{array}{l}\text { Camp Minden Training Site } \\
\text { (TS) }\end{array}$ & Subtropical Division & 3 & 2 & -1 \\
\hline $\begin{array}{l}\text { Camp Perry TS (Combat } \\
\text { Training Center [CTC]) }\end{array}$ & Hot Continental Division & 3 & 4 & 1 \\
\hline Camp Ripley & Hot Continental Division & 3 & 2 & -1 \\
\hline Camp Swift & Prairie Division & 3 & 3 & 0 \\
\hline Camp Villere TS & Subtropical Division & 2 & 1 & -1 \\
\hline Caswell Training Site & Warm Continental Division & 3 & 2 & -1 \\
\hline Florence & $\begin{array}{l}\text { Tropical/Subtropical Desert } \\
\text { Division }\end{array}$ & 1 & 1 & 0 \\
\hline Fort A P Hill & Subtropical Division & 3 & 2 & -1 \\
\hline Fort Benning & Subtropical Division & 3 & 3 & 0 \\
\hline Fort Bliss & $\begin{array}{l}\text { Tropical/Subtropical Steppe } \\
\text { Mountains }\end{array}$ & 2 & 3 & 1 \\
\hline Fort Bragg & Subtropical Division & 3 & 3 & 0 \\
\hline Fort Campbell & Hot Continental Division & 3 & 3 & 0 \\
\hline Fort Carson & Temperate Steppe Division & 2 & 3 & 1 \\
\hline $\begin{array}{l}\text { Fort Chaffee Maneuver } \\
\text { Training Center (MTC) }\end{array}$ & Hot Continental Mountains & 3 & 3 & 0 \\
\hline Fort Dix & Hot Continental Division & 3 & 3 & 0 \\
\hline Fort Drum & Warm Continental Division & 3 & 3 & 0 \\
\hline Fort Hood & $\begin{array}{l}\text { Tropical/Subtropical Steppe } \\
\text { Division }\end{array}$ & 2 & 3 & 1 \\
\hline Fort Huachuca & $\begin{array}{l}\text { Tropical/Subtropical Desert } \\
\text { Division }\end{array}$ & 1 & 2 & 1 \\
\hline
\end{tabular}




\begin{tabular}{|c|c|c|c|c|}
\hline Installation & Division & Doe & Combined & Change \\
\hline Fort Hunter-Liggett & Mediterranean Division & 2 & 1 & -1 \\
\hline Fort Indiantown Gap & Hot Continental Mountains & 3 & 3 & 0 \\
\hline Fort Jackson & Subtropical Division & 3 & 3 & 0 \\
\hline Fort Knox & Hot Continental Division & 3 & 3 & 0 \\
\hline Fort Leonard Wood & Hot Continental Division & 3 & 3 & 0 \\
\hline Fort Lewis & Marine Division & 4 & 1 & -3 \\
\hline Fort McCoy & Hot Continental Division & 3 & 2 & -1 \\
\hline Fort Pickett & Subtropical Division & 3 & 3 & 0 \\
\hline Fort Polk & Subtropical Division & 2 & 2 & 0 \\
\hline Fort Riley & Prairie Division & 4 & 4 & 0 \\
\hline Fort Rucker & Subtropical Division & 3 & 2 & -1 \\
\hline Fort Sill & $\begin{array}{l}\text { Tropical/Subtropical Steppe } \\
\text { Division }\end{array}$ & 2 & 4 & 2 \\
\hline Fort Stewart & Subtropical Division & 2 & 2 & 0 \\
\hline Fort Wolters & Prairie Division & 3 & 3 & 0 \\
\hline Hunter Army Airfield & Subtropical Division & 2 & 2 & 0 \\
\hline ITC Camp San Luis Obisbo & Mediterranean Division & 2 & 1 & -1 \\
\hline $\begin{array}{l}\text { Marseilles Military Training } \\
\text { Area (MTA) }\end{array}$ & Prairie Division & 4 & 3 & -1 \\
\hline $\begin{array}{l}\text { Milan Army Ammunition } \\
\text { Plant (AAP) }\end{array}$ & Hot Continental Division & 3 & 3 & 0 \\
\hline MTA Camp Crowder Neosho & Hot Continental Division & 3 & 3 & 0 \\
\hline MTA Camp Shelby & Subtropical Division & 2 & 2 & 0 \\
\hline MTC-H Camp Grayling & Warm Continental Division & 3 & 2 & -1 \\
\hline NTC and Fort Irwin & $\begin{array}{l}\text { Tropical/Subtropical Desert } \\
\text { Division }\end{array}$ & 1 & 1 & 0 \\
\hline Orchard Training Area & Temperate Desert Division & 1 & 2 & 1 \\
\hline $\begin{array}{l}\text { Parks Reserve Forces } \\
\text { Training Area }\end{array}$ & Mediterranean Division & 2 & 4 & 2 \\
\hline Pinon Canyon & Temperate Steppe Division & 2 & 4 & 2 \\
\hline $\begin{array}{l}\text { Ravenna Training and } \\
\text { Logistics Site }\end{array}$ & Hot Continental Division & 3 & 3 & 0 \\
\hline $\begin{array}{l}\text { Volunteer Training Site (VTS) } \\
\text { Catoosa }\end{array}$ & Hot Continental Division & 3 & 3 & 0 \\
\hline VTS Tullahoma & Hot Continental Division & 3 & 3 & 0 \\
\hline Wappapello Training Site & Hot Continental Division & 3 & 3 & 0 \\
\hline Yakima Training Center & Temperate Desert Division & 1 & 2 & 1 \\
\hline
\end{tabular}




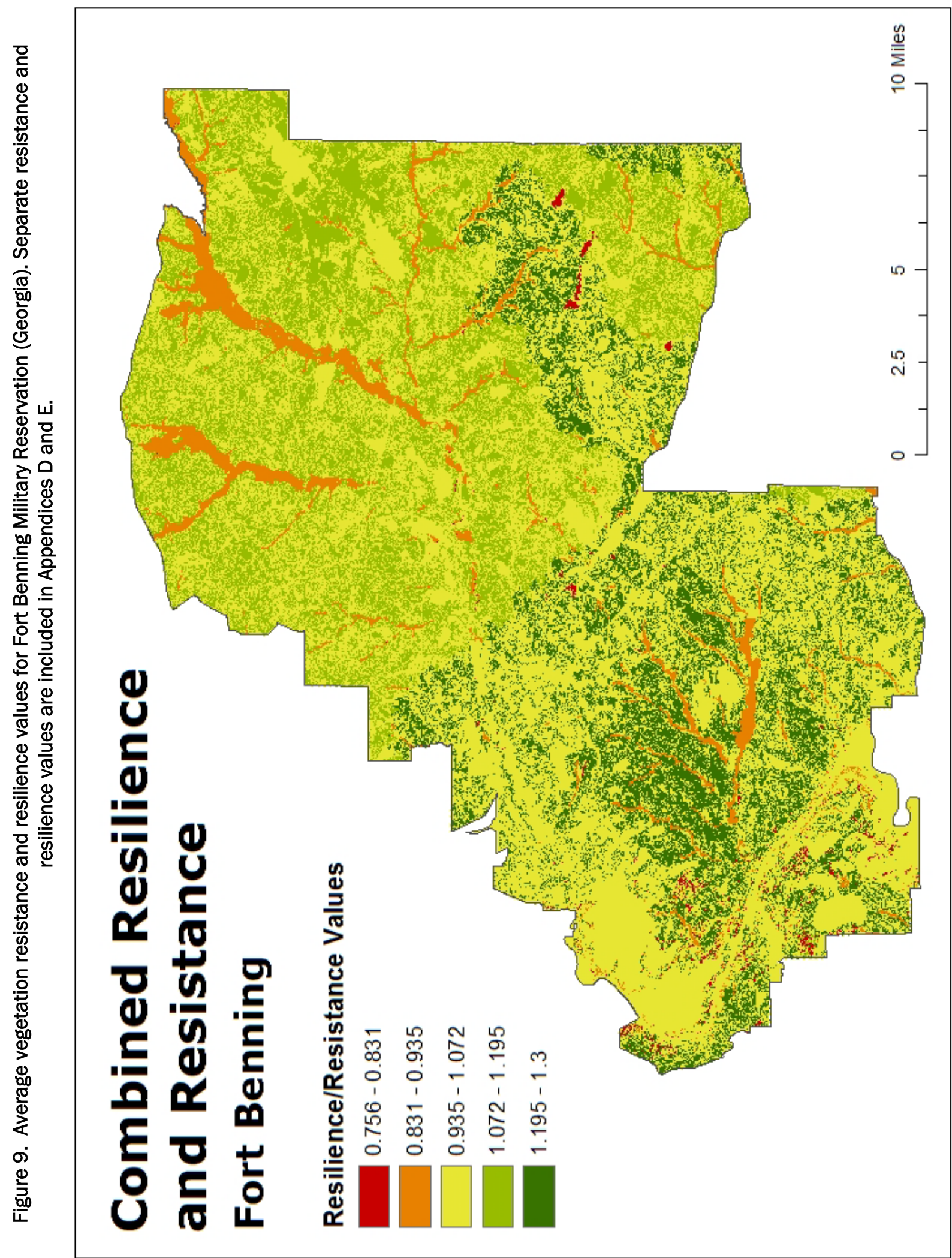




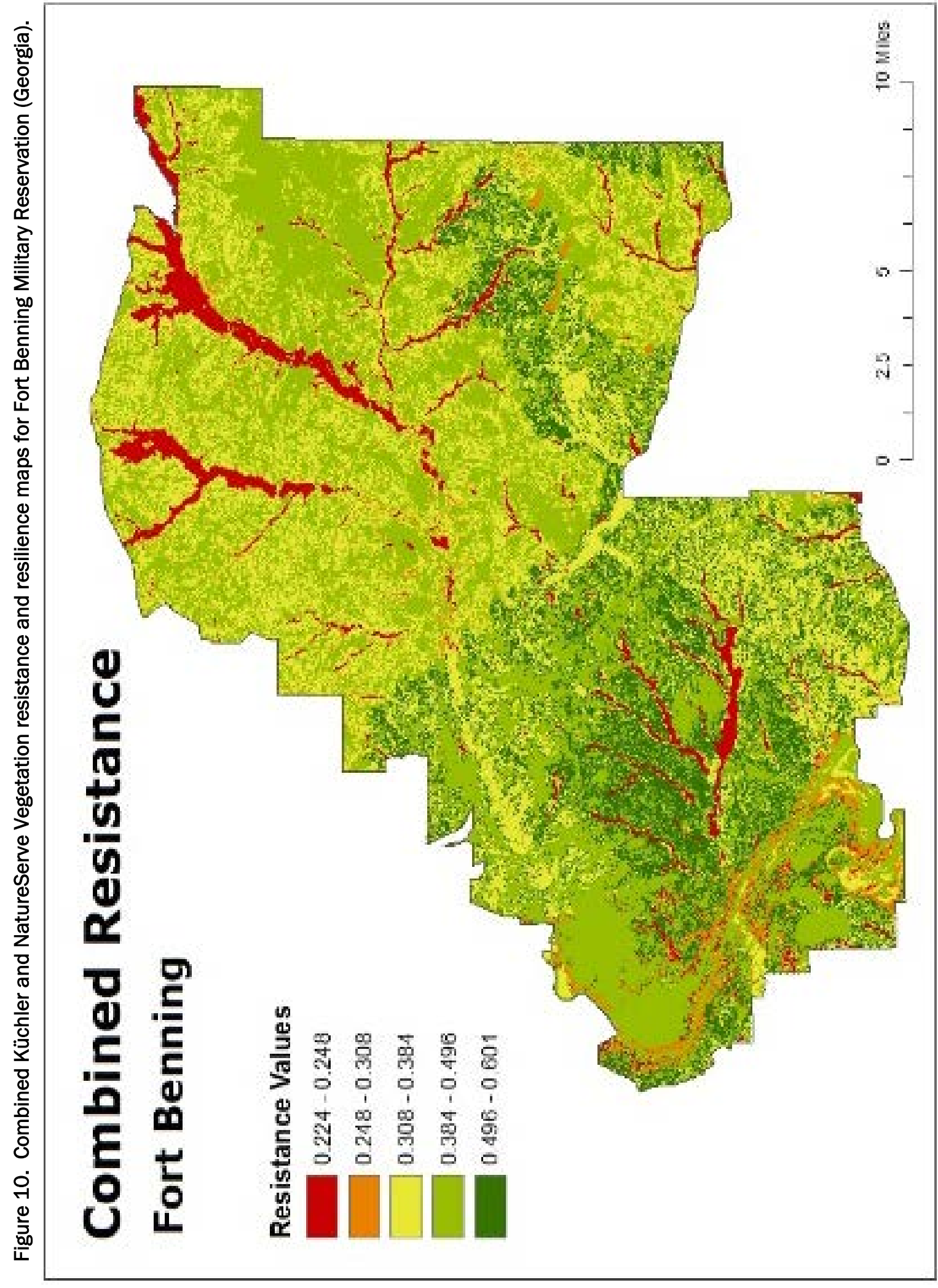




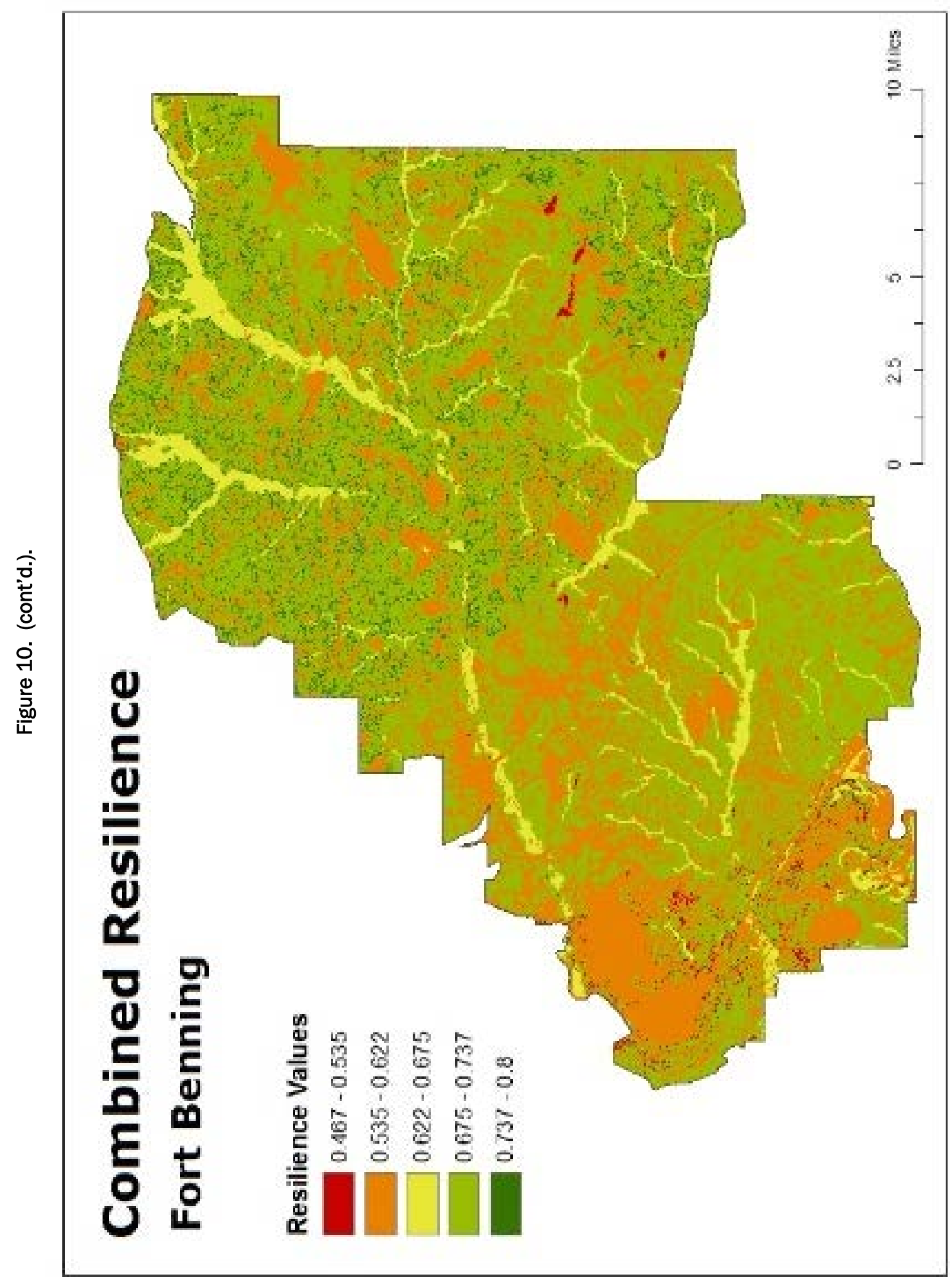


Table 4. Top 10 installations ranked according to the Combined RRVAL values; Küchler and NatureServe are provided for reference. Full data are available in Appendices $D$ and $E$.

\begin{tabular}{|l|c|c|c|}
\hline Installation & $\begin{array}{c}\text { Küchler } \\
\text { Rank }\end{array}$ & $\begin{array}{c}\text { NatureServe } \\
\text { Rank }\end{array}$ & $\begin{array}{c}\text { Combined } \\
\text { Rank }\end{array}$ \\
\hline Pinon Canyon & 1 & 1 & 1 \\
\hline Fort Riley & 2 & 2 & 2 \\
\hline Fort Sill & 3 & 7 & 3 \\
\hline Parks Reserve Forces Training Area & 4 & 8 & 4 \\
\hline Camp Perry TS (CTC) & 9 & 3 & 5 \\
\hline Camp Clark & 5 & 11 & 6 \\
\hline VTS Tullahoma & 10 & 4 & 7 \\
\hline Fort Wolters & 6 & 24 & 8 \\
\hline Fort Campbell & 7 & 12 & 9 \\
\hline Fort Knox & 8 & 22 & 10 \\
\hline
\end{tabular}




\section{Conclusions}

It is well-known that temperature and precipitation patterns are one of the main determinants of vegetation patterns (Whittaker 1975). This being the case, it is expected that projected climate change will have an impact on the spatial distribution of vegetation, both of individual species and community assemblages. These projected shifts in vegetation could lead to changes in training area resilience and recovery capabilities, which can, in turn, change the value of training lands to the Army.

This research defined a metric that is amenable to shifting vegetation patterns under projected global climate change. Specifically, dominant plant species for numerous plant communities, resistance and resilience values for plant functional groups, and national community vegetation maps were used to estimate resistance and resilience for the continental United States. Two different datasets were used:

1. NatureServe, a highly detailed dataset representing existing vegetation that contained categories for heavily altered lands with no discernible resistance and resilience values

2. Küchler, a more broadly defined dataset that contained potential vegetation, but provided resistance and resilience values for all land areas.

By combining these datasets, greater detail was obtained along with values for all land areas. The benefits of combining these datasets to develop resistance and resilience values lies in the fact that NatureServe (2015) is more contemporary and represents a snapshot of current plant community composition, whereas Küchler (1964) is an older, historic, and ecologically based dataset suggestive of potential plant community types irrespective of historical or current disturbance. This combination allows for plant community resistance and resilience values that better reflect the current status of Army training lands by capturing historical training disturbances in the context of current plant community composition.

The benefits of combining NatureServe and Küchler datasets provide an objective method to:

1. Assign resistance and resilience values to individual training areas within an installation (see Fort Benning example in Figure 10) to identify areas that may have training impacts 
2. Identify areas that are likely to have higher per-area maintenance costs based on resilience and resistance values

3. Estimate land rehabilitation and maintenance costs and potential training impacts for the purpose of supporting Army decisions on land usage and unit stationing

4. Provide comparable metrics across all Army locations, which makes it useful for installation portfolio analyses like strategic stationing studies.

This work correlated Combined RRVAL at the ecoregion scale with subject matter expert opinion estimates of resiliency (Doe et al. 1999), with a few notable exceptions. The Marine and Marine Mountain Divisions were estimated to have high Combined RRVAL; estimates by Doe et al. were high for both of these divisions. In addition, Doe et al. estimated part of the Temperate Steppe Division to have a low to moderate resiliency, while the entire Division had a high Combined RRVAL in the current study. The removal of these three outliers increased the correlation from 0.36 to 0.82 .

Individual installations can be valued and compared. For example, Pinon Canyon, CO, Fort Riley, KS, Fort Sill, OK, and Parks Reserve Forces Training Area, CA were in the top 10 of all installations, regardless of the data source, while NTC and Fort Irwin, CA, ITC Camp San Luis Obisbo, CA, Fort Lewis, WA, Florence Military Reservation, AZ, Fort Hunter-Liggett, CA, and MTC-H Camp Grayling, MI were in the bottom. Overall, 42 of the 56 installations had a change in rank of six or less between the Küchler, NatureServe, and Combined maps. Fort McCoy, WI, Fort Carson, CO, Camp Ashland, NE, and Fort Bragg, NC had the greatest change in rank, averaging $29,27,14$, and 13 , respectively.

This research provides a scientifically defensible RRVAL estimate for natural vegetation communities across the United States including CONUS military installations. Using the described methods, the Army could use the resulting RRVAL for comparative installations studies to determine the portfolio with the greatest training potential and MAC. 


\section{Appendix A: Summary Tables}

Table A-1. Resistance, resilience, and resistance and resilience values based on Küchler Potential Vegetation Map, NatureServe vegetation community map, and summation of the Küchler and NatureServe values.

\begin{tabular}{|c|c|c|c|c|c|c|c|c|c|}
\hline \multirow[b]{2}{*}{ Bailey Ecoregion } & \multicolumn{3}{|c|}{ Küchler } & \multicolumn{3}{|c|}{ NatureServe } & \multicolumn{3}{|c|}{ Combined } \\
\hline & Resist & Resil & Resist+Resil & Resist & Resil & Resist+Resil & Resist & Resil & Resist+Resil \\
\hline Hot Continental & 0.41 & 0.94 & 1.36 & 0.26 & 0.88 & 1.15 & 0.34 & 0.91 & 1.25 \\
\hline Hot Continental Mountains & 0.36 & 0.89 & 1.25 & 0.26 & 0.83 & 1.09 & 0.31 & 0.86 & 1.17 \\
\hline Marine & 0.52 & 0.13 & 0.65 & 0.31 & 0.29 & 0.60 & 0.42 & 0.21 & 0.63 \\
\hline Marine Mountains & 0.58 & 0.08 & 0.66 & 0.35 & 0.23 & 0.58 & 0.47 & 0.16 & 0.62 \\
\hline Mediterranean & 0.68 & 0.46 & 1.13 & 0.35 & 0.27 & 0.62 & 0.52 & 0.36 & 0.88 \\
\hline Mediterranean Mountains & 0.46 & 0.16 & 0.62 & 0.34 & 0.30 & 0.64 & 0.40 & 0.23 & 0.63 \\
\hline Prairie & 0.71 & 0.94 & 1.65 & 0.53 & 0.84 & 1.38 & 0.62 & 0.89 & 1.51 \\
\hline Savanna & 0.52 & 0.32 & 0.84 & 0.53 & 0.51 & 1.05 & 0.53 & 0.42 & 0.94 \\
\hline Subtropical & 0.44 & 0.60 & 1.04 & 0.31 & 0.71 & 1.02 & 0.37 & 0.66 & 1.03 \\
\hline Subtropical Mountains & 0.42 & 0.59 & 1.01 & 0.34 & 0.86 & 1.20 & 0.38 & 0.73 & 1.10 \\
\hline Temperate Desert & 0.37 & 0.32 & 0.69 & 0.26 & 0.39 & 0.65 & 0.32 & 0.35 & 0.67 \\
\hline $\begin{array}{l}\text { Temperate Desert } \\
\text { Mountains }\end{array}$ & 0.27 & 0.09 & 0.37 & 0.22 & 0.34 & 0.56 & 0.25 & 0.22 & 0.46 \\
\hline Temperate Steppe & 0.92 & 0.79 & 1.72 & 0.79 & 0.72 & 1.51 & 0.86 & 0.76 & 1.62 \\
\hline $\begin{array}{l}\text { Temperate Steppe } \\
\text { Mountains }\end{array}$ & 0.61 & 0.17 & 0.78 & 0.42 & 0.39 & 0.81 & 0.51 & 0.28 & 0.79 \\
\hline Tropical/Subtropical Desert & 0.21 & 0.31 & 0.52 & 0.40 & 0.60 & 1.00 & 0.30 & 0.45 & 0.76 \\
\hline Tropical/Subtropical Steppe & 0.67 & 0.61 & 1.28 & 0.52 & 0.61 & 1.13 & 0.60 & 0.61 & 1.20 \\
\hline $\begin{array}{l}\text { Tropical/Subtropical Steppe } \\
\text { Mountains }\end{array}$ & 0.62 & 0.21 & 0.83 & 0.47 & 0.47 & 0.94 & 0.54 & 0.34 & 0.89 \\
\hline Warm Continental & 0.45 & 0.47 & 0.92 & 0.25 & 0.69 & 0.94 & 0.35 & 0.58 & 0.93 \\
\hline $\begin{array}{l}\text { Warm Continental } \\
\text { Mountains }\end{array}$ & 0.42 & 0.58 & 1.00 & 0.28 & 0.73 & 1.02 & 0.35 & 0.66 & 1.01 \\
\hline
\end{tabular}

Table A-2. Resistance and resilience values for 56 U.S. Army installations based on predominant plant species as mapped according to Küchler, Nature Serve, and the average of the two vegetation maps.

\begin{tabular}{|l|c|c|c|c|c|c|c|c|c|}
\hline \multirow{2}{*}{ Installation } & \multicolumn{3}{|c|}{ Küchler } & \multicolumn{3}{c|}{ NatureServe } & \multicolumn{3}{c|}{ Combined } \\
\cline { 2 - 11 }$y$ & Resist & Resil & Resist+Resil & Resist & Resil & Resist+Resil & Resist & Resil & Resist+Resil \\
\hline Camp Ashland & 0.23 & 0.81 & 1.05 & 0.54 & 0.92 & 1.46 & 0.39 & 0.86 & 1.25 \\
\hline Camp Atterbury & 0.33 & 1.00 & 1.33 & 0.22 & 1.00 & 1.22 & 0.28 & 1.00 & 1.28 \\
\hline Camp Blanding & 0.44 & 0.50 & 0.94 & 0.38 & 0.43 & 0.81 & 0.41 & 0.46 & 0.88 \\
\hline Camp Bowie & 0.70 & 0.32 & 1.02 & 0.42 & 0.59 & 1.01 & 0.56 & 0.45 & 1.01 \\
\hline Camp Bullis & 0.70 & 0.32 & 1.02 & 0.45 & 0.54 & 0.99 & 0.57 & 0.43 & 1.00 \\
\hline Camp Clark & 0.67 & 0.98 & 1.65 & 0.24 & 0.99 & 1.23 & 0.45 & 0.99 & 1.44 \\
\hline Camp Dawson & 0.34 & 0.99 & 1.32 & 0.22 & 0.87 & 1.09 & 0.28 & 0.93 & 1.21 \\
\hline Camp Maxey & 0.33 & 1.00 & 1.33 & 0.29 & 0.74 & 1.03 & 0.31 & 0.87 & 1.18 \\
\hline Camp Minden TS & 0.42 & 0.60 & 1.02 & 0.31 & 0.68 & 1.00 & 0.37 & 0.64 & 1.01 \\
\hline Camp Perry TS (CTC) & 0.33 & 1.00 & 1.33 & 0.92 & 0.73 & 1.66 & 0.63 & 0.87 & 1.50 \\
\hline
\end{tabular}




\begin{tabular}{|c|c|c|c|c|c|c|c|c|c|}
\hline \multirow[b]{2}{*}{ Installation } & \multicolumn{3}{|c|}{ Küchler } & \multicolumn{3}{|c|}{ NatureServe } & \multicolumn{3}{|c|}{ Combined } \\
\hline & Resist & Resil & Resist+Resil & Resist & Resil & Resist+Resil & Resist & Resil & Resist+Resil \\
\hline Camp Ripley & 0.61 & 0.24 & 0.85 & 0.24 & 0.76 & 1.00 & 0.42 & 0.50 & 0.92 \\
\hline Camp Swift & 0.33 & 1.00 & 1.33 & 0.28 & 0.73 & 1.01 & 0.31 & 0.86 & 1.17 \\
\hline Camp Villere TS & 0.44 & 0.50 & 0.94 & 0.17 & 0.09 & 0.26 & 0.31 & 0.29 & 0.60 \\
\hline Caswell Training Site & 0.42 & 0.60 & 1.02 & 0.33 & 0.40 & 0.73 & 0.38 & 0.50 & 0.88 \\
\hline Florence & 0.00 & 0.25 & 0.25 & 0.12 & 0.83 & 0.95 & 0.06 & 0.54 & 0.60 \\
\hline Fort A P Hill & 0.42 & 0.60 & 1.02 & 0.20 & 0.84 & 1.04 & 0.31 & 0.72 & 1.03 \\
\hline Fort Benning & 0.42 & 0.60 & 1.02 & 0.40 & 0.81 & 1.21 & 0.41 & 0.71 & 1.12 \\
\hline Fort Bliss & 0.53 & 0.51 & 1.04 & 0.48 & 0.69 & 1.17 & 0.50 & 0.60 & 1.10 \\
\hline Fort Bragg & 0.44 & 0.50 & 0.94 & 0.40 & 0.79 & 1.19 & 0.42 & 0.65 & 1.07 \\
\hline Fort Campbell & 0.41 & 1.00 & 1.40 & 0.22 & 1.00 & 1.22 & 0.31 & 1.00 & 1.31 \\
\hline Fort Carson & 0.67 & 0.25 & 0.92 & 0.74 & 0.71 & 1.45 & 0.71 & 0.48 & 1.19 \\
\hline Fort Chaffee MTC & 0.36 & 0.89 & 1.25 & 0.34 & 0.93 & 1.26 & 0.35 & 0.91 & 1.26 \\
\hline Fort Dix & 0.38 & 0.79 & 1.18 & 0.27 & 0.82 & 1.09 & 0.33 & 0.80 & 1.13 \\
\hline Fort Drum & 0.39 & 0.74 & 1.13 & 0.31 & 0.74 & 1.06 & 0.35 & 0.74 & 1.10 \\
\hline Fort Hood & 0.70 & 0.32 & 1.02 & 0.46 & 0.59 & 1.05 & 0.58 & 0.45 & 1.03 \\
\hline Fort Huachuca & 0.57 & 0.41 & 0.98 & 0.42 & 0.54 & 0.96 & 0.50 & 0.48 & 0.97 \\
\hline Fort Hunter-Liggett & 0.36 & 0.24 & 0.60 & 0.26 & 0.45 & 0.71 & 0.31 & 0.34 & 0.65 \\
\hline Fort Indiantown Gap & 0.33 & 1.00 & 1.33 & 0.26 & 0.83 & 1.09 & 0.30 & 0.92 & 1.21 \\
\hline Fort Jackson & 0.42 & 0.60 & 1.02 & 0.40 & 0.80 & 1.20 & 0.41 & 0.70 & 1.11 \\
\hline Fort Knox & 0.40 & 1.00 & 1.40 & 0.21 & 0.97 & 1.18 & 0.31 & 0.99 & 1.29 \\
\hline Fort Leonard Wood & 0.33 & 1.00 & 1.33 & 0.23 & 0.99 & 1.22 & 0.28 & 0.99 & 1.28 \\
\hline Fort Lewis & 0.56 & 0.00 & 0.56 & 0.37 & 0.28 & 0.65 & 0.46 & 0.14 & 0.60 \\
\hline Fort McCoy & 0.56 & 0.00 & 0.56 & 0.43 & 0.88 & 1.32 & 0.50 & 0.44 & 0.94 \\
\hline Fort Pickett & 0.42 & 0.60 & 1.02 & 0.25 & 0.89 & 1.14 & 0.34 & 0.75 & 1.08 \\
\hline Fort Polk & 0.44 & 0.50 & 0.94 & 0.41 & 0.57 & 0.98 & 0.43 & 0.53 & 0.96 \\
\hline Fort Riley & 0.94 & 0.94 & 1.88 & 0.84 & 0.93 & 1.77 & 0.89 & 0.94 & 1.83 \\
\hline Fort Rucker & 0.44 & 0.52 & 0.96 & 0.28 & 0.76 & 1.04 & 0.36 & 0.64 & 1.00 \\
\hline Fort Sill & 0.79 & 0.97 & 1.76 & 0.62 & 0.81 & 1.43 & 0.70 & 0.89 & 1.59 \\
\hline Fort Stewart & 0.43 & 0.56 & 0.99 & 0.36 & 0.50 & 0.86 & 0.40 & 0.53 & 0.93 \\
\hline Fort Wolters & 0.56 & 0.99 & 1.54 & 0.36 & 0.80 & 1.16 & 0.46 & 0.89 & 1.35 \\
\hline Hunter Army Airfield & 0.44 & 0.50 & 0.94 & 0.36 & 0.50 & 0.86 & 0.40 & 0.50 & 0.90 \\
\hline ITC Camp San Luis Obisbo & 0.30 & 0.21 & 0.51 & 0.16 & 0.27 & 0.43 & 0.23 & 0.24 & 0.47 \\
\hline Marseilles MTA & 0.33 & 1.00 & 1.33 & 0.23 & 0.99 & 1.22 & 0.28 & 1.00 & 1.28 \\
\hline Milan AAP & 0.33 & 1.00 & 1.33 & 0.22 & 0.99 & 1.21 & 0.28 & 0.99 & 1.27 \\
\hline MTA Camp Crowder Neosho & 0.33 & 1.00 & 1.33 & 0.22 & 1.00 & 1.22 & 0.28 & 1.00 & 1.28 \\
\hline MTA Camp Shelby & 0.44 & 0.50 & 0.95 & 0.34 & 0.76 & 1.10 & 0.39 & 0.63 & 1.02 \\
\hline MTC-H Camp Grayling & 0.55 & 0.04 & 0.59 & 0.22 & 0.54 & 0.76 & 0.38 & 0.29 & 0.68 \\
\hline NTC and Fort Irwin & 0.00 & 0.10 & 0.10 & 0.16 & 0.53 & 0.69 & 0.08 & 0.32 & 0.39 \\
\hline Orchard Training Area & 0.46 & 0.40 & 0.86 & 0.44 & 0.53 & 0.96 & 0.45 & 0.46 & 0.91 \\
\hline Parks Reserve Forces Training Area & 1.00 & 0.70 & 1.70 & 0.76 & 0.56 & 1.32 & 0.88 & 0.63 & 1.51 \\
\hline Pinon Canyon & 1.00 & 0.95 & 1.95 & 0.93 & 0.88 & 1.81 & 0.97 & 0.91 & 1.88 \\
\hline Ravenna Training and Logistics Site & 0.33 & 1.00 & 1.33 & 0.25 & 0.88 & 1.13 & 0.29 & 0.94 & 1.23 \\
\hline VTS Catoosa & 0.40 & 0.71 & 1.11 & 0.26 & 0.84 & 1.10 & 0.33 & 0.77 & 1.10 \\
\hline VTS Tullahoma & 0.33 & 1.00 & 1.33 & 0.69 & 0.79 & 1.48 & 0.51 & 0.90 & 1.40 \\
\hline Wappapello Training Site & 0.33 & 1.00 & 1.33 & 0.22 & 1.00 & 1.22 & 0.28 & 1.00 & 1.28 \\
\hline Yakima Training Center & 0.50 & 0.40 & 0.90 & 0.41 & 0.42 & 0.83 & 0.45 & 0.41 & 0.87 \\
\hline
\end{tabular}


Table A-3. Fifty-six U.S. Army installations ranked according to Küchler, Nature Serve, and the average RRVAL.

\begin{tabular}{|c|c|c|c|}
\hline Installation & $\begin{array}{l}\text { Küchler } \\
\text { Rank }\end{array}$ & $\begin{array}{c}\text { NatureServe } \\
\text { Rank }\end{array}$ & $\begin{array}{l}\text { Combined } \\
\text { Rank }\end{array}$ \\
\hline Pinon Canyon & 1 & 1 & 1 \\
\hline Fort Riley & 2 & 2 & 2 \\
\hline Fort Sill & 3 & 7 & 3 \\
\hline Parks Reserve Forces Training Area & 4 & 8 & 4 \\
\hline Camp Perry TS (CTC) & 9 & 3 & 5 \\
\hline Camp Clark & 5 & 11 & 6 \\
\hline VTS Tullahoma & 10 & 4 & 7 \\
\hline Fort Wolters & 6 & 24 & 8 \\
\hline Fort Campbell & 7 & 12 & 9 \\
\hline Fort Knox & 8 & 22 & 10 \\
\hline Camp Atterbury & 11 & 13 & 11 \\
\hline MTA Camp Crowder Neosho & 12 & 14 & 12 \\
\hline Wappapello Training Site & 13 & 15 & 13 \\
\hline Marseilles MTA & 14 & 16 & 14 \\
\hline Fort Leonard Wood & 15 & 17 & 15 \\
\hline Milan AAP & 16 & 18 & 16 \\
\hline Fort Chaffee MTC & 22 & 10 & 17 \\
\hline Camp Ashland & 26 & 5 & 18 \\
\hline Ravenna Training and Logistics Site & 17 & 26 & 19 \\
\hline Fort Indiantown Gap & 18 & 29 & 20 \\
\hline Camp Dawson & 21 & 30 & 21 \\
\hline Fort Carson & 46 & 6 & 22 \\
\hline Camp Maxey & 19 & 36 & 23 \\
\hline Camp Swift & 20 & 37 & 24 \\
\hline Fort Dix & 23 & 31 & 25 \\
\hline Fort Benning & 28 & 19 & 26 \\
\hline Fort Jackson & 29 & 20 & 27 \\
\hline Fort Bliss & 27 & 23 & 28 \\
\hline VTS Catoosa & 25 & 27 & 29 \\
\hline Fort Drum & 24 & 32 & 30 \\
\hline Fort Pickett & 30 & 25 & 31 \\
\hline Fort Bragg & 41 & 21 & 32 \\
\hline Fort Hood & 31 & 33 & 33 \\
\hline Fort A P Hill & 32 & 34 & 34 \\
\hline MTA Camp Shelby & 40 & 28 & 35 \\
\hline Camp Bowie & 33 & 38 & 36 \\
\hline Camp Minden TS & 34 & 39 & 37 \\
\hline Fort Rucker & 39 & 35 & 38 \\
\hline Camp Bullis & 35 & 41 & 39 \\
\hline Fort Huachuca & 38 & 43 & 40 \\
\hline
\end{tabular}




\begin{tabular}{|l|c|c|c|}
\hline Fort Polk & 42 & 42 & 41 \\
\hline Fort McCoy & 52 & 9 & 42 \\
\hline Fort Stewart & 37 & 46 & 43 \\
\hline Camp Ripley & 49 & 40 & 44 \\
\hline Orchard Training Area & 48 & 44 & 45 \\
\hline Hunter Army Airfield & 43 & 47 & 46 \\
\hline Camp Blanding & 44 & 49 & 47 \\
\hline Caswell Training Site & 36 & 51 & 48 \\
\hline Yakima Training Center & 47 & 48 & 49 \\
\hline MTC-H Camp Grayling & 51 & 50 & 50 \\
\hline Fort Hunter-Liggett & 50 & 52 & 51 \\
\hline Florence & 55 & 45 & 52 \\
\hline Fort Lewis & 53 & 54 & 53 \\
\hline Camp Villere TS & 45 & 56 & 54 \\
\hline ITC Camp San Luis Obisbo & 54 & 55 & 55 \\
\hline NTC and Fort Irwin & 56 & 53 & 56 \\
\hline
\end{tabular}




\section{Appendix B: Dominant Species' Resistance and Resilience Values From USGS National Vegetation Classification Community Types}

Table B-1. Dominant species' resistance and resilience values from USGS national vegetation classification community types.

\begin{tabular}{|c|c|c|c|c|}
\hline Community & Species & Classification & Resistance & Resilience \\
\hline $\begin{array}{l}\text { North Pacific Dry-Mesic Silver Fir-Western } \\
\text { Hemlock-Douglas-fir Forest }\end{array}$ & Abies amabilis & Tree- Evergreen & 3.2 & 2.2 \\
\hline North Pacific Maritime Mesic Subalpine Parkland & Abies amabilis & Tree- Evergreen & 3.2 & 2.2 \\
\hline $\begin{array}{l}\text { North Pacific Mesic Western Hemlock-Silver Fir } \\
\text { Forest }\end{array}$ & Abies amabilis & Tree- Evergreen & 3.2 & 2.2 \\
\hline North Pacific Mountain Hemlock Forest & Abies amabilis & Tree- Evergreen & 3.2 & 2.2 \\
\hline $\begin{array}{l}\text { Acadian Low-Elevation Spruce-Fir-Hardwood } \\
\text { Forest }\end{array}$ & Abies balsamea & Tree- Evergreen & 3.2 & 2.2 \\
\hline Acadian-Appalachian Montane Spruce-Fir Forest & Abies balsamea & Tree- Evergreen & 3.2 & 2.2 \\
\hline High Allegheny Wetland & Abies balsamea & Tree- Evergreen & 3.2 & 2.2 \\
\hline Boreal White Spruce-Fir-Hardwood Forest & Abies balsamea & Tree- Evergreen & 3.2 & 2.2 \\
\hline $\begin{array}{l}\text { Eastern Hemi-Boreal Mesic Balsam Fir-Spruce } \\
\text { Forest }\end{array}$ & Abies balsamea & Tree- Evergreen & 3.2 & 2.2 \\
\hline $\begin{array}{l}\text { Great Basin Foothill and Lower Montane Riparian } \\
\text { Woodland and Shrubland }\end{array}$ & Abies concolor & Tree- Evergreen & 3.2 & 2.2 \\
\hline $\begin{array}{l}\text { Inter-Mountain Basins Aspen-Mixed Conifer Forest } \\
\text { and Woodland }\end{array}$ & Abies concolor & Tree- Evergreen & 3.2 & 2.2 \\
\hline $\begin{array}{l}\text { Madrean Lower Montane Pine-Oak Forest and } \\
\text { Woodland }\end{array}$ & Abies concolor & Tree- Evergreen & 3.2 & 2.2 \\
\hline $\begin{array}{l}\text { Madrean Upper Montane Conifer-Oak Forest and } \\
\text { Woodland }\end{array}$ & Abies concolor & Tree- Evergreen & 3.2 & 2.2 \\
\hline Mediterranean California Red Fir Forest & Abies concolor & Tree- Evergreen & 3.2 & 2.2 \\
\hline $\begin{array}{l}\text { North Pacific Montane Riparian Woodland and } \\
\text { Shrubland }\end{array}$ & Abies concolor & Tree- Evergreen & 3.2 & 2.2 \\
\hline Rocky Mountain Bigtooth Maple Ravine Woodland & Abies concolor & Tree- Evergreen & 3.2 & 2.2 \\
\hline $\begin{array}{l}\text { Sierran-Intermontane Desert Western White Pine- } \\
\text { White Fir Woodland }\end{array}$ & Abies concolor & Tree- Evergreen & 3.2 & 2.2 \\
\hline $\begin{array}{l}\text { Southern Rocky Mountain Dry-Mesic Montane } \\
\text { Mixed Conifer Forest and Woodland }\end{array}$ & Abies concolor & Tree- Evergreen & 3.2 & 2.2 \\
\hline $\begin{array}{l}\text { Southern Rocky Mountain Mesic Montane Mixed } \\
\text { Conifer Forest and Woodland }\end{array}$ & Abies concolor & Tree- Evergreen & 3.2 & 2.2 \\
\hline $\begin{array}{l}\text { Mediterranean California Mesic Mixed Conifer } \\
\text { Forest and Woodland }\end{array}$ & Abies concolor & Tree- Evergreen & 3.2 & 2.2 \\
\hline $\begin{array}{l}\text { Sierran-Intermontane Desert Western White Pine- } \\
\text { White Fir Woodland }\end{array}$ & Abies concolor & Tree- Evergreen & 3.2 & 2.2 \\
\hline $\begin{array}{l}\text { Central and Southern Appalachian Spruce-Fir } \\
\text { Forest }\end{array}$ & Abies fraseri & Tree- Evergreen & 3.2 & 2.2 \\
\hline $\begin{array}{l}\text { East Cascades Mesic Montane Mixed Conifer } \\
\text { Forest and Woodland }\end{array}$ & Abies grandis & Tree- Evergreen & 3.2 & 2.2 \\
\hline $\begin{array}{l}\text { North Pacific Lowland Mixed Hardwood-Conifer } \\
\text { Forest }\end{array}$ & Abies grandis & Tree- Evergreen & 3.2 & 2.2 \\
\hline
\end{tabular}




\begin{tabular}{|c|c|c|c|c|}
\hline Community & Species & Classification & Resistance & Resilience \\
\hline $\begin{array}{l}\text { North Pacific Maritime Dry-Mesic Douglas-fir- } \\
\text { Western Hemlock Forest }\end{array}$ & Abies grandis & Tree-Evergreen & 3.2 & 2.2 \\
\hline $\begin{array}{l}\text { Northern Rocky Mountain Dry-Mesic Montane } \\
\text { Mixed Conifer Forest }\end{array}$ & Abies grandis & Tree- Evergreen & 3.2 & 2.2 \\
\hline $\begin{array}{l}\text { Northern Rocky Mountain Lower Montane } \\
\text { Riparian Woodland and Shrubland }\end{array}$ & Abies grandis & Tree- Evergreen & 3.2 & 2.2 \\
\hline $\begin{array}{l}\text { Northern Rocky Mountain Mesic Montane Mixed } \\
\text { Conifer Forest }\end{array}$ & Abies grandis & Tree- Evergreen & 3.2 & 2.2 \\
\hline Northern Rocky Mountain Wooded Vernal Pool & Abies grandis & Tree-Evergreen & 3.2 & 2.2 \\
\hline $\begin{array}{l}\text { North Pacific Dry Douglas-fir-(Madrone) Forest and } \\
\text { Woodland }\end{array}$ & Abies grandis & Tree- Evergreen & 3.2 & 2.2 \\
\hline $\begin{array}{l}\text { Inter-Mountain Basins Aspen-Mixed Conifer Forest } \\
\text { and Woodland }\end{array}$ & Abies lasiocarpa & Tree- Evergreen & 3.2 & 2.2 \\
\hline North Pacific Maritime Mesic Subalpine Parkland & Abies lasiocarpa & Tree-Evergreen & 3.2 & 2.2 \\
\hline North Pacific Wooded Volcanic Flowage & Abies lasiocarpa & Tree- Evergreen & 3.2 & 2.2 \\
\hline $\begin{array}{l}\text { Northern Rocky Mountain Avalanche Chute } \\
\text { Shrubland }\end{array}$ & Abies lasiocarpa & Tree- Evergreen & 3.2 & 2.2 \\
\hline $\begin{array}{l}\text { Northern Rocky Mountain Subalpine Woodland } \\
\text { and Parkland }\end{array}$ & Abies lasiocarpa & Tree- Evergreen & 3.2 & 2.2 \\
\hline Northern Rocky Mountain Wooded Vernal Pool & Abies lasiocarpa & Tree- Evergreen & 3.2 & 2.2 \\
\hline $\begin{array}{l}\text { Rocky Mountain Subalpine Dry-Mesic Spruce-Fir } \\
\text { Forest and Woodland }\end{array}$ & Abies lasiocarpa & Tree- Evergreen & 3.2 & 2.2 \\
\hline $\begin{array}{l}\text { Rocky Mountain Subalpine Mesic-Wet Spruce-Fir } \\
\text { Forest and Woodland }\end{array}$ & Abies lasiocarpa & Tree- Evergreen & 3.2 & 2.2 \\
\hline $\begin{array}{l}\text { Rocky Mountain Subalpine-Montane Riparian } \\
\text { Woodland }\end{array}$ & Abies lasiocarpa & Tree- Evergreen & 3.2 & 2.2 \\
\hline $\begin{array}{l}\text { Rocky Mountain Subalpine Mesic-Wet Spruce-Fir } \\
\text { Forest and Woodland }\end{array}$ & Abies lasiocarpa & Tree- Evergreen & 3.2 & 2.2 \\
\hline $\begin{array}{l}\text { Inter-Mountain Basins Aspen-Mixed Conifer Forest } \\
\text { and Woodland }\end{array}$ & Abies magnifica & Tree- Evergreen & 3.2 & 2.2 \\
\hline Mediterranean California Red Fir Forest & Abies magnifica & Tree- Evergreen & 3.2 & 2.2 \\
\hline $\begin{array}{l}\text { North Pacific Montane Riparian Woodland and } \\
\text { Shrubland }\end{array}$ & Abies magnifica & Tree- Evergreen & 3.2 & 2.2 \\
\hline Mediterranean California Red Fir Forest & Abies procera & Tree- Evergreen & 3.2 & 2.2 \\
\hline Mediterranean California Red Fir Forest & Abies $x$ shastensis & Tree- Evergreen & 3.2 & 2.2 \\
\hline North Pacific Mountain Hemlock Forest & Abies $x$ shastensis & Tree- Evergreen & 3.2 & 2.2 \\
\hline Mediterranean California Southern Coastal Dune & Abronia maritima & Forb & 3 & 3.5 \\
\hline Mediterranean California Southern Coastal Dune & Abronia umbellata & Forb & 3 & 3.5 \\
\hline Tamaulipan Calcareous Thornscrub & Acacia berlandieri & Shrub- Deciduous & 2.7 & 3 \\
\hline Tamaulipan Mixed Deciduous Thornscrub & Acacia berlandieri & Shrub- Deciduous & 2.7 & 3 \\
\hline Chihuahuan Mixed Desert and Thornscrub & Acacia constricta & Shrub-Deciduous & 2.7 & 3 \\
\hline Apacherian-Chihuahuan Mesquite Upland Scrub & Acacia constricta & Tree-Deciduous & 3 & 4.2 \\
\hline Tamaulipan Mesquite Upland Scrub & Acacia constricta & Tree-Deciduous & 3 & 4.2 \\
\hline Tamaulipan Calcareous Thornscrub & Acacia farnesiana & Shrub- Deciduous & 2.7 & 3 \\
\hline Tamaulipan Mixed Deciduous Thornscrub & Acacia farnesiana & Shrub- Deciduous & 2.7 & 3 \\
\hline Tamaulipan Ramadero & Acacia farnesiana & Shrub- Evergreen & 2.7 & 2.4 \\
\hline Tamaulipan Floodplain & Acacia farnesiana & Tree-Evergreen & 3.2 & 2.2 \\
\hline Tamaulipan Palm Grove Riparian Forest & Acacia farnesiana & Tree- Evergreen & 3.2 & 2.2 \\
\hline $\begin{array}{l}\text { North American Warm Desert Riparian Mesquite } \\
\text { Bosque }\end{array}$ & Acacia greggii & Shrub- Deciduous & 2.7 & 3 \\
\hline North American Warm Desert Wash & Acacia greggii & Shrub- Deciduous & 2.7 & 3 \\
\hline Sonoran Paloverde-Mixed Cacti Desert Scrub & Acacia greggii & Shrub- Deciduous & 2.7 & 3 \\
\hline
\end{tabular}




\begin{tabular}{|c|c|c|c|c|}
\hline Community & Species & Classification & Resistance & Resilience \\
\hline Apacherian-Chihuahuan Mesquite Upland Scrub & Acacia neovernicosa & Shrub-Deciduous & 2.7 & 3 \\
\hline Chihuahuan Mixed Desert and Thornscrub & Acacia neovernicosa & Shrub- Deciduous & 2.7 & 3 \\
\hline Tamaulipan Mesquite Upland Scrub & Acacia neovernicosa & Shrub-Deciduous & 2.7 & 3 \\
\hline Tamaulipan Mixed Deciduous Thornscrub & Acacia rigidula & Shrub-Deciduous & 2.7 & 3 \\
\hline Tamaulipan Calcareous Thornscrub & Acacia rigidula & Tree-Deciduous & 3 & 4.2 \\
\hline Tamaulipan Mixed Deciduous Thornscrub & Acacia roemeriana & Shrub-Deciduous & 2.7 & 3 \\
\hline $\begin{array}{l}\text { East Gulf Coastal Plain Small Stream and River } \\
\text { Floodplain Forest }\end{array}$ & Acer barbatum & Tree- Deciduous & 3 & 4.2 \\
\hline Ozark-Ouachita Mesic Hardwood Forest & Acer barbatum & Tree-Deciduous & 3 & 4.2 \\
\hline Southern Coastal Plain Limestone Forest & Acer barbatum & Tree-Deciduous & 3 & 4.2 \\
\hline Southern Piedmont Mesic Forest & Acer barbatum & Tree-Deciduous & 3 & 4.2 \\
\hline West Gulf Coastal Plain Mesic Hardwood Forest & Acer barbatum & Tree-Deciduous & 3 & 4.2 \\
\hline North Pacific Avalanche Chute Shrubland & Acer circinatum & Shrub-Deciduous & 2.7 & 3 \\
\hline $\begin{array}{l}\text { North Pacific Lowland Mixed Hardwood-Conifer } \\
\text { Forest }\end{array}$ & Acer circinatum & Shrub-Deciduous & 2.7 & 3 \\
\hline $\begin{array}{l}\text { North Pacific Montane Riparian Woodland and } \\
\text { Shrubland }\end{array}$ & Acer circinatum & Shrub- Deciduous & 2.7 & 3 \\
\hline North Pacific Montane Shrubland & Acer circinatum & Shrub-Deciduous & 2.7 & 3 \\
\hline North Pacific Wooded Volcanic Flowage & Acer circinatum & Shrub- Deciduous & 2.7 & 3 \\
\hline $\begin{array}{l}\text { North Pacific Maritime Dry-Mesic Douglas-fir- } \\
\text { Western Hemlock Forest }\end{array}$ & Acer circinatum & Shrub- Evergreen & 2.7 & 2.4 \\
\hline North Pacific Montane Shrubland & Acer glabrum & Shrub-Deciduous & 2.7 & 3 \\
\hline $\begin{array}{l}\text { Northern Rocky Mountain Montane-Foothill } \\
\text { Deciduous Shrubland }\end{array}$ & Acer glabrum & Shrub- Deciduous & 2.7 & 3 \\
\hline $\begin{array}{l}\text { Northern Rocky Mountain Avalanche Chute } \\
\text { Shrubland }\end{array}$ & Acer glabrum & Tree-Deciduous & 3 & 4.2 \\
\hline Rocky Mountain Bigtooth Maple Ravine Woodland & Acer glabrum & Tree-Deciduous & 3 & 4.2 \\
\hline $\begin{array}{l}\text { Rocky Mountain Lower Montane-Foothill Riparian } \\
\text { Woodland and Shrubland }\end{array}$ & Acer glabrum & Tree-Deciduous & 3 & 4.2 \\
\hline $\begin{array}{l}\text { Inter-Mountain Basins Aspen-Mixed Conifer Forest } \\
\text { and Woodland }\end{array}$ & Acer grandidentatum & Tree-Deciduous & 3 & 4.2 \\
\hline Rocky Mountain Bigtooth Maple Ravine Woodland & Acer grandidentatum & Tree-Deciduous & 3 & 4.2 \\
\hline $\begin{array}{l}\text { Central and Southern California Mixed Evergreen } \\
\text { Woodland }\end{array}$ & Acer macrophyllum & Tree-Deciduous & 3 & 4.2 \\
\hline $\begin{array}{l}\text { Mediterranean California Mesic Mixed Conifer } \\
\text { Forest and Woodland }\end{array}$ & Acer macrophyllum & Tree-Deciduous & 3 & 4.2 \\
\hline $\begin{array}{l}\text { North Pacific Broadleaf Landslide Forest and } \\
\text { Shrubland }\end{array}$ & Acer macrophyllum & Tree- Deciduous & 3 & 4.2 \\
\hline $\begin{array}{l}\text { North Pacific Lowland Mixed Hardwood-Conifer } \\
\text { Forest }\end{array}$ & Acer macrophyllum & Tree- Deciduous & 3 & 4.2 \\
\hline $\begin{array}{l}\text { North Pacific Lowland Riparian Forest and } \\
\text { Shrubland }\end{array}$ & Acer macrophyllum & Tree-Deciduous & 3 & 4.2 \\
\hline $\begin{array}{l}\text { North Pacific Maritime Dry-Mesic Douglas-fir- } \\
\text { Western Hemlock Forest }\end{array}$ & Acer macrophyllum & Tree-Deciduous & 3 & 4.2 \\
\hline $\begin{array}{l}\text { North Pacific Maritime Mesic-Wet Douglas-fir- } \\
\text { Western Hemlock Forest }\end{array}$ & Acer macrophyllum & Tree-Deciduous & 3 & 4.2 \\
\hline $\begin{array}{l}\text { Mediterranean California Foothill and Lower } \\
\text { Montane Riparian Woodland and Shrubland }\end{array}$ & Acer macrophyllum & Tree-Deciduous & 3 & 4.2 \\
\hline $\begin{array}{l}\text { North Pacific Dry Douglas-fir-(Madrone) Forest and } \\
\text { Woodland }\end{array}$ & Acer macrophyllum & Tree-Deciduous & 3 & 4.2 \\
\hline $\begin{array}{l}\text { Atlantic Coastal Plain Small Brownwater River } \\
\text { Floodplain Forest }\end{array}$ & Acer negundo & Tree-Deciduous & 3 & 4.2 \\
\hline Central Appalachian Stream and Riparian & Acer negundo & Tree-Deciduous & 3 & 4.2 \\
\hline
\end{tabular}




\begin{tabular}{|c|c|c|c|c|}
\hline Community & Species & Classification & Resistance & Resilience \\
\hline Edwards Plateau Floodplain Terrace & Acer negundo & Tree- Deciduous & 3 & 4.2 \\
\hline $\begin{array}{l}\text { North American Warm Desert Riparian Woodland } \\
\text { and Shrubland }\end{array}$ & Acer negundo & Tree- Deciduous & 3 & 4.2 \\
\hline $\begin{array}{l}\text { Northwestern Great Plains-Black Hills Ponderosa } \\
\text { Pine Woodland and Savanna }\end{array}$ & Acer negundo & Tree-Deciduous & 3 & 4.2 \\
\hline Rocky Mountain Bigtooth Maple Ravine Woodland & Acer negundo & Tree-Deciduous & 3 & 4.2 \\
\hline $\begin{array}{l}\text { Rocky Mountain Lower Montane-Foothill Riparian } \\
\text { Woodland and Shrubland }\end{array}$ & Acer negundo & Tree-Deciduous & 3 & 4.2 \\
\hline Southeastern Great Plains Floodplain Forest & Acer negundo & Tree-Deciduous & 3 & 4.2 \\
\hline Southern Piedmont Large Floodplain Forest & Acer negundo & Tree-Deciduous & 3 & 4.2 \\
\hline $\begin{array}{l}\text { Southern Piedmont Small Floodplain and Riparian } \\
\text { Forest }\end{array}$ & Acer negundo & Tree- Deciduous & 3 & 4.2 \\
\hline $\begin{array}{l}\text { Mediterranean California Foothill and Lower } \\
\text { Montane Riparian Woodland and Shrubland }\end{array}$ & Acer negundo & Tree- Deciduous & 3 & 4.2 \\
\hline Southern Appalachian Northern Hardwood Forest & Acer pensylvanicum & Tree-Deciduous & 3 & 4.2 \\
\hline $\begin{array}{l}\text { Allegheny-Cumberland Dry Oak Forest and } \\
\text { Woodland }\end{array}$ & Acer rubrum & Tree- Deciduous & 3 & 4.2 \\
\hline Central Atlantic Coastal Plain Maritime Forest & Acer rubrum & Tree- Deciduous & 3 & 4.2 \\
\hline Great Lakes Wooded Dune and Swale & Acer rubrum & Tree-Deciduous & 3 & 4.2 \\
\hline $\begin{array}{l}\text { Laurentian-Acadian Alkaline Conifer-Hardwood } \\
\text { Swamp }\end{array}$ & Acer rubrum & Tree-Deciduous & 3 & 4.2 \\
\hline Laurentian-Acadian Northern Hardwood Forest & Acer rubrum & Tree- Deciduous & 3 & 4.2 \\
\hline $\begin{array}{l}\text { North-Central Interior and Appalachian Rich } \\
\text { Swamp }\end{array}$ & Acer rubrum & Tree-Deciduous & 3 & 4.2 \\
\hline North-Central Interior Wet Flatwoods & Acer rubrum & Tree- Deciduous & 3 & 4.2 \\
\hline $\begin{array}{l}\text { Northern Atlantic Coastal Plain Basin Swamp and } \\
\text { Wet Hardwood Forest }\end{array}$ & Acer rubrum & Tree- Deciduous & 3 & 4.2 \\
\hline Northern Atlantic Coastal Plain Maritime Forest & Acer rubrum & Tree-Deciduous & 3 & 4.2 \\
\hline Northern Atlantic Coastal Plain Tidal Swamp & Acer rubrum & Tree-Deciduous & 3 & 4.2 \\
\hline Piedmont Upland Depression Swamp & Acer rubrum & Tree-Deciduous & 3 & 4.2 \\
\hline $\begin{array}{l}\text { Southern Atlantic Coastal Plain Tidal Wooded } \\
\text { Swamp }\end{array}$ & Acer rubrum & Tree- Deciduous & 3 & 4.2 \\
\hline Southern Piedmont Mesic Forest & Acer rubrum & Tree-Deciduous & 3 & 4.2 \\
\hline $\begin{array}{l}\text { Southern Piedmont Small Floodplain and Riparian } \\
\text { Forest }\end{array}$ & Acer rubrum & Tree-Deciduous & 3 & 4.2 \\
\hline $\begin{array}{l}\text { Atlantic Coastal Plain Streamhead Seepage } \\
\text { Swamp, Pocosin and Baygall }\end{array}$ & Acer rubrum & Tree-Deciduous & 3 & 4.2 \\
\hline Laurentian-Acadian Northern Pine-(Oak) Forest & Acer rubrum & Tree- Deciduous & 3 & 4.2 \\
\hline $\begin{array}{l}\text { South-Central Interior / Upper Coastal Plain Wet } \\
\text { Flatwoods }\end{array}$ & Acer rubrum & Tree- Deciduous & 3 & 4.2 \\
\hline Southern Appalachian Oak Forest & Acer rubrum & Tree- Deciduous & 3 & 4.2 \\
\hline Mississippi River Bottomland Depression & Acer rubrum & Tree- Deciduous & 3 & 4.2 \\
\hline South-Central Interior Small Stream and Riparian & Acer rubrum & Tree- Deciduous & 3 & 4.2 \\
\hline Central Appalachian River Floodplain & Acer saccharinum & Tree-Deciduous & 3 & 4.2 \\
\hline Laurentian-Acadian Floodplain Forest & Acer saccharinum & Tree-Deciduous & 3 & 4.2 \\
\hline North-Central Interior Floodplain & Acer saccharinum & Tree-Deciduous & 3 & 4.2 \\
\hline South-Central Interior Large Floodplain & Acer saccharinum & Tree-Deciduous & 3 & 4.2 \\
\hline $\begin{array}{l}\text { Central Interior Highlands Calcareous Glade and } \\
\text { Barrens }\end{array}$ & Acer saccharum & Tree- Deciduous & 3 & 4.2 \\
\hline Crowley's Ridge Mesic Loess Slope Forest & Acer saccharum & Tree-Deciduous & 3 & 4.2 \\
\hline Laurentian-Acadian Northern Hardwood Forest & Acer saccharum & Tree-Deciduous & 3 & 4.2 \\
\hline
\end{tabular}




\begin{tabular}{|c|c|c|c|c|}
\hline Community & Species & Classification & Resistance & Resilience \\
\hline North-Central Interior Beech-Maple Forest & Acer saccharum & Tree-Deciduous & 3 & 4.2 \\
\hline North-Central Interior Maple-Basswood Forest & Acer saccharum & Tree-Deciduous & 3 & 4.2 \\
\hline Ozark-Ouachita Mesic Hardwood Forest & Acer saccharum & Tree-Deciduous & 3 & 4.2 \\
\hline South-Central Interior Mesophytic Forest & Acer saccharum & Tree-Deciduous & 3 & 4.2 \\
\hline Southern and Central Appalachian Cove Forest & Acer saccharum & Tree-Deciduous & 3 & 4.2 \\
\hline Southern Appalachian Northern Hardwood Forest & Acer saccharum & Tree- Deciduous & 3 & 4.2 \\
\hline Appalachian (Hemlock)-Northern Hardwood Forest & Acer saccharum & Tree-Deciduous & 3 & 4.2 \\
\hline Central Appalachian Alkaline Glade and Woodland & $\begin{array}{l}\text { Acer saccharum var. } \\
\text { saccharum }\end{array}$ & Tree-Deciduous & 3 & 4.2 \\
\hline Mediterranean California Subalpine Meadow & Achillea millefolium & Forb & 3 & 3.5 \\
\hline $\begin{array}{l}\text { North Pacific Maritime Dry-Mesic Douglas-fir- } \\
\text { Western Hemlock Forest }\end{array}$ & Achlys triphylla & Forb & 3 & 3.5 \\
\hline Chihuahuan Sandy Plains Semi-Desert Grassland & Achnatherum hymenoides & Graminoid $\mathrm{C}_{3}$ & 3.6 & 3.6 \\
\hline $\begin{array}{l}\text { Colorado Plateau Mixed Low Sagebrush } \\
\text { Shrubland }\end{array}$ & Achnatherum hymenoides & Graminoid $C_{3}$ & 3.6 & 3.6 \\
\hline Inter-Mountain Basins Big Sagebrush Steppe & Achnatherum hymenoides & Graminoid $C_{3}$ & 3.6 & 3.6 \\
\hline Inter-Mountain Basins Juniper Savanna & Achnatherum hymenoides & Graminoid $C_{3}$ & 3.6 & 3.6 \\
\hline Inter-Mountain Basins Semi-Desert Grassland & Achnatherum hymenoides & Graminoid $C_{3}$ & 3.6 & 3.6 \\
\hline Inter-Mountain Basins Semi-Desert Shrub-Steppe & Achnatherum hymenoides & Graminoid $\mathrm{C}_{3}$ & 3.6 & 3.6 \\
\hline $\begin{array}{l}\text { Southern Rocky Mountain Juniper Woodland and } \\
\text { Savanna }\end{array}$ & Achnatherum hymenoides & Graminoid $\mathrm{C}_{3}$ & 3.6 & 3.6 \\
\hline Klamath-Siskiyou Cliff and Outcrop & Achnatherum lemmonii & Graminoid $C_{3}$ & 3.6 & 3.6 \\
\hline North Pacific Herbaceous Bald and Bluff & Achnatherum lemmonii & Graminoid $C_{3}$ & 3.6 & 3.6 \\
\hline $\begin{array}{l}\text { Southern Rocky Mountain Montane-Subalpine } \\
\text { Grassland }\end{array}$ & Achnatherum lettermanii & Graminoid $C_{3}$ & 3.6 & 3.6 \\
\hline Klamath-Siskiyou Cliff and Outcrop & Achnatherum occidentale & Graminoid $\mathrm{C}_{3}$ & 3.6 & 3.6 \\
\hline $\begin{array}{l}\text { Northern Rocky Mountain Subalpine-Upper } \\
\text { Montane Grassland }\end{array}$ & Achnatherum occidentale & Graminoid $C_{3}$ & 3.6 & 3.6 \\
\hline $\begin{array}{l}\text { Northern Rocky Mountain Subalpine-Upper } \\
\text { Montane Grassland }\end{array}$ & Achnatherum richardsonii & Graminoid $C_{3}$ & 3.6 & 3.6 \\
\hline $\begin{array}{l}\text { Northern Rocky Mountain Lower Montane, Foothill } \\
\text { and Valley Grassland }\end{array}$ & Achnatherum richardsonii & Graminoid $C_{3}$ & 3.6 & 3.6 \\
\hline Great Basin Xeric Mixed Sagebrush Shrubland & Achnatherum thurberianum & Graminoid $C_{3}$ & 3.6 & 3.6 \\
\hline California Maritime Chaparral & Adenostoma fasciculatum & Shrub- Evergreen & 2.7 & 2.4 \\
\hline East Gulf Coastal Plain Dry Chalk Bluff & Adiantum capillus-veneris & Graminoid $\mathrm{C}_{3}$ & 3.6 & 3.6 \\
\hline Edwards Plateau Cliff & Adiantum capillus-veneris & Graminoid $C_{3}$ & 3.6 & 3.6 \\
\hline Edwards Plateau Mesic Canyon & Adiantum capillus-veneris & Graminoid $\mathrm{C}_{3}$ & 3.6 & 3.6 \\
\hline Southern and Central Appalachian Cove Forest & Aesculus flava & Tree- Deciduous & 3 & 4.2 \\
\hline Southern Appalachian Northern Hardwood Forest & Aesculus flava & Tree-Deciduous & 3 & 4.2 \\
\hline Chihuahuan Succulent Desert Scrub & Agave lechuguilla & Forb & 3 & 3.5 \\
\hline Chihuahuan Creosotebush Desert Scrub & Agave parryi & Forb & 3 & 3.5 \\
\hline Inter-Mountain Basins Playa & Allenrolfea occidentalis & Shrub- Deciduous & 2.7 & 3 \\
\hline North American Warm Desert Playa & Allenrolfea occidentalis & Shrub- Deciduous & 2.7 & 3 \\
\hline North Pacific Herbaceous Bald and Bluff & Allium cernuum & Forb & 3 & 3.5 \\
\hline $\begin{array}{l}\text { Great Basin Foothill and Lower Montane Riparian } \\
\text { Woodland and Shrubland }\end{array}$ & Alnus incana & Shrub- Deciduous & 2.7 & 3 \\
\hline Great Lakes Wooded Dune and Swale & Alnus incana & Shrub- Deciduous & 2.7 & 3 \\
\hline Laurentian-Acadian Wet Meadow-Shrub Swamp & Alnus incana & Shrub- Deciduous & 2.7 & 3 \\
\hline $\begin{array}{l}\text { Northern Rocky Mountain Lower Montane } \\
\text { Riparian Woodland and Shrubland }\end{array}$ & Alnus incana & Shrub- Deciduous & 2.7 & 3 \\
\hline
\end{tabular}




\begin{tabular}{|c|c|c|c|c|}
\hline Community & Species & Classification & Resistance & Resilience \\
\hline Northern Rocky Mountain Wooded Vernal Pool & Alnus incana & Shrub-Deciduous & 2.7 & 3 \\
\hline $\begin{array}{l}\text { Rocky Mountain Subalpine-Montane Riparian } \\
\text { Shrubland }\end{array}$ & Alnus incana & Shrub- Deciduous & 2.7 & 3 \\
\hline $\begin{array}{l}\text { Rocky Mountain Subalpine-Montane Riparian } \\
\text { Woodland }\end{array}$ & Alnus incana & Shrub- Deciduous & 2.7 & 3 \\
\hline $\begin{array}{l}\text { Rocky Mountain Lower Montane-Foothill Riparian } \\
\text { Woodland and Shrubland }\end{array}$ & Alnus incana & Tree-Deciduous & 3 & 4.2 \\
\hline $\begin{array}{l}\text { North Pacific Montane Riparian Woodland and } \\
\text { Shrubland }\end{array}$ & Alnus incana & Shrub-Deciduous & 2.7 & 3 \\
\hline North Pacific Shrub Swamp & Alnus incana & Shrub- Deciduous & 2.7 & 3 \\
\hline $\begin{array}{l}\text { North American Warm Desert Lower Montane } \\
\text { Riparian Woodland and Shrubland }\end{array}$ & Alnus oblongifolia & Shrub- Deciduous & 2.7 & 3 \\
\hline $\begin{array}{l}\text { Mediterranean California Foothill and Lower } \\
\text { Montane Riparian Woodland and Shrubland }\end{array}$ & Alnus rhombifolia & Tree-Deciduous & 3 & 4.2 \\
\hline $\begin{array}{l}\text { North Pacific Broadleaf Landslide Forest and } \\
\text { Shrubland }\end{array}$ & Alnus rubra & Shrub-Deciduous & 2.7 & 3 \\
\hline North Pacific Hardwood-Conifer Swamp & Alnus rubra & Shrub- Deciduous & 2.7 & 3 \\
\hline North Pacific Intertidal Freshwater Wetland & Alnus rubra & Shrub- Deciduous & 2.7 & 3 \\
\hline $\begin{array}{l}\text { North Pacific Lowland Mixed Hardwood-Conifer } \\
\text { Forest }\end{array}$ & Alnus rubra & Shrub- Deciduous & 2.7 & 3 \\
\hline $\begin{array}{l}\text { North Pacific Lowland Riparian Forest and } \\
\text { Shrubland }\end{array}$ & Alnus rubra & Shrub-Deciduous & 2.7 & 3 \\
\hline $\begin{array}{l}\text { North Pacific Maritime Mesic-Wet Douglas-fir- } \\
\text { Western Hemlock Forest }\end{array}$ & Alnus rubra & Shrub-Deciduous & 2.7 & 3 \\
\hline $\begin{array}{l}\text { North Pacific Montane Riparian Woodland and } \\
\text { Shrubland }\end{array}$ & Alnus rubra & Shrub- Deciduous & 2.7 & 3 \\
\hline $\begin{array}{l}\text { Mediterranean California Foothill and Lower } \\
\text { Montane Riparian Woodland and Shrubland }\end{array}$ & Alnus rubra & Tree-Deciduous & 3 & 4.2 \\
\hline Cumberland Riverscour & Alnus serrulata & Shrub-Deciduous & 2.7 & 3 \\
\hline $\begin{array}{l}\text { East Gulf Coastal Plain Small Stream and River } \\
\text { Floodplain Forest }\end{array}$ & Alnus serrulata & Shrub-Deciduous & 2.7 & 3 \\
\hline Northern Atlantic Coastal Plain Tidal Swamp & Alnus serrulata & Shrub- Deciduous & 2.7 & 3 \\
\hline South-Central Interior Small Stream and Riparian & Alnus serrulata & Shrub- Deciduous & 2.7 & 3 \\
\hline Southern and Central Appalachian Bog and Fen & Alnus serrulata & Shrub-Deciduous & 2.7 & 3 \\
\hline $\begin{array}{l}\text { Northern Atlantic Coastal Plain Fresh and } \\
\text { Oligohaline Tidal Marsh }\end{array}$ & Alnus serrulata & Shrub- Deciduous & 2.7 & 3 \\
\hline $\begin{array}{l}\text { North Pacific Active Volcanic Rock and Cinder } \\
\text { Land }\end{array}$ & Alnus viridis & Shrub-Deciduous & 2.7 & 3 \\
\hline $\begin{array}{l}\text { Northern Rocky Mountain Subalpine Deciduous } \\
\text { Shrubland }\end{array}$ & Alnus viridis & Shrub-Deciduous & 2.7 & 3 \\
\hline $\begin{array}{l}\text { North Pacific Montane Riparian Woodland and } \\
\text { Shrubland }\end{array}$ & Alnus viridis & Shrub-Deciduous & 2.7 & 3 \\
\hline North Pacific Shrub Swamp & Alnus viridis & Shrub-Deciduous & 2.7 & 3 \\
\hline Southern Appalachian Grass and Shrub Bald & Alnus viridis & Shrub- Deciduous & 2.7 & 3 \\
\hline North Pacific Avalanche Chute Shrubland & Alnus viridis & Shrub- Deciduous & 2.7 & 3 \\
\hline $\begin{array}{l}\text { North Pacific Montane Riparian Woodland and } \\
\text { Shrubland }\end{array}$ & Alnus viridis & Shrub-Deciduous & 2.7 & 3 \\
\hline North Pacific Shrub Swamp & Alnus viridis & Shrub- Deciduous & 2.7 & 3 \\
\hline $\begin{array}{l}\text { Northern Rocky Mountain Avalanche Chute } \\
\text { Shrubland }\end{array}$ & Alnus viridis & Shrub-Deciduous & 2.7 & 3 \\
\hline $\begin{array}{l}\text { Northern Atlantic Coastal Plain Fresh and } \\
\text { Oligohaline Tidal Marsh }\end{array}$ & Amaranthus cannabinus & Forb & 3 & 3.5 \\
\hline Sonoran Paloverde-Mixed Cacti Desert Scrub & Ambrosia deltoidea & Shrub- Deciduous & 2.7 & 3 \\
\hline
\end{tabular}




\begin{tabular}{|c|c|c|c|c|}
\hline Community & Species & Classification & Resistance & Resilience \\
\hline $\begin{array}{l}\text { North American Warm Desert Active and } \\
\text { Stabilized Dune }\end{array}$ & Ambrosia dumosa & Shrub- Deciduous & 2.7 & 3 \\
\hline $\begin{array}{l}\text { Sonora-Mojave Creosotebush-White Bursage } \\
\text { Desert Scrub }\end{array}$ & Ambrosia dumosa & Shrub- Deciduous & 2.7 & 3 \\
\hline Sonoran Mid-Elevation Desert Scrub & Ambrosia dumosa & Shrub-Deciduous & 2.7 & 3 \\
\hline Sonoran Paloverde-Mixed Cacti Desert Scrub & Ambrosia dumosa & Shrub- Deciduous & 2.7 & 3 \\
\hline Central Mixedgrass Prairie & Ambrosia psilostachya & Forb & 3 & 3.5 \\
\hline $\begin{array}{l}\text { Inter-Mountain Basins Aspen-Mixed Conifer Forest } \\
\text { and Woodland }\end{array}$ & Amelanchier alnifolia & Shrub- Deciduous & 2.7 & 3 \\
\hline $\begin{array}{l}\text { Northern Rocky Mountain Montane-Foothill } \\
\text { Deciduous Shrubland }\end{array}$ & Amelanchier alnifolia & Shrub- Deciduous & 2.7 & 3 \\
\hline $\begin{array}{l}\text { Northwestern Great Plains Aspen Forest and } \\
\text { Parkland }\end{array}$ & Amelanchier alnifolia & Shrub- Deciduous & 2.7 & 3 \\
\hline Rocky Mountain Bigtooth Maple Ravine Woodland & Amelanchier alnifolia & Shrub- Deciduous & 2.7 & 3 \\
\hline $\begin{array}{l}\text { Rocky Mountain Gambel Oak-Mixed Montane } \\
\text { Shrubland }\end{array}$ & Amelanchier alnifolia & Shrub- Deciduous & 2.7 & 3 \\
\hline Colorado Plateau Pinyon-Juniper Woodland & Amelanchier utahensis & Shrub- Deciduous & 2.7 & 3 \\
\hline $\begin{array}{l}\text { Rocky Mountain Gambel Oak-Mixed Montane } \\
\text { Shrubland }\end{array}$ & Amelanchier utahensis & Shrub-Deciduous & 2.7 & 3 \\
\hline $\begin{array}{l}\text { Rocky Mountain Lower Montane-Foothill } \\
\text { Shrubland }\end{array}$ & Amelanchier utahensis & Shrub- Deciduous & 2.7 & 3 \\
\hline Great Lakes Dune & Ammophila breviligulata & Graminoid $\mathrm{C}_{3}$ & 3.6 & 3.6 \\
\hline Great Lakes Wooded Dune and Swale & Ammophila breviligulata & Graminoid $\mathrm{C}_{3}$ & 3.6 & 3.6 \\
\hline Northern Atlantic Coastal Plain Dune and Swale & Ammophila breviligulata & Graminoid $\mathrm{C}_{3}$ & 3.6 & 3.6 \\
\hline Great Lakes Dune & Ammophila champlainensis & Graminoid $C_{3}$ & 3.6 & 3.6 \\
\hline Southeastern Great Plains Riparian Forest & Amorpha fruticosa & Shrub- Deciduous & 2.7 & 3 \\
\hline Tamaulipan Calcareous Thornscrub & Amyris madrensis & Shrub- Evergreen & 2.7 & 2.4 \\
\hline Tamaulipan Mixed Deciduous Thornscrub & Amyris madrensis & Shrub- Evergreen & 2.7 & 2.4 \\
\hline Tamaulipan Calcareous Thornscrub & Amyris texana & Shrub- Evergreen & 2.7 & 2.4 \\
\hline Tamaulipan Mixed Deciduous Thornscrub & Amyris texana & Shrub- Evergreen & 2.7 & 2.4 \\
\hline Central Florida Herbaceous Pondshore & Andropogon brachystachyus & Graminoid $\mathrm{C}_{4}$ & 3.6 & 4.1 \\
\hline Arkansas Valley Prairie and Woodland & Andropogon gerardii & Graminoid $\mathrm{C}_{4}$ & 3.6 & 4.1 \\
\hline Central Appalachian Alkaline Glade and Woodland & Andropogon gerardii & Graminoid $\mathrm{C}_{4}$ & 3.6 & 4.1 \\
\hline Cumberland Riverscour & Andropogon gerardii & Graminoid $\mathrm{C}_{4}$ & 3.6 & 4.1 \\
\hline Eastern Great Plains Tallgrass Aspen Parkland & Andropogon gerardii & Graminoid $\mathrm{C}_{4}$ & 3.6 & 4.1 \\
\hline Eastern Highland Rim Prairie and Barrens & Andropogon gerardii & Graminoid $\mathrm{C}_{4}$ & 3.6 & 4.1 \\
\hline Eastern Serpentine Woodland & Andropogon gerardii & Graminoid $\mathrm{C}_{4}$ & 3.6 & 4.1 \\
\hline Great Lakes Wet-Mesic Lakeplain Prairie & Andropogon gerardii & Graminoid $\mathrm{C}_{4}$ & 3.6 & 4.1 \\
\hline North-Central Interior Oak Savanna & Andropogon gerardii & Graminoid $\mathrm{C}_{4}$ & 3.6 & 4.1 \\
\hline $\begin{array}{l}\text { North-Central Interior Sand and Gravel Tallgrass } \\
\text { Prairie }\end{array}$ & Andropogon gerardii & Graminoid $\mathrm{C}_{4}$ & 3.6 & 4.1 \\
\hline North-Central Oak Barrens & Andropogon gerardii & Graminoid $\mathrm{C}_{4}$ & 3.6 & 4.1 \\
\hline Northern Tallgrass Prairie & Andropogon gerardii & Graminoid $\mathrm{C}_{4}$ & 3.6 & 4.1 \\
\hline Paleozoic Plateau Bluff and Talus & Andropogon gerardii & Graminoid $\mathrm{C}_{4}$ & 3.6 & 4.1 \\
\hline Southeastern Great Plains Tallgrass Prairie & Andropogon gerardii & Graminoid $\mathrm{C}_{4}$ & 3.6 & 4.1 \\
\hline $\begin{array}{l}\text { Southern Coastal Plain Blackland Prairie and } \\
\text { Woodland }\end{array}$ & Andropogon gerardii & Graminoid $\mathrm{C}_{4}$ & 3.6 & 4.1 \\
\hline Texas Blackland Tallgrass Prairie & Andropogon gerardii & Graminoid $\mathrm{C}_{4}$ & 3.6 & 4.1 \\
\hline Texas-Louisiana Coastal Prairie & Andropogon gerardii & Graminoid $\mathrm{C}_{4}$ & 3.6 & 4.1 \\
\hline
\end{tabular}




\begin{tabular}{|c|c|c|c|c|}
\hline Community & Species & Classification & Resistance & Resilience \\
\hline $\begin{array}{l}\text { Western Great Plains Foothill and Piedmont } \\
\text { Grassland }\end{array}$ & Andropogon gerardii & Graminoid $\mathrm{C}_{4}$ & 3.6 & 4.1 \\
\hline Western Great Plains Tallgrass Prairie & Andropogon gerardii & Graminoid $\mathrm{C}_{4}$ & 3.6 & 4.1 \\
\hline Central Tallgrass Prairie & Andropogon gerardii & Graminoid $\mathrm{C}_{4}$ & 3.6 & 4.1 \\
\hline Texas-Louisiana Coastal Prairie & Andropogon glomeratus & Graminoid $\mathrm{C}_{4}$ & 3.6 & 4.1 \\
\hline $\begin{array}{l}\text { West Gulf Coastal Plain Northern Calcareous } \\
\text { Prairie }\end{array}$ & Andropogon glomeratus & Graminoid $\mathrm{C}_{4}$ & 3.6 & 4.1 \\
\hline Southern Piedmont Glade and Barrens & Andropogon gyrans & Graminoid $\mathrm{C}_{4}$ & 3.6 & 4.1 \\
\hline Western Great Plains Sand Prairie & Andropogon hallii & Graminoid $\mathrm{C}_{4}$ & 3.6 & 4.1 \\
\hline $\begin{array}{l}\text { East Gulf Coastal Plain Interior Upland Longleaf } \\
\text { Pine Woodland }\end{array}$ & Andropogon ternarius & Graminoid $\mathrm{C}_{4}$ & 3.6 & 4.1 \\
\hline Florida Dry Prairie & Andropogon ternarius & Graminoid $\mathrm{C}_{4}$ & 3.6 & 4.1 \\
\hline Southeastern Coastal Plain Cliff & Andropogon virginicus & Graminoid $\mathrm{C}_{4}$ & 3.6 & 4.1 \\
\hline South Florida Cypress Dome & Annona glabra & Shrub- Evergreen & 2.7 & 2.4 \\
\hline $\begin{array}{l}\text { North Pacific Active Volcanic Rock and Cinder } \\
\text { Land }\end{array}$ & Antennaria lanata & Forb & 3 & 3.5 \\
\hline Rocky Mountain Alpine Bedrock and Scree & Aquilegia caerulea & Forb & 3 & 3.5 \\
\hline Rocky Mountain Alpine Bedrock and Scree & Aquilegia flavescens & Forb & 3 & 3.5 \\
\hline $\begin{array}{l}\text { Central and Southern California Mixed Evergreen } \\
\text { Woodland }\end{array}$ & Arbutus menziesii & Tree- Evergreen & 3.2 & 2.2 \\
\hline $\begin{array}{l}\text { North Pacific Dry Douglas-fir-(Madrone) Forest and } \\
\text { Woodland }\end{array}$ & Arbutus menziesii & Tree- Evergreen & 3.2 & 2.2 \\
\hline North Pacific Herbaceous Bald and Bluff & Arctostaphylos columbiana & Shrub- Evergreen & 2.7 & 2.4 \\
\hline California Montane Woodland and Chaparral & Arctostaphylos glandulosa & Shrub- Evergreen & 2.7 & 2.4 \\
\hline $\begin{array}{l}\text { Northern and Central California Dry-Mesic } \\
\text { Chaparral }\end{array}$ & Arctostaphylos glandulosa & Shrub- Evergreen & 2.7 & 2.4 \\
\hline California Xeric Serpentine Chaparral & Arctostaphylos glauca & Shrub- Evergreen & 2.7 & 2.4 \\
\hline $\begin{array}{l}\text { Mediterranean California Mesic Serpentine } \\
\text { Woodland and Chaparral }\end{array}$ & Arctostaphylos glauca & Shrub- Evergreen & 2.7 & 2.4 \\
\hline Sonora-Mojave Semi-Desert Chaparral & Arctostaphylos glauca & Shrub- Evergreen & 2.7 & 2.4 \\
\hline Southern California Coast Ranges Cliff and Canyon & Arctostaphylos glauca & Shrub- Evergreen & 2.7 & 2.4 \\
\hline California Montane Woodland and Chaparral & Arctostaphylos nevadensis & Shrub- Evergreen & 2.7 & 2.4 \\
\hline Great Basin Semi-Desert Chaparral & Arctostaphylos nevadensis & Shrub- Evergreen & 2.7 & 2.4 \\
\hline North Pacific Montane Shrubland & Arctostaphylos nevadensis & Shrub- Evergreen & 2.7 & 2.4 \\
\hline North Pacific Serpentine Barren & Arctostaphylos nevadensis & Shrub- Evergreen & 2.7 & 2.4 \\
\hline $\begin{array}{l}\text { Sierra Nevada Subalpine Lodgepole Pine Forest } \\
\text { and Woodland }\end{array}$ & Arctostaphylos nevadensis & Shrub- Evergreen & 2.7 & 2.4 \\
\hline Colorado Plateau Pinyon-Juniper Woodland & Arctostaphylos patula & Shrub- Evergreen & 2.7 & 2.4 \\
\hline Great Basin Pinyon-Juniper Woodland & Arctostaphylos patula & Shrub- Evergreen & 2.7 & 2.4 \\
\hline Great Basin Semi-Desert Chaparral & Arctostaphylos patula & Shrub- Evergreen & 2.7 & 2.4 \\
\hline $\begin{array}{l}\text { Rocky Mountain Gambel Oak-Mixed Montane } \\
\text { Shrubland }\end{array}$ & Arctostaphylos patula & Shrub- Evergreen & 2.7 & 2.4 \\
\hline $\begin{array}{l}\text { Sierra Nevada Subalpine Lodgepole Pine Forest } \\
\text { and Woodland }\end{array}$ & Arctostaphylos patula & Shrub- Evergreen & 2.7 & 2.4 \\
\hline Sonora-Mojave Semi-Desert Chaparral & Arctostaphylos patula & Shrub- Evergreen & 2.7 & 2.4 \\
\hline $\begin{array}{l}\text { Southern Rocky Mountain Ponderosa Pine } \\
\text { Woodland }\end{array}$ & Arctostaphylos patula & Shrub- Evergreen & 2.7 & 2.4 \\
\hline Mogollon Chaparral & Arctostaphylos pringlei & Shrub- Evergreen & 2.7 & 2.4 \\
\hline California Xeric Serpentine Chaparral & Arctostaphylos pungens & Shrub- Evergreen & 2.7 & 2.4 \\
\hline Mogollon Chaparral & Arctostaphylos pungens & Shrub- Evergreen & 2.7 & 2.4 \\
\hline Sonora-Mojave Semi-Desert Chaparral & Arctostaphylos pungens & Shrub- Evergreen & 2.7 & 2.4 \\
\hline
\end{tabular}




\begin{tabular}{|c|c|c|c|c|}
\hline Community & Species & Classification & Resistance & Resilience \\
\hline Great Lakes Dune & Arctostaphylos uva-ursi & Shrub & 2.7 & 2.8 \\
\hline $\begin{array}{l}\text { Northern Atlantic Coastal Plain Heathland and } \\
\text { Grassland }\end{array}$ & Arctostaphylos uva-ursi & Shrub & 2.7 & 2.8 \\
\hline $\begin{array}{l}\text { Northwestern Great Plains-Black Hills Ponderosa } \\
\text { Pine Woodland and Savanna }\end{array}$ & Arctostaphylos uva-ursi & Shrub & 2.7 & 2.8 \\
\hline Northern Rocky Mountain Western Larch Savanna & Arctostaphylos uva-ursi & Shrub- Evergreen & 2.7 & 2.4 \\
\hline $\begin{array}{l}\text { Rocky Mountain Foothill Limber Pine-Juniper } \\
\text { Woodland }\end{array}$ & Arctostaphylos uva-ursi & Shrub- Evergreen & 2.7 & 2.4 \\
\hline Rocky Mountain Lodgepole Pine Forest & Arctostaphylos uva-ursi & Shrub- Evergreen & 2.7 & 2.4 \\
\hline California Xeric Serpentine Chaparral & Arctostaphylos viscida & Shrub- Evergreen & 2.7 & 2.4 \\
\hline $\begin{array}{l}\text { Northern and Central California Dry-Mesic } \\
\text { Chaparral }\end{array}$ & Arctostaphylos viscida & Shrub- Evergreen & 2.7 & 2.4 \\
\hline $\begin{array}{l}\text { Mediterranean California Lower Montane Black } \\
\text { Oak-Conifer Forest and Woodland }\end{array}$ & Arctostaphylos viscida & Shrub- Evergreen & 2.7 & 2.4 \\
\hline Temperate Pacific Tidal Salt and Brackish Marsh & Argentina egedii & Forb & 3 & 3.5 \\
\hline $\begin{array}{l}\text { Atlantic Coastal Plain Fall-line Sandhills Longleaf } \\
\text { Pine Woodland }\end{array}$ & Aristida beyrichiana & Graminoid $\mathrm{C}_{4}$ & 3.6 & 4.1 \\
\hline Central Florida Pine Flatwoods & Aristida beyrichiana & Graminoid $\mathrm{C}_{4}$ & 3.6 & 4.1 \\
\hline $\begin{array}{l}\text { East Gulf Coastal Plain Interior Upland Longleaf } \\
\text { Pine Woodland }\end{array}$ & Aristida beyrichiana & Graminoid $\mathrm{C}_{4}$ & 3.6 & 4.1 \\
\hline East Gulf Coastal Plain Savanna and Wet Prairie & Aristida beyrichiana & Graminoid $\mathrm{C}_{4}$ & 3.6 & 4.1 \\
\hline Florida Dry Prairie & Aristida beyrichiana & Graminoid $\mathrm{C}_{4}$ & 3.6 & 4.1 \\
\hline South Florida Pine Flatwoods & Aristida beyrichiana & Graminoid $\mathrm{C}_{4}$ & 3.6 & 4.1 \\
\hline South-Central Saline Glade & Aristida dichotoma & Graminoid $\mathrm{C}_{4}$ & 3.6 & 4.1 \\
\hline South-Central Saline Glade & Aristida longespica & Graminoid $\mathrm{C}_{4}$ & 3.6 & 4.1 \\
\hline West Gulf Coastal Plain Catahoula Barrens & Aristida longespica & Graminoid $\mathrm{C}_{4}$ & 3.6 & 4.1 \\
\hline East Gulf Coastal Plain Depression Pond & Aristida palustris & Graminoid $\mathrm{C}_{4}$ & 3.6 & 4.1 \\
\hline West Gulf Coastal Plain Flatwoods Pond & Aristida palustris & Graminoid $\mathrm{C}_{4}$ & 3.6 & 4.1 \\
\hline West Gulf Coastal Plain Nepheline Syenite Glade & Aristida purpurascens & Graminoid $\mathrm{C}_{4}$ & 3.6 & 4.1 \\
\hline Eastern Highland Rim Prairie and Barrens & Aristida purpurascens & Graminoid $\mathrm{C}_{4}$ & 3.6 & 4.1 \\
\hline Inter-Mountain Basins Semi-Desert Grassland & Aristida purpurea & Graminoid $\mathrm{C}_{4}$ & 3.6 & 4.1 \\
\hline $\begin{array}{l}\text { Llano Estacado Caprock Escarpment and Breaks } \\
\text { Shrubland and Steppe }\end{array}$ & Aristida purpurea & Graminoid $\mathrm{C}_{4}$ & 3.6 & 4.1 \\
\hline Florida Dry Prairie & Aristida spiciformis & Graminoid $\mathrm{C}_{4}$ & 3.6 & 4.1 \\
\hline $\begin{array}{l}\text { Atlantic Coastal Plain Fall-line Sandhills Longleaf } \\
\text { Pine Woodland }\end{array}$ & Aristida stricta & Graminoid $\mathrm{C}_{4}$ & 3.6 & 4.1 \\
\hline $\begin{array}{l}\text { Atlantic Coastal Plain Upland Longleaf Pine } \\
\text { Woodland }\end{array}$ & Aristida stricta & Graminoid $\mathrm{C}_{4}$ & 3.6 & 4.1 \\
\hline $\begin{array}{l}\text { Central Atlantic Coastal Plain Wet Longleaf Pine } \\
\text { Savanna and Flatwoods }\end{array}$ & Aristida stricta & Graminoid $\mathrm{C}_{4}$ & 3.6 & 4.1 \\
\hline $\begin{array}{l}\text { East Gulf Coastal Plain Interior Upland Longleaf } \\
\text { Pine Woodland }\end{array}$ & Aristida stricta & Graminoid $\mathrm{C}_{4}$ & 3.6 & 4.1 \\
\hline Southern Coastal Plain Oak Dome and Hammock & Aristida stricta & Graminoid $\mathrm{C}_{4}$ & 3.6 & 4.1 \\
\hline Columbia Plateau Low Sagebrush Steppe & Artemisia arbuscula & Shrub- Evergreen & 2.7 & 2.4 \\
\hline Great Basin Pinyon-Juniper Woodland & Artemisia arbuscula & Shrub- Evergreen & 2.7 & 2.4 \\
\hline Great Basin Xeric Mixed Sagebrush Shrubland & Artemisia arbuscula & Shrub- Evergreen & 2.7 & 2.4 \\
\hline $\begin{array}{l}\text { Wyoming Basins Dwarf Sagebrush Shrubland and } \\
\text { Steppe }\end{array}$ & Artemisia arbuscula & Shrub- Evergreen & 2.7 & 2.4 \\
\hline Sierra Nevada Alpine Dwarf-Shrubland & Artemisia arbuscula & Tree- Deciduous & 3 & 4.2 \\
\hline Columbia Plateau Low Sagebrush Steppe & Artemisia arbuscula & Shrub- Evergreen & 2.7 & 2.4 \\
\hline
\end{tabular}




\begin{tabular}{|c|c|c|c|c|}
\hline Community & Species & Classification & Resistance & Resilience \\
\hline $\begin{array}{l}\text { Inter-Mountain Basins Montane Sagebrush } \\
\text { Steppe }\end{array}$ & Artemisia arbuscula & Shrub- Evergreen & 2.7 & 2.4 \\
\hline Great Basin Xeric Mixed Sagebrush Shrubland & Artemisia arbuscula & Shrub- Evergreen & 2.7 & 2.4 \\
\hline Columbia Plateau Low Sagebrush Steppe & Artemisia arbuscula & Shrub- Evergreen & 2.7 & 2.4 \\
\hline Great Basin Xeric Mixed Sagebrush Shrubland & Artemisia arbuscula & Shrub- Evergreen & 2.7 & 2.4 \\
\hline Inter-Mountain Basins Shale Badland & Artemisia arbuscula & Shrub- Evergreen & 2.7 & 2.4 \\
\hline $\begin{array}{l}\text { Wyoming Basins Dwarf Sagebrush Shrubland and } \\
\text { Steppe }\end{array}$ & Artemisia arbuscula & Shrub- Evergreen & 2.7 & 2.4 \\
\hline Rocky Mountain Alpine Turf & Artemisia arctica & Shrub & 2.7 & 2.8 \\
\hline $\begin{array}{l}\text { Colorado Plateau Mixed Low Sagebrush } \\
\text { Shrubland }\end{array}$ & Artemisia bigelovii & Shrub- Evergreen & 2.7 & 2.4 \\
\hline Northern California Coastal Scrub & Artemisia californica & Shrub- Deciduous & 2.7 & 3 \\
\hline Southern California Coastal Scrub & Artemisia californica & Shrub-Deciduous & 2.7 & 3 \\
\hline $\begin{array}{l}\text { Great Basin Foothill and Lower Montane Riparian } \\
\text { Woodland and Shrubland }\end{array}$ & Artemisia cana & Shrub- Evergreen & 2.7 & 2.4 \\
\hline $\begin{array}{l}\text { Columbia Plateau Silver Sagebrush Seasonally } \\
\text { Flooded Shrub-Steppe }\end{array}$ & Artemisia cana & Shrub- Evergreen & 2.7 & 2.4 \\
\hline Inter-Mountain Basins Big Sagebrush Steppe & Artemisia cana & Shrub- Evergreen & 2.7 & 2.4 \\
\hline Inter-Mountain Basins Greasewood Flat & Artemisia cana & Shrub- Evergreen & 2.7 & 2.4 \\
\hline Northwestern Great Plains Riparian & Artemisia cana & Shrub- Evergreen & 2.7 & 2.4 \\
\hline Western Great Plains Riparian & Artemisia cana & Shrub- Evergreen & 2.7 & 2.4 \\
\hline $\begin{array}{l}\text { Columbia Plateau Silver Sagebrush Seasonally } \\
\text { Flooded Shrub-Steppe }\end{array}$ & Artemisia cana & Shrub- Evergreen & 2.7 & 2.4 \\
\hline $\begin{array}{l}\text { Inter-Mountain Basins Montane Sagebrush } \\
\text { Steppe }\end{array}$ & Artemisia cana & Shrub- Evergreen & 2.7 & 2.4 \\
\hline $\begin{array}{l}\text { Chihuahuan Stabilized Coppice Dune and Sand } \\
\text { Flat Scrub }\end{array}$ & Artemisia filifolia & Shrub- Deciduous & 2.7 & 3 \\
\hline $\begin{array}{l}\text { Colorado Plateau Blackbrush-Mormon-tea } \\
\text { Shrubland }\end{array}$ & Artemisia filifolia & Shrub- Deciduous & 2.7 & 3 \\
\hline Inter-Mountain Basins Active and Stabilized Dune & Artemisia filifolia & Shrub- Deciduous & 2.7 & 3 \\
\hline $\begin{array}{l}\text { North American Warm Desert Active and } \\
\text { Stabilized Dune }\end{array}$ & Artemisia filifolia & Shrub- Deciduous & 2.7 & 3 \\
\hline Southern Colorado Plateau Sand Shrubland & Artemisia filifolia & Shrub- Deciduous & 2.7 & 3 \\
\hline Western Great Plains Sandhill Steppe & Artemisia filifolia & Shrub- Deciduous & 2.7 & 3 \\
\hline $\begin{array}{l}\text { Rocky Mountain Lower Montane-Foothill } \\
\text { Shrubland }\end{array}$ & Artemisia frigida & Shrub & 2.7 & 2.8 \\
\hline $\begin{array}{l}\text { Rocky Mountain Subalpine-Montane Limber- } \\
\text { Bristlecone Pine Woodland }\end{array}$ & Artemisia frigida & Shrub & 2.7 & 2.8 \\
\hline $\begin{array}{l}\text { Southern Rocky Mountain Pinyon-Juniper } \\
\text { Woodland }\end{array}$ & Artemisia frigida & Shrub & 2.7 & 2.8 \\
\hline Inter-Mountain Basins Mat Saltbush Shrubland & Artemisia longifolia & Shrub & 2.7 & 2.8 \\
\hline Western Great Plains Cliff and Outcrop & Artemisia longifolia & Shrub & 2.7 & 2.8 \\
\hline $\begin{array}{l}\text { Colorado Plateau Mixed Low Sagebrush } \\
\text { Shrubland }\end{array}$ & Artemisia nova & Shrub- Evergreen & 2.7 & 2.4 \\
\hline Colorado Plateau Pinyon-Juniper Woodland & Artemisia nova & Shrub- Evergreen & 2.7 & 2.4 \\
\hline Columbia Plateau Low Sagebrush Steppe & Artemisia nova & Shrub- Evergreen & 2.7 & 2.4 \\
\hline Great Basin Pinyon-Juniper Woodland & Artemisia nova & Shrub- Evergreen & 2.7 & 2.4 \\
\hline Great Basin Xeric Mixed Sagebrush Shrubland & Artemisia nova & Shrub- Evergreen & 2.7 & 2.4 \\
\hline $\begin{array}{l}\text { Wyoming Basins Dwarf Sagebrush Shrubland and } \\
\text { Steppe }\end{array}$ & Artemisia nova & Shrub- Evergreen & 2.7 & 2.4 \\
\hline Inter-Mountain Basins Mat Saltbush Shrubland & Artemisia pedatifida & Shrub & 2.7 & 2.8 \\
\hline Inter-Mountain Basins Shale Badland & Artemisia pedatifida & Shrub & 2.7 & 2.8 \\
\hline
\end{tabular}




\begin{tabular}{|c|c|c|c|c|}
\hline Community & Species & Classification & Resistance & Resilience \\
\hline Inter-Mountain Basins Shale Badland & Artemisia pygmaea & Shrub- Evergreen & 2.7 & 2.4 \\
\hline Columbia Plateau Scabland Shrubland & Artemisia rigida & Shrub & 2.7 & 2.8 \\
\hline $\begin{array}{l}\text { Colorado Plateau Mixed Low Sagebrush } \\
\text { Shrubland }\end{array}$ & Artemisia tridentata & Shrub- Evergreen & 2.7 & 2.4 \\
\hline Colorado Plateau Pinyon-Juniper Woodland & Artemisia tridentata & Shrub- Evergreen & 2.7 & 2.4 \\
\hline Columbia Plateau Ash and Tuff Badland & Artemisia tridentata & Shrub- Evergreen & 2.7 & 2.4 \\
\hline $\begin{array}{l}\text { Columbia Plateau Western Juniper Woodland and } \\
\text { Savanna }\end{array}$ & Artemisia tridentata & Shrub- Evergreen & 2.7 & 2.4 \\
\hline Inter-Mountain Basins Cliff and Canyon & Artemisia tridentata & Shrub- Evergreen & 2.7 & 2.4 \\
\hline Inter-Mountain Basins Mixed Salt Desert Scrub & Artemisia tridentata & Shrub- Evergreen & 2.7 & 2.4 \\
\hline Inter-Mountain Basins Semi-Desert Shrub-Steppe & Artemisia tridentata & Shrub- Evergreen & 2.7 & 2.4 \\
\hline $\begin{array}{l}\text { Northern Rocky Mountain Ponderosa Pine } \\
\text { Woodland and Savanna }\end{array}$ & Artemisia tridentata & Shrub- Evergreen & 2.7 & 2.4 \\
\hline $\begin{array}{l}\text { Rocky Mountain Gambel Oak-Mixed Montane } \\
\text { Shrubland }\end{array}$ & Artemisia tridentata & Shrub- Evergreen & 2.7 & 2.4 \\
\hline $\begin{array}{l}\text { Southern Rocky Mountain Juniper Woodland and } \\
\text { Savanna }\end{array}$ & Artemisia tridentata & Shrub- Evergreen & 2.7 & 2.4 \\
\hline $\begin{array}{l}\text { Southern Rocky Mountain Ponderosa Pine } \\
\text { Woodland }\end{array}$ & Artemisia tridentata & Shrub- Evergreen & 2.7 & 2.4 \\
\hline Western Great Plains Badlands & Artemisia tridentata & Shrub- Evergreen & 2.7 & 2.4 \\
\hline $\begin{array}{l}\text { Inter-Mountain Basins Montane Sagebrush } \\
\text { Steppe }\end{array}$ & Artemisia tridentata & Shrub- Evergreen & 2.7 & 2.4 \\
\hline $\begin{array}{l}\text { Columbia Plateau Silver Sagebrush Seasonally } \\
\text { Flooded Shrub-Steppe }\end{array}$ & Artemisia tridentata & Shrub- Evergreen & 2.7 & 2.4 \\
\hline Inter-Mountain Basins Active and Stabilized Dune & Artemisia tridentata & Shrub- Evergreen & 2.7 & 2.4 \\
\hline Inter-Mountain Basins Big Sagebrush Shrubland & Artemisia tridentata & Shrub- Evergreen & 2.7 & 2.4 \\
\hline Inter-Mountain Basins Big Sagebrush Steppe & Artemisia tridentata & Shrub- Evergreen & 2.7 & 2.4 \\
\hline Inter-Mountain Basins Greasewood Flat & Artemisia tridentata & Shrub- Evergreen & 2.7 & 2.4 \\
\hline Inter-Mountain Basins Semi-Desert Grassland & Artemisia tridentata & Shrub- Evergreen & 2.7 & 2.4 \\
\hline Inter-Mountain Basins Wash & Artemisia tridentata & Shrub- Evergreen & 2.7 & 2.4 \\
\hline $\begin{array}{l}\text { Columbia Plateau Silver Sagebrush Seasonally } \\
\text { Flooded Shrub-Steppe }\end{array}$ & Artemisia tridentata & Shrub- Evergreen & 2.7 & 2.4 \\
\hline $\begin{array}{l}\text { Inter-Mountain Basins Curl-leaf Mountain- } \\
\text { mahogany Woodland and Shrubland }\end{array}$ & Artemisia tridentata & Shrub- Evergreen & 2.7 & 2.4 \\
\hline Inter-Mountain Basins Juniper Savanna & Artemisia tridentata & Shrub- Evergreen & 2.7 & 2.4 \\
\hline $\begin{array}{l}\text { Inter-Mountain Basins Montane Sagebrush } \\
\text { Steppe }\end{array}$ & Artemisia tridentata & Shrub- Evergreen & 2.7 & 2.4 \\
\hline $\begin{array}{l}\text { Inter-Mountain Basins Volcanic Rock and Cinder } \\
\text { Land }\end{array}$ & Artemisia tridentata & Shrub- Evergreen & 2.7 & 2.4 \\
\hline $\begin{array}{l}\text { Colorado Plateau Mixed Low Sagebrush } \\
\text { Shrubland }\end{array}$ & Artemisia tridentata & Shrub- Evergreen & 2.7 & 2.4 \\
\hline Colorado Plateau Pinyon-Juniper Shrubland & Artemisia tridentata & Shrub- Evergreen & 2.7 & 2.4 \\
\hline $\begin{array}{l}\text { Columbia Plateau Silver Sagebrush Seasonally } \\
\text { Flooded Shrub-Steppe }\end{array}$ & Artemisia tridentata & Shrub- Evergreen & 2.7 & 2.4 \\
\hline Great Basin Xeric Mixed Sagebrush Shrubland & Artemisia tridentata & Shrub- Evergreen & 2.7 & 2.4 \\
\hline Inter-Mountain Basins Big Sagebrush Shrubland & Artemisia tridentata & Shrub- Evergreen & 2.7 & 2.4 \\
\hline Inter-Mountain Basins Big Sagebrush Steppe & Artemisia tridentata & Shrub- Evergreen & 2.7 & 2.4 \\
\hline Inter-Mountain Basins Greasewood Flat & Artemisia tridentata & Shrub- Evergreen & 2.7 & 2.4 \\
\hline Inter-Mountain Basins Juniper Savanna & Artemisia tridentata & Shrub- Evergreen & 2.7 & 2.4 \\
\hline Inter-Mountain Basins Mixed Salt Desert Scrub & Artemisia tridentata & Shrub- Evergreen & 2.7 & 2.4 \\
\hline $\begin{array}{l}\text { Inter-Mountain Basins Montane Sagebrush } \\
\text { Steppe }\end{array}$ & Artemisia tridentata & Shrub- Evergreen & 2.7 & 2.4 \\
\hline
\end{tabular}




\begin{tabular}{|c|c|c|c|c|}
\hline Community & Species & Classification & Resistance & Resilience \\
\hline Inter-Mountain Basins Semi-Desert Grassland & Artemisia tridentata & Shrub- Evergreen & 2.7 & 2.4 \\
\hline $\begin{array}{l}\text { Wyoming Basins Dwarf Sagebrush Shrubland and } \\
\text { Steppe }\end{array}$ & Artemisia tridentata & Shrub- Evergreen & 2.7 & 2.4 \\
\hline Inter-Mountain Basins Big Sagebrush Steppe & Artemisia tridentata & Shrub- Evergreen & 2.7 & 2.4 \\
\hline $\begin{array}{l}\text { Wyoming Basins Dwarf Sagebrush Shrubland and } \\
\text { Steppe }\end{array}$ & Artemisia tripartita & Shrub- Evergreen & 2.7 & 2.4 \\
\hline Inter-Mountain Basins Big Sagebrush Steppe & Artemisia tripartita & Shrub- Evergreen & 2.7 & 2.4 \\
\hline $\begin{array}{l}\text { East Gulf Coastal Plain Southern Loblolly- } \\
\text { Hardwood Flatwoods }\end{array}$ & Arundinaria gigantea & Graminoid $C_{3}$ & 3.6 & 3.6 \\
\hline $\begin{array}{l}\text { Atlantic Coastal Plain Streamhead Seepage } \\
\text { Swamp, Pocosin and Baygall }\end{array}$ & Arundinaria gigantea & Graminoid $C_{3}$ & 3.6 & 3.6 \\
\hline Southeastern Great Plains Floodplain Forest & Arundo donax & Graminoid $\mathrm{C}_{3}$ & 3.6 & 3.6 \\
\hline Tamaulipan Floodplain & Arundo donax & Graminoid $\mathrm{C}_{3}$ & 3.6 & 3.6 \\
\hline Southern Interior Calcareous Cliff & Asplenium ruta-muraria & Graminoid $C_{3}$ & 3.6 & 3.6 \\
\hline $\begin{array}{l}\text { Mediterranean California Alpine Bedrock and } \\
\text { Scree }\end{array}$ & Astragalus austiniae & Forb & 3 & 3.5 \\
\hline $\begin{array}{l}\text { Mediterranean California Alpine Bedrock and } \\
\text { Scree }\end{array}$ & Astragalus bolanderi & Forb & 3 & 3.5 \\
\hline $\begin{array}{l}\text { Mediterranean California Alpine Bedrock and } \\
\text { Scree }\end{array}$ & Astragalus kentrophyta & Forb & 3 & 3.5 \\
\hline North Pacific Intertidal Freshwater Wetland & Athyrium filix-femina & Graminoid $C_{3}$ & 3.6 & 3.6 \\
\hline Chihuahuan Gypsophilous Grassland and Steppe & Atriplex canescens & Shrub- Evergreen & 2.7 & 2.4 \\
\hline Chihuahuan Mixed Salt Desert Scrub & Atriplex canescens & Shrub- Evergreen & 2.7 & 2.4 \\
\hline Inter-Mountain Basins Active and Stabilized Dune & Atriplex canescens & Shrub- Evergreen & 2.7 & 2.4 \\
\hline Inter-Mountain Basins Greasewood Flat & Atriplex canescens & Shrub- Evergreen & 2.7 & 2.4 \\
\hline Inter-Mountain Basins Mixed Salt Desert Scrub & Atriplex canescens & Shrub- Evergreen & 2.7 & 2.4 \\
\hline Inter-Mountain Basins Wash & Atriplex canescens & Shrub- Evergreen & 2.7 & 2.4 \\
\hline Mojave Mid-Elevation Mixed Desert Scrub & Atriplex canescens & Shrub- Evergreen & 2.7 & 2.4 \\
\hline $\begin{array}{l}\text { North American Warm Desert Active and } \\
\text { Stabilized Dune }\end{array}$ & Atriplex canescens & Shrub- Evergreen & 2.7 & 2.4 \\
\hline Sonora-Mojave Mixed Salt Desert Scrub & Atriplex canescens & Shrub- Evergreen & 2.7 & 2.4 \\
\hline $\begin{array}{l}\text { Western Great Plains Mesquite Woodland and } \\
\text { Shrubland }\end{array}$ & Atriplex canescens & Shrub- Evergreen & 2.7 & 2.4 \\
\hline Inter-Mountain Basins Greasewood Flat & Atriplex confertifolia & Shrub-Deciduous & 2.7 & 3 \\
\hline Inter-Mountain Basins Mixed Salt Desert Scrub & Atriplex confertifolia & Shrub- Deciduous & 2.7 & 3 \\
\hline Mojave Mid-Elevation Mixed Desert Scrub & Atriplex confertifolia & Shrub- Deciduous & 2.7 & 3 \\
\hline Sonora-Mojave Mixed Salt Desert Scrub & Atriplex confertifolia & Shrub- Deciduous & 2.7 & 3 \\
\hline Inter-Mountain Basins Mat Saltbush Shrubland & Atriplex corrugata & Shrub- Deciduous & 2.7 & 3 \\
\hline Inter-Mountain Basins Shale Badland & Atriplex corrugata & Shrub- Deciduous & 2.7 & 3 \\
\hline Inter-Mountain Basins Shale Badland & Atriplex cuneata & Shrub- Deciduous & 2.7 & 3 \\
\hline Inter-Mountain Basins Greasewood Flat & Atriplex gardneri & Shrub- Deciduous & 2.7 & 3 \\
\hline Inter-Mountain Basins Mat Saltbush Shrubland & Atriplex gardneri & Shrub- Deciduous & 2.7 & 3 \\
\hline Inter-Mountain Basins Shale Badland & Atriplex gardneri & Shrub-Deciduous & 2.7 & 3 \\
\hline Western Great Plains Badlands & Atriplex gardneri & Shrub- Deciduous & 2.7 & 3 \\
\hline North American Warm Desert Badland & Atriplex hymenelytra & Shrub- Evergreen & 2.7 & 2.4 \\
\hline North American Warm Desert Volcanic Rockland & Atriplex hymenelytra & Shrub- Evergreen & 2.7 & 2.4 \\
\hline Mediterranean California Southern Coastal Dune & Atriplex leucophylla & Forb & 3 & 3.5 \\
\hline Chihuahuan Mixed Salt Desert Scrub & Atriplex obovata & Shrub- Deciduous & 2.7 & 3 \\
\hline Inter-Mountain Basins Mixed Salt Desert Scrub & Atriplex obovata & Shrub- Deciduous & 2.7 & 3 \\
\hline Inter-Mountain Basins Shale Badland & Atriplex obovata & Shrub-Deciduous & 2.7 & 3 \\
\hline
\end{tabular}




\begin{tabular}{|c|c|c|c|c|}
\hline Community & Species & Classification & Resistance & Resilience \\
\hline Chihuahuan Mixed Salt Desert Scrub & Atriplex polycarpa & Shrub- Deciduous & 2.7 & 3 \\
\hline Inter-Mountain Basins Mixed Salt Desert Scrub & Atriplex polycarpa & Shrub- Deciduous & 2.7 & 3 \\
\hline Mojave Mid-Elevation Mixed Desert Scrub & Atriplex polycarpa & Shrub- Deciduous & 2.7 & 3 \\
\hline North American Warm Desert Playa & Atriplex polycarpa & Shrub- Deciduous & 2.7 & 3 \\
\hline Sonora-Mojave Mixed Salt Desert Scrub & Atriplex polycarpa & Shrub- Deciduous & 2.7 & 3 \\
\hline Inter-Mountain Basins Mixed Salt Desert Scrub & Atriplex spinifera & Shrub- Deciduous & 2.7 & 3 \\
\hline Inter-Mountain Basins Playa & Atriplex spinifera & Shrub- Deciduous & 2.7 & 3 \\
\hline South Florida Mangrove Swamp & Avicennia germinans & Shrub- Evergreen & 2.7 & 2.4 \\
\hline South-Central Saline Glade & Baccharis halimifolia & Shrub- Evergreen & 2.7 & 2.4 \\
\hline $\begin{array}{l}\text { Southern Atlantic Coastal Plain Salt and Brackish } \\
\text { Tidal Marsh }\end{array}$ & Baccharis halimifolia & Shrub- Evergreen & 2.7 & 2.4 \\
\hline Mediterranean California Coastal Bluff & Baccharis pilularis & Shrub- Evergreen & 2.7 & 2.4 \\
\hline Southern California Coastal Scrub & Baccharis pilularis & Shrub- Evergreen & 2.7 & 2.4 \\
\hline $\begin{array}{l}\text { North American Warm Desert Lower Montane } \\
\text { Riparian Woodland and Shrubland }\end{array}$ & Baccharis salicifolia & Shrub- Evergreen & 2.7 & 2.4 \\
\hline $\begin{array}{l}\text { North American Warm Desert Riparian Mesquite } \\
\text { Bosque }\end{array}$ & Baccharis salicifolia & Shrub- Evergreen & 2.7 & 2.4 \\
\hline North American Warm Desert Wash & Baccharis sarothroides & Shrub- Evergreen & 2.7 & 2.4 \\
\hline North American Warm Desert Wash & Baccharis sergiloides & Shrub- Deciduous & 2.7 & 3 \\
\hline South Florida Slough, Gator Hole and Willow Head & Bacopa caroliniana & Forb & 3 & 3.5 \\
\hline $\begin{array}{l}\text { Rocky Mountain Subalpine-Montane Mesic } \\
\text { Meadow }\end{array}$ & Balsamorhiza sagittata & Forb & 3 & 3.5 \\
\hline South Texas Salt and Brackish Tidal Flat & Batis maritima & Shrub & 2.7 & 2.8 \\
\hline Texas Coast Salt and Brackish Tidal Marsh & Batis maritima & Shrub & 2.7 & 2.8 \\
\hline Florida Dry Prairie & Befaria racemosa & Shrub- Evergreen & 2.7 & 2.4 \\
\hline North American Warm Desert Cienega & Berula erecta & Forb & 3 & 3.5 \\
\hline Acadian-Appalachian Montane Spruce-Fir Forest & Betula alleghaniensis & Tree- Deciduous & 3 & 4.2 \\
\hline Laurentian-Acadian Northern Hardwood Forest & Betula alleghaniensis & Tree- Deciduous & 3 & 4.2 \\
\hline Southern Appalachian Northern Hardwood Forest & Betula alleghaniensis & Tree- Deciduous & 3 & 4.2 \\
\hline Appalachian (Hemlock)-Northern Hardwood Forest & Betula alleghaniensis & Tree- Deciduous & 3 & 4.2 \\
\hline Mediterranean California Subalpine-Montane Fen & Betula glandulosa & Shrub- Deciduous & 2.7 & 3 \\
\hline Rocky Mountain Subalpine-Montane Fen & Betula glandulosa & Shrub- Deciduous & 2.7 & 3 \\
\hline $\begin{array}{l}\text { Rocky Mountain Subalpine-Montane Riparian } \\
\text { Shrubland }\end{array}$ & Betula glandulosa & Shrub- Deciduous & 2.7 & 3 \\
\hline North-Central Appalachian Acidic Cliff and Talus & Betula lenta & Tree- Deciduous & 3 & 4.2 \\
\hline Northeastern Interior Dry-Mesic Oak Forest & Betula lenta & Tree- Deciduous & 3 & 4.2 \\
\hline Southern and Central Appalachian Cove Forest & Betula lenta & Tree- Deciduous & 3 & 4.2 \\
\hline Appalachian (Hemlock)-Northern Hardwood Forest & Betula lenta & Tree- Deciduous & 3 & 4.2 \\
\hline $\begin{array}{l}\text { Atlantic Coastal Plain Small Blackwater River } \\
\text { Floodplain Forest }\end{array}$ & Betula nigra & Tree- Deciduous & 3 & 4.2 \\
\hline Central Appalachian Stream and Riparian & Betula nigra & Tree-Deciduous & 3 & 4.2 \\
\hline Ozark-Ouachita Riparian & Betula nigra & Tree- Deciduous & 3 & 4.2 \\
\hline Southern Piedmont Large Floodplain Forest & Betula nigra & Tree- Deciduous & 3 & 4.2 \\
\hline $\begin{array}{l}\text { West Gulf Coastal Plain Small Stream and River } \\
\text { Forest }\end{array}$ & Betula nigra & Tree- Deciduous & 3 & 4.2 \\
\hline $\begin{array}{l}\text { Rocky Mountain Subalpine-Montane Riparian } \\
\text { Shrubland }\end{array}$ & Betula occidentalis & Shrub- Deciduous & 2.7 & 3 \\
\hline $\begin{array}{l}\text { Great Basin Foothill and Lower Montane Riparian } \\
\text { Woodland and Shrubland }\end{array}$ & Betula occidentalis & Tree- Deciduous & 3 & 4.2 \\
\hline
\end{tabular}




\begin{tabular}{|c|c|c|c|c|}
\hline Community & Species & Classification & Resistance & Resilience \\
\hline $\begin{array}{l}\text { Rocky Mountain Lower Montane-Foothill Riparian } \\
\text { Woodland and Shrubland }\end{array}$ & Betula occidentalis & Tree- Deciduous & 3 & 4.2 \\
\hline North Pacific Hardwood-Conifer Swamp & Betula papyrifera & Shrub-Deciduous & 2.7 & 3 \\
\hline $\begin{array}{l}\text { Acadian Low-Elevation Spruce-Fir-Hardwood } \\
\text { Forest }\end{array}$ & Betula papyrifera & Tree- Deciduous & 3 & 4.2 \\
\hline Laurentian-Acadian Northern Hardwood Forest & Betula papyrifera & Tree-Deciduous & 3 & 4.2 \\
\hline Northern Rocky Mountain Wooded Vernal Pool & Betula papyrifera & Tree-Deciduous & 3 & 4.2 \\
\hline $\begin{array}{l}\text { Northwestern Great Plains-Black Hills Ponderosa } \\
\text { Pine Woodland and Savanna }\end{array}$ & Betula papyrifera & Tree- Deciduous & 3 & 4.2 \\
\hline Eastern Hemi-Boreal Aspen-Birch Forest & Betula papyrifera & Tree- Deciduous & 3 & 4.2 \\
\hline Laurentian-Acadian Northern Pine-(Oak) Forest & Betula papyrifera & Tree-Deciduous & 3 & 4.2 \\
\hline Acadian-Appalachian Montane Spruce-Fir Forest & Betula papyrifera & Tree- Deciduous & 3 & 4.2 \\
\hline $\begin{array}{l}\text { Acadian-Appalachian Subalpine Woodland and } \\
\text { Heath-Krummholz }\end{array}$ & Betula papyrifera & Tree-Deciduous & 3 & 4.2 \\
\hline Northeastern Interior Pine Barrens & Betula populifolia & Tree-Deciduous & 3 & 4.2 \\
\hline West Gulf Coastal Plain Catahoula Barrens & Bigelowia nuttallii & Forb & 3 & 3.5 \\
\hline Laurentian-Acadian Floodplain Forest & Boehmeria cylindrica & Forb & 3 & 3.5 \\
\hline $\begin{array}{l}\text { Southern Rocky Mountain Juniper Woodland and } \\
\text { Savanna }\end{array}$ & Boerhavia gracillima & Forb & 3 & 3.5 \\
\hline South Texas Salt and Brackish Tidal Flat & Borrichia frutescens & Shrub & 2.7 & 2.8 \\
\hline $\begin{array}{l}\text { Southern Atlantic Coastal Plain Salt and Brackish } \\
\text { Tidal Marsh }\end{array}$ & Borrichia frutescens & Shrub & 2.7 & 2.8 \\
\hline Texas Coast Salt and Brackish Tidal Marsh & Borrichia frutescens & Shrub & 2.7 & 2.8 \\
\hline $\begin{array}{l}\text { Atlantic Coastal Plain Indian River Lagoon Tidal } \\
\text { Marsh }\end{array}$ & Borrichia frutescens & Shrub- Evergreen & 2.7 & 2.4 \\
\hline Tamaulipan Savanna Grassland & Bothriochloa barbinodis & Graminoid $\mathrm{C}_{4}$ & 3.6 & 4.1 \\
\hline Edwards Plateau Floodplain Terrace & Bothriochloa ischaemum & Graminoid $\mathrm{C}_{4}$ & 3.6 & 4.1 \\
\hline $\begin{array}{l}\text { Edwards Plateau Limestone Savanna and } \\
\text { Woodland }\end{array}$ & Bothriochloa ischaemum & Graminoid $\mathrm{C}_{4}$ & 3.6 & 4.1 \\
\hline Edwards Plateau Riparian & Bothriochloa ischaemum & Graminoid $\mathrm{C}_{4}$ & 3.6 & 4.1 \\
\hline Tamaulipan Calcareous Thornscrub & Bothriochloa ischaemum & Graminoid $\mathrm{C}_{4}$ & 3.6 & 4.1 \\
\hline Tamaulipan Floodplain & Bothriochloa ischaemum & Graminoid $\mathrm{C}_{4}$ & 3.6 & 4.1 \\
\hline Llano Uplift Acidic Forest, Woodland and Glade & Bothriochloa laguroides & Graminoid $\mathrm{C}_{4}$ & 3.6 & 4.1 \\
\hline $\begin{array}{l}\text { Apacherian-Chihuahuan Semi-Desert Grassland } \\
\text { and Steppe }\end{array}$ & Bouteloua curtipendula & Graminoid $\mathrm{C}_{4}$ & 3.6 & 4.1 \\
\hline Central Mixedgrass Prairie & Bouteloua curtipendula & Graminoid $\mathrm{C}_{4}$ & 3.6 & 4.1 \\
\hline Inter-Mountain Basins Juniper Savanna & Bouteloua curtipendula & Graminoid $\mathrm{C}_{4}$ & 3.6 & 4.1 \\
\hline Inter-Mountain Basins Semi-Desert Grassland & Bouteloua curtipendula & Graminoid $\mathrm{C}_{4}$ & 3.6 & 4.1 \\
\hline $\begin{array}{l}\text { Llano Estacado Caprock Escarpment and Breaks } \\
\text { Shrubland and Steppe }\end{array}$ & Bouteloua curtipendula & Graminoid $\mathrm{C}_{4}$ & 3.6 & 4.1 \\
\hline Madrean Encinal & Bouteloua curtipendula & Graminoid $\mathrm{C}_{4}$ & 3.6 & 4.1 \\
\hline Madrean Juniper Savanna & Bouteloua curtipendula & Graminoid $\mathrm{C}_{4}$ & 3.6 & 4.1 \\
\hline Madrean Oriental Chaparral & Bouteloua curtipendula & Graminoid $\mathrm{C}_{4}$ & 3.6 & 4.1 \\
\hline Paleozoic Plateau Bluff and Talus & Bouteloua curtipendula & Graminoid $\mathrm{C}_{4}$ & 3.6 & 4.1 \\
\hline Southwestern Great Plains Canyon & Bouteloua curtipendula & Graminoid $\mathrm{C}_{4}$ & 3.6 & 4.1 \\
\hline Western Great Plains Cliff and Outcrop & Bouteloua curtipendula & Graminoid $\mathrm{C}_{4}$ & 3.6 & 4.1 \\
\hline Central Tallgrass Prairie & Bouteloua curtipendula & Graminoid $\mathrm{C}_{4}$ & 3.6 & 4.1 \\
\hline Chihuahuan Gypsophilous Grassland and Steppe & Bouteloua eriopoda & Graminoid $\mathrm{C}_{4}$ & 3.6 & 4.1 \\
\hline Chihuahuan Loamy Plains Desert Grassland & Bouteloua eriopoda & Graminoid $\mathrm{C}_{4}$ & 3.6 & 4.1 \\
\hline Chihuahuan Sandy Plains Semi-Desert Grassland & Bouteloua eriopoda & Graminoid $\mathrm{C}_{4}$ & 3.6 & 4.1 \\
\hline
\end{tabular}




\begin{tabular}{|c|c|c|c|c|}
\hline Community & Species & Classification & Resistance & Resilience \\
\hline $\begin{array}{l}\text { Chihuahuan Stabilized Coppice Dune and Sand } \\
\text { Flat Scrub }\end{array}$ & Bouteloua eriopoda & Graminoid $\mathrm{C}_{4}$ & 3.6 & 4.1 \\
\hline Madrean Juniper Savanna & Bouteloua eriopoda & Graminoid $\mathrm{C}_{4}$ & 3.6 & 4.1 \\
\hline $\begin{array}{l}\text { Southern Rocky Mountain Juniper Woodland and } \\
\text { Savanna }\end{array}$ & Bouteloua eriopoda & Graminoid $\mathrm{C}_{4}$ & 3.6 & 4.1 \\
\hline $\begin{array}{l}\text { Apacherian-Chihuahuan Semi-Desert Grassland } \\
\text { and Steppe }\end{array}$ & Bouteloua gracilis & Graminoid $\mathrm{C}_{4}$ & 3.6 & 4.1 \\
\hline Central Mixedgrass Prairie & Bouteloua gracilis & Graminoid $\mathrm{C}_{4}$ & 3.6 & 4.1 \\
\hline Chihuahuan Loamy Plains Desert Grassland & Bouteloua gracilis & Graminoid $\mathrm{C}_{4}$ & 3.6 & 4.1 \\
\hline Chihuahuan Sandy Plains Semi-Desert Grassland & Bouteloua gracilis & Graminoid $\mathrm{C}_{4}$ & 3.6 & 4.1 \\
\hline $\begin{array}{l}\text { Colorado Plateau Mixed Low Sagebrush } \\
\text { Shrubland }\end{array}$ & Bouteloua gracilis & Graminoid $\mathrm{C}_{4}$ & 3.6 & 4.1 \\
\hline Colorado Plateau Pinyon-Juniper Woodland & Bouteloua gracilis & Graminoid $\mathrm{C}_{4}$ & 3.6 & 4.1 \\
\hline Inter-Mountain Basins Big Sagebrush Shrubland & Bouteloua gracilis & Graminoid $\mathrm{C}_{4}$ & 3.6 & 4.1 \\
\hline Inter-Mountain Basins Juniper Savanna & Bouteloua gracilis & Graminoid $\mathrm{C}_{4}$ & 3.6 & 4.1 \\
\hline Inter-Mountain Basins Mixed Salt Desert Scrub & Bouteloua gracilis & Graminoid $\mathrm{C}_{4}$ & 3.6 & 4.1 \\
\hline Inter-Mountain Basins Semi-Desert Grassland & Bouteloua gracilis & Graminoid $\mathrm{C}_{4}$ & 3.6 & 4.1 \\
\hline Inter-Mountain Basins Semi-Desert Shrub-Steppe & Bouteloua gracilis & Graminoid $\mathrm{C}_{4}$ & 3.6 & 4.1 \\
\hline $\begin{array}{l}\text { Llano Estacado Caprock Escarpment and Breaks } \\
\text { Shrubland and Steppe }\end{array}$ & Bouteloua gracilis & Graminoid $\mathrm{C}_{4}$ & 3.6 & 4.1 \\
\hline Madrean Encinal & Bouteloua gracilis & Graminoid $\mathrm{C}_{4}$ & 3.6 & 4.1 \\
\hline Madrean Juniper Savanna & Bouteloua gracilis & Graminoid $\mathrm{C}_{4}$ & 3.6 & 4.1 \\
\hline $\begin{array}{l}\text { North-Central Interior Sand and Gravel Tallgrass } \\
\text { Prairie }\end{array}$ & Bouteloua gracilis & Graminoid $\mathrm{C}_{4}$ & 3.6 & 4.1 \\
\hline Northwestern Great Plains Mixedgrass Prairie & Bouteloua gracilis & Graminoid $\mathrm{C}_{4}$ & 3.6 & 4.1 \\
\hline $\begin{array}{l}\text { Rocky Mountain Foothill Limber Pine-Juniper } \\
\text { Woodland }\end{array}$ & Bouteloua gracilis & Graminoid $C_{4}$ & 3.6 & 4.1 \\
\hline $\begin{array}{l}\text { Rocky Mountain Lower Montane-Foothill } \\
\text { Shrubland }\end{array}$ & Bouteloua gracilis & Graminoid $\mathrm{C}_{4}$ & 3.6 & 4.1 \\
\hline Southern Colorado Plateau Sand Shrubland & Bouteloua gracilis & Graminoid $\mathrm{C}_{4}$ & 3.6 & 4.1 \\
\hline $\begin{array}{l}\text { Southern Rocky Mountain Juniper Woodland and } \\
\text { Savanna }\end{array}$ & Bouteloua gracilis & Graminoid $\mathrm{C}_{4}$ & 3.6 & 4.1 \\
\hline $\begin{array}{l}\text { Southern Rocky Mountain Montane-Subalpine } \\
\text { Grassland }\end{array}$ & Bouteloua gracilis & Graminoid $\mathrm{C}_{4}$ & 3.6 & 4.1 \\
\hline $\begin{array}{l}\text { Southern Rocky Mountain Pinyon-Juniper } \\
\text { Woodland }\end{array}$ & Bouteloua gracilis & Graminoid $C_{4}$ & 3.6 & 4.1 \\
\hline $\begin{array}{l}\text { Southern Rocky Mountain Ponderosa Pine } \\
\text { Savanna }\end{array}$ & Bouteloua gracilis & Graminoid $\mathrm{C}_{4}$ & 3.6 & 4.1 \\
\hline $\begin{array}{l}\text { Southern Rocky Mountain Ponderosa Pine } \\
\text { Woodland }\end{array}$ & Bouteloua gracilis & Graminoid $C_{4}$ & 3.6 & 4.1 \\
\hline Western Great Plains Cliff and Outcrop & Bouteloua gracilis & Graminoid $\mathrm{C}_{4}$ & 3.6 & 4.1 \\
\hline $\begin{array}{l}\text { Western Great Plains Foothill and Piedmont } \\
\text { Grassland }\end{array}$ & Bouteloua gracilis & Graminoid $\mathrm{C}_{4}$ & 3.6 & 4.1 \\
\hline $\begin{array}{l}\text { Western Great Plains Mesquite Woodland and } \\
\text { Shrubland }\end{array}$ & Bouteloua gracilis & Graminoid $\mathrm{C}_{4}$ & 3.6 & 4.1 \\
\hline Western Great Plains Sandhill Steppe & Bouteloua gracilis & Graminoid $\mathrm{C}_{4}$ & 3.6 & 4.1 \\
\hline Western Great Plains Shortgrass Prairie & Bouteloua gracilis & Graminoid $\mathrm{C}_{4}$ & 3.6 & 4.1 \\
\hline $\begin{array}{l}\text { Wyoming Basins Dwarf Sagebrush Shrubland and } \\
\text { Steppe }\end{array}$ & Bouteloua gracilis & Graminoid $\mathrm{C}_{4}$ & 3.6 & 4.1 \\
\hline Chihuahuan Sandy Plains Semi-Desert Grassland & Bouteloua hirsuta & Graminoid $\mathrm{C}_{4}$ & 3.6 & 4.1 \\
\hline $\begin{array}{l}\text { Llano Estacado Caprock Escarpment and Breaks } \\
\text { Shrubland and Steppe }\end{array}$ & Bouteloua hirsuta & Graminoid $\mathrm{C}_{4}$ & 3.6 & 4.1 \\
\hline
\end{tabular}




\begin{tabular}{|c|c|c|c|c|}
\hline Community & Species & Classification & Resistance & Resilience \\
\hline Madrean Juniper Savanna & Bouteloua hirsuta & Graminoid $\mathrm{C}_{4}$ & 3.6 & 4.1 \\
\hline Madrean Oriental Chaparral & Bouteloua hirsuta & Graminoid $\mathrm{C}_{4}$ & 3.6 & 4.1 \\
\hline $\begin{array}{l}\text { Southern Rocky Mountain Juniper Woodland and } \\
\text { Savanna }\end{array}$ & Bouteloua hirsuta & Graminoid $\mathrm{C}_{4}$ & 3.6 & 4.1 \\
\hline $\begin{array}{l}\text { Apacherian-Chihuahuan Semi-Desert Grassland } \\
\text { and Steppe }\end{array}$ & Bouteloua ramosa & Graminoid $\mathrm{C}_{4}$ & 3.6 & 4.1 \\
\hline $\begin{array}{l}\text { Apacherian-Chihuahuan Semi-Desert Grassland } \\
\text { and Steppe }\end{array}$ & Bouteloua rothrockii & Graminoid $\mathrm{C}_{4}$ & 3.6 & 4.1 \\
\hline Madrean Encinal & Bouteloua rothrockii & Graminoid $\mathrm{C}_{4}$ & 3.6 & 4.1 \\
\hline Edwards Plateau Riparian & Brickellia laciniata & Shrub- Deciduous & 2.7 & 3 \\
\hline North American Warm Desert Wash & Brickellia laciniata & Shrub- Deciduous & 2.7 & 3 \\
\hline $\begin{array}{l}\text { Inter-Mountain Basins Aspen-Mixed Conifer Forest } \\
\text { and Woodland }\end{array}$ & Bromus carinatus & Graminoid $C_{3}$ & 3.6 & 3.6 \\
\hline Tamaulipan Floodplain & Bromus catharticus & Graminoid $C_{3}$ & 3.6 & 3.6 \\
\hline California Central Valley Mixed Oak Savanna & Bromus diandrus & Graminoid $\mathrm{C}_{3}$ & 3.6 & 3.6 \\
\hline California Central Valley Mixed Oak Savanna & Bromus hordeaceus & Graminoid $C_{3}$ & 3.6 & 3.6 \\
\hline Northwestern Great Plains Floodplain & Bromus inermis & Graminoid $\mathrm{C}_{3}$ & 3.6 & 3.6 \\
\hline $\begin{array}{l}\text { Northern Rocky Mountain Subalpine-Upper } \\
\text { Montane Grassland }\end{array}$ & Bromus inermis & Graminoid $C_{3}$ & 3.6 & 3.6 \\
\hline Sonora-Mojave Mixed Salt Desert Scrub & Bromus rubens & Graminoid $C_{3}$ & 3.6 & 3.6 \\
\hline $\begin{array}{l}\text { Columbia Basin Foothill and Canyon Dry } \\
\text { Grassland }\end{array}$ & Bromus tectorum & Graminoid $C_{3}$ & 3.6 & 3.6 \\
\hline Columbia Basin Palouse Prairie & Bromus tectorum & Graminoid $C_{3}$ & 3.6 & 3.6 \\
\hline Columbia Plateau Scabland Shrubland & Bromus tectorum & Graminoid $C_{3}$ & 3.6 & 3.6 \\
\hline Columbia Plateau Steppe and Grassland & Bromus tectorum & Graminoid $C_{3}$ & 3.6 & 3.6 \\
\hline Edwards Plateau Upland Depression & Buchloë dactyloides & Graminoid $\mathrm{C}_{4}$ & 3.6 & 4.1 \\
\hline Western Great Plains Closed Depression Wetland & Buchloë dactyloides & Graminoid $\mathrm{C}_{4}$ & 3.6 & 4.1 \\
\hline Western Great Plains Shortgrass Prairie & Buchloë dactyloides & Graminoid $\mathrm{C}_{4}$ & 3.6 & 4.1 \\
\hline $\begin{array}{l}\text { Western Great Plains Depressional Wetland } \\
\text { Systems }\end{array}$ & Buchloë dactyloides & Graminoid $\mathrm{C}_{4}$ & 3.6 & 4.1 \\
\hline Edwards Plateau Cliff & Buddleja racemosa & Shrub- Evergreen & 2.7 & 2.4 \\
\hline South Florida Hardwood Hammock & Bursera simaruba & Tree-Deciduous & 3 & 4.2 \\
\hline Northern Atlantic Coastal Plain Sandy Beach & Cakile edentula & Forb & 3 & 3.5 \\
\hline Southern Atlantic Coastal Plain Florida Beach & Cakile edentula & Forb & 3 & 3.5 \\
\hline Texas Coast Beach & Cakile geniculata & Forb & 3 & 3.5 \\
\hline Eastern Boreal Floodplain & Calamagrostis canadensis & Graminoid $C_{3}$ & 3.6 & 3.6 \\
\hline Eastern Great Plains Tallgrass Aspen Parkland & Calamagrostis canadensis & Graminoid $\mathrm{C}_{3}$ & 3.6 & 3.6 \\
\hline Great Lakes Wet-Mesic Lakeplain Prairie & Calamagrostis canadensis & Graminoid $\mathrm{C}_{3}$ & 3.6 & 3.6 \\
\hline Laurentian-Acadian Wet Meadow-Shrub Swamp & Calamagrostis canadensis & Graminoid $\mathrm{C}_{3}$ & 3.6 & 3.6 \\
\hline North-Central Interior Oak Savanna & Calamagrostis canadensis & Graminoid $C_{3}$ & 3.6 & 3.6 \\
\hline North-Central Interior Wet Meadow-Shrub Swamp & Calamagrostis canadensis & Graminoid $C_{3}$ & 3.6 & 3.6 \\
\hline Northern Great Lakes Coastal Marsh & Calamagrostis canadensis & Graminoid $C_{3}$ & 3.6 & 3.6 \\
\hline $\begin{array}{l}\text { Temperate Pacific Subalpine-Montane Wet } \\
\text { Meadow }\end{array}$ & Calamagrostis canadensis & Graminoid $C_{3}$ & 3.6 & 3.6 \\
\hline $\begin{array}{l}\text { Eastern Great Plains Wet Meadow, Prairie and } \\
\text { Marsh }\end{array}$ & Calamagrostis canadensis & Graminoid $C_{3}$ & 3.6 & 3.6 \\
\hline $\begin{array}{l}\text { North Pacific Hypermaritime Shrub and } \\
\text { Herbaceous Headland }\end{array}$ & Calamagrostis nutkaensis & Graminoid $C_{3}$ & 3.6 & 3.6 \\
\hline $\begin{array}{l}\text { Acadian-Appalachian Subalpine Woodland and } \\
\text { Heath-Krummholz }\end{array}$ & Calamagrostis pickeringii & Graminoid $C_{3}$ & 3.6 & 3.6 \\
\hline
\end{tabular}




\begin{tabular}{|c|c|c|c|c|}
\hline Community & Species & Classification & Resistance & Resilience \\
\hline $\begin{array}{l}\text { East Cascades Oak-Ponderosa Pine Forest and } \\
\text { Woodland }\end{array}$ & Calamagrostis rubescens & Graminoid $C_{3}$ & 3.6 & 3.6 \\
\hline $\begin{array}{l}\text { Inter-Mountain Basins Aspen-Mixed Conifer Forest } \\
\text { and Woodland }\end{array}$ & Calamagrostis rubescens & Graminoid $C_{3}$ & 3.6 & 3.6 \\
\hline $\begin{array}{l}\text { Middle Rocky Mountain Montane Douglas-fir } \\
\text { Forest and Woodland }\end{array}$ & Calamagrostis rubescens & Graminoid $C_{3}$ & 3.6 & 3.6 \\
\hline $\begin{array}{l}\text { Northern Rocky Mountain Dry-Mesic Montane } \\
\text { Mixed Conifer Forest }\end{array}$ & Calamagrostis rubescens & Graminoid $C_{3}$ & 3.6 & 3.6 \\
\hline $\begin{array}{l}\text { Northern Rocky Mountain Foothill Conifer Wooded } \\
\text { Steppe }\end{array}$ & Calamagrostis rubescens & Graminoid $C_{3}$ & 3.6 & 3.6 \\
\hline $\begin{array}{l}\text { Northern Rocky Mountain Ponderosa Pine } \\
\text { Woodland and Savanna }\end{array}$ & Calamagrostis rubescens & Graminoid $C_{3}$ & 3.6 & 3.6 \\
\hline Northern Rocky Mountain Western Larch Savanna & Calamagrostis rubescens & Graminoid $C_{3}$ & 3.6 & 3.6 \\
\hline $\begin{array}{l}\text { Northwestern Great Plains Aspen Forest and } \\
\text { Parkland }\end{array}$ & Calamagrostis rubescens & Graminoid $C_{3}$ & 3.6 & 3.6 \\
\hline Rocky Mountain Poor-Site Lodgepole Pine Forest & Calamagrostis rubescens & Graminoid $C_{3}$ & 3.6 & 3.6 \\
\hline Rocky Mountain Alpine-Montane Wet Meadow & Calamagrostis stricta & Graminoid $C_{3}$ & 3.6 & 3.6 \\
\hline Northern Atlantic Coastal Plain Pitch Pine Lowland & Calamovilfa brevipilis & Graminoid $\mathrm{C}_{4}$ & 3.6 & 4.1 \\
\hline Western Great Plains Cliff and Outcrop & Calamovilfa longifolia & Graminoid $\mathrm{C}_{4}$ & 3.6 & 4.1 \\
\hline Western Great Plains Sand Prairie & Calamovilfa longifolia & Graminoid $\mathrm{C}_{4}$ & 3.6 & 4.1 \\
\hline Western Great Plains Sandhill Steppe & Calamovilfa longifolia & Graminoid $\mathrm{C}_{4}$ & 3.6 & 4.1 \\
\hline Sonoran Paloverde-Mixed Cacti Desert Scrub & Calliandra eriophylla & Shrub- Evergreen & 2.7 & 2.4 \\
\hline $\begin{array}{l}\text { Klamath-Siskiyou Lower Montane Serpentine } \\
\text { Mixed Conifer Woodland }\end{array}$ & Calocedrus decurrens & Tree-Evergreen & 3.2 & 2.2 \\
\hline $\begin{array}{l}\text { Mediterranean California Dry-Mesic Mixed Conifer } \\
\text { Forest and Woodland }\end{array}$ & Calocedrus decurrens & Tree-Evergreen & 3.2 & 2.2 \\
\hline $\begin{array}{l}\text { Mediterranean California Mesic Mixed Conifer } \\
\text { Forest and Woodland }\end{array}$ & Calocedrus decurrens & Tree-Evergreen & 3.2 & 2.2 \\
\hline Rocky Mountain Alpine-Montane Wet Meadow & Caltha leptosepala & Forb & 3 & 3.5 \\
\hline $\begin{array}{l}\text { Temperate Pacific Subalpine-Montane Wet } \\
\text { Meadow }\end{array}$ & Caltha leptosepala & Forb & 3 & 3.5 \\
\hline North Pacific Herbaceous Bald and Bluff & Camassia leichtlinii & Forb & 3 & 3.5 \\
\hline North Pacific Herbaceous Bald and Bluff & Camassia quamash & Forb & 3 & 3.5 \\
\hline $\begin{array}{l}\text { Temperate Pacific Subalpine-Montane Wet } \\
\text { Meadow }\end{array}$ & Camassia quamash & Forb & 3 & 3.5 \\
\hline Willamette Valley Wet Prairie & Camassia quamash & Forb & 3 & 3.5 \\
\hline Great Lakes Acidic Rocky Shore and Cliff & Campanula rotundifolia & Forb & 3 & 3.5 \\
\hline Mediterranean California Serpentine Fen & Campanula wilkinsiana & Forb & 3 & 3.5 \\
\hline Southern Coastal Plain Oak Dome and Hammock & Campsis radicans & Forb & 3 & 3.5 \\
\hline Rocky Mountain Alpine-Montane Wet Meadow & Cardamine cordifolia & Forb & 3 & 3.5 \\
\hline Great Lakes Wet-Mesic Lakeplain Prairie & Carex aquatilis & Graminoid $C_{3}$ & 3.6 & 3.6 \\
\hline Great Lakes Wooded Dune and Swale & Carex aquatilis & Graminoid $C_{3}$ & 3.6 & 3.6 \\
\hline Rocky Mountain Subalpine-Montane Fen & Carex aquatilis & Graminoid $C_{3}$ & 3.6 & 3.6 \\
\hline North Pacific Shrub Swamp & Carex aquatilis & Graminoid $C_{3}$ & 3.6 & 3.6 \\
\hline Southern and Central Appalachian Bog and Fen & Carex atlantica & Graminoid $C_{3}$ & 3.6 & 3.6 \\
\hline Great Lakes Wet-Mesic Lakeplain Prairie & Carex bicknellii & Graminoid $\mathrm{C}_{3}$ & 3.6 & 3.6 \\
\hline Acadian-Appalachian Alpine Tundra & Carex bigelowii & Graminoid $\mathrm{C}_{3}$ & 3.6 & 3.6 \\
\hline $\begin{array}{l}\text { Temperate Pacific Subalpine-Montane Wet } \\
\text { Meadow }\end{array}$ & Carex bolanderi & Graminoid $\mathrm{C}_{3}$ & 3.6 & 3.6 \\
\hline Great Lakes Wet-Mesic Lakeplain Prairie & Carex buxbaumii & Graminoid $\mathrm{C}_{3}$ & 3.6 & 3.6 \\
\hline Mediterranean California Subalpine-Montane Fen & Carex buxbaumii & Graminoid $C_{3}$ & 3.6 & 3.6 \\
\hline
\end{tabular}




\begin{tabular}{|c|c|c|c|c|}
\hline Community & Species & Classification & Resistance & Resilience \\
\hline Rocky Mountain Subalpine-Montane Fen & Carex buxbaumii & Graminoid $C_{3}$ & 3.6 & 3.6 \\
\hline Mediterranean California Serpentine Fen & Carex californica & Graminoid $\mathrm{C}_{3}$ & 3.6 & 3.6 \\
\hline $\begin{array}{l}\text { Southern Coastal Plain Blackland Prairie and } \\
\text { Woodland }\end{array}$ & Carex cherokeensis & Graminoid $C_{3}$ & 3.6 & 3.6 \\
\hline Southern Coastal Plain Limestone Forest & Carex cherokeensis & Graminoid $\mathrm{C}_{3}$ & 3.6 & 3.6 \\
\hline $\begin{array}{l}\text { West Gulf Coastal Plain Northern Calcareous } \\
\text { Prairie }\end{array}$ & Carex cherokeensis & Graminoid $C_{3}$ & 3.6 & 3.6 \\
\hline $\begin{array}{l}\text { West Gulf Coastal Plain Southern Calcareous } \\
\text { Prairie }\end{array}$ & Carex cherokeensis & Graminoid $C_{3}$ & 3.6 & 3.6 \\
\hline Ozark-Ouachita Riparian & Carex crinita & Graminoid $C_{3}$ & 3.6 & 3.6 \\
\hline Willamette Valley Wet Prairie & Carex densa & Graminoid $C_{3}$ & 3.6 & 3.6 \\
\hline Columbia Plateau Vernal Pool & Carex douglasii & Graminoid $\mathrm{C}_{3}$ & 3.6 & 3.6 \\
\hline Mediterranean California Subalpine-Montane Fen & Carex echinata & Graminoid $\mathrm{C}_{3}$ & 3.6 & 3.6 \\
\hline Rocky Mountain Alpine Turf & Carex elynoides & Graminoid $C_{3}$ & 3.6 & 3.6 \\
\hline Mediterranean California Subalpine Meadow & Carex exserta & Graminoid $\mathrm{C}_{3}$ & 3.6 & 3.6 \\
\hline $\begin{array}{l}\text { Temperate Pacific Subalpine-Montane Wet } \\
\text { Meadow }\end{array}$ & Carex exsiccata & Graminoid $\mathrm{C}_{3}$ & 3.6 & 3.6 \\
\hline $\begin{array}{l}\text { Northern Rocky Mountain Lower Montane, Foothill } \\
\text { and Valley Grassland }\end{array}$ & Carex filifolia & Graminoid $\mathrm{C}_{3}$ & 3.6 & 3.6 \\
\hline $\begin{array}{l}\text { Eastern Great Plains Wet Meadow, Prairie and } \\
\text { Marsh }\end{array}$ & Carex frankii & Graminoid $\mathrm{C}_{3}$ & 3.6 & 3.6 \\
\hline $\begin{array}{l}\text { East Cascades Oak-Ponderosa Pine Forest and } \\
\text { Woodland }\end{array}$ & Carex geyeri & Graminoid $C_{3}$ & 3.6 & 3.6 \\
\hline $\begin{array}{l}\text { Inter-Mountain Basins Aspen-Mixed Conifer Forest } \\
\text { and Woodland }\end{array}$ & Carex geyeri & Graminoid $\mathrm{C}_{3}$ & 3.6 & 3.6 \\
\hline $\begin{array}{l}\text { Northern Rocky Mountain Dry-Mesic Montane } \\
\text { Mixed Conifer Forest }\end{array}$ & Carex geyeri & Graminoid $C_{3}$ & 3.6 & 3.6 \\
\hline $\begin{array}{l}\text { Northern Rocky Mountain Subalpine-Upper } \\
\text { Montane Grassland }\end{array}$ & Carex geyeri & Graminoid $\mathrm{C}_{3}$ & 3.6 & 3.6 \\
\hline Southern Coastal Plain Nonriverine Basin Swamp & Carex glaucescens & Graminoid $C_{3}$ & 3.6 & 3.6 \\
\hline $\begin{array}{l}\text { Northern Rocky Mountain Subalpine-Upper } \\
\text { Montane Grassland }\end{array}$ & Carex hoodii & Graminoid $\mathrm{C}_{3}$ & 3.6 & 3.6 \\
\hline $\begin{array}{l}\text { Eastern Great Plains Wet Meadow, Prairie and } \\
\text { Marsh }\end{array}$ & Carex hyalinolepis & Graminoid $C_{3}$ & 3.6 & 3.6 \\
\hline Rocky Mountain Alpine-Montane Wet Meadow & Carex illota & Graminoid $\mathrm{C}_{3}$ & 3.6 & 3.6 \\
\hline $\begin{array}{l}\text { East Cascades Oak-Ponderosa Pine Forest and } \\
\text { Woodland }\end{array}$ & Carex inops & Graminoid $\mathrm{C}_{3}$ & 3.6 & 3.6 \\
\hline Central Interior Acidic Cliff and Talus & Carex interior & Graminoid $\mathrm{C}_{3}$ & 3.6 & 3.6 \\
\hline Ozark-Ouachita Riparian & Carex interior & Graminoid $\mathrm{C}_{3}$ & 3.6 & 3.6 \\
\hline North-Central Interior Wet Meadow-Shrub Swamp & Carex lacustris & Graminoid $C_{3}$ & 3.6 & 3.6 \\
\hline Northern Great Lakes Coastal Marsh & Carex lacustris & Graminoid $\mathrm{C}_{3}$ & 3.6 & 3.6 \\
\hline $\begin{array}{l}\text { Boreal-Laurentian Conifer Acidic Swamp and } \\
\text { Treed Poor Fen }\end{array}$ & Carex lasiocarpa & Graminoid $\mathrm{C}_{3}$ & 3.6 & 3.6 \\
\hline Great Plains Prairie Pothole & Carex lasiocarpa & Graminoid $\mathrm{C}_{3}$ & 3.6 & 3.6 \\
\hline Rocky Mountain Subalpine-Montane Fen & Carex lasiocarpa & Graminoid $C_{3}$ & 3.6 & 3.6 \\
\hline Mediterranean California Subalpine-Montane Fen & Carex lenticularis & Graminoid $\mathrm{C}_{3}$ & 3.6 & 3.6 \\
\hline Central Interior Acidic Cliff and Talus & Carex leptalea & Graminoid $\mathrm{C}_{3}$ & 3.6 & 3.6 \\
\hline Mediterranean California Subalpine-Montane Fen & Carex limosa & Graminoid $C_{3}$ & 3.6 & 3.6 \\
\hline Rocky Mountain Subalpine-Montane Fen & Carex limosa & Graminoid $C_{3}$ & 3.6 & 3.6 \\
\hline Central Interior Acidic Cliff and Talus & Carex lurida & Graminoid $\mathrm{C}_{3}$ & 3.6 & 3.6 \\
\hline North Pacific Intertidal Freshwater Wetland & Carex lyngbyei & Graminoid $\mathrm{C}_{3}$ & 3.6 & 3.6 \\
\hline
\end{tabular}




\begin{tabular}{|c|c|c|c|c|}
\hline Community & Species & Classification & Resistance & Resilience \\
\hline Temperate Pacific Tidal Salt and Brackish Marsh & Carex lyngbyei & Graminoid $\mathrm{C}_{3}$ & 3.6 & 3.6 \\
\hline $\begin{array}{l}\text { North Pacific Alpine and Subalpine Bedrock and } \\
\text { Scree }\end{array}$ & Carex microchaeta & Graminoid $\mathrm{C}_{3}$ & 3.6 & 3.6 \\
\hline Rocky Mountain Alpine-Montane Wet Meadow & Carex microptera & Graminoid $\mathrm{C}_{3}$ & 3.6 & 3.6 \\
\hline Southern Appalachian Rocky Summit & Carex misera & Graminoid $\mathrm{C}_{3}$ & 3.6 & 3.6 \\
\hline Rocky Mountain Alpine Turf & Carex nardina & Graminoid $\mathrm{C}_{3}$ & 3.6 & 3.6 \\
\hline Rocky Mountain Alpine-Montane Wet Meadow & Carex nigricans & Graminoid $\mathrm{C}_{3}$ & 3.6 & 3.6 \\
\hline $\begin{array}{l}\text { Temperate Pacific Subalpine-Montane Wet } \\
\text { Meadow }\end{array}$ & Carex nigricans & Graminoid $\mathrm{C}_{3}$ & 3.6 & 3.6 \\
\hline $\begin{array}{l}\text { North Pacific Dry and Mesic Alpine Dwarf- } \\
\text { Shrubland, Fell-Field and Meadow }\end{array}$ & Carex nigricans & Graminoid $\mathrm{C}_{3}$ & 3.6 & 3.6 \\
\hline North Pacific Intertidal Freshwater Wetland & Carex obnupta & Graminoid $\mathrm{C}_{3}$ & 3.6 & 3.6 \\
\hline $\begin{array}{l}\text { Northern Rocky Mountain Subalpine-Upper } \\
\text { Montane Grassland }\end{array}$ & Carex obtusata & Graminoid $\mathrm{C}_{3}$ & 3.6 & 3.6 \\
\hline Great Plains Prairie Pothole & Carex oligosperma & Graminoid $\mathrm{C}_{3}$ & 3.6 & 3.6 \\
\hline Great Lakes Wet-Mesic Lakeplain Prairie & Carex pellita & Graminoid $\mathrm{C}_{3}$ & 3.6 & 3.6 \\
\hline Mediterranean California Subalpine-Montane Fen & Carex pellita & Graminoid $\mathrm{C}_{3}$ & 3.6 & 3.6 \\
\hline $\begin{array}{l}\text { Eastern Great Plains Wet Meadow, Prairie and } \\
\text { Marsh }\end{array}$ & Carex pellita & Graminoid $\mathrm{C}_{3}$ & 3.6 & 3.6 \\
\hline $\begin{array}{l}\text { Central and Southern Appalachian Montane Oak } \\
\text { Forest }\end{array}$ & Carex pensylvanica & Graminoid $\mathrm{C}_{3}$ & 3.6 & 3.6 \\
\hline North American Warm Desert Cienega & Carex praegracilis & Graminoid $\mathrm{C}_{3}$ & 3.6 & 3.6 \\
\hline $\begin{array}{l}\text { East Cascades Oak-Ponderosa Pine Forest and } \\
\text { Woodland }\end{array}$ & Carex rossii & Graminoid $\mathrm{C}_{3}$ & 3.6 & 3.6 \\
\hline North Pacific Coastal Cliff and Bluff & Carex rossii & Graminoid $\mathrm{C}_{3}$ & 3.6 & 3.6 \\
\hline $\begin{array}{l}\text { Northern Rocky Mountain Dry-Mesic Montane } \\
\text { Mixed Conifer Forest }\end{array}$ & Carex rossii & Graminoid $\mathrm{C}_{3}$ & 3.6 & 3.6 \\
\hline Temperate Pacific Freshwater Emergent Marsh & Carex rostrata & Graminoid $\mathrm{C}_{3}$ & 3.6 & 3.6 \\
\hline Rocky Mountain Alpine Turf & Carex rupestris & Graminoid $\mathrm{C}_{3}$ & 3.6 & 3.6 \\
\hline $\begin{array}{l}\text { Northern Rocky Mountain Subalpine-Upper } \\
\text { Montane Grassland }\end{array}$ & Carex scirpoidea & Graminoid $\mathrm{C}_{3}$ & 3.6 & 3.6 \\
\hline Rocky Mountain Alpine Turf & Carex scirpoidea & Graminoid $\mathrm{C}_{3}$ & 3.6 & 3.6 \\
\hline Rocky Mountain Alpine-Montane Wet Meadow & Carex scopulorum & Graminoid $\mathrm{C}_{3}$ & 3.6 & 3.6 \\
\hline Rocky Mountain Alpine Turf & Carex siccata & Graminoid $\mathrm{C}_{3}$ & 3.6 & 3.6 \\
\hline Rocky Mountain Subalpine-Montane Fen & Carex simulata & Graminoid $\mathrm{C}_{3}$ & 3.6 & 3.6 \\
\hline North Pacific Maritime Mesic Subalpine Parkland & Carex spectabilis & Graminoid $\mathrm{C}_{3}$ & 3.6 & 3.6 \\
\hline $\begin{array}{l}\text { North Pacific Dry and Mesic Alpine Dwarf- } \\
\text { Shrubland, Fell-Field and Meadow }\end{array}$ & Carex spectabilis & Graminoid $\mathrm{C}_{3}$ & 3.6 & 3.6 \\
\hline $\begin{array}{l}\text { Western Great Plains Dry Bur Oak Forest and } \\
\text { Woodland }\end{array}$ & Carex sprengelii & Graminoid $\mathrm{C}_{3}$ & 3.6 & 3.6 \\
\hline Southern Coastal Plain Nonriverine Basin Swamp & Carex striata & Graminoid $\mathrm{C}_{3}$ & 3.6 & 3.6 \\
\hline Great Lakes Wooded Dune and Swale & Carex stricta & Graminoid $\mathrm{C}_{3}$ & 3.6 & 3.6 \\
\hline Laurentian-Acadian Wet Meadow-Shrub Swamp & Carex stricta & Graminoid $\mathrm{C}_{3}$ & 3.6 & 3.6 \\
\hline $\begin{array}{l}\text { Southern Atlantic Coastal Plain Fresh and } \\
\text { Oligohaline Tidal Marsh }\end{array}$ & Carex stricta & Graminoid $\mathrm{C}_{3}$ & 3.6 & 3.6 \\
\hline High Allegheny Wetland & Carex trisperma & Graminoid $\mathrm{C}_{3}$ & 3.6 & 3.6 \\
\hline Willamette Valley Wet Prairie & Carex unilateralis & Graminoid $\mathrm{C}_{3}$ & 3.6 & 3.6 \\
\hline Inter-Mountain Basins Interdunal Swale Wetland & Carex utriculata & Graminoid $\mathrm{C}_{3}$ & 3.6 & 3.6 \\
\hline North Pacific Shrub Swamp & Carex utriculata & Graminoid $\mathrm{C}_{3}$ & 3.6 & 3.6 \\
\hline Rocky Mountain Alpine-Montane Wet Meadow & Carex utriculata & Graminoid $\mathrm{C}_{3}$ & 3.6 & 3.6 \\
\hline Rocky Mountain Subalpine-Montane Fen & Carex utriculata & Graminoid $\mathrm{C}_{3}$ & 3.6 & 3.6 \\
\hline
\end{tabular}




\begin{tabular}{|c|c|c|c|c|}
\hline Community & Species & Classification & Resistance & Resilience \\
\hline Temperate Pacific Freshwater Emergent Marsh & Carex utriculata & Graminoid $C_{3}$ & 3.6 & 3.6 \\
\hline $\begin{array}{l}\text { Temperate Pacific Subalpine-Montane Wet } \\
\text { Meadow }\end{array}$ & Carex utriculata & Graminoid $C_{3}$ & 3.6 & 3.6 \\
\hline Rocky Mountain Alpine-Montane Wet Meadow & Carex vernacula & Graminoid $C_{3}$ & 3.6 & 3.6 \\
\hline Mediterranean California Alpine Dry Tundra & Carex vernacula & Graminoid $\mathrm{C}_{3}$ & 3.6 & 3.6 \\
\hline Sonoran Paloverde-Mixed Cacti Desert Scrub & Carnegiea gigantea & Forb & 3 & 3.5 \\
\hline Mediterranean California Southern Coastal Dune & Carpobrotus chilensis & Forb & 3 & 3.5 \\
\hline $\begin{array}{l}\text { Allegheny-Cumberland Dry Oak Forest and } \\
\text { Woodland }\end{array}$ & Carya alba & Tree- Deciduous & 3 & 4.2 \\
\hline $\begin{array}{l}\text { East Gulf Coastal Plain Northern Loess Plain Oak- } \\
\text { Hickory Upland }\end{array}$ & Carya alba & Tree- Deciduous & 3 & 4.2 \\
\hline Lower Mississippi River Flatwoods & Carya alba & Tree-Deciduous & 3 & 4.2 \\
\hline $\begin{array}{l}\text { North-Central Interior Dry-Mesic Oak Forest and } \\
\text { Woodland }\end{array}$ & Carya alba & Tree- Deciduous & 3 & 4.2 \\
\hline Northeastern Interior Dry-Mesic Oak Forest & Carya alba & Tree- Deciduous & 3 & 4.2 \\
\hline $\begin{array}{l}\text { Ozark-Ouachita Shortleaf Pine-Bluestem } \\
\text { Woodland }\end{array}$ & Carya alba & Tree-Deciduous & 3 & 4.2 \\
\hline $\begin{array}{l}\text { Southern Interior Low Plateau Dry-Mesic Oak } \\
\text { Forest }\end{array}$ & Carya alba & Tree- Deciduous & 3 & 4.2 \\
\hline Southern Piedmont Dry Oak-(Pine) Forest & Carya alba & Tree- Deciduous & 3 & 4.2 \\
\hline Mississippi River Bottomland Depression & Carya aquatica & Tree- Deciduous & 3 & 4.2 \\
\hline $\begin{array}{l}\text { Mississippi River Low Floodplain (Bottomland) } \\
\text { Forest }\end{array}$ & Carya aquatica & Tree- Deciduous & 3 & 4.2 \\
\hline Southern Coastal Plain Limestone Forest & Carya carolinae-septentrionalis & Tree- Deciduous & 3 & 4.2 \\
\hline Crosstimbers Oak Forest and Woodland & Carya cordiformis & Tree-Deciduous & 3 & 4.2 \\
\hline $\begin{array}{l}\text { North-Central Interior Dry-Mesic Oak Forest and } \\
\text { Woodland }\end{array}$ & Carya cordiformis & Tree-Deciduous & 3 & 4.2 \\
\hline $\begin{array}{l}\text { Allegheny-Cumberland Dry Oak Forest and } \\
\text { Woodland }\end{array}$ & Carya glabra & Tree-Deciduous & 3 & 4.2 \\
\hline Appalachian Shale Barrens & Carya glabra & Tree- Deciduous & 3 & 4.2 \\
\hline East Gulf Coastal Plain Maritime Forest & Carya glabra & Tree-Deciduous & 3 & 4.2 \\
\hline $\begin{array}{l}\text { East Gulf Coastal Plain Northern Dry Upland } \\
\text { Hardwood Forest }\end{array}$ & Carya glabra & Tree-Deciduous & 3 & 4.2 \\
\hline Northeastern Interior Dry-Mesic Oak Forest & Carya glabra & Tree- Deciduous & 3 & 4.2 \\
\hline $\begin{array}{l}\text { Southern Coastal Plain Dry Upland Hardwood } \\
\text { Forest }\end{array}$ & Carya glabra & Tree- Deciduous & 3 & 4.2 \\
\hline Southern Piedmont Dry Oak-(Pine) Forest & Carya glabra & Tree-Deciduous & 3 & 4.2 \\
\hline Edwards Plateau Floodplain Terrace & Carya illinoinensis & Tree- Deciduous & 3 & 4.2 \\
\hline Southeastern Great Plains Floodplain Forest & Carya illinoinensis & Tree-Deciduous & 3 & 4.2 \\
\hline Southeastern Great Plains Riparian Forest & Carya illinoinensis & Tree- Deciduous & 3 & 4.2 \\
\hline Mississippi River Floodplain and Riparian Forest & Carya illinoinensis & Tree-Deciduous & 3 & 4.2 \\
\hline Mississippi River Riparian Forest & Carya illinoinensis & Tree-Deciduous & 3 & 4.2 \\
\hline $\begin{array}{l}\text { North-Central Interior Dry-Mesic Oak Forest and } \\
\text { Woodland }\end{array}$ & Carya ovata & Tree-Deciduous & 3 & 4.2 \\
\hline $\begin{array}{l}\text { Southern Interior Low Plateau Dry-Mesic Oak } \\
\text { Forest }\end{array}$ & Carya ovata & Tree- Deciduous & 3 & 4.2 \\
\hline $\begin{array}{l}\text { South-Central Interior / Upper Coastal Plain } \\
\text { Flatwoods }\end{array}$ & Carya ovata & Tree- Deciduous & 3 & 4.2 \\
\hline $\begin{array}{l}\text { South-Central Interior / Upper Coastal Plain Wet } \\
\text { Flatwoods }\end{array}$ & Carya ovata & Tree- Deciduous & 3 & 4.2 \\
\hline $\begin{array}{l}\text { Southern Ridge and Valley / Cumberland Dry } \\
\text { Calcareous Forest }\end{array}$ & Carya ovata & Tree- Deciduous & 3 & 4.2 \\
\hline
\end{tabular}




\begin{tabular}{|c|c|c|c|c|}
\hline Community & Species & Classification & Resistance & Resilience \\
\hline East Gulf Coastal Plain Maritime Forest & Carya pallida & Tree-Deciduous & 3 & 4.2 \\
\hline Crosstimbers Oak Forest and Woodland & Carya texana & Tree- Deciduous & 3 & 4.2 \\
\hline Crowley's Ridge Sand Forest & Carya texana & Tree-Deciduous & 3 & 4.2 \\
\hline $\begin{array}{l}\text { East-Central Texas Plains Post Oak Savanna and } \\
\text { Woodland }\end{array}$ & Carya texana & Tree- Deciduous & 3 & 4.2 \\
\hline $\begin{array}{l}\text { Mississippi River Alluvial Plain Dry-Mesic Loess } \\
\text { Slope Forest }\end{array}$ & Carya texana & Tree- Deciduous & 3 & 4.2 \\
\hline $\begin{array}{l}\text { Ozark-Ouachita Shortleaf Pine-Bluestem } \\
\text { Woodland }\end{array}$ & Carya texana & Tree- Deciduous & 3 & 4.2 \\
\hline West Gulf Coastal Plain Pine-Hardwood Forest & Carya texana & Tree-Deciduous & 3 & 4.2 \\
\hline $\begin{array}{l}\text { West Gulf Coastal Plain Stream Terrace Sandyland } \\
\text { Longleaf Pine Woodland }\end{array}$ & Carya texana & Tree-Deciduous & 3 & 4.2 \\
\hline Llano Uplift Acidic Forest, Woodland and Glade & Carya texana & Tree-Deciduous & 3 & 4.2 \\
\hline Rocky Mountain Alpine Dwarf-Shrubland & Cassiope mertensiana & Shrub & 2.7 & 2.8 \\
\hline North Pacific Maritime Mesic Subalpine Parkland & Cassiope mertensiana & Shrub- Evergreen & 2.7 & 2.4 \\
\hline $\begin{array}{l}\text { North Pacific Dry and Mesic Alpine Dwarf- } \\
\text { Shrubland, Fell-Field and Meadow }\end{array}$ & Cassiope mertensiana & Shrub- Evergreen & 2.7 & 2.4 \\
\hline Tamaulipan Calcareous Thornscrub & Castela erecta & Shrub- Deciduous & 2.7 & 3 \\
\hline Mediterranean California Alpine Dry Tundra & Castilleja nana & Forb & 3 & 3.5 \\
\hline California Montane Woodland and Chaparral & Ceanothus cordulatus & Shrub- Evergreen & 2.7 & 2.4 \\
\hline $\begin{array}{l}\text { Sierra Nevada Subalpine Lodgepole Pine Forest } \\
\text { and Woodland }\end{array}$ & Ceanothus cordulatus & Shrub- Evergreen & 2.7 & 2.4 \\
\hline Southern California Dry-Mesic Chaparral & Ceanothus crassifolius & Shrub- Evergreen & 2.7 & 2.4 \\
\hline California Xeric Serpentine Chaparral & Ceanothus cuneatus & Shrub- Evergreen & 2.7 & 2.4 \\
\hline $\begin{array}{l}\text { Mediterranean California Mesic Serpentine } \\
\text { Woodland and Chaparral }\end{array}$ & Ceanothus cuneatus & Shrub- Evergreen & 2.7 & 2.4 \\
\hline California Montane Woodland and Chaparral & Ceanothus diversifolius & Shrub- Evergreen & 2.7 & 2.4 \\
\hline Great Basin Semi-Desert Chaparral & Ceanothus greggii & Shrub- Evergreen & 2.7 & 2.4 \\
\hline Madrean Oriental Chaparral & Ceanothus greggii & Shrub- Evergreen & 2.7 & 2.4 \\
\hline Sonora-Mojave Semi-Desert Chaparral & Ceanothus greggii & Shrub- Evergreen & 2.7 & 2.4 \\
\hline Southern California Dry-Mesic Chaparral & Ceanothus greggii & Shrub- Evergreen & 2.7 & 2.4 \\
\hline California Montane Woodland and Chaparral & Ceanothus integerrimus & Shrub- Deciduous & 2.7 & 3 \\
\hline $\begin{array}{l}\text { Mediterranean California Lower Montane Black } \\
\text { Oak-Conifer Forest and Woodland }\end{array}$ & Ceanothus integerrimus & Shrub- Deciduous & 2.7 & 3 \\
\hline Southern California Dry-Mesic Chaparral & Ceanothus leucodermis & Shrub- Evergreen & 2.7 & 2.4 \\
\hline Southern California Dry-Mesic Chaparral & Ceanothus megacarpus & Shrub- Evergreen & 2.7 & 2.4 \\
\hline California Maritime Chaparral & Ceanothus oliganthus & Shrub- Evergreen & 2.7 & 2.4 \\
\hline Southern California Oak Woodland and Savanna & Ceanothus oliganthus & Shrub- Evergreen & 2.7 & 2.4 \\
\hline California Montane Woodland and Chaparral & Ceanothus sanguineus & Shrub- Evergreen & 2.7 & 2.4 \\
\hline Northern California Coastal Scrub & Ceanothus thyrsiflorus & Shrub- Evergreen & 2.7 & 2.4 \\
\hline Great Basin Semi-Desert Chaparral & Ceanothus velutinus & Shrub- Evergreen & 2.7 & 2.4 \\
\hline North Pacific Montane Shrubland & Ceanothus velutinus & Shrub- Evergreen & 2.7 & 2.4 \\
\hline Mississippi Delta Maritime Forest & Celtis laevigata & Shrub- Deciduous & 2.7 & 3 \\
\hline Southern Piedmont Large Floodplain Forest & Celtis laevigata & Shrub- Deciduous & 2.7 & 3 \\
\hline $\begin{array}{l}\text { Atlantic Coastal Plain Small Brownwater River } \\
\text { Floodplain Forest }\end{array}$ & Celtis laevigata & Tree-Deciduous & 3 & 4.2 \\
\hline Edwards Plateau Floodplain Terrace & Celtis laevigata & Tree-Deciduous & 3 & 4.2 \\
\hline Edwards Plateau Riparian & Celtis laevigata & Tree-Deciduous & 3 & 4.2 \\
\hline Southeastern Great Plains Floodplain Forest & Celtis laevigata & Tree-Deciduous & 3 & 4.2 \\
\hline
\end{tabular}




\begin{tabular}{|c|c|c|c|c|}
\hline Community & Species & Classification & Resistance & Resilience \\
\hline $\begin{array}{l}\text { West Gulf Coastal Plain Southern Calcareous } \\
\text { Prairie }\end{array}$ & Celtis laevigata & Tree- Deciduous & 3 & 4.2 \\
\hline $\begin{array}{l}\text { West Gulf Coastal Plain Chenier and Upper Texas } \\
\text { Coastal Fringe Forest and Woodland }\end{array}$ & Celtis laevigata & Tree- Deciduous & 3 & 4.2 \\
\hline Southeastern Great Plains Riparian Forest & Celtis laevigata & Tree- Deciduous & 3 & 4.2 \\
\hline $\begin{array}{l}\text { North American Warm Desert Riparian Woodland } \\
\text { and Shrubland }\end{array}$ & Celtis laevigata & Tree- Deciduous & 3 & 4.2 \\
\hline Southeastern Great Plains Riparian Forest & Celtis laevigata & Tree- Deciduous & 3 & 4.2 \\
\hline Tamaulipan Calcareous Thornscrub & Celtis pallida & Shrub- Deciduous & 2.7 & 3 \\
\hline Tamaulipan Mixed Deciduous Thornscrub & Celtis pallida & Shrub- Deciduous & 2.7 & 3 \\
\hline Tamaulipan Ramadero & Celtis pallida & Shrub- Deciduous & 2.7 & 3 \\
\hline $\begin{array}{l}\text { East Gulf Coastal Plain Small Stream and River } \\
\text { Floodplain Forest }\end{array}$ & Cephalanthus occidentalis & Shrub- Deciduous & 2.7 & 3 \\
\hline $\begin{array}{l}\text { East Gulf Coastal Plain Southern Loblolly- } \\
\text { Hardwood Flatwoods }\end{array}$ & Cephalanthus occidentalis & Shrub- Deciduous & 2.7 & 3 \\
\hline North-Central Interior Wet Meadow-Shrub Swamp & Cephalanthus occidentalis & Shrub- Deciduous & 2.7 & 3 \\
\hline Piedmont Upland Depression Swamp & Cephalanthus occidentalis & Shrub- Deciduous & 2.7 & 3 \\
\hline Southern Coastal Plain Nonriverine Cypress Dome & Cephalanthus occidentalis & Shrub- Deciduous & 2.7 & 3 \\
\hline $\begin{array}{l}\text { East Gulf Coastal Plain Dune and Coastal } \\
\text { Grassland }\end{array}$ & Ceratiola ericoides & Forb & 3 & 3.5 \\
\hline Southern Atlantic Coastal Plain Xeric River Dune & Ceratiola ericoides & Shrub & 2.7 & 2.8 \\
\hline Florida Peninsula Inland Scrub & Ceratiola ericoides & Shrub- Evergreen & 2.7 & 2.4 \\
\hline Nashville Basin Limestone Glade and Woodland & Cercis canadensis & Tree- Deciduous & 3 & 4.2 \\
\hline West Gulf Coastal Plain Weches Glade & Cercis canadensis & Tree-Deciduous & 3 & 4.2 \\
\hline Colorado Plateau Pinyon-Juniper Woodland & Cercocarpus intricatus & Shrub- Evergreen & 2.7 & 2.4 \\
\hline $\begin{array}{l}\text { Colorado Plateau Mixed Bedrock Canyon and } \\
\text { Tableland }\end{array}$ & Cercocarpus intricatus & Shrub- Evergreen & 2.7 & 2.4 \\
\hline $\begin{array}{l}\text { Columbia Plateau Western Juniper Woodland and } \\
\text { Savanna }\end{array}$ & Cercocarpus ledifolius & Shrub- Evergreen & 2.7 & 2.4 \\
\hline $\begin{array}{l}\text { Inter-Mountain Basins Curl-leaf Mountain- } \\
\text { mahogany Woodland and Shrubland }\end{array}$ & Cercocarpus ledifolius & Shrub- Evergreen & 2.7 & 2.4 \\
\hline $\begin{array}{l}\text { Inter-Mountain Basins Subalpine Limber- } \\
\text { Bristlecone Pine Woodland }\end{array}$ & Cercocarpus ledifolius & Shrub- Evergreen & 2.7 & 2.4 \\
\hline $\begin{array}{l}\text { Northwestern Great Plains-Black Hills Ponderosa } \\
\text { Pine Woodland and Savanna }\end{array}$ & Cercocarpus ledifolius & Shrub- Evergreen & 2.7 & 2.4 \\
\hline $\begin{array}{l}\text { Rocky Mountain Foothill Limber Pine-Juniper } \\
\text { Woodland }\end{array}$ & Cercocarpus ledifolius & Shrub- Evergreen & 2.7 & 2.4 \\
\hline Sierra Nevada Cliff and Canyon & Cercocarpus ledifolius & Shrub- Evergreen & 2.7 & 2.4 \\
\hline $\begin{array}{l}\text { Llano Estacado Caprock Escarpment and Breaks } \\
\text { Shrubland and Steppe }\end{array}$ & Cercocarpus montanus & Shrub- Evergreen & 2.7 & 2.4 \\
\hline Madrean Oriental Chaparral & Cercocarpus montanus & Shrub- Evergreen & 2.7 & 2.4 \\
\hline Mogollon Chaparral & Cercocarpus montanus & Shrub- Evergreen & 2.7 & 2.4 \\
\hline North American Warm Desert Volcanic Rockland & Cercocarpus montanus & Shrub- Evergreen & 2.7 & 2.4 \\
\hline $\begin{array}{l}\text { Northwestern Great Plains-Black Hills Ponderosa } \\
\text { Pine Woodland and Savanna }\end{array}$ & Cercocarpus montanus & Shrub- Evergreen & 2.7 & 2.4 \\
\hline $\begin{array}{l}\text { Rocky Mountain Foothill Limber Pine-Juniper } \\
\text { Woodland }\end{array}$ & Cercocarpus montanus & Shrub- Evergreen & 2.7 & 2.4 \\
\hline $\begin{array}{l}\text { Rocky Mountain Gambel Oak-Mixed Montane } \\
\text { Shrubland }\end{array}$ & Cercocarpus montanus & Shrub- Evergreen & 2.7 & 2.4 \\
\hline $\begin{array}{l}\text { Rocky Mountain Lower Montane-Foothill } \\
\text { Shrubland }\end{array}$ & Cercocarpus montanus & Shrub- Evergreen & 2.7 & 2.4 \\
\hline Southwestern Great Plains Canyon & Cercocarpus montanus & Shrub- Evergreen & 2.7 & 2.4 \\
\hline
\end{tabular}




\begin{tabular}{|c|c|c|c|c|}
\hline Community & Species & Classification & Resistance & Resilience \\
\hline California Mesic Chaparral & Cercocarpus montanus & Shrub- Evergreen & 2.7 & 2.4 \\
\hline Sonora-Mojave Semi-Desert Chaparral & Cercocarpus montanus & Shrub- Evergreen & 2.7 & 2.4 \\
\hline Southern California Coast Ranges Cliff and Canyon & Cercocarpus montanus & Shrub- Evergreen & 2.7 & 2.4 \\
\hline Mogollon Chaparral & Cercocarpus montanus & Shrub- Evergreen & 2.7 & 2.4 \\
\hline Edwards Plateau Upland Depression & Chaetopappa bellidifolia & Forb & 3 & 3.5 \\
\hline $\begin{array}{l}\text { North Pacific Maritime Mesic-Wet Douglas-fir- } \\
\text { Western Hemlock Forest }\end{array}$ & Chamaecyparis lawsoniana & Tree- Evergreen & 3.2 & 2.2 \\
\hline North Pacific Avalanche Chute Shrubland & Chamaecyparis nootkatensis & Tree- Evergreen & 3.2 & 2.2 \\
\hline $\begin{array}{l}\text { North Pacific Dry-Mesic Silver Fir-Western } \\
\text { Hemlock-Douglas-fir Forest }\end{array}$ & Chamaecyparis nootkatensis & Tree- Evergreen & 3.2 & 2.2 \\
\hline North Pacific Hardwood-Conifer Swamp & Chamaecyparis nootkatensis & Tree- Evergreen & 3.2 & 2.2 \\
\hline North Pacific Maritime Mesic Subalpine Parkland & Chamaecyparis nootkatensis & Tree- Evergreen & 3.2 & 2.2 \\
\hline $\begin{array}{l}\text { North Pacific Mesic Western Hemlock-Silver Fir } \\
\text { Forest }\end{array}$ & Chamaecyparis nootkatensis & Tree- Evergreen & 3.2 & 2.2 \\
\hline Northern Atlantic Coastal Plain Pitch Pine Lowland & Chamaecyparis thyoides & Tree- Evergreen & 3.2 & 2.2 \\
\hline $\begin{array}{l}\text { Southern Atlantic Coastal Plain Nonriverine } \\
\text { Swamp and Wet Hardwood Forest }\end{array}$ & Chamaecyparis thyoides & Tree- Evergreen & 3.2 & 2.2 \\
\hline Southern Coastal Plain Hydric Hammock & Chamaecyparis thyoides & Tree- Evergreen & 3.2 & 2.2 \\
\hline $\begin{array}{l}\text { Atlantic Coastal Plain Streamhead Seepage } \\
\text { Swamp, Pocosin and Baygall }\end{array}$ & Chamaecyparis thyoides & Tree- Evergreen & 3.2 & 2.2 \\
\hline Boreal-Laurentian Bog & Chamaedaphne calyculata & Shrub & 2.7 & 2.8 \\
\hline $\begin{array}{l}\text { Boreal-Laurentian Conifer Acidic Swamp and } \\
\text { Treed Poor Fen }\end{array}$ & Chamaedaphne calyculata & Shrub & 2.7 & 2.8 \\
\hline $\begin{array}{l}\text { North-Central Interior and Appalachian Acidic } \\
\text { Peatland }\end{array}$ & Chamaedaphne calyculata & Shrub & 2.7 & 2.8 \\
\hline Northern Atlantic Coastal Plain Sandy Beach & Chamaesyce polygonifolia & Forb & 3 & 3.5 \\
\hline Southern Atlantic Coastal Plain Florida Beach & Chamaesyce polygonifolia & Forb & 3 & 3.5 \\
\hline Southern Atlantic Coastal Plain Sea Island Beach & Chamaesyce polygonifolia & Forb & 3 & 3.5 \\
\hline $\begin{array}{l}\text { Rocky Mountain Subalpine-Montane Mesic } \\
\text { Meadow }\end{array}$ & Chamerion angustifolium & Forb & 3 & 3.5 \\
\hline Eastern Highland Rim Prairie and Barrens & Chasmanthium laxum & Graminoid $\mathrm{C}_{3}$ & 3.6 & 3.6 \\
\hline $\begin{array}{l}\text { East Gulf Coastal Plain Southern Loblolly- } \\
\text { Hardwood Flatwoods }\end{array}$ & Chasmanthium sessiliflorum & Graminoid $\mathrm{C}_{3}$ & 3.6 & 3.6 \\
\hline $\begin{array}{l}\text { West Gulf Coastal Plain Small Stream and River } \\
\text { Forest }\end{array}$ & Chasmanthium sessiliflorum & Graminoid $\mathrm{C}_{3}$ & 3.6 & 3.6 \\
\hline North American Warm Desert Wash & Chilopsis linearis & Tree- Deciduous & 3 & 4.2 \\
\hline Tamaulipan Savanna Grassland & Chloris pluriflora & Graminoid $\mathrm{C}_{4}$ & 3.6 & 4.1 \\
\hline California Montane Woodland and Chaparral & Chrysolepis sempervirens & Shrub- Evergreen & 2.7 & 2.4 \\
\hline $\begin{array}{l}\text { East Gulf Coastal Plain Dune and Coastal } \\
\text { Grassland }\end{array}$ & Chrysoma pauciflosculosa & Forb & 3 & 3.5 \\
\hline Southern Atlantic Coastal Plain Xeric River Dune & Chrysoma pauciflosculosa & Shrub & 2.7 & 2.8 \\
\hline Great Basin Xeric Mixed Sagebrush Shrubland & Chrysothamnus greenei & Shrub- Deciduous & 2.7 & 3 \\
\hline Inter-Mountain Basins Semi-Desert Shrub-Steppe & Chrysothamnus greenei & Shrub- Deciduous & 2.7 & 3 \\
\hline $\begin{array}{l}\text { Colorado Plateau Mixed Low Sagebrush } \\
\text { Shrubland }\end{array}$ & Chrysothamnus viscidiflorus & Shrub- Deciduous & 2.7 & 3 \\
\hline Colorado Plateau Pinyon-Juniper Shrubland & Chrysothamnus viscidiflorus & Shrub- Deciduous & 2.7 & 3 \\
\hline Columbia Plateau Low Sagebrush Steppe & Chrysothamnus viscidiflorus & Shrub- Deciduous & 2.7 & 3 \\
\hline Great Basin Xeric Mixed Sagebrush Shrubland & Chrysothamnus viscidiflorus & Shrub- Deciduous & 2.7 & 3 \\
\hline Inter-Mountain Basins Active and Stabilized Dune & Chrysothamnus viscidiflorus & Shrub- Deciduous & 2.7 & 3 \\
\hline Inter-Mountain Basins Greasewood Flat & Chrysothamnus viscidiflorus & Shrub-Deciduous & 2.7 & 3 \\
\hline Inter-Mountain Basins Mixed Salt Desert Scrub & Chrysothamnus viscidiflorus & Shrub- Deciduous & 2.7 & 3 \\
\hline
\end{tabular}




\begin{tabular}{|c|c|c|c|c|}
\hline Community & Species & Classification & Resistance & Resilience \\
\hline Southern Colorado Plateau Sand Shrubland & Chrysothamnus viscidiflorus & Shrub- Deciduous & 2.7 & 3 \\
\hline Rocky Mountain Alpine Bedrock and Scree & Cirsium scopulorum & Forb & 3 & 3.5 \\
\hline Tamaulipan Lomas & Citharexylum berlandieri & Shrub- Deciduous & 2.7 & 3 \\
\hline $\begin{array}{l}\text { Atlantic Coastal Plain Embayed Region Tidal } \\
\text { Freshwater Marsh }\end{array}$ & Cladium mariscus & Graminoid $\mathrm{C}_{3}$ & 3.6 & 3.6 \\
\hline South Florida Everglades Sawgrass Marsh & Cladium mariscus & Graminoid $\mathrm{C}_{3}$ & 3.6 & 3.6 \\
\hline $\begin{array}{l}\text { Southern Atlantic Coastal Plain Nonriverine } \\
\text { Swamp and Wet Hardwood Forest }\end{array}$ & Clethra alnifolia & Shrub- Deciduous & 2.7 & 3 \\
\hline Southern Coastal Plain Nonriverine Cypress Dome & Clethra alnifolia & Shrub- Deciduous & 2.7 & 3 \\
\hline $\begin{array}{l}\text { Atlantic Coastal Plain Streamhead Seepage } \\
\text { Swamp, Pocosin and Baygall }\end{array}$ & Clethra alnifolia & Shrub- Deciduous & 2.7 & 3 \\
\hline Southern Coastal Plain Nonriverine Basin Swamp & Cliftonia monophylla & Shrub- Evergreen & 2.7 & 2.4 \\
\hline $\begin{array}{l}\text { Southern Coastal Plain Seepage Swamp and } \\
\text { Baygall }\end{array}$ & Cliftonia monophylla & Shrub- Evergreen & 2.7 & 2.4 \\
\hline $\begin{array}{l}\text { Atlantic Coastal Plain Streamhead Seepage } \\
\text { Swamp, Pocosin and Baygall }\end{array}$ & Cliftonia monophylla & Shrub- Evergreen & 2.7 & 2.4 \\
\hline South Florida Hardwood Hammock & Coccoloba diversifolia & Tree- Evergreen & 3.2 & 2.2 \\
\hline $\begin{array}{l}\text { Southeast Florida Coastal Strand and Maritime } \\
\text { Hammock }\end{array}$ & Coccoloba uvifera & Shrub- Evergreen & 2.7 & 2.4 \\
\hline $\begin{array}{l}\text { Southwest Florida Coastal Strand and Maritime } \\
\text { Hammock }\end{array}$ & Coccoloba uvifera & Shrub- Evergreen & 2.7 & 2.4 \\
\hline Southwest Florida Dune and Coastal Grassland & Coccoloba uvifera & Shrub- Evergreen & 2.7 & 2.4 \\
\hline $\begin{array}{l}\text { Colorado Plateau Blackbrush-Mormon-tea } \\
\text { Shrubland }\end{array}$ & Coleogyne ramosissima & Shrub- Deciduous & 2.7 & 3 \\
\hline Colorado Plateau Pinyon-Juniper Woodland & Coleogyne ramosissima & Shrub- Deciduous & 2.7 & 3 \\
\hline Great Basin Pinyon-Juniper Woodland & Coleogyne ramosissima & Shrub- Deciduous & 2.7 & 3 \\
\hline Mojave Mid-Elevation Mixed Desert Scrub & Coleogyne ramosissima & Shrub- Deciduous & 2.7 & 3 \\
\hline South Florida Mangrove Swamp & Conocarpus erectus & Shrub- Evergreen & 2.7 & 2.4 \\
\hline Cumberland Riverscour & Coreopsis tripteris & Forb & 3 & 3.5 \\
\hline $\begin{array}{l}\text { Northern Atlantic Coastal Plain Fresh and } \\
\text { Oligohaline Tidal Marsh }\end{array}$ & Cornus amomum & Shrub- Deciduous & 2.7 & 3 \\
\hline Northern Atlantic Coastal Plain Tidal Swamp & Cornus amomum & Tree- Deciduous & 3 & 4.2 \\
\hline Central Atlantic Coastal Plain Maritime Forest & Cornus florida & Tree-Deciduous & 3 & 4.2 \\
\hline Southern Piedmont Dry Oak-(Pine) Forest & Cornus florida & Tree- Deciduous & 3 & 4.2 \\
\hline Central Atlantic Coastal Plain Maritime Forest & Cornus foemina & Tree- Deciduous & 3 & 4.2 \\
\hline $\begin{array}{l}\text { East Gulf Coastal Plain Southern Loblolly- } \\
\text { Hardwood Flatwoods }\end{array}$ & Cornus foemina & Tree-Deciduous & 3 & 4.2 \\
\hline $\begin{array}{l}\text { North Pacific Lowland Mixed Hardwood-Conifer } \\
\text { Forest }\end{array}$ & Cornus nuttallii & Tree-Deciduous & 3 & 4.2 \\
\hline $\begin{array}{l}\text { California Central Valley Riparian Woodland and } \\
\text { Shrubland }\end{array}$ & Cornus sericea & Shrub- Deciduous & 2.7 & 3 \\
\hline $\begin{array}{l}\text { Columbia Basin Foothill Riparian Woodland and } \\
\text { Shrubland }\end{array}$ & Cornus sericea & Shrub- Deciduous & 2.7 & 3 \\
\hline North Pacific Intertidal Freshwater Wetland & Cornus sericea & Shrub- Deciduous & 2.7 & 3 \\
\hline North Pacific Shrub Swamp & Cornus sericea & Shrub- Deciduous & 2.7 & 3 \\
\hline North-Central Interior Wet Meadow-Shrub Swamp & Cornus sericea & Shrub- Deciduous & 2.7 & 3 \\
\hline $\begin{array}{l}\text { Northern Rocky Mountain Lower Montane } \\
\text { Riparian Woodland and Shrubland }\end{array}$ & Cornus sericea & Shrub- Deciduous & 2.7 & 3 \\
\hline Northwestern Great Plains Riparian & Cornus sericea & Shrub- Deciduous & 2.7 & 3 \\
\hline $\begin{array}{l}\text { Rocky Mountain Lower Montane-Foothill Riparian } \\
\text { Woodland and Shrubland }\end{array}$ & Cornus sericea & Shrub- Deciduous & 2.7 & 3 \\
\hline
\end{tabular}




\begin{tabular}{|c|c|c|c|c|}
\hline Community & Species & Classification & Resistance & Resilience \\
\hline $\begin{array}{l}\text { Rocky Mountain Subalpine-Montane Riparian } \\
\text { Shrubland }\end{array}$ & Cornus sericea & Shrub- Deciduous & 2.7 & 3 \\
\hline $\begin{array}{l}\text { Great Basin Foothill and Lower Montane Riparian } \\
\text { Woodland and Shrubland }\end{array}$ & Cornus sericea & Tree- Deciduous & 3 & 4.2 \\
\hline $\begin{array}{l}\text { North Pacific Lowland Riparian Forest and } \\
\text { Shrubland }\end{array}$ & Cornus sericea & Tree- Deciduous & 3 & 4.2 \\
\hline Eastern Great Plains Tallgrass Aspen Parkland & Corylus americana & Shrub- Deciduous & 2.7 & 3 \\
\hline North-Central Interior Oak Savanna & Corylus americana & Shrub- Deciduous & 2.7 & 3 \\
\hline Laurentian Jack Pine-Red Pine Forest & Corylus cornuta & Shrub- Evergreen & 2.7 & 2.4 \\
\hline $\begin{array}{l}\text { Mediterranean California Mesic Mixed Conifer } \\
\text { Forest and Woodland }\end{array}$ & Corylus cornuta & Shrub- Evergreen & 2.7 & 2.4 \\
\hline Laurentian-Acadian Northern Pine-(Oak) Forest & Corylus cornuta & Shrub- Evergreen & 2.7 & 2.4 \\
\hline Temperate Pacific Freshwater Mudflat & Crassula aquatica & Forb & 3 & 3.5 \\
\hline Northwestern Great Plains Shrubland & Crataegus douglasii & Shrub- Deciduous & 2.7 & 3 \\
\hline $\begin{array}{l}\text { East Gulf Coastal Plain Southern Loblolly- } \\
\text { Hardwood Flatwoods }\end{array}$ & Crataegus opaca & Shrub- Deciduous & 2.7 & 3 \\
\hline $\begin{array}{l}\text { Rocky Mountain Lower Montane-Foothill Riparian } \\
\text { Woodland and Shrubland }\end{array}$ & Crataegus rivularis & Shrub- Deciduous & 2.7 & 3 \\
\hline $\begin{array}{l}\text { East Gulf Coastal Plain Southern Loblolly- } \\
\text { Hardwood Flatwoods }\end{array}$ & Crataegus viridis & Shrub- Deciduous & 2.7 & 3 \\
\hline Mediterranean California Southern Coastal Dune & Croton californicus & Forb & 3 & 3.5 \\
\hline South Florida Wet Marl Prairie & Croton linearis & Shrub- Evergreen & 2.7 & 2.4 \\
\hline West Gulf Coastal Plain Catahoula Barrens & Croton michauxii & Forb & 3 & 3.5 \\
\hline $\begin{array}{l}\text { Central Atlantic Coastal Plain Wet Longleaf Pine } \\
\text { Savanna and Flatwoods }\end{array}$ & Ctenium aromaticum & Graminoid $\mathrm{C}_{4}$ & 3.6 & 4.1 \\
\hline $\begin{array}{l}\text { Madrean Lower Montane Pine-Oak Forest and } \\
\text { Woodland }\end{array}$ & Cupressus arizonica & Tree- Evergreen & 3.2 & 2.2 \\
\hline $\begin{array}{l}\text { Mediterranean California Serpentine Foothill and } \\
\text { Lower Montane Riparian Woodland and Seep }\end{array}$ & Cupressus sargentii & Tree- Evergreen & 3.2 & 2.2 \\
\hline Edwards Plateau Floodplain Terrace & Cynodon dactylon & Graminoid $\mathrm{C}_{4}$ & 3.6 & 4.1 \\
\hline Edwards Plateau Riparian & Cynodon dactylon & Graminoid $\mathrm{C}_{4}$ & 3.6 & 4.1 \\
\hline Tamaulipan Floodplain & Cynodon dactylon & Graminoid $\mathrm{C}_{4}$ & 3.6 & 4.1 \\
\hline Tamaulipan Ramadero & Cynodon dactylon & Graminoid $\mathrm{C}_{4}$ & 3.6 & 4.1 \\
\hline $\begin{array}{l}\text { Atlantic Coastal Plain Peatland Pocosin and } \\
\text { Canebrake }\end{array}$ & Cyrilla racemiflora & Shrub- Evergreen & 2.7 & 2.4 \\
\hline Southeastern Coastal Plain Natural Lakeshore & Cyrilla racemiflora & Shrub- Evergreen & 2.7 & 2.4 \\
\hline Southern Coastal Plain Nonriverine Basin Swamp & Cyrilla racemiflora & Shrub- Evergreen & 2.7 & 2.4 \\
\hline $\begin{array}{l}\text { Southern Coastal Plain Seepage Swamp and } \\
\text { Baygall }\end{array}$ & Cyrilla racemiflora & Shrub- Evergreen & 2.7 & 2.4 \\
\hline $\begin{array}{l}\text { Atlantic Coastal Plain Streamhead Seepage } \\
\text { Swamp, Pocosin and Baygall }\end{array}$ & Cyrilla racemiflora & Shrub- Evergreen & 2.7 & 2.4 \\
\hline East Gulf Coastal Plain Near-Coast Pine Flatwoods & Cyrilla racemiflora & Shrub- Evergreen & 2.7 & 2.4 \\
\hline $\begin{array}{l}\text { Llano Estacado Caprock Escarpment and Breaks } \\
\text { Shrubland and Steppe }\end{array}$ & Dalea formosa & Shrub- Deciduous & 2.7 & 3 \\
\hline California Mesic Serpentine Grassland & Danthonia californica & Graminoid $\mathrm{C}_{3}$ & 3.6 & 3.6 \\
\hline California Northern Coastal Grassland & Danthonia californica & Graminoid $\mathrm{C}_{3}$ & 3.6 & 3.6 \\
\hline Mediterranean California Mixed Oak Woodland & Danthonia californica & Graminoid $C_{3}$ & 3.6 & 3.6 \\
\hline North Pacific Herbaceous Bald and Bluff & Danthonia californica & Graminoid $\mathrm{C}_{3}$ & 3.6 & 3.6 \\
\hline Willamette Valley Upland Prairie and Savanna & Danthonia californica & Graminoid $C_{3}$ & 3.6 & 3.6 \\
\hline Willamette Valley Wet Prairie & Danthonia californica & Graminoid $\mathrm{C}_{3}$ & 3.6 & 3.6 \\
\hline Southern Appalachian Grass and Shrub Bald & Danthonia compressa & Graminoid $\mathrm{C}_{3}$ & 3.6 & 3.6 \\
\hline
\end{tabular}




\begin{tabular}{|c|c|c|c|c|}
\hline Community & Species & Classification & Resistance & Resilience \\
\hline $\begin{array}{l}\text { Northwestern Great Plains-Black Hills Ponderosa } \\
\text { Pine Woodland and Savanna }\end{array}$ & Danthonia intermedia & Graminoid $C_{3}$ & 3.6 & 3.6 \\
\hline $\begin{array}{l}\text { Rocky Mountain Foothill Limber Pine-Juniper } \\
\text { Woodland }\end{array}$ & Danthonia intermedia & Graminoid $C_{3}$ & 3.6 & 3.6 \\
\hline $\begin{array}{l}\text { Southern Rocky Mountain Montane-Subalpine } \\
\text { Grassland }\end{array}$ & Danthonia intermedia & Graminoid $C_{3}$ & 3.6 & 3.6 \\
\hline $\begin{array}{l}\text { Northern Rocky Mountain Lower Montane, Foothill } \\
\text { and Valley Grassland }\end{array}$ & Danthonia intermedia & Graminoid $C_{3}$ & 3.6 & 3.6 \\
\hline $\begin{array}{l}\text { Inter-Mountain Basins Montane Sagebrush } \\
\text { Steppe }\end{array}$ & Danthonia parryi & Graminoid $\mathrm{C}_{3}$ & 3.6 & 3.6 \\
\hline $\begin{array}{l}\text { Southern Rocky Mountain Montane-Subalpine } \\
\text { Grassland }\end{array}$ & Danthonia parryi & Graminoid $\mathrm{C}_{3}$ & 3.6 & 3.6 \\
\hline Southern Appalachian Granitic Dome & Danthonia sericea & Graminoid $\mathrm{C}_{3}$ & 3.6 & 3.6 \\
\hline Southern Piedmont Glade and Barrens & Danthonia sericea & Graminoid $\mathrm{C}_{3}$ & 3.6 & 3.6 \\
\hline Central Appalachian Pine-Oak Rocky Woodland & Danthonia spicata & Graminoid $C_{3}$ & 3.6 & 3.6 \\
\hline Great Lakes Acidic Rocky Shore and Cliff & Danthonia spicata & Graminoid $\mathrm{C}_{3}$ & 3.6 & 3.6 \\
\hline Southern Piedmont Cliff & Danthonia spicata & Graminoid $\mathrm{C}_{3}$ & 3.6 & 3.6 \\
\hline $\begin{array}{l}\text { Columbia Plateau Silver Sagebrush Seasonally } \\
\text { Flooded Shrub-Steppe }\end{array}$ & Dasiphora fruticosa & Shrub- Evergreen & 2.7 & 2.4 \\
\hline Great Lakes Alvar & Dasiphora fruticosa & Shrub- Evergreen & 2.7 & 2.4 \\
\hline Northwestern Great Plains Shrubland & Dasiphora fruticosa & Shrub- Evergreen & 2.7 & 2.4 \\
\hline Rocky Mountain Alpine Dwarf-Shrubland & Dasiphora fruticosa & Shrub- Evergreen & 2.7 & 2.4 \\
\hline Chihuahuan Gypsophilous Grassland and Steppe & Dasyochloa pulchella & Graminoid $C_{4}$ & 3.6 & 4.1 \\
\hline $\begin{array}{l}\text { East Gulf Coastal Plain Small Stream and River } \\
\text { Floodplain Forest }\end{array}$ & Decodon verticillatus & Shrub-Deciduous & 2.7 & 3 \\
\hline $\begin{array}{l}\text { Central and Southern Appalachian Montane Oak } \\
\text { Forest }\end{array}$ & Dennstaedtia punctilobula & Graminoid $C_{3}$ & 3.6 & 3.6 \\
\hline $\begin{array}{l}\text { Columbia Plateau Silver Sagebrush Seasonally } \\
\text { Flooded Shrub-Steppe }\end{array}$ & Deschampsia caespitosa & Graminoid $C_{3}$ & 3.6 & 3.6 \\
\hline $\begin{array}{l}\text { Great Basin Foothill and Lower Montane Riparian } \\
\text { Woodland and Shrubland }\end{array}$ & Deschampsia caespitosa & Graminoid $C_{3}$ & 3.6 & 3.6 \\
\hline Mediterranean California Serpentine Fen & Deschampsia caespitosa & Graminoid $C_{3}$ & 3.6 & 3.6 \\
\hline Rocky Mountain Alpine Turf & Deschampsia caespitosa & Graminoid $\mathrm{C}_{3}$ & 3.6 & 3.6 \\
\hline Rocky Mountain Alpine-Montane Wet Meadow & Deschampsia caespitosa & Graminoid $\mathrm{C}_{3}$ & 3.6 & 3.6 \\
\hline Temperate Pacific Tidal Salt and Brackish Marsh & Deschampsia caespitosa & Graminoid $C_{3}$ & 3.6 & 3.6 \\
\hline Willamette Valley Wet Prairie & Deschampsia caespitosa & Graminoid $\mathrm{C}_{3}$ & 3.6 & 3.6 \\
\hline $\begin{array}{l}\text { Central and Southern Appalachian Montane Oak } \\
\text { Forest }\end{array}$ & Deschampsia flexuosa & Graminoid $\mathrm{C}_{3}$ & 3.6 & 3.6 \\
\hline Central Appalachian Pine-Oak Rocky Woodland & Deschampsia flexuosa & Graminoid $C_{3}$ & 3.6 & 3.6 \\
\hline Central Interior Acidic Cliff and Talus & Deschampsia flexuosa & Graminoid $C_{3}$ & 3.6 & 3.6 \\
\hline Ouachita Montane Oak Forest & Deschampsia flexuosa & Graminoid $C_{3}$ & 3.6 & 3.6 \\
\hline Acadian-Appalachian Alpine Tundra & Diapensia lapponica & Shrub & 2.7 & 2.8 \\
\hline Eastern Highland Rim Prairie and Barrens & Dichanthelium aciculare & Graminoid $C_{3}$ & 3.6 & 3.6 \\
\hline Eastern Highland Rim Prairie and Barrens & Dichanthelium dichotomum & Graminoid $\mathrm{C}_{3}$ & 3.6 & 3.6 \\
\hline Florida Dry Prairie & Dichanthelium dichotomum & Graminoid $C_{3}$ & 3.6 & 3.6 \\
\hline East Gulf Coastal Plain Depression Pond & Dichanthelium erectifolium & Graminoid $\mathrm{C}_{3}$ & 3.6 & 3.6 \\
\hline Florida Dry Prairie & Dichanthelium strigosum & Graminoid $\mathrm{C}_{3}$ & 3.6 & 3.6 \\
\hline $\begin{array}{l}\text { Atlantic Coastal Plain Clay-Based Carolina Bay } \\
\text { Wetland }\end{array}$ & Dichanthelium wrightianum & Graminoid $C_{3}$ & 3.6 & 3.6 \\
\hline Central Florida Herbaceous Pondshore & Dichanthelium wrightianum & Graminoid $C_{3}$ & 3.6 & 3.6 \\
\hline East Gulf Coastal Plain Depression Pond & Dichanthelium wrightianum & Graminoid $C_{3}$ & 3.6 & 3.6 \\
\hline
\end{tabular}




\begin{tabular}{|c|c|c|c|c|}
\hline Community & Species & Classification & Resistance & Resilience \\
\hline Southern Atlantic Coastal Plain Depression Pond & Dichanthelium wrightianum & Graminoid $C_{3}$ & 3.6 & 3.6 \\
\hline Madrean Encinal & Digitaria californica & Graminoid $\mathrm{C}_{4}$ & 3.6 & 4.1 \\
\hline Edwards Plateau Limestone Shrubland & Diospyros texana & Shrub- Deciduous & 2.7 & 3 \\
\hline Tamaulipan Floodplain & Diospyros texana & Tree-Deciduous & 3 & 4.2 \\
\hline Tamaulipan Palm Grove Riparian Forest & Diospyros texana & Tree-Deciduous & 3 & 4.2 \\
\hline $\begin{array}{l}\text { East Gulf Coastal Plain Southern Loblolly- } \\
\text { Hardwood Flatwoods }\end{array}$ & Diospyros virginiana & Tree- Deciduous & 3 & 4.2 \\
\hline Southern Coastal Plain Oak Dome and Hammock & Diospyros virginiana & Tree- Deciduous & 3 & 4.2 \\
\hline $\begin{array}{l}\text { South-Central Interior / Upper Coastal Plain Wet } \\
\text { Flatwoods }\end{array}$ & Diospyros virginiana & Tree-Deciduous & 3 & 4.2 \\
\hline Southern California Coastal Scrub & Diplacus aurantiacus & Shrub- Evergreen & 2.7 & 2.4 \\
\hline Acadian Coastal Salt Marsh & Distichlis spicata & Graminoid $\mathrm{C}_{4}$ & 3.6 & 4.1 \\
\hline $\begin{array}{l}\text { Atlantic Coastal Plain Embayed Region Tidal Salt } \\
\text { and Brackish Marsh }\end{array}$ & Distichlis spicata & Graminoid $\mathrm{C}_{4}$ & 3.6 & 4.1 \\
\hline Chihuahuan Mixed Salt Desert Scrub & Distichlis spicata & Graminoid $\mathrm{C}_{4}$ & 3.6 & 4.1 \\
\hline Florida Big Bend Salt and Brackish Tidal Marsh & Distichlis spicata & Graminoid $\mathrm{C}_{4}$ & 3.6 & 4.1 \\
\hline Inter-Mountain Basins Alkaline Closed Depression & Distichlis spicata & Graminoid $\mathrm{C}_{4}$ & 3.6 & 4.1 \\
\hline Inter-Mountain Basins Greasewood Flat & Distichlis spicata & Graminoid $\mathrm{C}_{4}$ & 3.6 & 4.1 \\
\hline Inter-Mountain Basins Semi-Desert Shrub-Steppe & Distichlis spicata & Graminoid $\mathrm{C}_{4}$ & 3.6 & 4.1 \\
\hline Inter-Mountain Basins Wash & Distichlis spicata & Graminoid $\mathrm{C}_{4}$ & 3.6 & 4.1 \\
\hline Mediterranean California Southern Coastal Dune & Distichlis spicata & Graminoid $\mathrm{C}_{4}$ & 3.6 & 4.1 \\
\hline North American Warm Desert Cienega & Distichlis spicata & Graminoid $\mathrm{C}_{4}$ & 3.6 & 4.1 \\
\hline North American Warm Desert Playa & Distichlis spicata & Graminoid $\mathrm{C}_{4}$ & 3.6 & 4.1 \\
\hline Northern Atlantic Coastal Plain Tidal Salt Marsh & Distichlis spicata & Graminoid $\mathrm{C}_{4}$ & 3.6 & 4.1 \\
\hline Sonora-Mojave Mixed Salt Desert Scrub & Distichlis spicata & Graminoid $\mathrm{C}_{4}$ & 3.6 & 4.1 \\
\hline $\begin{array}{l}\text { Southern Atlantic Coastal Plain Dune and } \\
\text { Maritime Grassland }\end{array}$ & Distichlis spicata & Graminoid $\mathrm{C}_{4}$ & 3.6 & 4.1 \\
\hline Temperate Pacific Tidal Salt and Brackish Marsh & Distichlis spicata & Graminoid $\mathrm{C}_{4}$ & 3.6 & 4.1 \\
\hline Texas Coast Salt and Brackish Tidal Marsh & Distichlis spicata & Graminoid $\mathrm{C}_{4}$ & 3.6 & 4.1 \\
\hline Western Great Plains Saline Depression Wetland & Distichlis spicata & Graminoid $\mathrm{C}_{4}$ & 3.6 & 4.1 \\
\hline $\begin{array}{l}\text { Western Great Plains Depressional Wetland } \\
\text { Systems }\end{array}$ & Distichlis spicata & Graminoid $\mathrm{C}_{4}$ & 3.6 & 4.1 \\
\hline $\begin{array}{l}\text { Temperate Pacific Subalpine-Montane Wet } \\
\text { Meadow }\end{array}$ & Dodecatheon jeffreyi & Forb & 3 & 3.5 \\
\hline Mediterranean California Alpine Fell-Field & Draba densifolia & Forb & 3 & 3.5 \\
\hline Rocky Mountain Alpine Dwarf-Shrubland & Dryas octopetala & Shrub & 2.7 & 2.8 \\
\hline Southern Coastal Plain Nonriverine Basin Swamp & Dulichium arundinaceum & Graminoid $C_{3}$ & 3.6 & 3.6 \\
\hline Tamaulipan Lomas & Ebenopsis ebano & Shrub- Evergreen & 2.7 & 2.4 \\
\hline Tamaulipan Floodplain & Ebenopsis ebano & Tree- Evergreen & 3.2 & 2.2 \\
\hline Central Mixedgrass Prairie & Echinacea angustifolia & Forb & 3 & 3.5 \\
\hline Tamaulipan Floodplain & Ehretia anacua & Tree- Evergreen & 3.2 & 2.2 \\
\hline Northwestern Great Plains Shrubland & Elaeagnus commutata & Shrub- Deciduous & 2.7 & 3 \\
\hline South Florida Everglades Sawgrass Marsh & Eleocharis cellulosa & Graminoid $C_{3}$ & 3.6 & 3.6 \\
\hline East Gulf Coastal Plain Depression Pond & Eleocharis elongata & Graminoid $C_{3}$ & 3.6 & 3.6 \\
\hline East Gulf Coastal Plain Depression Pond & Eleocharis equisetoides & Graminoid $\mathrm{C}_{3}$ & 3.6 & 3.6 \\
\hline East Gulf Coastal Plain Depression Pond & Eleocharis microcarpa & Graminoid $\mathrm{C}_{3}$ & 3.6 & 3.6 \\
\hline Temperate Pacific Freshwater Mudflat & Eleocharis obtusa & Graminoid $C_{3}$ & 3.6 & 3.6 \\
\hline $\begin{array}{l}\text { Columbia Plateau Silver Sagebrush Seasonally } \\
\text { Flooded Shrub-Steppe }\end{array}$ & Eleocharis palustris & Graminoid $C_{3}$ & 3.6 & 3.6 \\
\hline
\end{tabular}




\begin{tabular}{|c|c|c|c|c|}
\hline Community & Species & Classification & Resistance & Resilience \\
\hline Great Lakes Freshwater Estuary and Delta & Eleocharis palustris & Graminoid $\mathrm{C}_{3}$ & 3.6 & 3.6 \\
\hline North American Warm Desert Cienega & Eleocharis palustris & Graminoid $C_{3}$ & 3.6 & 3.6 \\
\hline North American Warm Desert Playa & Eleocharis palustris & Graminoid $C_{3}$ & 3.6 & 3.6 \\
\hline Northern Rocky Mountain Wooded Vernal Pool & Eleocharis palustris & Graminoid $\mathrm{C}_{3}$ & 3.6 & 3.6 \\
\hline Temperate Pacific Intertidal Flat & Eleocharis palustris & Graminoid $\mathrm{C}_{3}$ & 3.6 & 3.6 \\
\hline Temperate Pacific Tidal Salt and Brackish Marsh & Eleocharis palustris & Graminoid $C_{3}$ & 3.6 & 3.6 \\
\hline Western Great Plains Closed Depression Wetland & Eleocharis palustris & Graminoid $C_{3}$ & 3.6 & 3.6 \\
\hline $\begin{array}{l}\text { Western Great Plains Depressional Wetland } \\
\text { Systems }\end{array}$ & Eleocharis palustris & Graminoid $C_{3}$ & 3.6 & 3.6 \\
\hline Texas-Louisiana Coastal Prairie Pondshore & Eleocharis quadrangulata & Graminoid $\mathrm{C}_{3}$ & 3.6 & 3.6 \\
\hline Mediterranean California Serpentine Fen & Eleocharis quinqueflora & Graminoid $C_{3}$ & 3.6 & 3.6 \\
\hline Rocky Mountain Alpine-Montane Wet Meadow & Eleocharis quinqueflora & Graminoid $C_{3}$ & 3.6 & 3.6 \\
\hline Columbia Plateau Steppe and Grassland & Elymus elymoides & Graminoid $\mathrm{C}_{3}$ & 3.6 & 3.6 \\
\hline Inter-Mountain Basins Mat Saltbush Shrubland & Elymus elymoides & Graminoid $\mathrm{C}_{3}$ & 3.6 & 3.6 \\
\hline Inter-Mountain Basins Semi-Desert Shrub-Steppe & Elymus elymoides & Graminoid $C_{3}$ & 3.6 & 3.6 \\
\hline Klamath-Siskiyou Cliff and Outcrop & Elymus elymoides & Graminoid $C_{3}$ & 3.6 & 3.6 \\
\hline North Pacific Montane Grassland & Elymus elymoides & Graminoid $C_{3}$ & 3.6 & 3.6 \\
\hline $\begin{array}{l}\text { East Cascades Oak-Ponderosa Pine Forest and } \\
\text { Woodland }\end{array}$ & Elymus glaucus & Graminoid $C_{3}$ & 3.6 & 3.6 \\
\hline $\begin{array}{l}\text { Inter-Mountain Basins Aspen-Mixed Conifer Forest } \\
\text { and Woodland }\end{array}$ & Elymus glaucus & Graminoid $\mathrm{C}_{3}$ & 3.6 & 3.6 \\
\hline Mediterranean California Mixed Oak Woodland & Elymus glaucus & Graminoid $C_{3}$ & 3.6 & 3.6 \\
\hline North Pacific Coastal Cliff and Bluff & Elymus glaucus & Graminoid $C_{3}$ & 3.6 & 3.6 \\
\hline Inter-Mountain Basins Semi-Desert Shrub-Steppe & Elymus lanceolatus & Graminoid $C_{3}$ & 3.6 & 3.6 \\
\hline Inter-Mountain Basins Mat Saltbush Shrubland & Elymus lanceolatus & Graminoid $C_{3}$ & 3.6 & 3.6 \\
\hline Inter-Mountain Basins Mixed Salt Desert Scrub & Elymus lanceolatus & Graminoid $\mathrm{C}_{3}$ & 3.6 & 3.6 \\
\hline $\begin{array}{l}\text { Great Basin Foothill and Lower Montane Riparian } \\
\text { Woodland and Shrubland }\end{array}$ & Elymus trachycaulus & Graminoid $C_{3}$ & 3.6 & 3.6 \\
\hline $\begin{array}{l}\text { Northern Rocky Mountain Subalpine-Upper } \\
\text { Montane Grassland }\end{array}$ & Elymus trachycaulus & Graminoid $\mathrm{C}_{3}$ & 3.6 & 3.6 \\
\hline Northwestern Great Plains Riparian & Elymus trachycaulus & Graminoid $C_{3}$ & 3.6 & 3.6 \\
\hline $\begin{array}{l}\text { Southern Rocky Mountain Montane-Subalpine } \\
\text { Grassland }\end{array}$ & Elymus trachycaulus & Graminoid $C_{3}$ & 3.6 & 3.6 \\
\hline Acadian-Appalachian Alpine Tundra & Empetrum nigrum & Shrub & 2.7 & 2.8 \\
\hline Southern California Coastal Scrub & Encelia californica & Shrub- Deciduous & 2.7 & 3 \\
\hline Sonoran Mid-Elevation Desert Scrub & Encelia farinosa & Shrub- Deciduous & 2.7 & 3 \\
\hline Sonoran Paloverde-Mixed Cacti Desert Scrub & Encelia farinosa & Shrub- Deciduous & 2.7 & 3 \\
\hline $\begin{array}{l}\text { Colorado Plateau Blackbrush-Mormon-tea } \\
\text { Shrubland }\end{array}$ & Ephedra nevadensis & Shrub- Evergreen & 2.7 & 2.4 \\
\hline Inter-Mountain Basins Mixed Salt Desert Scrub & Ephedra nevadensis & Shrub- Evergreen & 2.7 & 2.4 \\
\hline Mojave Mid-Elevation Mixed Desert Scrub & Ephedra nevadensis & Shrub- Evergreen & 2.7 & 2.4 \\
\hline $\begin{array}{l}\text { Colorado Plateau Blackbrush-Mormon-tea } \\
\text { Shrubland }\end{array}$ & Ephedra torreyana & Shrub- Evergreen & 2.7 & 2.4 \\
\hline Inter-Mountain Basins Shale Badland & Ephedra torreyana & Shrub- Evergreen & 2.7 & 2.4 \\
\hline Southern Colorado Plateau Sand Shrubland & Ephedra torreyana & Shrub-Evergreen & 2.7 & 2.4 \\
\hline $\begin{array}{l}\text { Colorado Plateau Blackbrush-Mormon-tea } \\
\text { Shrubland }\end{array}$ & Ephedra viridis & Shrub- Evergreen & 2.7 & 2.4 \\
\hline Inter-Mountain Basins Active and Stabilized Dune & Ephedra viridis & Shrub- Evergreen & 2.7 & 2.4 \\
\hline Southern Colorado Plateau Sand Shrubland & Ephedra viridis & Shrub- Evergreen & 2.7 & 2.4 \\
\hline
\end{tabular}




\begin{tabular}{|c|c|c|c|c|}
\hline Community & Species & Classification & Resistance & Resilience \\
\hline $\begin{array}{l}\text { Colorado Plateau Blackbrush-Mormon-tea } \\
\text { Shrubland }\end{array}$ & Ephedra viridis & Shrub- Evergreen & 2.7 & 2.4 \\
\hline Southern Colorado Plateau Sand Shrubland & Ephedra viridis & Shrub- Evergreen & 2.7 & 2.4 \\
\hline North Pacific Shrub Swamp & Equisetum fluviatile & Graminoid $\mathrm{C}_{3}$ & 3.6 & 3.6 \\
\hline Temperate Pacific Freshwater Mudflat & Eragrostis hypnoides & Graminoid $\mathrm{C}_{4}$ & 3.6 & 4.1 \\
\hline Madrean Encinal & Eragrostis intermedia & Graminoid $\mathrm{C}_{4}$ & 3.6 & 4.1 \\
\hline Rocky Mountain Alpine Fell-Field & Eremogone capillaris & Forb & 3 & 3.5 \\
\hline Inter-Mountain Basins Mat Saltbush Shrubland & Eremogone hookeri & Forb & 3 & 3.5 \\
\hline Sierra Nevada Alpine Dwarf-Shrubland & Ericameria discoidea & Shrub-Deciduous & 2.7 & 3 \\
\hline North American Warm Desert Badland & Ericameria linearifolia & Shrub-Deciduous & 2.7 & 3 \\
\hline Sonoran Mid-Elevation Desert Scrub & Ericameria linearifolia & Shrub-Deciduous & 2.7 & 3 \\
\hline Inter-Mountain Basins Active and Stabilized Dune & Ericameria nauseosa & Shrub- Deciduous & 2.7 & 3 \\
\hline Inter-Mountain Basins Big Sagebrush Shrubland & Ericameria nauseosa & Shrub- Deciduous & 2.7 & 3 \\
\hline Inter-Mountain Basins Greasewood Flat & Ericameria nauseosa & Shrub- Deciduous & 2.7 & 3 \\
\hline Inter-Mountain Basins Mixed Salt Desert Scrub & Ericameria nauseosa & Shrub- Deciduous & 2.7 & 3 \\
\hline Inter-Mountain Basins Wash & Ericameria nauseosa & Shrub- Deciduous & 2.7 & 3 \\
\hline Southern Colorado Plateau Sand Shrubland & Ericameria nauseosa & Shrub-Deciduous & 2.7 & 3 \\
\hline Southern Coastal Plain Nonriverine Basin Swamp & Eriocaulon compressum & Forb & 3 & 3.5 \\
\hline Southern California Coastal Scrub & Eriogonum cinereum & Shrub- Deciduous & 2.7 & 3 \\
\hline North American Warm Desert Badland & Eriogonum fasciculatum & Forb & 3 & 3.5 \\
\hline Mojave Mid-Elevation Mixed Desert Scrub & Eriogonum fasciculatum & Shrub & 2.7 & 2.8 \\
\hline Southern California Coastal Scrub & Eriogonum fasciculatum & Shrub- Deciduous & 2.7 & 3 \\
\hline North American Warm Desert Badland & Eriogonum inflatum & Forb & 3 & 3.5 \\
\hline Mediterranean California Serpentine Barrens & Eriogonum nudum & Forb & 3 & 3.5 \\
\hline Sierra Nevada Alpine Dwarf-Shrubland & Eriogonum ovalifolium & Forb & 3 & 3.5 \\
\hline Columbia Plateau Scabland Shrubland & Eriogonum thymoides & Forb & 3 & 3.5 \\
\hline Mediterranean California Serpentine Barrens & Eriogonum ursinum & Forb & 3 & 3.5 \\
\hline $\begin{array}{l}\text { North-Central Interior and Appalachian Acidic } \\
\text { Peatland }\end{array}$ & Eriophorum virginicum & Graminoid $C_{3}$ & 3.6 & 3.6 \\
\hline Southern Coastal Plain Nonriverine Basin Swamp & Eriophorum virginicum & Graminoid $C_{3}$ & 3.6 & 3.6 \\
\hline Sonora-Mojave Mixed Salt Desert Scrub & Erodium cicutarium & Forb & 3 & 3.5 \\
\hline South Florida Hardwood Hammock & Eugenia axillaris & Shrub- Evergreen & 2.7 & 2.4 \\
\hline South Florida Hardwood Hammock & Eugenia foetida & Shrub- Evergreen & 2.7 & 2.4 \\
\hline Eastern Highland Rim Prairie and Barrens & Eurybia hemispherica & Forb & 3 & 3.5 \\
\hline Great Lakes Acidic Rocky Shore and Cliff & Euthamia graminifolia & Forb & 3 & 3.5 \\
\hline Tamaulipan Calcareous Thornscrub & Eysenhardtia texana & Shrub- Deciduous & 2.7 & 3 \\
\hline Crowley's Ridge Mesic Loess Slope Forest & Fagus grandifolia & Tree- Deciduous & 3 & 4.2 \\
\hline East Gulf Coastal Plain Northern Loess Bluff Forest & Fagus grandifolia & Tree- Deciduous & 3 & 4.2 \\
\hline $\begin{array}{l}\text { East Gulf Coastal Plain Northern Mesic Hardwood } \\
\text { Slope Forest }\end{array}$ & Fagus grandifolia & Tree- Deciduous & 3 & 4.2 \\
\hline $\begin{array}{l}\text { East Gulf Coastal Plain Southern Loess Bluff } \\
\text { Forest }\end{array}$ & Fagus grandifolia & Tree- Deciduous & 3 & 4.2 \\
\hline Laurentian-Acadian Northern Hardwood Forest & Fagus grandifolia & Tree- Deciduous & 3 & 4.2 \\
\hline North-Central Interior Beech-Maple Forest & Fagus grandifolia & Tree- Deciduous & 3 & 4.2 \\
\hline North-Central Interior Maple-Basswood Forest & Fagus grandifolia & Tree-Deciduous & 3 & 4.2 \\
\hline Northern Atlantic Coastal Plain Hardwood Forest & Fagus grandifolia & Tree- Deciduous & 3 & 4.2 \\
\hline Ozark-Ouachita Mesic Hardwood Forest & Fagus grandifolia & Tree- Deciduous & 3 & 4.2 \\
\hline South-Central Interior Mesophytic Forest & Fagus grandifolia & Tree-Deciduous & 3 & 4.2 \\
\hline
\end{tabular}




\begin{tabular}{|c|c|c|c|c|}
\hline Community & Species & Classification & Resistance & Resilience \\
\hline Southern and Central Appalachian Cove Forest & Fagus grandifolia & Tree-Deciduous & 3 & 4.2 \\
\hline Southern Appalachian Northern Hardwood Forest & Fagus grandifolia & Tree- Deciduous & 3 & 4.2 \\
\hline $\begin{array}{l}\text { Southern Atlantic Coastal Plain Mesic Hardwood } \\
\text { Forest }\end{array}$ & Fagus grandifolia & Tree- Deciduous & 3 & 4.2 \\
\hline Southern Piedmont Mesic Forest & Fagus grandifolia & Tree- Deciduous & 3 & 4.2 \\
\hline West Gulf Coastal Plain Mesic Hardwood Forest & Fagus grandifolia & Tree-Deciduous & 3 & 4.2 \\
\hline Appalachian (Hemlock)-Northern Hardwood Forest & Fagus grandifolia & Tree- Deciduous & 3 & 4.2 \\
\hline $\begin{array}{l}\text { Inter-Mountain Basins Volcanic Rock and Cinder } \\
\text { Land }\end{array}$ & Fallugia paradoxa & Shrub- Evergreen & 2.7 & 2.4 \\
\hline Inter-Mountain Basins Wash & Fallugia paradoxa & Shrub- Evergreen & 2.7 & 2.4 \\
\hline North American Warm Desert Wash & Fallugia paradoxa & Shrub-Evergreen & 2.7 & 2.4 \\
\hline $\begin{array}{l}\text { Inter-Mountain Basins Montane Sagebrush } \\
\text { Steppe }\end{array}$ & Festuca arizonica & Graminoid $C_{3}$ & 3.6 & 3.6 \\
\hline $\begin{array}{l}\text { Southern Rocky Mountain Montane-Subalpine } \\
\text { Grassland }\end{array}$ & Festuca arizonica & Graminoid $C_{3}$ & 3.6 & 3.6 \\
\hline Rocky Mountain Alpine Turf & Festuca brachyphylla & Graminoid $\mathrm{C}_{3}$ & 3.6 & 3.6 \\
\hline Mediterranean California Mixed Oak Woodland & Festuca californica & Graminoid $\mathrm{C}_{3}$ & 3.6 & 3.6 \\
\hline $\begin{array}{l}\text { Northern Rocky Mountain Subalpine-Upper } \\
\text { Montane Grassland }\end{array}$ & Festuca campestris & Graminoid $\mathrm{C}_{3}$ & 3.6 & 3.6 \\
\hline Northwestern Great Plains Mixedgrass Prairie & Festuca campestris & Graminoid $C_{3}$ & 3.6 & 3.6 \\
\hline $\begin{array}{l}\text { Rocky Mountain Foothill Limber Pine-Juniper } \\
\text { Woodland }\end{array}$ & Festuca campestris & Graminoid $C_{3}$ & 3.6 & 3.6 \\
\hline $\begin{array}{l}\text { Northern Rocky Mountain Lower Montane, Foothill } \\
\text { and Valley Grassland }\end{array}$ & Festuca campestris & Graminoid $C_{3}$ & 3.6 & 3.6 \\
\hline California Northern Coastal Grassland & Festuca idahoensis & Graminoid $C_{3}$ & 3.6 & 3.6 \\
\hline $\begin{array}{l}\text { Columbia Basin Foothill and Canyon Dry } \\
\text { Grassland }\end{array}$ & Festuca idahoensis & Graminoid $\mathrm{C}_{3}$ & 3.6 & 3.6 \\
\hline Columbia Basin Palouse Prairie & Festuca idahoensis & Graminoid $C_{3}$ & 3.6 & 3.6 \\
\hline $\begin{array}{l}\text { Columbia Plateau Silver Sagebrush Seasonally } \\
\text { Flooded Shrub-Steppe }\end{array}$ & Festuca idahoensis & Graminoid $\mathrm{C}_{3}$ & 3.6 & 3.6 \\
\hline $\begin{array}{l}\text { Inter-Mountain Basins Curl-leaf Mountain- } \\
\text { mahogany Woodland and Shrubland }\end{array}$ & Festuca idahoensis & Graminoid $\mathrm{C}_{3}$ & 3.6 & 3.6 \\
\hline Inter-Mountain Basins Semi-Desert Shrub-Steppe & Festuca idahoensis & Graminoid $\mathrm{C}_{3}$ & 3.6 & 3.6 \\
\hline Mediterranean California Mixed Oak Woodland & Festuca idahoensis & Graminoid $\mathrm{C}_{3}$ & 3.6 & 3.6 \\
\hline North Pacific Alpine and Subalpine Dry Grassland & Festuca idahoensis & Graminoid $C_{3}$ & 3.6 & 3.6 \\
\hline North Pacific Montane Grassland & Festuca idahoensis & Graminoid $C_{3}$ & 3.6 & 3.6 \\
\hline $\begin{array}{l}\text { Northern Rocky Mountain Subalpine-Upper } \\
\text { Montane Grassland }\end{array}$ & Festuca idahoensis & Graminoid $C_{3}$ & 3.6 & 3.6 \\
\hline Northwestern Great Plains Mixedgrass Prairie & Festuca idahoensis & Graminoid $\mathrm{C}_{3}$ & 3.6 & 3.6 \\
\hline Rocky Mountain Alpine Turf & Festuca idahoensis & Graminoid $C_{3}$ & 3.6 & 3.6 \\
\hline $\begin{array}{l}\text { Rocky Mountain Foothill Limber Pine-Juniper } \\
\text { Woodland }\end{array}$ & Festuca idahoensis & Graminoid $\mathrm{C}_{3}$ & 3.6 & 3.6 \\
\hline $\begin{array}{l}\text { Rocky Mountain Subalpine-Montane Mesic } \\
\text { Meadow }\end{array}$ & Festuca idahoensis & Graminoid $C_{3}$ & 3.6 & 3.6 \\
\hline $\begin{array}{l}\text { Southern Rocky Mountain Montane-Subalpine } \\
\text { Grassland }\end{array}$ & Festuca idahoensis & Graminoid $\mathrm{C}_{3}$ & 3.6 & 3.6 \\
\hline $\begin{array}{l}\text { Wyoming Basins Dwarf Sagebrush Shrubland and } \\
\text { Steppe }\end{array}$ & Festuca idahoensis & Graminoid $\mathrm{C}_{3}$ & 3.6 & 3.6 \\
\hline $\begin{array}{l}\text { Northern Rocky Mountain Lower Montane, Foothill } \\
\text { and Valley Grassland }\end{array}$ & Festuca idahoensis & Graminoid $C_{3}$ & 3.6 & 3.6 \\
\hline $\begin{array}{l}\text { Rocky Mountain Foothill Limber Pine-Juniper } \\
\text { Woodland }\end{array}$ & Festuca kingii & Graminoid $\mathrm{C}_{3}$ & 3.6 & 3.6 \\
\hline
\end{tabular}




\begin{tabular}{|c|c|c|c|c|}
\hline Community & Species & Classification & Resistance & Resilience \\
\hline North Pacific Alpine and Subalpine Dry Grassland & Festuca roemeri & Graminoid $C_{3}$ & 3.6 & 3.6 \\
\hline North Pacific Herbaceous Bald and Bluff & Festuca roemeri & Graminoid $\mathrm{C}_{3}$ & 3.6 & 3.6 \\
\hline Willamette Valley Upland Prairie and Savanna & Festuca roemeri & Graminoid $\mathrm{C}_{3}$ & 3.6 & 3.6 \\
\hline California Northern Coastal Grassland & Festuca rubra & Graminoid $C_{3}$ & 3.6 & 3.6 \\
\hline North Pacific Herbaceous Bald and Bluff & Festuca rubra & Graminoid $C_{3}$ & 3.6 & 3.6 \\
\hline $\begin{array}{l}\text { North Pacific Hypermaritime Shrub and } \\
\text { Herbaceous Headland }\end{array}$ & Festuca rubra & Graminoid $C_{3}$ & 3.6 & 3.6 \\
\hline $\begin{array}{l}\text { North Pacific Maritime Coastal Sand Dune and } \\
\text { Strand }\end{array}$ & Festuca rubra & Graminoid $C_{3}$ & 3.6 & 3.6 \\
\hline Temperate Pacific Tidal Salt and Brackish Marsh & Festuca rubra & Graminoid $\mathrm{C}_{3}$ & 3.6 & 3.6 \\
\hline $\begin{array}{l}\text { Inter-Mountain Basins Montane Sagebrush } \\
\text { Steppe }\end{array}$ & Festuca thurberi & Graminoid $C_{3}$ & 3.6 & 3.6 \\
\hline $\begin{array}{l}\text { Rocky Mountain Subalpine-Montane Limber- } \\
\text { Bristlecone Pine Woodland }\end{array}$ & Festuca thurberi & Graminoid $C_{3}$ & 3.6 & 3.6 \\
\hline $\begin{array}{l}\text { Southern Rocky Mountain Montane-Subalpine } \\
\text { Grassland }\end{array}$ & Festuca thurberi & Graminoid $C_{3}$ & 3.6 & 3.6 \\
\hline North Pacific Alpine and Subalpine Dry Grassland & Festuca viridula & Graminoid $\mathrm{C}_{3}$ & 3.6 & 3.6 \\
\hline $\begin{array}{l}\text { Northern Rocky Mountain Subalpine-Upper } \\
\text { Montane Grassland }\end{array}$ & Festuca viridula & Graminoid $\mathrm{C}_{3}$ & 3.6 & 3.6 \\
\hline Southeastern Coastal Plain Interdunal Wetland & Fimbristylis castanea & Graminoid $\mathrm{C}_{3}$ & 3.6 & 3.6 \\
\hline North American Warm Desert Cienega & Flaveria chlorifolia & Forb & 3 & 3.5 \\
\hline Chihuahuan Creosotebush Desert Scrub & Flourensia cernua & Shrub- Deciduous & 2.7 & 3 \\
\hline Chihuahuan Loamy Plains Desert Grassland & Flourensia cernua & Shrub-Deciduous & 2.7 & 3 \\
\hline Chihuahuan Mixed Desert and Thornscrub & Flourensia cernua & Shrub-Deciduous & 2.7 & 3 \\
\hline Chihuahuan Mixed Salt Desert Scrub & Flourensia cernua & Shrub-Deciduous & 2.7 & 3 \\
\hline Nashville Basin Limestone Glade and Woodland & Forestiera ligustrina & Shrub- Deciduous & 2.7 & 3 \\
\hline $\begin{array}{l}\text { Rocky Mountain Lower Montane-Foothill Riparian } \\
\text { Woodland and Shrubland }\end{array}$ & Forestiera pubescens & Shrub-Deciduous & 2.7 & 3 \\
\hline Northern California Coastal Scrub & Frangula californica & Shrub- Evergreen & 2.7 & 2.4 \\
\hline $\begin{array}{l}\text { Mediterranean California Serpentine Foothill and } \\
\text { Lower Montane Riparian Woodland and Seep }\end{array}$ & Frangula californica & Shrub- Evergreen & 2.7 & 2.4 \\
\hline $\begin{array}{l}\text { North Pacific Lowland Mixed Hardwood-Conifer } \\
\text { Forest }\end{array}$ & Frangula purshiana & Shrub-Deciduous & 2.7 & 3 \\
\hline Central Appalachian Alkaline Glade and Woodland & Fraxinus americana & Tree- Deciduous & 3 & 4.2 \\
\hline Crowley's Ridge Mesic Loess Slope Forest & Fraxinus americana & Tree-Deciduous & 3 & 4.2 \\
\hline Laurentian-Acadian Northern Hardwood Forest & Fraxinus americana & Tree-Deciduous & 3 & 4.2 \\
\hline $\begin{array}{l}\text { North-Central Appalachian Circumneutral Cliff and } \\
\text { Talus }\end{array}$ & Fraxinus americana & Tree- Deciduous & 3 & 4.2 \\
\hline Southeastern Great Plains Floodplain Forest & Fraxinus americana & Tree- Deciduous & 3 & 4.2 \\
\hline Southern and Central Appalachian Cove Forest & Fraxinus americana & Tree- Deciduous & 3 & 4.2 \\
\hline Southern Coastal Plain Limestone Forest & Fraxinus americana & Tree- Deciduous & 3 & 4.2 \\
\hline Southern Piedmont Mesic Forest & Fraxinus americana & Tree- Deciduous & 3 & 4.2 \\
\hline Tamaulipan Floodplain & Fraxinus berlandieriana & Tree- Deciduous & 3 & 4.2 \\
\hline Tamaulipan Palm Grove Riparian Forest & Fraxinus berlandieriana & Tree- Deciduous & 3 & 4.2 \\
\hline $\begin{array}{l}\text { East Gulf Coastal Plain Small Stream and River } \\
\text { Floodplain Forest }\end{array}$ & Fraxinus caroliniana & Tree- Deciduous & 3 & 4.2 \\
\hline California Mesic Chaparral & Fraxinus dipetala & Tree- Deciduous & 3 & 4.2 \\
\hline North Pacific Hardwood-Conifer Swamp & Fraxinus latifolia & Tree-Deciduous & 3 & 4.2 \\
\hline $\begin{array}{l}\text { North Pacific Lowland Riparian Forest and } \\
\text { Shrubland }\end{array}$ & Fraxinus latifolia & Tree- Deciduous & 3 & 4.2 \\
\hline North Pacific Shrub Swamp & Fraxinus latifolia & Tree- Deciduous & 3 & 4.2 \\
\hline
\end{tabular}




\begin{tabular}{|c|c|c|c|c|}
\hline Community & Species & Classification & Resistance & Resilience \\
\hline Northern Rocky Mountain Wooded Vernal Pool & Fraxinus latifolia & Tree-Deciduous & 3 & 4.2 \\
\hline Eastern Boreal Floodplain & Fraxinus nigra & Tree-Deciduous & 3 & 4.2 \\
\hline High Allegheny Wetland & Fraxinus nigra & Tree-Deciduous & 3 & 4.2 \\
\hline $\begin{array}{l}\text { Laurentian-Acadian Alkaline Conifer-Hardwood } \\
\text { Swamp }\end{array}$ & Fraxinus nigra & Tree- Deciduous & 3 & 4.2 \\
\hline $\begin{array}{l}\text { North-Central Interior and Appalachian Rich } \\
\text { Swamp }\end{array}$ & Fraxinus nigra & Tree- Deciduous & 3 & 4.2 \\
\hline $\begin{array}{l}\text { Atlantic Coastal Plain Small Brownwater River } \\
\text { Floodplain Forest }\end{array}$ & Fraxinus pennsylvanica & Tree- Deciduous & 3 & 4.2 \\
\hline Central Appalachian River Floodplain & Fraxinus pennsylvanica & Tree- Deciduous & 3 & 4.2 \\
\hline $\begin{array}{l}\text { East Gulf Coastal Plain Freshwater Tidal Wooded } \\
\text { Swamp }\end{array}$ & Fraxinus pennsylvanica & Tree- Deciduous & 3 & 4.2 \\
\hline $\begin{array}{l}\text { East Gulf Coastal Plain Large River Floodplain } \\
\text { Forest }\end{array}$ & Fraxinus pennsylvanica & Tree- Deciduous & 3 & 4.2 \\
\hline Edwards Plateau Riparian & Fraxinus pennsylvanica & Tree- Deciduous & 3 & 4.2 \\
\hline North-Central Interior Floodplain & Fraxinus pennsylvanica & Tree- Deciduous & 3 & 4.2 \\
\hline Northern Atlantic Coastal Plain Tidal Swamp & Fraxinus pennsylvanica & Tree- Deciduous & 3 & 4.2 \\
\hline Northwestern Great Plains Riparian & Fraxinus pennsylvanica & Tree-Deciduous & 3 & 4.2 \\
\hline $\begin{array}{l}\text { Northwestern Great Plains-Black Hills Ponderosa } \\
\text { Pine Woodland and Savanna }\end{array}$ & Fraxinus pennsylvanica & Tree-Deciduous & 3 & 4.2 \\
\hline Southeastern Great Plains Floodplain Forest & Fraxinus pennsylvanica & Tree- Deciduous & 3 & 4.2 \\
\hline Southeastern Great Plains Riparian Forest & Fraxinus pennsylvanica & Tree- Deciduous & 3 & 4.2 \\
\hline $\begin{array}{l}\text { Southern Atlantic Coastal Plain Tidal Wooded } \\
\text { Swamp }\end{array}$ & Fraxinus pennsylvanica & Tree-Deciduous & 3 & 4.2 \\
\hline Southern Piedmont Large Floodplain Forest & Fraxinus pennsylvanica & Tree-Deciduous & 3 & 4.2 \\
\hline $\begin{array}{l}\text { Southern Piedmont Small Floodplain and Riparian } \\
\text { Forest }\end{array}$ & Fraxinus pennsylvanica & Tree-Deciduous & 3 & 4.2 \\
\hline Great Plains Wooded Draw and Ravine & Fraxinus pennsylvanica & Tree- Deciduous & 3 & 4.2 \\
\hline $\begin{array}{l}\text { Edwards Plateau Dry-Mesic Slope Forest and } \\
\text { Woodland }\end{array}$ & Fraxinus texensis & Tree- Deciduous & 3 & 4.2 \\
\hline Edwards Plateau Floodplain Terrace & Fraxinus texensis & Tree-Deciduous & 3 & 4.2 \\
\hline $\begin{array}{l}\text { North American Warm Desert Lower Montane } \\
\text { Riparian Woodland and Shrubland }\end{array}$ & Fraxinus velutina & Tree- Deciduous & 3 & 4.2 \\
\hline $\begin{array}{l}\text { North American Warm Desert Riparian Woodland } \\
\text { and Shrubland }\end{array}$ & Fraxinus velutina & Tree-Deciduous & 3 & 4.2 \\
\hline East Gulf Coastal Plain Depression Pond & Fuirena scirpoidea & Graminoid $\mathrm{C}_{3}$ & 3.6 & 3.6 \\
\hline Southeastern Coastal Plain Interdunal Wetland & Fuirena scirpoidea & Graminoid $C_{3}$ & 3.6 & 3.6 \\
\hline California Mesic Chaparral & Garrya elliptica & Tree- Evergreen & 3.2 & 2.2 \\
\hline California Mesic Chaparral & Garrya flavescens & Shrub- Evergreen & 2.7 & 2.4 \\
\hline Sonora-Mojave Semi-Desert Chaparral & Garrya flavescens & Shrub- Evergreen & 2.7 & 2.4 \\
\hline Madrean Oriental Chaparral & Garrya wrightii & Shrub- Evergreen & 2.7 & 2.4 \\
\hline $\begin{array}{l}\text { North Pacific Hypermaritime Shrub and } \\
\text { Herbaceous Headland }\end{array}$ & Gaultheria shallon & Shrub- Evergreen & 2.7 & 2.4 \\
\hline $\begin{array}{l}\text { North Pacific Maritime Coastal Sand Dune and } \\
\text { Strand }\end{array}$ & Gaultheria shallon & Shrub- Evergreen & 2.7 & 2.4 \\
\hline North Pacific Montane Shrubland & Gaultheria shallon & Shrub- Evergreen & 2.7 & 2.4 \\
\hline $\begin{array}{l}\text { Northern Atlantic Coastal Plain Heathland and } \\
\text { Grassland }\end{array}$ & Gaylussacia baccata & Shrub- Deciduous & 2.7 & 3 \\
\hline $\begin{array}{l}\text { Southern Atlantic Coastal Plain Wet Pine Savanna } \\
\text { and Flatwoods }\end{array}$ & Gaylussacia frondosa & Shrub- Deciduous & 2.7 & 3 \\
\hline Southern Piedmont Granite Flatrock and Outcrop & Gelsemium sempervirens & Forb & 3 & 3.5 \\
\hline Rocky Mountain Alpine Fell-Field & Geum rossii & Forb & 3 & 3.5 \\
\hline
\end{tabular}




\begin{tabular}{|c|c|c|c|c|}
\hline Community & Species & Classification & Resistance & Resilience \\
\hline Rocky Mountain Alpine Turf & Geum rossii & Forb & 3 & 3.5 \\
\hline Rocky Mountain Alpine Turf & Geum rossii & Forb & 3 & 3.5 \\
\hline Temperate Pacific Intertidal Flat & Glaux maritima & Forb & 3 & 3.5 \\
\hline Southeastern Great Plains Riparian Forest & Gleditsia triacanthos & Tree-Deciduous & 3 & 4.2 \\
\hline $\begin{array}{l}\text { Great Basin Foothill and Lower Montane Riparian } \\
\text { Woodland and Shrubland }\end{array}$ & Glyceria striata & Graminoid $\mathrm{C}_{3}$ & 3.6 & 3.6 \\
\hline $\begin{array}{l}\text { Temperate Pacific Subalpine-Montane Wet } \\
\text { Meadow }\end{array}$ & Glyceria striata & Graminoid $\mathrm{C}_{3}$ & 3.6 & 3.6 \\
\hline Temperate Pacific Freshwater Mudflat & Gnaphalium palustre & Forb & 3 & 3.5 \\
\hline $\begin{array}{l}\text { Atlantic Coastal Plain Peatland Pocosin and } \\
\text { Canebrake }\end{array}$ & Gordonia lasianthus & Shrub- Evergreen & 2.7 & 2.4 \\
\hline $\begin{array}{l}\text { Colorado Plateau Blackbrush-Mormon-tea } \\
\text { Shrubland }\end{array}$ & Grayia spinosa & Shrub- Deciduous & 2.7 & 3 \\
\hline Columbia Plateau Ash and Tuff Badland & Grayia spinosa & Shrub- Deciduous & 2.7 & 3 \\
\hline Great Basin Xeric Mixed Sagebrush Shrubland & Grayia spinosa & Shrub-Deciduous & 2.7 & 3 \\
\hline Inter-Mountain Basins Big Sagebrush Shrubland & Grayia spinosa & Shrub- Deciduous & 2.7 & 3 \\
\hline Inter-Mountain Basins Mixed Salt Desert Scrub & Grayia spinosa & Shrub-Deciduous & 2.7 & 3 \\
\hline Inter-Mountain Basins Playa & Grayia spinosa & Shrub- Deciduous & 2.7 & 3 \\
\hline Inter-Mountain Basins Wash & Grayia spinosa & Shrub-Deciduous & 2.7 & 3 \\
\hline Mojave Mid-Elevation Mixed Desert Scrub & Grayia spinosa & Shrub- Deciduous & 2.7 & 3 \\
\hline Chihuahuan Creosotebush Desert Scrub & Gutierrezia sarothrae & Shrub & 2.7 & 2.8 \\
\hline $\begin{array}{l}\text { Chihuahuan Stabilized Coppice Dune and Sand } \\
\text { Flat Scrub }\end{array}$ & Gutierrezia sarothrae & Shrub- Deciduous & 2.7 & 3 \\
\hline $\begin{array}{l}\text { Colorado Plateau Mixed Low Sagebrush } \\
\text { Shrubland }\end{array}$ & Gutierrezia sarothrae & Shrub- Deciduous & 2.7 & 3 \\
\hline Inter-Mountain Basins Semi-Desert Grassland & Gutierrezia sarothrae & Shrub-Deciduous & 2.7 & 3 \\
\hline $\begin{array}{l}\text { Llano Estacado Caprock Escarpment and Breaks } \\
\text { Shrubland and Steppe }\end{array}$ & Gutierrezia sarothrae & Shrub- Deciduous & 2.7 & 3 \\
\hline Western Great Plains Badlands & Gutierrezia sarothrae & Shrub- Deciduous & 2.7 & 3 \\
\hline South Florida Hardwood Hammock & Gymnanthes lucida & Shrub- Evergreen & 2.7 & 2.4 \\
\hline Eastern Highland Rim Prairie and Barrens & Gymnopogon brevifolius & Graminoid $\mathrm{C}_{4}$ & 3.6 & 4.1 \\
\hline Southern and Central Appalachian Cove Forest & Halesia tetraptera & Shrub-Deciduous & 2.7 & 3 \\
\hline Tamaulipan Lomas & Havardia pallens & Shrub- Deciduous & 2.7 & 3 \\
\hline Eastern Highland Rim Prairie and Barrens & Helianthus angustifolius & Forb & 3 & 3.5 \\
\hline Tamaulipan Calcareous Thornscrub & Helietta parvifolia & Shrub- Evergreen & 2.7 & 2.4 \\
\hline California Mesic Serpentine Grassland & Hemizonia congesta & Forb & 3 & 3.5 \\
\hline $\begin{array}{l}\text { Colorado Plateau Mixed Low Sagebrush } \\
\text { Shrubland }\end{array}$ & Hesperostipa comata & Graminoid $\mathrm{C}_{3}$ & 3.6 & 3.6 \\
\hline Inter-Mountain Basins Active and Stabilized Dune & Hesperostipa comata & Graminoid $C_{3}$ & 3.6 & 3.6 \\
\hline Inter-Mountain Basins Big Sagebrush Steppe & Hesperostipa comata & Graminoid $\mathrm{C}_{3}$ & 3.6 & 3.6 \\
\hline Inter-Mountain Basins Juniper Savanna & Hesperostipa comata & Graminoid $\mathrm{C}_{3}$ & 3.6 & 3.6 \\
\hline Inter-Mountain Basins Mat Saltbush Shrubland & Hesperostipa comata & Graminoid $\mathrm{C}_{3}$ & 3.6 & 3.6 \\
\hline $\begin{array}{l}\text { Inter-Mountain Basins Montane Sagebrush } \\
\text { Steppe }\end{array}$ & Hesperostipa comata & Graminoid $C_{3}$ & 3.6 & 3.6 \\
\hline Inter-Mountain Basins Semi-Desert Grassland & Hesperostipa comata & Graminoid $C_{3}$ & 3.6 & 3.6 \\
\hline Inter-Mountain Basins Semi-Desert Shrub-Steppe & Hesperostipa comata & Graminoid $\mathrm{C}_{3}$ & 3.6 & 3.6 \\
\hline $\begin{array}{l}\text { Rocky Mountain Foothill Limber Pine-Juniper } \\
\text { Woodland }\end{array}$ & Hesperostipa comata & Graminoid $C_{3}$ & 3.6 & 3.6 \\
\hline $\begin{array}{l}\text { Western Great Plains Foothill and Piedmont } \\
\text { Grassland }\end{array}$ & Hesperostipa comata & Graminoid $\mathrm{C}_{3}$ & 3.6 & 3.6 \\
\hline
\end{tabular}




\begin{tabular}{|c|c|c|c|c|}
\hline Community & Species & Classification & Resistance & Resilience \\
\hline $\begin{array}{l}\text { Northern Rocky Mountain Lower Montane, Foothill } \\
\text { and Valley Grassland }\end{array}$ & Hesperostipa comata & Graminoid $\mathrm{C}_{3}$ & 3.6 & 3.6 \\
\hline Northwestern Great Plains Mixedgrass Prairie & Hesperostipa comata & Graminoid $\mathrm{C}_{3}$ & 3.6 & 3.6 \\
\hline Western Great Plains Sand Prairie & Hesperostipa comata & Graminoid $\mathrm{C}_{3}$ & 3.6 & 3.6 \\
\hline $\begin{array}{l}\text { Western Great Plains Foothill and Piedmont } \\
\text { Grassland }\end{array}$ & Hesperostipa neomexicana & Graminoid $\mathrm{C}_{3}$ & 3.6 & 3.6 \\
\hline $\begin{array}{l}\text { North-Central Interior Sand and Gravel Tallgrass } \\
\text { Prairie }\end{array}$ & Hesperostipa spartea & Graminoid $C_{3}$ & 3.6 & 3.6 \\
\hline Northern Tallgrass Prairie & Hesperostipa spartea & Graminoid $C_{3}$ & 3.6 & 3.6 \\
\hline Central Tallgrass Prairie & Hesperostipa spartea & Graminoid $\mathrm{C}_{3}$ & 3.6 & 3.6 \\
\hline California Mesic Chaparral & Heteromeles arbutifolia & Shrub- Evergreen & 2.7 & 2.4 \\
\hline Central Interior Calcareous Cliff and Talus & Heuchera americana & Forb & 3 & 3.5 \\
\hline Cumberland Acidic Cliff and Rockhouse & Heuchera parviflora & Forb & 3 & 3.5 \\
\hline Southern Appalachian Montane Cliff and Talus & Heuchera villosa & Forb & 3 & 3.5 \\
\hline $\begin{array}{l}\text { Northern Atlantic Coastal Plain Fresh and } \\
\text { Oligohaline Tidal Marsh }\end{array}$ & Hibiscus moscheutos & Forb & 3 & 3.5 \\
\hline $\begin{array}{l}\text { Apacherian-Chihuahuan Semi-Desert Grassland } \\
\text { and Steppe }\end{array}$ & Hilaria belangeri & Graminoid $\mathrm{C}_{4}$ & 3.6 & 4.1 \\
\hline Madrean Encinal & Hilaria belangeri & Graminoid $\mathrm{C}_{4}$ & 3.6 & 4.1 \\
\hline Madrean Juniper Savanna & Hilaria belangeri & Graminoid $\mathrm{C}_{4}$ & 3.6 & 4.1 \\
\hline California Montane Woodland and Chaparral & Holodiscus discolor & Shrub- Deciduous & 2.7 & 3 \\
\hline $\begin{array}{l}\text { Mediterranean California Dry-Mesic Mixed Conifer } \\
\text { Forest and Woodland }\end{array}$ & Holodiscus discolor & Shrub- Deciduous & 2.7 & 3 \\
\hline North Pacific Montane Shrubland & Holodiscus discolor & Shrub- Deciduous & 2.7 & 3 \\
\hline Northern California Coastal Scrub & Holodiscus discolor & Shrub- Deciduous & 2.7 & 3 \\
\hline $\begin{array}{l}\text { Northern Rocky Mountain Montane-Foothill } \\
\text { Deciduous Shrubland }\end{array}$ & Holodiscus discolor & Shrub- Deciduous & 2.7 & 3 \\
\hline Western Great Plains Closed Depression Wetland & Hordeum jubatum & Graminoid $\mathrm{C}_{3}$ & 3.6 & 3.6 \\
\hline Western Great Plains Saline Depression Wetland & Hordeum jubatum & Graminoid $\mathrm{C}_{3}$ & 3.6 & 3.6 \\
\hline $\begin{array}{l}\text { Western Great Plains Depressional Wetland } \\
\text { Systems }\end{array}$ & Hordeum jubatum & Graminoid $C_{3}$ & 3.6 & 3.6 \\
\hline $\begin{array}{l}\text { Western Great Plains Depressional Wetland } \\
\text { Systems }\end{array}$ & Hordeum jubatum & Graminoid $C_{3}$ & 3.6 & 3.6 \\
\hline Northern Atlantic Coastal Plain Dune and Swale & Hudsonia tomentosa & Shrub- Evergreen & 2.7 & 2.4 \\
\hline Southern Appalachian Montane Cliff and Talus & Hydrangea arborescens & Shrub- Deciduous & 2.7 & 3 \\
\hline $\begin{array}{l}\text { Central and South Texas Coastal Fringe Forest and } \\
\text { Woodland }\end{array}$ & Hydrocotyle bonariensis & Forb & 3 & 3.5 \\
\hline $\begin{array}{l}\text { Southern Atlantic Coastal Plain Dune and } \\
\text { Maritime Grassland }\end{array}$ & Hydrocotyle bonariensis & Forb & 3 & 3.5 \\
\hline South Florida Shell Hash Beach & Hymenocallis latifolia & Forb & 3 & 3.5 \\
\hline North American Warm Desert Wash & Hymenoclea monogyra & Shrub- Deciduous & 2.7 & 3 \\
\hline North American Warm Desert Wash & Hymenoclea salsola & Shrub & 2.7 & 2.8 \\
\hline Central Florida Herbaceous Pondshore & Hypericum edisonianum & Forb & 3 & 3.5 \\
\hline East Gulf Coastal Plain Savanna and Wet Prairie & Hypericum fasciculatum & Shrub- Evergreen & 2.7 & 2.4 \\
\hline Floridian Highlands Freshwater Marsh & Hypericum fasciculatum & Shrub- Evergreen & 2.7 & 2.4 \\
\hline $\begin{array}{l}\text { Atlantic Coastal Plain Peatland Pocosin and } \\
\text { Canebrake }\end{array}$ & Ilex coriacea & Shrub- Evergreen & 2.7 & 2.4 \\
\hline $\begin{array}{l}\text { Central Atlantic Coastal Plain Wet Longleaf Pine } \\
\text { Savanna and Flatwoods }\end{array}$ & Ilex coriacea & Shrub- Evergreen & 2.7 & 2.4 \\
\hline $\begin{array}{l}\text { West Gulf Coastal Plain Seepage Swamp and } \\
\text { Baygall }\end{array}$ & Ilex coriacea & Shrub- Evergreen & 2.7 & 2.4 \\
\hline
\end{tabular}




\begin{tabular}{|c|c|c|c|c|}
\hline Community & Species & Classification & Resistance & Resilience \\
\hline $\begin{array}{l}\text { East Gulf Coastal Plain Southern Loblolly- } \\
\text { Hardwood Flatwoods }\end{array}$ & Ilex decidua & Shrub- Deciduous & 2.7 & 3 \\
\hline $\begin{array}{l}\text { Atlantic Coastal Plain Peatland Pocosin and } \\
\text { Canebrake }\end{array}$ & Ilex glabra & Shrub- Evergreen & 2.7 & 2.4 \\
\hline Florida Dry Prairie & Ilex glabra & Shrub- Evergreen & 2.7 & 2.4 \\
\hline South Florida Pine Flatwoods & |llex glabra & Shrub- Evergreen & 2.7 & 2.4 \\
\hline $\begin{array}{l}\text { Southern Atlantic Coastal Plain Wet Pine Savanna } \\
\text { and Flatwoods }\end{array}$ & Ilex glabra & Shrub- Evergreen & 2.7 & 2.4 \\
\hline $\begin{array}{l}\text { Atlantic Coastal Plain Streamhead Seepage } \\
\text { Swamp, Pocosin and Baygall }\end{array}$ & Ilex glabra & Shrub- Evergreen & 2.7 & 2.4 \\
\hline Northern Atlantic Coastal Plain Maritime Forest & Ilex opaca & Shrub- Evergreen & 2.7 & 2.4 \\
\hline $\begin{array}{l}\text { Southern Atlantic Coastal Plain Mesic Hardwood } \\
\text { Forest }\end{array}$ & Ilex opaca & Shrub- Evergreen & 2.7 & 2.4 \\
\hline $\begin{array}{l}\text { East Gulf Coastal Plain Southern Loblolly- } \\
\text { Hardwood Flatwoods }\end{array}$ & Ilex opaca & Shrub- Evergreen & 2.7 & 2.4 \\
\hline West Gulf Coastal Plain Mesic Hardwood Forest & Ilex opaca & Shrub- Evergreen & 2.7 & 2.4 \\
\hline Central Atlantic Coastal Plain Maritime Forest & Ilex vomitoria & Shrub- Evergreen & 2.7 & 2.4 \\
\hline Southern Atlantic Coastal Plain Maritime Forest & Ilex vomitoria & Shrub- Evergreen & 2.7 & 2.4 \\
\hline Ozark-Ouachita Riparian & Impatiens capensis & Forb & 3 & 3.5 \\
\hline Gulf Coast Chenier Plain Beach & Ipomoea imperati & Forb & 3 & 3.5 \\
\hline Texas Coast Beach & Ipomoea imperati & Forb & 3 & 3.5 \\
\hline Gulf Coast Chenier Plain Beach & Ipomoea pes-caprae & Forb & 3 & 3.5 \\
\hline Southeast Florida Beach & Ipomoea pes-caprae & Forb & 3 & 3.5 \\
\hline Southwest Florida Beach & Ipomoea pes-caprae & Forb & 3 & 3.5 \\
\hline Texas Coast Beach & Ipomoea pes-caprae & Forb & 3 & 3.5 \\
\hline $\begin{array}{l}\text { Great Basin Foothill and Lower Montane Riparian } \\
\text { Woodland and Shrubland }\end{array}$ & Iris missouriensis & Forb & 3 & 3.5 \\
\hline Mediterranean California Southern Coastal Dune & Isocoma menziesii & Forb & 3 & 3.5 \\
\hline $\begin{array}{l}\text { East Gulf Coastal Plain Southern Loblolly- } \\
\text { Hardwood Flatwoods }\end{array}$ & Itea virginica & Shrub- Deciduous & 2.7 & 3 \\
\hline Southwest Florida Beach & Iva imbricata & Forb & 3 & 3.5 \\
\hline Southwest Florida Dune and Coastal Grassland & Iva imbricata & Forb & 3 & 3.5 \\
\hline Rocky Mountain Alpine Fell-Field & Ivesia gordonii & Forb & 3 & 3.5 \\
\hline Southern California Oak Woodland and Savanna & Juglans californica & Tree- Deciduous & 3 & 4.2 \\
\hline Edwards Plateau Floodplain Terrace & Juglans major & Tree-Deciduous & 3 & 4.2 \\
\hline $\begin{array}{l}\text { North American Warm Desert Lower Montane } \\
\text { Riparian Woodland and Shrubland }\end{array}$ & Juglans major & Tree-Deciduous & 3 & 4.2 \\
\hline $\begin{array}{l}\text { North American Warm Desert Riparian Woodland } \\
\text { and Shrubland }\end{array}$ & Juglans major & Tree-Deciduous & 3 & 4.2 \\
\hline Edwards Plateau Riparian & Juglans microcarpa & Tree- Deciduous & 3 & 4.2 \\
\hline North American Warm Desert Wash & Juglans microcarpa & Tree-Deciduous & 3 & 4.2 \\
\hline South-Central Interior Mesophytic Forest & Juglans nigra & Tree- Deciduous & 3 & 4.2 \\
\hline East Gulf Coastal Plain Depression Pond & Juncus abortivus & Graminoid $\mathrm{C}_{3}$ & 3.6 & 3.6 \\
\hline Inter-Mountain Basins Interdunal Swale Wetland & Juncus balticus & Graminoid $\mathrm{C}_{3}$ & 3.6 & 3.6 \\
\hline Temperate Pacific Tidal Salt and Brackish Marsh & Juncus balticus & Graminoid $C_{3}$ & 3.6 & 3.6 \\
\hline Rocky Mountain Alpine-Montane Wet Meadow & Juncus drummondii & Graminoid $\mathrm{C}_{3}$ & 3.6 & 3.6 \\
\hline East Gulf Coastal Plain Depression Pond & Juncus effusus & Graminoid $\mathrm{C}_{3}$ & 3.6 & 3.6 \\
\hline Acadian Coastal Salt Marsh & Juncus gerardii & Graminoid $C_{3}$ & 3.6 & 3.6 \\
\hline Northern Atlantic Coastal Plain Tidal Salt Marsh & Juncus gerardii & Graminoid $C_{3}$ & 3.6 & 3.6 \\
\hline
\end{tabular}




\begin{tabular}{|c|c|c|c|c|}
\hline Community & Species & Classification & Resistance & Resilience \\
\hline $\begin{array}{l}\text { Temperate Pacific Subalpine-Montane Wet } \\
\text { Meadow }\end{array}$ & Juncus nevadensis & Graminoid $C_{3}$ & 3.6 & 3.6 \\
\hline East Gulf Coastal Plain Depression Pond & Juncus repens & Graminoid $C_{3}$ & 3.6 & 3.6 \\
\hline $\begin{array}{l}\text { Atlantic Coastal Plain Embayed Region Tidal } \\
\text { Freshwater Marsh }\end{array}$ & Juncus roemerianus & Graminoid $C_{3}$ & 3.6 & 3.6 \\
\hline $\begin{array}{l}\text { Atlantic Coastal Plain Embayed Region Tidal Salt } \\
\text { and Brackish Marsh }\end{array}$ & Juncus roemerianus & Graminoid $C_{3}$ & 3.6 & 3.6 \\
\hline $\begin{array}{l}\text { Atlantic Coastal Plain Indian River Lagoon Tidal } \\
\text { Marsh }\end{array}$ & Juncus roemerianus & Graminoid $\mathrm{C}_{3}$ & 3.6 & 3.6 \\
\hline Florida Big Bend Salt and Brackish Tidal Marsh & Juncus roemerianus & Graminoid $\mathrm{C}_{3}$ & 3.6 & 3.6 \\
\hline $\begin{array}{l}\text { Gulf Coast Chenier Plain Salt and Brackish Tidal } \\
\text { Marsh }\end{array}$ & Juncus roemerianus & Graminoid $C_{3}$ & 3.6 & 3.6 \\
\hline $\begin{array}{l}\text { Southern Atlantic Coastal Plain Fresh and } \\
\text { Oligohaline Tidal Marsh }\end{array}$ & Juncus roemerianus & Graminoid $C_{3}$ & 3.6 & 3.6 \\
\hline $\begin{array}{l}\text { Southern Atlantic Coastal Plain Salt and Brackish } \\
\text { Tidal Marsh }\end{array}$ & Juncus roemerianus & Graminoid $C_{3}$ & 3.6 & 3.6 \\
\hline Texas Coast Salt and Brackish Tidal Marsh & Juncus roemerianus & Graminoid $\mathrm{C}_{3}$ & 3.6 & 3.6 \\
\hline Acadian-Appalachian Alpine Tundra & Juncus trifidus & Graminoid $C_{3}$ & 3.6 & 3.6 \\
\hline Edwards Plateau Floodplain Terrace & Juniperus ashei & Tree-Evergreen & 3.2 & 2.2 \\
\hline $\begin{array}{l}\text { Edwards Plateau Limestone Savanna and } \\
\text { Woodland }\end{array}$ & Juniperus ashei & Tree-Evergreen & 3.2 & 2.2 \\
\hline Edwards Plateau Limestone Shrubland & Juniperus ashei & Tree- Evergreen & 3.2 & 2.2 \\
\hline Apacherian-Chihuahuan Mesquite Upland Scrub & Juniperus coahuilensis & Tree-Evergreen & 3.2 & 2.2 \\
\hline Madrean Pinyon-Juniper Woodland & Juniperus coahuilensis & Tree- Evergreen & 3.2 & 2.2 \\
\hline Tamaulipan Mesquite Upland Scrub & Juniperus coahuilensis & Tree-Evergreen & 3.2 & 2.2 \\
\hline $\begin{array}{l}\text { Inter-Mountain Basins Aspen-Mixed Conifer Forest } \\
\text { and Woodland }\end{array}$ & Juniperus communis & Shrub- Evergreen & 2.7 & 2.4 \\
\hline Great Lakes Alvar & Juniperus communis & Tree- Evergreen & 3.2 & 2.2 \\
\hline Great Lakes Dune & Juniperus communis & Tree- Evergreen & 3.2 & 2.2 \\
\hline $\begin{array}{l}\text { Inter-Mountain Basins Subalpine Limber- } \\
\text { Bristlecone Pine Woodland }\end{array}$ & Juniperus communis & Tree- Evergreen & 3.2 & 2.2 \\
\hline Madrean Pinyon-Juniper Woodland & Juniperus deppeana & Tree-Evergreen & 3.2 & 2.2 \\
\hline Great Lakes Dune & Juniperus horizontalis & Shrub & 2.7 & 2.8 \\
\hline Great Lakes Wooded Dune and Swale & Juniperus horizontalis & Shrub & 2.7 & 2.8 \\
\hline Northwestern Great Plains Shrubland & Juniperus horizontalis & Shrub & 2.7 & 2.8 \\
\hline Apacherian-Chihuahuan Mesquite Upland Scrub & Juniperus monosperma & Tree- Evergreen & 3.2 & 2.2 \\
\hline Colorado Plateau Pinyon-Juniper Woodland & Juniperus monosperma & Tree- Evergreen & 3.2 & 2.2 \\
\hline $\begin{array}{l}\text { Inter-Mountain Basins Volcanic Rock and Cinder } \\
\text { Land }\end{array}$ & Juniperus monosperma & Tree-Evergreen & 3.2 & 2.2 \\
\hline Madrean Juniper Savanna & Juniperus monosperma & Tree-Evergreen & 3.2 & 2.2 \\
\hline Madrean Pinyon-Juniper Woodland & Juniperus monosperma & Tree-Evergreen & 3.2 & 2.2 \\
\hline $\begin{array}{l}\text { Southern Rocky Mountain Juniper Woodland and } \\
\text { Savanna }\end{array}$ & Juniperus monosperma & Tree- Evergreen & 3.2 & 2.2 \\
\hline $\begin{array}{l}\text { Southern Rocky Mountain Pinyon-Juniper } \\
\text { Woodland }\end{array}$ & Juniperus monosperma & Tree-Evergreen & 3.2 & 2.2 \\
\hline Southwestern Great Plains Canyon & Juniperus monosperma & Tree-Evergreen & 3.2 & 2.2 \\
\hline Tamaulipan Mesquite Upland Scrub & Juniperus monosperma & Tree-Evergreen & 3.2 & 2.2 \\
\hline $\begin{array}{l}\text { Columbia Plateau Western Juniper Woodland and } \\
\text { Savanna }\end{array}$ & Juniperus occidentalis & Tree- Evergreen & 3.2 & 2.2 \\
\hline Mediterranean California Subalpine Woodland & Juniperus occidentalis & Tree- Evergreen & 3.2 & 2.2 \\
\hline Colorado Plateau Pinyon-Juniper Shrubland & Juniperus osteosperma & Tree- Evergreen & 3.2 & 2.2 \\
\hline
\end{tabular}




\begin{tabular}{|c|c|c|c|c|}
\hline Community & Species & Classification & Resistance & Resilience \\
\hline Colorado Plateau Pinyon-Juniper Woodland & Juniperus osteosperma & Tree- Evergreen & 3.2 & 2.2 \\
\hline Great Basin Pinyon-Juniper Woodland & Juniperus osteosperma & Tree- Evergreen & 3.2 & 2.2 \\
\hline Inter-Mountain Basins Cliff and Canyon & Juniperus osteosperma & Tree- Evergreen & 3.2 & 2.2 \\
\hline Inter-Mountain Basins Juniper Savanna & Juniperus osteosperma & Tree- Evergreen & 3.2 & 2.2 \\
\hline $\begin{array}{l}\text { Rocky Mountain Foothill Limber Pine-Juniper } \\
\text { Woodland }\end{array}$ & Juniperus osteosperma & Tree- Evergreen & 3.2 & 2.2 \\
\hline Sierra Nevada Cliff and Canyon & Juniperus osteosperma & Tree- Evergreen & 3.2 & 2.2 \\
\hline $\begin{array}{l}\text { Colorado Plateau Mixed Bedrock Canyon and } \\
\text { Tableland }\end{array}$ & Juniperus osteosperma & Tree- Evergreen & 3.2 & 2.2 \\
\hline Edwards Plateau Limestone Shrubland & Juniperus pinchotii & Tree- Evergreen & 3.2 & 2.2 \\
\hline $\begin{array}{l}\text { Llano Estacado Caprock Escarpment and Breaks } \\
\text { Shrubland and Steppe }\end{array}$ & Juniperus pinchotii & Tree- Evergreen & 3.2 & 2.2 \\
\hline Madrean Pinyon-Juniper Woodland & Juniperus pinchotii & Tree- Evergreen & 3.2 & 2.2 \\
\hline Colorado Plateau Pinyon-Juniper Woodland & Juniperus scopulorum & Tree- Evergreen & 3.2 & 2.2 \\
\hline Inter-Mountain Basins Juniper Savanna & Juniperus scopulorum & Tree- Evergreen & 3.2 & 2.2 \\
\hline $\begin{array}{l}\text { Rocky Mountain Foothill Limber Pine-Juniper } \\
\text { Woodland }\end{array}$ & Juniperus scopulorum & Tree- Evergreen & 3.2 & 2.2 \\
\hline $\begin{array}{l}\text { Rocky Mountain Lower Montane-Foothill Riparian } \\
\text { Woodland and Shrubland }\end{array}$ & Juniperus scopulorum & Tree- Evergreen & 3.2 & 2.2 \\
\hline $\begin{array}{l}\text { Southern Rocky Mountain Dry-Mesic Montane } \\
\text { Mixed Conifer Forest and Woodland }\end{array}$ & Juniperus scopulorum & Tree- Evergreen & 3.2 & 2.2 \\
\hline $\begin{array}{l}\text { Southern Rocky Mountain Juniper Woodland and } \\
\text { Savanna }\end{array}$ & Juniperus scopulorum & Tree- Evergreen & 3.2 & 2.2 \\
\hline $\begin{array}{l}\text { Southern Rocky Mountain Pinyon-Juniper } \\
\text { Woodland }\end{array}$ & Juniperus scopulorum & Tree- Evergreen & 3.2 & 2.2 \\
\hline $\begin{array}{l}\text { Southern Rocky Mountain Ponderosa Pine } \\
\text { Woodland }\end{array}$ & Juniperus scopulorum & Tree- Evergreen & 3.2 & 2.2 \\
\hline Great Plains Wooded Draw and Ravine & Juniperus scopulorum & Tree- Evergreen & 3.2 & 2.2 \\
\hline Appalachian Shale Barrens & Juniperus virginiana & Tree-Evergreen & 3.2 & 2.2 \\
\hline Central Appalachian Alkaline Glade and Woodland & Juniperus virginiana & Tree- Evergreen & 3.2 & 2.2 \\
\hline $\begin{array}{l}\text { Central Interior Highlands Calcareous Glade and } \\
\text { Barrens }\end{array}$ & Juniperus virginiana & Tree- Evergreen & 3.2 & 2.2 \\
\hline $\begin{array}{l}\text { Central Interior Highlands Dry Acidic Glade and } \\
\text { Barrens }\end{array}$ & Juniperus virginiana & Tree- Evergreen & 3.2 & 2.2 \\
\hline Nashville Basin Limestone Glade and Woodland & Juniperus virginiana & Tree- Evergreen & 3.2 & 2.2 \\
\hline North-Central Appalachian Acidic Cliff and Talus & Juniperus virginiana & Tree- Evergreen & 3.2 & 2.2 \\
\hline Northern Atlantic Coastal Plain Maritime Forest & Juniperus virginiana & Tree- Evergreen & 3.2 & 2.2 \\
\hline $\begin{array}{l}\text { Southern Coastal Plain Blackland Prairie and } \\
\text { Woodland }\end{array}$ & Juniperus virginiana & Tree- Evergreen & 3.2 & 2.2 \\
\hline Southwestern Great Plains Canyon & Juniperus virginiana & Tree-Evergreen & 3.2 & 2.2 \\
\hline West Gulf Coastal Plain Weches Glade & Juniperus virginiana & Tree- Evergreen & 3.2 & 2.2 \\
\hline $\begin{array}{l}\text { Southern Ridge and Valley / Cumberland Dry } \\
\text { Calcareous Forest }\end{array}$ & Juniperus virginiana & Tree- Evergreen & 3.2 & 2.2 \\
\hline Central Atlantic Coastal Plain Maritime Forest & Juniperus virginiana & Tree- Evergreen & 3.2 & 2.2 \\
\hline Southern Atlantic Coastal Plain Maritime Forest & Juniperus virginiana & Tree-Evergreen & 3.2 & 2.2 \\
\hline $\begin{array}{l}\text { Southern Atlantic Coastal Plain Salt and Brackish } \\
\text { Tidal Marsh }\end{array}$ & Juniperus virginiana & Tree- Evergreen & 3.2 & 2.2 \\
\hline Crosstimbers Oak Forest and Woodland & Juniperus virginiana & Tree-Evergreen & 3.2 & 2.2 \\
\hline $\begin{array}{l}\text { East Gulf Coastal Plain Northern Loess Plain Oak- } \\
\text { Hickory Upland }\end{array}$ & Juniperus virginiana & Tree- Evergreen & 3.2 & 2.2 \\
\hline Southern Coastal Plain Limestone Forest & Juniperus virginiana & Tree-Evergreen & 3.2 & 2.2 \\
\hline Southern Interior Calcareous Cliff & Juniperus virginiana & Tree- Evergreen & 3.2 & 2.2 \\
\hline
\end{tabular}




\begin{tabular}{|c|c|c|c|c|}
\hline Community & Species & Classification & Resistance & Resilience \\
\hline Southern Piedmont Glade and Barrens & Juniperus virginiana & Tree- Evergreen & 3.2 & 2.2 \\
\hline Southern Piedmont Large Floodplain Forest & Justicia americana & Forb & 3 & 3.5 \\
\hline $\begin{array}{l}\text { Acadian-Appalachian Subalpine Woodland and } \\
\text { Heath-Krummholz }\end{array}$ & Kalmia angustifolia & Shrub- Evergreen & 2.7 & 2.4 \\
\hline Boreal-Laurentian Bog & Kalmia angustifolia & Shrub- Evergreen & 2.7 & 2.4 \\
\hline $\begin{array}{l}\text { Central and Southern Appalachian Montane Oak } \\
\text { Forest }\end{array}$ & Kalmia latifolia & Shrub- Evergreen & 2.7 & 2.4 \\
\hline Central Appalachian Dry Oak-Pine Forest & Kalmia latifolia & Shrub- Evergreen & 2.7 & 2.4 \\
\hline Southern Appalachian Grass and Shrub Bald & Kalmia latifolia & Shrub- Evergreen & 2.7 & 2.4 \\
\hline $\begin{array}{l}\text { Southern Appalachian Montane Pine Forest and } \\
\text { Woodland }\end{array}$ & Kalmia latifolia & Shrub- Evergreen & 2.7 & 2.4 \\
\hline Rocky Mountain Alpine Dwarf-Shrubland & Kalmia microphylla & Shrub- Evergreen & 2.7 & 2.4 \\
\hline Sierra Nevada Alpine Dwarf-Shrubland & Kalmia microphylla & Shrub- Evergreen & 2.7 & 2.4 \\
\hline Boreal-Laurentian Bog & Kalmia polifolia & Shrub- Evergreen & 2.7 & 2.4 \\
\hline Mediterranean California Subalpine-Montane Fen & Kobresia myosuroides & Graminoid $\mathrm{C}_{3}$ & 3.6 & 3.6 \\
\hline Rocky Mountain Alpine Turf & Kobresia myosuroides & Graminoid $C_{3}$ & 3.6 & 3.6 \\
\hline Rocky Mountain Subalpine-Montane Fen & Kobresia myosuroides & Graminoid $\mathrm{C}_{3}$ & 3.6 & 3.6 \\
\hline Rocky Mountain Subalpine-Montane Fen & Kobresia simpliciuscula & Graminoid $\mathrm{C}_{3}$ & 3.6 & 3.6 \\
\hline Tamaulipan Calcareous Thornscrub & Koeberlinia spinosa & Shrub-Deciduous & 2.7 & 3 \\
\hline Inter-Mountain Basins Semi-Desert Shrub-Steppe & Koeleria macrantha & Graminoid $\mathrm{C}_{3}$ & 3.6 & 3.6 \\
\hline North Pacific Herbaceous Bald and Bluff & Koeleria macrantha & Graminoid $\mathrm{C}_{3}$ & 3.6 & 3.6 \\
\hline $\begin{array}{l}\text { Northern Rocky Mountain Subalpine-Upper } \\
\text { Montane Grassland }\end{array}$ & Koeleria macrantha & Graminoid $\mathrm{C}_{3}$ & 3.6 & 3.6 \\
\hline Northwestern Great Plains Shrubland & Koeleria macrantha & Graminoid $C_{3}$ & 3.6 & 3.6 \\
\hline $\begin{array}{l}\text { Rocky Mountain Foothill Limber Pine-Juniper } \\
\text { Woodland }\end{array}$ & Koeleria macrantha & Graminoid $C_{3}$ & 3.6 & 3.6 \\
\hline Western Great Plains Sand Prairie & Koeleria macrantha & Graminoid $\mathrm{C}_{3}$ & 3.6 & 3.6 \\
\hline $\begin{array}{l}\text { Sonora-Mojave Creosotebush-White Bursage } \\
\text { Desert Scrub }\end{array}$ & Krameria grayi & Shrub- Deciduous & 2.7 & 3 \\
\hline Inter-Mountain Basins Greasewood Flat & Krascheninnikovia lanata & Shrub- Deciduous & 2.7 & 3 \\
\hline Inter-Mountain Basins Mixed Salt Desert Scrub & Krascheninnikovia lanata & Shrub-Deciduous & 2.7 & 3 \\
\hline Inter-Mountain Basins Semi-Desert Grassland & Krascheninnikovia lanata & Shrub- Deciduous & 2.7 & 3 \\
\hline Sonora-Mojave Mixed Salt Desert Scrub & Krascheninnikovia lanata & Shrub- Deciduous & 2.7 & 3 \\
\hline South Florida Mangrove Swamp & Laguncularia racemosa & Shrub- Evergreen & 2.7 & 2.4 \\
\hline Boreal-Laurentian Bog & Larix laricina & Tree- Evergreen & 3.2 & 2.2 \\
\hline $\begin{array}{l}\text { Boreal-Laurentian Conifer Acidic Swamp and } \\
\text { Treed Poor Fen }\end{array}$ & Larix laricina & Tree- Evergreen & 3.2 & 2.2 \\
\hline $\begin{array}{l}\text { Laurentian-Acadian Alkaline Conifer-Hardwood } \\
\text { Swamp }\end{array}$ & Larix laricina & Tree- Evergreen & 3.2 & 2.2 \\
\hline $\begin{array}{l}\text { North-Central Interior and Appalachian Acidic } \\
\text { Peatland }\end{array}$ & Larix laricina & Tree- Evergreen & 3.2 & 2.2 \\
\hline $\begin{array}{l}\text { North-Central Interior and Appalachian Rich } \\
\text { Swamp }\end{array}$ & Larix laricina & Tree- Evergreen & 3.2 & 2.2 \\
\hline $\begin{array}{l}\text { Northern Rocky Mountain Subalpine Woodland } \\
\text { and Parkland }\end{array}$ & Larix Iyallii & Tree- Evergreen & 3.2 & 2.2 \\
\hline $\begin{array}{l}\text { East Cascades Mesic Montane Mixed Conifer } \\
\text { Forest and Woodland }\end{array}$ & Larix occidentalis & Tree- Evergreen & 3.2 & 2.2 \\
\hline Northern Rocky Mountain Western Larch Savanna & Larix occidentalis & Tree- Evergreen & 3.2 & 2.2 \\
\hline Northern Rocky Mountain Wooded Vernal Pool & Larix occidentalis & Tree- Evergreen & 3.2 & 2.2 \\
\hline Chihuahuan Creosotebush Desert Scrub & Larrea tridentata & Shrub & 2.7 & 2.8 \\
\hline Chihuahuan Mixed Desert and Thornscrub & Larrea tridentata & Shrub & 2.7 & 2.8 \\
\hline
\end{tabular}




\begin{tabular}{|c|c|c|c|c|}
\hline Community & Species & Classification & Resistance & Resilience \\
\hline $\begin{array}{l}\text { North American Warm Desert Bedrock Cliff and } \\
\text { Outcrop }\end{array}$ & Larrea tridentata & Shrub & 2.7 & 2.8 \\
\hline North American Warm Desert Volcanic Rockland & Larrea tridentata & Shrub & 2.7 & 2.8 \\
\hline $\begin{array}{l}\text { Sonora-Mojave Creosotebush-White Bursage } \\
\text { Desert Scrub }\end{array}$ & Larrea tridentata & Shrub & 2.7 & 2.8 \\
\hline Sonoran Paloverde-Mixed Cacti Desert Scrub & Larrea tridentata & Shrub & 2.7 & 2.8 \\
\hline Apacherian-Chihuahuan Mesquite Upland Scrub & Larrea tridentata & Shrub- Evergreen & 2.7 & 2.4 \\
\hline Chihuahuan Mixed Salt Desert Scrub & Larrea tridentata & Shrub- Evergreen & 2.7 & 2.4 \\
\hline Mojave Mid-Elevation Mixed Desert Scrub & Larrea tridentata & Shrub- Evergreen & 2.7 & 2.4 \\
\hline $\begin{array}{l}\text { North American Warm Desert Active and } \\
\text { Stabilized Dune }\end{array}$ & Larrea tridentata & Shrub- Evergreen & 2.7 & 2.4 \\
\hline Sonoran Mid-Elevation Desert Scrub & Larrea tridentata & Shrub- Evergreen & 2.7 & 2.4 \\
\hline Tamaulipan Mesquite Upland Scrub & Larrea tridentata & Shrub- Evergreen & 2.7 & 2.4 \\
\hline Northern California Claypan Vernal Pool & Lasthenia glaberrima & Forb & 3 & 3.5 \\
\hline $\begin{array}{l}\text { Acadian-Appalachian Subalpine Woodland and } \\
\text { Heath-Krummholz }\end{array}$ & Ledum groenlandicum & Shrub & 2.7 & 2.8 \\
\hline Boreal-Laurentian Bog & Ledum groenlandicum & Shrub & 2.7 & 2.8 \\
\hline North Pacific Bog and Fen & Ledum groenlandicum & Shrub- Evergreen & 2.7 & 2.4 \\
\hline Texas-Louisiana Coastal Prairie Pondshore & Leersia hexandra & Graminoid $\mathrm{C}_{3}$ & 3.6 & 3.6 \\
\hline Madrean Encinal & Leptochloa dubia & Graminoid $\mathrm{C}_{4}$ & 3.6 & 4.1 \\
\hline Mediterranean California Alpine Fell-Field & Leptodactylon pungens & Forb & 3 & 3.5 \\
\hline Sierra Nevada Alpine Dwarf-Shrubland & Leptodactylon pungens & Forb & 3 & 3.5 \\
\hline Tamaulipan Calcareous Thornscrub & Leucophyllum frutescens & Shrub- Evergreen & 2.7 & 2.4 \\
\hline Tamaulipan Lomas & Leucophyllum frutescens & Shrub- Evergreen & 2.7 & 2.4 \\
\hline Tamaulipan Mixed Deciduous Thornscrub & Leucophyllum frutescens & Shrub- Evergreen & 2.7 & 2.4 \\
\hline $\begin{array}{l}\text { Southern Atlantic Coastal Plain Nonriverine } \\
\text { Swamp and Wet Hardwood Forest }\end{array}$ & Leucothoe axillaris & Shrub- Evergreen & 2.7 & 2.4 \\
\hline $\begin{array}{l}\text { Atlantic Coastal Plain Streamhead Seepage } \\
\text { Swamp, Pocosin and Baygall }\end{array}$ & Leucothoe axillaris & Shrub- Evergreen & 2.7 & 2.4 \\
\hline Inter-Mountain Basins Mixed Salt Desert Scrub & Leymus ambiguus & Graminoid $C_{3}$ & 3.6 & 3.6 \\
\hline $\begin{array}{l}\text { North Pacific Maritime Coastal Sand Dune and } \\
\text { Strand }\end{array}$ & Leymus arenarius & Graminoid $\mathrm{C}_{3}$ & 3.6 & 3.6 \\
\hline $\begin{array}{l}\text { Columbia Plateau Silver Sagebrush Seasonally } \\
\text { Flooded Shrub-Steppe }\end{array}$ & Leymus cinereus & Graminoid $\mathrm{C}_{3}$ & 3.6 & 3.6 \\
\hline Inter-Mountain Basins Semi-Desert Shrub-Steppe & Leymus cinereus & Graminoid $C_{3}$ & 3.6 & 3.6 \\
\hline $\begin{array}{l}\text { California Central Valley and Southern Coastal } \\
\text { Grassland }\end{array}$ & Leymus condensatus & Graminoid $C_{3}$ & 3.6 & 3.6 \\
\hline $\begin{array}{l}\text { Northern Rocky Mountain Subalpine-Upper } \\
\text { Montane Grassland }\end{array}$ & Leymus innovatus & Graminoid $C_{3}$ & 3.6 & 3.6 \\
\hline North Pacific Coastal Interdunal Wetland & Leymus mollis & Graminoid $C_{3}$ & 3.6 & 3.6 \\
\hline $\begin{array}{l}\text { North Pacific Maritime Coastal Sand Dune and } \\
\text { Strand }\end{array}$ & Leymus mollis & Graminoid $C_{3}$ & 3.6 & 3.6 \\
\hline $\begin{array}{l}\text { North Pacific Maritime Coastal Sand Dune and } \\
\text { Strand }\end{array}$ & Leymus mollis & Graminoid $C_{3}$ & 3.6 & 3.6 \\
\hline Inter-Mountain Basins Mat Saltbush Shrubland & Leymus salinus & Graminoid $C_{3}$ & 3.6 & 3.6 \\
\hline Inter-Mountain Basins Mixed Salt Desert Scrub & Leymus salinus & Graminoid $C_{3}$ & 3.6 & 3.6 \\
\hline Inter-Mountain Basins Semi-Desert Shrub-Steppe & Leymus salinus & Graminoid $C_{3}$ & 3.6 & 3.6 \\
\hline Inter-Mountain Basins Shale Badland & Leymus salinus & Graminoid $\mathrm{C}_{3}$ & 3.6 & 3.6 \\
\hline Inter-Mountain Basins Greasewood Flat & Leymus triticoides & Graminoid $C_{3}$ & 3.6 & 3.6 \\
\hline Cumberland Acidic Cliff and Rockhouse & Liatris microcephala & Forb & 3 & 3.5 \\
\hline Florida Longleaf Pine Sandhill & Licania michauxii & Shrub- Evergreen & 2.7 & 2.4 \\
\hline
\end{tabular}




\begin{tabular}{|c|c|c|c|c|}
\hline Community & Species & Classification & Resistance & Resilience \\
\hline Temperate Pacific Freshwater Mudflat & Lilaeopsis occidentalis & Forb & 3 & 3.5 \\
\hline Temperate Pacific Freshwater Mudflat & Limosella aquatica & Forb & 3 & 3.5 \\
\hline Southern Piedmont Large Floodplain Forest & Lindera benzoin & Shrub-Deciduous & 2.7 & 3 \\
\hline $\begin{array}{l}\text { North Pacific Maritime Dry-Mesic Douglas-fir- } \\
\text { Western Hemlock Forest }\end{array}$ & Linnaea borealis & Shrub & 2.7 & 2.8 \\
\hline Northern Rocky Mountain Western Larch Savanna & Linnaea borealis & Shrub & 2.7 & 2.8 \\
\hline $\begin{array}{l}\text { Atlantic Coastal Plain Small Blackwater River } \\
\text { Floodplain Forest }\end{array}$ & Liquidambar styraciflua & Tree-Deciduous & 3 & 4.2 \\
\hline $\begin{array}{l}\text { Atlantic Coastal Plain Small Brownwater River } \\
\text { Floodplain Forest }\end{array}$ & Liquidambar styraciflua & Tree-Deciduous & 3 & 4.2 \\
\hline $\begin{array}{l}\text { East Gulf Coastal Plain Large River Floodplain } \\
\text { Forest }\end{array}$ & Liquidambar styraciflua & Tree- Deciduous & 3 & 4.2 \\
\hline East Gulf Coastal Plain Northern Loess Bluff Forest & Liquidambar styraciflua & Tree-Deciduous & 3 & 4.2 \\
\hline $\begin{array}{l}\text { East Gulf Coastal Plain Northern Mesic Hardwood } \\
\text { Slope Forest }\end{array}$ & Liquidambar styraciflua & Tree-Deciduous & 3 & 4.2 \\
\hline $\begin{array}{l}\text { East Gulf Coastal Plain Small Stream and River } \\
\text { Floodplain Forest }\end{array}$ & Liquidambar styraciflua & Tree-Deciduous & 3 & 4.2 \\
\hline $\begin{array}{l}\text { East Gulf Coastal Plain Southern Loess Bluff } \\
\text { Forest }\end{array}$ & Liquidambar styraciflua & Tree-Deciduous & 3 & 4.2 \\
\hline Lower Mississippi River Flatwoods & Liquidambar styraciflua & Tree- Deciduous & 3 & 4.2 \\
\hline $\begin{array}{l}\text { Northern Atlantic Coastal Plain Basin Swamp and } \\
\text { Wet Hardwood Forest }\end{array}$ & Liquidambar styraciflua & Tree- Deciduous & 3 & 4.2 \\
\hline Ozark-Ouachita Mesic Hardwood Forest & Liquidambar styraciflua & Tree- Deciduous & 3 & 4.2 \\
\hline Ozark-Ouachita Riparian & Liquidambar styraciflua & Tree-Deciduous & 3 & 4.2 \\
\hline Piedmont Upland Depression Swamp & Liquidambar styraciflua & Tree-Deciduous & 3 & 4.2 \\
\hline South-Central Interior Large Floodplain & Liquidambar styraciflua & Tree- Deciduous & 3 & 4.2 \\
\hline South-Central Interior Small Stream and Riparian & Liquidambar styraciflua & Tree-Deciduous & 3 & 4.2 \\
\hline $\begin{array}{l}\text { Southern Atlantic Coastal Plain Dry and Dry-Mesic } \\
\text { Oak Forest }\end{array}$ & Liquidambar styraciflua & Tree-Deciduous & 3 & 4.2 \\
\hline $\begin{array}{l}\text { Southern Atlantic Coastal Plain Mesic Hardwood } \\
\text { Forest }\end{array}$ & Liquidambar styraciflua & Tree-Deciduous & 3 & 4.2 \\
\hline $\begin{array}{l}\text { Southern Atlantic Coastal Plain Nonriverine } \\
\text { Swamp and Wet Hardwood Forest }\end{array}$ & Liquidambar styraciflua & Tree-Deciduous & 3 & 4.2 \\
\hline Southern Coastal Plain Mesic Slope Forest & Liquidambar styraciflua & Tree-Deciduous & 3 & 4.2 \\
\hline Southern Coastal Plain Nonriverine Cypress Dome & Liquidambar styraciflua & Tree-Deciduous & 3 & 4.2 \\
\hline $\begin{array}{l}\text { Southern Coastal Plain Seepage Swamp and } \\
\text { Baygall }\end{array}$ & Liquidambar styraciflua & Tree-Deciduous & 3 & 4.2 \\
\hline Southern Piedmont Large Floodplain Forest & Liquidambar styraciflua & Tree-Deciduous & 3 & 4.2 \\
\hline $\begin{array}{l}\text { Southern Piedmont Small Floodplain and Riparian } \\
\text { Forest }\end{array}$ & Liquidambar styraciflua & Tree-Deciduous & 3 & 4.2 \\
\hline $\begin{array}{l}\text { West Gulf Coastal Plain Large River Floodplain } \\
\text { Forest }\end{array}$ & Liquidambar styraciflua & Tree- Deciduous & 3 & 4.2 \\
\hline $\begin{array}{l}\text { Mississippi River Low Floodplain (Bottomland) } \\
\text { Forest }\end{array}$ & Liquidambar styraciflua & Tree-Deciduous & 3 & 4.2 \\
\hline $\begin{array}{l}\text { South-Central Interior / Upper Coastal Plain Wet } \\
\text { Flatwoods }\end{array}$ & Liquidambar styraciflua & Tree-Deciduous & 3 & 4.2 \\
\hline East Gulf Coastal Plain Northern Loess Bluff Forest & Liriodendron tulipifera & Tree- Deciduous & 3 & 4.2 \\
\hline $\begin{array}{l}\text { East Gulf Coastal Plain Northern Mesic Hardwood } \\
\text { Slope Forest }\end{array}$ & Liriodendron tulipifera & Tree-Deciduous & 3 & 4.2 \\
\hline Northeastern Interior Dry-Mesic Oak Forest & Liriodendron tulipifera & Tree-Deciduous & 3 & 4.2 \\
\hline South-Central Interior Mesophytic Forest & Liriodendron tulipifera & Tree-Deciduous & 3 & 4.2 \\
\hline Southern and Central Appalachian Cove Forest & Liriodendron tulipifera & Tree- Deciduous & 3 & 4.2 \\
\hline
\end{tabular}




\begin{tabular}{|c|c|c|c|c|}
\hline Community & Species & Classification & Resistance & Resilience \\
\hline $\begin{array}{l}\text { Southern Atlantic Coastal Plain Nonriverine } \\
\text { Swamp and Wet Hardwood Forest }\end{array}$ & Liriodendron tulipifera & Tree- Deciduous & 3 & 4.2 \\
\hline $\begin{array}{l}\text { Southern Coastal Plain Seepage Swamp and } \\
\text { Baygall }\end{array}$ & Liriodendron tulipifera & Tree- Deciduous & 3 & 4.2 \\
\hline Southern Piedmont Large Floodplain Forest & Liriodendron tulipifera & Tree- Deciduous & 3 & 4.2 \\
\hline Southern Piedmont Mesic Forest & Liriodendron tulipifera & Tree- Deciduous & 3 & 4.2 \\
\hline $\begin{array}{l}\text { Southern Piedmont Small Floodplain and Riparian } \\
\text { Forest }\end{array}$ & Liriodendron tulipifera & Tree- Deciduous & 3 & 4.2 \\
\hline Appalachian (Hemlock)-Northern Hardwood Forest & Liriodendron tulipifera & Tree-Deciduous & 3 & 4.2 \\
\hline $\begin{array}{l}\text { Atlantic Coastal Plain Streamhead Seepage } \\
\text { Swamp, Pocosin and Baygall }\end{array}$ & Liriodendron tulipifera & Tree-Deciduous & 3 & 4.2 \\
\hline $\begin{array}{l}\text { Klamath-Siskiyou Lower Montane Serpentine } \\
\text { Mixed Conifer Woodland }\end{array}$ & Lithocarpus densiflorus & Shrub- Evergreen & 2.7 & 2.4 \\
\hline Acadian-Appalachian Alpine Tundra & Loiseleuria procumbens & Shrub & 2.7 & 2.8 \\
\hline North Pacific Coastal Cliff and Bluff & Lomatium martindalei & Forb & 3 & 3.5 \\
\hline North Pacific Herbaceous Bald and Bluff & Lomatium martindalei & Forb & 3 & 3.5 \\
\hline Southern California Coastal Scrub & Lotus scoparius & Shrub & 2.7 & 2.8 \\
\hline Texas-Louisiana Coastal Prairie Pondshore & Ludwigia glandulosa & Forb & 3 & 3.5 \\
\hline Texas-Louisiana Coastal Prairie Pondshore & Ludwigia linearis & Forb & 3 & 3.5 \\
\hline Temperate Pacific Freshwater Mudflat & Ludwigia palustris & Forb & 3 & 3.5 \\
\hline $\begin{array}{l}\text { North Pacific Dry and Mesic Alpine Dwarf- } \\
\text { Shrubland, Fell-Field and Meadow }\end{array}$ & Luetkea pectinata & Shrub- Evergreen & 2.7 & 2.4 \\
\hline North Pacific Maritime Mesic Subalpine Parkland & Lupinus arcticus & Forb & 3 & 3.5 \\
\hline Mediterranean California Southern Coastal Dune & Lupinus chamissonis & Shrub & 2.7 & 2.8 \\
\hline $\begin{array}{l}\text { Colorado Plateau Blackbrush-Mormon-tea } \\
\text { Shrubland }\end{array}$ & Lycium andersonii & Shrub- Deciduous & 2.7 & 3 \\
\hline Inter-Mountain Basins Mixed Salt Desert Scrub & Lycium andersonii & Shrub- Deciduous & 2.7 & 3 \\
\hline $\begin{array}{l}\text { Sonora-Mojave Creosotebush-White Bursage } \\
\text { Desert Scrub }\end{array}$ & Lycium andersonii & Shrub- Deciduous & 2.7 & 3 \\
\hline Sonoran Mid-Elevation Desert Scrub & Lycium andersonii & Shrub- Deciduous & 2.7 & 3 \\
\hline Central Mixedgrass Prairie & Lygodesmia juncea & Forb & 3 & 3.5 \\
\hline Florida Peninsula Inland Scrub & Lyonia ferruginea & Shrub- Evergreen & 2.7 & 2.4 \\
\hline Florida Dry Prairie & Lyonia fruticosa & Shrub- Evergreen & 2.7 & 2.4 \\
\hline $\begin{array}{l}\text { Atlantic Coastal Plain Streamhead Seepage } \\
\text { Swamp, Pocosin and Baygall }\end{array}$ & Lyonia ligustrina & Shrub- Deciduous & 2.7 & 3 \\
\hline $\begin{array}{l}\text { Atlantic Coastal Plain Peatland Pocosin and } \\
\text { Canebrake }\end{array}$ & Lyonia lucida & Shrub- Evergreen & 2.7 & 2.4 \\
\hline $\begin{array}{l}\text { Central Atlantic Coastal Plain Wet Longleaf Pine } \\
\text { Savanna and Flatwoods }\end{array}$ & Lyonia lucida & Shrub- Evergreen & 2.7 & 2.4 \\
\hline Florida Dry Prairie & Lyonia lucida & Shrub- Evergreen & 2.7 & 2.4 \\
\hline $\begin{array}{l}\text { Southern Atlantic Coastal Plain Wet Pine Savanna } \\
\text { and Flatwoods }\end{array}$ & Lyonia lucida & Shrub- Evergreen & 2.7 & 2.4 \\
\hline Southern Coastal Plain Herbaceous Seep and Bog & Lyonia lucida & Shrub- Evergreen & 2.7 & 2.4 \\
\hline Southern Coastal Plain Nonriverine Cypress Dome & Lyonia lucida & Shrub- Evergreen & 2.7 & 2.4 \\
\hline $\begin{array}{l}\text { Atlantic Coastal Plain Streamhead Seepage } \\
\text { Swamp, Pocosin and Baygall }\end{array}$ & Lyonia lucida & Shrub- Evergreen & 2.7 & 2.4 \\
\hline East Gulf Coastal Plain Near-Coast Pine Flatwoods & Lyonia lucida & Shrub- Evergreen & 2.7 & 2.4 \\
\hline $\begin{array}{l}\text { Atlantic Coastal Plain Peatland Pocosin and } \\
\text { Canebrake }\end{array}$ & Lyonia mariana & Shrub- Deciduous & 2.7 & 3 \\
\hline North Pacific Shrub Swamp & Lysichiton americanus & Forb & 3 & 3.5 \\
\hline $\begin{array}{l}\text { West Gulf Coastal Plain Northern Calcareous } \\
\text { Prairie }\end{array}$ & Maclura pomifera & Tree- Deciduous & 3 & 4.2 \\
\hline
\end{tabular}




\begin{tabular}{|c|c|c|c|c|}
\hline Community & Species & Classification & Resistance & Resilience \\
\hline South-Central Interior Mesophytic Forest & Magnolia acuminata & Tree-Deciduous & 3 & 4.2 \\
\hline Southern and Central Appalachian Cove Forest & Magnolia acuminata & Tree- Deciduous & 3 & 4.2 \\
\hline Southern and Central Appalachian Cove Forest & Magnolia fraseri & Tree- Deciduous & 3 & 4.2 \\
\hline $\begin{array}{l}\text { East Gulf Coastal Plain Southern Loess Bluff } \\
\text { Forest }\end{array}$ & Magnolia grandiflora & Tree- Evergreen & 3.2 & 2.2 \\
\hline Southern Coastal Plain Mesic Slope Forest & Magnolia grandiflora & Tree- Evergreen & 3.2 & 2.2 \\
\hline West Gulf Coastal Plain Mesic Hardwood Forest & Magnolia grandiflora & Tree- Evergreen & 3.2 & 2.2 \\
\hline $\begin{array}{l}\text { Atlantic Coastal Plain Blackwater Stream } \\
\text { Floodplain Forest }\end{array}$ & Magnolia virginiana & Tree-Evergreen & 3.2 & 2.2 \\
\hline $\begin{array}{l}\text { Atlantic Coastal Plain Peatland Pocosin and } \\
\text { Canebrake }\end{array}$ & Magnolia virginiana & Tree- Evergreen & 3.2 & 2.2 \\
\hline East Gulf Coastal Plain Maritime Forest & Magnolia virginiana & Tree- Evergreen & 3.2 & 2.2 \\
\hline South Florida Bayhead Swamp & Magnolia virginiana & Tree- Evergreen & 3.2 & 2.2 \\
\hline $\begin{array}{l}\text { Southern Coastal Plain Blackwater River } \\
\text { Floodplain Forest }\end{array}$ & Magnolia virginiana & Tree- Evergreen & 3.2 & 2.2 \\
\hline Southern Coastal Plain Hydric Hammock & Magnolia virginiana & Tree- Evergreen & 3.2 & 2.2 \\
\hline $\begin{array}{l}\text { Southern Coastal Plain Seepage Swamp and } \\
\text { Baygall }\end{array}$ & Magnolia virginiana & Tree- Evergreen & 3.2 & 2.2 \\
\hline $\begin{array}{l}\text { West Gulf Coastal Plain Seepage Swamp and } \\
\text { Baygall }\end{array}$ & Magnolia virginiana & Tree- Evergreen & 3.2 & 2.2 \\
\hline $\begin{array}{l}\text { Atlantic Coastal Plain Streamhead Seepage } \\
\text { Swamp, Pocosin and Baygall }\end{array}$ & Magnolia virginiana & Tree- Evergreen & 3.2 & 2.2 \\
\hline North American Warm Desert Volcanic Rockland & Mahonia haematocarpa & Shrub- Evergreen & 2.7 & 2.4 \\
\hline $\begin{array}{l}\text { Mediterranean California Dry-Mesic Mixed Conifer } \\
\text { Forest and Woodland }\end{array}$ & Mahonia nervosa & Shrub- Evergreen & 2.7 & 2.4 \\
\hline $\begin{array}{l}\text { North Pacific Maritime Dry-Mesic Douglas-fir- } \\
\text { Western Hemlock Forest }\end{array}$ & Mahonia nervosa & Shrub- Evergreen & 2.7 & 2.4 \\
\hline $\begin{array}{l}\text { Inter-Mountain Basins Aspen-Mixed Conifer Forest } \\
\text { and Woodland }\end{array}$ & Mahonia repens & Shrub- Evergreen & 2.7 & 2.4 \\
\hline $\begin{array}{l}\text { Acadian Low-Elevation Spruce-Fir-Hardwood } \\
\text { Forest }\end{array}$ & Maianthemum canadense & Forb & 3 & 3.5 \\
\hline $\begin{array}{l}\text { Laurentian-Acadian Pine-Hemlock-Hardwood } \\
\text { Forest }\end{array}$ & Maianthemum canadense & Forb & 3 & 3.5 \\
\hline $\begin{array}{l}\text { Great Basin Foothill and Lower Montane Riparian } \\
\text { Woodland and Shrubland }\end{array}$ & Maianthemum stellatum & Forb & 3 & 3.5 \\
\hline North Pacific Shrub Swamp & Malus fusca & Shrub- Deciduous & 2.7 & 3 \\
\hline Eastern Boreal Floodplain & Matteuccia struthiopteris & Graminoid $\mathrm{C}_{3}$ & 3.6 & 3.6 \\
\hline Laurentian-Acadian Floodplain Forest & Matteuccia struthiopteris & Graminoid $\mathrm{C}_{3}$ & 3.6 & 3.6 \\
\hline Mojave Mid-Elevation Mixed Desert Scrub & Menodora spinescens & Shrub- Deciduous & 2.7 & 3 \\
\hline $\begin{array}{l}\text { Northern Rocky Mountain Subalpine Deciduous } \\
\text { Shrubland }\end{array}$ & Menziesia ferruginea & Shrub- Deciduous & 2.7 & 3 \\
\hline $\begin{array}{l}\text { Rocky Mountain Subalpine Mesic-Wet Spruce-Fir } \\
\text { Forest and Woodland }\end{array}$ & Menziesia ferruginea & Shrub- Deciduous & 2.7 & 3 \\
\hline $\begin{array}{l}\text { Rocky Mountain Subalpine Mesic-Wet Spruce-Fir } \\
\text { Forest and Woodland }\end{array}$ & Menziesia ferruginea & Shrub- Deciduous & 2.7 & 3 \\
\hline South Florida Bayhead Swamp & Metopium toxiferum & Tree- Evergreen & 3.2 & 2.2 \\
\hline South Florida Hardwood Hammock & Metopium toxiferum & Tree- Evergreen & 3.2 & 2.2 \\
\hline Chihuahuan Mixed Desert and Thornscrub & Mimosa warnockii & Shrub- Deciduous & 2.7 & 3 \\
\hline Mogollon Chaparral & Mimosa warnockii & Shrub- Deciduous & 2.7 & 3 \\
\hline North Pacific Herbaceous Bald and Bluff & Mimulus guttatus & Forb & 3 & 3.5 \\
\hline $\begin{array}{l}\text { North Pacific Alpine and Subalpine Bedrock and } \\
\text { Scree }\end{array}$ & Minuartia arctica & Forb & 3 & 3.5 \\
\hline
\end{tabular}




\begin{tabular}{|c|c|c|c|c|}
\hline Community & Species & Classification & Resistance & Resilience \\
\hline Mediterranean California Alpine Fell-Field & Minuartia nuttallii & Forb & 3 & 3.5 \\
\hline South Texas Salt and Brackish Tidal Flat & Monanthochloe littoralis & Graminoid $\mathrm{C}_{4}$ & 3.6 & 4.1 \\
\hline $\begin{array}{l}\text { Atlantic Coastal Plain Streamhead Seepage } \\
\text { Swamp, Pocosin and Baygall }\end{array}$ & Morella caroliniensis & Shrub- Evergreen & 2.7 & 2.4 \\
\hline Central Atlantic Coastal Plain Maritime Forest & Morella cerifera & Shrub- Evergreen & 2.7 & 2.4 \\
\hline East Gulf Coastal Plain Maritime Forest & Morella cerifera & Shrub- Evergreen & 2.7 & 2.4 \\
\hline $\begin{array}{l}\text { East Gulf Coastal Plain Southern Loblolly- } \\
\text { Hardwood Flatwoods }\end{array}$ & Morella cerifera & Shrub- Evergreen & 2.7 & 2.4 \\
\hline $\begin{array}{l}\text { West Gulf Coastal Plain Wet Longleaf Pine } \\
\text { Savanna and Flatwoods }\end{array}$ & Morella cerifera & Shrub- Evergreen & 2.7 & 2.4 \\
\hline Northern Atlantic Coastal Plain Dune and Swale & Morella pensylvanica & Shrub- Evergreen & 2.7 & 2.4 \\
\hline Southeastern Great Plains Floodplain Forest & Morus rubra & Tree- Deciduous & 3 & 4.2 \\
\hline Chihuahuan Loamy Plains Desert Grassland & Muhlenbergia arenacea & Graminoid $\mathrm{C}_{4}$ & 3.6 & 4.1 \\
\hline Texas Saline Coastal Prairie & Muhlenbergia capillaris & Graminoid $\mathrm{C}_{4}$ & 3.6 & 4.1 \\
\hline South Florida Dwarf Cypress Savanna & Muhlenbergia capillaris & Graminoid $\mathrm{C}_{4}$ & 3.6 & 4.1 \\
\hline South Florida Pine Rockland & Muhlenbergia capillaris & Graminoid $\mathrm{C}_{4}$ & 3.6 & 4.1 \\
\hline South Florida Wet Marl Prairie & Muhlenbergia capillaris & Graminoid $\mathrm{C}_{4}$ & 3.6 & 4.1 \\
\hline Madrean Oriental Chaparral & Muhlenbergia emersleyi & Graminoid $\mathrm{C}_{4}$ & 3.6 & 4.1 \\
\hline $\begin{array}{l}\text { Southern Rocky Mountain Montane-Subalpine } \\
\text { Grassland }\end{array}$ & Muhlenbergia filiculmis & Graminoid $\mathrm{C}_{4}$ & 3.6 & 4.1 \\
\hline $\begin{array}{l}\text { Columbia Plateau Silver Sagebrush Seasonally } \\
\text { Flooded Shrub-Steppe }\end{array}$ & Muhlenbergia filiformis & Graminoid $\mathrm{C}_{4}$ & 3.6 & 4.1 \\
\hline $\begin{array}{l}\text { Rocky Mountain Lower Montane-Foothill } \\
\text { Shrubland }\end{array}$ & Muhlenbergia montana & Graminoid $\mathrm{C}_{4}$ & 3.6 & 4.1 \\
\hline $\begin{array}{l}\text { Rocky Mountain Subalpine-Montane Limber- } \\
\text { Bristlecone Pine Woodland }\end{array}$ & Muhlenbergia montana & Graminoid $\mathrm{C}_{4}$ & 3.6 & 4.1 \\
\hline $\begin{array}{l}\text { Western Great Plains Foothill and Piedmont } \\
\text { Grassland }\end{array}$ & Muhlenbergia montana & Graminoid $\mathrm{C}_{4}$ & 3.6 & 4.1 \\
\hline Chihuahuan Mixed Desert and Thornscrub & Muhlenbergia porteri & Graminoid $\mathrm{C}_{4}$ & 3.6 & 4.1 \\
\hline $\begin{array}{l}\text { Columbia Plateau Silver Sagebrush Seasonally } \\
\text { Flooded Shrub-Steppe }\end{array}$ & Muhlenbergia richardsonis & Graminoid $\mathrm{C}_{4}$ & 3.6 & 4.1 \\
\hline Columbia Plateau Vernal Pool & Muhlenbergia richardsonis & Graminoid $\mathrm{C}_{4}$ & 3.6 & 4.1 \\
\hline Inter-Mountain Basins Semi-Desert Shrub-Steppe & Muhlenbergia richardsonis & Graminoid $\mathrm{C}_{4}$ & 3.6 & 4.1 \\
\hline $\begin{array}{l}\text { Apacherian-Chihuahuan Semi-Desert Grassland } \\
\text { and Steppe }\end{array}$ & Muhlenbergia setifolia & Graminoid $\mathrm{C}_{4}$ & 3.6 & 4.1 \\
\hline North Pacific Bog and Fen & Myrica gale & Shrub- Evergreen & 2.7 & 2.4 \\
\hline North Pacific Montane Grassland & Nassella cernua & Graminoid $\mathrm{C}_{3}$ & 3.6 & 3.6 \\
\hline $\begin{array}{l}\text { Western Great Plains Foothill and Piedmont } \\
\text { Grassland }\end{array}$ & Nassella viridula & Graminoid $\mathrm{C}_{3}$ & 3.6 & 3.6 \\
\hline Chihuahuan Succulent Desert Scrub & Nolina microcarpa & Forb & 3 & 3.5 \\
\hline Madrean Oriental Chaparral & Nolina microcarpa & Forb & 3 & 3.5 \\
\hline $\begin{array}{l}\text { Atlantic Coastal Plain Small Brownwater River } \\
\text { Floodplain Forest }\end{array}$ & Nyssa aquatica & Tree- Deciduous & 3 & 4.2 \\
\hline $\begin{array}{l}\text { East Gulf Coastal Plain Freshwater Tidal Wooded } \\
\text { Swamp }\end{array}$ & Nyssa aquatica & Tree- Deciduous & 3 & 4.2 \\
\hline $\begin{array}{l}\text { East Gulf Coastal Plain Large River Floodplain } \\
\text { Forest }\end{array}$ & Nyssa aquatica & Tree- Deciduous & 3 & 4.2 \\
\hline Mississippi River Bottomland Depression & Nyssa aquatica & Tree- Deciduous & 3 & 4.2 \\
\hline Red River Large Floodplain Forest & Nyssa aquatica & Tree- Deciduous & 3 & 4.2 \\
\hline $\begin{array}{l}\text { Southern Atlantic Coastal Plain Nonriverine } \\
\text { Swamp and Wet Hardwood Forest }\end{array}$ & Nyssa aquatica & Tree- Deciduous & 3 & 4.2 \\
\hline
\end{tabular}




\begin{tabular}{|c|c|c|c|c|}
\hline Community & Species & Classification & Resistance & Resilience \\
\hline $\begin{array}{l}\text { Southern Atlantic Coastal Plain Tidal Wooded } \\
\text { Swamp }\end{array}$ & Nyssa aquatica & Tree- Deciduous & 3 & 4.2 \\
\hline $\begin{array}{l}\text { Southern Coastal Plain Blackwater River } \\
\text { Floodplain Forest }\end{array}$ & Nyssa aquatica & Tree-Deciduous & 3 & 4.2 \\
\hline $\begin{array}{l}\text { West Gulf Coastal Plain Large River Floodplain } \\
\text { Forest }\end{array}$ & Nyssa aquatica & Tree-Deciduous & 3 & 4.2 \\
\hline $\begin{array}{l}\text { West Gulf Coastal Plain Near-Coast Large River } \\
\text { Swamp }\end{array}$ & Nyssa aquatica & Tree-Deciduous & 3 & 4.2 \\
\hline $\begin{array}{l}\text { Atlantic Coastal Plain Blackwater Stream } \\
\text { Floodplain Forest }\end{array}$ & Nyssa biflora & Tree-Deciduous & 3 & 4.2 \\
\hline $\begin{array}{l}\text { Atlantic Coastal Plain Small Blackwater River } \\
\text { Floodplain Forest }\end{array}$ & Nyssa biflora & Tree- Deciduous & 3 & 4.2 \\
\hline Central Atlantic Coastal Plain Maritime Forest & Nyssa biflora & Tree-Deciduous & 3 & 4.2 \\
\hline $\begin{array}{l}\text { East Gulf Coastal Plain Freshwater Tidal Wooded } \\
\text { Swamp }\end{array}$ & Nyssa biflora & Tree- Deciduous & 3 & 4.2 \\
\hline Red River Large Floodplain Forest & Nyssa biflora & Tree-Deciduous & 3 & 4.2 \\
\hline $\begin{array}{l}\text { Southern Atlantic Coastal Plain Nonriverine } \\
\text { Swamp and Wet Hardwood Forest }\end{array}$ & Nyssa biflora & Tree-Deciduous & 3 & 4.2 \\
\hline $\begin{array}{l}\text { Southern Coastal Plain Blackwater River } \\
\text { Floodplain Forest }\end{array}$ & Nyssa biflora & Tree-Deciduous & 3 & 4.2 \\
\hline Southern Coastal Plain Nonriverine Basin Swamp & Nyssa biflora & Tree- Deciduous & 3 & 4.2 \\
\hline Southern Coastal Plain Nonriverine Cypress Dome & Nyssa biflora & Tree-Deciduous & 3 & 4.2 \\
\hline $\begin{array}{l}\text { Southern Coastal Plain Seepage Swamp and } \\
\text { Baygall }\end{array}$ & Nyssa biflora & Tree-Deciduous & 3 & 4.2 \\
\hline $\begin{array}{l}\text { West Gulf Coastal Plain Nonriverine Wet } \\
\text { Hardwood Flatwoods }\end{array}$ & Nyssa biflora & Tree- Deciduous & 3 & 4.2 \\
\hline $\begin{array}{l}\text { West Gulf Coastal Plain Seepage Swamp and } \\
\text { Baygall }\end{array}$ & Nyssa biflora & Tree- Deciduous & 3 & 4.2 \\
\hline $\begin{array}{l}\text { Atlantic Coastal Plain Streamhead Seepage } \\
\text { Swamp, Pocosin and Baygall }\end{array}$ & Nyssa biflora & Tree- Deciduous & 3 & 4.2 \\
\hline Lower Mississippi River Flatwoods & Nyssa sylvatica & Tree-Deciduous & 3 & 4.2 \\
\hline $\begin{array}{l}\text { Northern Atlantic Coastal Plain Basin Swamp and } \\
\text { Wet Hardwood Forest }\end{array}$ & Nyssa sylvatica & Tree-Deciduous & 3 & 4.2 \\
\hline West Gulf Coastal Plain Pine-Hardwood Flatwoods & Nyssa sylvatica & Tree- Deciduous & 3 & 4.2 \\
\hline $\begin{array}{l}\text { South-Central Interior / Upper Coastal Plain Wet } \\
\text { Flatwoods }\end{array}$ & Nyssa sylvatica & Tree- Deciduous & 3 & 4.2 \\
\hline Southern Appalachian Oak Forest & Nyssa sylvatica & Tree- Deciduous & 3 & 4.2 \\
\hline North American Warm Desert Wash & Olneya tesota & Tree-Evergreen & 3.2 & 2.2 \\
\hline Laurentian-Acadian Floodplain Forest & Onoclea sensibilis & Graminoid $C_{3}$ & 3.6 & 3.6 \\
\hline $\begin{array}{l}\text { North Pacific Montane Riparian Woodland and } \\
\text { Shrubland }\end{array}$ & Oplopanax horridus & Shrub- Deciduous & 2.7 & 3 \\
\hline Mojave Mid-Elevation Mixed Desert Scrub & Opuntia acanthocarpa & Forb & 3 & 3.5 \\
\hline $\begin{array}{l}\text { North American Warm Desert Bedrock Cliff and } \\
\text { Outcrop }\end{array}$ & Opuntia bigelovii & Forb & 3 & 3.5 \\
\hline North American Warm Desert Volcanic Rockland & Opuntia bigelovii & Forb & 3 & 3.5 \\
\hline Chihuahuan Succulent Desert Scrub & Opuntia engelmannii & Forb & 3 & 3.5 \\
\hline Tamaulipan Mixed Deciduous Thornscrub & Opuntia engelmannii & Forb & 3 & 3.5 \\
\hline Chihuahuan Creosotebush Desert Scrub & Opuntia imbricata & Forb & 3 & 3.5 \\
\hline Chihuahuan Creosotebush Desert Scrub & Opuntia kleiniae & Forb & 3 & 3.5 \\
\hline Southern California Coastal Scrub & Opuntia littoralis & Forb & 3 & 3.5 \\
\hline $\begin{array}{l}\text { Columbia Basin Foothill and Canyon Dry } \\
\text { Grassland }\end{array}$ & Opuntia polyacantha & Forb & 3 & 3.5 \\
\hline
\end{tabular}




\begin{tabular}{|c|c|c|c|c|}
\hline Community & Species & Classification & Resistance & Resilience \\
\hline $\begin{array}{l}\text { North American Warm Desert Bedrock Cliff and } \\
\text { Outcrop }\end{array}$ & Opuntia schottii & Forb & 3 & 3.5 \\
\hline Chihuahuan Succulent Desert Scrub & Opuntia spinosior & Forb & 3 & 3.5 \\
\hline $\begin{array}{l}\text { Northwestern Great Plains-Black Hills Ponderosa } \\
\text { Pine Woodland and Savanna }\end{array}$ & Oryzopsis asperifolia & Graminoid $C_{3}$ & 3.6 & 3.6 \\
\hline North-Central Interior Beech-Maple Forest & Osmorhiza claytonii & Forb & 3 & 3.5 \\
\hline $\begin{array}{l}\text { Southern Atlantic Coastal Plain Nonriverine } \\
\text { Swamp and Wet Hardwood Forest }\end{array}$ & Osmunda regalis & Graminoid $\mathrm{C}_{3}$ & 3.6 & 3.6 \\
\hline North-Central Interior Maple-Basswood Forest & Ostrya virginiana & Tree-Deciduous & 3 & 4.2 \\
\hline $\begin{array}{l}\text { Allegheny-Cumberland Dry Oak Forest and } \\
\text { Woodland }\end{array}$ & Oxydendrum arboreum & Tree-Deciduous & 3 & 4.2 \\
\hline $\begin{array}{l}\text { Central and Southern Appalachian Montane Oak } \\
\text { Forest }\end{array}$ & Oxydendrum arboreum & Tree-Deciduous & 3 & 4.2 \\
\hline Southern Appalachian Low-Elevation Pine Forest & Oxydendrum arboreum & Tree-Deciduous & 3 & 4.2 \\
\hline Southern Appalachian Oak Forest & Oxydendrum arboreum & Tree- Deciduous & 3 & 4.2 \\
\hline $\begin{array}{l}\text { Mediterranean California Alpine Bedrock and } \\
\text { Scree }\end{array}$ & Oxytropis borealis & Forb & 3 & 3.5 \\
\hline $\begin{array}{l}\text { Mediterranean California Alpine Bedrock and } \\
\text { Scree }\end{array}$ & Oxytropis parryi & Forb & 3 & 3.5 \\
\hline Central Florida Herbaceous Pondshore & Panicum abscissum & Graminoid $\mathrm{C}_{3}$ & 3.6 & 3.6 \\
\hline Central Florida Wet Prairie and Herbaceous Seep & Panicum abscissum & Graminoid $\mathrm{C}_{3}$ & 3.6 & 3.6 \\
\hline Texas Coast Dune and Coastal Grassland & Panicum amarum & Graminoid $\mathrm{C}_{4}$ & 3.6 & 4.1 \\
\hline South Texas Dune and Coastal Grassland & Panicum amarum & Graminoid $\mathrm{C}_{4}$ & 3.6 & 4.1 \\
\hline Southwest Florida Dune and Coastal Grassland & Panicum amarum & Graminoid $\mathrm{C}_{4}$ & 3.6 & 4.1 \\
\hline Eastern Highland Rim Prairie and Barrens & Panicum anceps & Graminoid $\mathrm{C}_{4}$ & 3.6 & 4.1 \\
\hline $\begin{array}{l}\text { Atlantic Coastal Plain Clay-Based Carolina Bay } \\
\text { Wetland }\end{array}$ & Panicum hemitomon & Graminoid $C_{3}$ & 3.6 & 3.6 \\
\hline Central Florida Herbaceous Pondshore & Panicum hemitomon & Graminoid $\mathrm{C}_{3}$ & 3.6 & 3.6 \\
\hline East Gulf Coastal Plain Depression Pond & Panicum hemitomon & Graminoid $\mathrm{C}_{3}$ & 3.6 & 3.6 \\
\hline Floridian Highlands Freshwater Marsh & Panicum hemitomon & Graminoid $\mathrm{C}_{3}$ & 3.6 & 3.6 \\
\hline $\begin{array}{l}\text { Gulf Coast Chenier Plain Fresh and Oligohaline } \\
\text { Tidal Marsh }\end{array}$ & Panicum hemitomon & Graminoid $C_{3}$ & 3.6 & 3.6 \\
\hline Southeastern Coastal Plain Natural Lakeshore & Panicum hemitomon & Graminoid $C_{3}$ & 3.6 & 3.6 \\
\hline Southern Coastal Plain Nonriverine Basin Swamp & Panicum hemitomon & Graminoid $C_{3}$ & 3.6 & 3.6 \\
\hline West Gulf Coastal Plain Flatwoods Pond & Panicum hemitomon & Graminoid $\mathrm{C}_{3}$ & 3.6 & 3.6 \\
\hline Chihuahuan Mixed Salt Desert Scrub & Panicum obtusum & Graminoid $\mathrm{C}_{4}$ & 3.6 & 4.1 \\
\hline Western Great Plains Riparian & Panicum obtusum & Graminoid $\mathrm{C}_{4}$ & 3.6 & 4.1 \\
\hline Eastern Highland Rim Prairie and Barrens & Panicum rigidulum & Graminoid $\mathrm{C}_{4}$ & 3.6 & 4.1 \\
\hline East Gulf Coastal Plain Depression Pond & Panicum verrucosum & Graminoid $C_{3}$ & 3.6 & 3.6 \\
\hline Eastern Highland Rim Prairie and Barrens & Panicum verrucosum & Graminoid $C_{3}$ & 3.6 & 3.6 \\
\hline Southern Atlantic Coastal Plain Depression Pond & Panicum verrucosum & Graminoid $\mathrm{C}_{3}$ & 3.6 & 3.6 \\
\hline Arkansas Valley Prairie and Woodland & Panicum virgatum & Graminoid $\mathrm{C}_{4}$ & 3.6 & 4.1 \\
\hline $\begin{array}{l}\text { Central and South Texas Coastal Fringe Forest and } \\
\text { Woodland }\end{array}$ & Panicum virgatum & Graminoid $\mathrm{C}_{4}$ & 3.6 & 4.1 \\
\hline Central Appalachian Stream and Riparian & Panicum virgatum & Graminoid $\mathrm{C}_{4}$ & 3.6 & 4.1 \\
\hline Great Lakes Wet-Mesic Lakeplain Prairie & Panicum virgatum & Graminoid $\mathrm{C}_{4}$ & 3.6 & 4.1 \\
\hline Northern Tallgrass Prairie & Panicum virgatum & Graminoid $\mathrm{C}_{4}$ & 3.6 & 4.1 \\
\hline Southeastern Great Plains Tallgrass Prairie & Panicum virgatum & Graminoid $\mathrm{C}_{4}$ & 3.6 & 4.1 \\
\hline Southern Atlantic Coastal Plain Depression Pond & Panicum virgatum & Graminoid $\mathrm{C}_{4}$ & 3.6 & 4.1 \\
\hline Texas Blackland Tallgrass Prairie & Panicum virgatum & Graminoid $\mathrm{C}_{4}$ & 3.6 & 4.1 \\
\hline
\end{tabular}




\begin{tabular}{|c|c|c|c|c|}
\hline Community & Species & Classification & Resistance & Resilience \\
\hline Texas-Louisiana Coastal Prairie & Panicum virgatum & Graminoid $\mathrm{C}_{4}$ & 3.6 & 4.1 \\
\hline West Gulf Coastal Plain Flatwoods Pond & Panicum virgatum & Graminoid $\mathrm{C}_{4}$ & 3.6 & 4.1 \\
\hline Western Great Plains Floodplain & Panicum virgatum & Graminoid $\mathrm{C}_{4}$ & 3.6 & 4.1 \\
\hline Western Great Plains Riparian & Panicum virgatum & Graminoid $\mathrm{C}_{4}$ & 3.6 & 4.1 \\
\hline Western Great Plains Tallgrass Prairie & Panicum virgatum & Graminoid $\mathrm{C}_{4}$ & 3.6 & 4.1 \\
\hline Central Tallgrass Prairie & Panicum virgatum & Graminoid $\mathrm{C}_{4}$ & 3.6 & 4.1 \\
\hline Tamaulipan Floodplain & Parkinsonia aculeata & Shrub- Evergreen & 2.7 & 2.4 \\
\hline North American Warm Desert Wash & Parkinsonia florida & Tree- Deciduous & 3 & 4.2 \\
\hline Sonoran Paloverde-Mixed Cacti Desert Scrub & Parkinsonia microphylla & Shrub- Deciduous & 2.7 & 3 \\
\hline Tamaulipan Mixed Deciduous Thornscrub & Parkinsonia texana & Shrub- Deciduous & 2.7 & 3 \\
\hline Tamaulipan Calcareous Thornscrub & Parkinsonia texana & Shrub- Deciduous & 2.7 & 3 \\
\hline Chihuahuan Creosotebush Desert Scrub & Parthenium incanum & Forb & 3 & 3.5 \\
\hline Southern Appalachian Montane Cliff and Talus & Parthenocissus quinquefolia & Shrub & 2.7 & 2.8 \\
\hline Central Mixedgrass Prairie & Pascopyrum smithii & Graminoid $C_{3}$ & 3.6 & 3.6 \\
\hline $\begin{array}{l}\text { Chihuahuan-Sonoran Desert Bottomland and } \\
\text { Swale Grassland }\end{array}$ & Pascopyrum smithii & Graminoid $C_{3}$ & 3.6 & 3.6 \\
\hline Inter-Mountain Basins Big Sagebrush Steppe & Pascopyrum smithii & Graminoid $\mathrm{C}_{3}$ & 3.6 & 3.6 \\
\hline Inter-Mountain Basins Greasewood Flat & Pascopyrum smithii & Graminoid $\mathrm{C}_{3}$ & 3.6 & 3.6 \\
\hline Inter-Mountain Basins Mat Saltbush Shrubland & Pascopyrum smithii & Graminoid $\mathrm{C}_{3}$ & 3.6 & 3.6 \\
\hline Inter-Mountain Basins Mixed Salt Desert Scrub & Pascopyrum smithii & Graminoid $C_{3}$ & 3.6 & 3.6 \\
\hline Inter-Mountain Basins Semi-Desert Shrub-Steppe & Pascopyrum smithii & Graminoid $C_{3}$ & 3.6 & 3.6 \\
\hline Northwestern Great Plains Floodplain & Pascopyrum smithii & Graminoid $\mathrm{C}_{3}$ & 3.6 & 3.6 \\
\hline Northwestern Great Plains Mixedgrass Prairie & Pascopyrum smithii & Graminoid $\mathrm{C}_{3}$ & 3.6 & 3.6 \\
\hline Northwestern Great Plains Riparian & Pascopyrum smithii & Graminoid $\mathrm{C}_{3}$ & 3.6 & 3.6 \\
\hline $\begin{array}{l}\text { Northwestern Great Plains-Black Hills Ponderosa } \\
\text { Pine Woodland and Savanna }\end{array}$ & Pascopyrum smithii & Graminoid $C_{3}$ & 3.6 & 3.6 \\
\hline Western Great Plains Closed Depression Wetland & Pascopyrum smithii & Graminoid $\mathrm{C}_{3}$ & 3.6 & 3.6 \\
\hline $\begin{array}{l}\text { Western Great Plains Foothill and Piedmont } \\
\text { Grassland }\end{array}$ & Pascopyrum smithii & Graminoid $\mathrm{C}_{3}$ & 3.6 & 3.6 \\
\hline Western Great Plains Riparian & Pascopyrum smithii & Graminoid $\mathrm{C}_{3}$ & 3.6 & 3.6 \\
\hline $\begin{array}{l}\text { Western Great Plains Depressional Wetland } \\
\text { Systems }\end{array}$ & Pascopyrum smithii & Graminoid $\mathrm{C}_{3}$ & 3.6 & 3.6 \\
\hline Texas Coast Dune and Coastal Grassland & Paspalum monostachyum & Graminoid $\mathrm{C}_{4}$ & 3.6 & 4.1 \\
\hline South Texas Dune and Coastal Grassland & Paspalum monostachyum & Graminoid $\mathrm{C}_{4}$ & 3.6 & 4.1 \\
\hline Texas-Louisiana Coastal Prairie & Paspalum plicatulum & Graminoid $\mathrm{C}_{4}$ & 3.6 & 4.1 \\
\hline South Texas Sand Sheet Grassland & Paspalum plicatulum & Graminoid $\mathrm{C}_{4}$ & 3.6 & 4.1 \\
\hline Florida Dry Prairie & Paspalum setaceum & Graminoid $\mathrm{C}_{4}$ & 3.6 & 4.1 \\
\hline $\begin{array}{l}\text { Gulf Coast Chenier Plain Fresh and Oligohaline } \\
\text { Tidal Marsh }\end{array}$ & Paspalum vaginatum & Graminoid $\mathrm{C}_{4}$ & 3.6 & 4.1 \\
\hline Southeastern Coastal Plain Interdunal Wetland & Paspalum vaginatum & Graminoid $\mathrm{C}_{4}$ & 3.6 & 4.1 \\
\hline Texas Coast Fresh and Oligohaline Tidal Marsh & Paspalum vaginatum & Graminoid $\mathrm{C}_{4}$ & 3.6 & 4.1 \\
\hline Southern Coastal Plain Nonriverine Basin Swamp & Peltandra virginica & Forb & 3 & 3.5 \\
\hline Tamaulipan Calcareous Thornscrub & Pennisetum ciliare & Graminoid $\mathrm{C}_{4}$ & 3.6 & 4.1 \\
\hline Tamaulipan Floodplain & Pennisetum ciliare & Graminoid $\mathrm{C}_{4}$ & 3.6 & 4.1 \\
\hline Tamaulipan Ramadero & Pennisetum ciliare & Graminoid $\mathrm{C}_{4}$ & 3.6 & 4.1 \\
\hline Tamaulipan Savanna Grassland & Pennisetum ciliare & Graminoid $\mathrm{C}_{4}$ & 3.6 & 4.1 \\
\hline Edwards Plateau Cliff & Penstemon baccharifolius & Forb & 3 & 3.5 \\
\hline $\begin{array}{l}\text { Atlantic Coastal Plain Peatland Pocosin and } \\
\text { Canebrake }\end{array}$ & Persea palustris & Shrub- Evergreen & 2.7 & 2.4 \\
\hline
\end{tabular}




\begin{tabular}{|c|c|c|c|c|}
\hline Community & Species & Classification & Resistance & Resilience \\
\hline Central Atlantic Coastal Plain Maritime Forest & Persea palustris & Shrub- Evergreen & 2.7 & 2.4 \\
\hline South Florida Bayhead Swamp & Persea palustris & Shrub-Evergreen & 2.7 & 2.4 \\
\hline South Florida Cypress Dome & Persea palustris & Shrub- Evergreen & 2.7 & 2.4 \\
\hline $\begin{array}{l}\text { Atlantic Coastal Plain Streamhead Seepage } \\
\text { Swamp, Pocosin and Baygall }\end{array}$ & Persea palustris & Shrub- Evergreen & 2.7 & 2.4 \\
\hline Rocky Mountain Alpine-Montane Wet Meadow & Phippsia algida & Graminoid $\mathrm{C}_{3}$ & 3.6 & 3.6 \\
\hline $\begin{array}{l}\text { Northern Rocky Mountain Subalpine-Upper } \\
\text { Montane Grassland }\end{array}$ & Phleum alpinum & Graminoid $C_{3}$ & 3.6 & 3.6 \\
\hline North Pacific Herbaceous Bald and Bluff & Phlox diffusa & Forb & 3 & 3.5 \\
\hline Inter-Mountain Basins Mat Saltbush Shrubland & Phlox hoodii & Forb & 3 & 3.5 \\
\hline Rocky Mountain Alpine Fell-Field & Phlox pulvinata & Forb & 3 & 3.5 \\
\hline Rocky Mountain Alpine Turf & Phlox pulvinata & Forb & 3 & 3.5 \\
\hline North-Central Interior Freshwater Marsh & Phragmites australis & Graminoid $\mathrm{C}_{3}$ & 3.6 & 3.6 \\
\hline Acadian-Appalachian Alpine Tundra & Phyllodoce caerulea & Shrub & 2.7 & 2.8 \\
\hline Rocky Mountain Alpine Dwarf-Shrubland & Phyllodoce empetriformis & Shrub & 2.7 & 2.8 \\
\hline North Pacific Maritime Mesic Subalpine Parkland & Phyllodoce empetriformis & Shrub- Evergreen & 2.7 & 2.4 \\
\hline $\begin{array}{l}\text { North Pacific Dry and Mesic Alpine Dwarf- } \\
\text { Shrubland, Fell-Field and Meadow }\end{array}$ & Phyllodoce empetriformis & Shrub- Evergreen & 2.7 & 2.4 \\
\hline $\begin{array}{l}\text { Middle Rocky Mountain Montane Douglas-fir } \\
\text { Forest and Woodland }\end{array}$ & Physocarpus malvaceus & Shrub- Deciduous & 2.7 & 3 \\
\hline $\begin{array}{l}\text { Northern Rocky Mountain Montane-Foothill } \\
\text { Deciduous Shrubland }\end{array}$ & Physocarpus malvaceus & Shrub-Deciduous & 2.7 & 3 \\
\hline $\begin{array}{l}\text { Southern Rocky Mountain Mesic Montane Mixed } \\
\text { Conifer Forest and Woodland }\end{array}$ & Physocarpus malvaceus & Shrub- Deciduous & 2.7 & 3 \\
\hline $\begin{array}{l}\text { Inter-Mountain Basins Aspen-Mixed Conifer Forest } \\
\text { and Woodland }\end{array}$ & Picea engelmannii & Tree-Evergreen & 3.2 & 2.2 \\
\hline $\begin{array}{l}\text { Inter-Mountain Basins Subalpine Limber- } \\
\text { Bristlecone Pine Woodland }\end{array}$ & Picea engelmannii & Tree- Evergreen & 3.2 & 2.2 \\
\hline Northern Rocky Mountain Conifer Swamp & Picea engelmannii & Tree- Evergreen & 3.2 & 2.2 \\
\hline Northern Rocky Mountain Wooded Vernal Pool & Picea engelmannii & Tree-Evergreen & 3.2 & 2.2 \\
\hline $\begin{array}{l}\text { Rocky Mountain Subalpine Dry-Mesic Spruce-Fir } \\
\text { Forest and Woodland }\end{array}$ & Picea engelmannii & Tree- Evergreen & 3.2 & 2.2 \\
\hline $\begin{array}{l}\text { Rocky Mountain Subalpine Mesic-Wet Spruce-Fir } \\
\text { Forest and Woodland }\end{array}$ & Picea engelmannii & Tree- Evergreen & 3.2 & 2.2 \\
\hline $\begin{array}{l}\text { Rocky Mountain Subalpine-Montane Limber- } \\
\text { Bristlecone Pine Woodland }\end{array}$ & Picea engelmannii & Tree- Evergreen & 3.2 & 2.2 \\
\hline $\begin{array}{l}\text { Rocky Mountain Subalpine-Montane Riparian } \\
\text { Woodland }\end{array}$ & Picea engelmannii & Tree- Evergreen & 3.2 & 2.2 \\
\hline $\begin{array}{l}\text { Rocky Mountain Subalpine Mesic-Wet Spruce-Fir } \\
\text { Forest and Woodland }\end{array}$ & Picea engelmannii & Tree- Evergreen & 3.2 & 2.2 \\
\hline Acadian-Appalachian Montane Spruce-Fir Forest & Picea glauca & Tree- Evergreen & 3.2 & 2.2 \\
\hline $\begin{array}{l}\text { Acadian-Appalachian Subalpine Woodland and } \\
\text { Heath-Krummholz }\end{array}$ & Picea glauca & Tree-Evergreen & 3.2 & 2.2 \\
\hline Eastern Boreal Floodplain & Picea glauca & Tree- Evergreen & 3.2 & 2.2 \\
\hline Boreal White Spruce-Fir-Hardwood Forest & Picea glauca & Tree- Evergreen & 3.2 & 2.2 \\
\hline $\begin{array}{l}\text { Eastern Hemi-Boreal Mesic Balsam Fir-Spruce } \\
\text { Forest }\end{array}$ & Picea glauca & Tree-Evergreen & 3.2 & 2.2 \\
\hline Acadian-Appalachian Montane Spruce-Fir Forest & Picea mariana & Tree-Evergreen & 3.2 & 2.2 \\
\hline $\begin{array}{l}\text { Acadian-Appalachian Subalpine Woodland and } \\
\text { Heath-Krummholz }\end{array}$ & Picea mariana & Tree- Evergreen & 3.2 & 2.2 \\
\hline Boreal-Laurentian Bog & Picea mariana & Tree-Evergreen & 3.2 & 2.2 \\
\hline
\end{tabular}




\begin{tabular}{|c|c|c|c|c|}
\hline Community & Species & Classification & Resistance & Resilience \\
\hline $\begin{array}{l}\text { Boreal-Laurentian Conifer Acidic Swamp and } \\
\text { Treed Poor Fen }\end{array}$ & Picea mariana & Tree- Evergreen & 3.2 & 2.2 \\
\hline Eastern Boreal Floodplain & Picea mariana & Tree-Evergreen & 3.2 & 2.2 \\
\hline $\begin{array}{l}\text { North-Central Interior and Appalachian Acidic } \\
\text { Peatland }\end{array}$ & Picea mariana & Tree-Evergreen & 3.2 & 2.2 \\
\hline $\begin{array}{l}\text { Eastern Hemi-Boreal Dry-Mesic Pine-Black Spruce- } \\
\text { Hardwood Forest }\end{array}$ & Picea mariana & Tree-Evergreen & 3.2 & 2.2 \\
\hline $\begin{array}{l}\text { Inter-Mountain Basins Aspen-Mixed Conifer Forest } \\
\text { and Woodland }\end{array}$ & Picea pungens & Tree-Evergreen & 3.2 & 2.2 \\
\hline $\begin{array}{l}\text { Rocky Mountain Lower Montane-Foothill Riparian } \\
\text { Woodland and Shrubland }\end{array}$ & Picea pungens & Tree- Evergreen & 3.2 & 2.2 \\
\hline $\begin{array}{l}\text { Southern Rocky Mountain Dry-Mesic Montane } \\
\text { Mixed Conifer Forest and Woodland }\end{array}$ & Picea pungens & Tree-Evergreen & 3.2 & 2.2 \\
\hline $\begin{array}{l}\text { Southern Rocky Mountain Mesic Montane Mixed } \\
\text { Conifer Forest and Woodland }\end{array}$ & Picea pungens & Tree-Evergreen & 3.2 & 2.2 \\
\hline $\begin{array}{l}\text { Acadian Low-Elevation Spruce-Fir-Hardwood } \\
\text { Forest }\end{array}$ & Picea rubens & Tree- Evergreen & 3.2 & 2.2 \\
\hline Acadian-Appalachian Montane Spruce-Fir Forest & Picea rubens & Tree-Evergreen & 3.2 & 2.2 \\
\hline $\begin{array}{l}\text { Central and Southern Appalachian Spruce-Fir } \\
\text { Forest }\end{array}$ & Picea rubens & Tree- Evergreen & 3.2 & 2.2 \\
\hline Eastern Boreal Floodplain & Picea rubens & Tree- Evergreen & 3.2 & 2.2 \\
\hline High Allegheny Wetland & Picea rubens & Tree- Evergreen & 3.2 & 2.2 \\
\hline North Pacific Hardwood-Conifer Swamp & Picea sitchensis & Tree-Evergreen & 3.2 & 2.2 \\
\hline North Pacific Intertidal Freshwater Wetland & Picea sitchensis & Tree-Evergreen & 3.2 & 2.2 \\
\hline $\begin{array}{l}\text { North Pacific Lowland Mixed Hardwood-Conifer } \\
\text { Forest }\end{array}$ & Picea sitchensis & Tree- Evergreen & 3.2 & 2.2 \\
\hline $\begin{array}{l}\text { North Pacific Lowland Riparian Forest and } \\
\text { Shrubland }\end{array}$ & Picea sitchensis & Tree-Evergreen & 3.2 & 2.2 \\
\hline $\begin{array}{l}\text { North Pacific Maritime Coastal Sand Dune and } \\
\text { Strand }\end{array}$ & Picea sitchensis & Tree- Evergreen & 3.2 & 2.2 \\
\hline North Pacific Seasonal Sitka Spruce Forest & Picea sitchensis & Tree-Evergreen & 3.2 & 2.2 \\
\hline Inter-Mountain Basins Mat Saltbush Shrubland & Picrothamnus desertorum & Shrub & 2.7 & 2.8 \\
\hline Inter-Mountain Basins Mixed Salt Desert Scrub & Picrothamnus desertorum & Shrub- Deciduous & 2.7 & 3 \\
\hline Mediterranean California Subalpine Woodland & Pinus albicaulis & Tree-Evergreen & 3.2 & 2.2 \\
\hline $\begin{array}{l}\text { Northern Rocky Mountain Subalpine Woodland } \\
\text { and Parkland }\end{array}$ & Pinus albicaulis & Tree-Evergreen & 3.2 & 2.2 \\
\hline $\begin{array}{l}\text { Rocky Mountain Subalpine-Montane Limber- } \\
\text { Bristlecone Pine Woodland }\end{array}$ & Pinus aristata & Tree-Evergreen & 3.2 & 2.2 \\
\hline $\begin{array}{l}\text { Klamath-Siskiyou Lower Montane Serpentine } \\
\text { Mixed Conifer Woodland }\end{array}$ & Pinus attenuata & Tree-Evergreen & 3.2 & 2.2 \\
\hline $\begin{array}{l}\text { Klamath-Siskiyou Xeromorphic Serpentine } \\
\text { Savanna and Chaparral }\end{array}$ & Pinus attenuata & Tree- Evergreen & 3.2 & 2.2 \\
\hline Mediterranean California Subalpine Woodland & Pinus balfouriana & Tree- Evergreen & 3.2 & 2.2 \\
\hline Great Lakes Wooded Dune and Swale & Pinus banksiana & Tree- Evergreen & 3.2 & 2.2 \\
\hline Laurentian Jack Pine-Red Pine Forest & Pinus banksiana & Tree- Evergreen & 3.2 & 2.2 \\
\hline Laurentian Pine-Oak Barrens & Pinus banksiana & Tree- Evergreen & 3.2 & 2.2 \\
\hline $\begin{array}{l}\text { Eastern Hemi-Boreal Dry-Mesic Pine-Black Spruce- } \\
\text { Hardwood Forest }\end{array}$ & Pinus banksiana & Tree- Evergreen & 3.2 & 2.2 \\
\hline Madrean Pinyon-Juniper Woodland & Pinus cembroides & Tree-Evergreen & 3.2 & 2.2 \\
\hline East Gulf Coastal Plain Maritime Forest & Pinus clausa & Tree- Evergreen & 3.2 & 2.2 \\
\hline Florida Peninsula Inland Scrub & Pinus clausa & Tree- Evergreen & 3.2 & 2.2 \\
\hline $\begin{array}{l}\text { East Cascades Mesic Montane Mixed Conifer } \\
\text { Forest and Woodland }\end{array}$ & Pinus contorta & Tree- Evergreen & 3.2 & 2.2 \\
\hline
\end{tabular}




\begin{tabular}{|c|c|c|c|c|}
\hline Community & Species & Classification & Resistance & Resilience \\
\hline $\begin{array}{l}\text { Inter-Mountain Basins Aspen-Mixed Conifer Forest } \\
\text { and Woodland }\end{array}$ & Pinus contorta & Tree- Evergreen & 3.2 & 2.2 \\
\hline North Pacific Hardwood-Conifer Swamp & Pinus contorta & Tree- Evergreen & 3.2 & 2.2 \\
\hline North Pacific Wooded Volcanic Flowage & Pinus contorta & Tree- Evergreen & 3.2 & 2.2 \\
\hline Northern Rocky Mountain Conifer Swamp & Pinus contorta & Tree- Evergreen & 3.2 & 2.2 \\
\hline $\begin{array}{l}\text { Northern Rocky Mountain Dry-Mesic Montane } \\
\text { Mixed Conifer Forest }\end{array}$ & Pinus contorta & Tree- Evergreen & 3.2 & 2.2 \\
\hline Northern Rocky Mountain Wooded Vernal Pool & Pinus contorta & Tree- Evergreen & 3.2 & 2.2 \\
\hline Rocky Mountain Lodgepole Pine Forest & Pinus contorta & Tree-Evergreen & 3.2 & 2.2 \\
\hline Rocky Mountain Poor-Site Lodgepole Pine Forest & Pinus contorta & Tree- Evergreen & 3.2 & 2.2 \\
\hline $\begin{array}{l}\text { Rocky Mountain Subalpine Dry-Mesic Spruce-Fir } \\
\text { Forest and Woodland }\end{array}$ & Pinus contorta & Tree- Evergreen & 3.2 & 2.2 \\
\hline $\begin{array}{l}\text { Sierra Nevada Subalpine Lodgepole Pine Forest } \\
\text { and Woodland }\end{array}$ & Pinus contorta & Tree- Evergreen & 3.2 & 2.2 \\
\hline $\begin{array}{l}\text { Southern Rocky Mountain Dry-Mesic Montane } \\
\text { Mixed Conifer Forest and Woodland }\end{array}$ & Pinus contorta & Tree- Evergreen & 3.2 & 2.2 \\
\hline $\begin{array}{l}\text { Southern Rocky Mountain Mesic Montane Mixed } \\
\text { Conifer Forest and Woodland }\end{array}$ & Pinus contorta & Tree-Evergreen & 3.2 & 2.2 \\
\hline $\begin{array}{l}\text { North Pacific Dry Douglas-fir-(Madrone) Forest and } \\
\text { Woodland }\end{array}$ & Pinus contorta & Tree- Evergreen & 3.2 & 2.2 \\
\hline $\begin{array}{l}\text { California Coastal Closed-Cone Conifer Forest and } \\
\text { Woodland }\end{array}$ & Pinus contorta & Tree- Evergreen & 3.2 & 2.2 \\
\hline $\begin{array}{l}\text { California Coastal Closed-Cone Conifer Forest and } \\
\text { Woodland }\end{array}$ & Pinus contorta & Tree- Evergreen & 3.2 & 2.2 \\
\hline North Pacific Hardwood-Conifer Swamp & Pinus contorta & Tree- Evergreen & 3.2 & 2.2 \\
\hline $\begin{array}{l}\text { North Pacific Maritime Coastal Sand Dune and } \\
\text { Strand }\end{array}$ & Pinus contorta & Tree-Evergreen & 3.2 & 2.2 \\
\hline Central California Coast Ranges Cliff and Canyon & Pinus contorta & Tree- Evergreen & 3.2 & 2.2 \\
\hline $\begin{array}{l}\text { Inter-Mountain Basins Aspen-Mixed Conifer Forest } \\
\text { and Woodland }\end{array}$ & Pinus contorta & Tree- Evergreen & 3.2 & 2.2 \\
\hline Mediterranean California Red Fir Forest & Pinus contorta & Tree- Evergreen & 3.2 & 2.2 \\
\hline Mediterranean California Subalpine Woodland & Pinus contorta & Tree-Evergreen & 3.2 & 2.2 \\
\hline $\begin{array}{l}\text { North Pacific Montane Riparian Woodland and } \\
\text { Shrubland }\end{array}$ & Pinus contorta & Tree- Evergreen & 3.2 & 2.2 \\
\hline Sierra Nevada Cliff and Canyon & Pinus contorta & Tree- Evergreen & 3.2 & 2.2 \\
\hline $\begin{array}{l}\text { Sierra Nevada Subalpine Lodgepole Pine Forest } \\
\text { and Woodland }\end{array}$ & Pinus contorta & Tree- Evergreen & 3.2 & 2.2 \\
\hline $\begin{array}{l}\text { California Coastal Closed-Cone Conifer Forest and } \\
\text { Woodland }\end{array}$ & Pinus coulteri & Tree- Evergreen & 3.2 & 2.2 \\
\hline $\begin{array}{l}\text { Central and Southern California Mixed Evergreen } \\
\text { Woodland }\end{array}$ & Pinus coulteri & Tree- Evergreen & 3.2 & 2.2 \\
\hline $\begin{array}{l}\text { Mediterranean California Mesic Serpentine } \\
\text { Woodland and Chaparral }\end{array}$ & Pinus coulteri & Tree- Evergreen & 3.2 & 2.2 \\
\hline Madrean Pinyon-Juniper Woodland & Pinus discolor & Tree-Evergreen & 3.2 & 2.2 \\
\hline Central Appalachian Dry Oak-Pine Forest & Pinus echinata & Tree- Evergreen & 3.2 & 2.2 \\
\hline Central Appalachian Pine-Oak Rocky Woodland & Pinus echinata & Tree- Evergreen & 3.2 & 2.2 \\
\hline Crowley's Ridge Sand Forest & Pinus echinata & Tree- Evergreen & 3.2 & 2.2 \\
\hline Cumberland Sandstone Glade and Barrens & Pinus echinata & Tree- Evergreen & 3.2 & 2.2 \\
\hline $\begin{array}{l}\text { East Gulf Coastal Plain Interior Shortleaf Pine-Oak } \\
\text { Forest }\end{array}$ & Pinus echinata & Tree- Evergreen & 3.2 & 2.2 \\
\hline $\begin{array}{l}\text { Ozark-Ouachita Shortleaf Pine-Bluestem } \\
\text { Woodland }\end{array}$ & Pinus echinata & Tree- Evergreen & 3.2 & 2.2 \\
\hline
\end{tabular}




\begin{tabular}{|c|c|c|c|c|}
\hline Community & Species & Classification & Resistance & Resilience \\
\hline $\begin{array}{l}\text { Ozark-Ouachita Shortleaf Pine-Oak Forest and } \\
\text { Woodland }\end{array}$ & Pinus echinata & Tree-Evergreen & 3.2 & 2.2 \\
\hline Southeastern Interior Longleaf Pine Woodland & Pinus echinata & Tree- Evergreen & 3.2 & 2.2 \\
\hline Southern Appalachian Low-Elevation Pine Forest & Pinus echinata & Tree- Evergreen & 3.2 & 2.2 \\
\hline West Gulf Coastal Plain Pine-Hardwood Forest & Pinus echinata & Tree- Evergreen & 3.2 & 2.2 \\
\hline $\begin{array}{l}\text { West Gulf Coastal Plain Sandhill Oak and Shortleaf } \\
\text { Pine Forest and Woodland }\end{array}$ & Pinus echinata & Tree- Evergreen & 3.2 & 2.2 \\
\hline $\begin{array}{l}\text { West Gulf Coastal Plain Stream Terrace Sandyland } \\
\text { Longleaf Pine Woodland }\end{array}$ & Pinus echinata & Tree- Evergreen & 3.2 & 2.2 \\
\hline Southern Piedmont Dry Oak-(Pine) Forest & Pinus echinata & Tree-Evergreen & 3.2 & 2.2 \\
\hline Colorado Plateau Pinyon-Juniper Shrubland & Pinus edulis & Tree- Evergreen & 3.2 & 2.2 \\
\hline Colorado Plateau Pinyon-Juniper Woodland & Pinus edulis & Tree- Evergreen & 3.2 & 2.2 \\
\hline Inter-Mountain Basins Cliff and Canyon & Pinus edulis & Tree-Evergreen & 3.2 & 2.2 \\
\hline Madrean Pinyon-Juniper Woodland & Pinus edulis & Tree-Evergreen & 3.2 & 2.2 \\
\hline $\begin{array}{l}\text { Southern Rocky Mountain Pinyon-Juniper } \\
\text { Woodland }\end{array}$ & Pinus edulis & Tree-Evergreen & 3.2 & 2.2 \\
\hline $\begin{array}{l}\text { Colorado Plateau Mixed Bedrock Canyon and } \\
\text { Tableland }\end{array}$ & Pinus edulis & Tree-Evergreen & 3.2 & 2.2 \\
\hline $\begin{array}{l}\text { Central Atlantic Coastal Plain Wet Longleaf Pine } \\
\text { Savanna and Flatwoods }\end{array}$ & Pinus elliottii & Tree- Evergreen & 3.2 & 2.2 \\
\hline Central Florida Pine Flatwoods & Pinus elliottii & Tree- Evergreen & 3.2 & 2.2 \\
\hline $\begin{array}{l}\text { Southern Atlantic Coastal Plain Wet Pine Savanna } \\
\text { and Flatwoods }\end{array}$ & Pinus elliottii & Tree-Evergreen & 3.2 & 2.2 \\
\hline East Gulf Coastal Plain Near-Coast Pine Flatwoods & Pinus elliottii & Tree- Evergreen & 3.2 & 2.2 \\
\hline South Florida Pine Flatwoods & Pinus elliottii & Tree- Evergreen & 3.2 & 2.2 \\
\hline South Florida Pine Rockland & Pinus elliottii & Tree-Evergreen & 3.2 & 2.2 \\
\hline East Gulf Coastal Plain Maritime Forest & Pinus elliottii & Tree- Evergreen & 3.2 & 2.2 \\
\hline Southern Atlantic Coastal Plain Maritime Forest & Pinus elliottii & Tree-Evergreen & 3.2 & 2.2 \\
\hline $\begin{array}{l}\text { Madrean Lower Montane Pine-Oak Forest and } \\
\text { Woodland }\end{array}$ & Pinus engelmannii & Tree- Evergreen & 3.2 & 2.2 \\
\hline $\begin{array}{l}\text { Inter-Mountain Basins Aspen-Mixed Conifer Forest } \\
\text { and Woodland }\end{array}$ & Pinus flexilis & Tree- Evergreen & 3.2 & 2.2 \\
\hline Inter-Mountain Basins Cliff and Canyon & Pinus flexilis & Tree-Evergreen & 3.2 & 2.2 \\
\hline $\begin{array}{l}\text { Inter-Mountain Basins Subalpine Limber- } \\
\text { Bristlecone Pine Woodland }\end{array}$ & Pinus flexilis & Tree- Evergreen & 3.2 & 2.2 \\
\hline $\begin{array}{l}\text { Inter-Mountain Basins Volcanic Rock and Cinder } \\
\text { Land }\end{array}$ & Pinus flexilis & Tree- Evergreen & 3.2 & 2.2 \\
\hline Mediterranean California Subalpine Woodland & Pinus flexilis & Tree-Evergreen & 3.2 & 2.2 \\
\hline $\begin{array}{l}\text { Rocky Mountain Foothill Limber Pine-Juniper } \\
\text { Woodland }\end{array}$ & Pinus flexilis & Tree- Evergreen & 3.2 & 2.2 \\
\hline $\begin{array}{l}\text { Rocky Mountain Subalpine-Montane Limber- } \\
\text { Bristlecone Pine Woodland }\end{array}$ & Pinus flexilis & Tree-Evergreen & 3.2 & 2.2 \\
\hline $\begin{array}{l}\text { Southern Rocky Mountain Dry-Mesic Montane } \\
\text { Mixed Conifer Forest and Woodland }\end{array}$ & Pinus flexilis & Tree- Evergreen & 3.2 & 2.2 \\
\hline $\begin{array}{l}\text { East Gulf Coastal Plain Southern Loblolly- } \\
\text { Hardwood Flatwoods }\end{array}$ & Pinus glabra & Tree-Evergreen & 3.2 & 2.2 \\
\hline $\begin{array}{l}\text { East Gulf Coastal Plain Southern Loess Bluff } \\
\text { Forest }\end{array}$ & Pinus glabra & Tree-Evergreen & 3.2 & 2.2 \\
\hline $\begin{array}{l}\text { Inter-Mountain Basins Aspen-Mixed Conifer Forest } \\
\text { and Woodland }\end{array}$ & Pinus jeffreyi & Tree- Evergreen & 3.2 & 2.2 \\
\hline $\begin{array}{l}\text { Klamath-Siskiyou Lower Montane Serpentine } \\
\text { Mixed Conifer Woodland }\end{array}$ & Pinus jeffreyi & Tree-Evergreen & 3.2 & 2.2 \\
\hline
\end{tabular}




\begin{tabular}{|c|c|c|c|c|}
\hline Community & Species & Classification & Resistance & Resilience \\
\hline $\begin{array}{l}\text { Klamath-Siskiyou Upper Montane Serpentine } \\
\text { Mixed Conifer Woodland }\end{array}$ & Pinus jeffreyi & Tree-Evergreen & 3.2 & 2.2 \\
\hline $\begin{array}{l}\text { Klamath-Siskiyou Xeromorphic Serpentine } \\
\text { Savanna and Chaparral }\end{array}$ & Pinus jeffreyi & Tree-Evergreen & 3.2 & 2.2 \\
\hline $\begin{array}{l}\text { Mediterranean California Mesic Serpentine } \\
\text { Woodland and Chaparral }\end{array}$ & Pinus jeffreyi & Tree- Evergreen & 3.2 & 2.2 \\
\hline Mediterranean California Red Fir Forest & Pinus jeffreyi & Tree- Evergreen & 3.2 & 2.2 \\
\hline Sierra Nevada Cliff and Canyon & Pinus jeffreyi & Tree-Evergreen & 3.2 & 2.2 \\
\hline $\begin{array}{l}\text { California Montane Jeffrey Pine-(Ponderosa Pine) } \\
\text { Woodland }\end{array}$ & Pinus jeffreyi & Tree-Evergreen & 3.2 & 2.2 \\
\hline $\begin{array}{l}\text { Klamath-Siskiyou Upper Montane Serpentine } \\
\text { Mixed Conifer Woodland }\end{array}$ & Pinus jeffreyi & Tree-Evergreen & 3.2 & 2.2 \\
\hline California Montane Woodland and Chaparral & Pinus lambertiana & Tree-Evergreen & 3.2 & 2.2 \\
\hline $\begin{array}{l}\text { Klamath-Siskiyou Lower Montane Serpentine } \\
\text { Mixed Conifer Woodland }\end{array}$ & Pinus lambertiana & Tree- Evergreen & 3.2 & 2.2 \\
\hline $\begin{array}{l}\text { Mediterranean California Mesic Mixed Conifer } \\
\text { Forest and Woodland }\end{array}$ & Pinus lambertiana & Tree-Evergreen & 3.2 & 2.2 \\
\hline $\begin{array}{l}\text { Madrean Lower Montane Pine-Oak Forest and } \\
\text { Woodland }\end{array}$ & Pinus leiophylla & Tree-Evergreen & 3.2 & 2.2 \\
\hline $\begin{array}{l}\text { Inter-Mountain Basins Subalpine Limber- } \\
\text { Bristlecone Pine Woodland }\end{array}$ & Pinus longaeva & Tree-Evergreen & 3.2 & 2.2 \\
\hline Great Basin Pinyon-Juniper Woodland & Pinus monophylla & Tree- Evergreen & 3.2 & 2.2 \\
\hline Inter-Mountain Basins Cliff and Canyon & Pinus monophylla & Tree-Evergreen & 3.2 & 2.2 \\
\hline Sierra Nevada Cliff and Canyon & Pinus monophylla & Tree- Evergreen & 3.2 & 2.2 \\
\hline $\begin{array}{l}\text { East Cascades Mesic Montane Mixed Conifer } \\
\text { Forest and Woodland }\end{array}$ & Pinus monticola & Tree-Evergreen & 3.2 & 2.2 \\
\hline $\begin{array}{l}\text { Klamath-Siskiyou Upper Montane Serpentine } \\
\text { Mixed Conifer Woodland }\end{array}$ & Pinus monticola & Tree-Evergreen & 3.2 & 2.2 \\
\hline Mediterranean California Red Fir Forest & Pinus monticola & Tree- Evergreen & 3.2 & 2.2 \\
\hline Mediterranean California Subalpine Woodland & Pinus monticola & Tree- Evergreen & 3.2 & 2.2 \\
\hline North Pacific Serpentine Barren & Pinus monticola & Tree- Evergreen & 3.2 & 2.2 \\
\hline North Pacific Wooded Volcanic Flowage & Pinus monticola & Tree- Evergreen & 3.2 & 2.2 \\
\hline $\begin{array}{l}\text { Sierran-Intermontane Desert Western White Pine- } \\
\text { White Fir Woodland }\end{array}$ & Pinus monticola & Tree-Evergreen & 3.2 & 2.2 \\
\hline $\begin{array}{l}\text { Klamath-Siskiyou Upper Montane Serpentine } \\
\text { Mixed Conifer Woodland }\end{array}$ & Pinus monticola & Tree-Evergreen & 3.2 & 2.2 \\
\hline $\begin{array}{l}\text { California Coastal Closed-Cone Conifer Forest and } \\
\text { Woodland }\end{array}$ & Pinus muricata & Tree-Evergreen & 3.2 & 2.2 \\
\hline $\begin{array}{l}\text { Atlantic Coastal Plain Fall-line Sandhills Longleaf } \\
\text { Pine Woodland }\end{array}$ & Pinus palustris & Tree-Evergreen & 3.2 & 2.2 \\
\hline $\begin{array}{l}\text { Atlantic Coastal Plain Upland Longleaf Pine } \\
\text { Woodland }\end{array}$ & Pinus palustris & Tree- Evergreen & 3.2 & 2.2 \\
\hline $\begin{array}{l}\text { Central Atlantic Coastal Plain Wet Longleaf Pine } \\
\text { Savanna and Flatwoods }\end{array}$ & Pinus palustris & Tree- Evergreen & 3.2 & 2.2 \\
\hline Central Florida Pine Flatwoods & Pinus palustris & Tree-Evergreen & 3.2 & 2.2 \\
\hline $\begin{array}{l}\text { East Gulf Coastal Plain Interior Upland Longleaf } \\
\text { Pine Woodland }\end{array}$ & Pinus palustris & Tree- Evergreen & 3.2 & 2.2 \\
\hline East Gulf Coastal Plain Maritime Forest & Pinus palustris & Tree-Evergreen & 3.2 & 2.2 \\
\hline Florida Longleaf Pine Sandhill & Pinus palustris & Tree-Evergreen & 3.2 & 2.2 \\
\hline Southeastern Interior Longleaf Pine Woodland & Pinus palustris & Tree-Evergreen & 3.2 & 2.2 \\
\hline Southern Atlantic Coastal Plain Maritime Forest & Pinus palustris & Tree-Evergreen & 3.2 & 2.2 \\
\hline $\begin{array}{l}\text { Southern Atlantic Coastal Plain Wet Pine Savanna } \\
\text { and Flatwoods }\end{array}$ & Pinus palustris & Tree-Evergreen & 3.2 & 2.2 \\
\hline
\end{tabular}




\begin{tabular}{|c|c|c|c|c|}
\hline Community & Species & Classification & Resistance & Resilience \\
\hline Southern Coastal Plain Oak Dome and Hammock & Pinus palustris & Tree- Evergreen & 3.2 & 2.2 \\
\hline West Gulf Coastal Plain Catahoula Barrens & Pinus palustris & Tree- Evergreen & 3.2 & 2.2 \\
\hline $\begin{array}{l}\text { West Gulf Coastal Plain Stream Terrace Sandyland } \\
\text { Longleaf Pine Woodland }\end{array}$ & Pinus palustris & Tree-Evergreen & 3.2 & 2.2 \\
\hline $\begin{array}{l}\text { West Gulf Coastal Plain Upland Longleaf Pine } \\
\text { Forest and Woodland }\end{array}$ & Pinus palustris & Tree- Evergreen & 3.2 & 2.2 \\
\hline $\begin{array}{l}\text { West Gulf Coastal Plain Wet Longleaf Pine } \\
\text { Savanna and Flatwoods }\end{array}$ & Pinus palustris & Tree- Evergreen & 3.2 & 2.2 \\
\hline $\begin{array}{l}\text { Atlantic Coastal Plain Fall-line Sandhills Longleaf } \\
\text { Pine Woodland - Offsite Hardwood Modifier }\end{array}$ & Pinus palustris & Tree- Evergreen & 3.2 & 2.2 \\
\hline East Gulf Coastal Plain Near-Coast Pine Flatwoods & Pinus palustris & Tree-Evergreen & 3.2 & 2.2 \\
\hline $\begin{array}{l}\text { East Cascades Oak-Ponderosa Pine Forest and } \\
\text { Woodland }\end{array}$ & Pinus ponderosa & Tree- Evergreen & 3.2 & 2.2 \\
\hline $\begin{array}{l}\text { Inter-Mountain Basins Aspen-Mixed Conifer Forest } \\
\text { and Woodland }\end{array}$ & Pinus ponderosa & Tree- Evergreen & 3.2 & 2.2 \\
\hline Inter-Mountain Basins Cliff and Canyon & Pinus ponderosa & Tree- Evergreen & 3.2 & 2.2 \\
\hline $\begin{array}{l}\text { Inter-Mountain Basins Volcanic Rock and Cinder } \\
\text { Land }\end{array}$ & Pinus ponderosa & Tree-Evergreen & 3.2 & 2.2 \\
\hline $\begin{array}{l}\text { Madrean Lower Montane Pine-Oak Forest and } \\
\text { Woodland }\end{array}$ & Pinus ponderosa & Tree- Evergreen & 3.2 & 2.2 \\
\hline $\begin{array}{l}\text { Mediterranean California Dry-Mesic Mixed Conifer } \\
\text { Forest and Woodland }\end{array}$ & Pinus ponderosa & Tree- Evergreen & 3.2 & 2.2 \\
\hline North Pacific Oak Woodland & Pinus ponderosa & Tree- Evergreen & 3.2 & 2.2 \\
\hline North Pacific Serpentine Barren & Pinus ponderosa & Tree- Evergreen & 3.2 & 2.2 \\
\hline $\begin{array}{l}\text { Northern Rocky Mountain Dry-Mesic Montane } \\
\text { Mixed Conifer Forest }\end{array}$ & Pinus ponderosa & Tree-Evergreen & 3.2 & 2.2 \\
\hline $\begin{array}{l}\text { Northern Rocky Mountain Foothill Conifer Wooded } \\
\text { Steppe }\end{array}$ & Pinus ponderosa & Tree- Evergreen & 3.2 & 2.2 \\
\hline $\begin{array}{l}\text { Northern Rocky Mountain Ponderosa Pine } \\
\text { Woodland and Savanna }\end{array}$ & Pinus ponderosa & Tree- Evergreen & 3.2 & 2.2 \\
\hline $\begin{array}{l}\text { Northwestern Great Plains-Black Hills Ponderosa } \\
\text { Pine Woodland and Savanna }\end{array}$ & Pinus ponderosa & Tree-Evergreen & 3.2 & 2.2 \\
\hline Sierra Nevada Cliff and Canyon & Pinus ponderosa & Tree- Evergreen & 3.2 & 2.2 \\
\hline $\begin{array}{l}\text { Sierran-Intermontane Desert Western White Pine- } \\
\text { White Fir Woodland }\end{array}$ & Pinus ponderosa & Tree-Evergreen & 3.2 & 2.2 \\
\hline $\begin{array}{l}\text { Southern Rocky Mountain Dry-Mesic Montane } \\
\text { Mixed Conifer Forest and Woodland }\end{array}$ & Pinus ponderosa & Tree-Evergreen & 3.2 & 2.2 \\
\hline $\begin{array}{l}\text { Southern Rocky Mountain Mesic Montane Mixed } \\
\text { Conifer Forest and Woodland }\end{array}$ & Pinus ponderosa & Tree-Evergreen & 3.2 & 2.2 \\
\hline $\begin{array}{l}\text { Southern Rocky Mountain Ponderosa Pine } \\
\text { Savanna }\end{array}$ & Pinus ponderosa & Tree-Evergreen & 3.2 & 2.2 \\
\hline $\begin{array}{l}\text { Southern Rocky Mountain Ponderosa Pine } \\
\text { Woodland }\end{array}$ & Pinus ponderosa & Tree- Evergreen & 3.2 & 2.2 \\
\hline $\begin{array}{l}\text { California Montane Jeffrey Pine-(Ponderosa Pine) } \\
\text { Woodland }\end{array}$ & Pinus ponderosa & Tree- Evergreen & 3.2 & 2.2 \\
\hline $\begin{array}{l}\text { Colorado Plateau Mixed Bedrock Canyon and } \\
\text { Tableland }\end{array}$ & Pinus ponderosa & Tree-Evergreen & 3.2 & 2.2 \\
\hline $\begin{array}{l}\text { Mediterranean California Lower Montane Black } \\
\text { Oak-Conifer Forest and Woodland }\end{array}$ & Pinus ponderosa & Tree-Evergreen & 3.2 & 2.2 \\
\hline $\begin{array}{l}\text { Northern Rocky Mountain Foothill Conifer Wooded } \\
\text { Steppe }\end{array}$ & Pinus ponderosa & Tree-Evergreen & 3.2 & 2.2 \\
\hline $\begin{array}{l}\text { Northern Rocky Mountain Ponderosa Pine } \\
\text { Woodland and Savanna }\end{array}$ & Pinus ponderosa & Tree-Evergreen & 3.2 & 2.2 \\
\hline $\begin{array}{l}\text { Southern Rocky Mountain Ponderosa Pine } \\
\text { Savanna }\end{array}$ & Pinus ponderosa & Tree-Evergreen & 3.2 & 2.2 \\
\hline
\end{tabular}




\begin{tabular}{|c|c|c|c|c|}
\hline Community & Species & Classification & Resistance & Resilience \\
\hline $\begin{array}{l}\text { Southern Rocky Mountain Ponderosa Pine } \\
\text { Woodland }\end{array}$ & Pinus ponderosa & Tree- Evergreen & 3.2 & 2.2 \\
\hline $\begin{array}{l}\text { Northern Rocky Mountain Foothill Conifer Wooded } \\
\text { Steppe }\end{array}$ & Pinus ponderosa & Tree- Evergreen & 3.2 & 2.2 \\
\hline $\begin{array}{l}\text { Southern Rocky Mountain Ponderosa Pine } \\
\text { Savanna }\end{array}$ & Pinus ponderosa & Tree- Evergreen & 3.2 & 2.2 \\
\hline $\begin{array}{l}\text { Southern Rocky Mountain Ponderosa Pine } \\
\text { Woodland }\end{array}$ & Pinus ponderosa & Tree- Evergreen & 3.2 & 2.2 \\
\hline $\begin{array}{l}\text { Southern Appalachian Montane Pine Forest and } \\
\text { Woodland }\end{array}$ & Pinus pungens & Tree- Evergreen & 3.2 & 2.2 \\
\hline $\begin{array}{l}\text { California Coastal Closed-Cone Conifer Forest and } \\
\text { Woodland }\end{array}$ & Pinus radiata & Tree- Evergreen & 3.2 & 2.2 \\
\hline $\begin{array}{l}\text { Edwards Plateau Limestone Savanna and } \\
\text { Woodland }\end{array}$ & Pinus remota & Tree- Evergreen & 3.2 & 2.2 \\
\hline Great Lakes Wooded Dune and Swale & Pinus resinosa & Tree- Evergreen & 3.2 & 2.2 \\
\hline Laurentian Jack Pine-Red Pine Forest & Pinus resinosa & Tree- Evergreen & 3.2 & 2.2 \\
\hline Laurentian Pine-Oak Barrens & Pinus resinosa & Tree- Evergreen & 3.2 & 2.2 \\
\hline Laurentian-Acadian Northern Pine-(Oak) Forest & Pinus resinosa & Tree- Evergreen & 3.2 & 2.2 \\
\hline Central Appalachian Dry Oak-Pine Forest & Pinus rigida & Tree- Evergreen & 3.2 & 2.2 \\
\hline Central Appalachian Pine-Oak Rocky Woodland & Pinus rigida & Tree- Evergreen & 3.2 & 2.2 \\
\hline Eastern Serpentine Woodland & Pinus rigida & Tree- Evergreen & 3.2 & 2.2 \\
\hline Northeastern Interior Pine Barrens & Pinus rigida & Tree- Evergreen & 3.2 & 2.2 \\
\hline Northern Atlantic Coastal Plain Pitch Pine Barrens & Pinus rigida & Tree- Evergreen & 3.2 & 2.2 \\
\hline Northern Atlantic Coastal Plain Pitch Pine Lowland & Pinus rigida & Tree- Evergreen & 3.2 & 2.2 \\
\hline Southern Appalachian Low-Elevation Pine Forest & Pinus rigida & Tree- Evergreen & 3.2 & 2.2 \\
\hline $\begin{array}{l}\text { Southern Appalachian Montane Pine Forest and } \\
\text { Woodland }\end{array}$ & Pinus rigida & Tree- Evergreen & 3.2 & 2.2 \\
\hline $\begin{array}{l}\text { Klamath-Siskiyou Lower Montane Serpentine } \\
\text { Mixed Conifer Woodland }\end{array}$ & Pinus sabiniana & Tree- Evergreen & 3.2 & 2.2 \\
\hline North Pacific Oak Woodland & Pinus sabiniana & Tree- Evergreen & 3.2 & 2.2 \\
\hline $\begin{array}{l}\text { Atlantic Coastal Plain Peatland Pocosin and } \\
\text { Canebrake }\end{array}$ & Pinus serotina & Tree- Evergreen & 3.2 & 2.2 \\
\hline $\begin{array}{l}\text { Central Atlantic Coastal Plain Wet Longleaf Pine } \\
\text { Savanna and Flatwoods }\end{array}$ & Pinus serotina & Tree- Evergreen & 3.2 & 2.2 \\
\hline Southern Atlantic Coastal Plain Maritime Forest & Pinus serotina & Tree- Evergreen & 3.2 & 2.2 \\
\hline $\begin{array}{l}\text { Southern Atlantic Coastal Plain Wet Pine Savanna } \\
\text { and Flatwoods }\end{array}$ & Pinus serotina & Tree- Evergreen & 3.2 & 2.2 \\
\hline $\begin{array}{l}\text { Atlantic Coastal Plain Streamhead Seepage } \\
\text { Swamp, Pocosin and Baygall }\end{array}$ & Pinus serotina & Tree- Evergreen & 3.2 & 2.2 \\
\hline Central Appalachian Dry Oak-Pine Forest & Pinus strobus & Tree- Evergreen & 3.2 & 2.2 \\
\hline Central Appalachian Pine-Oak Rocky Woodland & Pinus strobus & Tree- Evergreen & 3.2 & 2.2 \\
\hline Great Lakes Wooded Dune and Swale & Pinus strobus & Tree- Evergreen & 3.2 & 2.2 \\
\hline Laurentian Pine-Oak Barrens & Pinus strobus & Tree- Evergreen & 3.2 & 2.2 \\
\hline $\begin{array}{l}\text { Laurentian-Acadian Pine-Hemlock-Hardwood } \\
\text { Forest }\end{array}$ & Pinus strobus & Tree- Evergreen & 3.2 & 2.2 \\
\hline Northeastern Interior Dry-Mesic Oak Forest & Pinus strobus & Tree- Evergreen & 3.2 & 2.2 \\
\hline $\begin{array}{l}\text { Southern Appalachian Montane Pine Forest and } \\
\text { Woodland }\end{array}$ & Pinus strobus & Tree- Evergreen & 3.2 & 2.2 \\
\hline Laurentian-Acadian Northern Pine-(Oak) Forest & Pinus strobus & Tree- Evergreen & 3.2 & 2.2 \\
\hline Bastrop Lost Pines Forest and Woodland & Pinus taeda & Tree- Evergreen & 3.2 & 2.2 \\
\hline Central Atlantic Coastal Plain Maritime Forest & Pinus taeda & Tree- Evergreen & 3.2 & 2.2 \\
\hline
\end{tabular}




\begin{tabular}{|c|c|c|c|c|}
\hline Community & Species & Classification & Resistance & Resilience \\
\hline $\begin{array}{l}\text { East Gulf Coastal Plain Southern Loblolly- } \\
\text { Hardwood Flatwoods }\end{array}$ & Pinus taeda & Tree- Evergreen & 3.2 & 2.2 \\
\hline $\begin{array}{l}\text { East Gulf Coastal Plain Southern Loess Bluff } \\
\text { Forest }\end{array}$ & Pinus taeda & Tree- Evergreen & 3.2 & 2.2 \\
\hline Northern Atlantic Coastal Plain Maritime Forest & Pinus taeda & Tree- Evergreen & 3.2 & 2.2 \\
\hline Southeastern Interior Longleaf Pine Woodland & Pinus taeda & Tree- Evergreen & 3.2 & 2.2 \\
\hline $\begin{array}{l}\text { Southern Atlantic Coastal Plain Nonriverine } \\
\text { Swamp and Wet Hardwood Forest }\end{array}$ & Pinus taeda & Tree-Evergreen & 3.2 & 2.2 \\
\hline Southern Piedmont Large Floodplain Forest & Pinus taeda & Tree- Evergreen & 3.2 & 2.2 \\
\hline Southern Piedmont Mesic Forest & Pinus taeda & Tree- Evergreen & 3.2 & 2.2 \\
\hline $\begin{array}{l}\text { Southern Piedmont Small Floodplain and Riparian } \\
\text { Forest }\end{array}$ & Pinus taeda & Tree- Evergreen & 3.2 & 2.2 \\
\hline West Gulf Coastal Plain Pine-Hardwood Flatwoods & Pinus taeda & Tree- Evergreen & 3.2 & 2.2 \\
\hline West Gulf Coastal Plain Pine-Hardwood Forest & Pinus taeda & Tree- Evergreen & 3.2 & 2.2 \\
\hline $\begin{array}{l}\text { West Gulf Coastal Plain Upland Longleaf Pine } \\
\text { Forest and Woodland }\end{array}$ & Pinus taeda & Tree-Evergreen & 3.2 & 2.2 \\
\hline $\begin{array}{l}\text { West Gulf Coastal Plain Wet Longleaf Pine } \\
\text { Savanna and Flatwoods }\end{array}$ & Pinus taeda & Tree- Evergreen & 3.2 & 2.2 \\
\hline $\begin{array}{l}\text { California Coastal Closed-Cone Conifer Forest and } \\
\text { Woodland }\end{array}$ & Pinus torreyana & Tree- Evergreen & 3.2 & 2.2 \\
\hline $\begin{array}{l}\text { Allegheny-Cumberland Dry Oak Forest and } \\
\text { Woodland }\end{array}$ & Pinus virginiana & Tree- Evergreen & 3.2 & 2.2 \\
\hline Appalachian Shale Barrens & Pinus virginiana & Tree- Evergreen & 3.2 & 2.2 \\
\hline Central Appalachian Dry Oak-Pine Forest & Pinus virginiana & Tree- Evergreen & 3.2 & 2.2 \\
\hline Central Appalachian Pine-Oak Rocky Woodland & Pinus virginiana & Tree- Evergreen & 3.2 & 2.2 \\
\hline Cumberland Sandstone Glade and Barrens & Pinus virginiana & Tree- Evergreen & 3.2 & 2.2 \\
\hline Eastern Serpentine Woodland & Pinus virginiana & Tree- Evergreen & 3.2 & 2.2 \\
\hline Northeastern Interior Dry-Mesic Oak Forest & Pinus virginiana & Tree- Evergreen & 3.2 & 2.2 \\
\hline Southern Appalachian Low-Elevation Pine Forest & Pinus virginiana & Tree- Evergreen & 3.2 & 2.2 \\
\hline $\begin{array}{l}\text { Southern Appalachian Montane Pine Forest and } \\
\text { Woodland }\end{array}$ & Pinus virginiana & Tree- Evergreen & 3.2 & 2.2 \\
\hline Southern Piedmont Glade and Barrens & Pinus virginiana & Tree- Evergreen & 3.2 & 2.2 \\
\hline Southern Piedmont Granite Flatrock and Outcrop & Pinus virginiana & Tree- Evergreen & 3.2 & 2.2 \\
\hline Southern Piedmont Large Floodplain Forest & Pinus virginiana & Tree- Evergreen & 3.2 & 2.2 \\
\hline $\begin{array}{l}\text { Southern Piedmont Small Floodplain and Riparian } \\
\text { Forest }\end{array}$ & Pinus virginiana & Tree- Evergreen & 3.2 & 2.2 \\
\hline $\begin{array}{l}\text { Rocky Mountain Foothill Limber Pine-Juniper } \\
\text { Woodland }\end{array}$ & Piptatherum micranthum & Graminoid $\mathrm{C}_{3}$ & 3.6 & 3.6 \\
\hline Southeastern Interior Longleaf Pine Woodland & Piptochaetium avenaceum & Graminoid $\mathrm{C}_{3}$ & 3.6 & 3.6 \\
\hline West Gulf Coastal Plain Nepheline Syenite Glade & Piptochaetium avenaceum & Graminoid $C_{3}$ & 3.6 & 3.6 \\
\hline $\begin{array}{l}\text { Southwest Florida Coastal Strand and Maritime } \\
\text { Hammock }\end{array}$ & Piscidia piscipula & Shrub- Deciduous & 2.7 & 3 \\
\hline South Florida Hardwood Hammock & Piscidia piscipula & Tree-Deciduous & 3 & 4.2 \\
\hline South Florida Hardwood Hammock & Pithecellobium keyense & Tree- Evergreen & 3.2 & 2.2 \\
\hline $\begin{array}{l}\text { Atlantic Coastal Plain Small Blackwater River } \\
\text { Floodplain Forest }\end{array}$ & Planera aquatica & Tree- Deciduous & 3 & 4.2 \\
\hline Mediterranean California Coastal Bluff & Plantago maritima & Forb & 3 & 3.5 \\
\hline $\begin{array}{l}\text { Atlantic Coastal Plain Small Brownwater River } \\
\text { Floodplain Forest }\end{array}$ & Platanus occidentalis & Tree- Deciduous & 3 & 4.2 \\
\hline Central Appalachian River Floodplain & Platanus occidentalis & Tree- Deciduous & 3 & 4.2 \\
\hline Central Appalachian Stream and Riparian & Platanus occidentalis & Tree- Deciduous & 3 & 4.2 \\
\hline
\end{tabular}




\begin{tabular}{|c|c|c|c|c|}
\hline Community & Species & Classification & Resistance & Resilience \\
\hline Edwards Plateau Floodplain Terrace & Platanus occidentalis & Tree-Deciduous & 3 & 4.2 \\
\hline Edwards Plateau Riparian & Platanus occidentalis & Tree- Deciduous & 3 & 4.2 \\
\hline Ozark-Ouachita Riparian & Platanus occidentalis & Tree-Deciduous & 3 & 4.2 \\
\hline South-Central Interior Large Floodplain & Platanus occidentalis & Tree-Deciduous & 3 & 4.2 \\
\hline South-Central Interior Small Stream and Riparian & Platanus occidentalis & Tree-Deciduous & 3 & 4.2 \\
\hline Southeastern Great Plains Floodplain Forest & Platanus occidentalis & Tree-Deciduous & 3 & 4.2 \\
\hline Southeastern Great Plains Riparian Forest & Platanus occidentalis & Tree- Deciduous & 3 & 4.2 \\
\hline Southern Piedmont Large Floodplain Forest & Platanus occidentalis & Tree-Deciduous & 3 & 4.2 \\
\hline Mississippi River Floodplain and Riparian Forest & Platanus occidentalis & Tree-Deciduous & 3 & 4.2 \\
\hline Mississippi River Riparian Forest & Platanus occidentalis & Tree-Deciduous & 3 & 4.2 \\
\hline $\begin{array}{l}\text { North American Warm Desert Riparian Woodland } \\
\text { and Shrubland }\end{array}$ & Platanus racemosa & Tree- Deciduous & 3 & 4.2 \\
\hline $\begin{array}{l}\text { Mediterranean California Foothill and Lower } \\
\text { Montane Riparian Woodland and Shrubland }\end{array}$ & Platanus racemosa & Tree-Deciduous & 3 & 4.2 \\
\hline $\begin{array}{l}\text { North American Warm Desert Lower Montane } \\
\text { Riparian Woodland and Shrubland }\end{array}$ & Platanus wrightii & Tree-Deciduous & 3 & 4.2 \\
\hline North Pacific Herbaceous Bald and Bluff & Plectritis congesta & Forb & 3 & 3.5 \\
\hline Southern Coastal Plain Herbaceous Seep and Bog & Pleea tenuifolia & Forb & 3 & 3.5 \\
\hline Chihuahuan Sandy Plains Semi-Desert Grassland & Pleuraphis jamesii & Graminoid $\mathrm{C}_{4}$ & 3.6 & 4.1 \\
\hline Inter-Mountain Basins Big Sagebrush Shrubland & Pleuraphis jamesii & Graminoid $\mathrm{C}_{4}$ & 3.6 & 4.1 \\
\hline Inter-Mountain Basins Juniper Savanna & Pleuraphis jamesii & Graminoid $\mathrm{C}_{4}$ & 3.6 & 4.1 \\
\hline Inter-Mountain Basins Semi-Desert Grassland & Pleuraphis jamesii & Graminoid $\mathrm{C}_{4}$ & 3.6 & 4.1 \\
\hline Inter-Mountain Basins Semi-Desert Shrub-Steppe & Pleuraphis jamesii & Graminoid $\mathrm{C}_{4}$ & 3.6 & 4.1 \\
\hline Madrean Encinal & Pleuraphis jamesii & Graminoid $\mathrm{C}_{4}$ & 3.6 & 4.1 \\
\hline Southern Colorado Plateau Sand Shrubland & Pleuraphis jamesii & Graminoid $\mathrm{C}_{4}$ & 3.6 & 4.1 \\
\hline $\begin{array}{l}\text { Southern Rocky Mountain Juniper Woodland and } \\
\text { Savanna }\end{array}$ & Pleuraphis jamesii & Graminoid $\mathrm{C}_{4}$ & 3.6 & 4.1 \\
\hline Apacherian-Chihuahuan Mesquite Upland Scrub & Pleuraphis mutica & Graminoid $\mathrm{C}_{4}$ & 3.6 & 4.1 \\
\hline $\begin{array}{l}\text { Apacherian-Chihuahuan Semi-Desert Grassland } \\
\text { and Steppe }\end{array}$ & Pleuraphis mutica & Graminoid $\mathrm{C}_{4}$ & 3.6 & 4.1 \\
\hline Chihuahuan Creosotebush Desert Scrub & Pleuraphis mutica & Graminoid $\mathrm{C}_{4}$ & 3.6 & 4.1 \\
\hline Chihuahuan Loamy Plains Desert Grassland & Pleuraphis mutica & Graminoid $\mathrm{C}_{4}$ & 3.6 & 4.1 \\
\hline Chihuahuan Mixed Salt Desert Scrub & Pleuraphis mutica & Graminoid $\mathrm{C}_{4}$ & 3.6 & 4.1 \\
\hline $\begin{array}{l}\text { Chihuahuan-Sonoran Desert Bottomland and } \\
\text { Swale Grassland }\end{array}$ & Pleuraphis mutica & Graminoid $\mathrm{C}_{4}$ & 3.6 & 4.1 \\
\hline Edwards Plateau Upland Depression & Pleuraphis mutica & Graminoid $\mathrm{C}_{4}$ & 3.6 & 4.1 \\
\hline Madrean Juniper Savanna & Pleuraphis mutica & Graminoid $\mathrm{C}_{4}$ & 3.6 & 4.1 \\
\hline $\begin{array}{l}\text { Western Great Plains Mesquite Woodland and } \\
\text { Shrubland }\end{array}$ & Pleuraphis mutica & Graminoid $\mathrm{C}_{4}$ & 3.6 & 4.1 \\
\hline Tamaulipan Mesquite Upland Scrub & Pleuraphis mutica & Graminoid $\mathrm{C}_{4}$ & 3.6 & 4.1 \\
\hline Inter-Mountain Basins Mixed Salt Desert Scrub & Pleuraphis rigida & Graminoid $\mathrm{C}_{4}$ & 3.6 & 4.1 \\
\hline $\begin{array}{l}\text { North American Warm Desert Riparian Mesquite } \\
\text { Bosque }\end{array}$ & Pluchea sericea & Shrub-Deciduous & 2.7 & 3 \\
\hline $\begin{array}{l}\text { Columbia Plateau Silver Sagebrush Seasonally } \\
\text { Flooded Shrub-Steppe }\end{array}$ & Poa cusickii & Graminoid $\mathrm{C}_{3}$ & 3.6 & 3.6 \\
\hline Temperate Pacific Tidal Salt and Brackish Marsh & Poa eminens & Graminoid $\mathrm{C}_{3}$ & 3.6 & 3.6 \\
\hline $\begin{array}{l}\text { Inter-Mountain Basins Montane Sagebrush } \\
\text { Steppe }\end{array}$ & Poa fendleriana & Graminoid $C_{3}$ & 3.6 & 3.6 \\
\hline Inter-Mountain Basins Semi-Desert Shrub-Steppe & Poa fendleriana & Graminoid $\mathrm{C}_{3}$ & 3.6 & 3.6 \\
\hline
\end{tabular}




\begin{tabular}{|c|c|c|c|c|}
\hline Community & Species & Classification & Resistance & Resilience \\
\hline $\begin{array}{l}\text { Southern Rocky Mountain Montane-Subalpine } \\
\text { Grassland }\end{array}$ & Poa fendleriana & Graminoid $\mathrm{C}_{3}$ & 3.6 & 3.6 \\
\hline $\begin{array}{l}\text { Southern Rocky Mountain Ponderosa Pine } \\
\text { Savanna }\end{array}$ & Poa fendleriana & Graminoid $C_{3}$ & 3.6 & 3.6 \\
\hline $\begin{array}{l}\text { Southern Rocky Mountain Ponderosa Pine } \\
\text { Woodland }\end{array}$ & Poa fendleriana & Graminoid $\mathrm{C}_{3}$ & 3.6 & 3.6 \\
\hline Columbia Basin Palouse Prairie & Poa pratensis & Graminoid $C_{3}$ & 3.6 & 3.6 \\
\hline Rocky Mountain Aspen Forest and Woodland & Poa pratensis & Graminoid $C_{3}$ & 3.6 & 3.6 \\
\hline California Mesic Serpentine Grassland & Poa secunda & Graminoid $\mathrm{C}_{3}$ & 3.6 & 3.6 \\
\hline $\begin{array}{l}\text { Colorado Plateau Mixed Low Sagebrush } \\
\text { Shrubland }\end{array}$ & Poa secunda & Graminoid $C_{3}$ & 3.6 & 3.6 \\
\hline Columbia Plateau Scabland Shrubland & Poa secunda & Graminoid $C_{3}$ & 3.6 & 3.6 \\
\hline $\begin{array}{l}\text { Columbia Plateau Silver Sagebrush Seasonally } \\
\text { Flooded Shrub-Steppe }\end{array}$ & Poa secunda & Graminoid $C_{3}$ & 3.6 & 3.6 \\
\hline Columbia Plateau Steppe and Grassland & Poa secunda & Graminoid $\mathrm{C}_{3}$ & 3.6 & 3.6 \\
\hline Inter-Mountain Basins Mat Saltbush Shrubland & Poa secunda & Graminoid $C_{3}$ & 3.6 & 3.6 \\
\hline $\begin{array}{l}\text { Inter-Mountain Basins Montane Sagebrush } \\
\text { Steppe }\end{array}$ & Poa secunda & Graminoid $\mathrm{C}_{3}$ & 3.6 & 3.6 \\
\hline Inter-Mountain Basins Semi-Desert Grassland & Poa secunda & Graminoid $C_{3}$ & 3.6 & 3.6 \\
\hline Inter-Mountain Basins Semi-Desert Shrub-Steppe & Poa secunda & Graminoid $C_{3}$ & 3.6 & 3.6 \\
\hline Rocky Mountain Alpine Fell-Field & Poa secunda & Graminoid $\mathrm{C}_{3}$ & 3.6 & 3.6 \\
\hline $\begin{array}{l}\text { Rocky Mountain Foothill Limber Pine-Juniper } \\
\text { Woodland }\end{array}$ & Poa secunda & Graminoid $\mathrm{C}_{3}$ & 3.6 & 3.6 \\
\hline $\begin{array}{l}\text { Northern Rocky Mountain Lower Montane, Foothill } \\
\text { and Valley Grassland }\end{array}$ & Poa secunda & Graminoid $C_{3}$ & 3.6 & 3.6 \\
\hline $\begin{array}{l}\text { Sierra Nevada Subalpine Lodgepole Pine Forest } \\
\text { and Woodland }\end{array}$ & Poa wheeleri & Graminoid $C_{3}$ & 3.6 & 3.6 \\
\hline Rocky Mountain Alpine Bedrock and Scree & Polemonium viscosum & Forb & 3 & 3.5 \\
\hline $\begin{array}{l}\text { North American Warm Desert Bedrock Cliff and } \\
\text { Outcrop }\end{array}$ & Poliomintha incana & Shrub-Deciduous & 2.7 & 3 \\
\hline North Pacific Maritime Mesic Subalpine Parkland & Polygonum bistortoides & Forb & 3 & 3.5 \\
\hline Sierra Nevada Alpine Dwarf-Shrubland & Polygonum shastense & Forb & 3 & 3.5 \\
\hline $\begin{array}{l}\text { North Pacific Maritime Dry-Mesic Douglas-fir- } \\
\text { Western Hemlock Forest }\end{array}$ & Polystichum munitum & Graminoid $C_{3}$ & 3.6 & 3.6 \\
\hline Floridian Highlands Freshwater Marsh & Pontederia cordata & Forb & 3 & 3.5 \\
\hline Laurentian-Acadian Freshwater Marsh & Pontederia cordata & Forb & 3 & 3.5 \\
\hline $\begin{array}{l}\text { Great Basin Foothill and Lower Montane Riparian } \\
\text { Woodland and Shrubland }\end{array}$ & Populus angustifolia & Tree- Deciduous & 3 & 4.2 \\
\hline $\begin{array}{l}\text { North American Warm Desert Lower Montane } \\
\text { Riparian Woodland and Shrubland }\end{array}$ & Populus angustifolia & Tree-Deciduous & 3 & 4.2 \\
\hline $\begin{array}{l}\text { Rocky Mountain Lower Montane-Foothill Riparian } \\
\text { Woodland and Shrubland }\end{array}$ & Populus angustifolia & Tree- Deciduous & 3 & 4.2 \\
\hline Eastern Boreal Floodplain & Populus balsamifera & Tree- Deciduous & 3 & 4.2 \\
\hline Great Lakes Wooded Dune and Swale & Populus balsamifera & Tree-Deciduous & 3 & 4.2 \\
\hline $\begin{array}{l}\text { Northern Rocky Mountain Lower Montane } \\
\text { Riparian Woodland and Shrubland }\end{array}$ & Populus balsamifera & Tree- Deciduous & 3 & 4.2 \\
\hline $\begin{array}{l}\text { Great Basin Foothill and Lower Montane Riparian } \\
\text { Woodland and Shrubland }\end{array}$ & Populus balsamifera & Tree-Deciduous & 3 & 4.2 \\
\hline North Pacific Hardwood-Conifer Swamp & Populus balsamifera & Tree-Deciduous & 3 & 4.2 \\
\hline $\begin{array}{l}\text { North Pacific Lowland Riparian Forest and } \\
\text { Shrubland }\end{array}$ & Populus balsamifera & Tree-Deciduous & 3 & 4.2 \\
\hline $\begin{array}{l}\text { North Pacific Montane Riparian Woodland and } \\
\text { Shrubland }\end{array}$ & Populus balsamifera & Tree-Deciduous & 3 & 4.2 \\
\hline
\end{tabular}




\begin{tabular}{|c|c|c|c|c|}
\hline Community & Species & Classification & Resistance & Resilience \\
\hline $\begin{array}{l}\text { Northern Rocky Mountain Avalanche Chute } \\
\text { Shrubland }\end{array}$ & Populus balsamifera & Tree-Deciduous & 3 & 4.2 \\
\hline Northern Rocky Mountain Wooded Vernal Pool & Populus balsamifera & Tree-Deciduous & 3 & 4.2 \\
\hline Northwestern Great Plains Floodplain & Populus balsamifera & Tree-Deciduous & 3 & 4.2 \\
\hline Northwestern Great Plains Riparian & Populus balsamifera & Tree-Deciduous & 3 & 4.2 \\
\hline North-Central Interior Floodplain & Populus deltoides & Tree- Deciduous & 3 & 4.2 \\
\hline Northwestern Great Plains Floodplain & Populus deltoides & Tree-Deciduous & 3 & 4.2 \\
\hline Northwestern Great Plains Riparian & Populus deltoides & Tree-Deciduous & 3 & 4.2 \\
\hline $\begin{array}{l}\text { Rocky Mountain Lower Montane-Foothill Riparian } \\
\text { Woodland and Shrubland }\end{array}$ & Populus deltoides & Tree-Deciduous & 3 & 4.2 \\
\hline $\begin{array}{l}\text { West Gulf Coastal Plain Large River Floodplain } \\
\text { Forest }\end{array}$ & Populus deltoides & Tree-Deciduous & 3 & 4.2 \\
\hline Western Great Plains Floodplain & Populus deltoides & Tree-Deciduous & 3 & 4.2 \\
\hline Western Great Plains Riparian & Populus deltoides & Tree-Deciduous & 3 & 4.2 \\
\hline Mississippi River Floodplain and Riparian Forest & Populus deltoides & Tree-Deciduous & 3 & 4.2 \\
\hline Mississippi River Riparian Forest & Populus deltoides & Tree-Deciduous & 3 & 4.2 \\
\hline $\begin{array}{l}\text { North American Warm Desert Lower Montane } \\
\text { Riparian Woodland and Shrubland }\end{array}$ & Populus deltoides & Tree-Deciduous & 3 & 4.2 \\
\hline $\begin{array}{l}\text { Great Basin Foothill and Lower Montane Riparian } \\
\text { Woodland and Shrubland }\end{array}$ & Populus fremontii & Tree-Deciduous & 3 & 4.2 \\
\hline $\begin{array}{l}\text { North American Warm Desert Lower Montane } \\
\text { Riparian Woodland and Shrubland }\end{array}$ & Populus fremontii & Tree- Deciduous & 3 & 4.2 \\
\hline $\begin{array}{l}\text { North American Warm Desert Riparian Woodland } \\
\text { and Shrubland }\end{array}$ & Populus fremontii & Tree-Deciduous & 3 & 4.2 \\
\hline $\begin{array}{l}\text { Rocky Mountain Lower Montane-Foothill Riparian } \\
\text { Woodland and Shrubland }\end{array}$ & Populus fremontii & Tree-Deciduous & 3 & 4.2 \\
\hline $\begin{array}{l}\text { Mediterranean California Foothill and Lower } \\
\text { Montane Riparian Woodland and Shrubland }\end{array}$ & Populus fremontii & Tree-Deciduous & 3 & 4.2 \\
\hline Eastern Great Plains Tallgrass Aspen Parkland & Populus tremuloides & Tree-Deciduous & 3 & 4.2 \\
\hline $\begin{array}{l}\text { Inter-Mountain Basins Aspen-Mixed Conifer Forest } \\
\text { and Woodland }\end{array}$ & Populus tremuloides & Tree-Deciduous & 3 & 4.2 \\
\hline $\begin{array}{l}\text { Inter-Mountain Basins Subalpine Limber- } \\
\text { Bristlecone Pine Woodland }\end{array}$ & Populus tremuloides & Tree- Deciduous & 3 & 4.2 \\
\hline Laurentian-Acadian Northern Hardwood Forest & Populus tremuloides & Tree-Deciduous & 3 & 4.2 \\
\hline $\begin{array}{l}\text { North Pacific Montane Riparian Woodland and } \\
\text { Shrubland }\end{array}$ & Populus tremuloides & Tree-Deciduous & 3 & 4.2 \\
\hline $\begin{array}{l}\text { Northern Rocky Mountain Avalanche Chute } \\
\text { Shrubland }\end{array}$ & Populus tremuloides & Tree-Deciduous & 3 & 4.2 \\
\hline Northern Rocky Mountain Wooded Vernal Pool & Populus tremuloides & Tree-Deciduous & 3 & 4.2 \\
\hline $\begin{array}{l}\text { Northwestern Great Plains Aspen Forest and } \\
\text { Parkland }\end{array}$ & Populus tremuloides & Tree-Deciduous & 3 & 4.2 \\
\hline $\begin{array}{l}\text { Northwestern Great Plains-Black Hills Ponderosa } \\
\text { Pine Woodland and Savanna }\end{array}$ & Populus tremuloides & Tree-Deciduous & 3 & 4.2 \\
\hline Rocky Mountain Aspen Forest and Woodland & Populus tremuloides & Tree- Deciduous & 3 & 4.2 \\
\hline Rocky Mountain Bigtooth Maple Ravine Woodland & Populus tremuloides & Tree-Deciduous & 3 & 4.2 \\
\hline $\begin{array}{l}\text { Rocky Mountain Subalpine-Montane Limber- } \\
\text { Bristlecone Pine Woodland }\end{array}$ & Populus tremuloides & Tree- Deciduous & 3 & 4.2 \\
\hline $\begin{array}{l}\text { Rocky Mountain Subalpine-Montane Riparian } \\
\text { Woodland }\end{array}$ & Populus tremuloides & Tree-Deciduous & 3 & 4.2 \\
\hline Sierra Nevada Cliff and Canyon & Populus tremuloides & Tree-Deciduous & 3 & 4.2 \\
\hline $\begin{array}{l}\text { Southern Rocky Mountain Dry-Mesic Montane } \\
\text { Mixed Conifer Forest and Woodland }\end{array}$ & Populus tremuloides & Tree-Deciduous & 3 & 4.2 \\
\hline
\end{tabular}




\begin{tabular}{|c|c|c|c|c|}
\hline Community & Species & Classification & Resistance & Resilience \\
\hline $\begin{array}{l}\text { Southern Rocky Mountain Ponderosa Pine } \\
\text { Woodland }\end{array}$ & Populus tremuloides & Tree- Deciduous & 3 & 4.2 \\
\hline Boreal White Spruce-Fir-Hardwood Forest & Populus tremuloides & Tree- Deciduous & 3 & 4.2 \\
\hline Eastern Hemi-Boreal Aspen-Birch Forest & Populus tremuloides & Tree-Deciduous & 3 & 4.2 \\
\hline Laurentian-Acadian Northern Pine-(Oak) Forest & Populus tremuloides & Tree- Deciduous & 3 & 4.2 \\
\hline $\begin{array}{l}\text { Eastern Hemi-Boreal Dry-Mesic Pine-Black Spruce- } \\
\text { Hardwood Forest }\end{array}$ & Populus tremuloides & Tree- Deciduous & 3 & 4.2 \\
\hline Eastern Highland Rim Prairie and Barrens & Potentilla simplex & Forb & 3 & 3.5 \\
\hline $\begin{array}{l}\text { Chihuahuan Stabilized Coppice Dune and Sand } \\
\text { Flat Scrub }\end{array}$ & Prosopis glandulosa & Shrub- Deciduous & 2.7 & 3 \\
\hline $\begin{array}{l}\text { Chihuahuan-Sonoran Desert Bottomland and } \\
\text { Swale Grassland }\end{array}$ & Prosopis glandulosa & Shrub- Deciduous & 2.7 & 3 \\
\hline $\begin{array}{l}\text { North American Warm Desert Active and } \\
\text { Stabilized Dune }\end{array}$ & Prosopis glandulosa & Shrub- Deciduous & 2.7 & 3 \\
\hline Tamaulipan Mixed Deciduous Thornscrub & Prosopis glandulosa & Shrub- Deciduous & 2.7 & 3 \\
\hline Tamaulipan Ramadero & Prosopis glandulosa & Shrub- Deciduous & 2.7 & 3 \\
\hline Apacherian-Chihuahuan Mesquite Upland Scrub & Prosopis glandulosa & Tree-Deciduous & 3 & 4.2 \\
\hline $\begin{array}{l}\text { Llano Estacado Caprock Escarpment and Breaks } \\
\text { Shrubland and Steppe }\end{array}$ & Prosopis glandulosa & Tree-Deciduous & 3 & 4.2 \\
\hline $\begin{array}{l}\text { North American Warm Desert Riparian Mesquite } \\
\text { Bosque }\end{array}$ & Prosopis glandulosa & Tree- Deciduous & 3 & 4.2 \\
\hline North American Warm Desert Wash & Prosopis glandulosa & Tree-Deciduous & 3 & 4.2 \\
\hline $\begin{array}{l}\text { Western Great Plains Mesquite Woodland and } \\
\text { Shrubland }\end{array}$ & Prosopis glandulosa & Tree- Deciduous & 3 & 4.2 \\
\hline Llano Uplift Acidic Forest, Woodland and Glade & Prosopis glandulosa & Tree-Deciduous & 3 & 4.2 \\
\hline Tamaulipan Mesquite Upland Scrub & Prosopis glandulosa & Tree- Deciduous & 3 & 4.2 \\
\hline $\begin{array}{l}\text { Central and South Texas Coastal Fringe Forest and } \\
\text { Woodland }\end{array}$ & Prosopis glandulosa & Tree- Deciduous & 3 & 4.2 \\
\hline $\begin{array}{l}\text { North American Warm Desert Riparian Mesquite } \\
\text { Bosque }\end{array}$ & Prosopis glandulosa & Tree- Deciduous & 3 & 4.2 \\
\hline Tamaulipan Savanna Grassland & Prosopis glandulosa & Tree-Deciduous & 3 & 4.2 \\
\hline $\begin{array}{l}\text { North American Warm Desert Riparian Mesquite } \\
\text { Bosque }\end{array}$ & Prosopis glandulosa & Tree- Deciduous & 3 & 4.2 \\
\hline $\begin{array}{l}\text { North American Warm Desert Riparian Mesquite } \\
\text { Bosque }\end{array}$ & Prosopis pubescens & Tree- Deciduous & 3 & 4.2 \\
\hline Sonoran Paloverde-Mixed Cacti Desert Scrub & Prosopis velutina & Shrub- Deciduous & 2.7 & 3 \\
\hline Apacherian-Chihuahuan Mesquite Upland Scrub & Prosopis velutina & Tree- Deciduous & 3 & 4.2 \\
\hline $\begin{array}{l}\text { North American Warm Desert Riparian Mesquite } \\
\text { Bosque }\end{array}$ & Prosopis velutina & Tree- Deciduous & 3 & 4.2 \\
\hline Tamaulipan Mesquite Upland Scrub & Prosopis velutina & Tree- Deciduous & 3 & 4.2 \\
\hline California Montane Woodland and Chaparral & Prunus emarginata & Shrub- Deciduous & 2.7 & 3 \\
\hline $\begin{array}{l}\text { Northern Rocky Mountain Montane-Foothill } \\
\text { Deciduous Shrubland }\end{array}$ & Prunus emarginata & Shrub- Deciduous & 2.7 & 3 \\
\hline North American Warm Desert Wash & Prunus fasciculata & Shrub- Deciduous & 2.7 & 3 \\
\hline California Mesic Chaparral & Prunus ilicifolia & Shrub- Evergreen & 2.7 & 2.4 \\
\hline Southern Appalachian Rocky Summit & Prunus pensylvanica & Shrub- Deciduous & 2.7 & 3 \\
\hline Great Lakes Dune & Prunus pumila & Shrub- Deciduous & 2.7 & 3 \\
\hline Laurentian-Acadian Northern Hardwood Forest & Prunus serotina & Tree-Deciduous & 3 & 4.2 \\
\hline Southern and Central Appalachian Cove Forest & Prunus serotina & Tree-Deciduous & 3 & 4.2 \\
\hline Appalachian (Hemlock)-Northern Hardwood Forest & Prunus serotina & Tree- Deciduous & 3 & 4.2 \\
\hline $\begin{array}{l}\text { South-Central Interior / Upper Coastal Plain Wet } \\
\text { Flatwoods }\end{array}$ & Prunus serotina & Tree-Deciduous & 3 & 4.2 \\
\hline
\end{tabular}




\begin{tabular}{|c|c|c|c|c|}
\hline Community & Species & Classification & Resistance & Resilience \\
\hline Edwards Plateau Mesic Canyon & Prunus serotina & Tree- Deciduous & 3 & 4.2 \\
\hline California Montane Woodland and Chaparral & Prunus subcordata & Shrub- Deciduous & 2.7 & 3 \\
\hline California Montane Woodland and Chaparral & Prunus virginiana & Shrub- Deciduous & 2.7 & 3 \\
\hline $\begin{array}{l}\text { Columbia Basin Foothill Riparian Woodland and } \\
\text { Shrubland }\end{array}$ & Prunus virginiana & Shrub- Deciduous & 2.7 & 3 \\
\hline $\begin{array}{l}\text { Inter-Mountain Basins Aspen-Mixed Conifer Forest } \\
\text { and Woodland }\end{array}$ & Prunus virginiana & Shrub- Deciduous & 2.7 & 3 \\
\hline $\begin{array}{l}\text { Northern Rocky Mountain Montane-Foothill } \\
\text { Deciduous Shrubland }\end{array}$ & Prunus virginiana & Shrub- Deciduous & 2.7 & 3 \\
\hline Rocky Mountain Bigtooth Maple Ravine Woodland & Prunus virginiana & Shrub- Deciduous & 2.7 & 3 \\
\hline $\begin{array}{l}\text { Rocky Mountain Gambel Oak-Mixed Montane } \\
\text { Shrubland }\end{array}$ & Prunus virginiana & Tree- Deciduous & 3 & 4.2 \\
\hline $\begin{array}{l}\text { Rocky Mountain Lower Montane-Foothill Riparian } \\
\text { Woodland and Shrubland }\end{array}$ & Prunus virginiana & Tree- Deciduous & 3 & 4.2 \\
\hline $\begin{array}{l}\text { Western Great Plains Dry Bur Oak Forest and } \\
\text { Woodland }\end{array}$ & Prunus virginiana & Tree- Deciduous & 3 & 4.2 \\
\hline Great Plains Wooded Draw and Ravine & Prunus virginiana & Tree- Deciduous & 3 & 4.2 \\
\hline $\begin{array}{l}\text { Columbia Basin Foothill and Canyon Dry } \\
\text { Grassland }\end{array}$ & Pseudoroegneria spicata & Graminoid $\mathrm{C}_{3}$ & 3.6 & 3.6 \\
\hline Columbia Basin Palouse Prairie & Pseudoroegneria spicata & Graminoid $\mathrm{C}_{3}$ & 3.6 & 3.6 \\
\hline Columbia Plateau Low Sagebrush Steppe & Pseudoroegneria spicata & Graminoid $\mathrm{C}_{3}$ & 3.6 & 3.6 \\
\hline Columbia Plateau Steppe and Grassland & Pseudoroegneria spicata & Graminoid $\mathrm{C}_{3}$ & 3.6 & 3.6 \\
\hline Great Basin Pinyon-Juniper Woodland & Pseudoroegneria spicata & Graminoid $\mathrm{C}_{3}$ & 3.6 & 3.6 \\
\hline $\begin{array}{l}\text { Inter-Mountain Basins Curl-leaf Mountain- } \\
\text { mahogany Woodland and Shrubland }\end{array}$ & Pseudoroegneria spicata & Graminoid $\mathrm{C}_{3}$ & 3.6 & 3.6 \\
\hline Inter-Mountain Basins Mat Saltbush Shrubland & Pseudoroegneria spicata & Graminoid $\mathrm{C}_{3}$ & 3.6 & 3.6 \\
\hline Inter-Mountain Basins Mixed Salt Desert Scrub & Pseudoroegneria spicata & Graminoid $\mathrm{C}_{3}$ & 3.6 & 3.6 \\
\hline Inter-Mountain Basins Semi-Desert Grassland & Pseudoroegneria spicata & Graminoid $\mathrm{C}_{3}$ & 3.6 & 3.6 \\
\hline Inter-Mountain Basins Semi-Desert Shrub-Steppe & Pseudoroegneria spicata & Graminoid $\mathrm{C}_{3}$ & 3.6 & 3.6 \\
\hline Inter-Mountain Basins Shale Badland & Pseudoroegneria spicata & Graminoid $\mathrm{C}_{3}$ & 3.6 & 3.6 \\
\hline $\begin{array}{l}\text { Northern Rocky Mountain Dry-Mesic Montane } \\
\text { Mixed Conifer Forest }\end{array}$ & Pseudoroegneria spicata & Graminoid $\mathrm{C}_{3}$ & 3.6 & 3.6 \\
\hline Northwestern Great Plains Shrubland & Pseudoroegneria spicata & Graminoid $\mathrm{C}_{3}$ & 3.6 & 3.6 \\
\hline $\begin{array}{l}\text { Northwestern Great Plains-Black Hills Ponderosa } \\
\text { Pine Woodland and Savanna }\end{array}$ & Pseudoroegneria spicata & Graminoid $\mathrm{C}_{3}$ & 3.6 & 3.6 \\
\hline $\begin{array}{l}\text { Rocky Mountain Foothill Limber Pine-Juniper } \\
\text { Woodland }\end{array}$ & Pseudoroegneria spicata & Graminoid $\mathrm{C}_{3}$ & 3.6 & 3.6 \\
\hline $\begin{array}{l}\text { Southern Rocky Mountain Montane-Subalpine } \\
\text { Grassland }\end{array}$ & Pseudoroegneria spicata & Graminoid $\mathrm{C}_{3}$ & 3.6 & 3.6 \\
\hline $\begin{array}{l}\text { Southern Rocky Mountain Ponderosa Pine } \\
\text { Savanna }\end{array}$ & Pseudoroegneria spicata & Graminoid $\mathrm{C}_{3}$ & 3.6 & 3.6 \\
\hline $\begin{array}{l}\text { Wyoming Basins Dwarf Sagebrush Shrubland and } \\
\text { Steppe }\end{array}$ & Pseudoroegneria spicata & Graminoid $\mathrm{C}_{3}$ & 3.6 & 3.6 \\
\hline $\begin{array}{l}\text { Northern Rocky Mountain Lower Montane, Foothill } \\
\text { and Valley Grassland }\end{array}$ & Pseudoroegneria spicata & Graminoid $\mathrm{C}_{3}$ & 3.6 & 3.6 \\
\hline $\begin{array}{l}\text { Columbia Basin Foothill and Canyon Dry } \\
\text { Grassland }\end{array}$ & Pseudoroegneria spicata & Graminoid $\mathrm{C}_{3}$ & 3.6 & 3.6 \\
\hline $\begin{array}{l}\text { Columbia Plateau Western Juniper Woodland and } \\
\text { Savanna }\end{array}$ & Pseudoroegneria spicata & Graminoid $\mathrm{C}_{3}$ & 3.6 & 3.6 \\
\hline Great Basin Xeric Mixed Sagebrush Shrubland & Pseudoroegneria spicata & Graminoid $\mathrm{C}_{3}$ & 3.6 & 3.6 \\
\hline $\begin{array}{l}\text { Inter-Mountain Basins Curl-leaf Mountain- } \\
\text { mahogany Woodland and Shrubland }\end{array}$ & Pseudoroegneria spicata & Graminoid $\mathrm{C}_{3}$ & 3.6 & 3.6 \\
\hline
\end{tabular}




\begin{tabular}{|c|c|c|c|c|}
\hline Community & Species & Classification & Resistance & Resilience \\
\hline $\begin{array}{l}\text { Northern Rocky Mountain Foothill Conifer Wooded } \\
\text { Steppe }\end{array}$ & Pseudoroegneria spicata & Graminoid $C_{3}$ & 3.6 & 3.6 \\
\hline $\begin{array}{l}\text { Rocky Mountain Subalpine-Montane Mesic } \\
\text { Meadow }\end{array}$ & Pseudoroegneria spicata & Graminoid $\mathrm{C}_{3}$ & 3.6 & 3.6 \\
\hline Mediterranean California Mixed Evergreen Forest & Pseudotsuga macrocarpa & Tree- Evergreen & 3.2 & 2.2 \\
\hline California Coastal Redwood Forest & Pseudotsuga menziesii & Tree- Evergreen & 3.2 & 2.2 \\
\hline Central California Coast Ranges Cliff and Canyon & Pseudotsuga menziesii & Tree- Evergreen & 3.2 & 2.2 \\
\hline $\begin{array}{l}\text { East Cascades Mesic Montane Mixed Conifer } \\
\text { Forest and Woodland }\end{array}$ & Pseudotsuga menziesii & Tree-Evergreen & 3.2 & 2.2 \\
\hline $\begin{array}{l}\text { East Cascades Oak-Ponderosa Pine Forest and } \\
\text { Woodland }\end{array}$ & Pseudotsuga menziesii & Tree-Evergreen & 3.2 & 2.2 \\
\hline $\begin{array}{l}\text { Great Basin Foothill and Lower Montane Riparian } \\
\text { Woodland and Shrubland }\end{array}$ & Pseudotsuga menziesii & Tree-Evergreen & 3.2 & 2.2 \\
\hline $\begin{array}{l}\text { Inter-Mountain Basins Aspen-Mixed Conifer Forest } \\
\text { and Woodland }\end{array}$ & Pseudotsuga menziesii & Tree-Evergreen & 3.2 & 2.2 \\
\hline $\begin{array}{l}\text { Klamath-Siskiyou Lower Montane Serpentine } \\
\text { Mixed Conifer Woodland }\end{array}$ & Pseudotsuga menziesii & Tree-Evergreen & 3.2 & 2.2 \\
\hline $\begin{array}{l}\text { Madrean Lower Montane Pine-Oak Forest and } \\
\text { Woodland }\end{array}$ & Pseudotsuga menziesii & Tree- Evergreen & 3.2 & 2.2 \\
\hline $\begin{array}{l}\text { Madrean Upper Montane Conifer-Oak Forest and } \\
\text { Woodland }\end{array}$ & Pseudotsuga menziesii & Tree-Evergreen & 3.2 & 2.2 \\
\hline $\begin{array}{l}\text { Mediterranean California Dry-Mesic Mixed Conifer } \\
\text { Forest and Woodland }\end{array}$ & Pseudotsuga menziesii & Tree-Evergreen & 3.2 & 2.2 \\
\hline $\begin{array}{l}\text { Mediterranean California Mesic Mixed Conifer } \\
\text { Forest and Woodland }\end{array}$ & Pseudotsuga menziesii & Tree-Evergreen & 3.2 & 2.2 \\
\hline Mediterranean California Mixed Evergreen Forest & Pseudotsuga menziesii & Tree-Evergreen & 3.2 & 2.2 \\
\hline $\begin{array}{l}\text { Middle Rocky Mountain Montane Douglas-fir } \\
\text { Forest and Woodland }\end{array}$ & Pseudotsuga menziesii & Tree-Evergreen & 3.2 & 2.2 \\
\hline $\begin{array}{l}\text { North Pacific Dry-Mesic Silver Fir-Western } \\
\text { Hemlock-Douglas-fir Forest }\end{array}$ & Pseudotsuga menziesii & Tree-Evergreen & 3.2 & 2.2 \\
\hline $\begin{array}{l}\text { North Pacific Lowland Mixed Hardwood-Conifer } \\
\text { Forest }\end{array}$ & Pseudotsuga menziesii & Tree- Evergreen & 3.2 & 2.2 \\
\hline $\begin{array}{l}\text { North Pacific Lowland Riparian Forest and } \\
\text { Shrubland }\end{array}$ & Pseudotsuga menziesii & Tree-Evergreen & 3.2 & 2.2 \\
\hline $\begin{array}{l}\text { North Pacific Maritime Dry-Mesic Douglas-fir- } \\
\text { Western Hemlock Forest }\end{array}$ & Pseudotsuga menziesii & Tree-Evergreen & 3.2 & 2.2 \\
\hline $\begin{array}{l}\text { North Pacific Maritime Mesic-Wet Douglas-fir- } \\
\text { Western Hemlock Forest }\end{array}$ & Pseudotsuga menziesii & Tree-Evergreen & 3.2 & 2.2 \\
\hline $\begin{array}{l}\text { North Pacific Mesic Western Hemlock-Silver Fir } \\
\text { Forest }\end{array}$ & Pseudotsuga menziesii & Tree- Evergreen & 3.2 & 2.2 \\
\hline $\begin{array}{l}\text { North Pacific Montane Riparian Woodland and } \\
\text { Shrubland }\end{array}$ & Pseudotsuga menziesii & Tree-Evergreen & 3.2 & 2.2 \\
\hline North Pacific Oak Woodland & Pseudotsuga menziesii & Tree-Evergreen & 3.2 & 2.2 \\
\hline North Pacific Serpentine Barren & Pseudotsuga menziesii & Tree- Evergreen & 3.2 & 2.2 \\
\hline North Pacific Wooded Volcanic Flowage & Pseudotsuga menziesii & Tree-Evergreen & 3.2 & 2.2 \\
\hline $\begin{array}{l}\text { Northern Rocky Mountain Dry-Mesic Montane } \\
\text { Mixed Conifer Forest }\end{array}$ & Pseudotsuga menziesii & Tree- Evergreen & 3.2 & 2.2 \\
\hline $\begin{array}{l}\text { Northern Rocky Mountain Foothill Conifer Wooded } \\
\text { Steppe }\end{array}$ & Pseudotsuga menziesii & Tree-Evergreen & 3.2 & 2.2 \\
\hline $\begin{array}{l}\text { Northern Rocky Mountain Mesic Montane Mixed } \\
\text { Conifer Forest }\end{array}$ & Pseudotsuga menziesii & Tree- Evergreen & 3.2 & 2.2 \\
\hline Northern Rocky Mountain Wooded Vernal Pool & Pseudotsuga menziesii & Tree- Evergreen & 3.2 & 2.2 \\
\hline $\begin{array}{l}\text { Northwestern Great Plains-Black Hills Ponderosa } \\
\text { Pine Woodland and Savanna }\end{array}$ & Pseudotsuga menziesii & Tree- Evergreen & 3.2 & 2.2 \\
\hline
\end{tabular}




\begin{tabular}{|c|c|c|c|c|}
\hline Community & Species & Classification & Resistance & Resilience \\
\hline $\begin{array}{l}\text { Rocky Mountain Lower Montane-Foothill Riparian } \\
\text { Woodland and Shrubland }\end{array}$ & Pseudotsuga menziesii & Tree- Evergreen & 3.2 & 2.2 \\
\hline Sierra Nevada Cliff and Canyon & Pseudotsuga menziesii & Tree- Evergreen & 3.2 & 2.2 \\
\hline $\begin{array}{l}\text { Southern Rocky Mountain Dry-Mesic Montane } \\
\text { Mixed Conifer Forest and Woodland }\end{array}$ & Pseudotsuga menziesii & Tree- Evergreen & 3.2 & 2.2 \\
\hline $\begin{array}{l}\text { Southern Rocky Mountain Mesic Montane Mixed } \\
\text { Conifer Forest and Woodland }\end{array}$ & Pseudotsuga menziesii & Tree- Evergreen & 3.2 & 2.2 \\
\hline $\begin{array}{l}\text { Mediterranean California Foothill and Lower } \\
\text { Montane Riparian Woodland and Shrubland }\end{array}$ & Pseudotsuga menziesii & Tree- Evergreen & 3.2 & 2.2 \\
\hline $\begin{array}{l}\text { North Pacific Dry Douglas-fir-(Madrone) Forest and } \\
\text { Woodland }\end{array}$ & Pseudotsuga menziesii & Tree- Evergreen & 3.2 & 2.2 \\
\hline $\begin{array}{l}\text { North American Warm Desert Active and } \\
\text { Stabilized Dune }\end{array}$ & Psoralidium lanceolatum & Forb & 3 & 3.5 \\
\hline $\begin{array}{l}\text { Chihuahuan Stabilized Coppice Dune and Sand } \\
\text { Flat Scrub }\end{array}$ & Psorothamnus scoparius & Shrub- Deciduous & 2.7 & 3 \\
\hline North American Warm Desert Wash & Psorothamnus spinosus & Shrub-Deciduous & 2.7 & 3 \\
\hline $\begin{array}{l}\text { Rocky Mountain Gambel Oak-Mixed Montane } \\
\text { Shrubland }\end{array}$ & Ptelea trifoliata & Shrub- Deciduous & 2.7 & 3 \\
\hline Eastern Highland Rim Prairie and Barrens & Pteridium aquilinum & Graminoid $\mathrm{C}_{3}$ & 3.6 & 3.6 \\
\hline $\begin{array}{l}\text { Atlantic Coastal Plain Upland Longleaf Pine } \\
\text { Woodland }\end{array}$ & Pteridium aquilinum & Graminoid $\mathrm{C}_{3}$ & 3.6 & 3.6 \\
\hline Madrean Oriental Chaparral & Purshia mexicana & Shrub- Evergreen & 2.7 & 2.4 \\
\hline Mogollon Chaparral & Purshia stansburiana & Shrub- Evergreen & 2.7 & 2.4 \\
\hline $\begin{array}{l}\text { Rocky Mountain Gambel Oak-Mixed Montane } \\
\text { Shrubland }\end{array}$ & Purshia stansburiana & Shrub- Evergreen & 2.7 & 2.4 \\
\hline Great Basin Pinyon-Juniper Woodland & Purshia tridentata & Shrub- Evergreen & 2.7 & 2.4 \\
\hline Great Basin Semi-Desert Chaparral & Purshia tridentata & Shrub- Evergreen & 2.7 & 2.4 \\
\hline Inter-Mountain Basins Active and Stabilized Dune & Purshia tridentata & Shrub- Evergreen & 2.7 & 2.4 \\
\hline Inter-Mountain Basins Big Sagebrush Shrubland & Purshia tridentata & Shrub- Evergreen & 2.7 & 2.4 \\
\hline Inter-Mountain Basins Big Sagebrush Steppe & Purshia tridentata & Shrub- Evergreen & 2.7 & 2.4 \\
\hline Inter-Mountain Basins Juniper Savanna & Purshia tridentata & Shrub- Evergreen & 2.7 & 2.4 \\
\hline $\begin{array}{l}\text { Inter-Mountain Basins Volcanic Rock and Cinder } \\
\text { Land }\end{array}$ & Purshia tridentata & Shrub- Evergreen & 2.7 & 2.4 \\
\hline $\begin{array}{l}\text { Northern Rocky Mountain Ponderosa Pine } \\
\text { Woodland and Savanna }\end{array}$ & Purshia tridentata & Shrub- Evergreen & 2.7 & 2.4 \\
\hline $\begin{array}{l}\text { Rocky Mountain Foothill Limber Pine-Juniper } \\
\text { Woodland }\end{array}$ & Purshia tridentata & Shrub- Evergreen & 2.7 & 2.4 \\
\hline $\begin{array}{l}\text { Rocky Mountain Gambel Oak-Mixed Montane } \\
\text { Shrubland }\end{array}$ & Purshia tridentata & Shrub- Evergreen & 2.7 & 2.4 \\
\hline $\begin{array}{l}\text { Rocky Mountain Lower Montane-Foothill } \\
\text { Shrubland }\end{array}$ & Purshia tridentata & Shrub- Evergreen & 2.7 & 2.4 \\
\hline Rocky Mountain Poor-Site Lodgepole Pine Forest & Purshia tridentata & Shrub- Evergreen & 2.7 & 2.4 \\
\hline $\begin{array}{l}\text { Rocky Mountain Subalpine-Montane Limber- } \\
\text { Bristlecone Pine Woodland }\end{array}$ & Purshia tridentata & Shrub- Evergreen & 2.7 & 2.4 \\
\hline $\begin{array}{l}\text { Southern Rocky Mountain Pinyon-Juniper } \\
\text { Woodland }\end{array}$ & Purshia tridentata & Shrub- Evergreen & 2.7 & 2.4 \\
\hline California Central Valley Mixed Oak Savanna & Quercus agrifolia & Tree- Evergreen & 3.2 & 2.2 \\
\hline $\begin{array}{l}\text { California Coastal Live Oak Woodland and } \\
\text { Savanna }\end{array}$ & Quercus agrifolia & Tree- Evergreen & 3.2 & 2.2 \\
\hline Southern California Oak Woodland and Savanna & Quercus agrifolia & Tree- Evergreen & 3.2 & 2.2 \\
\hline $\begin{array}{l}\text { Mediterranean California Foothill and Lower } \\
\text { Montane Riparian Woodland and Shrubland }\end{array}$ & Quercus agrifolia & Tree- Evergreen & 3.2 & 2.2 \\
\hline
\end{tabular}




\begin{tabular}{|c|c|c|c|c|}
\hline Community & Species & Classification & Resistance & Resilience \\
\hline $\begin{array}{l}\text { Allegheny-Cumberland Dry Oak Forest and } \\
\text { Woodland }\end{array}$ & Quercus alba & Tree-Deciduous & 3 & 4.2 \\
\hline $\begin{array}{l}\text { Central and Southern Appalachian Montane Oak } \\
\text { Forest }\end{array}$ & Quercus alba & Tree- Deciduous & 3 & 4.2 \\
\hline Crowley's Ridge Mesic Loess Slope Forest & Quercus alba & Tree-Deciduous & 3 & 4.2 \\
\hline $\begin{array}{l}\text { East Gulf Coastal Plain Northern Dry Upland } \\
\text { Hardwood Forest }\end{array}$ & Quercus alba & Tree- Deciduous & 3 & 4.2 \\
\hline East Gulf Coastal Plain Northern Loess Bluff Forest & Quercus alba & Tree-Deciduous & 3 & 4.2 \\
\hline $\begin{array}{l}\text { East Gulf Coastal Plain Northern Loess Plain Oak- } \\
\text { Hickory Upland }\end{array}$ & Quercus alba & Tree-Deciduous & 3 & 4.2 \\
\hline $\begin{array}{l}\text { East Gulf Coastal Plain Northern Mesic Hardwood } \\
\text { Slope Forest }\end{array}$ & Quercus alba & Tree- Deciduous & 3 & 4.2 \\
\hline Eastern Highland Rim Prairie and Barrens & Quercus alba & Tree-Deciduous & 3 & 4.2 \\
\hline Eastern Serpentine Woodland & Quercus alba & Tree-Deciduous & 3 & 4.2 \\
\hline Lower Mississippi River Flatwoods & Quercus alba & Tree-Deciduous & 3 & 4.2 \\
\hline $\begin{array}{l}\text { Mississippi River Alluvial Plain Dry-Mesic Loess } \\
\text { Slope Forest }\end{array}$ & Quercus alba & Tree- Deciduous & 3 & 4.2 \\
\hline $\begin{array}{l}\text { North-Central Interior Dry Oak Forest and } \\
\text { Woodland }\end{array}$ & Quercus alba & Tree- Deciduous & 3 & 4.2 \\
\hline $\begin{array}{l}\text { North-Central Interior Dry-Mesic Oak Forest and } \\
\text { Woodland }\end{array}$ & Quercus alba & Tree- Deciduous & 3 & 4.2 \\
\hline North-Central Oak Barrens & Quercus alba & Tree-Deciduous & 3 & 4.2 \\
\hline Northeastern Interior Dry-Mesic Oak Forest & Quercus alba & Tree-Deciduous & 3 & 4.2 \\
\hline Northern Atlantic Coastal Plain Hardwood Forest & Quercus alba & Tree- Deciduous & 3 & 4.2 \\
\hline Ouachita Montane Oak Forest & Quercus alba & Tree-Deciduous & 3 & 4.2 \\
\hline Ozark-Ouachita Dry Oak Woodland & Quercus alba & Tree-Deciduous & 3 & 4.2 \\
\hline Ozark-Ouachita Dry-Mesic Oak Forest & Quercus alba & Tree- Deciduous & 3 & 4.2 \\
\hline Ozark-Ouachita Mesic Hardwood Forest & Quercus alba & Tree-Deciduous & 3 & 4.2 \\
\hline $\begin{array}{l}\text { Ozark-Ouachita Shortleaf Pine-Bluestem } \\
\text { Woodland }\end{array}$ & Quercus alba & Tree-Deciduous & 3 & 4.2 \\
\hline $\begin{array}{l}\text { Southern Atlantic Coastal Plain Dry and Dry-Mesic } \\
\text { Oak Forest }\end{array}$ & Quercus alba & Tree- Deciduous & 3 & 4.2 \\
\hline $\begin{array}{l}\text { Southern Atlantic Coastal Plain Mesic Hardwood } \\
\text { Forest }\end{array}$ & Quercus alba & Tree-Deciduous & 3 & 4.2 \\
\hline $\begin{array}{l}\text { Southern Coastal Plain Dry Upland Hardwood } \\
\text { Forest }\end{array}$ & Quercus alba & Tree- Deciduous & 3 & 4.2 \\
\hline Southern Coastal Plain Mesic Slope Forest & Quercus alba & Tree-Deciduous & 3 & 4.2 \\
\hline $\begin{array}{l}\text { Southern Interior Low Plateau Dry-Mesic Oak } \\
\text { Forest }\end{array}$ & Quercus alba & Tree-Deciduous & 3 & 4.2 \\
\hline Southern Piedmont Mesic Forest & Quercus alba & Tree- Deciduous & 3 & 4.2 \\
\hline West Gulf Coastal Plain Pine-Hardwood Flatwoods & Quercus alba & Tree-Deciduous & 3 & 4.2 \\
\hline West Gulf Coastal Plain Pine-Hardwood Forest & Quercus alba & Tree- Deciduous & 3 & 4.2 \\
\hline Appalachian (Hemlock)-Northern Hardwood Forest & Quercus alba & Tree-Deciduous & 3 & 4.2 \\
\hline $\begin{array}{l}\text { South-Central Interior / Upper Coastal Plain } \\
\text { Flatwoods }\end{array}$ & Quercus alba & Tree- Deciduous & 3 & 4.2 \\
\hline Southern Appalachian Oak Forest & Quercus alba & Tree-Deciduous & 3 & 4.2 \\
\hline Southern Piedmont Dry Oak-(Pine) Forest & Quercus alba & Tree- Deciduous & 3 & 4.2 \\
\hline $\begin{array}{l}\text { Southern Ridge and Valley / Cumberland Dry } \\
\text { Calcareous Forest }\end{array}$ & Quercus alba & Tree-Deciduous & 3 & 4.2 \\
\hline Madrean Encinal & Quercus arizonica & Tree-Evergreen & 3.2 & 2.2 \\
\hline Madrean Pinyon-Juniper Woodland & Quercus arizonica & Tree- Evergreen & 3.2 & 2.2 \\
\hline
\end{tabular}




\begin{tabular}{|c|c|c|c|c|}
\hline Community & Species & Classification & Resistance & Resilience \\
\hline $\begin{array}{l}\text { Madrean Upper Montane Conifer-Oak Forest and } \\
\text { Woodland }\end{array}$ & Quercus arizonica & Tree- Evergreen & 3.2 & 2.2 \\
\hline Sonora-Mojave Semi-Desert Chaparral & Quercus berberidifolia & Shrub- Evergreen & 2.7 & 2.4 \\
\hline California Mesic Chaparral & Quercus berberidifolia & Tree- Evergreen & 3.2 & 2.2 \\
\hline North-Central Interior Wet Flatwoods & Quercus bicolor & Tree-Deciduous & 3 & 4.2 \\
\hline $\begin{array}{l}\text { Edwards Plateau Dry-Mesic Slope Forest and } \\
\text { Woodland }\end{array}$ & Quercus buckleyi & Tree-Deciduous & 3 & 4.2 \\
\hline Edwards Plateau Mesic Canyon & Quercus buckleyi & Tree-Deciduous & 3 & 4.2 \\
\hline Florida Peninsula Inland Scrub & Quercus chapmanii & Tree- Evergreen & 3.2 & 2.2 \\
\hline Madrean Encinal & Quercus chihuahuensis & Tree- Deciduous & 3 & 4.2 \\
\hline Mediterranean California Mixed Evergreen Forest & Quercus chrysolepis & Tree- Evergreen & 3.2 & 2.2 \\
\hline Mediterranean California Mixed Oak Woodland & Quercus chrysolepis & Tree- Evergreen & 3.2 & 2.2 \\
\hline $\begin{array}{l}\text { Mediterranean California Lower Montane Black } \\
\text { Oak-Conifer Forest and Woodland }\end{array}$ & Quercus chrysolepis & Tree-Evergreen & 3.2 & 2.2 \\
\hline $\begin{array}{l}\text { Allegheny-Cumberland Dry Oak Forest and } \\
\text { Woodland }\end{array}$ & Quercus coccinea & Tree-Deciduous & 3 & 4.2 \\
\hline Central Appalachian Dry Oak-Pine Forest & Quercus coccinea & Tree- Deciduous & 3 & 4.2 \\
\hline $\begin{array}{l}\text { North-Central Interior Dry Oak Forest and } \\
\text { Woodland }\end{array}$ & Quercus coccinea & Tree- Deciduous & 3 & 4.2 \\
\hline Northeastern Interior Dry-Mesic Oak Forest & Quercus coccinea & Tree-Deciduous & 3 & 4.2 \\
\hline Northern Atlantic Coastal Plain Hardwood Forest & Quercus coccinea & Tree-Deciduous & 3 & 4.2 \\
\hline Ozark-Ouachita Dry Oak Woodland & Quercus coccinea & Tree-Deciduous & 3 & 4.2 \\
\hline Southeastern Interior Longleaf Pine Woodland & Quercus coccinea & Tree-Deciduous & 3 & 4.2 \\
\hline Southern Appalachian Oak Forest & Quercus coccinea & Tree-Deciduous & 3 & 4.2 \\
\hline Southern Piedmont Dry Oak-(Pine) Forest & Quercus coccinea & Tree-Deciduous & 3 & 4.2 \\
\hline Sonora-Mojave Semi-Desert Chaparral & Quercus cornelius-mulleri & Tree- Evergreen & 3.2 & 2.2 \\
\hline $\begin{array}{l}\text { California Lower Montane Blue Oak-Foothill Pine } \\
\text { Woodland and Savanna }\end{array}$ & Quercus douglasii & Tree-Deciduous & 3 & 4.2 \\
\hline Sonora-Mojave Semi-Desert Chaparral & Quercus dumosa & Shrub- Evergreen & 2.7 & 2.4 \\
\hline California Mesic Chaparral & Quercus dumosa & Tree- Evergreen & 3.2 & 2.2 \\
\hline California Xeric Serpentine Chaparral & Quercus durata & Tree-Evergreen & 3.2 & 2.2 \\
\hline Laurentian Pine-Oak Barrens & Quercus ellipsoidalis & Tree-Deciduous & 3 & 4.2 \\
\hline $\begin{array}{l}\text { North-Central Interior Dry Oak Forest and } \\
\text { Woodland }\end{array}$ & Quercus ellipsoidalis & Tree-Deciduous & 3 & 4.2 \\
\hline North-Central Oak Barrens & Quercus ellipsoidalis & Tree-Deciduous & 3 & 4.2 \\
\hline Madrean Encinal & Quercus emoryi & Tree- Evergreen & 3.2 & 2.2 \\
\hline Madrean Pinyon-Juniper Woodland & Quercus emoryi & Tree-Evergreen & 3.2 & 2.2 \\
\hline $\begin{array}{l}\text { Madrean Upper Montane Conifer-Oak Forest and } \\
\text { Woodland }\end{array}$ & Quercus emoryi & Tree- Evergreen & 3.2 & 2.2 \\
\hline Southern California Oak Woodland and Savanna & Quercus engelmannii & Tree- Evergreen & 3.2 & 2.2 \\
\hline $\begin{array}{l}\text { Allegheny-Cumberland Dry Oak Forest and } \\
\text { Woodland }\end{array}$ & Quercus falcata & Tree- Deciduous & 3 & 4.2 \\
\hline Crowley's Ridge Sand Forest & Quercus falcata & Tree- Deciduous & 3 & 4.2 \\
\hline $\begin{array}{l}\text { East Gulf Coastal Plain Interior Upland Longleaf } \\
\text { Pine Woodland }\end{array}$ & Quercus falcata & Tree-Deciduous & 3 & 4.2 \\
\hline $\begin{array}{l}\text { East Gulf Coastal Plain Northern Dry Upland } \\
\text { Hardwood Forest }\end{array}$ & Quercus falcata & Tree-Deciduous & 3 & 4.2 \\
\hline $\begin{array}{l}\text { East Gulf Coastal Plain Northern Loess Plain Oak- } \\
\text { Hickory Upland }\end{array}$ & Quercus falcata & Tree-Deciduous & 3 & 4.2 \\
\hline $\begin{array}{l}\text { Lower Mississippi River Dune Woodland and } \\
\text { Forest }\end{array}$ & Quercus falcata & Tree-Deciduous & 3 & 4.2 \\
\hline
\end{tabular}




\begin{tabular}{|c|c|c|c|c|}
\hline Community & Species & Classification & Resistance & Resilience \\
\hline $\begin{array}{l}\text { Mississippi River Alluvial Plain Dry-Mesic Loess } \\
\text { Slope Forest }\end{array}$ & Quercus falcata & Tree- Deciduous & 3 & 4.2 \\
\hline Southeastern Interior Longleaf Pine Woodland & Quercus falcata & Tree- Deciduous & 3 & 4.2 \\
\hline $\begin{array}{l}\text { Southern Atlantic Coastal Plain Dry and Dry-Mesic } \\
\text { Oak Forest }\end{array}$ & Quercus falcata & Tree- Deciduous & 3 & 4.2 \\
\hline $\begin{array}{l}\text { Southern Coastal Plain Dry Upland Hardwood } \\
\text { Forest }\end{array}$ & Quercus falcata & Tree- Deciduous & 3 & 4.2 \\
\hline West Gulf Coastal Plain Pine-Hardwood Forest & Quercus falcata & Tree-Deciduous & 3 & 4.2 \\
\hline $\begin{array}{l}\text { South-Central Interior / Upper Coastal Plain Wet } \\
\text { Flatwoods }\end{array}$ & Quercus falcata & Tree-Deciduous & 3 & 4.2 \\
\hline Southern Piedmont Dry Oak-(Pine) Forest & Quercus falcata & Tree- Deciduous & 3 & 4.2 \\
\hline $\begin{array}{l}\text { Central and South Texas Coastal Fringe Forest and } \\
\text { Woodland }\end{array}$ & Quercus fusiformis & Tree-Evergreen & 3.2 & 2.2 \\
\hline $\begin{array}{l}\text { East-Central Texas Plains Post Oak Savanna and } \\
\text { Woodland }\end{array}$ & Quercus fusiformis & Tree- Evergreen & 3.2 & 2.2 \\
\hline Edwards Plateau Floodplain Terrace & Quercus fusiformis & Tree-Evergreen & 3.2 & 2.2 \\
\hline $\begin{array}{l}\text { Edwards Plateau Limestone Savanna and } \\
\text { Woodland }\end{array}$ & Quercus fusiformis & Tree- Evergreen & 3.2 & 2.2 \\
\hline Edwards Plateau Limestone Shrubland & Quercus fusiformis & Tree- Evergreen & 3.2 & 2.2 \\
\hline Edwards Plateau Riparian & Quercus fusiformis & Tree- Evergreen & 3.2 & 2.2 \\
\hline Southeastern Great Plains Floodplain Forest & Quercus fusiformis & Tree-Evergreen & 3.2 & 2.2 \\
\hline Llano Uplift Acidic Forest, Woodland and Glade & Quercus fusiformis & Tree-Evergreen & 3.2 & 2.2 \\
\hline $\begin{array}{l}\text { Inter-Mountain Basins Curl-leaf Mountain- } \\
\text { mahogany Woodland and Shrubland }\end{array}$ & Quercus gambelii & Shrub- Deciduous & 2.7 & 3 \\
\hline Madrean Oriental Chaparral & Quercus gambelii & Shrub-Deciduous & 2.7 & 3 \\
\hline $\begin{array}{l}\text { Southern Rocky Mountain Pinyon-Juniper } \\
\text { Woodland }\end{array}$ & Quercus gambelii & Shrub- Deciduous & 2.7 & 3 \\
\hline $\begin{array}{l}\text { Southern Rocky Mountain Ponderosa Pine } \\
\text { Woodland }\end{array}$ & Quercus gambelii & Shrub- Deciduous & 2.7 & 3 \\
\hline Rocky Mountain Bigtooth Maple Ravine Woodland & Quercus gambelii & Tree- Deciduous & 3 & 4.2 \\
\hline $\begin{array}{l}\text { Rocky Mountain Gambel Oak-Mixed Montane } \\
\text { Shrubland }\end{array}$ & Quercus gambelii & Tree-Deciduous & 3 & 4.2 \\
\hline $\begin{array}{l}\text { Southern Rocky Mountain Dry-Mesic Montane } \\
\text { Mixed Conifer Forest and Woodland }\end{array}$ & Quercus gambelii & Tree- Deciduous & 3 & 4.2 \\
\hline $\begin{array}{l}\text { California Lower Montane Blue Oak-Foothill Pine } \\
\text { Woodland and Savanna }\end{array}$ & Quercus garryana & Tree- Deciduous & 3 & 4.2 \\
\hline $\begin{array}{l}\text { East Cascades Oak-Ponderosa Pine Forest and } \\
\text { Woodland }\end{array}$ & Quercus garryana & Tree- Deciduous & 3 & 4.2 \\
\hline Mediterranean California Mixed Oak Woodland & Quercus garryana & Tree- Deciduous & 3 & 4.2 \\
\hline $\begin{array}{l}\text { North Pacific Lowland Mixed Hardwood-Conifer } \\
\text { Forest }\end{array}$ & Quercus garryana & Tree-Deciduous & 3 & 4.2 \\
\hline North Pacific Oak Woodland & Quercus garryana & Tree- Deciduous & 3 & 4.2 \\
\hline $\begin{array}{l}\text { Mediterranean California Lower Montane Black } \\
\text { Oak-Conifer Forest and Woodland }\end{array}$ & Quercus garryana & Tree-Deciduous & 3 & 4.2 \\
\hline California Montane Woodland and Chaparral & Quercus garryana & Shrub-Deciduous & 2.7 & 3 \\
\hline Mediterranean California Mixed Oak Woodland & Quercus garryana & Tree-Deciduous & 3 & 4.2 \\
\hline Mediterranean California Mixed Oak Woodland & Quercus garryana & Tree-Deciduous & 3 & 4.2 \\
\hline $\begin{array}{l}\text { Southeast Florida Coastal Strand and Maritime } \\
\text { Hammock }\end{array}$ & Quercus geminata & Shrub- Evergreen & 2.7 & 2.4 \\
\hline Florida Peninsula Inland Scrub & Quercus geminata & Tree- Evergreen & 3.2 & 2.2 \\
\hline Southern Atlantic Coastal Plain Maritime Forest & Quercus geminata & Tree-Evergreen & 3.2 & 2.2 \\
\hline Southern Coastal Plain Oak Dome and Hammock & Quercus geminata & Tree- Evergreen & 3.2 & 2.2 \\
\hline
\end{tabular}




\begin{tabular}{|c|c|c|c|c|}
\hline Community & Species & Classification & Resistance & Resilience \\
\hline Madrean Encinal & Quercus grisea & Tree- Evergreen & 3.2 & 2.2 \\
\hline $\begin{array}{l}\text { Madrean Lower Montane Pine-Oak Forest and } \\
\text { Woodland }\end{array}$ & Quercus grisea & Tree-Evergreen & 3.2 & 2.2 \\
\hline Madrean Pinyon-Juniper Woodland & Quercus grisea & Tree- Evergreen & 3.2 & 2.2 \\
\hline $\begin{array}{l}\text { Madrean Upper Montane Conifer-Oak Forest and } \\
\text { Woodland }\end{array}$ & Quercus grisea & Tree- Evergreen & 3.2 & 2.2 \\
\hline $\begin{array}{l}\text { Atlantic Coastal Plain Upland Longleaf Pine } \\
\text { Woodland }\end{array}$ & Quercus hemisphaerica & Tree-Evergreen & 3.2 & 2.2 \\
\hline Central Atlantic Coastal Plain Maritime Forest & Quercus hemisphaerica & Tree- Evergreen & 3.2 & 2.2 \\
\hline $\begin{array}{l}\text { Southern Coastal Plain Dry Upland Hardwood } \\
\text { Forest }\end{array}$ & Quercus hemisphaerica & Tree- Evergreen & 3.2 & 2.2 \\
\hline Southern Coastal Plain Oak Dome and Hammock & Quercus hemisphaerica & Tree- Evergreen & 3.2 & 2.2 \\
\hline Madrean Encinal & Quercus hypoleucoides & Tree-Evergreen & 3.2 & 2.2 \\
\hline $\begin{array}{l}\text { Madrean Lower Montane Pine-Oak Forest and } \\
\text { Woodland }\end{array}$ & Quercus hypoleucoides & Tree- Evergreen & 3.2 & 2.2 \\
\hline $\begin{array}{l}\text { Madrean Upper Montane Conifer-Oak Forest and } \\
\text { Woodland }\end{array}$ & Quercus hypoleucoides & Tree- Evergreen & 3.2 & 2.2 \\
\hline Northeastern Interior Pine Barrens & Quercus ilicifolia & Tree-Deciduous & 3 & 4.2 \\
\hline Northern Atlantic Coastal Plain Pitch Pine Barrens & Quercus ilicifolia & Tree- Deciduous & 3 & 4.2 \\
\hline $\begin{array}{l}\text { Atlantic Coastal Plain Fall-line Sandhills Longleaf } \\
\text { Pine Woodland }\end{array}$ & Quercus incana & Tree-Deciduous & 3 & 4.2 \\
\hline $\begin{array}{l}\text { Atlantic Coastal Plain Upland Longleaf Pine } \\
\text { Woodland }\end{array}$ & Quercus incana & Tree- Deciduous & 3 & 4.2 \\
\hline East-Central Texas Plains Xeric Sandyland & Quercus incana & Tree-Deciduous & 3 & 4.2 \\
\hline $\begin{array}{l}\text { West Gulf Coastal Plain Sandhill Oak and Shortleaf } \\
\text { Pine Forest and Woodland }\end{array}$ & Quercus incana & Tree-Deciduous & 3 & 4.2 \\
\hline $\begin{array}{l}\text { West Gulf Coastal Plain Stream Terrace Sandyland } \\
\text { Longleaf Pine Woodland }\end{array}$ & Quercus incana & Tree-Deciduous & 3 & 4.2 \\
\hline $\begin{array}{l}\text { West Gulf Coastal Plain Upland Longleaf Pine } \\
\text { Forest and Woodland }\end{array}$ & Quercus incana & Tree-Deciduous & 3 & 4.2 \\
\hline $\begin{array}{l}\text { Atlantic Coastal Plain Fall-line Sandhills Longleaf } \\
\text { Pine Woodland - Offsite Hardwood Modifier }\end{array}$ & Quercus incana & Tree- Deciduous & 3 & 4.2 \\
\hline Central Florida Pine Flatwoods & Quercus inopina & Shrub- Evergreen & 2.7 & 2.4 \\
\hline Florida Peninsula Inland Scrub & Quercus inopina & Shrub- Evergreen & 2.7 & 2.4 \\
\hline Madrean Encinal & Quercus intricata & Tree-Evergreen & 3.2 & 2.2 \\
\hline Sonora-Mojave Semi-Desert Chaparral & Quercus john-tuckeri & Shrub- Evergreen & 2.7 & 2.4 \\
\hline $\begin{array}{l}\text { Central and Southern California Mixed Evergreen } \\
\text { Woodland }\end{array}$ & Quercus kelloggii & Tree-Deciduous & 3 & 4.2 \\
\hline Mediterranean California Mixed Oak Woodland & Quercus kelloggii & Tree-Deciduous & 3 & 4.2 \\
\hline Southern California Oak Woodland and Savanna & Quercus kelloggii & Tree-Deciduous & 3 & 4.2 \\
\hline $\begin{array}{l}\text { Mediterranean California Lower Montane Black } \\
\text { Oak-Conifer Forest and Woodland }\end{array}$ & Quercus kelloggii & Tree-Deciduous & 3 & 4.2 \\
\hline $\begin{array}{l}\text { Edwards Plateau Dry-Mesic Slope Forest and } \\
\text { Woodland }\end{array}$ & Quercus laceyi & Tree- Deciduous & 3 & 4.2 \\
\hline $\begin{array}{l}\text { Edwards Plateau Limestone Savanna and } \\
\text { Woodland }\end{array}$ & Quercus laceyi & Tree-Deciduous & 3 & 4.2 \\
\hline $\begin{array}{l}\text { Atlantic Coastal Plain Fall-line Sandhills Longleaf } \\
\text { Pine Woodland }\end{array}$ & Quercus laevis & Tree-Deciduous & 3 & 4.2 \\
\hline $\begin{array}{l}\text { Atlantic Coastal Plain Upland Longleaf Pine } \\
\text { Woodland }\end{array}$ & Quercus laevis & Tree-Deciduous & 3 & 4.2 \\
\hline Florida Longleaf Pine Sandhill & Quercus laevis & Tree-Deciduous & 3 & 4.2 \\
\hline Southern Atlantic Coastal Plain Xeric River Dune & Quercus laevis & Tree-Deciduous & 3 & 4.2 \\
\hline
\end{tabular}




\begin{tabular}{|c|c|c|c|c|}
\hline Community & Species & Classification & Resistance & Resilience \\
\hline $\begin{array}{l}\text { Atlantic Coastal Plain Fall-line Sandhills Longleaf } \\
\text { Pine Woodland - Offsite Hardwood Modifier }\end{array}$ & Quercus laevis & Tree- Deciduous & 3 & 4.2 \\
\hline $\begin{array}{l}\text { Atlantic Coastal Plain Brownwater Stream } \\
\text { Floodplain Forest }\end{array}$ & Quercus laurifolia & Tree- Evergreen & 3.2 & 2.2 \\
\hline $\begin{array}{l}\text { Atlantic Coastal Plain Small Brownwater River } \\
\text { Floodplain Forest }\end{array}$ & Quercus laurifolia & Tree- Evergreen & 3.2 & 2.2 \\
\hline Southern Coastal Plain Hydric Hammock & Quercus laurifolia & Tree-Evergreen & 3.2 & 2.2 \\
\hline $\begin{array}{l}\text { Southern Coastal Plain Seepage Swamp and } \\
\text { Baygall }\end{array}$ & Quercus laurifolia & Tree- Evergreen & 3.2 & 2.2 \\
\hline $\begin{array}{l}\text { West Gulf Coastal Plain Nonriverine Wet } \\
\text { Hardwood Flatwoods }\end{array}$ & Quercus laurifolia & Tree- Evergreen & 3.2 & 2.2 \\
\hline Mississippi River Bottomland Depression & Quercus Iyrata & Tree-Deciduous & 3 & 4.2 \\
\hline $\begin{array}{l}\text { West Gulf Coastal Plain Large River Floodplain } \\
\text { Forest }\end{array}$ & Quercus lyrata & Tree- Deciduous & 3 & 4.2 \\
\hline $\begin{array}{l}\text { Mississippi River Low Floodplain (Bottomland) } \\
\text { Forest }\end{array}$ & Quercus lyrata & Tree- Deciduous & 3 & 4.2 \\
\hline Eastern Great Plains Tallgrass Aspen Parkland & Quercus macrocarpa & Tree- Deciduous & 3 & 4.2 \\
\hline Edwards Plateau Floodplain Terrace & Quercus macrocarpa & Tree-Deciduous & 3 & 4.2 \\
\hline $\begin{array}{l}\text { North-Central Interior Dry Oak Forest and } \\
\text { Woodland }\end{array}$ & Quercus macrocarpa & Tree- Deciduous & 3 & 4.2 \\
\hline $\begin{array}{l}\text { North-Central Interior Dry-Mesic Oak Forest and } \\
\text { Woodland }\end{array}$ & Quercus macrocarpa & Tree- Deciduous & 3 & 4.2 \\
\hline North-Central Interior Oak Savanna & Quercus macrocarpa & Tree- Deciduous & 3 & 4.2 \\
\hline North-Central Oak Barrens & Quercus macrocarpa & Tree- Deciduous & 3 & 4.2 \\
\hline $\begin{array}{l}\text { Northwestern Great Plains-Black Hills Ponderosa } \\
\text { Pine Woodland and Savanna }\end{array}$ & Quercus macrocarpa & Tree- Deciduous & 3 & 4.2 \\
\hline Southeastern Great Plains Floodplain Forest & Quercus macrocarpa & Tree- Deciduous & 3 & 4.2 \\
\hline $\begin{array}{l}\text { Western Great Plains Dry Bur Oak Forest and } \\
\text { Woodland }\end{array}$ & Quercus macrocarpa & Tree- Deciduous & 3 & 4.2 \\
\hline $\begin{array}{l}\text { Atlantic Coastal Plain Fall-line Sandhills Longleaf } \\
\text { Pine Woodland }\end{array}$ & Quercus margarettiae & Tree- Deciduous & 3 & 4.2 \\
\hline $\begin{array}{l}\text { Atlantic Coastal Plain Upland Longleaf Pine } \\
\text { Woodland }\end{array}$ & Quercus margarettiae & Tree- Deciduous & 3 & 4.2 \\
\hline $\begin{array}{l}\text { Southern Coastal Plain Dry Upland Hardwood } \\
\text { Forest }\end{array}$ & Quercus margarettiae & Tree- Deciduous & 3 & 4.2 \\
\hline $\begin{array}{l}\text { West Gulf Coastal Plain Sandhill Oak and Shortleaf } \\
\text { Pine Forest and Woodland }\end{array}$ & Quercus margarettiae & Tree- Deciduous & 3 & 4.2 \\
\hline $\begin{array}{l}\text { West Gulf Coastal Plain Stream Terrace Sandyland } \\
\text { Longleaf Pine Woodland }\end{array}$ & Quercus margarettiae & Tree- Deciduous & 3 & 4.2 \\
\hline $\begin{array}{l}\text { Atlantic Coastal Plain Fall-line Sandhills Longleaf } \\
\text { Pine Woodland - Offsite Hardwood Modifier }\end{array}$ & Quercus margarettiae & Tree- Deciduous & 3 & 4.2 \\
\hline $\begin{array}{l}\text { Atlantic Coastal Plain Fall-line Sandhills Longleaf } \\
\text { Pine Woodland }\end{array}$ & Quercus marilandica & Tree- Deciduous & 3 & 4.2 \\
\hline Bastrop Lost Pines Forest and Woodland & Quercus marilandica & Tree- Deciduous & 3 & 4.2 \\
\hline $\begin{array}{l}\text { Central Interior Highlands Dry Acidic Glade and } \\
\text { Barrens }\end{array}$ & Quercus marilandica & Tree- Deciduous & 3 & 4.2 \\
\hline Crosstimbers Oak Forest and Woodland & Quercus marilandica & Tree- Deciduous & 3 & 4.2 \\
\hline $\begin{array}{l}\text { East Gulf Coastal Plain Northern Loess Plain Oak- } \\
\text { Hickory Upland }\end{array}$ & Quercus marilandica & Tree- Deciduous & 3 & 4.2 \\
\hline $\begin{array}{l}\text { East-Central Texas Plains Post Oak Savanna and } \\
\text { Woodland }\end{array}$ & Quercus marilandica & Tree- Deciduous & 3 & 4.2 \\
\hline Eastern Serpentine Woodland & Quercus marilandica & Tree- Deciduous & 3 & 4.2 \\
\hline Northern Atlantic Coastal Plain Pitch Pine Barrens & Quercus marilandica & Tree-Deciduous & 3 & 4.2 \\
\hline Ozark-Ouachita Dry Oak Woodland & Quercus marilandica & Tree- Deciduous & 3 & 4.2 \\
\hline
\end{tabular}




\begin{tabular}{|c|c|c|c|c|}
\hline Community & Species & Classification & Resistance & Resilience \\
\hline $\begin{array}{l}\text { Ozark-Ouachita Shortleaf Pine-Bluestem } \\
\text { Woodland }\end{array}$ & Quercus marilandica & Tree-Deciduous & 3 & 4.2 \\
\hline $\begin{array}{l}\text { West Gulf Coastal Plain Sandhill Oak and Shortleaf } \\
\text { Pine Forest and Woodland }\end{array}$ & Quercus marilandica & Tree-Deciduous & 3 & 4.2 \\
\hline $\begin{array}{l}\text { Atlantic Coastal Plain Fall-line Sandhills Longleaf } \\
\text { Pine Woodland - Offsite Hardwood Modifier }\end{array}$ & Quercus marilandica & Tree-Deciduous & 3 & 4.2 \\
\hline Llano Uplift Acidic Forest, Woodland and Glade & Quercus marilandica & Tree-Deciduous & 3 & 4.2 \\
\hline $\begin{array}{l}\text { Atlantic Coastal Plain Small Brownwater River } \\
\text { Floodplain Forest }\end{array}$ & Quercus michauxii & Tree- Deciduous & 3 & 4.2 \\
\hline Lower Mississippi River Flatwoods & Quercus michauxii & Tree-Deciduous & 3 & 4.2 \\
\hline Southern Piedmont Large Floodplain Forest & Quercus michauxii & Tree- Deciduous & 3 & 4.2 \\
\hline $\begin{array}{l}\text { West Gulf Coastal Plain Nonriverine Wet } \\
\text { Hardwood Flatwoods }\end{array}$ & Quercus michauxii & Tree-Deciduous & 3 & 4.2 \\
\hline $\begin{array}{l}\text { West Gulf Coastal Plain Small Stream and River } \\
\text { Forest }\end{array}$ & Quercus michauxii & Tree- Deciduous & 3 & 4.2 \\
\hline $\begin{array}{l}\text { South-Central Interior / Upper Coastal Plain Wet } \\
\text { Flatwoods }\end{array}$ & Quercus michauxii & Tree- Deciduous & 3 & 4.2 \\
\hline Florida Dry Prairie & Quercus minima & Shrub- Evergreen & 2.7 & 2.4 \\
\hline Edwards Plateau Limestone Shrubland & Quercus mohriana & Shrub- Evergreen & 2.7 & 2.4 \\
\hline $\begin{array}{l}\text { Llano Estacado Caprock Escarpment and Breaks } \\
\text { Shrubland and Steppe }\end{array}$ & Quercus mohriana & Tree- Evergreen & 3.2 & 2.2 \\
\hline Madrean Pinyon-Juniper Woodland & Quercus mohriana & Tree-Evergreen & 3.2 & 2.2 \\
\hline Central Appalachian Alkaline Glade and Woodland & Quercus muehlenbergii & Tree-Deciduous & 3 & 4.2 \\
\hline $\begin{array}{l}\text { Central Interior Highlands Calcareous Glade and } \\
\text { Barrens }\end{array}$ & Quercus muehlenbergii & Tree-Deciduous & 3 & 4.2 \\
\hline Edwards Plateau Mesic Canyon & Quercus muehlenbergii & Tree-Deciduous & 3 & 4.2 \\
\hline Ozark-Ouachita Mesic Hardwood Forest & Quercus muehlenbergii & Tree- Deciduous & 3 & 4.2 \\
\hline Southern Coastal Plain Limestone Forest & Quercus muehlenbergii & Tree-Deciduous & 3 & 4.2 \\
\hline $\begin{array}{l}\text { Southern Interior Low Plateau Dry-Mesic Oak } \\
\text { Forest }\end{array}$ & Quercus muehlenbergii & Tree- Deciduous & 3 & 4.2 \\
\hline $\begin{array}{l}\text { Southern Ridge and Valley / Cumberland Dry } \\
\text { Calcareous Forest }\end{array}$ & Quercus muhlenbergii & Tree- Deciduous & 3 & 4.2 \\
\hline $\begin{array}{l}\text { East Gulf Coastal Plain Dune and Coastal } \\
\text { Grassland }\end{array}$ & Quercus myrtifolia & Shrub- Evergreen & 2.7 & 2.4 \\
\hline Florida Longleaf Pine Sandhill & Quercus myrtifolia & Tree- Evergreen & 3.2 & 2.2 \\
\hline Florida Peninsula Inland Scrub & Quercus myrtifolia & Tree-Evergreen & 3.2 & 2.2 \\
\hline Southern Atlantic Coastal Plain Xeric River Dune & Quercus myrtifolia & Tree- Evergreen & 3.2 & 2.2 \\
\hline $\begin{array}{l}\text { Atlantic Coastal Plain Small Blackwater River } \\
\text { Floodplain Forest }\end{array}$ & Quercus nigra & Tree-Deciduous & 3 & 4.2 \\
\hline $\begin{array}{l}\text { East Gulf Coastal Plain Southern Loblolly- } \\
\text { Hardwood Flatwoods }\end{array}$ & Quercus nigra & Tree-Deciduous & 3 & 4.2 \\
\hline Lower Mississippi River Flatwoods & Quercus nigra & Tree-Deciduous & 3 & 4.2 \\
\hline Mississippi Delta Maritime Forest & Quercus nigra & Tree- Deciduous & 3 & 4.2 \\
\hline Southeastern Great Plains Floodplain Forest & Quercus nigra & Tree-Deciduous & 3 & 4.2 \\
\hline Southeastern Great Plains Riparian Forest & Quercus nigra & Tree-Deciduous & 3 & 4.2 \\
\hline $\begin{array}{l}\text { Southern Atlantic Coastal Plain Dry and Dry-Mesic } \\
\text { Oak Forest }\end{array}$ & Quercus nigra & Tree-Deciduous & 3 & 4.2 \\
\hline Southern Coastal Plain Oak Dome and Hammock & Quercus nigra & Tree-Deciduous & 3 & 4.2 \\
\hline $\begin{array}{l}\text { West Gulf Coastal Plain Nonriverine Wet } \\
\text { Hardwood Flatwoods }\end{array}$ & Quercus nigra & Tree-Deciduous & 3 & 4.2 \\
\hline Madrean Encinal & Quercus oblongifolia & Tree- Evergreen & 3.2 & 2.2 \\
\hline
\end{tabular}




\begin{tabular}{|c|c|c|c|c|}
\hline Community & Species & Classification & Resistance & Resilience \\
\hline $\begin{array}{l}\text { Madrean Lower Montane Pine-Oak Forest and } \\
\text { Woodland }\end{array}$ & Quercus oblongifolia & Tree-Evergreen & 3.2 & 2.2 \\
\hline $\begin{array}{l}\text { Atlantic Coastal Plain Small Brownwater River } \\
\text { Floodplain Forest }\end{array}$ & Quercus pagoda & Tree-Deciduous & 3 & 4.2 \\
\hline $\begin{array}{l}\text { East Gulf Coastal Plain Northern Mesic Hardwood } \\
\text { Slope Forest }\end{array}$ & Quercus pagoda & Tree-Deciduous & 3 & 4.2 \\
\hline $\begin{array}{l}\text { East Gulf Coastal Plain Southern Loess Bluff } \\
\text { Forest }\end{array}$ & Quercus pagoda & Tree- Deciduous & 3 & 4.2 \\
\hline Lower Mississippi River Flatwoods & Quercus pagoda & Tree-Deciduous & 3 & 4.2 \\
\hline $\begin{array}{l}\text { Mississippi River Alluvial Plain Dry-Mesic Loess } \\
\text { Slope Forest }\end{array}$ & Quercus pagoda & Tree-Deciduous & 3 & 4.2 \\
\hline Southern Coastal Plain Limestone Forest & Quercus pagoda & Tree-Deciduous & 3 & 4.2 \\
\hline Southern Piedmont Large Floodplain Forest & Quercus pagoda & Tree-Deciduous & 3 & 4.2 \\
\hline Mississippi River Floodplain and Riparian Forest & Quercus pagoda & Tree-Deciduous & 3 & 4.2 \\
\hline Mississippi River Riparian Forest & Quercus pagoda & Tree-Deciduous & 3 & 4.2 \\
\hline $\begin{array}{l}\text { South-Central Interior / Upper Coastal Plain Wet } \\
\text { Flatwoods }\end{array}$ & Quercus pagoda & Tree-Deciduous & 3 & 4.2 \\
\hline North-Central Interior Wet Flatwoods & Quercus palustris & Tree-Deciduous & 3 & 4.2 \\
\hline $\begin{array}{l}\text { South-Central Interior / Upper Coastal Plain Wet } \\
\text { Flatwoods }\end{array}$ & Quercus palustris & Tree-Deciduous & 3 & 4.2 \\
\hline $\begin{array}{l}\text { Atlantic Coastal Plain Brownwater Stream } \\
\text { Floodplain Forest }\end{array}$ & Quercus phellos & Tree- Deciduous & 3 & 4.2 \\
\hline $\begin{array}{l}\text { East Gulf Coastal Plain Southern Loblolly- } \\
\text { Hardwood Flatwoods }\end{array}$ & Quercus phellos & Tree- Deciduous & 3 & 4.2 \\
\hline Piedmont Upland Depression Swamp & Quercus phellos & Tree- Deciduous & 3 & 4.2 \\
\hline Southeastern Great Plains Riparian Forest & Quercus phellos & Tree-Deciduous & 3 & 4.2 \\
\hline $\begin{array}{l}\text { West Gulf Coastal Plain Nonriverine Wet } \\
\text { Hardwood Flatwoods }\end{array}$ & Quercus phellos & Tree- Deciduous & 3 & 4.2 \\
\hline $\begin{array}{l}\text { West Gulf Coastal Plain Small Stream and River } \\
\text { Forest }\end{array}$ & Quercus phellos & Tree- Deciduous & 3 & 4.2 \\
\hline $\begin{array}{l}\text { Allegheny-Cumberland Dry Oak Forest and } \\
\text { Woodland }\end{array}$ & Quercus prinus & Tree- Deciduous & 3 & 4.2 \\
\hline Appalachian Shale Barrens & Quercus prinus & Tree- Deciduous & 3 & 4.2 \\
\hline $\begin{array}{l}\text { Central and Southern Appalachian Montane Oak } \\
\text { Forest }\end{array}$ & Quercus prinus & Tree-Deciduous & 3 & 4.2 \\
\hline Central Appalachian Dry Oak-Pine Forest & Quercus prinus & Tree-Deciduous & 3 & 4.2 \\
\hline Central Appalachian Pine-Oak Rocky Woodland & Quercus prinus & Tree-Deciduous & 3 & 4.2 \\
\hline $\begin{array}{l}\text { Central Interior Highlands Dry Acidic Glade and } \\
\text { Barrens }\end{array}$ & Quercus prinus & Tree-Deciduous & 3 & 4.2 \\
\hline Northern Atlantic Coastal Plain Hardwood Forest & Quercus prinus & Tree- Deciduous & 3 & 4.2 \\
\hline Southeastern Interior Longleaf Pine Woodland & Quercus prinus & Tree- Deciduous & 3 & 4.2 \\
\hline Southern Appalachian Oak Forest & Quercus prinus & Tree-Deciduous & 3 & 4.2 \\
\hline Southern Piedmont Dry Oak-(Pine) Forest & Quercus prinus & Tree-Deciduous & 3 & 4.2 \\
\hline $\begin{array}{l}\text { Central and Southern Appalachian Montane Oak } \\
\text { Forest }\end{array}$ & Quercus rubra & Tree-Deciduous & 3 & 4.2 \\
\hline Crowley's Ridge Sand Forest & Quercus rubra & Tree- Deciduous & 3 & 4.2 \\
\hline Great Lakes Wooded Dune and Swale & Quercus rubra & Tree- Deciduous & 3 & 4.2 \\
\hline $\begin{array}{l}\text { Laurentian-Acadian Pine-Hemlock-Hardwood } \\
\text { Forest }\end{array}$ & Quercus rubra & Tree-Deciduous & 3 & 4.2 \\
\hline $\begin{array}{l}\text { Mississippi River Alluvial Plain Dry-Mesic Loess } \\
\text { Slope Forest }\end{array}$ & Quercus rubra & Tree-Deciduous & 3 & 4.2 \\
\hline North-Central Interior Beech-Maple Forest & Quercus rubra & Tree-Deciduous & 3 & 4.2 \\
\hline
\end{tabular}




\begin{tabular}{|c|c|c|c|c|}
\hline Community & Species & Classification & Resistance & Resilience \\
\hline $\begin{array}{l}\text { North-Central Interior Dry-Mesic Oak Forest and } \\
\text { Woodland }\end{array}$ & Quercus rubra & Tree-Deciduous & 3 & 4.2 \\
\hline North-Central Interior Maple-Basswood Forest & Quercus rubra & Tree-Deciduous & 3 & 4.2 \\
\hline Northeastern Interior Dry-Mesic Oak Forest & Quercus rubra & Tree-Deciduous & 3 & 4.2 \\
\hline Ozark-Ouachita Dry-Mesic Oak Forest & Quercus rubra & Tree- Deciduous & 3 & 4.2 \\
\hline Ozark-Ouachita Mesic Hardwood Forest & Quercus rubra & Tree-Deciduous & 3 & 4.2 \\
\hline South-Central Interior Mesophytic Forest & Quercus rubra & Tree- Deciduous & 3 & 4.2 \\
\hline Southern Piedmont Mesic Forest & Quercus rubra & Tree-Deciduous & 3 & 4.2 \\
\hline Appalachian (Hemlock)-Northern Hardwood Forest & Quercus rubra & Tree-Deciduous & 3 & 4.2 \\
\hline Laurentian-Acadian Northern Pine-(Oak) Forest & Quercus rubra & Tree- Deciduous & 3 & 4.2 \\
\hline Southern Appalachian Oak Forest & Quercus rubra & Tree-Deciduous & 3 & 4.2 \\
\hline Southern Piedmont Dry Oak-(Pine) Forest & Quercus rubra & Tree-Deciduous & 3 & 4.2 \\
\hline $\begin{array}{l}\text { Southern Ridge and Valley / Cumberland Dry } \\
\text { Calcareous Forest }\end{array}$ & Quercus rubra & Tree-Deciduous & 3 & 4.2 \\
\hline $\begin{array}{l}\text { Madrean Lower Montane Pine-Oak Forest and } \\
\text { Woodland }\end{array}$ & Quercus rugosa & Tree- Evergreen & 3.2 & 2.2 \\
\hline $\begin{array}{l}\text { Madrean Upper Montane Conifer-Oak Forest and } \\
\text { Woodland }\end{array}$ & Quercus rugosa & Tree- Evergreen & 3.2 & 2.2 \\
\hline $\begin{array}{l}\text { Klamath-Siskiyou Xeromorphic Serpentine } \\
\text { Savanna and Chaparral }\end{array}$ & Quercus sadleriana & Shrub- Evergreen & 2.7 & 2.4 \\
\hline $\begin{array}{l}\text { Mississippi River Alluvial Plain Dry-Mesic Loess } \\
\text { Slope Forest }\end{array}$ & Quercus shumardii & Tree- Deciduous & 3 & 4.2 \\
\hline Southern Coastal Plain Limestone Forest & Quercus shumardii & Tree- Deciduous & 3 & 4.2 \\
\hline Red River Large Floodplain Forest & Quercus similis & Tree- Deciduous & 3 & 4.2 \\
\hline Edwards Plateau Limestone Shrubland & Quercus sinuata & Tree- Deciduous & 3 & 4.2 \\
\hline Arkansas Valley Prairie and Woodland & Quercus stellata & Tree- Deciduous & 3 & 4.2 \\
\hline Bastrop Lost Pines Forest and Woodland & Quercus stellata & Tree- Deciduous & 3 & 4.2 \\
\hline $\begin{array}{l}\text { Central Interior Highlands Dry Acidic Glade and } \\
\text { Barrens }\end{array}$ & Quercus stellata & Tree- Deciduous & 3 & 4.2 \\
\hline Crosstimbers Oak Forest and Woodland & Quercus stellata & Tree- Deciduous & 3 & 4.2 \\
\hline Crowley's Ridge Sand Forest & Quercus stellata & Tree- Deciduous & 3 & 4.2 \\
\hline $\begin{array}{l}\text { East Gulf Coastal Plain Interior Shortleaf Pine-Oak } \\
\text { Forest }\end{array}$ & Quercus stellata & Tree- Deciduous & 3 & 4.2 \\
\hline $\begin{array}{l}\text { East Gulf Coastal Plain Northern Dry Upland } \\
\text { Hardwood Forest }\end{array}$ & Quercus stellata & Tree- Deciduous & 3 & 4.2 \\
\hline $\begin{array}{l}\text { East Gulf Coastal Plain Northern Loess Plain Oak- } \\
\text { Hickory Upland }\end{array}$ & Quercus stellata & Tree-Deciduous & 3 & 4.2 \\
\hline $\begin{array}{l}\text { East-Central Texas Plains Post Oak Savanna and } \\
\text { Woodland }\end{array}$ & Quercus stellata & Tree-Deciduous & 3 & 4.2 \\
\hline Eastern Serpentine Woodland & Quercus stellata & Tree- Deciduous & 3 & 4.2 \\
\hline $\begin{array}{l}\text { Edwards Plateau Limestone Savanna and } \\
\text { Woodland }\end{array}$ & Quercus stellata & Tree- Deciduous & 3 & 4.2 \\
\hline $\begin{array}{l}\text { Lower Mississippi River Dune Woodland and } \\
\text { Forest }\end{array}$ & Quercus stellata & Tree- Deciduous & 3 & 4.2 \\
\hline $\begin{array}{l}\text { Mississippi River Alluvial Plain Dry-Mesic Loess } \\
\text { Slope Forest }\end{array}$ & Quercus stellata & Tree-Deciduous & 3 & 4.2 \\
\hline Ouachita Montane Oak Forest & Quercus stellata & Tree- Deciduous & 3 & 4.2 \\
\hline Ozark-Ouachita Dry Oak Woodland & Quercus stellata & Tree- Deciduous & 3 & 4.2 \\
\hline Ozark-Ouachita Dry-Mesic Oak Forest & Quercus stellata & Tree- Deciduous & 3 & 4.2 \\
\hline $\begin{array}{l}\text { Ozark-Ouachita Shortleaf Pine-Bluestem } \\
\text { Woodland }\end{array}$ & Quercus stellata & Tree- Deciduous & 3 & 4.2 \\
\hline
\end{tabular}




\begin{tabular}{|c|c|c|c|c|}
\hline Community & Species & Classification & Resistance & Resilience \\
\hline $\begin{array}{l}\text { Ozark-Ouachita Shortleaf Pine-Oak Forest and } \\
\text { Woodland }\end{array}$ & Quercus stellata & Tree- Deciduous & 3 & 4.2 \\
\hline South-Central Saline Glade & Quercus stellata & Tree- Deciduous & 3 & 4.2 \\
\hline Southeastern Interior Longleaf Pine Woodland & Quercus stellata & Tree- Deciduous & 3 & 4.2 \\
\hline $\begin{array}{l}\text { Southern Atlantic Coastal Plain Dry and Dry-Mesic } \\
\text { Oak Forest }\end{array}$ & Quercus stellata & Tree- Deciduous & 3 & 4.2 \\
\hline $\begin{array}{l}\text { Southern Coastal Plain Dry Upland Hardwood } \\
\text { Forest }\end{array}$ & Quercus stellata & Tree- Deciduous & 3 & 4.2 \\
\hline Southern Coastal Plain Limestone Forest & Quercus stellata & Tree- Deciduous & 3 & 4.2 \\
\hline West Gulf Coastal Plain Catahoula Barrens & Quercus stellata & Tree-Deciduous & 3 & 4.2 \\
\hline West Gulf Coastal Plain Nepheline Syenite Glade & Quercus stellata & Tree- Deciduous & 3 & 4.2 \\
\hline West Gulf Coastal Plain Pine-Hardwood Flatwoods & Quercus stellata & Tree- Deciduous & 3 & 4.2 \\
\hline West Gulf Coastal Plain Pine-Hardwood Forest & Quercus stellata & Tree- Deciduous & 3 & 4.2 \\
\hline Llano Uplift Acidic Forest, Woodland and Glade & Quercus stellata & Tree-Deciduous & 3 & 4.2 \\
\hline $\begin{array}{l}\text { South-Central Interior / Upper Coastal Plain } \\
\text { Flatwoods }\end{array}$ & Quercus stellata & Tree-Deciduous & 3 & 4.2 \\
\hline $\begin{array}{l}\text { South-Central Interior / Upper Coastal Plain Wet } \\
\text { Flatwoods }\end{array}$ & Quercus stellata & Tree-Deciduous & 3 & 4.2 \\
\hline Southern Piedmont Dry Oak-(Pine) Forest & Quercus stellata & Tree- Deciduous & 3 & 4.2 \\
\hline Mogollon Chaparral & Quercus toumeyi & Shrub- Evergreen & 2.7 & 2.4 \\
\hline Madrean Encinal & Quercus toumeyi & Tree- Evergreen & 3.2 & 2.2 \\
\hline $\begin{array}{l}\text { Madrean Lower Montane Pine-Oak Forest and } \\
\text { Woodland }\end{array}$ & Quercus toumeyi & Tree- Evergreen & 3.2 & 2.2 \\
\hline $\begin{array}{l}\text { Madrean Upper Montane Conifer-Oak Forest and } \\
\text { Woodland }\end{array}$ & Quercus toumeyi & Tree-Evergreen & 3.2 & 2.2 \\
\hline Mogollon Chaparral & Quercus turbinella & Shrub- Evergreen & 2.7 & 2.4 \\
\hline Great Basin Pinyon-Juniper Woodland & Quercus turbinella & Tree- Evergreen & 3.2 & 2.2 \\
\hline $\begin{array}{l}\text { Klamath-Siskiyou Xeromorphic Serpentine } \\
\text { Savanna and Chaparral }\end{array}$ & Quercus vacciniifolia & Shrub- Evergreen & 2.7 & 2.4 \\
\hline $\begin{array}{l}\text { Edwards Plateau Limestone Savanna and } \\
\text { Woodland }\end{array}$ & Quercus vaseyana & Tree- Evergreen & 3.2 & 2.2 \\
\hline Edwards Plateau Limestone Shrubland & Quercus vaseyana & Tree- Evergreen & 3.2 & 2.2 \\
\hline Crowley's Ridge Sand Forest & Quercus velutina & Tree-Deciduous & 3 & 4.2 \\
\hline $\begin{array}{l}\text { East Gulf Coastal Plain Northern Loess Plain Oak- } \\
\text { Hickory Upland }\end{array}$ & Quercus velutina & Tree- Deciduous & 3 & 4.2 \\
\hline $\begin{array}{l}\text { Mississippi River Alluvial Plain Dry-Mesic Loess } \\
\text { Slope Forest }\end{array}$ & Quercus velutina & Tree- Deciduous & 3 & 4.2 \\
\hline $\begin{array}{l}\text { North-Central Interior Dry Oak Forest and } \\
\text { Woodland }\end{array}$ & Quercus velutina & Tree- Deciduous & 3 & 4.2 \\
\hline North-Central Oak Barrens & Quercus velutina & Tree- Deciduous & 3 & 4.2 \\
\hline Northeastern Interior Dry-Mesic Oak Forest & Quercus velutina & Tree- Deciduous & 3 & 4.2 \\
\hline Ozark-Ouachita Dry Oak Woodland & Quercus velutina & Tree-Deciduous & 3 & 4.2 \\
\hline Ozark-Ouachita Dry-Mesic Oak Forest & Quercus velutina & Tree- Deciduous & 3 & 4.2 \\
\hline $\begin{array}{l}\text { Ozark-Ouachita Shortleaf Pine-Oak Forest and } \\
\text { Woodland }\end{array}$ & Quercus velutina & Tree- Deciduous & 3 & 4.2 \\
\hline Southeastern Interior Longleaf Pine Woodland & Quercus velutina & Tree- Deciduous & 3 & 4.2 \\
\hline Southern Appalachian Oak Forest & Quercus velutina & Tree- Deciduous & 3 & 4.2 \\
\hline Southern Piedmont Dry Oak-(Pine) Forest & Quercus velutina & Tree- Deciduous & 3 & 4.2 \\
\hline Central Atlantic Coastal Plain Maritime Forest & Quercus virginiana & Tree- Evergreen & 3.2 & 2.2 \\
\hline East Gulf Coastal Plain Maritime Forest & Quercus virginiana & Tree- Evergreen & 3.2 & 2.2 \\
\hline Mississippi Delta Maritime Forest & Quercus virginiana & Tree- Evergreen & 3.2 & 2.2 \\
\hline
\end{tabular}




\begin{tabular}{|c|c|c|c|c|}
\hline Community & Species & Classification & Resistance & Resilience \\
\hline $\begin{array}{l}\text { Southeast Florida Coastal Strand and Maritime } \\
\text { Hammock }\end{array}$ & Quercus virginiana & Tree- Evergreen & 3.2 & 2.2 \\
\hline Southeastern Great Plains Floodplain Forest & Quercus virginiana & Tree- Evergreen & 3.2 & 2.2 \\
\hline Southern Atlantic Coastal Plain Maritime Forest & Quercus virginiana & Tree- Evergreen & 3.2 & 2.2 \\
\hline Southern Coastal Plain Hydric Hammock & Quercus virginiana & Tree- Evergreen & 3.2 & 2.2 \\
\hline Southern Coastal Plain Oak Dome and Hammock & Quercus virginiana & Tree- Evergreen & 3.2 & 2.2 \\
\hline $\begin{array}{l}\text { Southwest Florida Coastal Strand and Maritime } \\
\text { Hammock }\end{array}$ & Quercus virginiana & Tree- Evergreen & 3.2 & 2.2 \\
\hline $\begin{array}{l}\text { West Gulf Coastal Plain Chenier and Upper Texas } \\
\text { Coastal Fringe Forest and Woodland }\end{array}$ & Quercus virginiana & Tree-Evergreen & 3.2 & 2.2 \\
\hline California Central Valley Mixed Oak Savanna & Quercus wislizeni & Tree- Evergreen & 3.2 & 2.2 \\
\hline $\begin{array}{l}\text { California Lower Montane Blue Oak-Foothill Pine } \\
\text { Woodland and Savanna }\end{array}$ & Quercus wislizeni & Tree-Evergreen & 3.2 & 2.2 \\
\hline Mediterranean California Mixed Oak Woodland & Quercus wislizeni & Tree- Evergreen & 3.2 & 2.2 \\
\hline Southern California Oak Woodland and Savanna & Quercus wislizeni & Tree- Evergreen & 3.2 & 2.2 \\
\hline $\begin{array}{l}\text { Mediterranean California Lower Montane Black } \\
\text { Oak-Conifer Forest and Woodland }\end{array}$ & Quercus wislizeni & Tree-Evergreen & 3.2 & 2.2 \\
\hline California Mesic Chaparral & Quercus wislizeni & Tree-Evergreen & 3.2 & 2.2 \\
\hline $\begin{array}{l}\text { Northern Rocky Mountain Subalpine Deciduous } \\
\text { Shrubland }\end{array}$ & Rhamnus alnifolia & Shrub-Deciduous & 2.7 & 3 \\
\hline California Mesic Chaparral & Rhamnus crocea & Shrub- Evergreen & 2.7 & 2.4 \\
\hline California Mesic Chaparral & Rhamnus crocea & Shrub- Evergreen & 2.7 & 2.4 \\
\hline South Florida Mangrove Swamp & Rhizophora mangle & Shrub- Evergreen & 2.7 & 2.4 \\
\hline $\begin{array}{l}\text { Northern Rocky Mountain Subalpine Deciduous } \\
\text { Shrubland }\end{array}$ & Rhododendron albiflorum & Shrub-Deciduous & 2.7 & 3 \\
\hline Southern Appalachian Grass and Shrub Bald & Rhododendron carolinianum & Shrub- Evergreen & 2.7 & 2.4 \\
\hline Southern Appalachian Grass and Shrub Bald & Rhododendron catawbiense & Shrub- Evergreen & 2.7 & 2.4 \\
\hline $\begin{array}{l}\text { North Pacific Maritime Dry-Mesic Douglas-fir- } \\
\text { Western Hemlock Forest }\end{array}$ & Rhododendron macrophyllum & Shrub- Evergreen & 2.7 & 2.4 \\
\hline $\begin{array}{l}\text { Northern Rocky Mountain Montane-Foothill } \\
\text { Deciduous Shrubland }\end{array}$ & Rhus glabra & Shrub-Deciduous & 2.7 & 3 \\
\hline Madrean Oriental Chaparral & Rhus microphylla & Shrub- Deciduous & 2.7 & 3 \\
\hline North American Warm Desert Wash & Rhus microphylla & Shrub-Deciduous & 2.7 & 3 \\
\hline Mogollon Chaparral & Rhus ovata & Shrub- Evergreen & 2.7 & 2.4 \\
\hline $\begin{array}{l}\text { Llano Estacado Caprock Escarpment and Breaks } \\
\text { Shrubland and Steppe }\end{array}$ & Rhus trilobata & Shrub-Deciduous & 2.7 & 3 \\
\hline $\begin{array}{l}\text { Rocky Mountain Lower Montane-Foothill Riparian } \\
\text { Woodland and Shrubland }\end{array}$ & Rhus trilobata & Shrub- Deciduous & 2.7 & 3 \\
\hline $\begin{array}{l}\text { Rocky Mountain Lower Montane-Foothill } \\
\text { Shrubland }\end{array}$ & Rhus trilobata & Shrub- Deciduous & 2.7 & 3 \\
\hline Western Great Plains Cliff and Outcrop & Rhus trilobata & Shrub- Deciduous & 2.7 & 3 \\
\hline Edwards Plateau Limestone Shrubland & Rhus virens & Shrub- Evergreen & 2.7 & 2.4 \\
\hline Madrean Oriental Chaparral & Rhus virens & Shrub- Evergreen & 2.7 & 2.4 \\
\hline East Gulf Coastal Plain Depression Pond & Rhynchospora chapmanii & Graminoid $\mathrm{C}_{3}$ & 3.6 & 3.6 \\
\hline $\begin{array}{l}\text { Southern Coastal Plain Blackland Prairie and } \\
\text { Woodland }\end{array}$ & Rhynchospora colorata & Graminoid $C_{3}$ & 3.6 & 3.6 \\
\hline East Gulf Coastal Plain Depression Pond & Rhynchospora corniculata & Graminoid $C_{3}$ & 3.6 & 3.6 \\
\hline East Gulf Coastal Plain Savanna and Wet Prairie & Rhynchospora corniculata & Graminoid $C_{3}$ & 3.6 & 3.6 \\
\hline West Gulf Coastal Plain Flatwoods Pond & Rhynchospora corniculata & Graminoid $C_{3}$ & 3.6 & 3.6 \\
\hline East Gulf Coastal Plain Depression Pond & Rhynchospora filifolia & Graminoid $C_{3}$ & 3.6 & 3.6 \\
\hline East Gulf Coastal Plain Depression Pond & Rhynchospora harperi & Graminoid $C_{3}$ & 3.6 & 3.6 \\
\hline
\end{tabular}




\begin{tabular}{|c|c|c|c|c|}
\hline Community & Species & Classification & Resistance & Resilience \\
\hline $\begin{array}{l}\text { Atlantic Coastal Plain Clay-Based Carolina Bay } \\
\text { Wetland }\end{array}$ & Rhynchospora inundata & Graminoid $C_{3}$ & 3.6 & 3.6 \\
\hline East Gulf Coastal Plain Depression Pond & Rhynchospora inundata & Graminoid $C_{3}$ & 3.6 & 3.6 \\
\hline Southern Atlantic Coastal Plain Depression Pond & Rhynchospora inundata & Graminoid $C_{3}$ & 3.6 & 3.6 \\
\hline Southern Coastal Plain Herbaceous Seep and Bog & Rhynchospora macra & Graminoid $C_{3}$ & 3.6 & 3.6 \\
\hline South Florida Dwarf Cypress Savanna & Rhynchospora microcarpa & Graminoid $C_{3}$ & 3.6 & 3.6 \\
\hline South Florida Wet Marl Prairie & Rhynchospora microcarpa & Graminoid $\mathrm{C}_{3}$ & 3.6 & 3.6 \\
\hline Southern Coastal Plain Herbaceous Seep and Bog & Rhynchospora stenophylla & Graminoid $\mathrm{C}_{3}$ & 3.6 & 3.6 \\
\hline East Gulf Coastal Plain Depression Pond & Rhynchospora tracyi & Graminoid $C_{3}$ & 3.6 & 3.6 \\
\hline $\begin{array}{l}\text { North Pacific Montane Riparian Woodland and } \\
\text { Shrubland }\end{array}$ & Ribes bracteosum & Shrub-Deciduous & 2.7 & 3 \\
\hline $\begin{array}{l}\text { Rocky Mountain Lower Montane-Foothill } \\
\text { Shrubland }\end{array}$ & Ribes cereum & Shrub- Deciduous & 2.7 & 3 \\
\hline $\begin{array}{l}\text { Southern Rocky Mountain Ponderosa Pine } \\
\text { Woodland }\end{array}$ & Ribes cereum & Shrub- Deciduous & 2.7 & 3 \\
\hline $\begin{array}{l}\text { Northern Rocky Mountain Subalpine Deciduous } \\
\text { Shrubland }\end{array}$ & Ribes lacustre & Shrub- Deciduous & 2.7 & 3 \\
\hline $\begin{array}{l}\text { Rocky Mountain Gambel Oak-Mixed Montane } \\
\text { Shrubland }\end{array}$ & Robinia neomexicana & Tree-Deciduous & 3 & 4.2 \\
\hline Rocky Mountain Alpine-Montane Wet Meadow & Rorippa alpina & Forb & 3 & 3.5 \\
\hline Southern Appalachian Grass and Shrub Bald & Rubus allegheniensis & Shrub-Deciduous & 2.7 & 3 \\
\hline $\begin{array}{l}\text { Central and Southern Appalachian Spruce-Fir } \\
\text { Forest }\end{array}$ & Rubus canadensis & Shrub- Deciduous & 2.7 & 3 \\
\hline $\begin{array}{l}\text { California Central Valley Riparian Woodland and } \\
\text { Shrubland }\end{array}$ & Rubus discolor & Shrub & 2.7 & 2.8 \\
\hline North Pacific Avalanche Chute Shrubland & Rubus parviflorus & Shrub- Deciduous & 2.7 & 3 \\
\hline North Pacific Montane Shrubland & Rubus parviflorus & Shrub- Deciduous & 2.7 & 3 \\
\hline $\begin{array}{l}\text { Northern Rocky Mountain Subalpine Deciduous } \\
\text { Shrubland }\end{array}$ & Rubus parviflorus & Shrub- Deciduous & 2.7 & 3 \\
\hline $\begin{array}{l}\text { North Pacific Hypermaritime Shrub and } \\
\text { Herbaceous Headland }\end{array}$ & Rubus spectabilis & Shrub- Deciduous & 2.7 & 3 \\
\hline $\begin{array}{l}\text { North Pacific Lowland Riparian Forest and } \\
\text { Shrubland }\end{array}$ & Rubus spectabilis & Shrub- Deciduous & 2.7 & 3 \\
\hline $\begin{array}{l}\text { North Pacific Montane Riparian Woodland and } \\
\text { Shrubland }\end{array}$ & Rubus spectabilis & Shrub- Deciduous & 2.7 & 3 \\
\hline $\begin{array}{l}\text { California Central Valley Riparian Woodland and } \\
\text { Shrubland }\end{array}$ & Rubus ursinus & Shrub & 2.7 & 2.8 \\
\hline $\begin{array}{l}\text { California Coastal Live Oak Woodland and } \\
\text { Savanna }\end{array}$ & Rubus ursinus & Shrub- Deciduous & 2.7 & 3 \\
\hline $\begin{array}{l}\text { East Gulf Coastal Plain Southern Loblolly- } \\
\text { Hardwood Flatwoods }\end{array}$ & Sabal minor & Shrub-Deciduous & 2.7 & 3 \\
\hline Central Atlantic Coastal Plain Maritime Forest & Sabal palmetto & Shrub- Deciduous & 2.7 & 3 \\
\hline East Gulf Coastal Plain Maritime Forest & Sabal palmetto & Shrub- Deciduous & 2.7 & 3 \\
\hline South Florida Pine Rockland & Sabal palmetto & Tree- Deciduous & 3 & 4.2 \\
\hline Southern Coastal Plain Hydric Hammock & Sabal palmetto & Tree- Deciduous & 3 & 4.2 \\
\hline $\begin{array}{l}\text { Southwest Florida Coastal Strand and Maritime } \\
\text { Hammock }\end{array}$ & Sabal palmetto & Tree- Deciduous & 3 & 4.2 \\
\hline East Gulf Coastal Plain Depression Pond & Sabatia angularis & Forb & 3 & 3.5 \\
\hline $\begin{array}{l}\text { Florida Big Bend Fresh and Oligohaline Tidal } \\
\text { Marsh }\end{array}$ & Sagittaria lancifolia & Forb & 3 & 3.5 \\
\hline Texas-Louisiana Coastal Prairie Pondshore & Sagittaria longiloba & Forb & 3 & 3.5 \\
\hline Mojave Mid-Elevation Mixed Desert Scrub & Salazaria mexicana & Shrub- Deciduous & 2.7 & 3 \\
\hline
\end{tabular}




\begin{tabular}{|c|c|c|c|c|}
\hline Community & Species & Classification & Resistance & Resilience \\
\hline North American Warm Desert Wash & Salazaria mexicana & Shrub- Deciduous & 2.7 & 3 \\
\hline Temperate Pacific Tidal Salt and Brackish Marsh & Salicornia virginica & Forb & 3 & 3.5 \\
\hline Western Great Plains Floodplain & Salix amygdaloides & Shrub-Deciduous & 2.7 & 3 \\
\hline $\begin{array}{l}\text { Rocky Mountain Lower Montane-Foothill Riparian } \\
\text { Woodland and Shrubland }\end{array}$ & Salix amygdaloides & Tree- Deciduous & 3 & 4.2 \\
\hline Rocky Mountain Alpine Dwarf-Shrubland & Salix arctica & Shrub- Deciduous & 2.7 & 3 \\
\hline $\begin{array}{l}\text { Rocky Mountain Subalpine-Montane Riparian } \\
\text { Shrubland }\end{array}$ & Salix bebbiana & Shrub-Deciduous & 2.7 & 3 \\
\hline $\begin{array}{l}\text { Rocky Mountain Subalpine-Montane Riparian } \\
\text { Shrubland }\end{array}$ & Salix boothii & Shrub- Deciduous & 2.7 & 3 \\
\hline $\begin{array}{l}\text { Rocky Mountain Subalpine-Montane Riparian } \\
\text { Shrubland }\end{array}$ & Salix brachycarpa & Shrub- Deciduous & 2.7 & 3 \\
\hline $\begin{array}{l}\text { Atlantic Coastal Plain Small Blackwater River } \\
\text { Floodplain Forest }\end{array}$ & Salix caroliniana & Shrub- Deciduous & 2.7 & 3 \\
\hline Central Atlantic Coastal Plain Maritime Forest & Salix caroliniana & Shrub- Deciduous & 2.7 & 3 \\
\hline South Florida Everglades Sawgrass Marsh & Salix caroliniana & Shrub- Deciduous & 2.7 & 3 \\
\hline South Florida Slough, Gator Hole and Willow Head & Salix caroliniana & Shrub- Deciduous & 2.7 & 3 \\
\hline Great Lakes Dune & Salix cordata & Shrub- Deciduous & 2.7 & 3 \\
\hline $\begin{array}{l}\text { Rocky Mountain Lower Montane-Foothill Riparian } \\
\text { Woodland and Shrubland }\end{array}$ & Salix drummondiana & Shrub-Deciduous & 2.7 & 3 \\
\hline $\begin{array}{l}\text { Rocky Mountain Subalpine-Montane Riparian } \\
\text { Shrubland }\end{array}$ & Salix drummondiana & Shrub- Deciduous & 2.7 & 3 \\
\hline $\begin{array}{l}\text { Rocky Mountain Subalpine-Montane Riparian } \\
\text { Shrubland }\end{array}$ & Salix eriocephala & Shrub-Deciduous & 2.7 & 3 \\
\hline $\begin{array}{l}\text { California Central Valley Riparian Woodland and } \\
\text { Shrubland }\end{array}$ & Salix exigua & Shrub- Deciduous & 2.7 & 3 \\
\hline $\begin{array}{l}\text { Great Basin Foothill and Lower Montane Riparian } \\
\text { Woodland and Shrubland }\end{array}$ & Salix exigua & Shrub- Deciduous & 2.7 & 3 \\
\hline $\begin{array}{l}\text { North American Warm Desert Lower Montane } \\
\text { Riparian Woodland and Shrubland }\end{array}$ & Salix exigua & Shrub- Deciduous & 2.7 & 3 \\
\hline $\begin{array}{l}\text { North American Warm Desert Riparian Mesquite } \\
\text { Bosque }\end{array}$ & Salix exigua & Shrub- Deciduous & 2.7 & 3 \\
\hline $\begin{array}{l}\text { North American Warm Desert Riparian Woodland } \\
\text { and Shrubland }\end{array}$ & Salix exigua & Shrub-Deciduous & 2.7 & 3 \\
\hline Northwestern Great Plains Floodplain & Salix exigua & Shrub- Deciduous & 2.7 & 3 \\
\hline $\begin{array}{l}\text { Rocky Mountain Lower Montane-Foothill Riparian } \\
\text { Woodland and Shrubland }\end{array}$ & Salix exigua & Shrub-Deciduous & 2.7 & 3 \\
\hline Western Great Plains Floodplain & Salix exigua & Shrub-Deciduous & 2.7 & 3 \\
\hline $\begin{array}{l}\text { Mediterranean California Foothill and Lower } \\
\text { Montane Riparian Woodland and Shrubland }\end{array}$ & Salix exigua & Shrub- Deciduous & 2.7 & 3 \\
\hline $\begin{array}{l}\text { North American Warm Desert Riparian Woodland } \\
\text { and Shrubland }\end{array}$ & Salix geyeriana & Shrub- Deciduous & 2.7 & 3 \\
\hline $\begin{array}{l}\text { Rocky Mountain Subalpine-Montane Riparian } \\
\text { Shrubland }\end{array}$ & Salix geyeriana & Shrub- Deciduous & 2.7 & 3 \\
\hline $\begin{array}{l}\text { Great Basin Foothill and Lower Montane Riparian } \\
\text { Woodland and Shrubland }\end{array}$ & Salix gooddingii & Shrub- Deciduous & 2.7 & 3 \\
\hline $\begin{array}{l}\text { North American Warm Desert Lower Montane } \\
\text { Riparian Woodland and Shrubland }\end{array}$ & Salix gooddingii & Shrub- Deciduous & 2.7 & 3 \\
\hline $\begin{array}{l}\text { North American Warm Desert Riparian Woodland } \\
\text { and Shrubland }\end{array}$ & Salix gooddingii & Tree- Deciduous & 3 & 4.2 \\
\hline $\begin{array}{l}\text { Mediterranean California Foothill and Lower } \\
\text { Montane Riparian Woodland and Shrubland }\end{array}$ & Salix gooddingii & Tree-Deciduous & 3 & 4.2 \\
\hline $\begin{array}{l}\text { Rocky Mountain Lower Montane-Foothill Riparian } \\
\text { Woodland and Shrubland }\end{array}$ & Salix irrorata & Shrub- Deciduous & 2.7 & 3 \\
\hline
\end{tabular}




\begin{tabular}{|c|c|c|c|c|}
\hline Community & Species & Classification & Resistance & Resilience \\
\hline $\begin{array}{l}\text { Great Basin Foothill and Lower Montane Riparian } \\
\text { Woodland and Shrubland }\end{array}$ & Salix laevigata & Shrub- Deciduous & 2.7 & 3 \\
\hline $\begin{array}{l}\text { Mediterranean California Foothill and Lower } \\
\text { Montane Riparian Woodland and Shrubland }\end{array}$ & Salix laevigata & Shrub- Deciduous & 2.7 & 3 \\
\hline $\begin{array}{l}\text { Great Basin Foothill and Lower Montane Riparian } \\
\text { Woodland and Shrubland }\end{array}$ & Salix lasiolepis & Shrub- Deciduous & 2.7 & 3 \\
\hline $\begin{array}{l}\text { Mediterranean California Foothill and Lower } \\
\text { Montane Riparian Woodland and Shrubland }\end{array}$ & Salix lasiolepis & Shrub- Deciduous & 2.7 & 3 \\
\hline $\begin{array}{l}\text { North American Warm Desert Riparian Woodland } \\
\text { and Shrubland }\end{array}$ & Salix lasiolepis & Tree- Deciduous & 3 & 4.2 \\
\hline $\begin{array}{l}\text { Great Basin Foothill and Lower Montane Riparian } \\
\text { Woodland and Shrubland }\end{array}$ & Salix lemmonii & Shrub- Deciduous & 2.7 & 3 \\
\hline $\begin{array}{l}\text { Rocky Mountain Lower Montane-Foothill Riparian } \\
\text { Woodland and Shrubland }\end{array}$ & Salix lucida & Shrub- Deciduous & 2.7 & 3 \\
\hline $\begin{array}{l}\text { North Pacific Lowland Riparian Forest and } \\
\text { Shrubland }\end{array}$ & Salix lucida & Shrub- Deciduous & 2.7 & 3 \\
\hline $\begin{array}{l}\text { Great Basin Foothill and Lower Montane Riparian } \\
\text { Woodland and Shrubland }\end{array}$ & Salix lutea & Shrub- Deciduous & 2.7 & 3 \\
\hline $\begin{array}{l}\text { Rocky Mountain Lower Montane-Foothill Riparian } \\
\text { Woodland and Shrubland }\end{array}$ & Salix monticola & Shrub- Deciduous & 2.7 & 3 \\
\hline $\begin{array}{l}\text { Rocky Mountain Subalpine-Montane Riparian } \\
\text { Shrubland }\end{array}$ & Salix monticola & Shrub- Deciduous & 2.7 & 3 \\
\hline Great Lakes Dune & Salix myricoides & Shrub- Deciduous & 2.7 & 3 \\
\hline $\begin{array}{l}\text { Atlantic Coastal Plain Small Blackwater River } \\
\text { Floodplain Forest }\end{array}$ & Salix nigra & Shrub- Deciduous & 2.7 & 3 \\
\hline South-Central Interior Large Floodplain & Salix nigra & Shrub- Deciduous & 2.7 & 3 \\
\hline Southeastern Great Plains Floodplain Forest & Salix nigra & Shrub- Deciduous & 2.7 & 3 \\
\hline Southeastern Great Plains Riparian Forest & Salix nigra & Shrub- Deciduous & 2.7 & 3 \\
\hline Southern Piedmont Large Floodplain Forest & Salix nigra & Shrub- Deciduous & 2.7 & 3 \\
\hline Rocky Mountain Alpine Dwarf-Shrubland & Salix nivalis & Shrub- Deciduous & 2.7 & 3 \\
\hline $\begin{array}{l}\text { Rocky Mountain Subalpine-Montane Riparian } \\
\text { Shrubland }\end{array}$ & Salix planifolia & Shrub- Deciduous & 2.7 & 3 \\
\hline Rocky Mountain Alpine Dwarf-Shrubland & Salix reticulata & Shrub- Deciduous & 2.7 & 3 \\
\hline $\begin{array}{l}\text { North Pacific Alpine and Subalpine Bedrock and } \\
\text { Scree }\end{array}$ & Salix rotundifolia & Shrub- Deciduous & 2.7 & 3 \\
\hline $\begin{array}{l}\text { North Pacific Lowland Riparian Forest and } \\
\text { Shrubland }\end{array}$ & Salix sitchensis & Shrub- Deciduous & 2.7 & 3 \\
\hline Rocky Mountain Alpine Dwarf-Shrubland & Salix vestita & Shrub- Deciduous & 2.7 & 3 \\
\hline $\begin{array}{l}\text { Rocky Mountain Subalpine-Montane Riparian } \\
\text { Shrubland }\end{array}$ & Salix wolfii & Shrub- Deciduous & 2.7 & 3 \\
\hline Northern Atlantic Coastal Plain Sandy Beach & Salsola kali & Forb & 3 & 3.5 \\
\hline Southern California Coastal Scrub & Salvia apiana & Shrub & 2.7 & 2.8 \\
\hline $\begin{array}{l}\text { Colorado Plateau Blackbrush-Mormon-tea } \\
\text { Shrubland }\end{array}$ & Salvia dorrii & Shrub- Deciduous & 2.7 & 3 \\
\hline Columbia Plateau Ash and Tuff Badland & Salvia dorrii & Shrub- Deciduous & 2.7 & 3 \\
\hline Southern California Coastal Scrub & Salvia leucophylla & Shrub- Deciduous & 2.7 & 3 \\
\hline Southern California Coastal Scrub & Salvia mellifera & Shrub- Deciduous & 2.7 & 3 \\
\hline $\begin{array}{l}\text { North American Warm Desert Lower Montane } \\
\text { Riparian Woodland and Shrubland }\end{array}$ & Sapindus saponaria & Shrub- Evergreen & 2.7 & 2.4 \\
\hline Southeastern Great Plains Riparian Forest & Sapindus saponaria & Shrub- Evergreen & 2.7 & 2.4 \\
\hline Southeastern Great Plains Floodplain Forest & Sapindus saponaria & Shrub- Evergreen & 2.7 & 2.4 \\
\hline $\begin{array}{l}\text { West Gulf Coastal Plain Southern Calcareous } \\
\text { Prairie }\end{array}$ & Sapindus saponaria & Shrub- Evergreen & 2.7 & 2.4 \\
\hline
\end{tabular}




\begin{tabular}{|c|c|c|c|c|}
\hline Community & Species & Classification & Resistance & Resilience \\
\hline Inter-Mountain Basins Active and Stabilized Dune & Sarcobatus vermiculatus & Shrub- Deciduous & 2.7 & 3 \\
\hline Inter-Mountain Basins Greasewood Flat & Sarcobatus vermiculatus & Shrub-Deciduous & 2.7 & 3 \\
\hline Inter-Mountain Basins Wash & Sarcobatus vermiculatus & Shrub- Deciduous & 2.7 & 3 \\
\hline North American Warm Desert Wash & Sarcobatus vermiculatus & Shrub- Deciduous & 2.7 & 3 \\
\hline Western Great Plains Badlands & Sarcobatus vermiculatus & Shrub-Deciduous & 2.7 & 3 \\
\hline Western Great Plains Saline Depression Wetland & Sarcobatus vermiculatus & Shrub- Deciduous & 2.7 & 3 \\
\hline $\begin{array}{l}\text { Western Great Plains Depressional Wetland } \\
\text { Systems }\end{array}$ & Sarcobatus vermiculatus & Shrub- Deciduous & 2.7 & 3 \\
\hline Texas Coast Salt and Brackish Tidal Marsh & Sarcocornia pacifica & Forb & 3 & 3.5 \\
\hline South Texas Salt and Brackish Tidal Flat & Sarcocornia perennis & Forb & 3 & 3.5 \\
\hline North American Warm Desert Playa & Sarcocornia utahensis & Forb & 3 & 3.5 \\
\hline $\begin{array}{l}\text { North Pacific Alpine and Subalpine Bedrock and } \\
\text { Scree }\end{array}$ & Saxifraga bronchialis & Forb & 3 & 3.5 \\
\hline Southern Appalachian Rocky Summit & Saxifraga michauxii & Forb & 3 & 3.5 \\
\hline $\begin{array}{l}\text { North Pacific Alpine and Subalpine Bedrock and } \\
\text { Scree }\end{array}$ & Saxifraga sibirica & Forb & 3 & 3.5 \\
\hline Southern Piedmont Cliff & Saxifraga virginiensis & Forb & 3 & 3.5 \\
\hline Madrean Encinal & Schizachyrium cirratum & Graminoid $\mathrm{C}_{4}$ & 3.6 & 4.1 \\
\hline Florida Panhandle Beach Vegetation & Schizachyrium maritimum & Graminoid $\mathrm{C}_{4}$ & 3.6 & 4.1 \\
\hline Arkansas Valley Prairie and Woodland & Schizachyrium scoparium & Graminoid $\mathrm{C}_{4}$ & 3.6 & 4.1 \\
\hline Central Appalachian Alkaline Glade and Woodland & Schizachyrium scoparium & Graminoid $C_{4}$ & 3.6 & 4.1 \\
\hline Central Interior Calcareous Cliff and Talus & Schizachyrium scoparium & Graminoid $\mathrm{C}_{4}$ & 3.6 & 4.1 \\
\hline $\begin{array}{l}\text { Central Interior Highlands Calcareous Glade and } \\
\text { Barrens }\end{array}$ & Schizachyrium scoparium & Graminoid $\mathrm{C}_{4}$ & 3.6 & 4.1 \\
\hline $\begin{array}{l}\text { Central Interior Highlands Dry Acidic Glade and } \\
\text { Barrens }\end{array}$ & Schizachyrium scoparium & Graminoid $\mathrm{C}_{4}$ & 3.6 & 4.1 \\
\hline Central Mixedgrass Prairie & Schizachyrium scoparium & Graminoid $\mathrm{C}_{4}$ & 3.6 & 4.1 \\
\hline Crosstimbers Oak Forest and Woodland & Schizachyrium scoparium & Graminoid $\mathrm{C}_{4}$ & 3.6 & 4.1 \\
\hline Cumberland Sandstone Glade and Barrens & Schizachyrium scoparium & Graminoid $\mathrm{C}_{4}$ & 3.6 & 4.1 \\
\hline $\begin{array}{l}\text { East Gulf Coastal Plain Interior Upland Longleaf } \\
\text { Pine Woodland }\end{array}$ & Schizachyrium scoparium & Graminoid $\mathrm{C}_{4}$ & 3.6 & 4.1 \\
\hline East-Central Texas Plains Xeric Sandyland & Schizachyrium scoparium & Graminoid $\mathrm{C}_{4}$ & 3.6 & 4.1 \\
\hline Eastern Serpentine Woodland & Schizachyrium scoparium & Graminoid $\mathrm{C}_{4}$ & 3.6 & 4.1 \\
\hline $\begin{array}{l}\text { Edwards Plateau Limestone Savanna and } \\
\text { Woodland }\end{array}$ & Schizachyrium scoparium & Graminoid $\mathrm{C}_{4}$ & 3.6 & 4.1 \\
\hline Great Lakes Alvar & Schizachyrium scoparium & Graminoid $\mathrm{C}_{4}$ & 3.6 & 4.1 \\
\hline Great Lakes Wet-Mesic Lakeplain Prairie & Schizachyrium scoparium & Graminoid $\mathrm{C}_{4}$ & 3.6 & 4.1 \\
\hline $\begin{array}{l}\text { Llano Estacado Caprock Escarpment and Breaks } \\
\text { Shrubland and Steppe }\end{array}$ & Schizachyrium scoparium & Graminoid $\mathrm{C}_{4}$ & 3.6 & 4.1 \\
\hline $\begin{array}{l}\text { Lower Mississippi River Dune Woodland and } \\
\text { Forest }\end{array}$ & Schizachyrium scoparium & Graminoid $\mathrm{C}_{4}$ & 3.6 & 4.1 \\
\hline Nashville Basin Limestone Glade and Woodland & Schizachyrium scoparium & Graminoid $\mathrm{C}_{4}$ & 3.6 & 4.1 \\
\hline North-Central Interior Oak Savanna & Schizachyrium scoparium & Graminoid $\mathrm{C}_{4}$ & 3.6 & 4.1 \\
\hline $\begin{array}{l}\text { North-Central Interior Sand and Gravel Tallgrass } \\
\text { Prairie }\end{array}$ & Schizachyrium scoparium & Graminoid $\mathrm{C}_{4}$ & 3.6 & 4.1 \\
\hline North-Central Oak Barrens & Schizachyrium scoparium & Graminoid $\mathrm{C}_{4}$ & 3.6 & 4.1 \\
\hline Northeastern Interior Pine Barrens & Schizachyrium scoparium & Graminoid $\mathrm{C}_{4}$ & 3.6 & 4.1 \\
\hline $\begin{array}{l}\text { Northern Atlantic Coastal Plain Heathland and } \\
\text { Grassland }\end{array}$ & Schizachyrium scoparium & Graminoid $\mathrm{C}_{4}$ & 3.6 & 4.1 \\
\hline Northern Atlantic Coastal Plain Pitch Pine Barrens & Schizachyrium scoparium & Graminoid $\mathrm{C}_{4}$ & 3.6 & 4.1 \\
\hline
\end{tabular}




\begin{tabular}{|c|c|c|c|c|}
\hline Community & Species & Classification & Resistance & Resilience \\
\hline Northern Tallgrass Prairie & Schizachyrium scoparium & Graminoid $\mathrm{C}_{4}$ & 3.6 & 4.1 \\
\hline Ouachita Montane Oak Forest & Schizachyrium scoparium & Graminoid $\mathrm{C}_{4}$ & 3.6 & 4.1 \\
\hline $\begin{array}{l}\text { Ozark-Ouachita Shortleaf Pine-Bluestem } \\
\text { Woodland }\end{array}$ & Schizachyrium scoparium & Graminoid $\mathrm{C}_{4}$ & 3.6 & 4.1 \\
\hline $\begin{array}{l}\text { Ozark-Ouachita Shortleaf Pine-Oak Forest and } \\
\text { Woodland }\end{array}$ & Schizachyrium scoparium & Graminoid $\mathrm{C}_{4}$ & 3.6 & 4.1 \\
\hline Paleozoic Plateau Bluff and Talus & Schizachyrium scoparium & Graminoid $\mathrm{C}_{4}$ & 3.6 & 4.1 \\
\hline Southeastern Great Plains Tallgrass Prairie & Schizachyrium scoparium & Graminoid $\mathrm{C}_{4}$ & 3.6 & 4.1 \\
\hline Southeastern Interior Longleaf Pine Woodland & Schizachyrium scoparium & Graminoid $\mathrm{C}_{4}$ & 3.6 & 4.1 \\
\hline Southern Appalachian Granitic Dome & Schizachyrium scoparium & Graminoid $\mathrm{C}_{4}$ & 3.6 & 4.1 \\
\hline $\begin{array}{l}\text { Southern Coastal Plain Blackland Prairie and } \\
\text { Woodland }\end{array}$ & Schizachyrium scoparium & Graminoid $\mathrm{C}_{4}$ & 3.6 & 4.1 \\
\hline Southern Coastal Plain Limestone Forest & Schizachyrium scoparium & Graminoid $\mathrm{C}_{4}$ & 3.6 & 4.1 \\
\hline Southern Interior Calcareous Cliff & Schizachyrium scoparium & Graminoid $\mathrm{C}_{4}$ & 3.6 & 4.1 \\
\hline Southern Piedmont Cliff & Schizachyrium scoparium & Graminoid $\mathrm{C}_{4}$ & 3.6 & 4.1 \\
\hline Southern Piedmont Granite Flatrock and Outcrop & Schizachyrium scoparium & Graminoid $\mathrm{C}_{4}$ & 3.6 & 4.1 \\
\hline $\begin{array}{l}\text { Southern Rocky Mountain Juniper Woodland and } \\
\text { Savanna }\end{array}$ & Schizachyrium scoparium & Graminoid $\mathrm{C}_{4}$ & 3.6 & 4.1 \\
\hline $\begin{array}{l}\text { Southern Rocky Mountain Ponderosa Pine } \\
\text { Savanna }\end{array}$ & Schizachyrium scoparium & Graminoid $\mathrm{C}_{4}$ & 3.6 & 4.1 \\
\hline Texas Blackland Tallgrass Prairie & Schizachyrium scoparium & Graminoid $\mathrm{C}_{4}$ & 3.6 & 4.1 \\
\hline Texas-Louisiana Coastal Prairie & Schizachyrium scoparium & Graminoid $\mathrm{C}_{4}$ & 3.6 & 4.1 \\
\hline West Gulf Coastal Plain Catahoula Barrens & Schizachyrium scoparium & Graminoid $\mathrm{C}_{4}$ & 3.6 & 4.1 \\
\hline West Gulf Coastal Plain Nepheline Syenite Glade & Schizachyrium scoparium & Graminoid $\mathrm{C}_{4}$ & 3.6 & 4.1 \\
\hline $\begin{array}{l}\text { West Gulf Coastal Plain Upland Longleaf Pine } \\
\text { Forest and Woodland }\end{array}$ & Schizachyrium scoparium & Graminoid $\mathrm{C}_{4}$ & 3.6 & 4.1 \\
\hline $\begin{array}{l}\text { West Gulf Coastal Plain Wet Longleaf Pine } \\
\text { Savanna and Flatwoods }\end{array}$ & Schizachyrium scoparium & Graminoid $\mathrm{C}_{4}$ & 3.6 & 4.1 \\
\hline $\begin{array}{l}\text { Western Great Plains Foothill and Piedmont } \\
\text { Grassland }\end{array}$ & Schizachyrium scoparium & Graminoid $\mathrm{C}_{4}$ & 3.6 & 4.1 \\
\hline Western Great Plains Riparian & Schizachyrium scoparium & Graminoid $\mathrm{C}_{4}$ & 3.6 & 4.1 \\
\hline Western Great Plains Sandhill Steppe & Schizachyrium scoparium & Graminoid $\mathrm{C}_{4}$ & 3.6 & 4.1 \\
\hline Western Great Plains Tallgrass Prairie & Schizachyrium scoparium & Graminoid $\mathrm{C}_{4}$ & 3.6 & 4.1 \\
\hline Central Tallgrass Prairie & Schizachyrium scoparium & Graminoid $\mathrm{C}_{4}$ & 3.6 & 4.1 \\
\hline Llano Uplift Acidic Forest, Woodland and Glade & Schizachyrium scoparium & Graminoid $\mathrm{C}_{4}$ & 3.6 & 4.1 \\
\hline South Texas Dune and Coastal Grassland & Schizachyrium scoparium & Graminoid $\mathrm{C}_{4}$ & 3.6 & 4.1 \\
\hline South Texas Sand Sheet Grassland & Schizachyrium scoparium & Graminoid $\mathrm{C}_{4}$ & 3.6 & 4.1 \\
\hline Texas Coast Dune and Coastal Grassland & Schizachyrium scoparium & Graminoid $\mathrm{C}_{4}$ & 3.6 & 4.1 \\
\hline Texas Saline Coastal Prairie & Schizachyrium scoparium & Graminoid $\mathrm{C}_{4}$ & 3.6 & 4.1 \\
\hline Florida Dry Prairie & Schizachyrium stoloniferum & Graminoid $\mathrm{C}_{4}$ & 3.6 & 4.1 \\
\hline Great Lakes Freshwater Estuary and Delta & Schoenoplectus acutus & Graminoid $C_{3}$ & 3.6 & 3.6 \\
\hline Great Plains Prairie Pothole & Schoenoplectus acutus & Graminoid $\mathrm{C}_{3}$ & 3.6 & 3.6 \\
\hline North-Central Interior Freshwater Marsh & Schoenoplectus acutus & Graminoid $C_{3}$ & 3.6 & 3.6 \\
\hline Northern Great Lakes Coastal Marsh & Schoenoplectus acutus & Graminoid $C_{3}$ & 3.6 & 3.6 \\
\hline Temperate Pacific Freshwater Emergent Marsh & Schoenoplectus acutus & Graminoid $\mathrm{C}_{3}$ & 3.6 & 3.6 \\
\hline Temperate Pacific Tidal Salt and Brackish Marsh & Schoenoplectus acutus & Graminoid $\mathrm{C}_{3}$ & 3.6 & 3.6 \\
\hline $\begin{array}{l}\text { Western Great Plains Open Freshwater } \\
\text { Depression Wetland }\end{array}$ & Schoenoplectus acutus & Graminoid $C_{3}$ & 3.6 & 3.6 \\
\hline $\begin{array}{l}\text { Western Great Plains Depressional Wetland } \\
\text { Systems }\end{array}$ & Schoenoplectus acutus & Graminoid $C_{3}$ & 3.6 & 3.6 \\
\hline
\end{tabular}




\begin{tabular}{|c|c|c|c|c|}
\hline Community & Species & Classification & Resistance & Resilience \\
\hline Inter-Mountain Basins Alkaline Closed Depression & Schoenoplectus americanus & Graminoid $\mathrm{C}_{3}$ & 3.6 & 3.6 \\
\hline Inter-Mountain Basins Interdunal Swale Wetland & Schoenoplectus americanus & Graminoid $\mathrm{C}_{3}$ & 3.6 & 3.6 \\
\hline Laurentian-Acadian Freshwater Marsh & Schoenoplectus americanus & Graminoid $\mathrm{C}_{3}$ & 3.6 & 3.6 \\
\hline North American Warm Desert Cienega & Schoenoplectus americanus & Graminoid $\mathrm{C}_{3}$ & 3.6 & 3.6 \\
\hline Temperate Pacific Tidal Salt and Brackish Marsh & Schoenoplectus americanus & Graminoid $\mathrm{C}_{3}$ & 3.6 & 3.6 \\
\hline Texas Coast Salt and Brackish Tidal Marsh & Schoenoplectus americanus & Graminoid $C_{3}$ & 3.6 & 3.6 \\
\hline $\begin{array}{l}\text { Florida Big Bend Fresh and Oligohaline Tidal } \\
\text { Marsh }\end{array}$ & Schoenoplectus californicus & Graminoid $\mathrm{C}_{3}$ & 3.6 & 3.6 \\
\hline North American Arid West Emergent Marsh & Schoenoplectus californicus & Graminoid $\mathrm{C}_{3}$ & 3.6 & 3.6 \\
\hline Great Plains Prairie Pothole & Schoenoplectus fluviatilis & Graminoid $\mathrm{C}_{3}$ & 3.6 & 3.6 \\
\hline Inter-Mountain Basins Alkaline Closed Depression & Schoenoplectus maritimus & Graminoid $C_{3}$ & 3.6 & 3.6 \\
\hline Temperate Pacific Tidal Salt and Brackish Marsh & Schoenoplectus maritimus & Graminoid $C_{3}$ & 3.6 & 3.6 \\
\hline Acadian Estuary Marsh & Schoenoplectus pungens & Graminoid $C_{3}$ & 3.6 & 3.6 \\
\hline $\begin{array}{l}\text { Atlantic Coastal Plain Embayed Region Tidal } \\
\text { Freshwater Marsh }\end{array}$ & Schoenoplectus pungens & Graminoid $\mathrm{C}_{3}$ & 3.6 & 3.6 \\
\hline Great Lakes Freshwater Estuary and Delta & Schoenoplectus pungens & Graminoid $\mathrm{C}_{3}$ & 3.6 & 3.6 \\
\hline $\begin{array}{l}\text { Southern Atlantic Coastal Plain Fresh and } \\
\text { Oligohaline Tidal Marsh }\end{array}$ & Schoenoplectus pungens & Graminoid $\mathrm{C}_{3}$ & 3.6 & 3.6 \\
\hline Temperate Pacific Freshwater Emergent Marsh & Schoenoplectus pungens & Graminoid $\mathrm{C}_{3}$ & 3.6 & 3.6 \\
\hline Acadian Estuary Marsh & $\begin{array}{l}\text { Schoenoplectus } \\
\text { tabernaemontani }\end{array}$ & Graminoid $\mathrm{C}_{3}$ & 3.6 & 3.6 \\
\hline Temperate Pacific Freshwater Emergent Marsh & $\begin{array}{l}\text { Schoenoplectus } \\
\text { tabernaemontani }\end{array}$ & Graminoid $\mathrm{C}_{3}$ & 3.6 & 3.6 \\
\hline $\begin{array}{l}\text { Western Great Plains Open Freshwater } \\
\text { Depression Wetland }\end{array}$ & $\begin{array}{l}\text { Schoenoplectus } \\
\text { tabernaemontani }\end{array}$ & Graminoid $\mathrm{C}_{3}$ & 3.6 & 3.6 \\
\hline $\begin{array}{l}\text { Northern Atlantic Coastal Plain Fresh and } \\
\text { Oligohaline Tidal Marsh }\end{array}$ & $\begin{array}{l}\text { Schoenoplectus } \\
\text { tabernaemontani }\end{array}$ & Graminoid $\mathrm{C}_{3}$ & 3.6 & 3.6 \\
\hline South Florida Slough, Gator Hole and Willow Head & $\begin{array}{l}\text { Schoenoplectus } \\
\text { tabernaemontani }\end{array}$ & Graminoid $\mathrm{C}_{3}$ & 3.6 & 3.6 \\
\hline $\begin{array}{l}\text { Western Great Plains Depressional Wetland } \\
\text { Systems }\end{array}$ & $\begin{array}{l}\text { Schoenoplectus } \\
\text { tabernaemontani }\end{array}$ & Graminoid $\mathrm{C}_{3}$ & 3.6 & 3.6 \\
\hline South Florida Wet Marl Prairie & Schoenus nigricans & Graminoid $\mathrm{C}_{3}$ & 3.6 & 3.6 \\
\hline Piedmont Upland Depression Swamp & Scirpus cyperinus & Graminoid $\mathrm{C}_{3}$ & 3.6 & 3.6 \\
\hline $\begin{array}{l}\text { Southern Coastal Plain Blackland Prairie and } \\
\text { Woodland }\end{array}$ & Scleria verticillata & Graminoid $\mathrm{C}_{3}$ & 3.6 & 3.6 \\
\hline Chihuahuan Loamy Plains Desert Grassland & Scleropogon brevifolius & Graminoid $\mathrm{C}_{4}$ & 3.6 & 4.1 \\
\hline Edwards Plateau Upland Depression & Sedum nuttallianum & Forb & 3 & 3.5 \\
\hline Edwards Plateau Upland Depression & Sedum pulchellum & Forb & 3 & 3.5 \\
\hline Rocky Mountain Alpine-Montane Wet Meadow & Senecio triangularis & Forb & 3 & 3.5 \\
\hline Central Florida Pine Flatwoods & Serenoa repens & Shrub- Deciduous & 2.7 & 3 \\
\hline East Gulf Coastal Plain Maritime Forest & Serenoa repens & Shrub- Deciduous & 2.7 & 3 \\
\hline Florida Dry Prairie & Serenoa repens & Shrub- Deciduous & 2.7 & 3 \\
\hline Florida Peninsula Inland Scrub & Serenoa repens & Shrub- Deciduous & 2.7 & 3 \\
\hline South Florida Pine Flatwoods & Serenoa repens & Shrub- Deciduous & 2.7 & 3 \\
\hline South Florida Pine Rockland & Serenoa repens & Shrub-Deciduous & 2.7 & 3 \\
\hline $\begin{array}{l}\text { Southeast Florida Coastal Strand and Maritime } \\
\text { Hammock }\end{array}$ & Serenoa repens & Shrub- Deciduous & 2.7 & 3 \\
\hline Southern Atlantic Coastal Plain Maritime Forest & Serenoa repens & Shrub- Deciduous & 2.7 & 3 \\
\hline Central Atlantic Coastal Plain Sandy Beach & Sesuvium portulacastrum & Forb & 3 & 3.5 \\
\hline Southern Atlantic Coastal Plain Florida Beach & Sesuvium portulacastrum & Forb & 3 & 3.5 \\
\hline
\end{tabular}




\begin{tabular}{|c|c|c|c|c|}
\hline Community & Species & Classification & Resistance & Resilience \\
\hline Southern Atlantic Coastal Plain Sea Island Beach & Sesuvium portulacastrum & Forb & 3 & 3.5 \\
\hline $\begin{array}{l}\text { North American Warm Desert Riparian Woodland } \\
\text { and Shrubland }\end{array}$ & Shepherdia argentea & Shrub-Deciduous & 2.7 & 3 \\
\hline Northwestern Great Plains Shrubland & Shepherdia argentea & Shrub-Deciduous & 2.7 & 3 \\
\hline $\begin{array}{l}\text { Rocky Mountain Lower Montane-Foothill Riparian } \\
\text { Woodland and Shrubland }\end{array}$ & Shepherdia argentea & Shrub- Deciduous & 2.7 & 3 \\
\hline North Pacific Montane Shrubland & Shepherdia canadensis & Shrub- Deciduous & 2.7 & 3 \\
\hline $\begin{array}{l}\text { Rocky Mountain Foothill Limber Pine-Juniper } \\
\text { Woodland }\end{array}$ & Shepherdia canadensis & Shrub-Deciduous & 2.7 & 3 \\
\hline $\begin{array}{l}\text { North Pacific Alpine and Subalpine Bedrock and } \\
\text { Scree }\end{array}$ & Sibbaldia procumbens & Forb & 3 & 3.5 \\
\hline Rocky Mountain Alpine Fell-Field & Sibbaldia procumbens & Forb & 3 & 3.5 \\
\hline Sierra Nevada Alpine Dwarf-Shrubland & Sibbaldia procumbens & Forb & 3 & 3.5 \\
\hline Southern Appalachian Rocky Summit & Sibbaldiopsis tridentata & Forb & 3 & 3.5 \\
\hline West Gulf Coastal Plain Weches Glade & Sideroxylon lanuginosum & Shrub- Evergreen & 2.7 & 2.4 \\
\hline Rocky Mountain Alpine Fell-Field & Silene acaulis & Forb & 3 & 3.5 \\
\hline $\begin{array}{l}\text { Atlantic Coastal Plain Peatland Pocosin and } \\
\text { Canebrake }\end{array}$ & Smilax laurifolia & Shrub & 2.7 & 2.8 \\
\hline $\begin{array}{l}\text { West Gulf Coastal Plain Seepage Swamp and } \\
\text { Baygall }\end{array}$ & Smilax laurifolia & Shrub & 2.7 & 2.8 \\
\hline $\begin{array}{l}\text { Atlantic Coastal Plain Streamhead Seepage } \\
\text { Swamp, Pocosin and Baygall }\end{array}$ & Smilax laurifolia & Shrub & 2.7 & 2.8 \\
\hline Eastern Highland Rim Prairie and Barrens & Solidago odora & Forb & 3 & 3.5 \\
\hline Eastern Highland Rim Prairie and Barrens & Solidago rugosa & Forb & 3 & 3.5 \\
\hline Northern Atlantic Coastal Plain Dune and Swale & Solidago sempervirens & Forb & 3 & 3.5 \\
\hline Edwards Plateau Limestone Shrubland & Sophora secundiflora & Shrub- Evergreen & 2.7 & 2.4 \\
\hline Tamaulipan Calcareous Thornscrub & Sophora secundiflora & Shrub- Evergreen & 2.7 & 2.4 \\
\hline $\begin{array}{l}\text { Central and Southern Appalachian Spruce-Fir } \\
\text { Forest }\end{array}$ & Sorbus americana & Shrub- Deciduous & 2.7 & 3 \\
\hline Southern Appalachian Grass and Shrub Bald & Sorbus americana & Shrub-Deciduous & 2.7 & 3 \\
\hline $\begin{array}{l}\text { Northern Rocky Mountain Subalpine Deciduous } \\
\text { Shrubland }\end{array}$ & Sorbus scopulina & Shrub- Deciduous & 2.7 & 3 \\
\hline $\begin{array}{l}\text { Northern Rocky Mountain Subalpine Deciduous } \\
\text { Shrubland }\end{array}$ & Sorbus sitchensis & Shrub-Deciduous & 2.7 & 3 \\
\hline Arkansas Valley Prairie and Woodland & Sorghastrum nutans & Graminoid $\mathrm{C}_{4}$ & 3.6 & 4.1 \\
\hline $\begin{array}{l}\text { Central Interior Highlands Dry Acidic Glade and } \\
\text { Barrens }\end{array}$ & Sorghastrum nutans & Graminoid $\mathrm{C}_{4}$ & 3.6 & 4.1 \\
\hline Cumberland Riverscour & Sorghastrum nutans & Graminoid $\mathrm{C}_{4}$ & 3.6 & 4.1 \\
\hline Eastern Highland Rim Prairie and Barrens & Sorghastrum nutans & Graminoid $\mathrm{C}_{4}$ & 3.6 & 4.1 \\
\hline Eastern Serpentine Woodland & Sorghastrum nutans & Graminoid $\mathrm{C}_{4}$ & 3.6 & 4.1 \\
\hline Great Lakes Wet-Mesic Lakeplain Prairie & Sorghastrum nutans & Graminoid $\mathrm{C}_{4}$ & 3.6 & 4.1 \\
\hline $\begin{array}{l}\text { Lower Mississippi River Dune Woodland and } \\
\text { Forest }\end{array}$ & Sorghastrum nutans & Graminoid $\mathrm{C}_{4}$ & 3.6 & 4.1 \\
\hline Northern Tallgrass Prairie & Sorghastrum nutans & Graminoid $\mathrm{C}_{4}$ & 3.6 & 4.1 \\
\hline Southeastern Great Plains Tallgrass Prairie & Sorghastrum nutans & Graminoid $\mathrm{C}_{4}$ & 3.6 & 4.1 \\
\hline $\begin{array}{l}\text { Southern Coastal Plain Blackland Prairie and } \\
\text { Woodland }\end{array}$ & Sorghastrum nutans & Graminoid $\mathrm{C}_{4}$ & 3.6 & 4.1 \\
\hline Texas Blackland Tallgrass Prairie & Sorghastrum nutans & Graminoid $\mathrm{C}_{4}$ & 3.6 & 4.1 \\
\hline Texas-Louisiana Coastal Prairie & Sorghastrum nutans & Graminoid $\mathrm{C}_{4}$ & 3.6 & 4.1 \\
\hline $\begin{array}{l}\text { West Gulf Coastal Plain Northern Calcareous } \\
\text { Prairie }\end{array}$ & Sorghastrum nutans & Graminoid $\mathrm{C}_{4}$ & 3.6 & 4.1 \\
\hline
\end{tabular}




\begin{tabular}{|c|c|c|c|c|}
\hline Community & Species & Classification & Resistance & Resilience \\
\hline $\begin{array}{l}\text { West Gulf Coastal Plain Southern Calcareous } \\
\text { Prairie }\end{array}$ & Sorghastrum nutans & Graminoid $\mathrm{C}_{4}$ & 3.6 & 4.1 \\
\hline Central Tallgrass Prairie & Sorghastrum nutans & Graminoid $\mathrm{C}_{4}$ & 3.6 & 4.1 \\
\hline Florida Dry Prairie & Sorghastrum secundum & Graminoid $\mathrm{C}_{4}$ & 3.6 & 4.1 \\
\hline Acadian Coastal Salt Marsh & Spartina alterniflora & Graminoid $\mathrm{C}_{4}$ & 3.6 & 4.1 \\
\hline Acadian Estuary Marsh & Spartina alterniflora & Graminoid $\mathrm{C}_{4}$ & 3.6 & 4.1 \\
\hline $\begin{array}{l}\text { Atlantic Coastal Plain Embayed Region Tidal Salt } \\
\text { and Brackish Marsh }\end{array}$ & Spartina alterniflora & Graminoid $\mathrm{C}_{4}$ & 3.6 & 4.1 \\
\hline $\begin{array}{l}\text { Atlantic Coastal Plain Indian River Lagoon Tidal } \\
\text { Marsh }\end{array}$ & Spartina alterniflora & Graminoid $\mathrm{C}_{4}$ & 3.6 & 4.1 \\
\hline Florida Big Bend Salt and Brackish Tidal Marsh & Spartina alterniflora & Graminoid $\mathrm{C}_{4}$ & 3.6 & 4.1 \\
\hline $\begin{array}{l}\text { Gulf Coast Chenier Plain Salt and Brackish Tidal } \\
\text { Marsh }\end{array}$ & Spartina alterniflora & Graminoid $\mathrm{C}_{4}$ & 3.6 & 4.1 \\
\hline Northern Atlantic Coastal Plain Tidal Salt Marsh & Spartina alterniflora & Graminoid $\mathrm{C}_{4}$ & 3.6 & 4.1 \\
\hline $\begin{array}{l}\text { Southern Atlantic Coastal Plain Salt and Brackish } \\
\text { Tidal Marsh }\end{array}$ & Spartina alterniflora & Graminoid $\mathrm{C}_{4}$ & 3.6 & 4.1 \\
\hline $\begin{array}{l}\text { Atlantic Coastal Plain Indian River Lagoon Tidal } \\
\text { Marsh }\end{array}$ & Spartina bakeri & Graminoid $\mathrm{C}_{4}$ & 3.6 & 4.1 \\
\hline $\begin{array}{l}\text { Southern Atlantic Coastal Plain Fresh and } \\
\text { Oligohaline Tidal Marsh }\end{array}$ & Spartina cynosuroides & Graminoid $\mathrm{C}_{4}$ & 3.6 & 4.1 \\
\hline Acadian Coastal Salt Marsh & Spartina patens & Graminoid $\mathrm{C}_{4}$ & 3.6 & 4.1 \\
\hline Acadian Estuary Marsh & Spartina patens & Graminoid $\mathrm{C}_{4}$ & 3.6 & 4.1 \\
\hline $\begin{array}{l}\text { Atlantic Coastal Plain Embayed Region Tidal } \\
\text { Freshwater Marsh }\end{array}$ & Spartina patens & Graminoid $\mathrm{C}_{4}$ & 3.6 & 4.1 \\
\hline $\begin{array}{l}\text { Central and South Texas Coastal Fringe Forest and } \\
\text { Woodland }\end{array}$ & Spartina patens & Graminoid $\mathrm{C}_{4}$ & 3.6 & 4.1 \\
\hline $\begin{array}{l}\text { East Gulf Coastal Plain Dune and Coastal } \\
\text { Grassland }\end{array}$ & Spartina patens & Graminoid $\mathrm{C}_{4}$ & 3.6 & 4.1 \\
\hline Gulf Coast Chenier Plain Beach & Spartina patens & Graminoid $\mathrm{C}_{4}$ & 3.6 & 4.1 \\
\hline $\begin{array}{l}\text { Gulf Coast Chenier Plain Fresh and Oligohaline } \\
\text { Tidal Marsh }\end{array}$ & Spartina patens & Graminoid $\mathrm{C}_{4}$ & 3.6 & 4.1 \\
\hline $\begin{array}{l}\text { Gulf Coast Chenier Plain Salt and Brackish Tidal } \\
\text { Marsh }\end{array}$ & Spartina patens & Graminoid $\mathrm{C}_{4}$ & 3.6 & 4.1 \\
\hline Louisiana Beach & Spartina patens & Graminoid $\mathrm{C}_{4}$ & 3.6 & 4.1 \\
\hline Northern Atlantic Coastal Plain Tidal Salt Marsh & Spartina patens & Graminoid $\mathrm{C}_{4}$ & 3.6 & 4.1 \\
\hline $\begin{array}{l}\text { Southern Atlantic Coastal Plain Dune and } \\
\text { Maritime Grassland }\end{array}$ & Spartina patens & Graminoid $\mathrm{C}_{4}$ & 3.6 & 4.1 \\
\hline $\begin{array}{l}\text { Southern Atlantic Coastal Plain Fresh and } \\
\text { Oligohaline Tidal Marsh }\end{array}$ & Spartina patens & Graminoid $\mathrm{C}_{4}$ & 3.6 & 4.1 \\
\hline Texas Coast Beach & Spartina patens & Graminoid $\mathrm{C}_{4}$ & 3.6 & 4.1 \\
\hline Texas Coast Dune and Coastal Grassland & Spartina patens & Graminoid $\mathrm{C}_{4}$ & 3.6 & 4.1 \\
\hline Texas Coast Fresh and Oligohaline Tidal Marsh & Spartina patens & Graminoid $\mathrm{C}_{4}$ & 3.6 & 4.1 \\
\hline Texas Coast Salt and Brackish Tidal Marsh & Spartina patens & Graminoid $\mathrm{C}_{4}$ & 3.6 & 4.1 \\
\hline Texas Saline Coastal Prairie & Spartina patens & Graminoid $\mathrm{C}_{4}$ & 3.6 & 4.1 \\
\hline South Texas Dune and Coastal Grassland & Spartina patens & Graminoid $\mathrm{C}_{4}$ & 3.6 & 4.1 \\
\hline Great Lakes Wet-Mesic Lakeplain Prairie & Spartina pectinata & Graminoid $\mathrm{C}_{4}$ & 3.6 & 4.1 \\
\hline $\begin{array}{l}\text { Western Great Plains Open Freshwater } \\
\text { Depression Wetland }\end{array}$ & Spartina pectinata & Graminoid $\mathrm{C}_{4}$ & 3.6 & 4.1 \\
\hline $\begin{array}{l}\text { Eastern Great Plains Wet Meadow, Prairie and } \\
\text { Marsh }\end{array}$ & Spartina pectinata & Graminoid $\mathrm{C}_{4}$ & 3.6 & 4.1 \\
\hline $\begin{array}{l}\text { Western Great Plains Depressional Wetland } \\
\text { Systems }\end{array}$ & Spartina pectinata & Graminoid $\mathrm{C}_{4}$ & 3.6 & 4.1 \\
\hline
\end{tabular}




\begin{tabular}{|c|c|c|c|c|}
\hline Community & Species & Classification & Resistance & Resilience \\
\hline South Texas Salt and Brackish Tidal Flat & Spartina spartinae & Graminoid $\mathrm{C}_{4}$ & 3.6 & 4.1 \\
\hline Texas Saline Coastal Prairie & Spartina spartinae & Graminoid $\mathrm{C}_{4}$ & 3.6 & 4.1 \\
\hline Northern California Claypan Vernal Pool & Spergularia salina & Forb & 3 & 3.5 \\
\hline Laurentian-Acadian Wet Meadow-Shrub Swamp & Spiraea alba & Shrub- Deciduous & 2.7 & 3 \\
\hline Northern Rocky Mountain Western Larch Savanna & Spiraea betulifolia & Shrub- Deciduous & 2.7 & 3 \\
\hline Rocky Mountain Lodgepole Pine Forest & Spiraea betulifolia & Shrub- Deciduous & 2.7 & 3 \\
\hline North Pacific Shrub Swamp & Spiraea douglasii & Shrub- Deciduous & 2.7 & 3 \\
\hline Chihuahuan Gypsophilous Grassland and Steppe & Sporobolus airoides & Graminoid $\mathrm{C}_{4}$ & 3.6 & 4.1 \\
\hline Chihuahuan Mixed Salt Desert Scrub & Sporobolus airoides & Graminoid $\mathrm{C}_{4}$ & 3.6 & 4.1 \\
\hline $\begin{array}{l}\text { Chihuahuan-Sonoran Desert Bottomland and } \\
\text { Swale Grassland }\end{array}$ & Sporobolus airoides & Graminoid $\mathrm{C}_{4}$ & 3.6 & 4.1 \\
\hline Inter-Mountain Basins Greasewood Flat & Sporobolus airoides & Graminoid $\mathrm{C}_{4}$ & 3.6 & 4.1 \\
\hline Inter-Mountain Basins Semi-Desert Grassland & Sporobolus airoides & Graminoid $\mathrm{C}_{4}$ & 3.6 & 4.1 \\
\hline Inter-Mountain Basins Semi-Desert Shrub-Steppe & Sporobolus airoides & Graminoid $\mathrm{C}_{4}$ & 3.6 & 4.1 \\
\hline North American Warm Desert Cienega & Sporobolus airoides & Graminoid $\mathrm{C}_{4}$ & 3.6 & 4.1 \\
\hline Western Great Plains Saline Depression Wetland & Sporobolus airoides & Graminoid $\mathrm{C}_{4}$ & 3.6 & 4.1 \\
\hline $\begin{array}{l}\text { Western Great Plains Depressional Wetland } \\
\text { Systems }\end{array}$ & Sporobolus airoides & Graminoid $\mathrm{C}_{4}$ & 3.6 & 4.1 \\
\hline West Gulf Coastal Plain Nepheline Syenite Glade & Sporobolus clandestinus & Graminoid $\mathrm{C}_{4}$ & 3.6 & 4.1 \\
\hline $\begin{array}{l}\text { Columbia Basin Foothill and Canyon Dry } \\
\text { Grassland }\end{array}$ & Sporobolus cryptandrus & Graminoid $\mathrm{C}_{4}$ & 3.6 & 4.1 \\
\hline Inter-Mountain Basins Semi-Desert Shrub-Steppe & Sporobolus cryptandrus & Graminoid $\mathrm{C}_{4}$ & 3.6 & 4.1 \\
\hline $\begin{array}{l}\text { Southern Rocky Mountain Juniper Woodland and } \\
\text { Savanna }\end{array}$ & Sporobolus cryptandrus & Graminoid $\mathrm{C}_{4}$ & 3.6 & 4.1 \\
\hline $\begin{array}{l}\text { Western Great Plains Foothill and Piedmont } \\
\text { Grassland }\end{array}$ & Sporobolus cryptandrus & Graminoid $\mathrm{C}_{4}$ & 3.6 & 4.1 \\
\hline $\begin{array}{l}\text { Western Great Plains Mesquite Woodland and } \\
\text { Shrubland }\end{array}$ & Sporobolus cryptandrus & Graminoid $\mathrm{C}_{4}$ & 3.6 & 4.1 \\
\hline Western Great Plains Riparian & Sporobolus cryptandrus & Graminoid $\mathrm{C}_{4}$ & 3.6 & 4.1 \\
\hline Western Great Plains Sandhill Steppe & Sporobolus cryptandrus & Graminoid $\mathrm{C}_{4}$ & 3.6 & 4.1 \\
\hline Western Great Plains Shortgrass Prairie & Sporobolus cryptandrus & Graminoid $\mathrm{C}_{4}$ & 3.6 & 4.1 \\
\hline Chihuahuan Sandy Plains Semi-Desert Grassland & Sporobolus flexuosus & Graminoid $\mathrm{C}_{4}$ & 3.6 & 4.1 \\
\hline $\begin{array}{l}\text { Chihuahuan Stabilized Coppice Dune and Sand } \\
\text { Flat Scrub }\end{array}$ & Sporobolus flexuosus & Graminoid $\mathrm{C}_{4}$ & 3.6 & 4.1 \\
\hline East Gulf Coastal Plain Savanna and Wet Prairie & Sporobolus floridanus & Graminoid $\mathrm{C}_{4}$ & 3.6 & 4.1 \\
\hline Western Great Plains Sandhill Steppe & Sporobolus giganteus & Graminoid $\mathrm{C}_{4}$ & 3.6 & 4.1 \\
\hline Great Lakes Alvar & Sporobolus heterolepis & Graminoid $\mathrm{C}_{4}$ & 3.6 & 4.1 \\
\hline Western Great Plains Tallgrass Prairie & Sporobolus heterolepis & Graminoid $\mathrm{C}_{4}$ & 3.6 & 4.1 \\
\hline Chihuahuan Gypsophilous Grassland and Steppe & Sporobolus nealleyi & Graminoid $\mathrm{C}_{4}$ & 3.6 & 4.1 \\
\hline $\begin{array}{l}\text { Central Atlantic Coastal Plain Wet Longleaf Pine } \\
\text { Savanna and Flatwoods }\end{array}$ & Sporobolus pinetorum & Graminoid $\mathrm{C}_{4}$ & 3.6 & 4.1 \\
\hline West Gulf Coastal Plain Catahoula Barrens & Sporobolus silveanus & Graminoid $\mathrm{C}_{4}$ & 3.6 & 4.1 \\
\hline $\begin{array}{l}\text { Central Atlantic Coastal Plain Wet Longleaf Pine } \\
\text { Savanna and Flatwoods }\end{array}$ & Sporobolus teretifolius & Graminoid $\mathrm{C}_{4}$ & 3.6 & 4.1 \\
\hline Edwards Plateau Upland Depression & Sporobolus vaginiflorus & Graminoid $\mathrm{C}_{4}$ & 3.6 & 4.1 \\
\hline West Gulf Coastal Plain Weches Glade & Sporobolus vaginiflorus & Graminoid $\mathrm{C}_{4}$ & 3.6 & 4.1 \\
\hline Louisiana Beach & Sporobolus virginicus & Graminoid $\mathrm{C}_{4}$ & 3.6 & 4.1 \\
\hline $\begin{array}{l}\text { Chihuahuan-Sonoran Desert Bottomland and } \\
\text { Swale Grassland }\end{array}$ & Sporobolus wrightii & Graminoid $\mathrm{C}_{4}$ & 3.6 & 4.1 \\
\hline
\end{tabular}




\begin{tabular}{|c|c|c|c|c|}
\hline Community & Species & Classification & Resistance & Resilience \\
\hline $\begin{array}{l}\text { North-Central Appalachian Circumneutral Cliff and } \\
\text { Talus }\end{array}$ & Staphylea trifolia & Shrub- Deciduous & 2.7 & 3 \\
\hline Columbia Plateau Scabland Shrubland & Stenotus stenophyllus & Forb & 3 & 3.5 \\
\hline Southern Coastal Plain Oak Dome and Hammock & Stylisma humistrata & Forb & 3 & 3.5 \\
\hline $\begin{array}{l}\text { East Gulf Coastal Plain Southern Loblolly- } \\
\text { Hardwood Flatwoods }\end{array}$ & Styrax americanus & Shrub- Deciduous & 2.7 & 3 \\
\hline Southern Coastal Plain Nonriverine Cypress Dome & Styrax americanus & Shrub- Deciduous & 2.7 & 3 \\
\hline North American Warm Desert Playa & Suaeda calceoliformis & Forb & 3 & 3.5 \\
\hline South Florida Hardwood Hammock & Swietenia mahagoni & Tree- Evergreen & 3.2 & 2.2 \\
\hline $\begin{array}{l}\text { Middle Rocky Mountain Montane Douglas-fir } \\
\text { Forest and Woodland }\end{array}$ & Symphoricarpos albus & Shrub- Deciduous & 2.7 & 3 \\
\hline $\begin{array}{l}\text { Northern Rocky Mountain Montane-Foothill } \\
\text { Deciduous Shrubland }\end{array}$ & Symphoricarpos albus & Shrub- Deciduous & 2.7 & 3 \\
\hline $\begin{array}{l}\text { Northern Rocky Mountain Ponderosa Pine } \\
\text { Woodland and Savanna }\end{array}$ & Symphoricarpos albus & Shrub- Deciduous & 2.7 & 3 \\
\hline $\begin{array}{l}\text { Northwestern Great Plains Aspen Forest and } \\
\text { Parkland }\end{array}$ & Symphoricarpos albus & Shrub- Deciduous & 2.7 & 3 \\
\hline Rocky Mountain Aspen Forest and Woodland & Symphoricarpos albus & Shrub- Deciduous & 2.7 & 3 \\
\hline $\begin{array}{l}\text { Rocky Mountain Foothill Limber Pine-Juniper } \\
\text { Woodland }\end{array}$ & Symphoricarpos albus & Shrub- Deciduous & 2.7 & 3 \\
\hline $\begin{array}{l}\text { California Coastal Live Oak Woodland and } \\
\text { Savanna }\end{array}$ & Symphoricarpos mollis & Shrub- Deciduous & 2.7 & 3 \\
\hline Northwestern Great Plains Shrubland & Symphoricarpos occidentalis & Shrub- Deciduous & 2.7 & 3 \\
\hline $\begin{array}{l}\text { Western Great Plains Dry Bur Oak Forest and } \\
\text { Woodland }\end{array}$ & Symphoricarpos occidentalis & Shrub- Deciduous & 2.7 & 3 \\
\hline Great Plains Wooded Draw and Ravine & Symphoricarpos occidentalis & Shrub- Deciduous & 2.7 & 3 \\
\hline $\begin{array}{l}\text { Inter-Mountain Basins Aspen-Mixed Conifer Forest } \\
\text { and Woodland }\end{array}$ & Symphoricarpos oreophilus & Shrub- Deciduous & 2.7 & 3 \\
\hline $\begin{array}{l}\text { Inter-Mountain Basins Montane Sagebrush } \\
\text { Steppe }\end{array}$ & Symphoricarpos oreophilus & Shrub- Deciduous & 2.7 & 3 \\
\hline $\begin{array}{l}\text { Inter-Mountain Basins Subalpine Limber- } \\
\text { Bristlecone Pine Woodland }\end{array}$ & Symphoricarpos oreophilus & Shrub- Deciduous & 2.7 & 3 \\
\hline $\begin{array}{l}\text { Northern Rocky Mountain Montane-Foothill } \\
\text { Deciduous Shrubland }\end{array}$ & Symphoricarpos oreophilus & Shrub- Deciduous & 2.7 & 3 \\
\hline Rocky Mountain Aspen Forest and Woodland & Symphoricarpos oreophilus & Shrub- Deciduous & 2.7 & 3 \\
\hline Rocky Mountain Bigtooth Maple Ravine Woodland & Symphoricarpos oreophilus & Shrub- Deciduous & 2.7 & 3 \\
\hline $\begin{array}{l}\text { Rocky Mountain Foothill Limber Pine-Juniper } \\
\text { Woodland }\end{array}$ & Symphoricarpos oreophilus & Shrub- Deciduous & 2.7 & 3 \\
\hline $\begin{array}{l}\text { Rocky Mountain Gambel Oak-Mixed Montane } \\
\text { Shrubland }\end{array}$ & Symphoricarpos oreophilus & Shrub- Deciduous & 2.7 & 3 \\
\hline $\begin{array}{l}\text { Rocky Mountain Lower Montane-Foothill } \\
\text { Shrubland }\end{array}$ & Symphoricarpos oreophilus & Shrub- Deciduous & 2.7 & 3 \\
\hline $\begin{array}{l}\text { Southern Rocky Mountain Ponderosa Pine } \\
\text { Woodland }\end{array}$ & Symphoricarpos oreophilus & Shrub- Deciduous & 2.7 & 3 \\
\hline $\begin{array}{l}\text { Rocky Mountain Gambel Oak-Mixed Montane } \\
\text { Shrubland }\end{array}$ & Symphoricarpos rotundifolius & Shrub- Deciduous & 2.7 & 3 \\
\hline Eastern Highland Rim Prairie and Barrens & Symphyotrichum dumosum & Forb & 3 & 3.5 \\
\hline Temperate Pacific Tidal Salt and Brackish Marsh & Symphyotrichum subspicatum & Forb & 3 & 3.5 \\
\hline $\begin{array}{l}\text { Atlantic Coastal Plain Clay-Based Carolina Bay } \\
\text { Wetland }\end{array}$ & Taxodium ascendens & Tree- Evergreen & 3.2 & 2.2 \\
\hline $\begin{array}{l}\text { Atlantic Coastal Plain Small Blackwater River } \\
\text { Floodplain Forest }\end{array}$ & Taxodium ascendens & Tree- Evergreen & 3.2 & 2.2 \\
\hline East Gulf Coastal Plain Maritime Forest & Taxodium ascendens & Tree- Evergreen & 3.2 & 2.2 \\
\hline
\end{tabular}




\begin{tabular}{|c|c|c|c|c|}
\hline Community & Species & Classification & Resistance & Resilience \\
\hline South Florida Cypress Dome & Taxodium ascendens & Tree-Evergreen & 3.2 & 2.2 \\
\hline South Florida Dwarf Cypress Savanna & Taxodium ascendens & Tree-Evergreen & 3.2 & 2.2 \\
\hline Southeastern Coastal Plain Natural Lakeshore & Taxodium ascendens & Tree-Evergreen & 3.2 & 2.2 \\
\hline Southern Coastal Plain Nonriverine Cypress Dome & Taxodium ascendens & Tree- Evergreen & 3.2 & 2.2 \\
\hline $\begin{array}{l}\text { Atlantic Coastal Plain Streamhead Seepage } \\
\text { Swamp, Pocosin and Baygall }\end{array}$ & Taxodium ascendens & Tree- Evergreen & 3.2 & 2.2 \\
\hline $\begin{array}{l}\text { Atlantic Coastal Plain Blackwater Stream } \\
\text { Floodplain Forest }\end{array}$ & Taxodium distichum & Tree- Evergreen & 3.2 & 2.2 \\
\hline $\begin{array}{l}\text { Atlantic Coastal Plain Brownwater Stream } \\
\text { Floodplain Forest }\end{array}$ & Taxodium distichum & Tree- Evergreen & 3.2 & 2.2 \\
\hline $\begin{array}{l}\text { Atlantic Coastal Plain Small Blackwater River } \\
\text { Floodplain Forest }\end{array}$ & Taxodium distichum & Tree-Evergreen & 3.2 & 2.2 \\
\hline $\begin{array}{l}\text { Atlantic Coastal Plain Small Brownwater River } \\
\text { Floodplain Forest }\end{array}$ & Taxodium distichum & Tree- Evergreen & 3.2 & 2.2 \\
\hline Central Atlantic Coastal Plain Maritime Forest & Taxodium distichum & Tree- Evergreen & 3.2 & 2.2 \\
\hline $\begin{array}{l}\text { East Gulf Coastal Plain Freshwater Tidal Wooded } \\
\text { Swamp }\end{array}$ & Taxodium distichum & Tree- Evergreen & 3.2 & 2.2 \\
\hline $\begin{array}{l}\text { East Gulf Coastal Plain Large River Floodplain } \\
\text { Forest }\end{array}$ & Taxodium distichum & Tree- Evergreen & 3.2 & 2.2 \\
\hline Edwards Plateau Riparian & Taxodium distichum & Tree-Evergreen & 3.2 & 2.2 \\
\hline Red River Large Floodplain Forest & Taxodium distichum & Tree- Evergreen & 3.2 & 2.2 \\
\hline South Florida Bayhead Swamp & Taxodium distichum & Tree-Evergreen & 3.2 & 2.2 \\
\hline Southeastern Coastal Plain Natural Lakeshore & Taxodium distichum & Tree-Evergreen & 3.2 & 2.2 \\
\hline $\begin{array}{l}\text { Southern Atlantic Coastal Plain Nonriverine } \\
\text { Swamp and Wet Hardwood Forest }\end{array}$ & Taxodium distichum & Tree- Evergreen & 3.2 & 2.2 \\
\hline $\begin{array}{l}\text { Southern Atlantic Coastal Plain Tidal Wooded } \\
\text { Swamp }\end{array}$ & Taxodium distichum & Tree- Evergreen & 3.2 & 2.2 \\
\hline $\begin{array}{l}\text { Southern Coastal Plain Blackwater River } \\
\text { Floodplain Forest }\end{array}$ & Taxodium distichum & Tree- Evergreen & 3.2 & 2.2 \\
\hline Southern Coastal Plain Nonriverine Basin Swamp & Taxodium distichum & Tree- Evergreen & 3.2 & 2.2 \\
\hline $\begin{array}{l}\text { West Gulf Coastal Plain Large River Floodplain } \\
\text { Forest }\end{array}$ & Taxodium distichum & Tree- Evergreen & 3.2 & 2.2 \\
\hline $\begin{array}{l}\text { West Gulf Coastal Plain Near-Coast Large River } \\
\text { Swamp }\end{array}$ & Taxodium distichum & Tree- Evergreen & 3.2 & 2.2 \\
\hline Tamaulipan Ramadero & Tecoma stans & Shrub- Evergreen & 2.7 & 2.4 \\
\hline Inter-Mountain Basins Active and Stabilized Dune & Tetradymia tetrameres & Shrub- Deciduous & 2.7 & 3 \\
\hline $\begin{array}{l}\text { Great Basin Foothill and Lower Montane Riparian } \\
\text { Woodland and Shrubland }\end{array}$ & Thalictrum fendleri & Forb & 3 & 3.5 \\
\hline Edwards Plateau Cliff & Thelypteris ovata & Graminoid $\mathrm{C}_{3}$ & 3.6 & 3.6 \\
\hline Edwards Plateau Mesic Canyon & Thelypteris ovata & Graminoid $\mathrm{C}_{3}$ & 3.6 & 3.6 \\
\hline Central Interior Calcareous Cliff and Talus & Thuja occidentalis & Tree- Evergreen & 3.2 & 2.2 \\
\hline Great Lakes Wooded Dune and Swale & Thuja occidentalis & Tree-Evergreen & 3.2 & 2.2 \\
\hline $\begin{array}{l}\text { Laurentian-Acadian Alkaline Conifer-Hardwood } \\
\text { Swamp }\end{array}$ & Thuja occidentalis & Tree- Evergreen & 3.2 & 2.2 \\
\hline Southern Interior Calcareous Cliff & Thuja occidentalis & Tree- Evergreen & 3.2 & 2.2 \\
\hline $\begin{array}{l}\text { East Cascades Mesic Montane Mixed Conifer } \\
\text { Forest and Woodland }\end{array}$ & Thuja plicata & Tree-Evergreen & 3.2 & 2.2 \\
\hline North Pacific Hardwood-Conifer Swamp & Thuja plicata & Tree-Evergreen & 3.2 & 2.2 \\
\hline $\begin{array}{l}\text { North Pacific Hypermaritime Western Red-cedar- } \\
\text { Western Hemlock Forest }\end{array}$ & Thuja plicata & Tree- Evergreen & 3.2 & 2.2 \\
\hline $\begin{array}{l}\text { North Pacific Lowland Mixed Hardwood-Conifer } \\
\text { Forest }\end{array}$ & Thuja plicata & Tree-Evergreen & 3.2 & 2.2 \\
\hline
\end{tabular}




\begin{tabular}{|c|c|c|c|c|}
\hline Community & Species & Classification & Resistance & Resilience \\
\hline $\begin{array}{l}\text { North Pacific Maritime Dry-Mesic Douglas-fir- } \\
\text { Western Hemlock Forest }\end{array}$ & Thuja plicata & Tree- Evergreen & 3.2 & 2.2 \\
\hline $\begin{array}{l}\text { North Pacific Maritime Mesic-Wet Douglas-fir- } \\
\text { Western Hemlock Forest }\end{array}$ & Thuja plicata & Tree- Evergreen & 3.2 & 2.2 \\
\hline Northern Rocky Mountain Conifer Swamp & Thuja plicata & Tree- Evergreen & 3.2 & 2.2 \\
\hline $\begin{array}{l}\text { Northern Rocky Mountain Lower Montane } \\
\text { Riparian Woodland and Shrubland }\end{array}$ & Thuja plicata & Tree-Evergreen & 3.2 & 2.2 \\
\hline $\begin{array}{l}\text { Northern Rocky Mountain Mesic Montane Mixed } \\
\text { Conifer Forest }\end{array}$ & Thuja plicata & Tree- Evergreen & 3.2 & 2.2 \\
\hline $\begin{array}{l}\text { North-Central Appalachian Circumneutral Cliff and } \\
\text { Talus }\end{array}$ & Tilia americana & Tree- Deciduous & 3 & 4.2 \\
\hline North-Central Interior Maple-Basswood Forest & Tilia americana & Tree- Deciduous & 3 & 4.2 \\
\hline Ozark-Ouachita Mesic Hardwood Forest & Tilia americana & Tree-Deciduous & 3 & 4.2 \\
\hline South-Central Interior Mesophytic Forest & Tilia americana & Tree- Deciduous & 3 & 4.2 \\
\hline Southern and Central Appalachian Cove Forest & Tilia americana & Tree- Deciduous & 3 & 4.2 \\
\hline Southern Coastal Plain Limestone Forest & Tilia americana & Tree-Deciduous & 3 & 4.2 \\
\hline Southern and Central Appalachian Cove Forest & Tilia americana & Tree- Deciduous & 3 & 4.2 \\
\hline Southern Coastal Plain Limestone Forest & Tilia americana & Tree- Deciduous & 3 & 4.2 \\
\hline Chihuahuan Gypsophilous Grassland and Steppe & Tiquilia canescens & Shrub & 2.7 & 2.8 \\
\hline Chihuahuan Gypsophilous Grassland and Steppe & Tiquilia hispidissima & Shrub & 2.7 & 2.8 \\
\hline California Central Valley Mixed Oak Savanna & Toxicodendron diversilobum & Shrub & 2.7 & 2.8 \\
\hline $\begin{array}{l}\text { California Central Valley Riparian Woodland and } \\
\text { Shrubland }\end{array}$ & Toxicodendron diversilobum & Shrub & 2.7 & 2.8 \\
\hline $\begin{array}{l}\text { California Coastal Live Oak Woodland and } \\
\text { Savanna }\end{array}$ & Toxicodendron diversilobum & Shrub & 2.7 & 2.8 \\
\hline California Mesic Chaparral & Toxicodendron diversilobum & Shrub & 2.7 & 2.8 \\
\hline Mediterranean California Mixed Evergreen Forest & Toxicodendron diversilobum & Shrub & 2.7 & 2.8 \\
\hline Northern California Coastal Scrub & Toxicodendron diversilobum & Shrub & 2.7 & 2.8 \\
\hline Southern California Oak Woodland and Savanna & Toxicodendron diversilobum & Shrub & 2.7 & 2.8 \\
\hline $\begin{array}{l}\text { Mediterranean California Lower Montane Black } \\
\text { Oak-Conifer Forest and Woodland }\end{array}$ & Toxicodendron diversilobum & Shrub & 2.7 & 2.8 \\
\hline Central Appalachian River Floodplain & Toxicodendron radicans & Shrub & 2.7 & 2.8 \\
\hline North-Central Appalachian Acidic Cliff and Talus & Toxicodendron radicans & Shrub & 2.7 & 2.8 \\
\hline Southern Appalachian Montane Cliff and Talus & Toxicodendron radicans & Shrub & 2.7 & 2.8 \\
\hline Edwards Plateau Cliff & Toxicodendron radicans & Shrub & 2.7 & 2.8 \\
\hline $\begin{array}{l}\text { Atlantic Coastal Plain Streamhead Seepage } \\
\text { Swamp, Pocosin and Baygall }\end{array}$ & Toxicodendron vernix & Shrub-Deciduous & 2.7 & 3 \\
\hline Rocky Mountain Subalpine-Montane Fen & Trichophorum pumilum & Graminoid $\mathrm{C}_{3}$ & 3.6 & 3.6 \\
\hline Edwards Plateau Upland Depression & Tridens albescens & Graminoid $\mathrm{C}_{4}$ & 3.6 & 4.1 \\
\hline Rocky Mountain Alpine Turf & Trifolium dasyphyllum & Forb & 3 & 3.5 \\
\hline Rocky Mountain Alpine Fell-Field & Trifolium parryi & Forb & 3 & 3.5 \\
\hline Rocky Mountain Alpine Turf & Trifolium parryi & Forb & 3 & 3.5 \\
\hline Rocky Mountain Alpine-Montane Wet Meadow & Trifolium paryi & Forb & 3 & 3.5 \\
\hline Temperate Pacific Tidal Salt and Brackish Marsh & Triglochin maritima & Graminoid $\mathrm{C}_{3}$ & 3.6 & 3.6 \\
\hline Texas Blackland Tallgrass Prairie & Tripsacum dactyloides & Graminoid $\mathrm{C}_{4}$ & 3.6 & 4.1 \\
\hline Texas-Louisiana Coastal Prairie & Tripsacum dactyloides & Graminoid $\mathrm{C}_{4}$ & 3.6 & 4.1 \\
\hline Texas-Louisiana Coastal Prairie Pondshore & Tripsacum dactyloides & Graminoid $\mathrm{C}_{4}$ & 3.6 & 4.1 \\
\hline $\begin{array}{l}\text { Eastern Great Plains Wet Meadow, Prairie and } \\
\text { Marsh }\end{array}$ & Tripsacum dactyloides & Graminoid $\mathrm{C}_{4}$ & 3.6 & 4.1 \\
\hline
\end{tabular}




\begin{tabular}{|c|c|c|c|c|}
\hline Community & Species & Classification & Resistance & Resilience \\
\hline $\begin{array}{l}\text { Northern Rocky Mountain Subalpine-Upper } \\
\text { Montane Grassland }\end{array}$ & Trisetum spicatum & Graminoid $C_{3}$ & 3.6 & 3.6 \\
\hline Mediterranean California Alpine Dry Tundra & Trisetum spicatum & Graminoid $\mathrm{C}_{3}$ & 3.6 & 3.6 \\
\hline North Pacific Herbaceous Bald and Bluff & Triteleia hyacinthina & Forb & 3 & 3.5 \\
\hline Rocky Mountain Alpine-Montane Wet Meadow & Trollius laxus & Forb & 3 & 3.5 \\
\hline $\begin{array}{l}\text { Laurentian-Acadian Pine-Hemlock-Hardwood } \\
\text { Forest }\end{array}$ & Tsuga canadensis & Tree- Evergreen & 3.2 & 2.2 \\
\hline $\begin{array}{l}\text { North-Central Interior and Appalachian Rich } \\
\text { Swamp }\end{array}$ & Tsuga canadensis & Tree-Evergreen & 3.2 & 2.2 \\
\hline Southern and Central Appalachian Cove Forest & Tsuga canadensis & Tree- Evergreen & 3.2 & 2.2 \\
\hline Southern Appalachian Northern Hardwood Forest & Tsuga canadensis & Tree-Evergreen & 3.2 & 2.2 \\
\hline Appalachian (Hemlock)-Northern Hardwood Forest & Tsuga canadensis & Tree-Evergreen & 3.2 & 2.2 \\
\hline California Coastal Redwood Forest & Tsuga heterophylla & Tree-Evergreen & 3.2 & 2.2 \\
\hline $\begin{array}{l}\text { East Cascades Mesic Montane Mixed Conifer } \\
\text { Forest and Woodland }\end{array}$ & Tsuga heterophylla & Tree-Evergreen & 3.2 & 2.2 \\
\hline $\begin{array}{l}\text { North Pacific Dry-Mesic Silver Fir-Western } \\
\text { Hemlock-Douglas-fir Forest }\end{array}$ & Tsuga heterophylla & Tree- Evergreen & 3.2 & 2.2 \\
\hline North Pacific Hardwood-Conifer Swamp & Tsuga heterophylla & Tree-Evergreen & 3.2 & 2.2 \\
\hline $\begin{array}{l}\text { North Pacific Hypermaritime Western Red-cedar- } \\
\text { Western Hemlock Forest }\end{array}$ & Tsuga heterophylla & Tree-Evergreen & 3.2 & 2.2 \\
\hline $\begin{array}{l}\text { North Pacific Lowland Mixed Hardwood-Conifer } \\
\text { Forest }\end{array}$ & Tsuga heterophylla & Tree-Evergreen & 3.2 & 2.2 \\
\hline $\begin{array}{l}\text { North Pacific Maritime Dry-Mesic Douglas-fir- } \\
\text { Western Hemlock Forest }\end{array}$ & Tsuga heterophylla & Tree-Evergreen & 3.2 & 2.2 \\
\hline $\begin{array}{l}\text { North Pacific Maritime Mesic-Wet Douglas-fir- } \\
\text { Western Hemlock Forest }\end{array}$ & Tsuga heterophylla & Tree-Evergreen & 3.2 & 2.2 \\
\hline $\begin{array}{l}\text { North Pacific Mesic Western Hemlock-Silver Fir } \\
\text { Forest }\end{array}$ & Tsuga heterophylla & Tree- Evergreen & 3.2 & 2.2 \\
\hline $\begin{array}{l}\text { North Pacific Montane Riparian Woodland and } \\
\text { Shrubland }\end{array}$ & Tsuga heterophylla & Tree- Evergreen & 3.2 & 2.2 \\
\hline North Pacific Seasonal Sitka Spruce Forest & Tsuga heterophylla & Tree- Evergreen & 3.2 & 2.2 \\
\hline $\begin{array}{l}\text { Northern Rocky Mountain Lower Montane } \\
\text { Riparian Woodland and Shrubland }\end{array}$ & Tsuga heterophylla & Tree-Evergreen & 3.2 & 2.2 \\
\hline $\begin{array}{l}\text { Northern Rocky Mountain Mesic Montane Mixed } \\
\text { Conifer Forest }\end{array}$ & Tsuga heterophylla & Tree-Evergreen & 3.2 & 2.2 \\
\hline Mediterranean California Red Fir Forest & Tsuga mertensiana & Tree-Evergreen & 3.2 & 2.2 \\
\hline North Pacific Hardwood-Conifer Swamp & Tsuga mertensiana & Tree- Evergreen & 3.2 & 2.2 \\
\hline North Pacific Maritime Mesic Subalpine Parkland & Tsuga mertensiana & Tree-Evergreen & 3.2 & 2.2 \\
\hline North Pacific Mountain Hemlock Forest & Tsuga mertensiana & Tree-Evergreen & 3.2 & 2.2 \\
\hline Northern California Mesic Subalpine Woodland & Tsuga mertensiana & Tree-Evergreen & 3.2 & 2.2 \\
\hline Acadian Estuary Marsh & Typha angustifolia & Graminoid $\mathrm{C}_{3}$ & 3.6 & 3.6 \\
\hline $\begin{array}{l}\text { Atlantic Coastal Plain Embayed Region Tidal } \\
\text { Freshwater Marsh }\end{array}$ & Typha angustifolia & Graminoid $C_{3}$ & 3.6 & 3.6 \\
\hline North Pacific Intertidal Freshwater Wetland & Typha angustifolia & Graminoid $\mathrm{C}_{3}$ & 3.6 & 3.6 \\
\hline $\begin{array}{l}\text { Western Great Plains Open Freshwater } \\
\text { Depression Wetland }\end{array}$ & Typha angustifolia & Graminoid $C_{3}$ & 3.6 & 3.6 \\
\hline $\begin{array}{l}\text { Western Great Plains Depressional Wetland } \\
\text { Systems }\end{array}$ & Typha angustifolia & Graminoid $\mathrm{C}_{3}$ & 3.6 & 3.6 \\
\hline $\begin{array}{l}\text { Florida Big Bend Fresh and Oligohaline Tidal } \\
\text { Marsh }\end{array}$ & Typha domingensis & Graminoid $C_{3}$ & 3.6 & 3.6 \\
\hline Inter-Mountain Basins Interdunal Swale Wetland & Typha domingensis & Graminoid $\mathrm{C}_{3}$ & 3.6 & 3.6 \\
\hline North American Arid West Emergent Marsh & Typha domingensis & Graminoid $C_{3}$ & 3.6 & 3.6 \\
\hline
\end{tabular}




\begin{tabular}{|c|c|c|c|c|}
\hline Community & Species & Classification & Resistance & Resilience \\
\hline Southeastern Coastal Plain Interdunal Wetland & Typha domingensis & Graminoid $C_{3}$ & 3.6 & 3.6 \\
\hline $\begin{array}{l}\text { Atlantic Coastal Plain Embayed Region Tidal } \\
\text { Freshwater Marsh }\end{array}$ & Typha latifolia & Graminoid $C_{3}$ & 3.6 & 3.6 \\
\hline Great Lakes Freshwater Estuary and Delta & Typha latifolia & Graminoid $\mathrm{C}_{3}$ & 3.6 & 3.6 \\
\hline Inter-Mountain Basins Interdunal Swale Wetland & Typha latifolia & Graminoid $\mathrm{C}_{3}$ & 3.6 & 3.6 \\
\hline Laurentian-Acadian Freshwater Marsh & Typha latifolia & Graminoid $C_{3}$ & 3.6 & 3.6 \\
\hline North-Central Interior Floodplain & Typha latifolia & Graminoid $\mathrm{C}_{3}$ & 3.6 & 3.6 \\
\hline North-Central Interior Freshwater Marsh & Typha latifolia & Graminoid $\mathrm{C}_{3}$ & 3.6 & 3.6 \\
\hline Temperate Pacific Freshwater Emergent Marsh & Typha latifolia & Graminoid $C_{3}$ & 3.6 & 3.6 \\
\hline Texas Coast Fresh and Oligohaline Tidal Marsh & Typha latifolia & Graminoid $\mathrm{C}_{3}$ & 3.6 & 3.6 \\
\hline $\begin{array}{l}\text { South-Central Interior / Upper Coastal Plain } \\
\text { Flatwoods }\end{array}$ & Ulmus alata & Tree- Deciduous & 3 & 4.2 \\
\hline $\begin{array}{l}\text { South-Central Interior / Upper Coastal Plain Wet } \\
\text { Flatwoods }\end{array}$ & Ulmus alata & Tree- Deciduous & 3 & 4.2 \\
\hline Edwards Plateau Floodplain Terrace & Ulmus americana & Tree-Deciduous & 3 & 4.2 \\
\hline $\begin{array}{l}\text { Northwestern Great Plains-Black Hills Ponderosa } \\
\text { Pine Woodland and Savanna }\end{array}$ & Ulmus americana & Tree-Deciduous & 3 & 4.2 \\
\hline Southeastern Great Plains Floodplain Forest & Ulmus americana & Tree-Deciduous & 3 & 4.2 \\
\hline Southern Coastal Plain Hydric Hammock & Ulmus americana & Tree-Deciduous & 3 & 4.2 \\
\hline Great Plains Wooded Draw and Ravine & Ulmus americana & Tree-Deciduous & 3 & 4.2 \\
\hline $\begin{array}{l}\text { South-Central Interior / Upper Coastal Plain Wet } \\
\text { Flatwoods }\end{array}$ & Ulmus americana & Tree-Deciduous & 3 & 4.2 \\
\hline $\begin{array}{l}\text { Edwards Plateau Dry-Mesic Slope Forest and } \\
\text { Woodland }\end{array}$ & Ulmus crassifolia & Tree-Deciduous & 3 & 4.2 \\
\hline Edwards Plateau Floodplain Terrace & Ulmus crassifolia & Tree-Deciduous & 3 & 4.2 \\
\hline $\begin{array}{l}\text { Edwards Plateau Limestone Savanna and } \\
\text { Woodland }\end{array}$ & Ulmus crassifolia & Tree- Deciduous & 3 & 4.2 \\
\hline Edwards Plateau Riparian & Ulmus crassifolia & Tree-Deciduous & 3 & 4.2 \\
\hline Southeastern Great Plains Floodplain Forest & Ulmus crassifolia & Tree- Deciduous & 3 & 4.2 \\
\hline Southeastern Great Plains Riparian Forest & Ulmus crassifolia & Tree- Deciduous & 3 & 4.2 \\
\hline Tamaulipan Floodplain & Ulmus crassifolia & Tree- Deciduous & 3 & 4.2 \\
\hline Tamaulipan Palm Grove Riparian Forest & Ulmus crassifolia & Tree-Deciduous & 3 & 4.2 \\
\hline Llano Uplift Acidic Forest, Woodland and Glade & Ulmus crassifolia & Tree-Deciduous & 3 & 4.2 \\
\hline Great Plains Wooded Draw and Ravine & Ulmus rubra & Tree-Deciduous & 3 & 4.2 \\
\hline $\begin{array}{l}\text { California Coastal Live Oak Woodland and } \\
\text { Savanna }\end{array}$ & Umbellularia californica & Tree-Evergreen & 3.2 & 2.2 \\
\hline $\begin{array}{l}\text { Mediterranean California Mesic Serpentine } \\
\text { Woodland and Chaparral }\end{array}$ & Umbellularia californica & Tree- Evergreen & 3.2 & 2.2 \\
\hline Mediterranean California Mixed Evergreen Forest & Umbellularia californica & Tree- Evergreen & 3.2 & 2.2 \\
\hline $\begin{array}{l}\text { Mediterranean California Serpentine Foothill and } \\
\text { Lower Montane Riparian Woodland and Seep }\end{array}$ & Umbellularia californica & Tree- Evergreen & 3.2 & 2.2 \\
\hline Edwards Plateau Cliff & Ungnadia speciosa & Shrub- Deciduous & 2.7 & 3 \\
\hline Florida Panhandle Beach Vegetation & Uniola paniculata & Graminoid $\mathrm{C}_{4}$ & 3.6 & 4.1 \\
\hline South Florida Shell Hash Beach & Uniola paniculata & Graminoid $\mathrm{C}_{4}$ & 3.6 & 4.1 \\
\hline $\begin{array}{l}\text { Southern Atlantic Coastal Plain Dune and } \\
\text { Maritime Grassland }\end{array}$ & Uniola paniculata & Graminoid $\mathrm{C}_{4}$ & 3.6 & 4.1 \\
\hline Southern Atlantic Coastal Plain Florida Beach & Uniola paniculata & Graminoid $\mathrm{C}_{4}$ & 3.6 & 4.1 \\
\hline Southwest Florida Beach & Uniola paniculata & Graminoid $\mathrm{C}_{4}$ & 3.6 & 4.1 \\
\hline Southwest Florida Dune and Coastal Grassland & Uniola paniculata & Graminoid $\mathrm{C}_{4}$ & 3.6 & 4.1 \\
\hline Tamaulipan Floodplain & Urochloa maxima & Graminoid $\mathrm{C}_{4}$ & 3.6 & 4.1 \\
\hline
\end{tabular}




\begin{tabular}{|c|c|c|c|c|}
\hline Community & Species & Classification & Resistance & Resilience \\
\hline Tamaulipan Mixed Deciduous Thornscrub & Urochloa maxima & Graminoid $\mathrm{C}_{4}$ & 3.6 & 4.1 \\
\hline Tamaulipan Ramadero & Urochloa maxima & Graminoid $\mathrm{C}_{4}$ & 3.6 & 4.1 \\
\hline Laurentian Jack Pine-Red Pine Forest & Vaccinium angustifolium & Shrub & 2.7 & 2.8 \\
\hline Laurentian Pine-Oak Barrens & Vaccinium angustifolium & Shrub & 2.7 & 2.8 \\
\hline $\begin{array}{l}\text { Northern Atlantic Coastal Plain Heathland and } \\
\text { Grassland }\end{array}$ & Vaccinium angustifolium & Shrub & 2.7 & 2.8 \\
\hline Northern Atlantic Coastal Plain Pitch Pine Barrens & Vaccinium angustifolium & Shrub & 2.7 & 2.8 \\
\hline Cumberland Sandstone Glade and Barrens & Vaccinium arboreum & Shrub- Evergreen & 2.7 & 2.4 \\
\hline Southern Piedmont Granite Flatrock and Outcrop & Vaccinium arboreum & Shrub- Evergreen & 2.7 & 2.4 \\
\hline Northern Rocky Mountain Western Larch Savanna & Vaccinium caespitosum & Shrub-Deciduous & 2.7 & 3 \\
\hline Rocky Mountain Alpine Dwarf-Shrubland & Vaccinium caespitosum & Shrub- Deciduous & 2.7 & 3 \\
\hline $\begin{array}{l}\text { Northern Atlantic Coastal Plain Basin Swamp and } \\
\text { Wet Hardwood Forest }\end{array}$ & Vaccinium corymbosum & Shrub- Deciduous & 2.7 & 3 \\
\hline Northern Atlantic Coastal Plain Pitch Pine Lowland & Vaccinium corymbosum & Shrub- Deciduous & 2.7 & 3 \\
\hline Florida Dry Prairie & Vaccinium darrowii & Shrub- Evergreen & 2.7 & 2.4 \\
\hline North Pacific Maritime Mesic Subalpine Parkland & Vaccinium deliciosum & Shrub- Deciduous & 2.7 & 3 \\
\hline $\begin{array}{l}\text { North Pacific Dry and Mesic Alpine Dwarf- } \\
\text { Shrubland, Fell-Field and Meadow }\end{array}$ & Vaccinium deliciosum & Shrub- Deciduous & 2.7 & 3 \\
\hline Southern Appalachian Granitic Dome & Vaccinium erythrocarpum & Shrub- Deciduous & 2.7 & 3 \\
\hline North Pacific Montane Shrubland & Vaccinium membranaceum & Shrub- Deciduous & 2.7 & 3 \\
\hline North Pacific Wooded Volcanic Flowage & Vaccinium membranaceum & Shrub- Deciduous & 2.7 & 3 \\
\hline $\begin{array}{l}\text { Northern Rocky Mountain Subalpine Deciduous } \\
\text { Shrubland }\end{array}$ & Vaccinium membranaceum & Shrub- Deciduous & 2.7 & 3 \\
\hline $\begin{array}{l}\text { Rocky Mountain Subalpine Mesic-Wet Spruce-Fir } \\
\text { Forest and Woodland }\end{array}$ & Vaccinium membranaceum & Shrub- Deciduous & 2.7 & 3 \\
\hline $\begin{array}{l}\text { Southern Rocky Mountain Mesic Montane Mixed } \\
\text { Conifer Forest and Woodland }\end{array}$ & Vaccinium membranaceum & Shrub- Deciduous & 2.7 & 3 \\
\hline $\begin{array}{l}\text { Rocky Mountain Subalpine Mesic-Wet Spruce-Fir } \\
\text { Forest and Woodland }\end{array}$ & Vaccinium membranaceum & Shrub- Deciduous & 2.7 & 3 \\
\hline Florida Dry Prairie & Vaccinium myrsinites & Shrub- Evergreen & 2.7 & 2.4 \\
\hline $\begin{array}{l}\text { Northern Rocky Mountain Subalpine Deciduous } \\
\text { Shrubland }\end{array}$ & Vaccinium myrtillus & Shrub- Deciduous & 2.7 & 3 \\
\hline $\begin{array}{l}\text { Southern Rocky Mountain Mesic Montane Mixed } \\
\text { Conifer Forest and Woodland }\end{array}$ & Vaccinium myrtillus & Shrub- Deciduous & 2.7 & 3 \\
\hline $\begin{array}{l}\text { North Pacific Mesic Western Hemlock-Silver Fir } \\
\text { Forest }\end{array}$ & Vaccinium ovalifolium & Shrub- Deciduous & 2.7 & 3 \\
\hline $\begin{array}{l}\text { North Pacific Hypermaritime Shrub and } \\
\text { Herbaceous Headland }\end{array}$ & Vaccinium ovatum & Shrub- Evergreen & 2.7 & 2.4 \\
\hline $\begin{array}{l}\text { North Pacific Maritime Coastal Sand Dune and } \\
\text { Strand }\end{array}$ & Vaccinium ovatum & Shrub- Evergreen & 2.7 & 2.4 \\
\hline $\begin{array}{l}\text { North Pacific Maritime Dry-Mesic Douglas-fir- } \\
\text { Western Hemlock Forest }\end{array}$ & Vaccinium ovatum & Shrub- Evergreen & 2.7 & 2.4 \\
\hline Northern Atlantic Coastal Plain Pitch Pine Barrens & Vaccinium pallidum & Shrub- Deciduous & 2.7 & 3 \\
\hline $\begin{array}{l}\text { Northern Rocky Mountain Subalpine Deciduous } \\
\text { Shrubland }\end{array}$ & Vaccinium scoparium & Shrub- Deciduous & 2.7 & 3 \\
\hline $\begin{array}{l}\text { Northern Rocky Mountain Subalpine Woodland } \\
\text { and Parkland }\end{array}$ & Vaccinium scoparium & Shrub- Deciduous & 2.7 & 3 \\
\hline Rocky Mountain Alpine Dwarf-Shrubland & Vaccinium scoparium & Shrub- Deciduous & 2.7 & 3 \\
\hline Rocky Mountain Lodgepole Pine Forest & Vaccinium scoparium & Shrub- Deciduous & 2.7 & 3 \\
\hline Rocky Mountain Poor-Site Lodgepole Pine Forest & Vaccinium scoparium & Shrub- Deciduous & 2.7 & 3 \\
\hline $\begin{array}{l}\text { Rocky Mountain Subalpine Dry-Mesic Spruce-Fir } \\
\text { Forest and Woodland }\end{array}$ & Vaccinium scoparium & Shrub- Deciduous & 2.7 & 3 \\
\hline
\end{tabular}




\begin{tabular}{|c|c|c|c|c|}
\hline Community & Species & Classification & Resistance & Resilience \\
\hline $\begin{array}{l}\text { North Pacific Dry and Mesic Alpine Dwarf- } \\
\text { Shrubland, Fell-Field and Meadow }\end{array}$ & Vaccinium scoparium & Shrub- Deciduous & 2.7 & 3 \\
\hline Acadian-Appalachian Alpine Tundra & Vaccinium uliginosum & Shrub & 2.7 & 2.8 \\
\hline North Pacific Bog and Fen & Vaccinium uliginosum & Shrub- Deciduous & 2.7 & 3 \\
\hline North Pacific Maritime Mesic Subalpine Parkland & Valeriana sitchensis & Forb & 3 & 3.5 \\
\hline $\begin{array}{l}\text { Temperate Pacific Subalpine-Montane Wet } \\
\text { Meadow }\end{array}$ & Veratrum californicum & Forb & 3 & 3.5 \\
\hline Northern California Claypan Vernal Pool & Veronica peregrina & Forb & 3 & 3.5 \\
\hline $\begin{array}{l}\text { East Gulf Coastal Plain Southern Loblolly- } \\
\text { Hardwood Flatwoods }\end{array}$ & Viburnum dentatum & Shrub-Deciduous & 2.7 & 3 \\
\hline $\begin{array}{l}\text { Atlantic Coastal Plain Streamhead Seepage } \\
\text { Swamp, Pocosin and Baygall }\end{array}$ & Viburnum nudum & Shrub- Deciduous & 2.7 & 3 \\
\hline Southern and Central Appalachian Bog and Fen & Viburnum nudum & Shrub- Deciduous & 2.7 & 3 \\
\hline Mojave Mid-Elevation Mixed Desert Scrub & Viguiera parishii & Shrub-Deciduous & 2.7 & 3 \\
\hline North Pacific Coastal Cliff and Bluff & Viola adunca & Forb & 3 & 3.5 \\
\hline $\begin{array}{l}\text { California Central Valley Riparian Woodland and } \\
\text { Shrubland }\end{array}$ & Vitis californica & Shrub & 2.7 & 2.8 \\
\hline California Northern Coastal Grassland & Vulpia myuros & Graminoid $\mathrm{C}_{3}$ & 3.6 & 3.6 \\
\hline $\begin{array}{l}\text { Southern Atlantic Coastal Plain Nonriverine } \\
\text { Swamp and Wet Hardwood Forest }\end{array}$ & Woodwardia areolata & Graminoid $C_{3}$ & 3.6 & 3.6 \\
\hline Southern Coastal Plain Nonriverine Basin Swamp & Woodwardia virginica & Graminoid $\mathrm{C}_{3}$ & 3.6 & 3.6 \\
\hline North Pacific Montane Shrubland & Xerophyllum tenax & Forb & 3 & 3.5 \\
\hline North Pacific Wooded Volcanic Flowage & Xerophyllum tenax & Forb & 3 & 3.5 \\
\hline Northern Rocky Mountain Western Larch Savanna & Xerophyllum tenax & Forb & 3 & 3.5 \\
\hline Southern California Coast Ranges Cliff and Canyon & Xylococcus bicolor & Shrub- Evergreen & 2.7 & 2.4 \\
\hline Mojave Mid-Elevation Mixed Desert Scrub & Yucca brevifolia & Shrub & 2.7 & 2.8 \\
\hline $\begin{array}{l}\text { Rocky Mountain Lower Montane-Foothill } \\
\text { Shrubland }\end{array}$ & Yucca glauca & Shrub & 2.7 & 2.8 \\
\hline Mojave Mid-Elevation Mixed Desert Scrub & Yucca schidigera & Shrub & 2.7 & 2.8 \\
\hline Southern California Coastal Scrub & Yucca whipplei & Shrub & 2.7 & 2.8 \\
\hline South Florida Hardwood Hammock & Zanthoxylum fagara & Shrub- Evergreen & 2.7 & 2.4 \\
\hline $\begin{array}{l}\text { Atlantic Coastal Plain Peatland Pocosin and } \\
\text { Canebrake }\end{array}$ & Zenobia pulverulenta & Shrub- Evergreen & 2.7 & 2.4 \\
\hline $\begin{array}{l}\text { Southern Atlantic Coastal Plain Fresh and } \\
\text { Oligohaline Tidal Marsh }\end{array}$ & Zizania aquatica & Graminoid $C_{3}$ & 3.6 & 3.6 \\
\hline $\begin{array}{l}\text { Northern Atlantic Coastal Plain Fresh and } \\
\text { Oligohaline Tidal Marsh }\end{array}$ & Zizania aquatica & Graminoid $\mathrm{C}_{3}$ & 3.6 & 3.6 \\
\hline $\begin{array}{l}\text { Florida Big Bend Fresh and Oligohaline Tidal } \\
\text { Marsh }\end{array}$ & Zizaniopsis miliacea & Graminoid $C_{3}$ & 3.6 & 3.6 \\
\hline $\begin{array}{l}\text { Gulf Coast Chenier Plain Fresh and Oligohaline } \\
\text { Tidal Marsh }\end{array}$ & Zizaniopsis miliacea & Graminoid $C_{3}$ & 3.6 & 3.6 \\
\hline $\begin{array}{l}\text { Southern Atlantic Coastal Plain Fresh and } \\
\text { Oligohaline Tidal Marsh }\end{array}$ & Zizaniopsis miliacea & Graminoid $C_{3}$ & 3.6 & 3.6 \\
\hline South Florida Slough, Gator Hole and Willow Head & Zizaniopsis miliacea & Graminoid $C_{3}$ & 3.6 & 3.6 \\
\hline $\begin{array}{l}\text { Western Great Plains Mesquite Woodland and } \\
\text { Shrubland }\end{array}$ & Ziziphus obtusifolia & Shrub- Deciduous & 2.7 & 3 \\
\hline
\end{tabular}




\section{Appendix C: Dominant Species' Resistance and Resilience Values from Küchler Potential Vegetation Community Types}

\section{Table C-1. Dominant Species' Resistance and Resilience Values from Küchler Potential Vegetation Community Types.}

\begin{tabular}{|c|c|c|c|c|}
\hline Community & Species & Classification & Resistance & Resilience \\
\hline Silver fir - Douglas-fir forest & Abies amabilis & Tree - Evergreen & 3.2 & 2.2 \\
\hline Northern hardwoods - fir forest & Abies balsamea & Tree - Evergreen & 3.2 & 2.2 \\
\hline Great lakes spruce - fir forest & Abies balsamea & Tree - Evergreen & 3.2 & 2.2 \\
\hline Northeastern spruce - fir forest & Abies balsamea & Tree - Evergreen & 3.2 & 2.2 \\
\hline Spruce - fir - Douglas-fir forest & Abies concolor & Tree - Evergreen & 3.2 & 2.2 \\
\hline Mixed conifer forest & Abies concolor & Tree - Evergreen & 3.2 & 2.2 \\
\hline Northeastern spruce - fir forest & Abies fraseri & Tree - Evergreen & 3.2 & 2.2 \\
\hline Grand fir - Douglas-fir forest & Abies grandis & Tree - Evergreen & 3.2 & 2.2 \\
\hline Western spruce - fir forest & Abies lasiocarpa & Tree - Evergreen & 3.2 & 2.2 \\
\hline Fir - hemlock forest & Abies lasiocarpa & Tree - Evergreen & 3.2 & 2.2 \\
\hline Southwestern spruce - fir forest & Abies lasiocarpa & Tree - Evergreen & 3.2 & 2.2 \\
\hline Red fir forest & Abies magnifica & Tree - Evergreen & 3.2 & 2.2 \\
\hline Mesquite - acacia savanna & Acacia rigidula & Shrub - Deciduous & 2.7 & 3 \\
\hline Beech - maple forest & Acer saccharum & Tree - Deciduous & 3 & 4.2 \\
\hline Mixed mesophytic forest & Acer saccharum & Tree - Deciduous & 3 & 4.2 \\
\hline Northern hardwoods & Acer saccharum & Tree - Deciduous & 3 & 4.2 \\
\hline Northern hardwoods - fir forest & Acer saccharum & Tree - Deciduous & 3 & 4.2 \\
\hline Northern hardwood - spruce forest & Acer saccharum & Tree - Deciduous & 3 & 4.2 \\
\hline Maple- basswood forest & Acer saccharum & Tree - Deciduous & 3 & 4.2 \\
\hline Chaparral & Adenostoma fasciculatum & Shrub - Evergreen & 2.7 & 2.4 \\
\hline Mixed mesophytic forest & Aesculus flava & Tree - Deciduous & 3 & 4.2 \\
\hline Alpine meadows and barren & Agrostis scabra & Graminoid $-\mathrm{C}_{3}$ & 3.6 & 3.6 \\
\hline Alder-ash forest & Alnus rubra & Shrub - Deciduous & 2.7 & 3 \\
\hline Creosote bush - bur sage & Ambrosia dumosa & Shrub - Deciduous & 2.7 & 3 \\
\hline Wheatgrass - bluestem - needlegrass & Andropogon gerardi & Graminoid $-\mathrm{C}_{4}$ & 3.6 & 4.1 \\
\hline Bluestem prairie & Andropogon gerardi & Graminoid - $\mathrm{C}_{4}$ & 3.6 & 4.1 \\
\hline Nebraska sandhills prairie & Andropogon gerardi & Graminoid $-\mathrm{C}_{4}$ & 3.6 & 4.1 \\
\hline Oak savanna & Andropogon gerardi & Graminoid $-\mathrm{C}_{4}$ & 3.6 & 4.1 \\
\hline Sandsage - bluestem prairie & Andropogon hallii & Graminoid $-\mathrm{C}_{4}$ & 3.6 & 4.1 \\
\hline Nebraska sandhills prairie & Andropogon hallii & Graminoid $-\mathrm{C}_{4}$ & 3.6 & 4.1 \\
\hline Mesquite - acacia savanna & Andropogon littoralis & Graminoid $-\mathrm{C}_{4}$ & 3.6 & 4.1 \\
\hline Mesquite - live oak savanna & Andropogon littoralis & Graminoid $-\mathrm{C}_{4}$ & 3.6 & 4.1 \\
\hline Sea oats prairie & Andropogon littoralis & Graminoid $-\mathrm{C}_{4}$ & 3.6 & 4.1 \\
\hline Bluestem - sacahuista prairie & Andropogon littoralis & Graminoid $-\mathrm{C}_{4}$ & 3.6 & 4.1 \\
\hline California mixed evergreen forest & Arbutus menziesii & Tree - Evergreen & 3.2 & 2.2 \\
\hline Montane chaparra & Arctostaphylos patula & Shrub - Evergreen & 2.7 & 2.4 \\
\hline Chaparral & Arctostaphylos spp. & Shrub - Evergreen & 2.7 & 2.4 \\
\hline Cypress savanna & Aristida affinis & Graminoid $-\mathrm{C}_{4}$ & 3.6 & 4.1 \\
\hline
\end{tabular}




\begin{tabular}{|c|c|c|c|c|}
\hline Community & Species & Classification & Resistance & Resilience \\
\hline Galleta - three awn shrub-steppe & Aristida longiseta & Graminoid - $\mathrm{C}_{4}$ & 3.6 & 4.1 \\
\hline Cypress savanna & Aristida patula & Graminoid - $\mathrm{C}_{4}$ & 3.6 & 4.1 \\
\hline Palmetto prairie & Aristida stricta & Graminoid - $\mathrm{C}_{4}$ & 3.6 & 4.1 \\
\hline Galleta - three awn shrub-steppe & Artemisia filifolia & Shrub - Deciduous & 2.7 & 3 \\
\hline Sandsage - bluestem prairie & Artemisia filifolia & Shrub - Deciduous & 2.7 & 3 \\
\hline Juniper steppe woodland & Artemisia tridentata & Shrub - Evergreen & 2.7 & 2.4 \\
\hline Great Basin sagebrush & Artemisia tridentata & Shrub - Evergreen & 2.7 & 2.4 \\
\hline Sagebrush steppe & Artemisia tridentata & Shrub - Evergreen & 2.7 & 2.4 \\
\hline Wheatgrass - needlegrass shrub-steppe & Artemisia tridentata & Shrub - Evergreen & 2.7 & 2.4 \\
\hline Saltbush - greasewood & Atriplex confertifolia & Shrub - Deciduous & 2.7 & 3 \\
\hline Mangrove & Avicennia germinans & Shrub - Evergreen & 2.7 & 2.4 \\
\hline Northern hardwoods & Betula allegheniensis & Tree - Deciduous & 3 & 4.2 \\
\hline Northern hardwood - spruce forest & Betula allegheniensis & Tree - Deciduous & 3 & 4.2 \\
\hline Northern hardwoods - fir forest & Betula papyrifera & Tree-Deciduous & 3 & 4.2 \\
\hline Bluestem - grama prairie & Bouteloua curtipendula & Graminoid - $\mathrm{C}_{4}$ & 3.6 & 4.1 \\
\hline Grama - tobosa shrub-steppe & Bouteloua eriopoda & Graminoid - $\mathrm{C}_{4}$ & 3.6 & 4.1 \\
\hline Grama - galleta steppe & Bouteloua gracilis & Graminoid - $\mathrm{C}_{4}$ & 3.6 & 4.1 \\
\hline Grama - tobosa prairie & Bouteloua gracilis & Graminoid - $\mathrm{C}_{4}$ & 3.6 & 4.1 \\
\hline Grama - needlegrass - wheatgrass & Bouteloua gracilis & Graminoid - $\mathrm{C}_{4}$ & 3.6 & 4.1 \\
\hline Grama - buffalo grass & Bouteloua gracilis & Graminoid - $\mathrm{C}_{4}$ & 3.6 & 4.1 \\
\hline Wheatgrass - needlegrass & Bouteloua gracilis & Graminoid - $\mathrm{C}_{4}$ & 3.6 & 4.1 \\
\hline Wheatgrass - grama - buffalo grass & Bouteloua gracilis & Graminoid - $\mathrm{C}_{4}$ & 3.6 & 4.1 \\
\hline Bluestem - grama prairie & Bouteloua gracilis & Graminoid - $\mathrm{C}_{4}$ & 3.6 & 4.1 \\
\hline Sandsage - bluestem prairie & Bouteloua hirsuta & Graminoid - $\mathrm{C}_{4}$ & 3.6 & 4.1 \\
\hline Grama - buffalo grass & Buchloë dactyloides & Graminoid - $\mathrm{C}_{4}$ & 3.6 & 4.1 \\
\hline Wheatgrass - grama - buffalo grass & Buchloë dactyloides & Graminoid - $\mathrm{C}_{4}$ & 3.6 & 4.1 \\
\hline Mesquite - buffalo grass & Buchloë dactyloides & Graminoid - $\mathrm{C}_{4}$ & 3.6 & 4.1 \\
\hline Fayette prairie & Buchloë dactyloides & Graminoid - $\mathrm{C}_{4}$ & 3.6 & 4.1 \\
\hline Nebraska sandhills prairie & Calamovilfa longifolia & Graminoid - $\mathrm{C}_{4}$ & 3.6 & 4.1 \\
\hline Alpine meadows and barren & Carex spp. & Graminoid $-\mathrm{C}_{3}$ & 3.6 & 3.6 \\
\hline Fescue - oatgrass & Carex tumulicola & Graminoid - $\mathrm{C}_{3}$ & 3.6 & 3.6 \\
\hline Oak-hickory forest & Carya cordiformis & Tree - Deciduous & 3 & 4.2 \\
\hline Oak-hickory forest & Carya ovata & Tree - Deciduous & 3 & 4.2 \\
\hline Oak - hickory - pine forest & Carya ovata & Tree - Deciduous & 3 & 4.2 \\
\hline Montane chaparra & Ceanothus cordulatus & Shrub - Evergreen & 2.7 & 2.4 \\
\hline Chaparral & Ceanothus spp. & Shrub - Evergreen & 2.7 & 2.4 \\
\hline Cedar glades & Celtis laevigata & Tree - Deciduous & 3 & 4.2 \\
\hline Mountain-mahogany - oak scrub & Cercocarpus ledifolius & Shrub - Evergreen & 2.7 & 2.4 \\
\hline California mixed evergreen forest & Chrysolepis chrysophylla & Tree - Evergreen & 3.2 & 2.2 \\
\hline Montane chaparra & Chrysolepis sempervirens & Shrub - Evergreen & 2.7 & 2.4 \\
\hline Marl everglades & Cladium mariscus & Graminoid - $\mathrm{C}_{3}$ & 3.6 & 3.6 \\
\hline Everglades & Cladium mariscus & Graminoid - $\mathrm{C}_{3}$ & 3.6 & 3.6 \\
\hline Black-brush & Coleogyne ramosissima & Shrub - Deciduous & 2.7 & 3 \\
\hline Fescue - oatgrass & Danthonia californica & Graminoid - $C_{3}$ & 3.6 & 3.6 \\
\hline Alpine meadows and barren & Deschampsia caespitosa & Graminoid $-\mathrm{C}_{3}$ & 3.6 & 3.6 \\
\hline Fescue - oatgrass & Deschampsia holciformis & Graminoid - $\mathrm{C}_{3}$ & 3.6 & 3.6 \\
\hline Northern cordgrass prairie & Distichlis spicata & Graminoid - $\mathrm{C}_{4}$ & 3.6 & 4.1 \\
\hline
\end{tabular}




\begin{tabular}{|c|c|c|c|c|}
\hline Community & Species & Classification & Resistance & Resilience \\
\hline Galleta - three awn shrub-steppe & Ephedra viridis & Shrub - Evergreen & 2.7 & 2.4 \\
\hline Coastal sagebrush & Eriogonum fasciculatum & Shrub - Evergreen & 2.7 & 2.4 \\
\hline Beech - maple forest & Fagus grandifolia & Tree - Deciduous & 3 & 4.2 \\
\hline Mixed mesophytic forest & Fagus grandifolia & Tree - Deciduous & 3 & 4.2 \\
\hline Northern hardwoods & Fagus grandifolia & Tree - Deciduous & 3 & 4.2 \\
\hline Northern hardwood - spruce forest & Fagus grandifolia & Tree - Deciduous & 3 & 4.2 \\
\hline Southern mixed forest & Fagus grandifolia & Tree - Deciduous & 3 & 4.2 \\
\hline Fescue - oatgrass & Festuca idahoensis & Graminoid - $\mathrm{C}_{3}$ & 3.6 & 3.6 \\
\hline Fescue - wheatgrass & Festuca idahoensis & Graminoid - $\mathrm{C}_{3}$ & 3.6 & 3.6 \\
\hline Wheatgrass bluegrass & Festuca idahoensis & Graminoid - $C_{3}$ & 3.6 & 3.6 \\
\hline Foothills prairie & Festuca idahoensis & Graminoid - $\mathrm{C}_{3}$ & 3.6 & 3.6 \\
\hline Foothills prairie & Festuca scabrella & Graminoid - $\mathrm{C}_{3}$ & 3.6 & 3.6 \\
\hline Alpine meadows and barren & Festuca viridula & Graminoid - $\mathrm{C}_{3}$ & 3.6 & 3.6 \\
\hline Creosote bush - tarbush & Flourensia cernua & Shrub - Deciduous & 2.7 & 3 \\
\hline Trans - pecos shrub savanna & Flourensia cernua & Shrub - Deciduous & 2.7 & 3 \\
\hline Alder - ash forest & Fraxinus latifolia & Tree - Deciduous & 3 & 4.2 \\
\hline Elm - ash forest & Fraxinus pennsylvanica & Tree-Deciduous & 3 & 4.2 \\
\hline Pine - cypress forest & Hesperocyparis goveniana & Tree - Evergreen & 3.2 & 2.2 \\
\hline Pine - cypress forest & Hesperocyparis macrocarpa & Tree - Evergreen & 3.2 & 2.2 \\
\hline Wheatgrass - needlegrass shrub-steppe & Hesperostipa comata & Graminoid - $\mathrm{C}_{3}$ & 3.6 & 3.6 \\
\hline Foothills prairie & Hesperostipa comata & Graminoid - $\mathrm{C}_{3}$ & 3.6 & 3.6 \\
\hline Grama - needlegrass - wheatgrass & Hesperostipa comata & Graminoid $-\mathrm{C}_{3}$ & 3.6 & 3.6 \\
\hline Wheatgrass - needlegrass & Hesperostipa comata & Graminoid $-\mathrm{C}_{3}$ & 3.6 & 3.6 \\
\hline Nebraska sandhills prairie & Hesperostipa comata & Graminoid - $\mathrm{C}_{3}$ & 3.6 & 3.6 \\
\hline Wheatgrass - bluestem - needlegrass & Hesperostipa spartea & Graminoid - $\mathrm{C}_{3}$ & 3.6 & 3.6 \\
\hline Mesquite savanna & Hilaria belangeri & Graminoid - $\mathrm{C}_{4}$ & 3.6 & 4.1 \\
\hline Grama - galleta steppe & Hilaria jamesii & Graminoid - $\mathrm{C}_{4}$ & 3.6 & 4.1 \\
\hline Galleta - three awn shrub-steppe & Hilaria jamesii & Graminoid - $\mathrm{C}_{4}$ & 3.6 & 4.1 \\
\hline Grama - tobosa prairie & Hilaria mutica & Graminoid - $\mathrm{C}_{4}$ & 3.6 & 4.1 \\
\hline Grama - tobosa shrub-steppe & Hilaria mutica & Graminoid - $\mathrm{C}_{4}$ & 3.6 & 4.1 \\
\hline Mesquite savanna & Hilaria mutica & Graminoid - $\mathrm{C}_{4}$ & 3.6 & 4.1 \\
\hline Pocosin & Ilex glabra & Shrub - Evergreen & 2.7 & 2.4 \\
\hline Juniper - oak savanna & Juniperus ashei & Tree - Evergreen & 3.2 & 2.2 \\
\hline Oak-juniper woodland & Juniperus deppeana & Tree - Evergreen & 3.2 & 2.2 \\
\hline Juniper - pinyon woodland & Juniperus monosperma & Tree - Evergreen & 3.2 & 2.2 \\
\hline Oak - juniper woodland & Juniperus monosperma & Tree - Evergreen & 3.2 & 2.2 \\
\hline Juniper steppe woodland & Juniperus occidentalis & Tree - Evergreen & 3.2 & 2.2 \\
\hline Juniper - pinyon woodland & Juniperus osteosperma & Tree - Evergreen & 3.2 & 2.2 \\
\hline Cedar glades & Juniperus virginiana & Tree - Evergreen & 3.2 & 2.2 \\
\hline Blackbelt & Juniperus virginiana & Tree - Evergreen & 3.2 & 2.2 \\
\hline Conifer bog & Larix laricina & Tree - Evergreen & 3.2 & 2.2 \\
\hline Creosote bush & Larrea divaricata & Shrub - Evergreen & 2.7 & 2.4 \\
\hline Creosote bush - bur sage & Larrea divaricata & Shrub - Evergreen & 2.7 & 2.4 \\
\hline Creosote bush - tarbush & Larrea divaricata & Shrub - Evergreen & 2.7 & 2.4 \\
\hline Ceniza shrub & Larrea divaricata & Shrub - Evergreen & 2.7 & 2.4 \\
\hline Grama - tobosa shrub-steppe & Larrea divaricata & Shrub - Evergreen & 2.7 & 2.4 \\
\hline Trans - pecos shrub savanna & Larrea divaricata & Shrub - Evergreen & 2.7 & 2.4 \\
\hline
\end{tabular}




\begin{tabular}{|c|c|c|c|c|}
\hline Community & Species & Classification & Resistance & Resilience \\
\hline Ceniza shrub & Leucophyllum frutescens & Shrub - Evergreen & 2.7 & 2.4 \\
\hline Mixed conifer forest & Libocedrus decurrens & Tree - Evergreen & 3.2 & 2.2 \\
\hline Southern mixed forest & Liquidambar styraciflua & Tree - Deciduous & 3 & 4.2 \\
\hline Blackbelt & Liquidambar styraciflua & Tree - Deciduous & 3 & 4.2 \\
\hline Mixed mesophytic forest & Liriodendron tulipifera & Tree - Deciduous & 3 & 4.2 \\
\hline California mixed evergreen forest & Lithocarpus densiflorus & Shrub - Evergreen & 2.7 & 2.4 \\
\hline Alpine meadows and barren & Luzula spicata & Graminoid - $C_{3}$ & 3.6 & 3.6 \\
\hline Southern mixed forest & Magnolia grandiflora & Tree - Evergreen & 3.2 & 2.2 \\
\hline Everglades & Magnolia virginiana & Tree - Evergreen & 3.2 & 2.2 \\
\hline California steppe & Nassela cernua & Graminoid $-C_{3}$ & 3.6 & 3.6 \\
\hline Blackland prairie & Nassela leucotricha & Graminoid - $C_{3}$ & 3.6 & 3.6 \\
\hline California steppe & Nassela pulchra & Graminoid $-C_{3}$ & 3.6 & 3.6 \\
\hline Wheatgrass - needlegrass & Nassela viridula & Graminoid - $C_{3}$ & 3.6 & 3.6 \\
\hline Southern floodplain forest & Nyssa aquatica & Tree - Deciduous & 3 & 4.2 \\
\hline Palo verde - cactus shrub & Opuntia spp. & Forb & 3 & 3.5 \\
\hline Bluestem prairie & Panicum virgatum & Graminoid - $\mathrm{C}_{4}$ & 3.6 & 4.1 \\
\hline Palo verde - cactus shrub & Parkinsonia microphyllum & Shrub - Deciduous & 2.7 & 3 \\
\hline Wheatgrass - needlegrass shrub-steppe & Pascopyrum smithii & Graminoid - $C_{3}$ & 3.6 & 3.6 \\
\hline Grama - needlegrass - wheatgrass & Pascopyrum smithii & Graminoid - $\mathrm{C}_{3}$ & 3.6 & 3.6 \\
\hline Wheatgrass - needlegrass & Pascopyrum smithii & Graminoid - $C_{3}$ & 3.6 & 3.6 \\
\hline Wheatgrass - bluestem - needlegrass & Pascopyrum smithii & Graminoid - $\mathrm{C}_{3}$ & 3.6 & 3.6 \\
\hline Wheatgrass - grama - buffalo grass & Pascopyrum smithii & Graminoid - $C_{3}$ & 3.6 & 3.6 \\
\hline Marl everglades & Persea borbonia & Shrub - Evergreen & 2.7 & 2.4 \\
\hline Everglades & Persea borbonia & Shrub - Evergreen & 2.7 & 2.4 \\
\hline Alpine meadows and barren & Phleum alpinum & Graminoid - $\mathrm{C}_{3}$ & 3.6 & 3.6 \\
\hline Western spruce - fir forest & Picea engelmannii & Tree - Evergreen & 3.2 & 2.2 \\
\hline Southwestern spruce - fir forest & Picea engelmannii & Tree - Evergreen & 3.2 & 2.2 \\
\hline Great lakes spruce - fir forest & Picea glauca & Tree - Evergreen & 3.2 & 2.2 \\
\hline Conifer bog & Picea mariana & Tree - Evergreen & 3.2 & 2.2 \\
\hline Spruce - fir - Douglas-fir forest & Picea pungens & Tree - Evergreen & 3.2 & 2.2 \\
\hline Northern hardwood - spruce forest & Picea rubens & Tree - Evergreen & 3.2 & 2.2 \\
\hline Northeastern spruce - fir forest & Picea rubens & Tree - Evergreen & 3.2 & 2.2 \\
\hline Northeastern spruce - fir forest & Picea rubens & Tree - Evergreen & 3.2 & 2.2 \\
\hline Spruce - cedar - hemlock forest & Picea sitchensis & Tree - Evergreen & 3.2 & 2.2 \\
\hline Lodgepole pine - subalpine forest & Pinus albicaulis & Tree - Evergreen & 3.2 & 2.2 \\
\hline Lodgepole pine - subalpine forest & Pinus alfouriana & Tree - Evergreen & 3.2 & 2.2 \\
\hline Great Basin pine forest & Pinus aristata & Tree - Evergreen & 3.2 & 2.2 \\
\hline Great lakes pine forest & Pinus banksiana & Tree - Evergreen & 3.2 & 2.2 \\
\hline Sand pine scrub & Pinus clausa & Tree - Evergreen & 3.2 & 2.2 \\
\hline Lodgepole pine - subalpine forest & Pinus contorta & Tree - Evergreen & 3.2 & 2.2 \\
\hline Pine - cypress forest & Pinus contorta & Tree - Evergreen & 3.2 & 2.2 \\
\hline California oak - woods & Pinus Coulteri & Tree - Evergreen & 3.2 & 2.2 \\
\hline Oak - hickory - pine forest & Pinus echinata & Tree - Evergreen & 3.2 & 2.2 \\
\hline Juniper - pinyon woodland & Pinus edulis & Tree - Evergreen & 3.2 & 2.2 \\
\hline Sub - tropical pine forest & Pinus elliottii & Tree - Evergreen & 3.2 & 2.2 \\
\hline Great Basin pine forest & Pinus flexilis & Tree - Evergreen & 3.2 & 2.2 \\
\hline Mixed conifer forest & Pinus lambertiana & Tree - Evergreen & 3.2 & 2.2 \\
\hline
\end{tabular}




\begin{tabular}{|c|c|c|c|c|}
\hline Community & Species & Classification & Resistance & Resilience \\
\hline Juniper - pinyon woodland & Pinus monophylla & Tree - Evergreen & 3.2 & 2.2 \\
\hline Cedar - hemlock - pine forest & Pinus monticola & Tree - Evergreen & 3.2 & 2.2 \\
\hline Pine - cypress forest & Pinus muricata & Tree - Evergreen & 3.2 & 2.2 \\
\hline Ponderosa - shrub forest & Pinus ponderosa & Tree - Evergreen & 3.2 & 2.2 \\
\hline Western ponderosa forest & Pinus ponderosa & Tree - Evergreen & 3.2 & 2.2 \\
\hline Eastern ponderosa forest & Pinus ponderosa & Tree - Evergreen & 3.2 & 2.2 \\
\hline Black Hills pine forest & Pinus ponderosa & Tree - Evergreen & 3.2 & 2.2 \\
\hline Pine - Douglas-fir forest & Pinus ponderosa & Tree - Evergreen & 3.2 & 2.2 \\
\hline Arizona pine forest & Pinus ponderosa & Tree - Evergreen & 3.2 & 2.2 \\
\hline Mixed conifer forest & Pinus ponderosa & Tree - Evergreen & 3.2 & 2.2 \\
\hline Pine - cypress forest & Pinus radiata & Tree - Evergreen & 3.2 & 2.2 \\
\hline Great lakes pine forest & Pinus resinosa & Tree - Evergreen & 3.2 & 2.2 \\
\hline Northeastern oak - pine forest & Pinus rigida & Tree - Evergreen & 3.2 & 2.2 \\
\hline California oak - woods & Pinus sabiniana & Tree - Evergreen & 3.2 & 2.2 \\
\hline Pocosin & Pinus serotina & Tree - Evergreen & 3.2 & 2.2 \\
\hline Great lakes pine forest & Pinus strobus & Tree - Evergreen & 3.2 & 2.2 \\
\hline Oak - hickory - pine forest & Pinus taeda & Tree - Evergreen & 3.2 & 2.2 \\
\hline Southern mixed forest & Pinus taeda & Tree - Evergreen & 3.2 & 2.2 \\
\hline Wheatgrass - needlegrass shrub-steppe & Poa arida & Graminoid - $\mathrm{C}_{3}$ & 3.6 & 3.6 \\
\hline Wheatgrass bluegrass & Poa secunda & Graminoid - $C_{3}$ & 3.6 & 3.6 \\
\hline Alpine meadows and barren & Poa spp. & Graminoid $-\mathrm{C}_{3}$ & 3.6 & 3.6 \\
\hline Northern floodplain forest & Populus deltoides & Tree - Deciduous & 3 & 4.2 \\
\hline Ceniza shrub & Prosopis juliflora & Shrub - Deciduous & 2.7 & 3 \\
\hline Mesquite savanna & Prosopis juliflora & Shrub - Deciduous & 2.7 & 3 \\
\hline Mesquite - acacia savanna & Prosopis juliflora & Shrub - Deciduous & 2.7 & 3 \\
\hline Mesquite - live oak savanna & Prosopis juliflora & Shrub - Deciduous & 2.7 & 3 \\
\hline Mesquite - buffalo grass & Prosopis juliflora & Shrub - Deciduous & 2.7 & 3 \\
\hline Mesquite - oak savanna & Prosopis juliflora & Shrub - Deciduous & 2.7 & 3 \\
\hline Mesquite bosques & Prosopis juliflora & Shrub - Deciduous & 2.7 & 3 \\
\hline Douglas-fir forest & Pseudotsuga menziesii & Tree - Evergreen & 3.2 & 2.2 \\
\hline Grand fir - Douglas-fir forest & Pseudotsuga menziesii & Tree - Evergreen & 3.2 & 2.2 \\
\hline Pine - Douglas-fir forest & Pseudotsuga menziesii & Tree - Evergreen & 3.2 & 2.2 \\
\hline Cedar - hemlock - Douglas-fir forest & Pseudotsuga menziesii & Tree - Evergreen & 3.2 & 2.2 \\
\hline Spruce - fir - Douglas-fir forest & Pseudotsuga menziesii & Tree - Evergreen & 3.2 & 2.2 \\
\hline California mixed evergreen forest & Pseudotsuga menziesii & Tree - Evergreen & 3.2 & 2.2 \\
\hline Silver fir - Douglas-fir forest & Pseudotsuga menziesii & Tree - Evergreen & 3.2 & 2.2 \\
\hline Mixed conifer forest & Pseudotsuga menziesii & Tree - Evergreen & 3.2 & 2.2 \\
\hline Redwood forest & Pseudotsuga menziesii & Tree - Evergreen & 3.2 & 2.2 \\
\hline Juniper steppe woodland & Psuedoroegneria spicata & Graminoid - $\mathrm{C}_{3}$ & 3.6 & 3.6 \\
\hline Fescue - wheatgrass & Psuedoroegneria spicata & Graminoid - $\mathrm{C}_{3}$ & 3.6 & 3.6 \\
\hline Wheatgrass bluegrass & Psuedoroegneria spicata & Graminoid $-\mathrm{C}_{3}$ & 3.6 & 3.6 \\
\hline Sagebrush steppe & Psuedoroegneria spicata & Graminoid $-C_{3}$ & 3.6 & 3.6 \\
\hline Foothills prairie & Psuedoroegneria spicata & Graminoid - $\mathrm{C}_{3}$ & 3.6 & 3.6 \\
\hline California oak - woods & Quercus agrifolia & Tree - Evergreen & 3.2 & 2.2 \\
\hline Oak - hickory forest & Quercus alba & Tree - Deciduous & 3 & 4.2 \\
\hline Mixed mesophytic forest & Quercus alba & Tree - Deciduous & 3 & 4.2 \\
\hline Appalachian oak forest & Quercus alba & Tree-Deciduous & 3 & 4.2 \\
\hline
\end{tabular}




\begin{tabular}{|c|c|c|c|c|}
\hline Community & Species & Classification & Resistance & Resilience \\
\hline Oak - hickory - pine forest & Quercus alba & Tree - Deciduous & 3 & 4.2 \\
\hline Southern mixed forest & Quercus alba & Tree - Deciduous & 3 & 4.2 \\
\hline Sand pine scrub & Quercus chapmanii & Tree - Evergreen & 3.2 & 2.2 \\
\hline California mixed evergreen forest & Quercus chrysolepis & Tree - Evergreen & 3.2 & 2.2 \\
\hline California oak - woods & Quercus chrysolepis & Tree - Evergreen & 3.2 & 2.2 \\
\hline Northeastern oak - pine forest & Quercus coccinea & Tree - Deciduous & 3 & 4.2 \\
\hline California oak - woods & Quercus douglasii & Tree - Deciduous & 3 & 4.2 \\
\hline Oak - juniper woodland & Quercus emoryi & Tree - Evergreen & 3.2 & 2.2 \\
\hline Mountain-mahogany - oak scrub & Quercus gambelii & Shrub - Deciduous & 2.7 & 3 \\
\hline Oregon oak woods & Quercus garryana & Tree - Deciduous & 3 & 4.2 \\
\hline Southern mixed forest & Quercus laurifolia & Tree - Evergreen & 3.2 & 2.2 \\
\hline California oak - woods & Quercus lobata & Tree - Deciduous & 3 & 4.2 \\
\hline Oak savanna & Quercus macrocarpa & Tree - Deciduous & 3 & 4.2 \\
\hline Cross timbers & Quercus marilandica & Tree - Deciduous & 3 & 4.2 \\
\hline Shinnery & Quercus mohriana & Tree - Evergreen & 3.2 & 2.2 \\
\hline Sand pine scrub & Quercus myrtifolia & Tree - Evergreen & 3.2 & 2.2 \\
\hline Oak - juniper woodland & Quercus oblongifolia & Tree - Evergreen & 3.2 & 2.2 \\
\hline Oak - hickory forest & Quercus rubra & Tree - Deciduous & 3 & 4.2 \\
\hline Mixed mesophytic forest & Quercus rubra & Tree - Deciduous & 3 & 4.2 \\
\hline Appalachian oak forest & Quercus rubra & Tree - Deciduous & 3 & 4.2 \\
\hline Southern floodplain forest & Quercus spp. & Tree - Deciduous & 3 & 4.2 \\
\hline Mesquite - oak savanna & Quercus spp. & Tree - Deciduous & 3 & 4.2 \\
\hline Oak - hickory - pine forest & Quercus stellata & Tree - Deciduous & 3 & 4.2 \\
\hline Cedar glades & Quercus stellata & Tree - Deciduous & 3 & 4.2 \\
\hline Cross timbers & Quercus stellata & Tree - Deciduous & 3 & 4.2 \\
\hline Blackbelt & Quercus stellata & Tree - Deciduous & 3 & 4.2 \\
\hline Oak - hickory forest & Quercus velutina & Tree - Deciduous & 3 & 4.2 \\
\hline Northeastern oak - pine forest & Quercus velutina & Tree - Deciduous & 3 & 4.2 \\
\hline Juniper - oak savanna & Quercus virginiana & Tree - Evergreen & 3.2 & 2.2 \\
\hline Sand pine scrub & Quercus virginiana & Tree - Evergreen & 3.2 & 2.2 \\
\hline Mesquite - live oak savanna & Quercus virginiana & Tree - Evergreen & 3.2 & 2.2 \\
\hline Live oak - sea oats & Quercus virginiana & Tree - Evergreen & 3.2 & 2.2 \\
\hline California mixed evergreen forest & Quercus wislizenii & Tree - Evergreen & 3.2 & 2.2 \\
\hline California oak - woods & Quercus wislizenii & Tree - Evergreen & 3.2 & 2.2 \\
\hline Mangrove & Rhizophora mangle & Shrub - Evergreen & 2.7 & 2.4 \\
\hline Northern floodplain forest & Salix nigra & Shrub - Deciduous & 2.7 & 3 \\
\hline Coastal sagebrush & Salvia apiana & Shrub & 2.7 & 2.8 \\
\hline Coastal sagebrush & Salvia mellifera & Shrub - Deciduous & 2.7 & 3 \\
\hline Saltbush - greasewood & Sarcobatus vermiculatus & Shrub-Deciduous & 2.7 & 3 \\
\hline Bluestem - grama prairie & Schizachyrium scoparium & Graminoid- $\mathrm{C}_{4}$ & 3.6 & 4.1 \\
\hline Sandsage - bluestem prairie & Schizachyrium scoparium & Graminoid - $\mathrm{C}_{4}$ & 3.6 & 4.1 \\
\hline Shinnery & Schizachyrium scoparium & Graminoid - $\mathrm{C}_{4}$ & 3.6 & 4.1 \\
\hline Bluestem prairie & Schizachyrium scoparium & Graminoid - $\mathrm{C}_{4}$ & 3.6 & 4.1 \\
\hline Nebraska sandhills prairie & Schizachyrium scoparium & Graminoid - $\mathrm{C}_{4}$ & 3.6 & 4.1 \\
\hline Blackland prairie & Schizachyrium scoparium & Graminoid - $\mathrm{C}_{4}$ & 3.6 & 4.1 \\
\hline Oak savanna & Schizachyrium scoparium & Graminoid - $\mathrm{C}_{4}$ & 3.6 & 4.1 \\
\hline Cross timbers & Schizachyrium scoparium & Graminoid - $\mathrm{C}_{4}$ & 3.6 & 4.1 \\
\hline
\end{tabular}




\begin{tabular}{|c|c|c|c|c|}
\hline Community & Species & Classification & Resistance & Resilience \\
\hline Juniper - oak savanna & Schizachyrium scoparium & Graminoid - $\mathrm{C}_{4}$ & 3.6 & 4.1 \\
\hline Mesquite - oak savanna & Schizachyrium scoparium & Graminoid - $\mathrm{C}_{4}$ & 3.6 & 4.1 \\
\hline Fayette prairie & Schizachyrium scoparium & Graminoid - $\mathrm{C}_{4}$ & 3.6 & 4.1 \\
\hline Tule marshes & Scirpus acutus & Graminoid - $C_{3}$ & 3.6 & 3.6 \\
\hline Tule marshes & Scirpus californicus & Graminoid - $C_{3}$ & 3.6 & 3.6 \\
\hline Tule marshes & Scirpus olneyi & Graminoid $-C_{3}$ & 3.6 & 3.6 \\
\hline Tule marshes & Scirpus validus & Graminoid $-C_{3}$ & 3.6 & 3.6 \\
\hline Redwood forest & Sequoia sempervirens & Tree - Evergreen & 3.2 & 2.2 \\
\hline Palmetto prairie & Serenoa repens & Shrub - Deciduous & 2.7 & 3 \\
\hline Mesquite - acacia savanna & Setaria macrostachya & Graminoid - $\mathrm{C}_{4}$ & 3.6 & 4.1 \\
\hline Bluestem prairie & Sorghastrum nutans & Graminoid - $\mathrm{C}_{4}$ & 3.6 & 4.1 \\
\hline Northern cordgrass prairie & Spartina alterniflora & Graminoid - $\mathrm{C}_{4}$ & 3.6 & 4.1 \\
\hline Southern cordgrass prairie & Spartina alterniflora & Graminoid - $\mathrm{C}_{4}$ & 3.6 & 4.1 \\
\hline Northern cordgrass prairie & Spartina patens & Graminoid - $\mathrm{C}_{4}$ & 3.6 & 4.1 \\
\hline Bluestem - sacahuista prairie & Spartina spartinae & Graminoid - $\mathrm{C}_{4}$ & 3.6 & 4.1 \\
\hline Cedar glades & Sporobolus neglectus & Graminoid - $\mathrm{C}_{4}$ & 3.6 & 4.1 \\
\hline Southern floodplain forest & Taxodium distichum & Tree - Evergreen & 3.2 & 2.2 \\
\hline Marl everglades & Taxodium distichum & Tree - Evergreen & 3.2 & 2.2 \\
\hline Cypress savanna & Taxodium distichum & Tree - Evergreen & 3.2 & 2.2 \\
\hline Conifer bog & Thuja occidentalis & Tree - Evergreen & 3.2 & 2.2 \\
\hline Spruce - cedar- hemlock forest & Thuja plicata & Tree - Evergreen & 3.2 & 2.2 \\
\hline Cedar - hemlock - pine forest & Thuja plicata & Tree - Evergreen & 3.2 & 2.2 \\
\hline Cedar - hemlock - Douglas-fir forest & Thuja plicata & Tree-Evergreen & 3.2 & 2.2 \\
\hline Maple - basswood forest & Tilia americana & Tree - Deciduous & 3 & 4.2 \\
\hline Mixed mesophytic forest & Tilia heterophylla & Tree - Deciduous & 3 & 4.2 \\
\hline Alpine meadows and barren & Trisetum spicatum & Graminoid $-C_{3}$ & 3.6 & 3.6 \\
\hline Northern hardwoods & Tsuga canadensis & Tree - Evergreen & 3.2 & 2.2 \\
\hline Northern hardwoods - fir forest & Tsuga canadensis & Tree - Evergreen & 3.2 & 2.2 \\
\hline Northern hardwood - spruce forest & Tsuga canadensis & Tree - Evergreen & 3.2 & 2.2 \\
\hline Spruce - cedar - hemlock forest & Tsuga heterophylla & Tree - Evergreen & 3.2 & 2.2 \\
\hline Cedar - hemlock - pine forest & Tsuga heterophylla & Tree - Evergreen & 3.2 & 2.2 \\
\hline Cedar-hemlock - Douglas-fir forest & Tsuga heterophylla & Tree - Evergreen & 3.2 & 2.2 \\
\hline Fir - hemlock forest & Tsuga mertensiana & Tree - Evergreen & 3.2 & 2.2 \\
\hline Lodgepole pine - subalpine forest & Tsuga mertensiana & Tree-Evergreen & 3.2 & 2.2 \\
\hline Tule marshes & Typha domingensis & Graminoid $-\mathrm{C}_{3}$ & 3.6 & 3.6 \\
\hline Tule marshes & Typha latifolia & Graminoid - $C_{3}$ & 3.6 & 3.6 \\
\hline Cedar glades & Ulmus alata & Tree - Deciduous & 3 & 4.2 \\
\hline Elm - ash forest & Ulmus americana & Tree - Deciduous & 3 & 4.2 \\
\hline Northern floodplain forest & Ulmus americana & Tree - Deciduous & 3 & 4.2 \\
\hline California mixed evergreen forest & Umbellularia californica & Tree - Evergreen & 3.2 & 2.2 \\
\hline Sea oats prairie & Uniola paniculata & Graminoid - $\mathrm{C}_{4}$ & 3.6 & 4.1 \\
\hline Live oak - sea oats & Uniola paniculata & Graminoid - $\mathrm{C}_{4}$ & 3.6 & 4.1 \\
\hline
\end{tabular}




\section{Appendix D: Resistance and Resilience Values from USGS National Vegetation Classification Community Types}

Table D-1. Resistance and resilience values from USGS National Vegetation Classification Community Types.

\begin{tabular}{|c|c|c|c|c|c|}
\hline Community & \begin{tabular}{c|} 
Mean \\
Resistance
\end{tabular} & $\begin{array}{l}\text { Scaled } \\
\text { Resistance }\end{array}$ & $\begin{array}{c}\text { Mean } \\
\text { Resilience }\end{array}$ & $\begin{array}{c}\text { Scaled } \\
\text { Resilience }\end{array}$ & $\begin{array}{c}\text { Net } \\
\text { Persistence }\end{array}$ \\
\hline Acadian Coastal Salt Marsh & 3.60 & 1.00 & 3.98 & 0.89 & 1.89 \\
\hline Acadian Estuary Marsh & 3.60 & 1.00 & 3.80 & 0.80 & 1.80 \\
\hline Acadian Low-Elevation Spruce-Fir-Hardwood Forest & 3.10 & 0.35 & 3.03 & 0.41 & 0.76 \\
\hline Acadian-Appalachian Alpine Tundra & 2.96 & 0.17 & 3.03 & 0.42 & 0.58 \\
\hline Acadian-Appalachian Montane Spruce-Fir Forest & 3.13 & 0.39 & 2.87 & 0.34 & 0.72 \\
\hline Acadian-Appalachian Subalpine Woodland and Heath-Krummholz & 3.07 & 0.31 & 2.90 & 0.35 & 0.66 \\
\hline Agriculture - Cultivated Crops and Irrigated Agriculture & 0.00 & 0.00 & 0.00 & 0.00 & 0.00 \\
\hline Agriculture - Pasture/Hay & 0.00 & 0.00 & 0.00 & 0.00 & 0.00 \\
\hline Allegheny-Cumberland Dry Oak Forest and Woodland & 3.02 & 0.25 & 3.98 & 0.89 & 1.14 \\
\hline Apacherian-Chihuahuan Mesquite Upland Scrub & 3.05 & 0.29 & 3.31 & 0.56 & 0.84 \\
\hline Apacherian-Chihuahuan Semi-Desert Grassland and Steppe & 3.60 & 1.00 & 4.10 & 0.95 & 1.95 \\
\hline Appalachian (Hemlock)-Northern Hardwood Forest & 3.02 & 0.25 & 3.98 & 0.89 & 1.14 \\
\hline Appalachian Shale Barrens & 3.10 & 0.35 & 3.20 & 0.50 & 0.85 \\
\hline Arkansas Valley Prairie and Woodland & 3.48 & 0.84 & 4.12 & 0.96 & 1.80 \\
\hline Atlantic Coastal Plain Blackwater Stream Floodplain Forest & 3.13 & 0.39 & 2.87 & 0.34 & 0.72 \\
\hline Atlantic Coastal Plain Brownwater Stream Floodplain Forest & 3.13 & 0.39 & 2.87 & 0.34 & 0.72 \\
\hline Atlantic Coastal Plain Clay-Based Carolina Bay Wetland & 3.50 & 0.87 & 3.25 & 0.53 & 1.40 \\
\hline Atlantic Coastal Plain Embayed Region Tidal Freshwater Marsh & 3.60 & 1.00 & 3.68 & 0.74 & 1.74 \\
\hline Atlantic Coastal Plain Embayed Region Tidal Salt and Brackish Marsh & 3.60 & 1.00 & 3.93 & 0.87 & 1.87 \\
\hline Atlantic Coastal Plain Fall-line Sandhills Longleaf Pine Woodland & 3.20 & 0.48 & 3.89 & 0.85 & 1.33 \\
\hline $\begin{array}{l}\text { Atlantic Coastal Plain Fall-line Sandhills Longleaf Pine Woodland - Offsite } \\
\text { Hardwood Modifier }\end{array}$ & 3.04 & 0.27 & 3.80 & 0.80 & 1.07 \\
\hline Atlantic Coastal Plain Indian River Lagoon Tidal Marsh & 3.38 & 0.71 & 3.55 & 0.68 & 1.39 \\
\hline Atlantic Coastal Plain Small Blackwater River Floodplain Forest & 2.98 & 0.19 & 3.49 & 0.65 & 0.84 \\
\hline Atlantic Coastal Plain Small Brownwater River Floodplain Forest & 3.04 & 0.27 & 3.80 & 0.80 & 1.07 \\
\hline Atlantic Coastal Plain Streamhead Seepage Swamp, Pocosin and Baygall & 2.89 & 0.08 & 2.83 & 0.32 & 0.39 \\
\hline Atlantic Coastal Plain Upland Longleaf Pine Woodland & 3.23 & 0.52 & 3.53 & 0.67 & 1.18 \\
\hline Bastrop Lost Pines Forest and Woodland & 3.07 & 0.31 & 3.53 & 0.67 & 0.98 \\
\hline Boreal White Spruce-Fir-Hardwood Forest & 3.13 & 0.39 & 2.87 & 0.34 & 0.72 \\
\hline Boreal-Laurentian Bog & 2.87 & 0.05 & 2.47 & 0.14 & 0.19 \\
\hline Boreal-Laurentian Conifer Acidic Swamp and Treed Poor Fen & 3.18 & 0.45 & 2.70 & 0.25 & 0.70 \\
\hline California Annual Grassland & 0.00 & 0.00 & 0.00 & 0.00 & 0.00 \\
\hline California Central Valley and Southern Coastal Grassland & 3.60 & 1.00 & 3.60 & 0.70 & 1.70 \\
\hline California Central Valley Mixed Oak Savanna & 3.26 & 0.56 & 2.88 & 0.34 & 0.90 \\
\hline California Coastal Closed-Cone Conifer Forest and Woodland & 3.20 & 0.48 & 2.20 & 0.00 & 0.48 \\
\hline California Coastal Live Oak Woodland and Savanna & 2.90 & 0.09 & 2.64 & 0.22 & 0.31 \\
\hline California Coastal Redwood Forest & 3.20 & 0.48 & 2.20 & 0.00 & 0.48 \\
\hline California Lower Montane Blue Oak-Foothill Pine Woodland and Savanna & 3.07 & 0.31 & 3.53 & 0.67 & 0.98 \\
\hline
\end{tabular}




\begin{tabular}{|c|c|c|c|c|c|}
\hline Community & $\begin{array}{c}\text { Mean } \\
\text { Resistance }\end{array}$ & $\begin{array}{c}\text { Scaled } \\
\text { Resistance }\end{array}$ & $\begin{array}{c}\text { Mean } \\
\text { Resilience }\end{array}$ & $\begin{array}{c}\text { Scaled } \\
\text { Resilience }\end{array}$ & $\begin{array}{c}\text { Net } \\
\text { Persistence }\end{array}$ \\
\hline California Mesic Chaparral & 2.89 & 0.08 & 2.52 & 0.16 & 0.24 \\
\hline California Mesic Serpentine Grassland & 3.40 & 0.74 & 3.57 & 0.69 & 1.43 \\
\hline California Montane Jeffrey Pine-(Ponderosa Pine) Woodland & 3.20 & 0.48 & 2.20 & 0.00 & 0.48 \\
\hline California Northern Coastal Grassland & 3.60 & 1.00 & 3.60 & 0.70 & 1.70 \\
\hline Central and South Texas Coastal Fringe Forest and Woodland & 3.28 & 0.58 & 3.62 & 0.71 & 1.29 \\
\hline Central and Southern Appalachian Montane Oak Forest & 3.19 & 0.47 & 3.75 & 0.78 & 1.24 \\
\hline Central and Southern Appalachian Spruce-Fir Forest & 2.95 & 0.16 & 2.60 & 0.20 & 0.36 \\
\hline Central and Southern California Mixed Evergreen Woodland & 3.10 & 0.35 & 3.20 & 0.50 & 0.85 \\
\hline Central Appalachian Alkaline Glade and Woodland & 3.23 & 0.52 & 3.83 & 0.82 & 1.33 \\
\hline Central Appalachian Dry Oak-Pine Forest & 3.07 & 0.31 & 2.80 & 0.30 & 0.61 \\
\hline Central Appalachian Pine-Oak Rocky Woodland & 3.29 & 0.60 & 2.89 & 0.35 & 0.94 \\
\hline Central Appalachian River Floodplain & 2.93 & 0.13 & 3.85 & 0.83 & 0.95 \\
\hline Central Appalachian Stream and Riparian & 3.15 & 0.42 & 4.18 & 0.99 & 1.41 \\
\hline Central Atlantic Coastal Plain Maritime Forest & 2.96 & 0.17 & 2.93 & 0.37 & 0.53 \\
\hline Central Atlantic Coastal Plain Sandy Beach & 3.00 & 0.22 & 3.50 & 0.65 & 0.87 \\
\hline Central Atlantic Coastal Plain Wet Longleaf Pine Savanna and Flatwoods & 3.27 & 0.57 & 3.09 & 0.45 & 1.02 \\
\hline Central California Coast Ranges Cliff and Canyon & 3.20 & 0.48 & 2.20 & 0.00 & 0.48 \\
\hline Central Florida Herbaceous Pondshore & 3.48 & 0.84 & 3.68 & 0.74 & 1.58 \\
\hline Central Florida Pine Flatwoods & 3.08 & 0.32 & 2.78 & 0.29 & 0.61 \\
\hline Central Florida Wet Prairie and Herbaceous Seep & 3.60 & 1.00 & 3.60 & 0.70 & 1.70 \\
\hline Central Interior Acidic Cliff and Talus & 3.60 & 1.00 & 3.60 & 0.70 & 1.70 \\
\hline Central Interior Calcareous Cliff and Talus & 3.27 & 0.57 & 3.27 & 0.54 & 1.11 \\
\hline Central Interior Highlands Calcareous Glade and Barrens & 3.20 & 0.48 & 3.68 & 0.74 & 1.22 \\
\hline Central Interior Highlands Dry Acidic Glade and Barrens & 3.23 & 0.52 & 3.83 & 0.82 & 1.33 \\
\hline Central Mixedgrass Prairie & 3.34 & 0.66 & 3.77 & 0.79 & 1.45 \\
\hline Central Tallgrass Prairie & 3.60 & 1.00 & 4.02 & 0.91 & 1.91 \\
\hline Chihuahuan Creosotebush Desert Scrub & 2.96 & 0.17 & 3.34 & 0.57 & 0.74 \\
\hline Chihuahuan Gypsophilous Grassland and Steppe & 3.21 & 0.49 & 3.49 & 0.65 & 1.14 \\
\hline Chihuahuan Loamy Plains Desert Grassland & 3.45 & 0.81 & 3.92 & 0.86 & 1.67 \\
\hline Chihuahuan Mixed Desert and Thornscrub & 2.85 & 0.03 & 3.15 & 0.48 & 0.50 \\
\hline Chihuahuan Mixed Salt Desert Scrub & 3.10 & 0.35 & 3.36 & 0.58 & 0.93 \\
\hline Chihuahuan Sandy Plains Semi-Desert Grassland & 3.60 & 1.00 & 4.02 & 0.91 & 1.91 \\
\hline Chihuahuan Stabilized Coppice Dune and Sand Flat Scrub & 3.00 & 0.22 & 3.37 & 0.59 & 0.81 \\
\hline Chihuahuan Succulent Desert Scrub & 3.00 & 0.22 & 3.50 & 0.65 & 0.87 \\
\hline Chihuahuan-Sonoran Desert Bottomland and Swale Grassland & 3.42 & 0.77 & 3.78 & 0.79 & 1.56 \\
\hline Clearcut - Grassland/Herbaceous & 0.00 & 0.00 & 0.00 & 0.00 & 0.00 \\
\hline Colorado Plateau Mixed Bedrock Canyon and Tableland & 3.08 & 0.32 & 2.25 & 0.02 & 0.35 \\
\hline Colorado Plateau Mixed Low Sagebrush Shrubland & 3.06 & 0.30 & 3.05 & 0.43 & 0.72 \\
\hline Colorado Plateau Pinyon-Juniper Shrubland & 2.95 & 0.16 & 2.45 & 0.13 & 0.28 \\
\hline Colorado Plateau Pinyon-Juniper Woodland & 2.96 & 0.17 & 2.59 & 0.20 & 0.36 \\
\hline Columbia Basin Foothill and Canyon Dry Grassland & 3.50 & 0.87 & 3.67 & 0.74 & 1.61 \\
\hline Columbia Basin Palouse Prairie & 3.60 & 1.00 & 3.60 & 0.70 & 1.70 \\
\hline Columbia Plateau Low Sagebrush Steppe & 2.85 & 0.03 & 2.70 & 0.25 & 0.28 \\
\hline Columbia Plateau Scabland Shrubland & 3.18 & 0.45 & 3.40 & 0.60 & 1.05 \\
\hline Columbia Plateau Silver Sagebrush Seasonally Flooded Shrub-Steppe & 3.21 & 0.49 & 3.16 & 0.48 & 0.97 \\
\hline Columbia Plateau Steppe and Grassland & 3.60 & 1.00 & 3.60 & 0.70 & 1.70 \\
\hline
\end{tabular}




\begin{tabular}{|c|c|c|c|c|c|}
\hline Community & $\begin{array}{c}\text { Mean } \\
\text { Resistance }\end{array}$ & $\begin{array}{c}\text { Scaled } \\
\text { Resistance }\end{array}$ & $\begin{array}{c}\text { Mean } \\
\text { Resilience }\end{array}$ & $\begin{array}{c}\text { Scaled } \\
\text { Resilience }\end{array}$ & $\begin{array}{c}\text { Net } \\
\text { Persistence }\end{array}$ \\
\hline Columbia Plateau Vernal Pool & 3.60 & 1.00 & 3.85 & 0.83 & 1.83 \\
\hline Columbia Plateau Western Juniper Woodland and Savanna & 3.05 & 0.29 & 2.65 & 0.23 & 0.51 \\
\hline Crosstimbers Oak Forest and Woodland & 3.13 & 0.39 & 3.85 & 0.83 & 1.21 \\
\hline Crowley's Ridge Mesic Loess Slope Forest & 3.00 & 0.22 & 4.20 & 1.00 & 1.22 \\
\hline Crowley's Ridge Sand Forest & 3.03 & 0.26 & 3.87 & 0.84 & 1.09 \\
\hline Cumberland Acidic Cliff and Rockhouse & 3.00 & 0.22 & 3.50 & 0.65 & 0.87 \\
\hline Cumberland Riverscour & 3.23 & 0.52 & 3.68 & 0.74 & 1.26 \\
\hline Cumberland Sandstone Glade and Barrens & 3.18 & 0.45 & 2.73 & 0.27 & 0.72 \\
\hline Developed-High Intensity & 0.00 & 0.00 & 0.00 & 0.00 & 0.00 \\
\hline Developed-Low Intensity & 0.00 & 0.00 & 0.00 & 0.00 & 0.00 \\
\hline Developed-Medium Intensity & 0.00 & 0.00 & 0.00 & 0.00 & 0.00 \\
\hline Developed-Open Space & 0.00 & 0.00 & 0.00 & 0.00 & 0.00 \\
\hline East Cascades Mesic Montane Mixed Conifer Forest and Woodland & 3.20 & 0.48 & 2.20 & 0.00 & 0.48 \\
\hline East Cascades Oak-Ponderosa Pine Forest and Woodland & 3.43 & 0.78 & 3.33 & 0.57 & 1.34 \\
\hline East Gulf Coastal Plain Depression Pond & 3.57 & 0.96 & 3.62 & 0.71 & 1.67 \\
\hline East Gulf Coastal Plain Dry Chalk Bluff & 3.60 & 1.00 & 3.60 & 0.70 & 1.70 \\
\hline East Gulf Coastal Plain Dune and Coastal Grassland & 3.08 & 0.32 & 3.38 & 0.59 & 0.91 \\
\hline East Gulf Coastal Plain Freshwater Tidal Wooded Swamp & 3.05 & 0.29 & 3.70 & 0.75 & 1.04 \\
\hline East Gulf Coastal Plain Interior Shortleaf Pine-Oak Forest & 3.10 & 0.35 & 3.20 & 0.50 & 0.85 \\
\hline East Gulf Coastal Plain Interior Upland Longleaf Pine Woodland & 3.43 & 0.78 & 3.80 & 0.80 & 1.58 \\
\hline East Gulf Coastal Plain Large River Floodplain Forest & 3.05 & 0.29 & 3.70 & 0.75 & 1.04 \\
\hline East Gulf Coastal Plain Maritime Forest & 3.03 & 0.26 & 2.73 & 0.27 & 0.52 \\
\hline East Gulf Coastal Plain Near-Coast Pine Flatwoods & 2.95 & 0.16 & 2.30 & 0.05 & 0.21 \\
\hline East Gulf Coastal Plain Northern Dry Upland Hardwood Forest & 3.00 & 0.22 & 4.20 & 1.00 & 1.22 \\
\hline East Gulf Coastal Plain Northern Loess Bluff Forest & 3.00 & 0.22 & 4.20 & 1.00 & 1.22 \\
\hline East Gulf Coastal Plain Northern Loess Plain Oak-Hickory Upland & 3.03 & 0.26 & 3.91 & 0.86 & 1.11 \\
\hline East Gulf Coastal Plain Northern Mesic Hardwood Slope Forest & 3.00 & 0.22 & 4.20 & 1.00 & 1.22 \\
\hline East Gulf Coastal Plain Savanna and Wet Prairie & 3.38 & 0.71 & 3.55 & 0.68 & 1.39 \\
\hline East Gulf Coastal Plain Small Stream and River Floodplain Forest & 2.85 & 0.03 & 3.60 & 0.70 & 0.73 \\
\hline East Gulf Coastal Plain Southern Loblolly-Hardwood Flatwoods & 2.92 & 0.12 & 3.18 & 0.49 & 0.61 \\
\hline East Gulf Coastal Plain Southern Loess Bluff Forest & 3.10 & 0.35 & 3.20 & 0.50 & 0.85 \\
\hline East-Central Texas Plains Post Oak Savanna and Woodland & 3.05 & 0.29 & 3.70 & 0.75 & 1.04 \\
\hline East-Central Texas Plains Xeric Sandyland & 3.30 & 0.61 & 4.15 & 0.98 & 1.59 \\
\hline Eastern Boreal Floodplain & 3.26 & 0.56 & 3.17 & 0.49 & 1.04 \\
\hline Eastern Great Plains Tallgrass Aspen Parkland & 3.18 & 0.45 & 3.82 & 0.81 & 1.26 \\
\hline Eastern Great Plains Wet Meadow, Prairie and Marsh & 3.60 & 1.00 & 3.77 & 0.79 & 1.79 \\
\hline Eastern Hemi-Boreal Aspen-Birch Forest & 3.00 & 0.22 & 4.20 & 1.00 & 1.22 \\
\hline Eastern Hemi-Boreal Dry-Mesic Pine-Black Spruce-Hardwood Forest & 3.13 & 0.39 & 2.87 & 0.34 & 0.72 \\
\hline Eastern Hemi-Boreal Mesic Balsam Fir-Spruce Forest & 3.20 & 0.48 & 2.20 & 0.00 & 0.48 \\
\hline Eastern Highland Rim Prairie and Barrens & 3.37 & 0.70 & 3.77 & 0.79 & 1.49 \\
\hline Eastern Serpentine Woodland & 3.28 & 0.58 & 3.66 & 0.73 & 1.31 \\
\hline Edwards Plateau Cliff & 3.05 & 0.29 & 3.15 & 0.48 & 0.76 \\
\hline Edwards Plateau Dry-Mesic Slope Forest and Woodland & 3.00 & 0.22 & 4.20 & 1.00 & 1.22 \\
\hline Edwards Plateau Floodplain Terrace & 3.12 & 0.38 & 3.88 & 0.84 & 1.22 \\
\hline Edwards Plateau Limestone Savanna and Woodland & 3.22 & 0.51 & 3.29 & 0.55 & 1.05 \\
\hline Edwards Plateau Limestone Shrubland & 2.96 & 0.17 & 2.58 & 0.19 & 0.36 \\
\hline
\end{tabular}




\begin{tabular}{|c|c|c|c|c|c|}
\hline Community & $\begin{array}{c}\text { Mean } \\
\text { Resistance }\end{array}$ & $\begin{array}{c}\text { Scaled } \\
\text { Resistance }\end{array}$ & $\begin{array}{c}\text { Mean } \\
\text { Resilience }\end{array}$ & $\begin{array}{c}\text { Scaled } \\
\text { Resilience }\end{array}$ & $\begin{array}{c}\text { Net } \\
\text { Persistence }\end{array}$ \\
\hline Edwards Plateau Mesic Canyon & 3.24 & 0.53 & 3.96 & 0.88 & 1.41 \\
\hline Edwards Plateau Riparian & 3.13 & 0.39 & 3.66 & 0.73 & 1.12 \\
\hline Edwards Plateau Upland Depression & 3.34 & 0.66 & 3.84 & 0.82 & 1.48 \\
\hline Florida Big Bend Fresh and Oligohaline Tidal Marsh & 3.45 & 0.81 & 3.58 & 0.69 & 1.50 \\
\hline Florida Big Bend Salt and Brackish Tidal Marsh & 3.60 & 1.00 & 3.93 & 0.87 & 1.87 \\
\hline Florida Dry Prairie & 3.15 & 0.42 & 3.23 & 0.52 & 0.93 \\
\hline Florida Longleaf Pine Sandhill & 3.03 & 0.26 & 2.75 & 0.28 & 0.53 \\
\hline Florida Panhandle Beach Vegetation & 3.60 & 1.00 & 4.10 & 0.95 & 1.95 \\
\hline Florida Peninsula Inland Scrub & 2.95 & 0.16 & 2.38 & 0.09 & 0.25 \\
\hline Floridian Highlands Freshwater Marsh & 3.10 & 0.35 & 3.17 & 0.49 & 0.84 \\
\hline Great Basin Foothill and Lower Montane Riparian Woodland and Shrubland & 2.99 & 0.21 & 3.34 & 0.57 & 0.78 \\
\hline Great Basin Pinyon-Juniper Woodland & 2.97 & 0.18 & 2.53 & 0.17 & 0.35 \\
\hline Great Basin Xeric Mixed Sagebrush Shrubland & 2.88 & 0.06 & 2.82 & 0.31 & 0.37 \\
\hline Great Lakes Acidic Rocky Shore and Cliff & 3.20 & 0.48 & 3.53 & 0.67 & 1.15 \\
\hline Great Lakes Alvar & 3.28 & 0.58 & 3.20 & 0.50 & 1.08 \\
\hline Great Lakes Dune & 2.99 & 0.21 & 3.00 & 0.40 & 0.61 \\
\hline Great Lakes Freshwater Estuary and Delta & 3.60 & 1.00 & 3.60 & 0.70 & 1.70 \\
\hline Great Lakes Wet-Mesic Lakeplain Prairie & 3.60 & 1.00 & 3.85 & 0.83 & 1.83 \\
\hline Great Lakes Wooded Dune and Swale & 3.17 & 0.44 & 3.17 & 0.49 & 0.93 \\
\hline Great Plains Prairie Pothole & 3.60 & 1.00 & 3.60 & 0.70 & 1.70 \\
\hline Great Plains Wooded Draw and Ravine & 2.98 & 0.19 & 3.67 & 0.74 & 0.93 \\
\hline Gulf Coast Chenier Plain Beach & 3.20 & 0.48 & 3.70 & 0.75 & 1.23 \\
\hline Gulf Coast Chenier Plain Fresh and Oligohaline Tidal Marsh & 3.60 & 1.00 & 3.85 & 0.83 & 1.83 \\
\hline Gulf Coast Chenier Plain Salt and Brackish Tidal Marsh & 3.60 & 1.00 & 3.93 & 0.87 & 1.87 \\
\hline Harvested Forest-Grass Regeneration & 0.00 & 0.00 & 0.00 & 0.00 & 0.00 \\
\hline Harvested Forest-herbaceous Regeneration & 0.00 & 0.00 & 0.00 & 0.00 & 0.00 \\
\hline High Allegheny Wetland & 3.25 & 0.55 & 3.05 & 0.43 & 0.97 \\
\hline Inter-Mountain Basins Alkaline Closed Depression & 3.60 & 1.00 & 3.77 & 0.79 & 1.79 \\
\hline Inter-Mountain Basins Aspen-Mixed Conifer Forest and Woodland & 3.14 & 0.40 & 2.76 & 0.28 & 0.68 \\
\hline Inter-Mountain Basins Big Sagebrush Shrubland & 2.96 & 0.17 & 3.06 & 0.43 & 0.60 \\
\hline Inter-Mountain Basins Big Sagebrush Steppe & 3.00 & 0.22 & 2.80 & 0.30 & 0.52 \\
\hline Inter-Mountain Basins Cliff and Canyon & 3.12 & 0.38 & 2.23 & 0.01 & 0.39 \\
\hline $\begin{array}{l}\text { Inter-Mountain Basins Curl-leaf Mountain-mahogany Woodland and } \\
\text { Shrubland }\end{array}$ & 3.15 & 0.42 & 3.10 & 0.45 & 0.87 \\
\hline Inter-Mountain Basins Greasewood Flat & 2.96 & 0.17 & 3.07 & 0.44 & 0.60 \\
\hline Inter-Mountain Basins Interdunal Swale Wetland & 3.60 & 1.00 & 3.60 & 0.70 & 1.70 \\
\hline Inter-Mountain Basins Juniper Savanna & 3.25 & 0.55 & 3.11 & 0.46 & 1.00 \\
\hline Inter-Mountain Basins Mat Saltbush Shrubland & 3.19 & 0.47 & 3.33 & 0.57 & 1.03 \\
\hline Inter-Mountain Basins Mixed Salt Desert Scrub & 3.00 & 0.22 & 3.13 & 0.47 & 0.69 \\
\hline Inter-Mountain Basins Montane Sagebrush Steppe & 3.15 & 0.42 & 3.05 & 0.43 & 0.84 \\
\hline Inter-Mountain Basins Semi-Desert Grassland & 3.23 & 0.52 & 3.52 & 0.66 & 1.18 \\
\hline Inter-Mountain Basins Semi-Desert Shrub-Steppe & 3.51 & 0.88 & 3.66 & 0.73 & 1.61 \\
\hline Inter-Mountain Basins Shale Badland & 2.88 & 0.06 & 2.92 & 0.36 & 0.42 \\
\hline Inter-Mountain Basins Subalpine Limber-Bristlecone Pine Woodland & 3.03 & 0.26 & 2.63 & 0.22 & 0.47 \\
\hline Inter-Mountain Basins Volcanic Rock and Cinder Land & 2.95 & 0.16 & 2.30 & 0.05 & 0.21 \\
\hline Introduced Riparian Vegetation & 0.00 & 0.00 & 0.00 & 0.00 & 0.00 \\
\hline
\end{tabular}




\begin{tabular}{|c|c|c|c|c|c|}
\hline Community & $\begin{array}{c}\text { Mean } \\
\text { Resistance }\end{array}$ & $\begin{array}{c}\text { Scaled } \\
\text { Resistance }\end{array}$ & $\begin{array}{c}\text { Mean } \\
\text { Resilience }\end{array}$ & $\begin{array}{c}\text { Scaled } \\
\text { Resilience }\end{array}$ & $\begin{array}{c}\text { Net } \\
\text { Persistence }\end{array}$ \\
\hline Introduced Upland Vegetation - Annual and Biennial Forbland & 0.00 & 0.00 & 0.00 & 0.00 & 0.00 \\
\hline Introduced Upland Vegetation - Annual Grassland & 0.00 & 0.00 & 0.00 & 0.00 & 0.00 \\
\hline Introduced Upland Vegetation - Perennial Grassland and Forbland & 0.00 & 0.00 & 0.00 & 0.00 & 0.00 \\
\hline Introduced Upland Vegetation - Shrub & 0.00 & 0.00 & 0.00 & 0.00 & 0.00 \\
\hline Introduced Upland Vegetation - Treed & 0.00 & 0.00 & 0.00 & 0.00 & 0.00 \\
\hline Introduced Wetland Vegetation & 0.00 & 0.00 & 0.00 & 0.00 & 0.00 \\
\hline Klamath-Siskiyou Cliff and Outcrop & 3.60 & 1.00 & 3.60 & 0.70 & 1.70 \\
\hline Klamath-Siskiyou Lower Montane Serpentine Mixed Conifer Woodland & 3.14 & 0.40 & 2.22 & 0.01 & 0.41 \\
\hline Klamath-Siskiyou Upper Montane Serpentine Mixed Conifer Woodland & 3.20 & 0.48 & 2.20 & 0.00 & 0.48 \\
\hline Klamath-Siskiyou Xeromorphic Serpentine Savanna and Chaparral & 2.95 & 0.16 & 2.30 & 0.05 & 0.21 \\
\hline Laurentian Jack Pine-Red Pine Forest & 2.95 & 0.16 & 2.40 & 0.10 & 0.26 \\
\hline Laurentian Pine-Oak Barrens & 3.06 & 0.30 & 2.72 & 0.26 & 0.56 \\
\hline Laurentian-Acadian Alkaline Conifer-Hardwood Swamp & 3.10 & 0.35 & 3.20 & 0.50 & 0.85 \\
\hline Laurentian-Acadian Floodplain Forest & 3.30 & 0.61 & 3.73 & 0.77 & 1.38 \\
\hline Laurentian-Acadian Freshwater Marsh & 3.40 & 0.74 & 3.57 & 0.69 & 1.43 \\
\hline Laurentian-Acadian Northern Hardwood Forest & 3.00 & 0.22 & 4.20 & 1.00 & 1.22 \\
\hline Laurentian-Acadian Northern Pine-(Oak) Forest & 3.01 & 0.23 & 3.37 & 0.59 & 0.82 \\
\hline Laurentian-Acadian Pine-Hemlock-Hardwood Forest & 3.10 & 0.35 & 3.03 & 0.42 & 0.77 \\
\hline Laurentian-Acadian Wet Meadow-Shrub Swamp & 3.15 & 0.42 & 3.30 & 0.55 & 0.97 \\
\hline Llano Estacado Caprock Escarpment and Breaks Shrubland and Steppe & 3.18 & 0.45 & 3.38 & 0.59 & 1.04 \\
\hline Llano Uplift Acidic Forest, Woodland and Glade & 3.18 & 0.45 & 3.93 & 0.87 & 1.32 \\
\hline Louisiana Beach & 3.60 & 1.00 & 4.10 & 0.95 & 1.95 \\
\hline Lower Mississippi River Dune Woodland and Forest & 3.30 & 0.61 & 4.15 & 0.98 & 1.59 \\
\hline Lower Mississippi River Flatwoods & 3.00 & 0.22 & 4.20 & 1.00 & 1.22 \\
\hline Madrean Encinal & 3.40 & 0.74 & 3.32 & 0.56 & 1.30 \\
\hline Madrean Juniper Savanna & 3.54 & 0.92 & 3.83 & 0.82 & 1.74 \\
\hline Madrean Lower Montane Pine-Oak Forest and Woodland & 3.20 & 0.48 & 2.20 & 0.00 & 0.48 \\
\hline Madrean Oriental Chaparral & 2.97 & 0.18 & 3.07 & 0.44 & 0.62 \\
\hline Madrean Pinyon-Juniper Woodland & 3.20 & 0.48 & 2.20 & 0.00 & 0.48 \\
\hline Madrean Upper Montane Conifer-Oak Forest and Woodland & 3.20 & 0.48 & 2.20 & 0.00 & 0.48 \\
\hline Managed Tree Plantation & 0.00 & 0.00 & 0.00 & 0.00 & 0.00 \\
\hline Mediterranean California Alpine Bedrock and Scree & 3.00 & 0.22 & 3.50 & 0.65 & 0.87 \\
\hline Mediterranean California Alpine Dry Tundra & 3.40 & 0.74 & 3.57 & 0.69 & 1.43 \\
\hline Mediterranean California Alpine Fell-Field & 3.00 & 0.22 & 3.50 & 0.65 & 0.87 \\
\hline Mediterranean California Coastal Bluff & 2.85 & 0.03 & 2.95 & 0.38 & 0.40 \\
\hline Mediterranean California Dry-Mesic Mixed Conifer Forest and Woodland & 3.00 & 0.22 & 2.40 & 0.10 & 0.32 \\
\hline $\begin{array}{l}\text { Mediterranean California Foothill and Lower Montane Riparian Woodland } \\
\text { and Shrubland }\end{array}$ & 2.96 & 0.17 & 3.57 & 0.69 & 0.85 \\
\hline $\begin{array}{l}\text { Mediterranean California Lower Montane Black Oak-Conifer Forest and } \\
\text { Woodland }\end{array}$ & 2.96 & 0.17 & 2.90 & 0.35 & 0.52 \\
\hline Mediterranean California Mesic Mixed Conifer Forest and Woodland & 3.08 & 0.32 & 2.57 & 0.19 & 0.51 \\
\hline Mediterranean California Mesic Serpentine Woodland and Chaparral & 3.00 & 0.22 & 2.28 & 0.04 & 0.26 \\
\hline Mediterranean California Mixed Evergreen Forest & 3.10 & 0.35 & 2.32 & 0.06 & 0.41 \\
\hline Mediterranean California Mixed Oak Woodland & 3.28 & 0.58 & 3.56 & 0.68 & 1.26 \\
\hline Mediterranean California Red Fir Forest & 3.20 & 0.48 & 2.20 & 0.00 & 0.48 \\
\hline Mediterranean California Serpentine Barrens & 3.00 & 0.22 & 3.50 & 0.65 & 0.87 \\
\hline Mediterranean California Serpentine Fen & 3.45 & 0.81 & 3.58 & 0.69 & 1.50 \\
\hline
\end{tabular}




\begin{tabular}{|c|c|c|c|c|c|}
\hline Community & $\begin{array}{c}\text { Mean } \\
\text { Resistance }\end{array}$ & $\begin{array}{c}\text { Scaled } \\
\text { Resistance }\end{array}$ & $\begin{array}{c}\text { Mean } \\
\text { Resilience }\end{array}$ & $\begin{array}{l}\text { Scaled } \\
\text { Resilience }\end{array}$ & $\begin{array}{c}\text { Net } \\
\text { Persistence }\end{array}$ \\
\hline $\begin{array}{l}\text { Mediterranean California Serpentine Foothill and Lower Montane Riparian } \\
\text { Woodland and Seep }\end{array}$ & 3.03 & 0.26 & 2.27 & 0.03 & 0.29 \\
\hline Mediterranean California Southern Coastal Dune & 3.04 & 0.27 & 3.49 & 0.65 & 0.92 \\
\hline Mediterranean California Subalpine Meadow & 3.30 & 0.61 & 3.55 & 0.68 & 1.29 \\
\hline Mediterranean California Subalpine Woodland & 3.20 & 0.48 & 2.20 & 0.00 & 0.48 \\
\hline Mediterranean California Subalpine-Montane Fen & 3.47 & 0.83 & 3.51 & 0.66 & 1.49 \\
\hline Middle Rocky Mountain Montane Douglas-fir Forest and Woodland & 3.05 & 0.29 & 2.95 & 0.38 & 0.66 \\
\hline Mississippi Delta Maritime Forest & 2.97 & 0.18 & 3.13 & 0.47 & 0.65 \\
\hline Mississippi River Alluvial Plain Dry-Mesic Loess Slope Forest & 3.00 & 0.22 & 4.20 & 1.00 & 1.22 \\
\hline Mississippi River Bottomland Depression & 3.00 & 0.22 & 4.20 & 1.00 & 1.22 \\
\hline Mississippi River Floodplain and Riparian Forest & 3.00 & 0.22 & 4.20 & 1.00 & 1.22 \\
\hline Mississippi River Low Floodplain (Bottomland) Forest & 3.00 & 0.22 & 4.20 & 1.00 & 1.22 \\
\hline Mississippi River Riparian Forest & 3.00 & 0.22 & 4.20 & 1.00 & 1.22 \\
\hline Modified/Managed Southern Tall Grassland & 0.00 & 0.00 & 0.00 & 0.00 & 0.00 \\
\hline Nashville Basin Limestone Glade and Woodland & 3.13 & 0.39 & 3.38 & 0.59 & 0.98 \\
\hline Non-Specific Disturbed & 0.00 & 0.00 & 0.00 & 0.00 & 0.00 \\
\hline North American Arid West Emergent Marsh & 3.60 & 1.00 & 3.60 & 0.70 & 1.70 \\
\hline North American Warm Desert Badland & 2.85 & 0.03 & 3.10 & 0.45 & 0.48 \\
\hline North American Warm Desert Bedrock Cliff and Outcrop & 2.85 & 0.03 & 3.20 & 0.50 & 0.53 \\
\hline North American Warm Desert Cienega & 3.43 & 0.78 & 3.71 & 0.76 & 1.53 \\
\hline $\begin{array}{l}\text { North American Warm Desert Lower Montane Riparian Woodland and } \\
\text { Shrubland }\end{array}$ & 2.86 & 0.04 & 3.55 & 0.68 & 0.71 \\
\hline North American Warm Desert Pavement & 0.00 & 0.00 & 0.00 & 0.00 & 0.00 \\
\hline North American Warm Desert Playa & 3.10 & 0.35 & 3.45 & 0.63 & 0.98 \\
\hline North American Warm Desert Riparian Mesquite Bosque & 2.87 & 0.05 & 3.60 & 0.70 & 0.75 \\
\hline North American Warm Desert Riparian Woodland and Shrubland & 2.92 & 0.12 & 3.87 & 0.84 & 0.95 \\
\hline North Pacific Active Volcanic Rock and Cinder Land & 2.85 & 0.03 & 3.25 & 0.53 & 0.55 \\
\hline North Pacific Alpine and Subalpine Bedrock and Scree & 3.05 & 0.29 & 3.43 & 0.62 & 0.90 \\
\hline North Pacific Alpine and Subalpine Dry Grassland & 3.60 & 1.00 & 3.60 & 0.70 & 1.70 \\
\hline North Pacific Avalanche Chute Shrubland & 2.83 & 0.00 & 2.80 & 0.30 & 0.30 \\
\hline North Pacific Broadleaf Landslide Forest and Shrubland & 2.85 & 0.03 & 3.60 & 0.70 & 0.73 \\
\hline North Pacific Coastal Cliff and Bluff & 3.30 & 0.61 & 3.55 & 0.68 & 1.29 \\
\hline North Pacific Coastal Interdunal Wetland & 3.60 & 1.00 & 3.60 & 0.70 & 1.70 \\
\hline North Pacific Dry and Mesic Alpine Dwarf-Shrubland, Fell-Field and Meadow & 2.96 & 0.17 & 2.91 & 0.36 & 0.52 \\
\hline North Pacific Dry Douglas-fir-(Madrone) Forest and Woodland & 3.16 & 0.43 & 2.60 & 0.20 & 0.63 \\
\hline North Pacific Dry-Mesic Silver Fir-Western Hemlock-Douglas-fir Forest & 3.20 & 0.48 & 2.20 & 0.00 & 0.48 \\
\hline North Pacific Hardwood-Conifer Swamp & 3.07 & 0.31 & 2.71 & 0.26 & 0.57 \\
\hline North Pacific Herbaceous Bald and Bluff & 3.19 & 0.47 & 3.46 & 0.63 & 1.10 \\
\hline North Pacific Hypermaritime Shrub and Herbaceous Headland & 3.06 & 0.30 & 3.00 & 0.40 & 0.70 \\
\hline North Pacific Hypermaritime Western Red-cedar-Western Hemlock Forest & 3.20 & 0.48 & 2.20 & 0.00 & 0.48 \\
\hline North Pacific Intertidal Freshwater Wetland & 3.29 & 0.60 & 3.23 & 0.52 & 1.11 \\
\hline North Pacific Lowland Mixed Hardwood-Conifer Forest & 3.01 & 0.23 & 2.96 & 0.38 & 0.61 \\
\hline North Pacific Lowland Riparian Forest and Shrubland & 2.92 & 0.12 & 3.32 & 0.56 & 0.68 \\
\hline North Pacific Maritime Coastal Sand Dune and Strand & 3.28 & 0.58 & 2.95 & 0.38 & 0.96 \\
\hline North Pacific Maritime Dry-Mesic Douglas-fir-Western Hemlock Forest & 2.99 & 0.21 & 2.71 & 0.26 & 0.46 \\
\hline North Pacific Maritime Mesic Subalpine Parkland & 3.05 & 0.29 & 2.79 & 0.30 & 0.58 \\
\hline North Pacific Maritime Mesic-Wet Douglas-fir-Western Hemlock Forest & 3.08 & 0.32 & 2.67 & 0.24 & 0.56 \\
\hline
\end{tabular}




\begin{tabular}{|c|c|c|c|c|c|}
\hline Community & $\begin{array}{c}\text { Mean } \\
\text { Resistance }\end{array}$ & $\begin{array}{c}\text { Scaled } \\
\text { Resistance }\end{array}$ & $\begin{array}{c}\text { Mean } \\
\text { Resilience }\end{array}$ & $\begin{array}{c}\text { Scaled } \\
\text { Resilience }\end{array}$ & $\begin{array}{c}\text { Net } \\
\text { Persistence }\end{array}$ \\
\hline North Pacific Mesic Western Hemlock-Silver Fir Forest & 3.10 & 0.35 & 2.36 & 0.08 & 0.43 \\
\hline North Pacific Montane Grassland & 3.60 & 1.00 & 3.60 & 0.70 & 1.70 \\
\hline North Pacific Montane Massive Bedrock, Cliff and Talus & 0.00 & 0.00 & 0.00 & 0.00 & 0.00 \\
\hline North Pacific Montane Riparian Woodland and Shrubland & 2.91 & 0.10 & 2.89 & 0.35 & 0.45 \\
\hline North Pacific Mountain Hemlock Forest & 3.20 & 0.48 & 2.20 & 0.00 & 0.48 \\
\hline North Pacific Oak Woodland & 3.15 & 0.42 & 2.70 & 0.25 & 0.67 \\
\hline North Pacific Seasonal Sitka Spruce Forest & 3.20 & 0.48 & 2.20 & 0.00 & 0.48 \\
\hline North Pacific Serpentine Barren & 3.08 & 0.32 & 2.25 & 0.02 & 0.35 \\
\hline North Pacific Shrub Swamp & 3.00 & 0.22 & 3.32 & 0.56 & 0.78 \\
\hline North Pacific Wooded Volcanic Flowage & 3.03 & 0.26 & 2.61 & 0.21 & 0.46 \\
\hline North-Central Appalachian Acidic Cliff and Talus & 2.97 & 0.18 & 3.07 & 0.44 & 0.62 \\
\hline North-Central Appalachian Circumneutral Cliff and Talus & 2.90 & 0.09 & 3.80 & 0.80 & 0.89 \\
\hline North-Central Interior and Appalachian Acidic Peatland & 3.18 & 0.45 & 2.70 & 0.25 & 0.70 \\
\hline North-Central Interior and Appalachian Rich Swamp & 3.10 & 0.35 & 3.20 & 0.50 & 0.85 \\
\hline North-Central Interior Beech-Maple Forest & 3.00 & 0.22 & 4.03 & 0.92 & 1.14 \\
\hline North-Central Interior Dry Oak Forest and Woodland & 3.00 & 0.22 & 4.20 & 1.00 & 1.22 \\
\hline North-Central Interior Dry-Mesic Oak Forest and Woodland & 3.00 & 0.22 & 4.20 & 1.00 & 1.22 \\
\hline North-Central Interior Floodplain & 3.15 & 0.42 & 4.05 & 0.93 & 1.34 \\
\hline North-Central Interior Freshwater Marsh & 3.60 & 1.00 & 3.60 & 0.70 & 1.70 \\
\hline North-Central Interior Maple-Basswood Forest & 3.00 & 0.22 & 4.20 & 1.00 & 1.22 \\
\hline North-Central Interior Oak Savanna & 3.30 & 0.61 & 3.80 & 0.80 & 1.41 \\
\hline North-Central Interior Sand and Gravel Tallgrass Prairie & 3.60 & 1.00 & 3.98 & 0.89 & 1.89 \\
\hline North-Central Interior Wet Flatwoods & 3.00 & 0.22 & 4.20 & 1.00 & 1.22 \\
\hline North-Central Interior Wet Meadow-Shrub Swamp & 3.15 & 0.42 & 3.30 & 0.55 & 0.97 \\
\hline North-Central Oak Barrens & 3.20 & 0.48 & 4.17 & 0.99 & 1.47 \\
\hline Northeastern Interior Dry-Mesic Oak Forest & 3.04 & 0.27 & 3.80 & 0.80 & 1.07 \\
\hline Northeastern Interior Pine Barrens & 3.20 & 0.48 & 3.68 & 0.74 & 1.22 \\
\hline Northern Atlantic Coastal Plain Basin Swamp and Wet Hardwood Forest & 2.93 & 0.13 & 3.90 & 0.85 & 0.98 \\
\hline Northern Atlantic Coastal Plain Dune and Swale & 3.00 & 0.22 & 2.98 & 0.39 & 0.61 \\
\hline Northern Atlantic Coastal Plain Fresh and Oligohaline Tidal Marsh & 3.10 & 0.35 & 3.37 & 0.59 & 0.94 \\
\hline Northern Atlantic Coastal Plain Hardwood Forest & 3.00 & 0.22 & 4.20 & 1.00 & 1.22 \\
\hline Northern Atlantic Coastal Plain Heathland and Grassland & 2.93 & 0.13 & 3.18 & 0.49 & 0.62 \\
\hline Northern Atlantic Coastal Plain Maritime Forest & 3.03 & 0.26 & 2.75 & 0.28 & 0.53 \\
\hline Northern Atlantic Coastal Plain Pitch Pine Barrens & 3.03 & 0.26 & 3.42 & 0.61 & 0.87 \\
\hline Northern Atlantic Coastal Plain Pitch Pine Lowland & 3.18 & 0.45 & 2.88 & 0.34 & 0.79 \\
\hline Northern Atlantic Coastal Plain Sandy Beach & 3.00 & 0.22 & 3.50 & 0.65 & 0.87 \\
\hline Northern Atlantic Coastal Plain Stream and River & 0.00 & 0.00 & 0.00 & 0.00 & 0.00 \\
\hline Northern Atlantic Coastal Plain Tidal Salt Marsh & 3.60 & 1.00 & 3.98 & 0.89 & 1.89 \\
\hline Northern Atlantic Coastal Plain Tidal Swamp & 2.93 & 0.13 & 3.90 & 0.85 & 0.98 \\
\hline Northern California Claypan Vernal Pool & 3.00 & 0.22 & 3.50 & 0.65 & 0.87 \\
\hline Northern California Mesic Subalpine Woodland & 3.20 & 0.48 & 2.20 & 0.00 & 0.48 \\
\hline Northern Great Lakes Coastal Marsh & 3.60 & 1.00 & 3.60 & 0.70 & 1.70 \\
\hline Northern Rocky Mountain Avalanche Chute Shrubland & 2.98 & 0.19 & 3.56 & 0.68 & 0.87 \\
\hline Northern Rocky Mountain Conifer Swamp & 3.20 & 0.48 & 2.20 & 0.00 & 0.48 \\
\hline Northern Rocky Mountain Dry-Mesic Montane Mixed Conifer Forest & 3.40 & 0.74 & 2.90 & 0.35 & 1.09 \\
\hline Northern Rocky Mountain Foothill Conifer Wooded Steppe & 3.33 & 0.65 & 2.67 & 0.24 & 0.88 \\
\hline
\end{tabular}




\begin{tabular}{|c|c|c|c|c|c|}
\hline Community & $\begin{array}{c}\text { Mean } \\
\text { Resistance }\end{array}$ & $\begin{array}{c}\text { Scaled } \\
\text { Resistance }\end{array}$ & $\begin{array}{c}\text { Mean } \\
\text { Resilience }\end{array}$ & $\begin{array}{c}\text { Scaled } \\
\text { Resilience }\end{array}$ & $\begin{array}{l}\text { Net } \\
\text { Persistence }\end{array}$ \\
\hline $\begin{array}{l}\text { Northern Rocky Mountain Lower Montane Riparian Woodland and } \\
\text { Shrubland }\end{array}$ & 3.00 & 0.22 & 2.80 & 0.30 & 0.52 \\
\hline Northern Rocky Mountain Lower Montane, Foothill and Valley Grassland & 3.60 & 1.00 & 3.60 & 0.70 & 1.70 \\
\hline Northern Rocky Mountain Mesic Montane Mixed Conifer Forest & 3.20 & 0.48 & 2.20 & 0.00 & 0.48 \\
\hline Northern Rocky Mountain Ponderosa Pine Woodland and Savanna & 3.02 & 0.25 & 2.63 & 0.22 & 0.46 \\
\hline Northern Rocky Mountain Subalpine Woodland and Parkland & 3.08 & 0.32 & 2.40 & 0.10 & 0.42 \\
\hline Northern Rocky Mountain Subalpine-Upper Montane Grassland & 3.60 & 1.00 & 3.60 & 0.70 & 1.70 \\
\hline Northern Rocky Mountain Western Larch Savanna & 2.94 & 0.14 & 2.93 & 0.37 & 0.51 \\
\hline Northern Rocky Mountain Wooded Vernal Pool & 3.13 & 0.39 & 3.05 & 0.43 & 0.81 \\
\hline Northern Tallgrass Prairie & 3.60 & 1.00 & 4.00 & 0.90 & 1.90 \\
\hline Northwestern Great Plains Aspen Forest and Parkland & 3.00 & 0.22 & 3.45 & 0.63 & 0.85 \\
\hline Northwestern Great Plains Floodplain & 3.18 & 0.45 & 3.72 & 0.76 & 1.21 \\
\hline Northwestern Great Plains Mixedgrass Prairie & 3.60 & 1.00 & 3.70 & 0.75 & 1.75 \\
\hline Northwestern Great Plains Riparian & 3.09 & 0.34 & 3.60 & 0.70 & 1.04 \\
\hline Northwestern Great Plains Shrubland & 2.93 & 0.13 & 3.05 & 0.43 & 0.55 \\
\hline $\begin{array}{l}\text { Northwestern Great Plains-Black Hills Ponderosa Pine Woodland and } \\
\text { Savanna }\end{array}$ & 3.13 & 0.39 & 3.44 & 0.62 & 1.01 \\
\hline Open Water & 0.00 & 0.00 & 0.00 & 0.00 & 0.00 \\
\hline Ouachita Montane Oak Forest & 3.30 & 0.61 & 4.03 & 0.92 & 1.53 \\
\hline Ozark-Ouachita Dry Oak Woodland & 3.00 & 0.22 & 4.20 & 1.00 & 1.22 \\
\hline Ozark-Ouachita Dry-Mesic Oak Forest & 3.00 & 0.22 & 4.20 & 1.00 & 1.22 \\
\hline Ozark-Ouachita Mesic Hardwood Forest & 3.00 & 0.22 & 4.20 & 1.00 & 1.22 \\
\hline Ozark-Ouachita Riparian & 3.20 & 0.48 & 3.88 & 0.84 & 1.32 \\
\hline Ozark-Ouachita Shortleaf Pine-Bluestem Woodland & 3.11 & 0.36 & 3.90 & 0.85 & 1.21 \\
\hline Ozark-Ouachita Shortleaf Pine-Oak Forest and Woodland & 3.20 & 0.48 & 3.68 & 0.74 & 1.22 \\
\hline Paleozoic Plateau Bluff and Talus & 3.60 & 1.00 & 4.10 & 0.95 & 1.95 \\
\hline Piedmont Upland Depression Swamp & 3.06 & 0.30 & 3.84 & 0.82 & 1.12 \\
\hline Quarries/Strip Mines/Gravel Pits & 0.00 & 0.00 & 0.00 & 0.00 & 0.00 \\
\hline Recently Burned & 0.00 & 0.00 & 0.00 & 0.00 & 0.00 \\
\hline Recently Burned Herbaceous & 0.00 & 0.00 & 0.00 & 0.00 & 0.00 \\
\hline Recently Logged Timberland & 0.00 & 0.00 & 0.00 & 0.00 & 0.00 \\
\hline Recently Logged Timberland-Herbaceous Cover & 0.00 & 0.00 & 0.00 & 0.00 & 0.00 \\
\hline Recently Logged Timberland-Shrubland Cover & 0.00 & 0.00 & 0.00 & 0.00 & 0.00 \\
\hline Red River Large Floodplain Forest & 3.05 & 0.29 & 3.70 & 0.75 & 1.04 \\
\hline Rocky Mountain Alpine Bedrock and Scree & 3.00 & 0.22 & 3.50 & 0.65 & 0.87 \\
\hline Rocky Mountain Alpine Fell-Field & 3.08 & 0.32 & 3.51 & 0.66 & 0.98 \\
\hline Rocky Mountain Alpine Turf & 3.34 & 0.66 & 3.51 & 0.66 & 1.32 \\
\hline Rocky Mountain Alpine-Montane Wet Meadow & 3.39 & 0.73 & 3.56 & 0.68 & 1.41 \\
\hline Rocky Mountain Aspen Forest and Woodland & 3.00 & 0.22 & 3.45 & 0.63 & 0.85 \\
\hline Rocky Mountain Bigtooth Maple Ravine Woodland & 2.92 & 0.12 & 3.58 & 0.69 & 0.81 \\
\hline Rocky Mountain Cliff, Canyon and Massive Bedrock & 0.00 & 0.00 & 0.00 & 0.00 & 0.00 \\
\hline Rocky Mountain Foothill Limber Pine-Juniper Woodland & 3.23 & 0.52 & 3.09 & 0.45 & 0.96 \\
\hline Rocky Mountain Lodgepole Pine Forest & 2.83 & 0.00 & 2.65 & 0.23 & 0.23 \\
\hline Rocky Mountain Lower Montane-Foothill Riparian Woodland and Shrubland & 2.89 & 0.08 & 3.38 & 0.59 & 0.67 \\
\hline Rocky Mountain Lower Montane-Foothill Shrubland & 2.88 & 0.06 & 3.06 & 0.43 & 0.49 \\
\hline Rocky Mountain Poor-Site Lodgepole Pine Forest & 3.05 & 0.29 & 2.80 & 0.30 & 0.59 \\
\hline Rocky Mountain Subalpine Dry-Mesic Spruce-Fir Forest and Woodland & 3.01 & 0.23 & 2.50 & 0.15 & 0.38 \\
\hline
\end{tabular}




\begin{tabular}{|c|c|c|c|c|c|}
\hline Community & $\begin{array}{c}\text { Mean } \\
\text { Resistance }\end{array}$ & $\begin{array}{c}\text { Scaled } \\
\text { Resistance }\end{array}$ & $\begin{array}{c}\text { Mean } \\
\text { Resilience }\end{array}$ & $\begin{array}{c}\text { Scaled } \\
\text { Resilience }\end{array}$ & $\begin{array}{c}\text { Net } \\
\text { Persistence }\end{array}$ \\
\hline Rocky Mountain Subalpine Mesic-Wet Spruce-Fir Forest and Woodland & 2.95 & 0.16 & 2.60 & 0.20 & 0.36 \\
\hline Rocky Mountain Subalpine-Montane Fen & 3.51 & 0.88 & 3.54 & 0.67 & 1.55 \\
\hline Rocky Mountain Subalpine-Montane Limber-Bristlecone Pine Woodland & 3.15 & 0.42 & 2.96 & 0.38 & 0.80 \\
\hline Rocky Mountain Subalpine-Montane Mesic Meadow & 3.30 & 0.61 & 3.55 & 0.68 & 1.29 \\
\hline Rocky Mountain Subalpine-Montane Riparian Woodland & 3.03 & 0.26 & 2.90 & 0.35 & 0.61 \\
\hline Ruderal Forest & 0.00 & 0.00 & 0.00 & 0.00 & 0.00 \\
\hline Ruderal Upland - Old Field & 0.00 & 0.00 & 0.00 & 0.00 & 0.00 \\
\hline Sierra Nevada Alpine Dwarf-Shrubland & 2.91 & 0.10 & 3.37 & 0.59 & 0.69 \\
\hline Sierra Nevada Cliff and Canyon & 3.11 & 0.36 & 2.48 & 0.14 & 0.50 \\
\hline Sierra Nevada Subalpine Lodgepole Pine Forest and Woodland & 3.04 & 0.27 & 2.53 & 0.17 & 0.44 \\
\hline Sierran-Intermontane Desert Western White Pine-White Fir Woodland & 3.20 & 0.48 & 2.20 & 0.00 & 0.48 \\
\hline Sonora-Mojave Mixed Salt Desert Scrub & 3.00 & 0.22 & 3.23 & 0.52 & 0.74 \\
\hline South Florida Bayhead Swamp & 3.08 & 0.32 & 2.25 & 0.02 & 0.35 \\
\hline South Florida Cypress Dome & 2.87 & 0.05 & 2.33 & 0.06 & 0.12 \\
\hline South Florida Dwarf Cypress Savanna & 3.47 & 0.83 & 3.30 & 0.55 & 1.38 \\
\hline South Florida Everglades Sawgrass Marsh & 3.30 & 0.61 & 3.40 & 0.60 & 1.21 \\
\hline South Florida Hardwood Hammock & 2.96 & 0.17 & 2.68 & 0.24 & 0.41 \\
\hline South Florida Pine Flatwoods & 3.05 & 0.29 & 2.93 & 0.37 & 0.65 \\
\hline South Florida Pine Rockland & 3.13 & 0.39 & 3.38 & 0.59 & 0.98 \\
\hline South Florida Shell Hash Beach & 3.30 & 0.61 & 3.80 & 0.80 & 1.41 \\
\hline South Florida Slough, Gator Hole and Willow Head & 3.23 & 0.52 & 3.43 & 0.62 & 1.13 \\
\hline South Florida Wet Marl Prairie & 3.38 & 0.71 & 3.43 & 0.62 & 1.33 \\
\hline South Texas Dune and Coastal Grassland & 3.60 & 1.00 & 4.10 & 0.95 & 1.95 \\
\hline South Texas Salt and Brackish Tidal Flat & 3.12 & 0.38 & 3.46 & 0.63 & 1.01 \\
\hline South Texas Sand Sheet Grassland & 3.60 & 1.00 & 4.10 & 0.95 & 1.95 \\
\hline South-Central Interior / Upper Coastal Plain Flatwoods & 3.00 & 0.22 & 4.20 & 1.00 & 1.22 \\
\hline South-Central Interior / Upper Coastal Plain Wet Flatwoods & 3.00 & 0.22 & 4.20 & 1.00 & 1.22 \\
\hline South-Central Interior Large Floodplain & 2.93 & 0.13 & 3.90 & 0.85 & 0.98 \\
\hline South-Central Interior Mesophytic Forest & 3.00 & 0.22 & 4.20 & 1.00 & 1.22 \\
\hline South-Central Interior Small Stream and Riparian & 2.93 & 0.13 & 3.90 & 0.85 & 0.98 \\
\hline South-Central Saline Glade & 3.23 & 0.52 & 3.70 & 0.75 & 1.27 \\
\hline Southeast Florida Beach & 3.00 & 0.22 & 3.50 & 0.65 & 0.87 \\
\hline Southeast Florida Coastal Strand and Maritime Hammock & 2.83 & 0.00 & 2.50 & 0.15 & 0.15 \\
\hline Southeastern Coastal Plain Cliff & 3.60 & 1.00 & 4.10 & 0.95 & 1.95 \\
\hline Southeastern Coastal Plain Interdunal Wetland & 3.60 & 1.00 & 3.73 & 0.77 & 1.77 \\
\hline Southeastern Coastal Plain Natural Lakeshore & 3.18 & 0.45 & 2.60 & 0.20 & 0.65 \\
\hline Southeastern Great Plains Floodplain Forest & 3.03 & 0.26 & 3.73 & 0.77 & 1.02 \\
\hline Southeastern Great Plains Riparian Forest & 2.93 & 0.13 & 3.85 & 0.83 & 0.95 \\
\hline Southeastern Great Plains Tallgrass Prairie & 3.60 & 1.00 & 4.10 & 0.95 & 1.95 \\
\hline Southeastern Interior Longleaf Pine Woodland & 3.18 & 0.45 & 3.53 & 0.67 & 1.12 \\
\hline Southern and Central Appalachian Bog and Fen & 3.10 & 0.35 & 3.20 & 0.50 & 0.85 \\
\hline Southern and Central Appalachian Cove Forest & 2.99 & 0.21 & 3.95 & 0.88 & 1.08 \\
\hline Southern Appalachian Granitic Dome & 3.30 & 0.61 & 3.57 & 0.69 & 1.30 \\
\hline Southern Appalachian Grass and Shrub Bald & 2.83 & 0.00 & 2.83 & 0.32 & 0.32 \\
\hline Southern Appalachian Low-Elevation Pine Forest & 3.15 & 0.42 & 2.70 & 0.25 & 0.67 \\
\hline Southern Appalachian Montane Pine Forest and Woodland & 3.10 & 0.35 & 2.24 & 0.02 & 0.37 \\
\hline
\end{tabular}




\begin{tabular}{|c|c|c|c|c|c|}
\hline Community & $\begin{array}{c}\text { Mean } \\
\text { Resistance }\end{array}$ & $\begin{array}{c}\text { Scaled } \\
\text { Resistance }\end{array}$ & $\begin{array}{c}\text { Mean } \\
\text { Resilience }\end{array}$ & $\begin{array}{c}\text { Scaled } \\
\text { Resilience }\end{array}$ & $\begin{array}{l}\text { Net } \\
\text { Persistence }\end{array}$ \\
\hline Southern Appalachian Northern Hardwood Forest & 3.03 & 0.26 & 3.87 & 0.84 & 1.09 \\
\hline Southern Appalachian Oak Forest & 3.00 & 0.22 & 4.20 & 1.00 & 1.22 \\
\hline Southern Appalachian Rocky Summit & 3.08 & 0.32 & 3.40 & 0.60 & 0.92 \\
\hline Southern Atlantic Coastal Plain Depression Pond & 3.60 & 1.00 & 3.73 & 0.77 & 1.77 \\
\hline Southern Atlantic Coastal Plain Dry and Dry-Mesic Oak Forest & 3.00 & 0.22 & 4.20 & 1.00 & 1.22 \\
\hline Southern Atlantic Coastal Plain Dune and Maritime Grassland & 3.45 & 0.81 & 3.95 & 0.88 & 1.68 \\
\hline Southern Atlantic Coastal Plain Florida Beach & 3.15 & 0.42 & 3.65 & 0.73 & 1.14 \\
\hline Southern Atlantic Coastal Plain Fresh and Oligohaline Tidal Marsh & 3.60 & 1.00 & 3.74 & 0.77 & 1.77 \\
\hline Southern Atlantic Coastal Plain Maritime Forest & 3.08 & 0.32 & 2.33 & 0.06 & 0.39 \\
\hline Southern Atlantic Coastal Plain Mesic Hardwood Forest & 2.93 & 0.13 & 3.75 & 0.78 & 0.90 \\
\hline $\begin{array}{l}\text { Southern Atlantic Coastal Plain Nonriverine Swamp and Wet Hardwood } \\
\text { Forest }\end{array}$ & 3.11 & 0.36 & 3.27 & 0.54 & 0.90 \\
\hline Southern Atlantic Coastal Plain Salt and Brackish Tidal Marsh & 3.16 & 0.43 & 3.02 & 0.41 & 0.84 \\
\hline Southern Atlantic Coastal Plain Sea Island Beach & 3.00 & 0.22 & 3.50 & 0.65 & 0.87 \\
\hline Southern Atlantic Coastal Plain Tidal Wooded Swamp & 3.05 & 0.29 & 3.70 & 0.75 & 1.04 \\
\hline Southern Atlantic Coastal Plain Wet Pine Savanna and Flatwoods & 2.95 & 0.16 & 2.40 & 0.10 & 0.26 \\
\hline Southern Atlantic Coastal Plain Xeric River Dune & 2.90 & 0.09 & 3.00 & 0.40 & 0.49 \\
\hline Southern California Oak Woodland and Savanna & 3.00 & 0.22 & 2.89 & 0.35 & 0.57 \\
\hline Southern Coastal Plain Blackland Prairie and Woodland & 3.54 & 0.92 & 3.61 & 0.71 & 1.63 \\
\hline Southern Coastal Plain Blackwater River Floodplain Forest & 3.10 & 0.35 & 3.20 & 0.50 & 0.85 \\
\hline Southern Coastal Plain Dry Upland Hardwood Forest & 3.03 & 0.26 & 3.87 & 0.84 & 1.09 \\
\hline Southern Coastal Plain Herbaceous Seep and Bog & 3.23 & 0.52 & 3.28 & 0.54 & 1.06 \\
\hline Southern Coastal Plain Hydric Hammock & 3.13 & 0.39 & 2.87 & 0.34 & 0.72 \\
\hline Southern Coastal Plain Limestone Forest & 3.12 & 0.38 & 3.98 & 0.89 & 1.27 \\
\hline Southern Coastal Plain Mesic Slope Forest & 3.07 & 0.31 & 3.53 & 0.67 & 0.98 \\
\hline Southern Coastal Plain Nonriverine Basin Swamp & 3.27 & 0.57 & 3.32 & 0.56 & 1.13 \\
\hline Southern Coastal Plain Nonriverine Cypress Dome & 2.86 & 0.04 & 3.14 & 0.47 & 0.51 \\
\hline Southern Coastal Plain Oak Dome and Hammock & 3.16 & 0.43 & 3.14 & 0.47 & 0.90 \\
\hline Southern Coastal Plain Seepage Swamp and Baygall & 2.97 & 0.18 & 3.11 & 0.46 & 0.64 \\
\hline Southern Colorado Plateau Sand Shrubland & 2.93 & 0.13 & 3.05 & 0.43 & 0.55 \\
\hline Southern Interior Calcareous Cliff & 3.40 & 0.74 & 3.03 & 0.42 & 1.16 \\
\hline Southern Interior Low Plateau Dry-Mesic Oak Forest & 3.00 & 0.22 & 4.20 & 1.00 & 1.22 \\
\hline Southern Piedmont Cliff & 3.40 & 0.74 & 3.73 & 0.77 & 1.51 \\
\hline Southern Piedmont Dry Oak-(Pine) Forest & 3.02 & 0.25 & 4.02 & 0.91 & 1.16 \\
\hline Southern Piedmont Glade and Barrens & 3.40 & 0.74 & 3.03 & 0.42 & 1.16 \\
\hline Southern Piedmont Granite Flatrock and Outcrop & 3.13 & 0.39 & 3.05 & 0.43 & 0.81 \\
\hline Southern Piedmont Large Floodplain Forest & 2.96 & 0.17 & 3.61 & 0.71 & 0.87 \\
\hline Southern Piedmont Mesic Forest & 3.03 & 0.26 & 3.95 & 0.88 & 1.13 \\
\hline Southern Piedmont Small Floodplain and Riparian Forest & 3.06 & 0.30 & 3.63 & 0.72 & 1.01 \\
\hline Southern Ridge and Valley / Cumberland Dry Calcareous Forest & 3.04 & 0.27 & 3.80 & 0.80 & 1.07 \\
\hline $\begin{array}{l}\text { Southern Rocky Mountain Dry-Mesic Montane Mixed Conifer Forest and } \\
\text { Woodland }\end{array}$ & 3.16 & 0.43 & 2.64 & 0.22 & 0.65 \\
\hline Southern Rocky Mountain Juniper Woodland and Savanna & 3.39 & 0.73 & 3.50 & 0.65 & 1.38 \\
\hline $\begin{array}{l}\text { Southern Rocky Mountain Mesic Montane Mixed Conifer Forest and } \\
\text { Woodland }\end{array}$ & 3.01 & 0.23 & 2.50 & 0.15 & 0.38 \\
\hline Southern Rocky Mountain Montane-Subalpine Grassland & 3.60 & 1.00 & 3.69 & 0.75 & 1.75 \\
\hline Southern Rocky Mountain Pinyon-Juniper Woodland & 3.04 & 0.27 & 2.70 & 0.25 & 0.52 \\
\hline
\end{tabular}




\begin{tabular}{|c|c|c|c|c|c|}
\hline Community & $\begin{array}{c}\text { Mean } \\
\text { Resistance }\end{array}$ & $\begin{array}{c}\text { Scaled } \\
\text { Resistance }\end{array}$ & $\begin{array}{c}\text { Mean } \\
\text { Resilience }\end{array}$ & $\begin{array}{c}\text { Scaled } \\
\text { Resilience }\end{array}$ & $\begin{array}{c}\text { Net } \\
\text { Persistence }\end{array}$ \\
\hline Southern Rocky Mountain Ponderosa Pine Savanna & 3.43 & 0.78 & 3.14 & 0.47 & 1.25 \\
\hline Southern Rocky Mountain Ponderosa Pine Woodland & 3.04 & 0.27 & 2.88 & 0.34 & 0.61 \\
\hline Southwest Florida Beach & 3.20 & 0.48 & 3.70 & 0.75 & 1.23 \\
\hline Southwest Florida Coastal Strand and Maritime Hammock & 2.90 & 0.09 & 2.95 & 0.38 & 0.47 \\
\hline Southwest Florida Dune and Coastal Grassland & 3.23 & 0.52 & 3.53 & 0.67 & 1.18 \\
\hline Southwestern Great Plains Canyon & 3.18 & 0.45 & 2.73 & 0.27 & 0.72 \\
\hline Successional Shrub/Scrub & 0.00 & 0.00 & 0.00 & 0.00 & 0.00 \\
\hline Successional Shrub/Scrub (Clear Cut) & 0.00 & 0.00 & 0.00 & 0.00 & 0.00 \\
\hline Tamaulipan Calcareous Thornscrub & 2.84 & 0.01 & 3.03 & 0.42 & 0.43 \\
\hline Tamaulipan Floodplain & 3.30 & 0.61 & 3.48 & 0.64 & 1.25 \\
\hline Tamaulipan Mesquite Upland Scrub & 3.05 & 0.29 & 3.31 & 0.56 & 0.84 \\
\hline Tamaulipan Palm Grove Riparian Forest & 3.05 & 0.29 & 3.70 & 0.75 & 1.04 \\
\hline Tamaulipan Ramadero & 3.09 & 0.34 & 3.30 & 0.55 & 0.89 \\
\hline Tamaulipan Savanna Grassland & 3.45 & 0.81 & 4.13 & 0.97 & 1.77 \\
\hline Temperate Pacific Freshwater Aquatic Bed & 0.00 & 0.00 & 0.00 & 0.00 & 0.00 \\
\hline Temperate Pacific Freshwater Emergent Marsh & 3.60 & 1.00 & 3.60 & 0.70 & 1.70 \\
\hline Temperate Pacific Freshwater Mudflat & 3.17 & 0.44 & 3.60 & 0.70 & 1.14 \\
\hline Temperate Pacific Intertidal Flat & 3.30 & 0.61 & 3.55 & 0.68 & 1.29 \\
\hline Temperate Pacific Subalpine-Montane Wet Meadow & 3.38 & 0.71 & 3.56 & 0.68 & 1.39 \\
\hline Temperate Pacific Tidal Salt and Brackish Marsh & 3.47 & 0.83 & 3.61 & 0.71 & 1.54 \\
\hline Texas Blackland Tallgrass Prairie & 3.60 & 1.00 & 4.10 & 0.95 & 1.95 \\
\hline Texas Coast Beach & 3.15 & 0.42 & 3.65 & 0.73 & 1.14 \\
\hline Texas Coast Dune and Coastal Grassland & 3.60 & 1.00 & 4.10 & 0.95 & 1.95 \\
\hline Texas Coast Fresh and Oligohaline Tidal Marsh & 3.60 & 1.00 & 3.93 & 0.87 & 1.87 \\
\hline Texas Coast Salt and Brackish Tidal Marsh & 3.26 & 0.56 & 3.50 & 0.65 & 1.21 \\
\hline Texas Saline Coastal Prairie & 3.60 & 1.00 & 4.10 & 0.95 & 1.95 \\
\hline Texas-Louisiana Coastal Prairie & 3.60 & 1.00 & 4.10 & 0.95 & 1.95 \\
\hline Texas-Louisiana Coastal Prairie Pondshore & 3.30 & 0.61 & 3.63 & 0.72 & 1.33 \\
\hline West Gulf Coastal Plain Catahoula Barrens & 3.29 & 0.60 & 3.67 & 0.74 & 1.33 \\
\hline $\begin{array}{l}\text { West Gulf Coastal Plain Chenier and Upper Texas Coastal Fringe Forest and } \\
\text { Woodland }\end{array}$ & 3.10 & 0.35 & 3.20 & 0.50 & 0.85 \\
\hline West Gulf Coastal Plain Flatwoods Pond & 3.60 & 1.00 & 3.85 & 0.83 & 1.83 \\
\hline West Gulf Coastal Plain Large River Floodplain Forest & 3.04 & 0.27 & 3.80 & 0.80 & 1.07 \\
\hline West Gulf Coastal Plain Mesic Hardwood Forest & 2.98 & 0.19 & 3.25 & 0.53 & 0.72 \\
\hline West Gulf Coastal Plain Near-Coast Large River Swamp & 3.10 & 0.35 & 3.20 & 0.50 & 0.85 \\
\hline West Gulf Coastal Plain Nepheline Syenite Glade & 3.48 & 0.84 & 4.02 & 0.91 & 1.75 \\
\hline West Gulf Coastal Plain Nonriverine Wet Hardwood Flatwoods & 3.04 & 0.27 & 3.80 & 0.80 & 1.07 \\
\hline West Gulf Coastal Plain Northern Calcareous Prairie & 3.45 & 0.81 & 4.00 & 0.90 & 1.71 \\
\hline West Gulf Coastal Plain Pine-Hardwood Flatwoods & 3.05 & 0.29 & 3.70 & 0.75 & 1.04 \\
\hline West Gulf Coastal Plain Pine-Hardwood Forest & 3.07 & 0.31 & 3.53 & 0.67 & 0.98 \\
\hline $\begin{array}{l}\text { West Gulf Coastal Plain Sandhill Oak and Shortleaf Pine Forest and } \\
\text { Woodland }\end{array}$ & 3.05 & 0.29 & 3.70 & 0.75 & 1.04 \\
\hline West Gulf Coastal Plain Seepage Swamp and Baygall & 2.90 & 0.09 & 2.90 & 0.35 & 0.44 \\
\hline West Gulf Coastal Plain Small Stream and River Forest & 3.15 & 0.42 & 4.05 & 0.93 & 1.34 \\
\hline West Gulf Coastal Plain Southern Calcareous Prairie & 3.23 & 0.52 & 3.58 & 0.69 & 1.21 \\
\hline West Gulf Coastal Plain Stream Terrace Sandyland Longleaf Pine Woodland & 3.08 & 0.32 & 3.40 & 0.60 & 0.92 \\
\hline West Gulf Coastal Plain Upland Longleaf Pine Forest and Woodland & 3.25 & 0.55 & 3.18 & 0.49 & 1.04 \\
\hline
\end{tabular}




\begin{tabular}{|l|c|c|c|c|c|}
\hline Community & $\begin{array}{c}\text { Mean } \\
\text { Resistance }\end{array}$ & $\begin{array}{c}\text { Scaled } \\
\text { Resistance }\end{array}$ & $\begin{array}{c}\text { Mean } \\
\text { Resilience }\end{array}$ & $\begin{array}{c}\text { Scaled } \\
\text { Resilience }\end{array}$ & $\begin{array}{c}\text { Net } \\
\text { Persistence }\end{array}$ \\
\hline West Gulf Coastal Plain Weches Glade & 3.13 & 0.39 & 3.23 & 0.52 & 0.90 \\
\hline West Gulf Coastal Plain Wet Longleaf Pine Savanna and Flatwoods & 3.18 & 0.45 & 2.73 & 0.27 & 0.72 \\
\hline Western Great Plains Cliff and Outcrop & 3.24 & 0.53 & 3.62 & 0.71 & 1.24 \\
\hline Western Great Plains Closed Depression Wetland & 3.60 & 1.00 & 3.73 & 0.77 & 1.77 \\
\hline Western Great Plains Depressional Wetland Systems & 3.53 & 0.91 & 3.72 & 0.76 & 1.67 \\
\hline Western Great Plains Dry Bur Oak Forest and Woodland & 3.08 & 0.32 & 3.75 & 0.78 & 1.10 \\
\hline Western Great Plains Floodplain & 3.00 & 0.22 & 3.58 & 0.69 & 0.91 \\
\hline Western Great Plains Foothill and Piedmont Grassland & 3.60 & 1.00 & 3.88 & 0.84 & 1.84 \\
\hline Western Great Plains Mesquite Woodland and Shrubland & 3.20 & 0.48 & 3.65 & 0.73 & 1.21 \\
\hline Western Great Plains Open Freshwater Depression Wetland & 3.60 & 1.00 & 3.73 & 0.77 & 1.77 \\
\hline Western Great Plains Riparian & 3.39 & 0.73 & 3.80 & 0.80 & 1.53 \\
\hline Western Great Plains Saline Depression Wetland & 3.38 & 0.71 & 3.70 & 0.75 & 1.46 \\
\hline Western Great Plains Sand Prairie & 3.60 & 1.00 & 3.85 & 0.83 & 1.83 \\
\hline Western Great Plains Sandhill Steppe & 3.45 & 0.81 & 3.92 & 0.86 & 1.67 \\
\hline Western Great Plains Shortgrass Prairie & 3.60 & 1.00 & 4.10 & 0.95 & 1.95 \\
\hline Western Great Plains Tallgrass Prairie & 3.60 & 1.00 & 4.10 & 0.95 & 1.95 \\
\hline Willamette Valley Upland Prairie and Savanna & 3.60 & 1.00 & 3.60 & 0.70 & 1.70 \\
\hline Willamette Valley Wet Prairie & 3.48 & 0.84 & 3.58 & 0.69 & 1.53 \\
\hline Wyoming Basins Dwarf Sagebrush Shrubland and Steppe & 3.04 & 0.27 & 2.91 & 0.36 & 0.63 \\
\hline
\end{tabular}




\section{Appendix E: Resistance and Resilience Values from Küchler Potential Vegetation Community Types}

Table E-1. Resistance and Resilience Values from Küchler Potential Vegetation Community Types.

\begin{tabular}{|c|c|c|c|c|c|}
\hline Community & $\begin{array}{c}\text { Mean } \\
\text { Resistance }\end{array}$ & $\begin{array}{c}\text { Scaled } \\
\text { Resistance }\end{array}$ & $\begin{array}{c}\text { Mean } \\
\text { Resilience }\end{array}$ & $\begin{array}{c}\text { Scaled } \\
\text { Resilience }\end{array}$ & $\begin{array}{c}\text { Net } \\
\text { Persistence }\end{array}$ \\
\hline Mangrove & 2.70 & 0.00 & 2.40 & 0.10 & 0.10 \\
\hline Mesquite bosques & 2.70 & 0.00 & 3.00 & 0.40 & 0.40 \\
\hline Chaparral & 2.70 & 0.00 & 2.40 & 0.10 & 0.10 \\
\hline Montane chaparral & 2.70 & 0.00 & 2.40 & 0.10 & 0.10 \\
\hline Coastal sagebrush & 2.70 & 0.00 & 2.73 & 0.27 & 0.27 \\
\hline Mountain-mahogany - oak scrub & 2.70 & 0.00 & 2.70 & 0.25 & 0.25 \\
\hline Great Basin sagebrush & 2.70 & 0.00 & 2.40 & 0.10 & 0.10 \\
\hline Black - brush & 2.70 & 0.00 & 3.00 & 0.40 & 0.40 \\
\hline Saltbush - greasewood & 2.70 & 0.00 & 3.00 & 0.40 & 0.40 \\
\hline Creosote bush & 2.70 & 0.00 & 2.40 & 0.10 & 0.10 \\
\hline Creosote bush - bur sage & 2.70 & 0.00 & 2.70 & 0.25 & 0.25 \\
\hline Creosote bush - tarbush & 2.70 & 0.00 & 2.70 & 0.25 & 0.25 \\
\hline Ceniza shrub & 2.70 & 0.00 & 2.60 & 0.20 & 0.20 \\
\hline Trans - pecos shrub savanna & 2.70 & 0.00 & 2.70 & 0.25 & 0.25 \\
\hline Alder - ash forest & 2.85 & 0.17 & 3.60 & 0.70 & 0.87 \\
\hline Palo verde - cactus shrub & 2.85 & 0.17 & 3.25 & 0.53 & 0.69 \\
\hline Northern floodplain forest & 2.90 & 0.22 & 3.80 & 0.80 & 1.02 \\
\hline Pocosin & 2.95 & 0.28 & 2.30 & 0.05 & 0.33 \\
\hline Oak - hickory forest & 3.00 & 0.33 & 4.20 & 1.00 & 1.33 \\
\hline Elm - ash forest & 3.00 & 0.33 & 4.20 & 1.00 & 1.33 \\
\hline Beech - maple forest & 3.00 & 0.33 & 4.20 & 1.00 & 1.33 \\
\hline Mixed mesophytic forest & 3.00 & 0.33 & 4.20 & 1.00 & 1.33 \\
\hline Appalachian oak forest & 3.00 & 0.33 & 4.20 & 1.00 & 1.33 \\
\hline Oregon oak woods & 3.00 & 0.33 & 4.20 & 1.00 & 1.33 \\
\hline Maple - basswood forest & 3.00 & 0.33 & 4.20 & 1.00 & 1.33 \\
\hline Northern hardwoods & 3.05 & 0.39 & 3.70 & 0.75 & 1.14 \\
\hline Northeastern oak - pine forest & 3.07 & 0.41 & 3.53 & 0.67 & 1.08 \\
\hline Southern floodplain forest & 3.07 & 0.41 & 3.53 & 0.67 & 1.08 \\
\hline Blackbelt & 3.07 & 0.41 & 3.53 & 0.67 & 1.08 \\
\hline Northern hardwood - spruce forest & 3.08 & 0.42 & 3.40 & 0.60 & 1.02 \\
\hline Oak - hickory - pine forest & 3.08 & 0.42 & 3.40 & 0.60 & 1.02 \\
\hline Northern hardwoods - fir forest & 3.10 & 0.44 & 3.20 & 0.50 & 0.94 \\
\hline Southern mixed forest & 3.10 & 0.44 & 3.20 & 0.50 & 0.94 \\
\hline Mesquite - oak savanna & 3.10 & 0.44 & 3.77 & 0.79 & 1.23 \\
\hline California mixed evergreen forest & 3.13 & 0.48 & 2.29 & 0.04 & 0.52 \\
\hline California oak - woods & 3.14 & 0.49 & 2.77 & 0.29 & 0.77 \\
\hline Sagebrush steppe & 3.15 & 0.50 & 3.00 & 0.40 & 0.90 \\
\hline
\end{tabular}




\begin{tabular}{|c|c|c|c|c|c|}
\hline Community & $\begin{array}{c}\text { Mean } \\
\text { Resistance }\end{array}$ & $\begin{array}{c}\text { Scaled } \\
\text { Resistance }\end{array}$ & $\begin{array}{c}\text { Mean } \\
\text { Resilience }\end{array}$ & $\begin{array}{c}\text { Scaled } \\
\text { Resilience }\end{array}$ & $\begin{array}{c}\text { Net } \\
\text { Persistence }\end{array}$ \\
\hline Galleta - three awn shrub-steppe & 3.15 & 0.50 & 3.40 & 0.60 & 1.10 \\
\hline Mesquite - acacia savanna & 3.15 & 0.50 & 3.55 & 0.68 & 1.18 \\
\hline Palmetto prairie & 3.15 & 0.50 & 3.55 & 0.68 & 1.18 \\
\hline Mesquite - buffalo grass & 3.15 & 0.50 & 3.55 & 0.68 & 1.18 \\
\hline Cedar glades & 3.16 & 0.51 & 3.78 & 0.79 & 1.30 \\
\hline Juniper steppe woodland & 3.17 & 0.52 & 2.73 & 0.27 & 0.79 \\
\hline Mesquite - live oak savanna & 3.17 & 0.52 & 3.10 & 0.45 & 0.97 \\
\hline Marl everglades & 3.17 & 0.52 & 2.73 & 0.27 & 0.79 \\
\hline Everglades & 3.17 & 0.52 & 2.73 & 0.27 & 0.79 \\
\hline Spruce - cedar-hemlock forest & 3.20 & 0.56 & 2.20 & 0.00 & 0.56 \\
\hline Ponderosa - shrub forest & 3.20 & 0.56 & 2.20 & 0.00 & 0.56 \\
\hline Western ponderosa forest & 3.20 & 0.56 & 2.20 & 0.00 & 0.56 \\
\hline Sand pine scrub & 3.20 & 0.56 & 2.20 & 0.00 & 0.56 \\
\hline Sub - tropical pine forest & 3.20 & 0.56 & 2.20 & 0.00 & 0.56 \\
\hline Douglas-fir forest & 3.20 & 0.56 & 2.20 & 0.00 & 0.56 \\
\hline Cedar - hemlock - pine forest & 3.20 & 0.56 & 2.20 & 0.00 & 0.56 \\
\hline Grand fir - Douglas-fir forest & 3.20 & 0.56 & 2.20 & 0.00 & 0.56 \\
\hline Western spruce - fir forest & 3.20 & 0.56 & 2.20 & 0.00 & 0.56 \\
\hline Eastern ponderosa forest & 3.20 & 0.56 & 2.20 & 0.00 & 0.56 \\
\hline Black Hills pine forest & 3.20 & 0.56 & 2.20 & 0.00 & 0.56 \\
\hline Pine - Douglas-fir forest & 3.20 & 0.56 & 2.20 & 0.00 & 0.56 \\
\hline Arizona pine forest & 3.20 & 0.56 & 2.20 & 0.00 & 0.56 \\
\hline Cedar - hemlock - Douglas-fir forest & 3.20 & 0.56 & 2.20 & 0.00 & 0.56 \\
\hline Spruce - fir - Douglas-fir forest & 3.20 & 0.56 & 2.20 & 0.00 & 0.56 \\
\hline Southwestern spruce - fir forest & 3.20 & 0.56 & 2.20 & 0.00 & 0.56 \\
\hline Great Basin pine forest & 3.20 & 0.56 & 2.20 & 0.00 & 0.56 \\
\hline Juniper - pinyon woodland & 3.20 & 0.56 & 2.20 & 0.00 & 0.56 \\
\hline Silver fir - Douglas-fir forest & 3.20 & 0.56 & 2.20 & 0.00 & 0.56 \\
\hline Oak-juniper woodland & 3.20 & 0.56 & 2.20 & 0.00 & 0.56 \\
\hline Fir-hemlock forest & 3.20 & 0.56 & 2.20 & 0.00 & 0.56 \\
\hline Mixed conifer forest & 3.20 & 0.56 & 2.20 & 0.00 & 0.56 \\
\hline Redwood forest & 3.20 & 0.56 & 2.20 & 0.00 & 0.56 \\
\hline Red fir forest & 3.20 & 0.56 & 2.20 & 0.00 & 0.56 \\
\hline Lodgepole pine - subalpine forest & 3.20 & 0.56 & 2.20 & 0.00 & 0.56 \\
\hline Cross timbers & 3.20 & 0.56 & 4.17 & 0.99 & 1.54 \\
\hline Pine - cypress forest & 3.20 & 0.56 & 2.20 & 0.00 & 0.56 \\
\hline Great lakes spruce - fir forest & 3.20 & 0.56 & 2.20 & 0.00 & 0.56 \\
\hline Conifer bog & 3.20 & 0.56 & 2.20 & 0.00 & 0.56 \\
\hline Great lakes pine forest & 3.20 & 0.56 & 2.20 & 0.00 & 0.56 \\
\hline Northeastern spruce - fir forest & 3.20 & 0.56 & 2.20 & 0.00 & 0.56 \\
\hline Southeastern spruce - fir forest & 3.20 & 0.56 & 2.20 & 0.00 & 0.56 \\
\hline Grama - tobosa shrub-steppe & 3.30 & 0.67 & 3.53 & 0.67 & 1.33 \\
\hline Mesquite savanna & 3.30 & 0.67 & 3.73 & 0.77 & 1.43 \\
\hline Juniper - oak savanna & 3.33 & 0.70 & 2.83 & 0.32 & 1.02 \\
\hline Wheatgrass - needlegrass shrub-steppe & 3.38 & 0.76 & 3.30 & 0.55 & 1.31 \\
\hline Sandsage - bluestem prairie & 3.38 & 0.76 & 3.83 & 0.82 & 1.57 \\
\hline
\end{tabular}




\begin{tabular}{|l|c|c|c|c|c|}
\hline Community & $\begin{array}{c}\text { Mean } \\
\text { Resistance }\end{array}$ & $\begin{array}{c}\text { Scaled } \\
\text { Resistance }\end{array}$ & $\begin{array}{c}\text { Mean } \\
\text { Resilience }\end{array}$ & $\begin{array}{c}\text { Scaled } \\
\text { Resilience }\end{array}$ & $\begin{array}{c}\text { Net } \\
\text { Persistence }\end{array}$ \\
\hline Shinnery & 3.40 & 0.78 & 3.15 & 0.48 & 1.25 \\
\hline Oak savanna & 3.40 & 0.78 & 4.13 & 0.97 & 1.74 \\
\hline Live oak - sea oats & 3.40 & 0.78 & 3.15 & 0.48 & 1.25 \\
\hline Cypress savanna & 3.47 & 0.86 & 3.47 & 0.64 & 1.49 \\
\hline Fescue - oatgrass & 3.60 & 1.00 & 3.60 & 0.70 & 1.70 \\
\hline California steppe & 3.60 & 1.00 & 3.60 & 0.70 & 1.70 \\
\hline Tule marshes & 3.60 & 1.00 & 3.60 & 0.70 & 1.70 \\
\hline Fescue - wheatgrass & 3.60 & 1.00 & 3.60 & 0.70 & 1.70 \\
\hline Wheatgrass bluegrass & 3.60 & 1.00 & 3.60 & 0.70 & 1.70 \\
\hline Alpine meadows and barren & 3.60 & 1.00 & 3.60 & 0.70 & 1.70 \\
\hline Grama - galleta steppe & 3.60 & 1.00 & 4.10 & 0.95 & 1.95 \\
\hline Grama - tobosa prairie & 3.60 & 1.00 & 4.10 & 0.95 & 1.95 \\
\hline Foothills prairie & 3.60 & 1.00 & 3.60 & 0.70 & 1.70 \\
\hline Grama - needlegrass - wheatgrass & 3.60 & 1.00 & 3.77 & 0.79 & 1.79 \\
\hline Grama - buffalo grass & 3.60 & 1.00 & 4.10 & 0.95 & 1.95 \\
\hline Wheatgrass - needlegrass & 3.60 & 1.00 & 3.73 & 0.77 & 1.77 \\
\hline Wheatgrass - bluestem - needlegrass & 3.60 & 1.00 & 3.77 & 0.79 & 1.79 \\
\hline Wheatgrass - grama - buffalo grass & 3.60 & 1.00 & 3.93 & 0.87 & 1.87 \\
\hline Bluestem - grama prairie & 3.60 & 1.00 & 4.10 & 0.95 & 1.95 \\
\hline Sea oats prairie & 3.60 & 1.00 & 4.10 & 0.95 & 1.95 \\
\hline Northern cordgrass prairie & 3.60 & 1.00 & 4.10 & 0.95 & 1.95 \\
\hline Bluestem prairie & 3.60 & 1.00 & 4.10 & 0.95 & 1.95 \\
\hline Nebraska sandhills prairie & 3.60 & 1.00 & 4.00 & 0.90 & 1.90 \\
\hline Blackland prairie & 3.60 & 1.00 & 3.85 & 0.83 & 1.83 \\
\hline Bluestem - sacahuista prairie & 3.60 & 1.00 & 4.10 & 0.95 & 1.95 \\
\hline Southern cordgrass prairie & 3.60 & 1.00 & 4.10 & 0.95 & 1.95 \\
\hline Fayette prairie & 3.60 & 1.00 & 4.10 & 0.95 & 1.95 \\
\hline
\end{tabular}




\section{References}

Andrés-Abellán, Manuela, Francisco R. López-Serrano, Francisco A. García Morote, and Antonio Del Cerro-Barja. 2006. Assessment of trampling simulation impacts on native vegetation in Mediterranean Sclerophyllous forest. Environmental Monitoring and Assessment 120(1-3):93-107.

Bailey, Robert G. 1980. Description of the Ecoregions of the United States. 1391. Washington, DC: U.S. Department of Agriculture, Forest Service.

Ballantyne, Mark, and Catherine Marina Pickering. 2013. Tourism and recreation: A common threat to IUCN red-listed vascular plants in Europe. Biodiversity and Conservation 22(13-14):3027-3044.

Barros, Agustina, J orge Gonnet, and Catherine Pickering. 2013. Impacts of informal trails on vegetation and soils in the highest protected area in the Southern Hemisphere. J ournal of Environmental Management 127:50-60.

Bell, Katherine L., and Lawrence C. Bliss. 1973. Alpine disturbance studies: Olympic National Park, USA. Biological Conservation 5(1):25- 32.

Bernhardt-Römermann, Markus, Alan Gray, Adam J . Vanbergen, Laurent Bergès, Andreas Bohner, Rob W. Brooker, Luc De Bruyn, Bruno De Cinti, Thomas Dirnböck, Ulf Grandin, Alison J . Hester, Róbert Kanka, Stefan Klotz, Grégory Loucougaray, Lars Lundin, Giorgio Matteucci, Ilona Mészáros, Viktor Oláh, Elena Preda, Bernard Prévosto, J uha Pykälä, Wolfgang Schmidt, Michele E. Taylor, Angheluta Vadineanu, Theresa Waldmann, and J utta Stadler.2011. Functional traits and local environment predict vegetation responses to disturbance: A pan-European multi-site experiment. J ournal of Ecology 99(3):777- 787.

Bratton, Susan Power. 1985. Effects of disturbance by visitors on two woodland orchid species in Great Smoky Mountains National Park, USA. Biological Conservation 31(3):211- 227.

Cole, David N. 1995. Experimental trampling of vegetation. II. Predictors of resistance and resilience. J ournal of Applied Ecology 32:15- 224.

2004. Impacts of hiking and camping on soils and vegetation: A review. Environmental Impacts of Ecotourism 41:60.

Crisfield, Varina E., S. Ellen Macdonald, and A. J oyce Gould. 2012. Effects of recreational traffic on Alpine plant communities in the Northern Canadian Rockies. Arctic, Antarctic, and Alpine Research 44(3):277- 287.

Department of Defense (DoD). 2014. Department of Defense Report to the Defense Base Closure and Realignment Commission, Department of the Army, Analysis and Recommendations, BRAC 2005, Volume III.

2011. Instruction No. 4715.03. Subject: Natural Resources Conservation Program. Washington, DC: Office of the Under Secretary of Defense for Acquisition, Technology and Logistics (USD[AT\&L]), http://www.dtic.mil/whs/directives/corres/pdf/471503p.pdf 
Doe, William W., Robert B. Shaw, Robert G. Bailey, David S. J ones, and Thomas E. Macia. 1999. Locations and environments of US Army training and testing lands: An ecoregional framework for assessment. Federal Facilities Environmental J ournal 10(3):9- 26.

Duarte, Carlos M., Timothy M. Lenton, Peter Wadhams, and Paul Wassmann. 2012. Abrupt climate change in the Arctic. Nature Climate Change 2(2):60-62.

Dzwonko, Zbigniew, and Stefania Loster. 1997. Effects of dominant trees and anthropogenic disturbances on species richness and floristic composition of secondary communities in Southern Poland. J ournal of Applied Ecology 34(4):861-870.

Elmqvist, Thomas, Carl Folke, Magnus Nyström, Garry Peterson, J an Bengtsson, Brian Walker, and J on Norberg. 2003. Response diversity, ecosystem change, and resilience. Frontiers in Ecology and the Environment 1(9):488- 494.

Environmental Systems Research Institute, Inc. (ESRI). 2011. ArcGIS Desktop: Release 10. Redlands, CA: Environmental Systems Research Institute.

Ewing, P., W. Tarantino, and G. Parnell. 2006. Use of decision analysis in the Army base realignment and closure (BRAC) 2005 military value analysis. Decision Analysis 3(1):33- 49 .

Fischer, E. M., and R. Knutti. 2015. Anthropogenic contribution to global occurrence of heavy-precipitation and high-temperature extremes. Nature Climate Change 5:560564. doi:10.1038/nclimate2617, http://www.nature.com/doifinder/10.1038/nclimate2617

Forbes, B. C., A. Tolvanen, K. Laine, and F. E. Wielgolaski. 2005. Rates and processes of natural regeneration in disturbed habitats. Plant Ecology, Herbivory, and Human Impact in Nordic Mountain Birch Forests, pp 193-202. Springer, http://link.springer.com/chapter/10.1007/3-540-26595-3_14

Fortunel, Claire, Eric Garnier, Richard J offre, Elena Kazakou, Helen Quested, Karl Grigulis, Sandra Lavorel, et al. 2009. Leaf traits capture the effects of land use changes and climate on litter decomposability of grasslands across Europe. Ecology 90(3):598- 611.

Francis, Robert A., Angela M. Gurnell, Geoffrey E. Petts, and Peter J . Edwards. 2005. Survival and growth responses of Populus Nigra, Salix Elaeagnos and Alnus Incana cuttings to varying levels of hydric stress. Forest Ecology and Management 210(1):291-301.

Gallet, Sébastien, and Françoise ROzÉ. 2001. Resistance of Atlantic heathlands to trampling in Brittany (France): Influence of vegetation type, season and weather conditions. Biological Conservation 97(2):189-198.

Greenberg, Cathryn H., Daniel G. Neary, Lawrence D. Harris, and Steven P. Linda. 1995. Vegetation recovery following high-intensity wildfire and silvicultural treatments in sand pine scrub. American Midland Naturalist 133(1):149- 163.

Grime, J . P., and B. D. Campbell. 1991. Growth rate, habitat productivity and plant strategy as predictors of stress response. Response of Plants to Multiple Stresses. New York: Academic Press, pp 143- 159 
Hamberg, Leena, Minna Malmivaara-Lämsä, Susanna Lehvävirta, Robert B. O’Hara, and D. J ohan Kotze. 2010. Quantifying the effects of trampling and habitat edges on forest understory vegetation- A field experiment. J ournal of Environmental Management 91(9):1811-1820.

Headquarters, Department of the Army (HQDA). 2013. Guide to Army Real Property Category Codes. Department of the Army Pamphlet (DA PAM) 415-28. Washington, DC: HQDA.

Hill, Rachel, and Catherine Pickering. 2009. Differences in resistance of three subtropical vegetation types to experimental trampling. J ournal of Environmental Management 90(2):1305-1312.

Küchler, August Wilhelm. 1964. Potential Natural Vegetation of the Conterminous United States. Vol. 36. New York: American Geographical Society, http://library.wur.nl/WebQuery/clc/357106

Kuss, Fred R., and Christine N. Hall. 1991. Ground flora trampling studies: Five years after closure. Environmental Management 15(5):715-727.

Kutiel, P., E. Eden, and Y. Zhevelev. 2000. Effect of experimental trampling and off-road motorcycle traffic on soil and vegetation of stabilized coastal dunes, Israel. Environmental Conservation 27(01):14-23.

Lane, Diana R., Debra P. Coffin, and William K. Lauenroth. 1998. Effects of soil texture and precipitation on above-ground net primary productivity and vegetation structure across the central grassland region of the United States. J ournal of Vegetation Science 9(2):239-250.

Liddle, Michael J . 1975. A selective review of the ecological effects of human trampling on natural ecosystems. Biological Conservation 7(1):17- 36.

Liddle, Michael. 1997. Recreation Ecology: The Ecological Impact of Outdoor Recreation and Ecotourism. London: Chapman \& Hall Ltd.

Li, Mei-Rong, David A. Wedin, and Larry L. Tieszen. 1999. $\mathrm{C}_{3}$ and $\mathrm{C}_{4}$ photosynthesis in Cyperus (Cyperaceae) in temperate Eastern North America. Canadian J ournal of Botany 77(2):209-218.

Lucas-Borja, M. E., F. Bastida, J . L. Moreno, César Nicolás, M. Andres, F. R. Lopez, and A. Del Cerro. 2011. The effects of human trampling on the microbiological properties of soil and vegetation in Mediterranean mountain areas. Land Degradation \& Development 22(4):383- 394.

Ludwig, J ohn A., Michael B. Coughenour, Adam C. Liedloff, and Rodd Dyer. 2001. Modelling the resilience of Australian savanna systems to grazing impacts. Environment International 27(2):167- 172.

MacGillivray, C. W., J . P. Grime, and the Integrated Screening Programme (ISP) Team. 1995. Testing predictions of the resistance and resilience of vegetation subjected to extreme events. Functional Ecology: 9:640-649. 
Mangan, J ennifer M., J onathan T. Overpeck, Robert S. Webb, Carol Wessman, and Alexander F. H. Goetz. 2004. Response of Nebraska Sand Hills natural vegetation to drought, fire, grazing, and plant functional type shifts as simulated by the century model. Climatic Change 63(1-2):49-90.

Mason, S., D. Newsome, S. Moore, and R. Admiraal. 2015. Recreational trampling negatively impacts vegetation structure of an Australian biodiversity hotspot. Biodiversity and Conservation 24(11):2685- 2707.

Monz, Christopher A., David N. Cole, Yu-Fai Leung, and J effrey L. Marion. 2010. Sustaining visitor use in protected areas: Future opportunities in recreation ecology research based on the USA experience. Environmental Management 45(3):551- 562.

NatureServe. 2015. NatureServe Explorer: An Online Encyclopedia of Life. Web application. Version 7.1, http://www.natureserve.org/explorer

Newsome, David, Susan A. Moore, and Ross Kingston Dowling. 2012. Natural Area Tourism: Ecology, Impacts and Management. Vol. 58. Buffalo, NY: Channel View Publications.

Papaik, M. J., and C. D. Canham. 2006. Species resistance and community response to wind disturbance regimes in northern temperate forests. J ournal of Ecology 94(5):1011- 1026.

Pertierra, L. R., F. Lara, P. Tejedo, A. Quesada, and J . Benayas. 2013. Rapid denudation processes in cryptogamic communities from maritime Antarctica subjected to human trampling. Antarctic Science 25(2):318- 328.

Pescott, Oliver L., and Gavin B. Stewart. 2014. Assessing the impact of human trampling on vegetation: A systematic review and meta-analysis of experimental evidence. PeerJ 2:e360.

Pickering, Catherine Marina. 2010. Ten factors that affect the severity of environmental impacts of visitors in protected areas. Ambio 39(1):70- 77.

Pickering, Catherine Marina, and Wendy Hill. 2007. Impacts of recreation and tourism on plant biodiversity and vegetation in protected areas in Australia. J ournal of Environmental Management 85(4):791- 800.

Pickett, S. T. A., and P. S. White. 1985. The ecology of natural disturbance and patch dynamics, http://library.wur.nl/WebQuery/clc/231473

Potito, Aaron P., and Susan W. Beatty. 2005. Impacts of recreation trails on exotic and ruderal species distribution in grassland areas along the Colorado front range. Environmental Management 36(2):230-236.

Roovers, Pieter, Sara Baeten, and Martin Hermy. 2004. Plant species variation across path ecotones in a variety of common vegetation types. Plant Ecology 170(1):107- 119 .

Sala, O. E., W. J oyce Parton, L. A. J oyce, and W. K. Lauenroth. 1988. Primary production of the central grassland region of the United States. Ecology 69(1):40-45. 
Speed, James D. M., Elisabeth J . Cooper, Ingibjörg S. J ónsdóttir, René Van Der Wal, and Sarah J. Woodin. 2010. Plant community properties predict vegetation resilience to herbivore disturbance in the Arctic. J ournal of Ecology 98(5):1002- 1013.

Sun, Dan, and M. J . Liddle. 1993. The morphological responses of some Australian tussock grasses and the importance of tiller number in their resistance to trampling. Biological Conservation 65(1):43-49.

Tolvanen, A., B. C. Forbes, K. E. Rytkönen, and K. Laine. 2001. Regeneration of dominant plants after short-term pedestrian trampling in subarctic plant communities. Nordic Mountain Birch Ecosystems. Paris: UNESCO, pp 359- 368, http://www.cabdirect.org/abstracts/20013133595.html

U.S. Department of Agriculture (USDA). 2015. PLANTS Database. Web site. Greensboro, NC: USDA, Natural Resources Conservation Service (NRCS), National Plant Data Team, http://plants.usda.gov

U.S. Fish and Wildlife Service (USFWS). 2015. National Wetlands Inventory. Web Site. Washington, DC: USFWS, http://www.fws.gov/wetlands/

Vetter, Susanne. 2009. Drought, change and resilience in South Africa's arid and semi-arid rangelands. South African J ournal of Science 105(1-2):29- 33.

Waller, S. S., and J. K. Lewis. 1979. Occurrence of $\mathrm{C}_{3}$ and $\mathrm{C}_{4}$ photosynthetic pathways in North American grasses. J ournal of Range Management 32(1):12- 28.

Whinam, J ennie, and Nicole M. Chilcott. 2003. Impacts after four years of experimental trampling on Alpine/ sub-Alpine environments in Western Tasmania. J ournal of Environmental Management 67(4):339- 351.

Whittaker, Robert Harding. 1975. Communities and Ecosystems. NewYork: MacMillan. http://www.cabdirect.org/abstracts/19740615709.html

Willard, Beatrice E., David J . Cooper, and Bruce C. Forbes. 2007. Natural regeneration of Alpine tundra vegetation after human trampling: A 42-year dataset from Rocky Mountain National Park, Colorado, USA. Arctic, Antarctic, and Alpine Research 39(1):177-183.

Yorks, Terence Preston, Neil E. West, Richard J . Mueller, and Steven D. Warren. 1997. Toleration of traffic by vegetation: Life form conclusions and summary extracts from a comprehensive data base. Environmental Management 21(1):121-131.

Zoller, Nancy. 2016. Military Value Analysis VI. Final Report, CAA-TBD. Fort Belvoir, VA: Center for Army Analysis. 


\section{Acronyms and Abbreviations}

\begin{tabular}{|c|c|}
\hline $\begin{array}{l}\text { Term } \\
\text { AAP }\end{array}$ & $\begin{array}{l}\text { Definition } \\
\text { Army Ammunition Plant }\end{array}$ \\
\hline ANSI & American National Standards Institute \\
\hline BRAC & Base Realignment and Closure \\
\hline CAA & Center for Army Analysis \\
\hline CEERD & US Army Corps of Engineers, Engineer Research and Development Center \\
\hline CERL & Construction Engineering Research Laboratory \\
\hline CNN & Cable News Network \\
\hline CONUS & Continental United States \\
\hline СТC & Combat Training Center \\
\hline DA PAM & Department of the Army Pamphlet \\
\hline DoD & U.S. Department of Defense \\
\hline ERDC & U.S. Army Engineer Research and Development Center \\
\hline ERDC-CERL & $\begin{array}{l}\text { Engineer Research and Development Center, Construction Engineering } \\
\text { Research Laboratory }\end{array}$ \\
\hline ESRI & Environmental Systems Research Institute, Inc. \\
\hline FMR & Florence Military Reservation \\
\hline GIS & Geographic Information System \\
\hline HQDA & Headquarters, Department of the Army \\
\hline HQUSACE & Headquarters, U.S. Army Corps of Engineers \\
\hline ISP & Installation Sustainability Planning \\
\hline ITC & Installation Training Capacity \\
\hline IUCN & International Union for Conservation of Nature and Natural Resources \\
\hline MAC & Maneuver Area Capacity \\
\hline MTA & Military Training Area \\
\hline MTC & Maneuver Training Center \\
\hline MTC-H & Maneuver Training Center-H \\
\hline MVA & Military Value Analysis \\
\hline NRCS & Natural Resources Conservation Service \\
\hline NSN & National Supply Number \\
\hline NTC & National Training Center \\
\hline NW & Northwest \\
\hline OCONUS & Outside Continental United States \\
\hline OMB & Office of Management and Budget \\
\hline PO & Post Office \\
\hline RRVAL & Resistance and Resilience Values \\
\hline SAR & Same As Report \\
\hline SF & Standard Form \\
\hline TS & Training Site \\
\hline UNESCO & United Nations Educational, Scientific, and Cultural Organization \\
\hline
\end{tabular}




$\begin{array}{ll}\text { Term } & \text { Definition } \\ \text { USDA } & \begin{array}{l}\text { U.S. Department of Agriculture } \\ \text { Office of the Under Secretary of Defense for Acquisition, Technology and } \\ \text { USD(AT\&L) }\end{array} \\ \text { Logistics } \\ \text { USFWS } & \text { US Fish and Wildlife Service } \\ \text { USGS } & \text { U.S. Geological Survey } \\ \text { VTS } & \text { Volunteer Training Site }\end{array}$




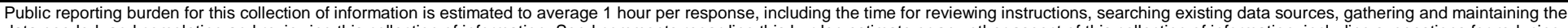

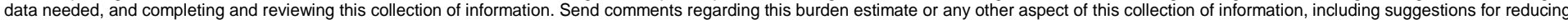

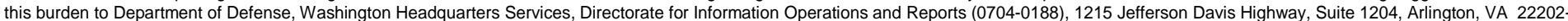

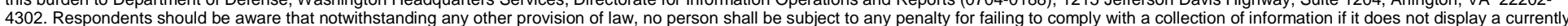
4302. Respondents should be aware that notwithstanding any other provision of law, no person sha
valid OMB control number. PLEASE DO NOT RETURN YOUR FORM TO THE ABOVE ADDRESS.

\begin{tabular}{|l|l} 
valid OMB control number. PLEASE DO NOT RETURN YOUR FORM TO THE ABOVE ADDRESS. \\
\hline 1. REPORT DATE (DD-MM-YYYY) 2. REPOR
\end{tabular}
1. REPORT DATE
$05 / 17 / 2017$
2. REPORT TYPE
Final

4. TITLE AND SUBTITLE

Estimating Resistance and Resilience of Military Lands Using Vegetation Indices

3. DATES COVERED (From - To)

5a. CONTRACT NUMBER

5b. GRANT NUMBER

5c. PROGRAM ELEMENT

622720A896

\section{AUTHOR(S)}

Ryan R. Busby, Dick L. Gebhart, Steven J . Oxley, William D. Tarantino, and Wade A. Wall

\section{5d. PROJECT NUMBER}

402188

5e. TASK NUMBER

5f. WORK UNIT NUMBER

7. PERFORMING ORGANIZATION NAME(S) AND ADDRESS(ES)

U.S. Army Engineer Research and Development Center (ERDC)

Construction Engineering Research Laboratory (CERL)

PO Box 9005,

Champaign, IL 61826-9005

8. PERFORMING ORGANIZATION REPORT NUMBER

ERDC/ CERL TR-17-12

\section{SPONSORING I MONITORING AGENCY NAME(S) AND ADDRESS(ES)}

Headquarters, U.S. Army Corps of Engineers (HQUSACE)

$441 \mathrm{G}$ St., NW

Washington, DC 20314-1000

10. SPONSOR/MONITOR'S ACRONYM(S)

CAA

11. SPONSOR/MONITOR'S REPORT NUMBER(S)

\section{DISTRIBUTION I AVAILABILITY STATEMENT}

Approved for public release; distribution is unlimited.

\section{SUPPLEMENTARY NOTES}

\section{ABSTRACT}

Military training inevitably leads to land degradation; however, some ecosystems have higher resistance and resilience to training based on the functional traits of existing vegetation, making them preferred for long-term use. This work estimated resistance and resilience for the continental United States using dominant plant species for numerous plant communities, resistance and resilience values for plant functional groups, and national community vegetation maps. Two datasets were combined to obtain greater detail and values for all land area. Results indicate that graminoid communities have the highest resistance values, and shrublands the lowest; and that eastern deciduous forests and prairies have the highest resilience values, and evergreen forests and shrublands the lowest. This lists the resistance and resilience values of a selection of Army installations using both datasets and a new combined metric. This new method will help the Army determine the portfolio of installations that will best meet its future training land requirements.

\section{SUBJECT TERMS}

Military bases, Environmental protection-Management, Land degradation, Resilience (Ecology), Vegetation mapping

\section{SECURITY CLASSIFICATION OF:}

\section{a. REPORT}

Unclassified

\section{b. ABSTRACT}

Unclassified

\section{LIMITATION} OF ABSTRACT

c. THIS PAGE

Unclassified
18. NUMBER OF PAGES

163 19a. NAME OF RESPONSIBLE PERSON

19b. TELEPHONE NUMBER

(include area code) 